\title{
Pressure and Stress Transients in Autoinjector Devices
}

\author{
Thesis by \\ Jean-Christophe Veilleux \\ In Partial Fulfillment of the Requirements for the \\ Degree of \\ Doctor of Philosophy

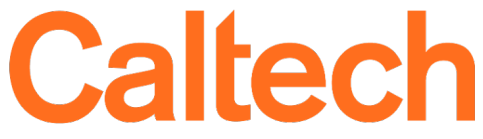

\section{CALIFORNIA INSTITUTE OF TECHNOLOGY}

Pasadena, California

2019

Defended February 6, 2019 
(C) 2019

Jean-Christophe Veilleux ORCID: 0000-0002-5420-9411

All rights reserved 


\section{ACKNOWLEDGEMENTS}

First and foremost, I would like to express my gratitude to Professor Joseph E. Shepherd, my advisor and mentor. Despite having an important commitment as Vice President of Student Affairs, Prof. Shepherd has always been available to discuss and help with my research. With his tremendous experience, he was always able to offer guidance throughout the course of my Ph.D, whether it was about research or my future endeavors. I am extremely thankful for all the help, support, and encouragement I received from Prof. Shepherd, and I will remain indebted to him for all he has done to make sure I become a successful researcher and engineer.

I would also like to extend my gratitude to the members of my thesis committee, Professors Morteza Gharib, Ravi Ravichandran, and Tim Colonius. I would also like to thank Prof. Tim Colonius for allowing me to use his computational resources and his numerical code, and for offering guidance and advising with regards to some of the numerical simulations I performed during my Ph.D. A special thank you to former and present members of his group, Drs. Kazuki Maeda and Kevin Schmidmayer, for their help with some of the numerical simulations.

I would like to thank Amgen for their financial support. Over the past few years, Amgen has offered Prof. Shepherd and myself the opportunity to work on a very exciting research project which combines a number of practical engineering issues, as well as a number of more fundamental challenges. In particular, I would like to thank Dr. Bruce Eu and Julian Jazayeri, with whom I had the opportunity to interact on a regular basis regarding my research project. Both have shown great interest in my research and progress. Bruce and Julian also provided me with invaluable technical information regarding autoinjector devices.

I am grateful and indebted to the Natural Sciences and Engineering Research Council of Canada for partial financial support of my graduate studies.

A special thank you to all former and current members of the Shepherd research group: Barbara McKinzie Slater, Dr. Remy Mevel, Dr. Stephanie Coronel, Dr. Neil Bitter, Dr. Bryan Schmidt, Dr. Josue Melguizo Gavilanes, Dr. Lorenz Boeck, Silken Jones, Conor Martin, and Dr. Bohoon Kim. In particular, a very special thank you to Stephanie, Josue, and Silken, with whom I had fruitful discussions and collaborations throughout my $\mathrm{Ph} . \mathrm{D}$. 
I am grateful to my parents, Sylvie and Marcel, for their loving support. It would not have been possible to accomplish my graduate studies abroad without their unconditional love and encouragement.

A loving thank you to Audrey, my wonderful wife. I am very grateful that you embarked on this journey abroad with me. With your love, support, and encouragement you gave me the strength to accomplish much more than I could have hoped for, both personally and professionally. I cannot wait to embrace our next adventure together, and see what the future holds. 


\section{ABSTRACT}

The viscosity of drug solutions delivered parenterally has been increasing over the years. Injecting viscous drug solutions using spring-actuated autoinjector devices is challenging due to a number of technical and human factor constraints. Some of the related challenges are investigated in this thesis.

Actuation of autoinjector devices powered using stiff springs can create deleterious pressure and stress transients which are not needed to achieve the normal functions of the device. Experimental measurements have shown that peak pressures and stresses substantially larger than what is needed to achieve the normal device function can occur during the actuation phase, creating unnecessary potential for device failure.

The acceleration of the syringe during actuation can be very large, often creating transient cavitation in the cone region. The occurrence or absence of cavitation is determined by the relative timing of syringe pressurization and syringe acceleration, which is affected by several factors such as the presence, location, and size of an air gap inside the syringe, and the friction between the plunger-stopper and the syringe.

Experiments and numerical simulations have shown that sharp pressure waves traveling inside the syringe can be amplified within the cone terminating the syringe. Despite the potential for shock focusing, the impulsive pressurization and the rapid deceleration of pre-filled syringes create a potential for failure which is localized in the syringe shoulder and at the junction between the flange and the barrel, not inside the cone. The cavitation events, on the other hand, create a potential for failure which is limited to a region in close proximity of the bubble upon collapse. The collapse of cavitation bubbles located within the syringe cone can be enhanced due to geometrical effects, and the resulting stresses can be large enough to cause syringe failure.

This thesis demonstrates that static and quasi-static analyses do not provide accurate estimates of the peak pressures and stresses occurring within the device. The pressure and stresses created by the highly dynamic events occurring during actuation need to be accounted for during device design in order to improve device reliability, the user's experience, and patient's adherence to prescribed treatments. The findings discussed in this work provide insights and guidance as to how the transient events can be mitigated. 


\section{PUBLISHED CONTENT AND CONTRIBUTIONS}

Veilleux, J.-C. and Shepherd, J. E. (2019). "Impulsive Motion in a Cylindrical FluidFilled Tube Terminated by a Converging Section". In: ASME Journal of Pressure Vessel Technology. Accepted Manuscript. DoI: 10.1115/1.4042799.

Veilleux, J.C., Maeda, K., et al. (2018). "Transient Cavitation in Pre-Filled Syringes During Autoinjector Actuation”. In: Proceedings of the 10th International Symposium on Cavitation. (Baltimore, MD). Dor: 10.1115/1.861851_ch203.

Veilleux, J.-C. and Shepherd, J.E. (2018). "Pressure and Stress Transients in Autoinjector Devices". In: Journal of Drug Delivery and Translational Research 8 (5), pp. 1238-1253. DOI: 10.1007/s13346-018-0568-7.

Veilleux, J.-C. and Shepherd, J.E. (2017a). "Dampers and Methods for Performing Measurements in an Autoinjector". Patent application 20180015224 (US). URL: https://www . lens.org/lens/patent/123-673-900-361-650.

Veilleux, J.-C. and Shepherd, J.E. (2017b). "Impulsively-Generated Pressure Transients and Strains in a Cylindrical Fluid-Filled Tube Terminated by a Converging Section". In: Proceedings of the ASME 2017 Pressure Vessels and Piping Conference. (Waikoloa, HI). Vol. 4. Paper PVP2017-65471. Dor: 10. 1115/PVP2017 $-65471$. 


\section{TABLE OF CONTENTS}

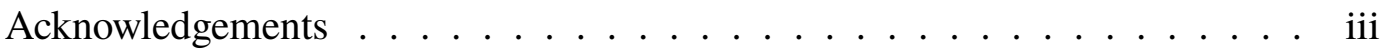

Abstract ......................

Published Content and Contributions . . . . . . . . . . . . . . vi

Table of Contents . . . . . . . . . . . . . . . . vii

List of Illustrations . . . . . . . . . . . . . . . . . . . $\mathrm{x}$

List of Tables . . . . . . . . . . . . . . . . . . X Xx

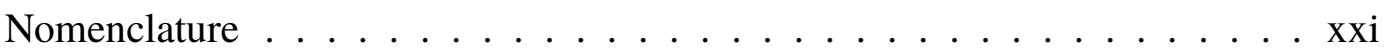

Chapter I: Introduction . . . . . . . . . . . . . . . . . 1

1.1 Motivation . . . . . . . . . . . . . . . 1

1.2 Objectives and Structure . . . . . . . . . . . . . 6

Chapter II: Dynamic Events in Autoinjector Devices . . . . . . . . . . . . . . 9

2.1 Actuation Sequence of a Typical Spring-Actuated Autoinjector . . . . 9

2.2 Transient Events During Autoinjector Actuation . . . . . . . . . . 13

2.2.1 Acoustics and Wave Dynamics . . . . . . . . . . . . . . 14

2.2.2 Event 1: Syringe Acceleration . . . . . . . . . . . 16

2.2.3 Event 2: Syringe Deceleration . . . . . . . . . . 25

2.2.4 Event 3: Impact of the Driving Rod on the Plunger-Stopper . 37

2.3 SureClick Autoinjector . . . . . . . . . . . . . . . . . . 41

Chapter III: In Situ Measurements . . . . . . . . . . . . . . . . . . . . . . . . . . . . . . . . . . . . 45

3.1 Methodology and Material . . . . . . . . . . . . . . . . . 45

3.1.1 Step 1: Modifying the Shell . . . . . . . . . . . . 46

3.1.2 Step 2: Modifying the Syringe Carrier . . . . . . . . . . . . 47

3.1.3 Step 3: Removing the Needle . . . . . . . . . . . . . . 47

3.1.4 Step 4: Mounting the Pressure Transducer . . . . . . . . . . 48

3.1.5 Step 5: Filling the Syringe . . . . . . . . . . . . 50

3.1.6 Step 6: Preparing the Syringe Surface . . . . . . . . . . 51

3.1.7 Step 7: Installing the Strain Gauges . . . . . . . . . . . 51

3.1.8 Step 8: Assembling the Autoinjector . . . . . . . . . . 53

3.1.9 Step 9: Mounting the Autoinjector on a Support . . . . . . . 53

3.1.10 Step 10: Final Preparation . . . . . . . . . . . . . . 55

3.1 .11 Limitations . . . . . . . . . . . . . . 56

3.2 Results and Discussion . . . . . . . . . . . . . . . 57

3.2.1 Configuration 1: Glass Syringe Without an Air Gap . . . . . 59

3.2.2 Configuration 2: Glass Syringe With an Air Gap . . . . . . 68

3.2.3 Configuration 3: Plastic Syringe Without an Air Gap . . . . 74

3.2.4 Configuration 4: Plastic Syringe With an Air Gap . . . . . . 79

3.2.5 Friction Estimates . . . . . . . . . . . . . . . . 84

Chapter IV: Large Scale Model Autoinjector . . . . . . . . . . . . . . . . 87

4.1 Static Large-Scale Model Autoinjector . . . . . . . . . . . . . . 88 
4.1.1 Experimental Setup . . . . . . . . . . . . . . . . . . . . 88

4.1.2 LS-DYNA Numerical Model . . . . . . . . . . . . . . . 92

4.1 .3 Results . . . . . . . . . . . . . . . . . . . . . 994

4.1.3.1 Case $1 \ldots \ldots \ldots$. . . . . . . . . . . . 94

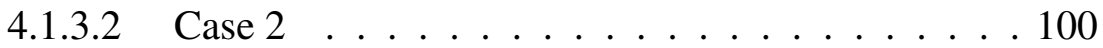

4.1.3.3 Case $3 \ldots \ldots$. . . . . . . . . . . . . . . . . . . . . .

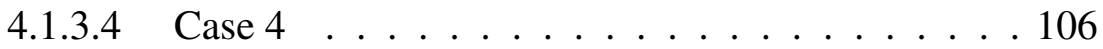

4.1.3.5 Case $5 \ldots \ldots$. . . . . . . . . . . . . . . 109

4.1.4 Conclusion on Cases 1 to $5 \ldots$. . . . . . . . . . 111

4.2 Dynamic Large-Scale Model Autoinjector . . . . . . . . . . 113

4.2 .1 Experimental Setup . . . . . . . . . . . . . . . . . 113

4.2.2 LS-DYNA Numerical Model . . . . . . . . . . . . . . . . 118

4.2.3 Results: Syringe Acceleration and Pressurization . . . . . 119

4.2.3.1 Case 6 . . . . . . . . . . . . . . . . . . . 120

4.2 .3 .2 Case $7 \ldots \ldots \ldots 131$

4.2 .3 .3 Case $8 \ldots \ldots \ldots \ldots$

4.2.3.4 Case $9 \ldots \ldots$. . . . . . . . . . . . . . . . 135

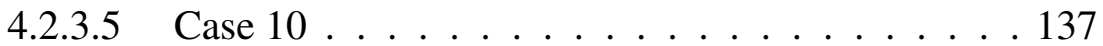

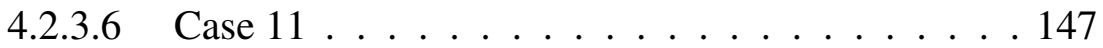

4.2 .3 .7 Case $12 \ldots \ldots \ldots$

4.2.3.8 Summary of Cases 6 to $12 \ldots \ldots$. . . . . . . . . 153

4.2.4 Results and Discussion: Syringe Deceleration . . . . . . . 156

4.2.4.1 Case $13 \ldots \ldots \ldots \ldots$. . . . . . . . . . . . . . . . . . . . . . . .

4.2 .4 .2 Case $14 \ldots \ldots \ldots \ldots \ldots$

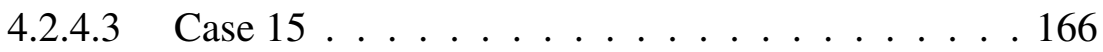

4.2.4.4 Summary of Cases 13 to $15 \ldots 168$

Chapter V: Numerical Simulations of Shock Focusing and Syringe Stresses . 170

5.1 Shock Focusing . . . . . . . . . . . . . . . . . . 170

5.1 .1 Numerical Model . . . . . . . . . . . . . . . . . . . . 171

5.1.2 Shock Focusing in a Straight Cone . . . . . . . . . . . 172

5.1.3 Effect of the Half-Angle . . . . . . . . . . . . . . . 177

5.1.4 Effect of the Rise Time . . . . . . . . . . . . . . . . . 179

5.1.5 Effect of the Acoustic Impedance . . . . . . . . . . . . 181

5.1.6 Focusing in the Cone of a Syringe . . . . . . . . . . . . 184

5.1.7 Summary of Shock Wave Focusing . . . . . . . . . . . . 185

5.2 Wall Stresses . . . . . . . . . . . . . . . . . . . . 187

$5.2 .1 \quad$ Numerical Model . . . . . . . . . . . . . . . . . . . . . 187

5.2.2 Static Internal Loading . . . . . . . . . . . . . . . . . . 189

5.2.2.1 Simply Supported Flange _. . . . . . . . . . 189

5.2.2.2 Simply Supported Shoulder . . . . . . . . . . . . 192

5.2.2.3 Summary of Static Stresses _ . . . . . . . . 196

5.2 .3 Dynamic Internal Loading . . . . . . . . . . . . . . . . 198

5.2.3.1 Simply Supported Flange _. . . . . . . . . . . . 199

5.2.3.2 Simply Supported Shoulder . . . . . . . . . . . 202

5.2.3.3 Summary of Dynamic Loading Stresses . . . . . . 203 
5.2.4 Syringe Deceleration . . . . . . . . . . . . . . . . 205

5.2.4.1 Simply Supported Flange _. . . . . . . . . . . 206

5.2.4.2 Simply Supported Shoulder . . . . . . . . . . 207

5.2.4.3 Summary of Stresses Created by Syringe Decel-

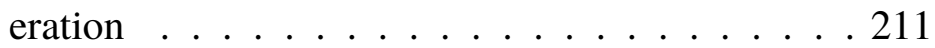

5.2 .5 Weibull Curves . . . . . . . . . . . . . . . 211

5.2 .6 Bubble Collapse . . . . . . . . . . . . . . . . . . . . . . 214

Chapter VI: Bubble Dynamics in a Cone . . . . . . . . . . . . . . . . 219

6.1 Collapse of a Cavitation Bubble . . . . . . . . . . . . . . 219

6.2 Numerical Setup . . . . . . . . . . . . . . . . . . 221

6.3 Bubble Collapse Time . . . . . . . . . . . . . . . . . . . 225

6.4 Pressure in the Tip . . . . . . . . . . . . . . . . . 231

6.5 Pressure in a Syringe Geometry . . . . . . . . . . . . . . . . . . . . . . . . . . . . . .

6.6 Summary . . . . . . . . . . . . . . . . . . . 241

Chapter VII: Mitigation of the Pressure and Stress Transients . . . . . . . . . 243

7.1 Dampers for Autoinjector Devices . . . . . . . . . . . . . . 245

7.2 Maximum Allowable Syringe Acceleration . . . . . . . . . . . . . 249

Chapter VIII: Summary \& Future Work . . . . . . . . . . . . . . . . . . . . . . . . . . . . .

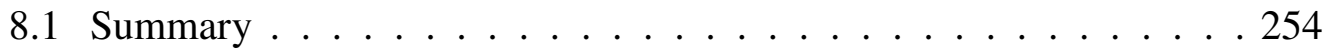

8.2 Future Work . . . . . . . . . . . . . . . . . . . 258

Bibliography . . . . . . . . . . . . . . . . . . 261

Appendix A: Acoustics and Method-of-Characteristics . . . . . . . . . 269

A.1 Introduction . . . . . . . . . . . . . . . . . . . . . . 269

A.2 Acoustics and Fluid Mechanics . . . . . . . . . . . . . 270

A.3 Characteristics . . . . . . . . . . . . . . . . . . 272

A.4 The Wave Equation . . . . . . . . . . . . . . . . . . 274

A.5 The Acoustic Limit of the Method-of-Characteristics . . . . . . . . 276

A.5.1 Boundary Conditions . . . . . . . . . . . . . . 278

A.5.2 Initial Conditions . . . . . . . . . . . . . . . . . 279

A.6 Cavitation Model for the Method-of-Characteristics . . . . . . . 280

A.6.1 Qualitative Description of DVCM . . . . . . . . . . . 280

A.6.2 Mathematical Formulation of DVCM . . . . . . . . . . . 281

A.6.3 Numerical Implementation of DVCM _. . . . . . . . . . 282

A.7 Impact Between Solid Parts . . . . . . . . . . . . . . . . . 284

Appendix B: Test Matrices . . . . . . . . . . . . . . . . . . . 287 


\section{LIST OF ILLUSTRATIONS}

Number Page

1.1 Concentration of FDA approved monoclonal antibodies over the years. 3

1.2 Flow in the barrel and the needle of a syringe resulting from a force $F$ applied on the plunger-stopper. . . . . . . . . . . . 4

1.3 Example of a broken glass syringe. . . . . . . . . . . . . 6

2.1 Simplified schematic of the actuation sequence of a typical springactuated autoinjector device. . . . . . . . . . . . . . . 10

2.2 Examples of pre-filled, glass syringes. . . . . . . . . . . . 11

2.3 Schematic of a pre-filled syringe in a vertical, tip-down configuration with and without an air gap. . . . . . . . . . . . . . 12

2.4 Reflection and transmission of pressure/stress waves at the interface between two media. . . . . . . . . . . . . . . . 15

2.5 Schematic of the growth and collapse of a cavity shortly after the abrupt acceleration of a liquid-filled syringe. . . . . . . . . . . 18

2.6 Pressure at the bottom wall of a liquid-filled syringe which is impulsively accelerated. . . . . . . . . . . . . . 18

2.7 Space-time diagram of the pressure transient created by the impulsive acceleration of the syringe. The syringe contains an ideal fluid which can sustain tension. . . . . . . . . . . . . . . . . 20

2.8 Space-time diagram of the pressure transient created by the impulsive acceleration of the syringe. The syringe contains an ideal fluid which can sustain tension. Results obtained with the method-ofcharacteristics. . . . . . . . . . . . . . 21

2.9 Space-time diagram of the pressure transient created by the impulsive acceleration of the syringe. The syringe contains a real fluid which does not sustain tension. . . . . . . . . . . . . . . . 23

2.10 Space-time diagram of the pressure transient generated upon the impulsive acceleration of the syringe. The syringe contains a real fluid which does not sustain tension. Results obtained with the methodof-characteristics in combination with a cavitation model. . . . . . . 24

2.11 Simplified schematic showing the rapid deceleration of the syringe. . 27 
2.12 Space-time diagram of the pressure transient in the liquid created by the impulsive deceleration of the syringe. The syringe contains an ideal fluid, and cavitation does not occur. . . . . . . . . . . . . 29

2.13 Space-time diagram of the stress transient in the syringe wall created by the impulsive deceleration of the syringe. The syringe contains an ideal fluid, and cavitation does not occur. . . . . . . . . . . . 29

2.14 Space-time diagram of the pressure transient (without cavitation) or the stress transient created by the impulsive deceleration of the syringe. Results obtained using the method-of-characteristics. . . . . 31

2.15 Space-time diagram of the pressure transient (with cavitation) created by the impulsive deceleration of the syringe. Results obtained using the method-of-characteristics. . . . . . . . . . . . . . . 31

2.16 Deformation of the syringe wall resulting from its interaction with a pressure wave propagating in the liquid. . . . . . . . . . . . 32

2.17 Velocity of the syringe during a deceleration of constant magnitude over a time $\Delta t . \ldots \ldots$. . . . . . . . . . . . . . 33

2.18 Peak magnitude of the pressure or stress created by a constant deceleration of the syringe over a time $\Delta t \ldots \ldots$. . . . . . . . . . 37

2.19 Pressure history at a fixed location along the barrel of the syringe upon application of a force $F$ on the plunger-stopper. . . . . . . . . . 40

2.20 SureClick autoinjector device. . . . . . . . . . . . . . . 42

2.21 Schematic of the key features of a SureClick autoinjector device. . . . 43

3.1 Schematic of a syringe carrier with an oblong slit for the leadwires of the strain gauges to be mounted on the syringe. . . . . . . . . . . 47

3.2 PCB 138M186 tourmaline underwater blast pressure sensor. . . . . . 48

3.3 Schematic of a PCB 138M186 tourmaline underwater blast pressure sensor mounted inside a syringe. . . . . . . . . . . . 50

3.4 Schematic of a syringe instrumented with C2A-06-015LW-120 strain gauges and a PCB 138M186 pressure transducer. . . . . . . . . . 53

3.5 Schematic of an instrumented syringe mounted into a syringe carrier. 53

3.6 Schematic of a spring-actuated autoinjector device instrumented with strain gauges and a pressure transducer. . . . . . . . . . . . 54

3.7 Schematic of an instrumented autoinjector device mounted in aluminum blocks on a T-slot extrusion. . . . . . . . . . . . . . 54

3.8 Schematic of an autoinjector device instrumented with strain gauges, a pressure transducer, and a high speed camera. . . . . . . . . . 56 
3.9 Position and velocity of the moving components in a SureClick autoinjector - glass syringe without an air gap (test SC-033) . . . . . . . 60

3.10 Liquid pressure in a SureClick autoinjector - glass syringe without an air gap (test SC-033). . . . . . . . . . . . . . . 61

3.11 Hoop and axial strains on the barrel of the syringe in a SureClick autoinjector - glass syringe without an air gap (test SC-033) . . . . . . 61

3.12 Position and velocity of the moving components in a SureClick autoinjector - glass syringe with an air gap (test SC-178). . . . . . . . 69

3.13 Liquid pressure in a SureClick autoinjector - glass syringe with an air gap (test SC-178). . . . . . . . . . . . . . . . . 70

3.14 Hoop and axial strains on the barrel of the syringe in a SureClick autoinjector - glass syringe with an air gap (test SC-178). . . . . . . 70

3.15 Growth and collapse of a bubble in the cone area of a glass syringe when an air gap is present (test SC-0137). . . . . . . . . . . 73

3.16 Growth and collapse of bubbles along the barrel of a glass syringe when an air gap is present (test SC-0137). . . . . . . . . . . . 74

3.17 Position and velocity of the moving components in a SureClick autoinjector - plastic syringe without an air gap (test SC-200). . . . . . 76

3.18 Liquid pressure in a SureClick autoinjector - plastic syringe without an air gap (test SC-200). . . . . . . . . . . . . . . . 77

3.19 Hoop and axial strains on the barrel of the syringe in a SureClick autoinjector - plastic syringe without an air gap (test SC-200). . . . . 77

3.20 Position and velocity of the moving components in a SureClick autoinjector - plastic syringe with an air gap (test SC-201) . . . . . . 80

3.21 Liquid pressure in a SureClick autoinjector - plastic syringe with an air gap (test SC-201) . . . . . . . . . . . . . . . . 81

3.22 Hoop and axial strains on the barrel of the syringe in a SureClick autoinjector - plastic syringe with an air gap (test SC-201). . . . . . . 81

3.23 Motion of the driving rod, the plunger-stopper and the syringe (plastic

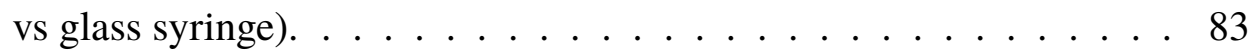

3.24 Position and velocity of the moving components in a SureClick autoinjector with an empty syringe. . . . . . . . . . . 85

4.1 Schematic of the static, large-scale model autoinjector experimental setup. ....................... 88

4.2 Schematic of the base fixtures used with the static, large-scale model autoinjector. ..................... 90 
4.3 LS-DYNA model of the static, large-scale model autoinjector. . . . . 92

4.4 Pressure at the bottom end for case 1 of the static, large-scale model autoinjector experiments (test GG-0030) . . . . . . . . . . . . 96

4.5 Wave dynamics in the static, large-scale model autoinjector test setup (adapted from Inaba and Shepherd (2010) with permission). . . . . . 96

4.6 Hoop and axial strains for case 1 of the static, large-scale model autoinjector experiments (test GG-0030) . . . . . . . . . . . . . . . 98

4.7 Motion of the buffer and the projectile with a space-time pressure plot (LS-DYNA) for case 1 of the static, large-scale model autoinjector experiments (test GG-0030). . . . . . . . . . . . . . . . . 99

4.8 Pressure at the bottom end for case 2 of the static, large-scale model autoinjector experiments (test GG-0240). . . . . . . . . . . . . 101

4.9 Sequence of images showing distributed cavitation for case $2 b$ of the static, large-scale model autoinjector experiments (test GG-0215). . . 102

4.10 Pressure at the bottom end for case 3 of the static, large-scale model autoinjector experiments (test GG-0033). . . . . . . . . . . . 104

4.11 Motion of the buffer and the projectile with a space-time pressure plot (LS-DYNA) for case 3 of the static, large-scale model autoinjector experiments (test GG-0033) . . . . . . . . . . . . . 105

4.12 Pressure at the bottom end for case 4 of the static, large-scale model autoinjector experiments (test GG-0034) . . . . . . . . . . . 106

4.13 Hoop strains for case 4 of the static, large-scale model autoinjector experiments (test GG-0034) . . . . . . . . . . . . . . 107

4.14 Motion of the buffer and the projectile with a space-time pressure plot (LS-DYNA) for case 4 of the static, large-scale model autoinjector experiments (test GG-0034) . . . . . . . . . . . . . . 107

4.15 Pressure at the bottom end for case 5 of the static, large-scale model autoinjector experiments (test GG-0099). . . . . . . . . . . . . 109

4.16 Sequence of images showing cavitation at the tip of the cone for tests performed with a large air gap (test GG-0235). . . . . . . . . . . 111

4.17 Schematic of the dynamic, large-scale model autoinjector experimental setup. . . . . . . . . . . . . . . . . . 114

4.18 Schematic of the decelerators used to stop the syringe in the dynamic, large-scale model autoinjector experimental setup. . . . . . . . . . 116 
4.19 LS-DYNA model used to simulate the transient events created by the impact of the reversed outer projectile on the buffer of the syringe in the dynamic, large-scale model autoinjector. . . . . . . . . . . . . 118

4.20 LS-DYNA model used to simulate the transient events created by the deceleration of the syringe in the dynamic, large-scale model autoinjector.

4.21 Velocity of the syringe and the buffer for case 6 of the dynamic, large-scale model autoinjector experiments (test LS-0353) . . . . . 121

4.22 Pressure at the bottom wall for case 6 of the dynamic, large-scale model autoinjector experiments (test LS-0353). . . . . . . . . . . 122

4.23 Reflection of a pressure pulse on an accelerated, rigid wall. . . . . . 123

4.24 Hoop and axial strains for case 6 of the dynamic, large-scale model autoinjector experiments (test LS-0353). . . . . . . . . . . . 126

4.25 Peak pressure in the syringe tip as a function of initial air gap size. The outer projectile impacts on the buffer of the syringe in the dynamic, large-scale model autoinjector. . . . . . . . . . . . . . . . . . . . . 128

4.26 Schematic of the simplified model of the isentropic compression of an air gap. . . . . . . . . . . . . . . . . . . . 128

4.27 Peak air gap pressure and air gap compression time obtained with the simplified model. . . . . . . . . . . . . . . . . . . 129

4.28 Velocity of the syringe and the buffer for case 7 of the dynamic, large-scale model autoinjector experiments (test LS-0345). . . . . . . 131

4.29 Pressure at the bottom wall for case 7 of the dynamic, large-scale model autoinjector experiments (test LS-0345) . . . . . . . . . . 132

4.30 Hoop and axial strains for case 7 of the dynamic, large-scale model autoinjector experiments (test LS-0345). . . . . . . . . . . . . 134

4.31 Pressure at the bottom wall for case 8 of the dynamic, large-scale model autoinjector experiments (test LS-0208). . . . . . . . . . 135

4.32 Velocity of the syringe and the buffer for case 9 of the dynamic, large-scale model autoinjector experiments (test LS-0329). . . . . . . 136

4.33 Pressure at the bottom wall for case 9 of the dynamic, large-scale model autoinjector experiments (test LS-0329). . . . . . . . . . . . 136

4.34 Velocity of the syringe for case 10 of the dynamic, large-scale model autoinjector experiments (test LS-0322). . . . . . . . . . . . . 138

4.35 Pressure at the bottom wall for case 10 of the dynamic, large-scale model autoinjector experiments (test LS-0322) . . . . . . . . . . . 139 
4.36 Hoop and axial strains for case 10 of the dynamic, large-scale model autoinjector experiments (test LS-0322). . . . . . . . . . . . . 142

4.37 Pressure at the bottom wall for case $10 \mathrm{~b}$ of the dynamic, large-scale model autoinjector experiments. . . . . . . . . . . . . . . 144

4.38 Sequence of images showing the cavitation event at the bottom of the syringe for test $10 \mathrm{~b}$ of the dynamic, large-scale model autoinjector experiments (test LS-0382). . . . . . . . . . . . . . . . . 145

4.39 Peak pressure in the syringe tip as a function of initial air gap size. The outer projectile impacts on the syringe wall. This is for the dynamic, large-scale model autoinjector test setup with a flat tip. . . . 146

4.40 Final stage of bubble collapse for tests cases $10 \mathrm{~b}$ and $10 \mathrm{c}$ performed without a buffer. This is for the dynamic, large-scale model autoinjector. 147

4.41 Pressure at the bottom wall for case 11 of the dynamic, large-scale model autoinjector experiments (test LS-0318). . . . . . . . . . . . 148

4.42 Peak pressure in the syringe tip as a function of initial air gap size. The outer projectile impacts on the syringe wall. This is for the dynamic, large-scale model autoinjector test setup with a conical tip. . 150

4.43 Velocity of the syringe for case 12 of the dynamic, large-scale model autoinjector experiments (test LS-0263). . . . . . . . . . . . 150

4.44 Pressure at the bottom wall for case 12 of the dynamic, large-scale model autoinjector experiments (test LS-0263). . . . . . . . . . . 151

4.45 Hoop and axial strains for case 12 of the dynamic, large-scale model autoinjector experiments (test LS-0263). . . . . . . . . . . 152

4.46 Pressure at the bottom wall for case $12 \mathrm{~b}$ of the dynamic, large-scale model autoinjector experiments (test LS-0402). . . . . . . . . . . 153

4.47 Sequence of images showing the cavitation event at the bottom of the syringe for test $12 \mathrm{~b}$ of the dynamic, large-scale model autoinjector experiments (test LS-0402). . . . . . . . . . . . . . . . 154

4.48 Final stage of bubble collapse for test $12 \mathrm{~b}$ of the dynamic, large-scale model autoinjector experiments (test LS-0402). . . . . . . . . . . . 154

4.49 Velocity of the syringe for case 13 of the dynamic, large-scale model autoinjector experiments (test LS-0296) . . . . . . . . . . . . . 157

4.50 Sequence of images showing the motion of the projectile, the buffer and the syringe for case 13 of the dynamic, large-scale model autoinjector experiments (test LS-0296) . . . . . . . . . . . . 158 
4.51 Pressure at the bottom wall for case 13 of the dynamic, large-scale model autoinjector experiments (test LS-0296). . . . . . . . . . . . 159

4.52 Hoop and axial strains for case 13 of the dynamic, large-scale model autoinjector experiments (test LS-0296). . . . . . . . . . . . 160

4.53 Schematic of the wave mechanics in the syringe wall and the liquid column during and after the syringe deceleration for case 13. . . . . 161

4.54 Sequence of images of the cavitation events for case 13b of the dynamic, large-scale model autoinjector experiments (test LS-0417). . 164

4.55 Velocity of the syringe for case 14 of the dynamic, large-scale model autoinjector experiments (test LS-0319). . . . . . . . . . . . . . 164

4.56 Pressure at the bottom wall for case 14 of the dynamic, large-scale model autoinjector experiments (test LS-0319). . . . . . . . . . . 165

4.57 Box plot of the peak pressure at the flat wall of the tip during the rapid deceleration of the syringe in the dynamic, large-scale model autoinjector experiments. . . . . . . . . . . . . . . 166

4.58 Velocity of the syringe for case 15 of the dynamic, large-scale model autoinjector experiments (test LS-0227). . . . . . . . . . . 167

4.59 Pressure at the bottom wall for case 15 of the dynamic, large-scale model autoinjector experiments (test LS-0227). . . . . . . . . 168

5.1 Cross-sectional view of the geometry used in the numerical simulations on shock focusing in a straight cone. . . . . . . . . . . . 171

5.2 Amplification of a sharp pressure wave entering a straight, rigid cone with a half-angle of 30 degrees. . . . . . . . . . . . . . . 175

5.3 Pressure history on the cone axis and the tip wall for a sharp pressure wave entering a straight, rigid cone with a half-angle of 30 degrees. . 176

5.4 Evolution of the amplification factor as a function of the half-angle $\alpha .178$

5.5 Spherical convergence of an acoustic wave. . . . . . . . 178

5.6 Evolution of the amplification factor as a function of the normalized rise time of the incident pressure wave. . . . . . . . . . 180

5.7 History of the average tip pressure for a pressure wave entering a straight, rigid cone with a half-angle of 30 degrees. The average tip pressure is shown for different rise times of the incident wave. . . . 181

5.8 Evolution of the amplification factor of the cone as a function of the reflection coefficient. . . . . . . . . . . . . . . 183

5.9 Amplification of a sharp pressure wave entering the cone of a BD Hyfloe $1 \mathrm{~mL}$ pre-filled syringe. . . . . . . . . . . . . . . 185 
5.10 Pressure history in the narrower section of the syringe cone. . . . . 186

5.11 LS-DYNA syringe model of a $1 \mathrm{ml}$ BD Hypack syringe. . . . . . . 188

5.12 Schematic representation of the boundary conditions used in the LSDYNA numerical model. . . . . . . . . . . . . . . . . . . . . 189

5.13 Maximum principal stress in the syringe wall for a constant and uniform liquid pressure of $1 \mathrm{MPa}$. The syringe is supported at the flange. . . . . . . . . . . . . . . . . . 190

5.14 Stresses at three different stations in the syringe wall for a constant and uniform liquid pressure. The syringe is supported at the flange. . 191

5.15 Stresses along the inner and the outer surface of the syringe for a constant and uniform liquid pressure. The syringe is supported at the flange. . . . . . . . . . . . . . . . . . . 193

5.16 Maximum principal stress in the syringe wall for a constant and uniform liquid pressure of $1 \mathrm{MPa}$. The syringe is supported at the shoulder. . . . . . . . . . . . . . . . . . . . 195

5.17 Stresses at three different stations in the syringe wall for a constant and uniform liquid pressure. The syringe is supported at the shoulder. 196

5.18 Stresses along the inner and the outer surface of the syringe for a constant and uniform liquid pressure. The syringe is supported at the shoulder. . . . . . . . . . . . . . . . . . . . . . 197

5.19 Maximum pressure along the inner surface of the syringe. The syringe is supported at the flange, and dynamically pressurized with a sharp pressure wave. . . . . . . . . . . . . . . . 200

5.20 Stresses along the inner and the outer surface of the syringe. The syringe is supported at the flange, and dynamically pressurized with a sharp pressure wave. . . . . . . . . . . . . . . . . 201

5.21 Maximum pressure along the inner surface of the syringe. The syringe is supported at the shoulder, and dynamically pressurized with a sharp pressure wave. . . . . . . . . . . . . . . . . 202

5.22 Stresses along the inner and the outer surface of the syringe. The syringe is supported at the shoulder, and dynamically pressurized with a sharp pressure wave. . . . . . . . . . . . . . . . . 204

5.23 Maximum pressure along the inner surface of the syringe. The syringe and its liquid content are initially traveling downward, and the syringe is rapidly decelerated using a support at the flange. . . . . . 207 
5.24 Stresses along the inner and the outer surface of the syringe. The syringe and its liquid content are initially traveling downward, and the syringe is rapidly decelerated using a support at the flange. . . . . 208

5.25 Maximum pressure along the inner surface of the syringe. The syringe and its liquid content are initially traveling downward, and the syringe is rapidly decelerated using a support at the shoulder. . . . . 209

5.26 Stresses along the inner and the outer surface of the syringe. The syringe and its liquid content are initially traveling downward, and the syringe is rapidly decelerated using a support at the shoulder. . . . 210

5.27 Schematic of the Ram-Rod testing facility used to construct Weibull curves of various pre-filled syringes. . . . . . . . . . . . . 212

5.28 Probability of failure as a function of the peak magnitude of the pressure wave created under the plunger-stopper (Weibull curve) for a $1 \mathrm{~mL}$ BD Hypack pre-filled syringe. . . . . . . . . . . . . . . 213

5.29 Probability of failure as a function of the peak magnitude of the maximum principal stress in the syringe shoulder (Weibull curve) for a $1 \mathrm{~mL}$ BD Hypack pre-filled syringe. The main plot uses double logarithmic coordinates so that the regime of small failure probability is visible. . . . . . . . . . . . . . . . . . . . . . . 214

5.30 Initial pressure source (left \& center) and spherical shock wave (right) used in the LS-DYNA simulations to predict wall stresses. . . . . . . 215

5.31 Pressure and stresses along the inner surface of the syringe. The pressure and stresses are created by a spherical pressure wave similar to the wave created by a bubble collapsing in the cone. . . . . . . . 217

6.1 Geometry used in the numerical simulations of a bubble collapse inside a straight cone. . . . . . . . . . . . . . . . . . 222

6.2 Effect of the grid resolution on the evolution of bubble volume and peak pressure on the wall. The grid resolutions are reported in Table 6.1.223

6.3 Collapse time as a function of the initial location of the bubble and the half-angle of the cone. . . . . . . . . . . . . . 225

6.4 Evolution of the bubble volume during collapse in a 45-degree cone. . 227

6.5 Shock-induced collapse of a bubble initially located at $\Lambda / R_{0}=2.8$ in a 45-degree cone. . . . . . . . . . . . . . . . . 228

6.6 Placement of the bubble in a cone with $\Lambda / R_{0}=1$ for a small and a

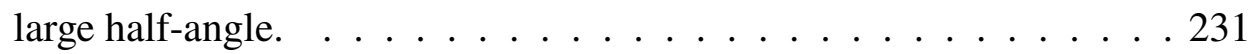


6.7 Peak wall pressure as a function of the initial location of the bubble and the half-angle of the cone. . . . . . . . . . . . . . . 232

6.8 Peak wall pressure when no bubble is present in the cone (i.e., effect of shock focusing alone). . . . . . . . . . . . . . . . . . 234

6.9 Amplification of the peak wall pressure due to the collapse of a bubble inside the cone. . . . . . . . . . . . . . . . . . 235

6.10 Initial configuration used in the numerical simulations performed with MFC and ECOGEN. . . . . . . . . . . . . . . . . . 237

6.11 Collapse of a bubble inside the cone of a pre-filled syringe. Results obtained using ECOGEN. . . . . . . . . . . . . . . . . . 239

6.12 Evolution of the bubble volume during collapse in the cone of a syringe. Results obtained using ECOGEN. . . . . . . . . . . . . 240

6.13 Evolution of the peak pressure during collapse in the cone of a syringe. Results obtained using ECOGEN. . . . . . . . . . . . . . . . . . 241

7.1 Schematic of a SureClick autoinjector with damping material. . . . . 246

7.2 Velocity of the moving components in a SureClick autoinjector with damping material. . . . . . . . . . . . . . . . 247

7.3 Liquid pressure in a SureClick autoinjector with damping material. . 248

7.4 Hoop and axial strains on the barrel of the syringe in a SureClick autoinjector with damping material. . . . . . . . . . . . . . . 248

7.5 Syringe model acceleration with memory foam between the outer projectile and the syringe wall. . . . . . . . . . . . . . . 251

A.1 Characteristic directions at a point in the flow. . . . . . . . . . 272

A.2 Geometry of characteristics. . . . . . . . . . . . . . . 273

A.3 Network of characteristics in the acoustic limit. . . . . . . . . . 277

A.4 Schematic representing the idea behind the DVCM model. . . . . . . 281

A.5 Representation of the penalty coupling approach to model impact events. . . . . . . . . . . . . . . . . . . . 285 


\section{LIST OF TABLES}

Number

Page

2.1 Reflection of pressure/stress waves at the interface between two media - magnitude of the reflected $\Delta P_{r}$ and transmitted waves $\Delta P_{t} \ldots \ldots 16$

2.2 Density and sound speed of fluids and solids which are relevant to this thesis. . . . . . . . . . . . . . . . 16

2.3 Axial stress and velocity within regions 0 to 7 identified in Figure 2.13. 30

3.1 Estimated physical and geometrical properties of a BD HyPack and a Daikyo Crystal Zenith pre-filled syringe. . . . . . . . . . . . . 58

4.1 Axial location of the pressure transducers in the static, large-scale model autoinjector. . . . . . . . . . . . . . . . . . 91

4.2 Axial location of the strain gauges in the static, large-scale model autoinjector. . . . . . . . . . . . . . . . 991

4.3 Parameters used in the equations of state used in the LS-DYNA model. 93

4.4 Rise time of the second incident wave for case 4 of the static, largescale model autoinjector experiments (test GG-0034). . . . . . . . 108

4.5 Nominal axial location of the strain gauges mounted on the outer surface of the syringe used in the dynamic, large-scale model autoinjector experimental setup. . . . . . . . . . . . . . . 117

6.1 Grid resolutions used to investigate the effect of the mesh on the shock-induced collapse of a bubble in the vicinity of a flat wall. . . . 224

6.2 Axial and radial location of the vertices in the simplified syringe geometry used in ECOGEN and MFC. . . . . . . . . . . . . . 237

7.1 Experimental verification of the maximum allowable model syringe acceleration to eliminate cavitation - large-scale model syringe with a flat tip. . . . . . . . . . . . . . . . . . 251

7.2 Experimental verification of the maximum allowable model syringe acceleration to eliminate cavitation - large-scale model syringe with a conical tip. . . . . . . . . . . . . . . . . 252

B.1 Test matrix of the successful in situ experiments. . . . . . . . . . . . 288

B.2 Test matrix for the experiments performed with the static, large-scale model autoinjector. . . . . . . . . . . . . . . . 295

B.3 Test matrix for the experiments performed with the dynamic, largescale model autoinjector. . . . . . . . . . . . . . . . . . . . . . . 298 


\section{NOMENCLATURE}

\section{Greek symbols}

$\alpha \quad$ Void fraction (-) or half-angle (degree)

$\beta \quad$ Fluid structure interaction coupling parameter (-)

$\delta \quad$ Air gap size $(\mathrm{mm})$

$\Delta($ ) Variation of

$\epsilon \quad$ Strain (-)

$\gamma \quad$ Ratio of the specific heats (-)

$\Lambda \quad$ Minimum distance between the bubble center and the cone wall ( $\mathrm{mm})$

$\lambda \quad$ Amplification factor (-) or relative position of the inner projectile within the outer projectile $(\mathrm{mm})$

$\mu \quad$ Dynamic viscosity of the syringe content ( $\mathrm{P}$ or $\mathrm{cP}$ )

$v \quad$ Poisson's ratio (-)

$\phi \quad$ Acoustic potential $\left(\mathrm{m}^{2} / \mathrm{s}\right)$

$\psi \quad$ Integration weighting factor (-)

$\rho \quad$ Density $\left(\mathrm{kg} / \mathrm{m}^{3}\right)$

$\sigma \quad$ Stress (MPa)

$\tau \quad$ Non-dimensional time (-)

$\tau_{c} \quad$ Non-dimensional bubble collapse time (-)

$\zeta \quad$ Axial coordinate (stationary frame) ( $\mathrm{mm}$ or $\mathrm{m}$ )

\section{Roman (uppercase)}

A Cross-sectional area $\left(\mathrm{mm}^{2}\right.$ or $\left.\mathrm{m}^{2}\right)$

$C^{ \pm}, C^{0} \quad$ Characteristic directions (-)

$D \quad$ Diameter (mm)

E Young's modulus of elasticity (GPa)

$F \quad$ Syringeability or force applied on the plunger-stopper (N)

$H \quad$ Distance between the bubble center and the tip of the cone (mm)

$K \quad$ Bulk modulus (GPa)

$L \quad$ Axial length (mm)

$P \quad$ Pressure $(\mathrm{Pa}, \mathrm{kPa}$ or $\mathrm{MPa})$

$P_{s} \quad$ Pressure downstream of a shock wave (MPa)

$P_{\text {vap }} \quad$ Vapor pressure $(\mathrm{Pa})$

$Q, \mathcal{R} \quad$ Riemann invariants $(\mathrm{m} / \mathrm{s})$

$R \quad$ Radius (mm)

$T \quad$ Extrusion time or period of oscillation (s, ms or $\mu \mathrm{s}$ ) or temperature (K) 
$V \quad$ Volume $(\mathrm{mL})$ or velocity $(\mathrm{m} / \mathrm{s})$

$V_{c a v} \quad$ Volume of vapor cavities $\left(\mathrm{m}^{-3}\right)$

$Z \quad$ Acoustic impedance $\left(\mathrm{kg} \mathrm{m}^{-1} \mathrm{~s}^{-2}\right)$

\section{Roman (lowercase)}

$\begin{array}{ll}a & \text { Acceleration }\left(\mathrm{m} / \mathrm{s}^{2}\right) \\ c & \text { Sound speed }(\mathrm{m} / \mathrm{s}) \\ c_{K} & \text { Korteweg speed }(\mathrm{m} / \mathrm{s}) \\ d_{t i p, i} & \text { Diameter of the truncated tip of a straight cone }(\mathrm{mm}) \\ e & \text { Internal energy }(\mathrm{J}) \\ f & \text { Friction force }(\mathrm{N}) \\ h & \text { Wall thickness }(\mathrm{m} \text { or } \mathrm{mm}) \\ m & \text { Mass }(\text { kg or g) } \\ r & \text { Radial coordinate }(\mathrm{m} \text { or } \mathrm{mm}) \\ s & \text { Entropy }(\mathrm{J} / \mathrm{K}) \\ t & \text { Time }(\mathrm{s} \text { or } \mathrm{ms}) \\ u & \text { Velocity }(\mathrm{m} / \mathrm{s}) \\ x, y, z & \text { Spatial coordinates }(\mathrm{m} \text { or } \mathrm{mm})\end{array}$

\section{Subscripts}

0 Ambient or initial condition

$\theta \quad$ Hoop

$b \quad$ Syringe barrel or bubble

bf Buffer

c Cone

$d r \quad$ Driving rod

gap Air gap

$i \quad$ Inner or incident

$l \quad$ Liquid or syringe liquid content

$n \quad$ Needle

$o \quad$ Outer

ps Plunger-stopper

qs Quasi-static

$r \quad$ Radial, radial coordinate or reflected

$s \quad$ Syringe or solid

$t \quad$ Transmitted

$w \quad$ Wall

$z \quad$ Axial 
Chapter 1

\section{INTRODUCTION}

\subsection{Motivation}

Autoinjectors were first developed and introduced for military application in the 1970s for the delivery of emergency drugs in the field of combat (Lange and Thompson, 2013). Today, autoinjectors are also used by civilians for emergency purposes (e.g., epinephrine), but they are also extensively used for long-term treatments requiring the frequent subcutaneous injection of biopharmaceuticals (e.g., etanercept, adalimumab, and darbepoetin alfa) (Akers, 2010).

Even if autoinjectors are already ubiquitous in the pharmaceutical industry, the market for those devices is still continuously growing at a very fast pace (Akers, 2010; Thompson, 2006). According to some recent market studies (Future Market Insights, 2017) the Compound Annual Growth Rate (CAGR) of the autoinjector industry could exceed $15 \%$ for the next decade, and over US\$6.8G worth of autoinjectors could be annually manufactured by the year 2026. Autoinjector devices will therefore receive significant attention for years to come, and we can expect an increasing number of parenteral drugs to be administered using autoinjector devices.

The continuously increasing demand for autoinjectors is attributed to a variety of factors. From a technical standpoint, there is a clear trend toward biological drugs which cannot be administered orally, and the relative importance of those drugs is expected to grow further (Lange and Thompson, 2013; Thompson, 2006; Thompson and Lange, 2013). Oral delivery of biological drugs is often not possible because they tend to degrade substantially in the gastrointestinal tract. Furthermore, oral delivery generally results in the poor absorption of the molecules into the system. For those reasons, the preferred route of administration is injection (Singh, Singh, and Lillard, 2008). There are multiple types of injection, such as intravenous (IV), subcunatenous (SC), and intramuscular (IM). Only the subcutaneous delivery of injectables is considered herein.

From a human factor standpoint, there are several advantages to the use of subcunatenous autoinjectors when compared to more traditional means of parenteral

delivery, such as syringes and vials. A large number of human factor studies were conducted over the years to better understand how autoinjectors can be beneficial, 
and improve the quality and efficacy of treatments. Some of the most important and recurrent reasons cited to explain the undeniable success and the large popularity of autoinjectors are the compactness of the device, its ease of use, an improved dosage accuracy, and the increased treatment adherence. (Akers, 2010; Cadranel et al., 2007; French and Collins, 2010; Limmroth and Gerbershagen, 2014; Pozzilli et al., 2011; Schiff et al., 2014; Shire, Shahrokh, and Liu, 2004). Another advantage is that autoinjectors generally reduce the number of required visits to a health-care provider because they are typically approved for use at home (Turner and Balu-Iyer, 2018).

There are mechanical, physiological, and human factor constraints which need to be accounted for during the design and development of subcutaneous autoinjector devices. The volume of a subcutaneous injection is generally limited to approximately 1.0-1.5 mL because subcutaneous tissues can only absorb a finite quantity of liquid, and because larger injection volumes generally create more discomfort and pain (Adler, 2012; Arendt-Nielsen, Egekvist, and Bjerring, 2006; French and Collins, 2010; Jorgensen et al., 1996; Shire, Shahrokh, and Liu, 2004; Yadav, Shire, and Kalonia, 2010). ${ }^{1}$ For this reason, typical autoinjectors currently available on the market use a $1 \mathrm{~mL}$ syringe (French and Collins, 2010; Stout and Vilivalam, 2009). Furthermore, the maximum injection time a patient is willing to tolerate is typically around 10 to 15 seconds (French and Collins, 2010; Fry, 2014). Lastly, larger diameter needles are typically associated with more painful injections (Arendt-Nielsen, Egekvist, and Bjerring, 2006; Harvinder and Prausnitz, 2007), meaning that small diameter needles are preferred.

Monoclonal antibodies are one example of injectables which are often delivered subcutaneously. Monoclonal antibodies generally have a low potency, meaning that large doses have to be delivered (Warne, 2011; Yadav, Shire, and Kalonia, 2010). Figure 1.1 is a plot of the concentration of monoclonal antibodies approved by the FDA between 1996 and 2017. Figure 1.1 indicates there is a trend toward more concentrated drug solutions. The upper limit of drug concentrations appears to be increasing over time, and there is a substantial spread in the concentrations of newly developed drug solutions. The increase in drug concentration is partially attributed to the limited injection volume; because the injection volume is limited to $1-1.5 \mathrm{~mL}$, as explained above, controlling the drug dosage is primarily achieved through variations in the drug concentration.

\footnotetext{
${ }^{1}$ As highlighted by Heise et al. (2014), published data on the relation between pain and injection volume is conflicting. This is still an active field of research.
} 


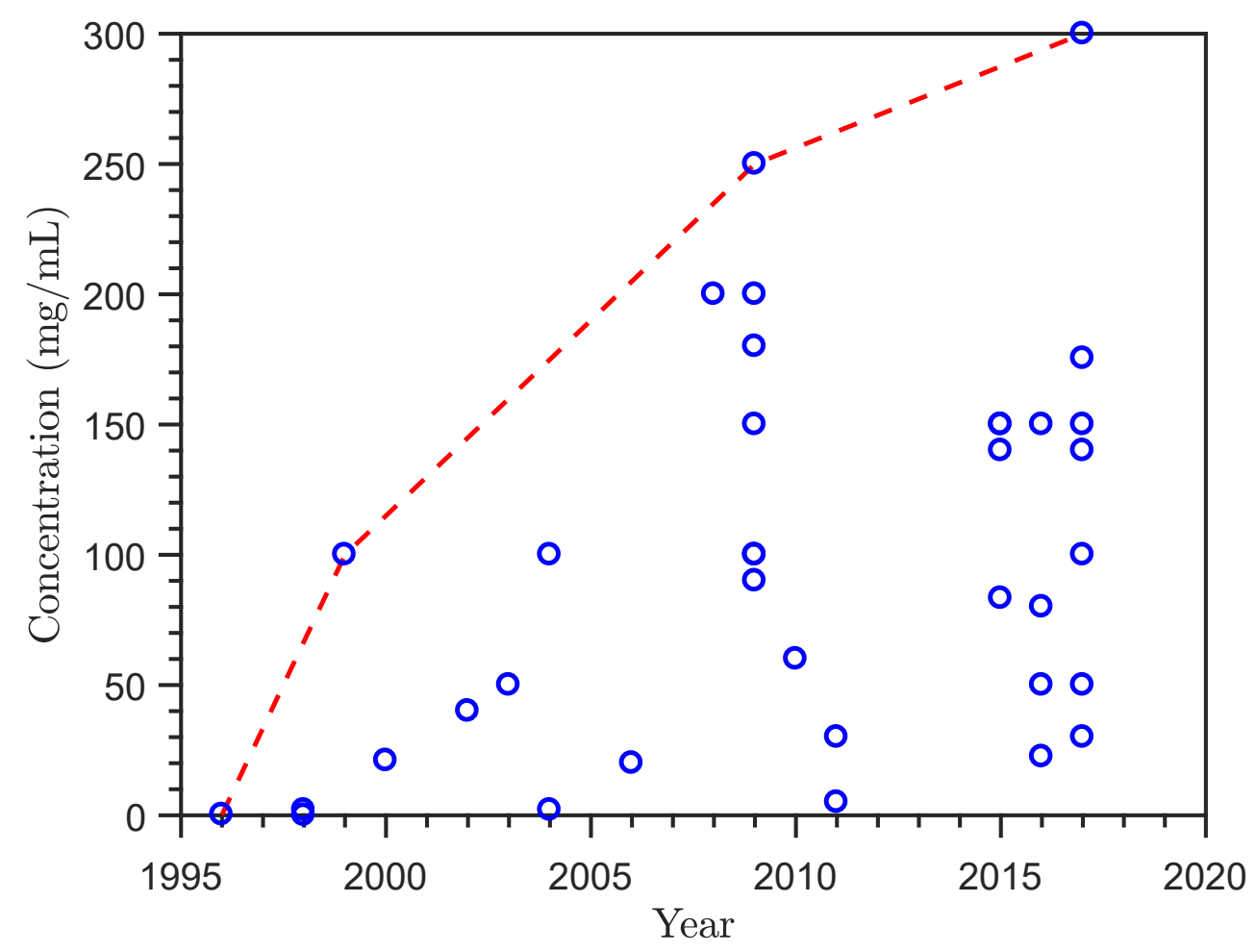

Figure 1.1: Concentration of FDA approved monoclonal antibodies over the years. Compilation prepared by Amgen (private communication). Data obtained from www . newdrugapprovals. com and www. fda.gov.

There is a direct correlation between the concentration and the viscosity of drug solutions. As expected, a more concentrated drug solution tends to be more viscous. The viscosity of drug solutions can increase much faster than linearly with concentration, as is the case for solutions containing monoclonal antibodies ( $\mathrm{Li}$ et al., 2014; Yadav, Shire, and Kalonia, 2010). By extension, Figure 1.1 indicates the upper bound on the viscosity of drug solutions is increasing over time.

The increasing spread in viscosity and concentration of parenteral drug solutions has a significant implication: it is becoming increasingly difficult to develop a "universal" or "platform" autoinjector device which can be used to effectively and safely inject all the parenteral drugs developed by one pharmaceutical corporation. Instead, multiple autoinjector devices need to be designed, each targeting a narrower range of drug viscosities. Furthermore, challenges arise in the development of reliable autoinjectors for the delivery of the more concentrated and viscous drug solutions. 


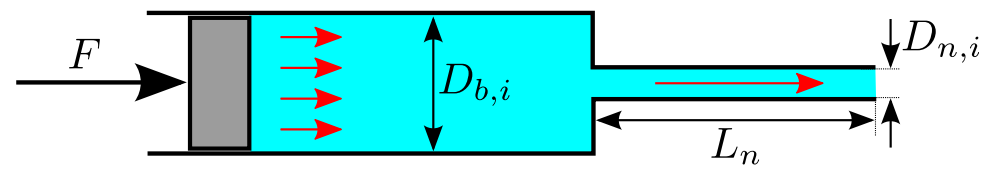

Figure 1.2: Flow in the barrel and the needle of a syringe resulting from a force $F$ applied on the plunger-stopper.

The force required to extrude a volume $V$ of drug solution within a time $T$ can be estimated by assuming a steady Poiseuille flow through the syringe needle (Kundu, Cohen, and Dowling, 2012). The required force $F$, which is also commonly called syringeability, is the force which must be applied on the plunger-stopper or piston in order to extrude the drug solution (see Figure 1.2):

$$
F=32 \mu L_{n}\left(\frac{D_{b, i}^{2}}{D_{n, i}^{4}}\right)\left(\frac{V}{T}\right),
$$

where $\mu$ is the viscosity of the drug solution, $D_{b, i}$ is the inner diameter of the syringe's barrel, and $D_{n, i}$ and $L_{n}$ are respectively the inner diameter and the length of the needle. This equation assumes no friction between the plunger-stopper and the syringe barrel, which is not true in general.

Equation 1.1 shows that for a given combination of syringe, needle, injection time and volume, the required force $F$ increases linearly with viscosity. This has significant implications for device design for high concentration drug formulations with high viscosities. The constraints previously mentioned on injection time, injection volume, and needle size mean that increasing the force $F$ is the only practical alternative to inject newly developed drug solutions with viscosities as large as 25-30 cP (French and Collins, 2010). However, applying an increasingly large force $F$ on the plunger-stopper to extrude the drug solution comes with the risk of damaging the autoinjector device due to the large pressures and stresses this produces in the syringe. The jerking motion of the autoinjector created by large spring forces could also affect negatively the user's experience.

Even if the specific design of each autoinjector device may differ, in most devices currently available on the market the mechanism is spring actuated (French and Collins, 2010; Thompson, 2006; Thompson and Lange, 2013). Activation of the device may result in mechanical impacts between the moving components of the autoinjector mechanism, and large accelerations/decelerations of the moving components may occur. This can be an issue when very viscous drug solutions are to be injected: the large syringeability of the drug means that stiff springs must be 
used to power the autoinjector. When this is the case, the large spring forces can result in impact velocities and accelerations/decelerations which are large enough to cause failure of the device (Fry, 2014; Stout and Vilivalam, 2009). Peak pressures in excess of 6.9 $\mathrm{MPa}$ are believed to exist within certain autoinjectors upon actuation (Schiff et al., 2014).

Although the potential issues associated with the delivery of very viscous drug solutions using autoinjectors have been acknowledged in the literature, no detailed study of the pressure and stress transients created upon actuation has been published. It is possible that some aspects of the pressure and stress transients in autoinjector devices have been studied within the pharmaceutical industry, but those studies have not been published to be part of public knowledge. Some authors previously acknowledged there is a lack of detailed understanding of how the key design parameters affect the device performances (Wilkins and Simpson, 2012), and further research and development is needed to extend the range of operation of autoinjectors with respect to viscosity (Adler, 2012). There is also a need for physical measurements on actual devices (Thompson and Lange, 2013).

More recently, some pharmaceutical companies started to experience reliability issues with autoinjector devices under development for the delivery of very viscous drug solutions. This is the case of Amgen, the sponsor of this research project, who adapted an autoinjector device they had successfully used in the past. Adaptation of the device was necessary to make the autoinjector usable for the delivery of a newer, more viscous parenteral drug solution. The primary modification consisted of stiffening the spring mounted inside the device to increase the force $F$ applied on the plunger-stopper. This modification resulted in a low but detectable failure rate of about $30 \mathrm{ppm}$ (parts per million) of the glass syringes during clinical trials (private communication). A $30 \mathrm{ppm}$ failure rate may appear as negligible, but this is far from being the case. A $30 \mathrm{ppm}$ failure rate is generally sufficiently large for regulators to become concerned.

Images of a broken syringe are shown in Figure 1.3. The images are from a report on the forensic analysis of the glass syringes which broke during clinical trials (American Glass Research, 2016). Forensic analysis of the broken syringes indicates that failure consistently originated on the inner surface of the conical section located near the tip of the syringe. The study concluded there were no visible flaws in the glass, and an excessive hoop stress associated with a very large liquid pressure inside the syringe was the root cause of the observed failures. Unfortunately, the limited 

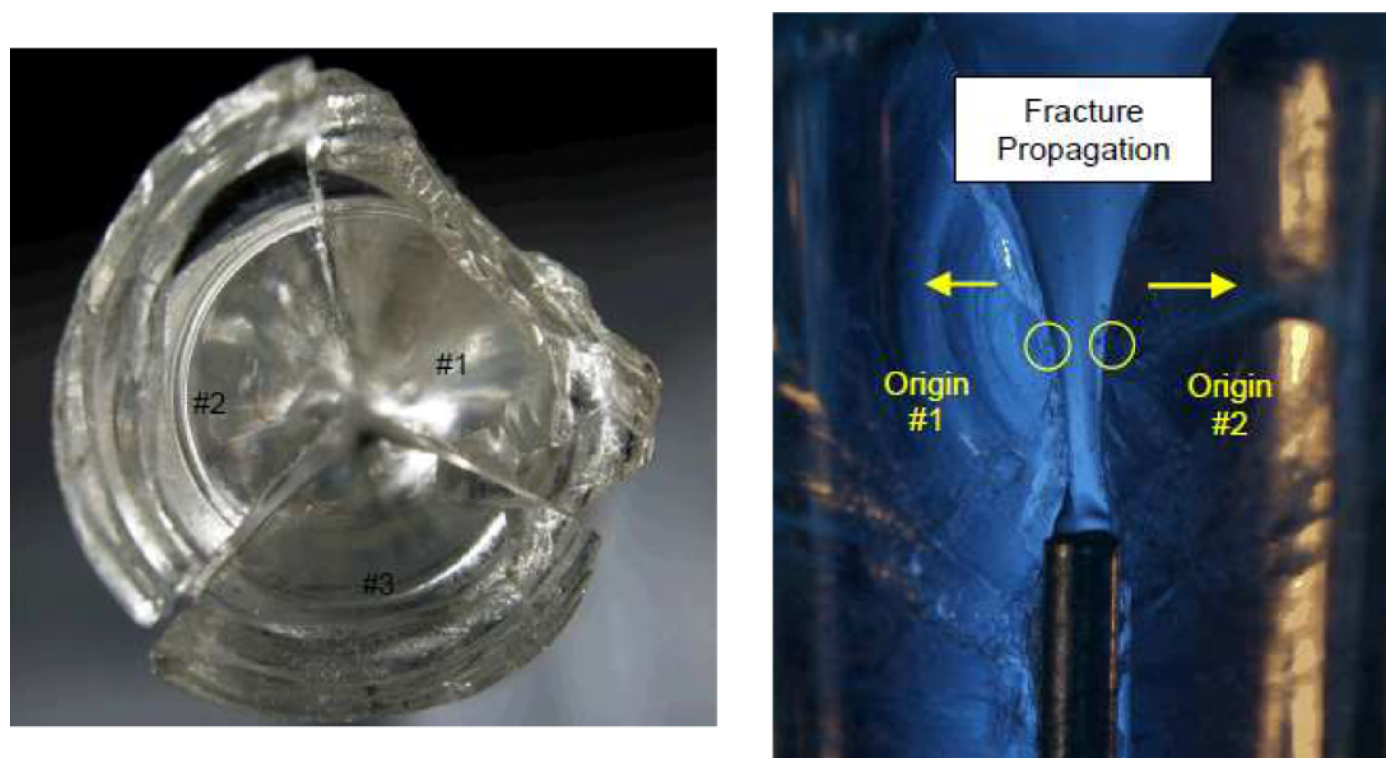

Figure 1.3: Example of a broken glass syringe. Failure originated on the inner surface of the cone area due to an excessive hoop stress. Reproduced from Veilleux, Jazayeri, et al. (2017) with permission.

knowledge and understanding of the mechanics of autoinjector devices could not explain the source of the large hoop stress and liquid pressure. ${ }^{2}$

\subsection{Objectives and Structure}

The unexplained cone failures and the lack of understanding of the dynamic events in an autoinjector has led to this investigation of the pressure and stress transients in the device during actuation. To the author's knowledge this is the first time an extensive study of the pressure and stress transients in an autoinjector device is performed and published. The main objectives of the present work can be summarized as follows:

1. to explain and characterize the dynamic events taking place in an autoinjector during actuation, and to investigate the resulting pressure and stress transients;

2. to develop novel experimental methods and numerical models which can be used to explain, understand, and quantify the pressure and stress transients during actuation;

3. to understand if and how the presence, location, and size of an air gap in the syringe can affect the pressure and stress transients;

\footnotetext{
${ }^{2}$ The reliability issue experienced by Amgen has been entirely resolved.
} 
4. to develop a better understanding of transient cavitation and bubble dynamics within the cone area of the syringe;

5. to investigate and understand the effect of relevant design parameters, such as syringe material and syringe lubrication, on the pressure and stress transients;

6. to determine more precisely the root cause of the large hoop stress in the cone which caused the failures observed by Amgen during clinical trials;

7. to propose, investigate and assess the efficacy of potential ways to mitigate the pressure and stress transients.

The overall goal of this research is to develop a better understanding of the failure modes of autoinjector devices in order to improve upon the current designs, and to make autoinjectors more robust and reliable. Ultimately, it is the author's hope that this deeper understanding of the mechanics of autoinjectors will make it possible to use those devices to safely inject the increasingly viscous drug solutions which are currently under development.

This thesis is structured as follows. The actuation sequence of typical springactuated autoinjector devices is introduced in Chapter 2. The various dynamic events which can occur and potentially create a pressure and stress transient are also introduced in an idealized, theoretical context. Simple models are developed and used to explain in simple terms the effect of the various transient events.

The novel experimental methods developed to measure the pressure and stress transients in a minimally modified autoinjector during actuation are described in Chapter 3. Typical results obtained using the in situ methods are shown and discussed. The results are used to further illustrate how the dynamic events which occur upon actuation can create deleterious pressure and stress transients in the device. Both glass and plastic syringes are investigated in this chapter to better understand the effect of syringe material and lubrication on the transient events.

Experiments performed on a scaled-up model autoinjector are introduced and discussed in Chapter 4. The first part of this chapter details the experiments performed with a scaled-up model which does not include the translational motion of the syringe. This setup is particularly useful to investigate the interaction between the plunger-stopper, the air gap (if any), and the liquid contained in the syringe. The effect of the cone area on the pressure transient is also investigated. The second 
part of this chapter is on the experiments performed with a scaled-up model which includes the translational motion of the syringe. This setup is particularly useful to investigate the effect of the large acceleration and deceleration of the syringe during actuation. In particular, this setup makes it possible to better understand the role of the large syringe acceleration along with the effect of the cone area on the cavitation events which can occur inside the syringe.

Numerical results obtained using the LS-DYNA finite-element analysis platform are shown and discussed in Chapter 5. LS-DYNA is used to study numerically the shock focusing effect resulting in the substantial amplification of pressure waves entering the cone area of the syringe. LS-DYNA is also used to predict the liquid pressure and the wall stresses during the different dynamic events occurring during autoinjector actuation. The numerical simulations are particularly useful to identify the root cause of the failures observed by Amgen during clinical trials.

Chapter 6 is about the shock-induced collapse of a bubble inside a cone. Numerical simulations are performed with two different in-house codes to determine if and how the collapse of a bubble inside the cone region of a syringe is different from the collapse of a bubble in the vicinity of a flat-wall or in a free field. The results are particularly useful to determine whether or not a cavitation event within the cone area of the syringe could cause failure of the glass.

Simple ideas and device modifications which can be used to mitigate the pressure and stress transients upon actuation are introduced in Chapter 7. The efficacy of some of the ideas proposed in this chapter is assessed experimentally.

The most important findings of this thesis are summarized in Chapter 8. The suggested future work is also discussed in this final chapter. 
Chapter 2

\section{DYNAMIC EVENTS IN AUTOINJECTOR DEVICES}

There is a large variety of autoinjector designs available on the market. This thesis focuses on spring-actuated autoinjector devices which are responsible for two main functions:

1. insertion of the needle into the patient;

2. delivery of the medicament to the patient.

The actuation sequence of a typical spring-actuated autoinjector device is discussed in the first section of this chapter. The second section discusses the dynamic events along with the resulting pressure and stress transients in the syringe caused by device actuation. The third and last section introduces the SureClick autoinjector, a device which is extensively discussed in this thesis.

The primary objective of this chapter is to introduce the origin and essence of each transient event in a generalized context, and to describe how each event could potentially cause failure of a syringe system. Discussions of the various scientific challenges one can expect to encounter in developing a full understanding of the transient events is presented along the way. The content of this chapter is paramount to the remainder of this thesis; subsequent chapters build upon the knowledge introduced herein.

\subsection{Actuation Sequence of a Typical Spring-Actuated Autoinjector}

Figure 2.1 is a simplified schematic of the internal components and actuation sequence of a typical spring-actuated autoinjector device. Note that only the key components relevant to the present discussion are represented for simplicity.

Only panel A of Figure 2.1 is considered at this time, where the device is in its initial state just before actuation. The device bottom features are in contact with the patient's skin. The key component is the syringe mounted inside the autoinjector. It is frequently referred to as a pre-filled syringe (PFS) due to the fact that it is often pre-filled with the medicament in the factory, prior to being mounted inside the autoinjector, and prior to being sold to a patient. The syringe can be fabricated with 

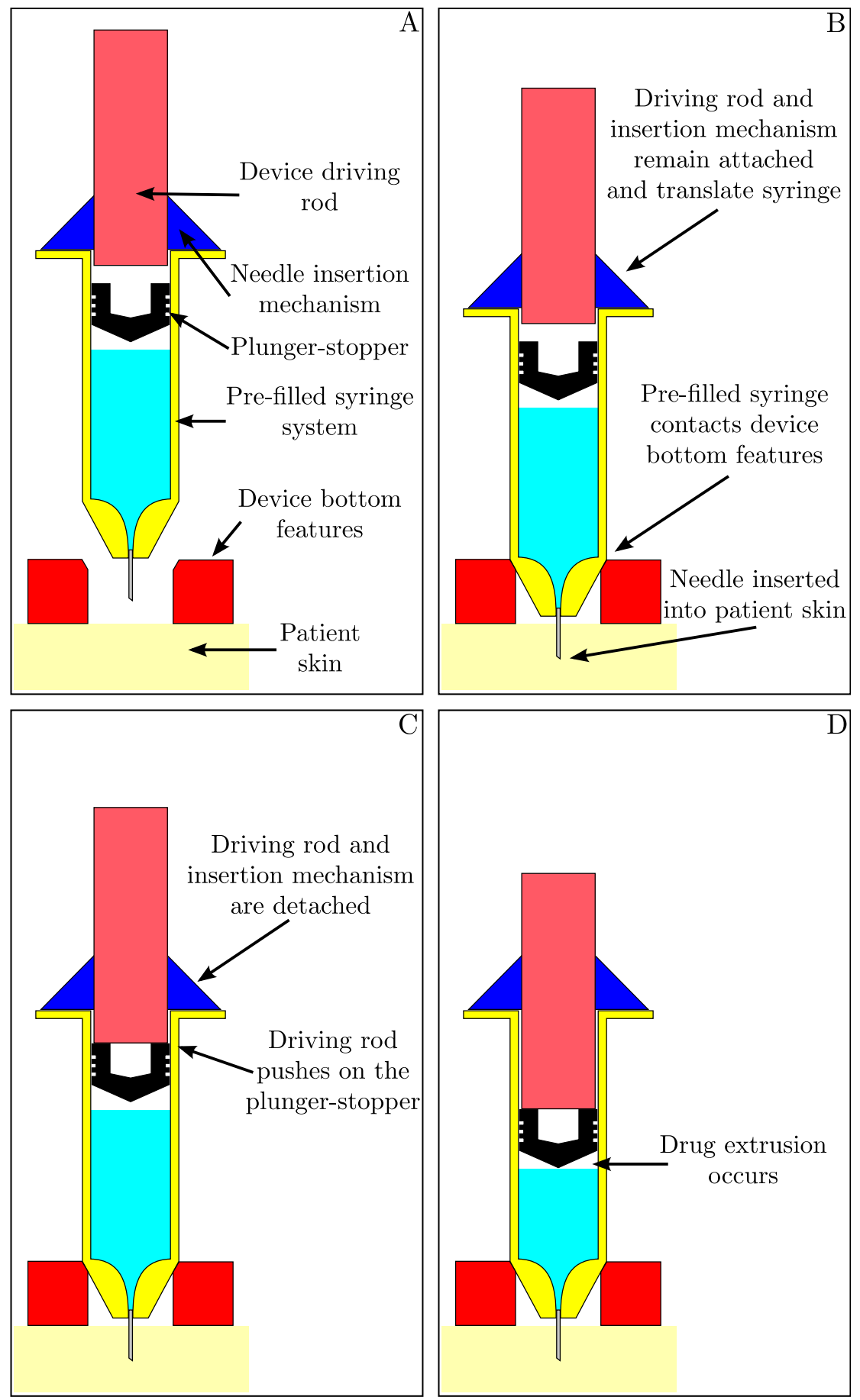

Figure 2.1: Simplified schematic of the actuation sequence of a typical springactuated autoinjector device. 


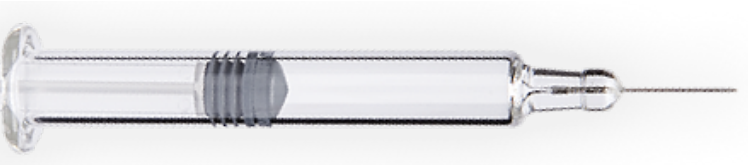

(a) BD HyPak - pre-filled syringe with a pre-attached needle.

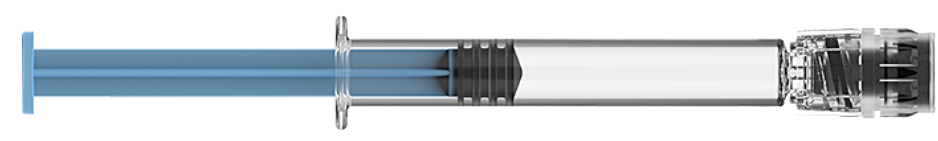

(b) BD HyLok - pre-filled syringe without a pre-attached needle.

Figure 2.2: Examples of pre-filled, glass syringes from BD. Reproduced from http://drugdeliverysystems.bd.com.

plastic, but most autoinjector devices currently available on the market make use of glass syringes. A review paper by Sacha, Rogers, and Miller (2015) provides more information on pre-filled syringes.

A needle, as shown in Figure 2.1, is attached to the syringe. The root of the needle is located at the bottom end or tip of the syringe. The needle can be pre-attached to the syringe in the factory, as shown in Figure 2.2a. In other cases the user needs to attach the needle to the syringe prior to using the autoinjector device, as shown in Figure 2.2b (Sacha, Rogers, and Miller, 2015).

The top end of the syringe typically has a flange. The internal and external geometry of the syringe can vary largely from one model to the other, especially in the vicinity of the tip. In some syringes the transition from the barrel to the needle is achieved with a smooth converging section, as depicted in Figure 2.1. In other cases the syringe ends with a flat wall which has a narrow channel at the center for the liquid to enter the needle, similar to the schematic shown in Figure 1.2. In all cases the tip of the syringe is sealed during storage. Adequate sealing is necessary to prevent the ingress of contaminants toward the inside of the syringe.

The syringe is sealed at its other end using a plunger-stopper. This is depicted in Figures 2.1 and 2.2. The plunger-stopper is typically fabricated with an elastomer, and it serves two important purposes (Sacha, Rogers, and Miller, 2015):

1. it seals the syringe content during storage to avoid drug contamination;

2. it serves as a piston used to pressurize the syringe and extrude the medicament into the patient. 


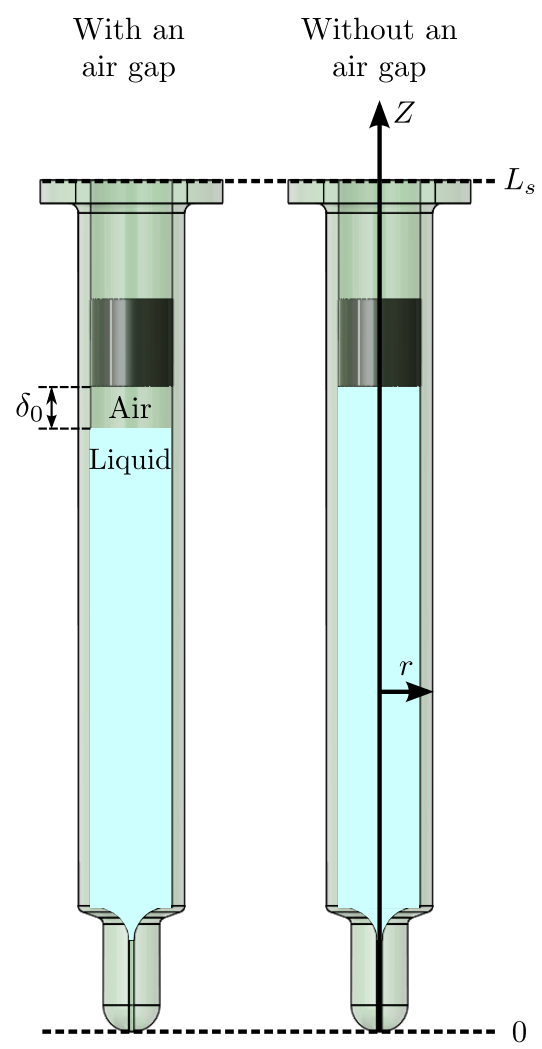

Figure 2.3: Schematic of a pre-filled syringe in a vertical, tip-down configuration with (left) and without (right) an air gap.

It is common for the syringe to not only contain the liquid drug solution, but to also contain an air gap or headspace (Sacha, Rogers, and Miller, 2015). This is shown in Figures 2.1 and 2.3 for the case of a syringe in a vertical, tip-down configuration. The presence of an air gap typically results from the syringe filling method. In some cases the presence of an air gap is a necessity, as is the case for drug solutions which contain suspensions. The air gap has important consequences on the transient events during device actuation. This is discussed further in the remainder of this thesis.

There are different means of supporting the syringe within the device. One approach is to support the syringe using its flange. Another approach is to support the syringe using the shoulder located in the vicinity of the tip. Sometimes a combination of both is used. In the configuration shown in Figure 2.1, the shoulder is used to decelerate the syringe, and the flange is used to accelerate the syringe by the means of a needle insertion mechanism.

The power pack is only partially shown in Figure 2.1. The power pack is responsible for actuation of the device. It consists primarily of an actuation button (not shown), 
a spring (not shown), and a driving rod. The spring is often contained within the driving rod, and the spring force is applied directly on the tip of the driving rod which, in turn, applies a force on the plunger-stopper of the syringe. The needle insertion mechanism is initially attached to the driving rod.

The actuation sequence shown in Figure 2.1 is now discussed using panels A through D. In panel A the device is in its initial state, just before actuation: the unshielded needle is attached to the syringe tip, the device bottom features are in contact with the patient's skin, and the pre-filled syringe is mounted inside the device and sealed by a plunger-stopper.

In panel B the device has been activated, and the spring-actuated driving rod is moving forward. The insertion mechanism attached to the driving rod is in contact with the flange of the syringe, and this accelerates the syringe assembly forward. The forward motion of the syringe inserts the needle into the patient. The syringe is decelerated to a complete stop once the needle has reached the adequate depth for injection. The deceleration of the syringe results from the contact of the syringe shoulder on a device bottom feature which is part of the enclosing shell (not shown). In panel $\mathrm{C}$ the driving rod is moving independently from the insertion mechanism, and it impacts on the plunger-stopper. The impact velocity and the force exerted by the spring-actuated driving rod on the plunger-stopper pressurizes the syringe, and this forces the medicament to be extruded through the needle and into the patient, as shown in panel $\mathrm{D}$.

\subsection{Transient Events During Autoinjector Actuation}

In the sequence of events described in the previous section, there are three dynamic events which are capable of producing deleterious pressure and stress transients during device actuation:

- event 1: the syringe acceleration;

- event 2: the syringe deceleration;

- event 3: the impact of the spring-actuated driving rod on the plunger-stopper.

The three dynamic events are introduced in an idealized context in this section. All three events are assumed to be entirely decoupled from one another. Other relevant simplifying assumptions are introduced to facilitate the discussion; those 
assumptions are introduced when appropriate. The objective of this section is to provide the reader with basic information about the physics at play during the transient events, but without getting into all the intricacies of the dynamic events. The next several chapters build upon the basic knowledge introduced herein.

\subsubsection{Acoustics and Wave Dynamics}

Before diving into the discussion of the dynamic events it is necessary to introduce or review a few concepts related to acoustics and wave dynamics. First and foremost, acoustics is extensively used in this thesis, starting with the present chapter. Using acoustics is justified because the magnitude of motion $u$ of the solid and fluid elements is small (i.e., less than $10 \mathrm{~m} / \mathrm{s}$ ) compared to the sound speed $c$ of pressure/stress waves in the materials: $u \ll c$. This is equivalent to saying that the peak magnitude of the pressure/stress waves created during the dynamic events is relatively weak. In the context of acoustics, a pressure/stress wave is weak if its magnitude $\Delta P$ is much smaller than $\rho c^{2}$, where $\rho$ and $c$ are respectively the density and sound speed of the medium into which the pressure/stress wave propagates.

For a liquid with properties similar to that of water, $\rho c^{2}$ is well over $2 \mathrm{GPa}$. The value of $\rho c^{2}$ is even larger for most solids. As it will later be seen, all pressure and stress waves considered in this thesis have peak magnitudes which remain below $100 \mathrm{MPa}$. Therefore, $\Delta P \ll \rho c^{2}$, and using acoustics is justified.

Acoustic theory and the acoustic version of the method-of-characteristics are thoroughly introduced in Appendix A. A cavitation model which can be used within the acoustic version of the method-of-characteristics is also described in Appendix A. The reader is urged to review this appendix if not entirely familiar with those concepts.

Concepts of wave dynamics are also extensively used in this thesis. The reflection of pressure and stress waves at an interface between two media is reviewed. For illustration it is assumed that a pressure or stress wave is traveling from left to right in medium 1. Medium 1 is in contact with medium 2, and the interface between the two media is straight and sharp. Both media have a different acoustic impedance $Z=\rho c$. Partial reflection and transmission of the pressure or stress wave occurs when it arrives at the interface. Figure 2.4 depicts this physical situation: the lefthand side is a schematic of the situation before reflection occurs, and the right-hand side depicts the situation after reflection has occurred. After reflection, a transmitted 


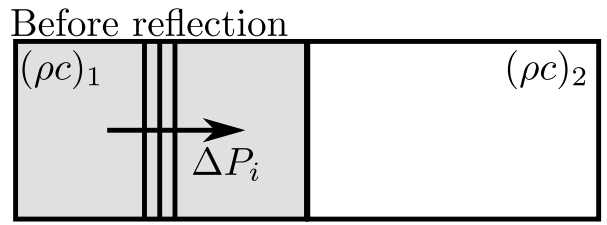

After reflection

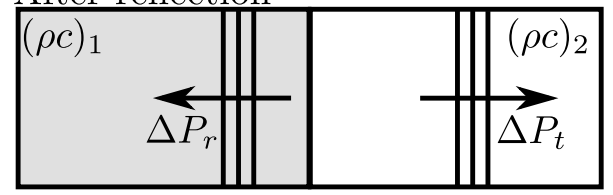

Figure 2.4: Reflection and transmission of pressure/stress waves at the interface between two media.

wave has formed and propagates from left to right in medium 2, and a reflected wave has formed and propagates from right to left in medium 1.

The magnitude of the transmitted $\left(\Delta P_{t}\right)$ and the reflected $\left(\Delta P_{r}\right)$ waves depends on the acoustic impedances $Z=\rho c$ of the two media. Enforcing compatibility at the interface (i.e., continuity of the pressure and velocity fields) yields (Pierce, 1989):

$$
\Delta P_{r}=\left[\frac{Z_{2}-Z_{1}}{Z_{2}+Z_{1}}\right] \Delta P_{i},
$$

and

$$
\Delta P_{t}=\Delta P_{r}+\Delta P_{i}
$$

The general result expressed using Equations 2.1 and 2.2 can be specialized to a few cases which are relevant to the syringe situation. First, the case of a wave reflecting on a rigid wall is considered. Medium 2 is the rigid wall, and therefore it has an infinite acoustic impedance. Taking the limit of Equation 2.1 for $Z_{2}$ which tends to infinity indicates that $\Delta P_{r}=\Delta P_{i}$ : the incident wave is entirely reflected back into medium 1. This important result can also be obtained using the method-ofcharacteristics introduced in Appendix A.

Second, the case of a wave reflecting on a free surface is considered. The pressure at a free surface is constant. Therefore, the magnitude of the transmitted wave in medium 2 is zero. Equation 2.2 indicates that $\Delta P_{r}=-\Delta P_{i}$ : the incident wave is reflected into medium 1, but the sign of the reflected wave is changed. In other words, a compression wave reflecting on a free surface becomes a tension or rarefaction wave, and vice versa. The same result is obtained when taking the limit of Equation 2.1 for $Z_{2}$ which tends to zero. This important result can also be obtained using the method-of-characteristics introduced in Appendix A.

Third, the case where medium 1 is water, and medium 2 is air is considered. In this case, $Z_{2} / Z_{1} \approx 0.0003$. Equations 2.1 and 2.2 indicate that $\Delta P_{r} \approx-\Delta P_{i}$, and 
Table 2.1: Reflection of pressure/stress waves at the interface between two media - magnitude of the reflected $\Delta P_{r}$ and transmitted waves $\Delta P_{t}$. The reflection and transmission coefficients are calculated using Equations 2.1 and 2.2 and the data shown in Table 2.2.

\begin{tabular}{lllcl}
\hline Case & Medium 1 & Medium 2 & $\Delta P_{r} / \Delta P_{i}$ & $\Delta P_{t} / \Delta P_{i}$ \\
\hline 1 & Arbitrary & Rigid wall & 1.00 & 2.00 \\
2 & Arbitrary & Vacuum & -1.00 & 0.00 \\
3 & Water & Air & -1.00 & 0.00 \\
4 & Water & Glass & 0.80 & 1.80 \\
5 & Water & Aluminum & 0.81 & 1.81 \\
6 & Water & Polycarbonate & 0.30 & 1.30 \\
\hline
\end{tabular}

Table 2.2: Density and sound speed of fluids and solids which are relevant to this thesis.

\begin{tabular}{llll}
\hline Medium & $\begin{array}{l}\text { Density } \\
\left(\mathrm{kg} / \mathrm{m}^{3}\right)\end{array}$ & $\begin{array}{l}\text { Sound speed } \\
(\mathrm{m} / \mathrm{s})\end{array}$ & Reference \\
\hline Air & 1.2 & 343 & Shepherd and Inaba (2010) \\
Water & 1000 & 1482 & Shepherd and Inaba (2010) \\
Glass (pyrex) & 2320 & 5640 & Lide (1990) \\
Aluminum & 2700 & 5100 & Shepherd and Inaba (2010) \\
Polycarbonate & 1200 & 2300 & Selfridge (1985) \\
\hline
\end{tabular}

$\Delta P_{t} \approx 0$. This result is practically the same as the one previously obtained for the reflection of a wave on a free surface. This is because the acoustic impedance of air is negligible compared to the acoustic impedance of water. The three results obtained above are summarized in Table 2.1, along with a few more cases relevant to this thesis. The density and sound speed of fluids and solids which are relevant to this thesis are summarized in Table 2.2.

\subsubsection{Event 1: Syringe Acceleration}

Actuation of the autoinjector results in the acceleration of the driving rod. The needle insertion mechanism is initially attached to the driving rod, and it is also accelerated forward. The needle insertion mechanism engages the flange and accelerates the syringe forward. The acceleration of the syringe can be substantial, $10^{3}$ to $10^{4} \mathrm{~m} / \mathrm{s}^{2}$ (see Chapter 3). 
The acceleration of the syringe occurs over a time scale of approximately $0.25-0.5 \mathrm{~ms}$ (see Chapter 3). Although the syringe is accelerated though the propagation of stress waves created at the contact between the insertion mechanism and the syringe wall, the syringe appears to be accelerated as a rigid body at this time scale. This is because the stress waves can complete several round trips (approximately 15 to 30) within the syringe wall in $0.25-0.5 \mathrm{~ms}$. This has the effect of accelerating forward and equilibrating the motion of the solid elements, making the rigid body approximation adequate.

Although the syringe is rapidly accelerated when the driving rod and the insertion mechanism are released, the liquid contained inside the syringe is not; the acceleration of the liquid is lagging behind the acceleration of the syringe. The explanation for the delayed acceleration is provided later in this section and supported using results obtained with the method-of-characteristics. The delay in accelerating the liquid contained inside the syringe means that the liquid can't keep up with the motion of the syringe, and a large cavity forms at the bottom of the container; the liquid begins to cavitate.

The mechanism for creating cavitation is schematically depicted using Figure 2.5 where a liquid-filled container with a flat bottom wall is used for illustration. The wave mechanics in the wall of the container is neglected; the container is assumed to be a rigid body. In frame A a large force is applied on the vertical wall of the container. The large force rapidly accelerates the container, but not the liquid. This is because the liquid, contrary to the solid wall, can't sustain tensile forces. ${ }^{1}$ There is a loss of contact between the container and the liquid, and a cavity forms. The pressure inside the cavity forming at the bottom wall is sub-atmospheric, and tension waves are created in the liquid. The tension waves slowly but progressively accelerate the liquid. Later the growth of the cavity stops, the cavity then starts diminishing in size, and finally collapses with great intensity. The collapse of the cavity produces a relatively large and sharp pressure increase as the liquid impacts or crashes into the bottom wall of the container.

A qualitative example of the liquid pressure at the bottom of the container is shown in Figure 2.6. The large and momentarily increase in liquid pressure at collapse (time "D") creates stresses and strains in the wall of the container. One common example of this type of pressure and stress transient is when a beer bottle is broken upon its abrupt acceleration (Daily et al., 2012). The abrupt acceleration can be

\footnotetext{
${ }^{1}$ This is discussed in greater detail later in this chapter.
} 

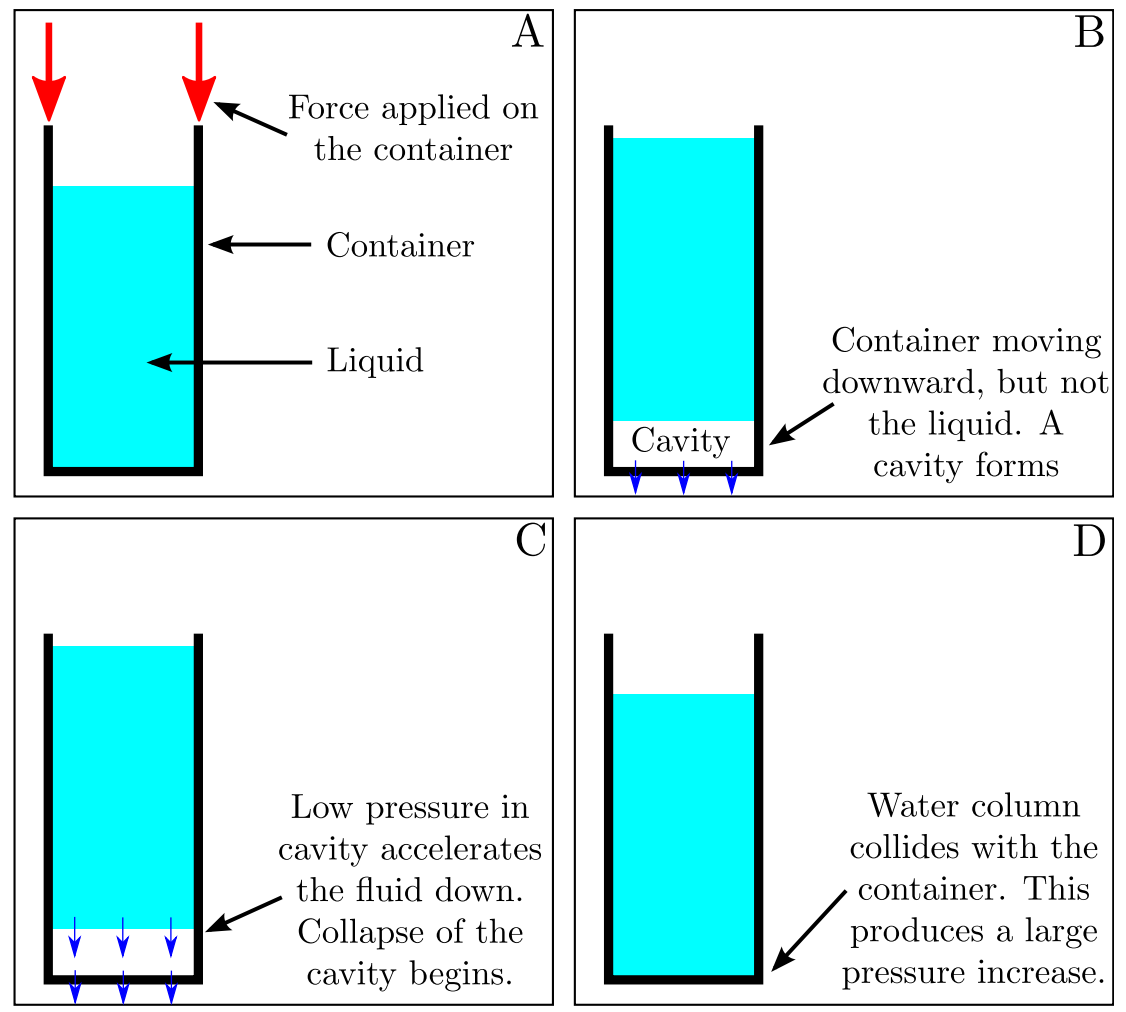

Figure 2.5: Schematic of the growth and collapse of a cavity shortly after the abrupt acceleration of a liquid-filled syringe.

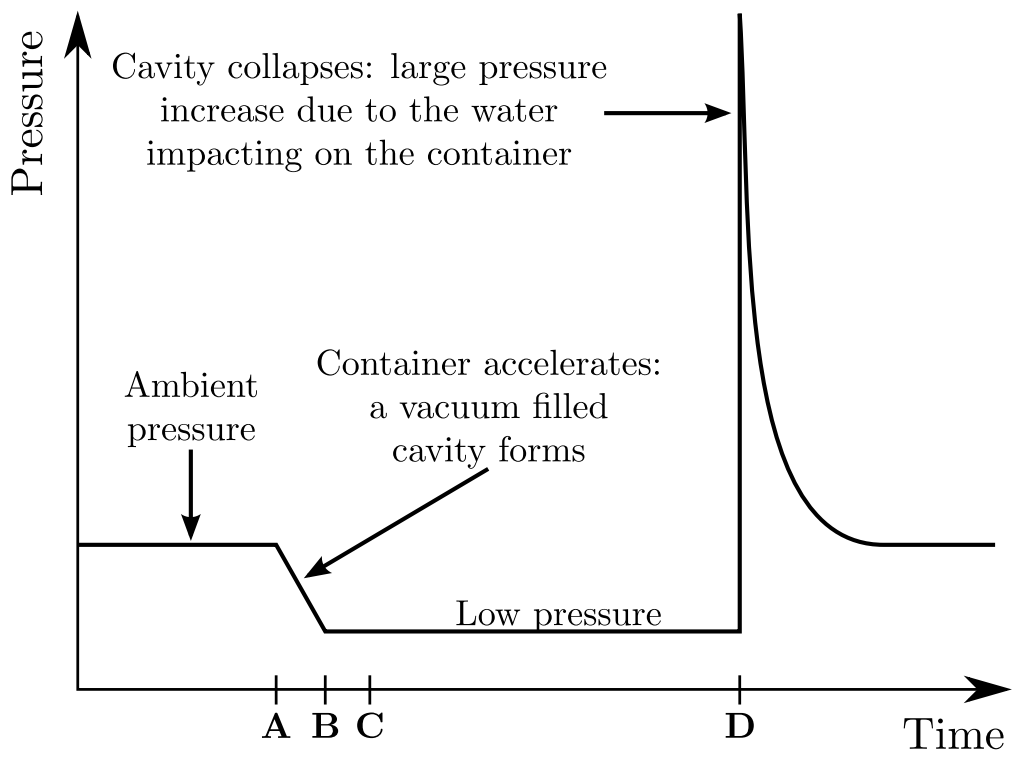

Figure 2.6: Pressure at the bottom wall of a liquid-filled syringe which is impulsively accelerated. The events labeled from A through D correspond to the events identified in Figure 2.5. 
generated by holding the open bottle with one hand, and impacting on the lip of the bottle with the other hand. This practical example indicates the pressure and stress transients generated by the cavitation event created by the rapid acceleration of a container, even when using bare hands, is large enough to break glass.

The above explanation of the pressure and stress transient during the first event is rather intuitive. A more thorough explanation where the wave dynamics within the liquid is taken into account in a more consistent manner is presented below.

The rapid acceleration of the syringe is partially analogous to the well known gas dynamics problem of a piston withdrawal (Liepmann and Roshko, 2002), where the bottom wall of the syringe is analog to the piston. The rapid withdrawal of a piston from a tube filled with gas creates an expansion fan. In the present situation the tension waves originate at the bottom of the syringe in the vicinity of the cone area. This situation is represented in Figure 2.7 using a space-time diagram. There are several assumptions behind this space-time diagram to simplify the discussion:

1. the interaction between the air gap and the plunger-stopper is neglected (i.e., the top end of the liquid column is assumed to be a free end where a constant pressure is maintained);

2. the bottom wall of the syringe is assumed to be flat and rigid (i.e., this is assumed to be a one dimensional problem);

3. the wave mechanics within the syringe wall is neglected (this assumption is justified by the much larger sound speed in the solid wall than in the liquid);

4. the liquid inside the syringe is an ideal fluid which can withstand tension without undergoing cavitation;

5. the acceleration of the syringe is assumed to be impulsive.

We now return to the space-time diagram shown in Figure 2.7. The bottom wall of the syringe is impulsively accelerated to a velocity $u$. Note that $u<0$, meaning the bottom wall is accelerated toward negative $z$. This results in the production of a tension wave which propagates upward in the syringe liquid content. The magnitude of the tension wave can be estimated using acoustic theory (see Appendix A):

$$
\Delta P=\rho_{l} c_{l} u,
$$




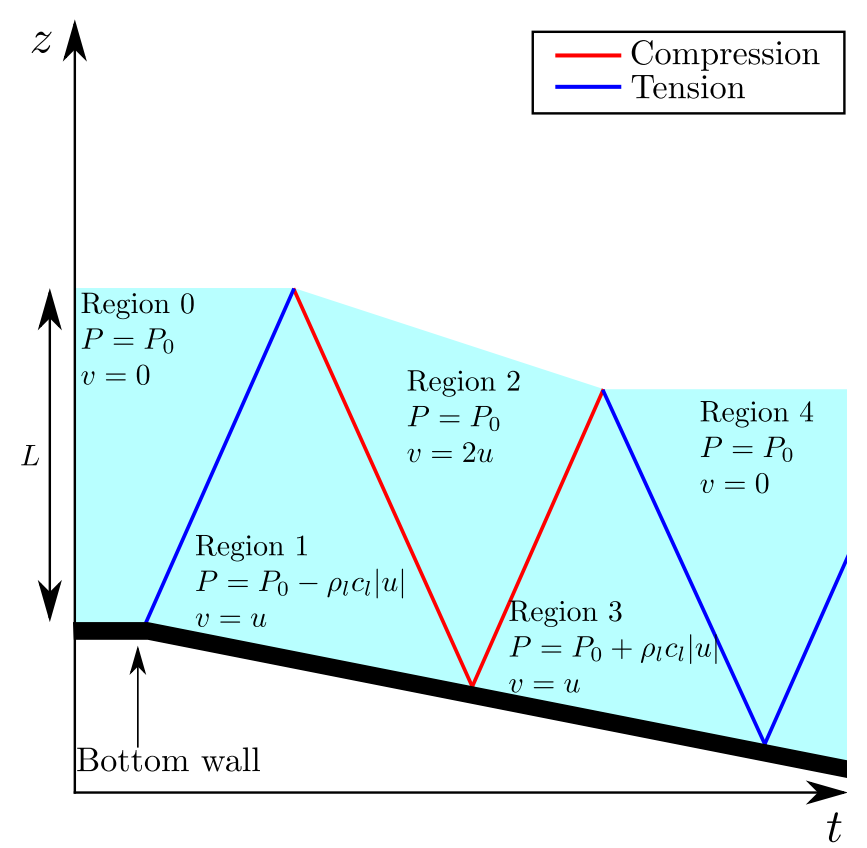

Figure 2.7: Space-time diagram of the pressure transient created by the impulsive acceleration of the syringe. The syringe contains an ideal fluid which can sustain tension.

where $\Delta P$ is the change in pressure, $\rho_{l}$ is the density of the liquid, and $c_{l}$ is the sound speed in the liquid. $\Delta P$ is negative, which indicates the pressure wave created upon the acceleration of the syringe is a tension wave, not a compression wave.

The liquid pressure is initially $P_{0}$ before the transient event begins (region 0 ). The tension wave, upon its arrival at a given location, reduces the liquid pressure. The pressure after the passage of the tension wave (region 1) is $P=P_{0}+\Delta P$ or $P=P_{0}-\rho_{l} c_{l}|u|$. The tension wave also accelerates the fluid downward, such that the velocity of the liquid after the passage of the tension wave (region 1) is the same as the velocity of the bottom wall.

The tension wave eventually arrives at the free surface located at the top end of the syringe. There it reflects as a compression wave, and travels down into the liquid. The wave subsequently arrives at the bottom wall of the syringe. Assuming the bottom wall is rigid, the compression wave is entirely reflected as a compression wave. If dissipation is absent, those reverberations of the wave within the liquid continue until an external force is applied and the velocity of the syringe is modified. Each passage of a compression or tension wave is associated with a pressure and velocity change. Different regions have been identified in Figure 2.7. The pressure and velocity for each region is indicated. 


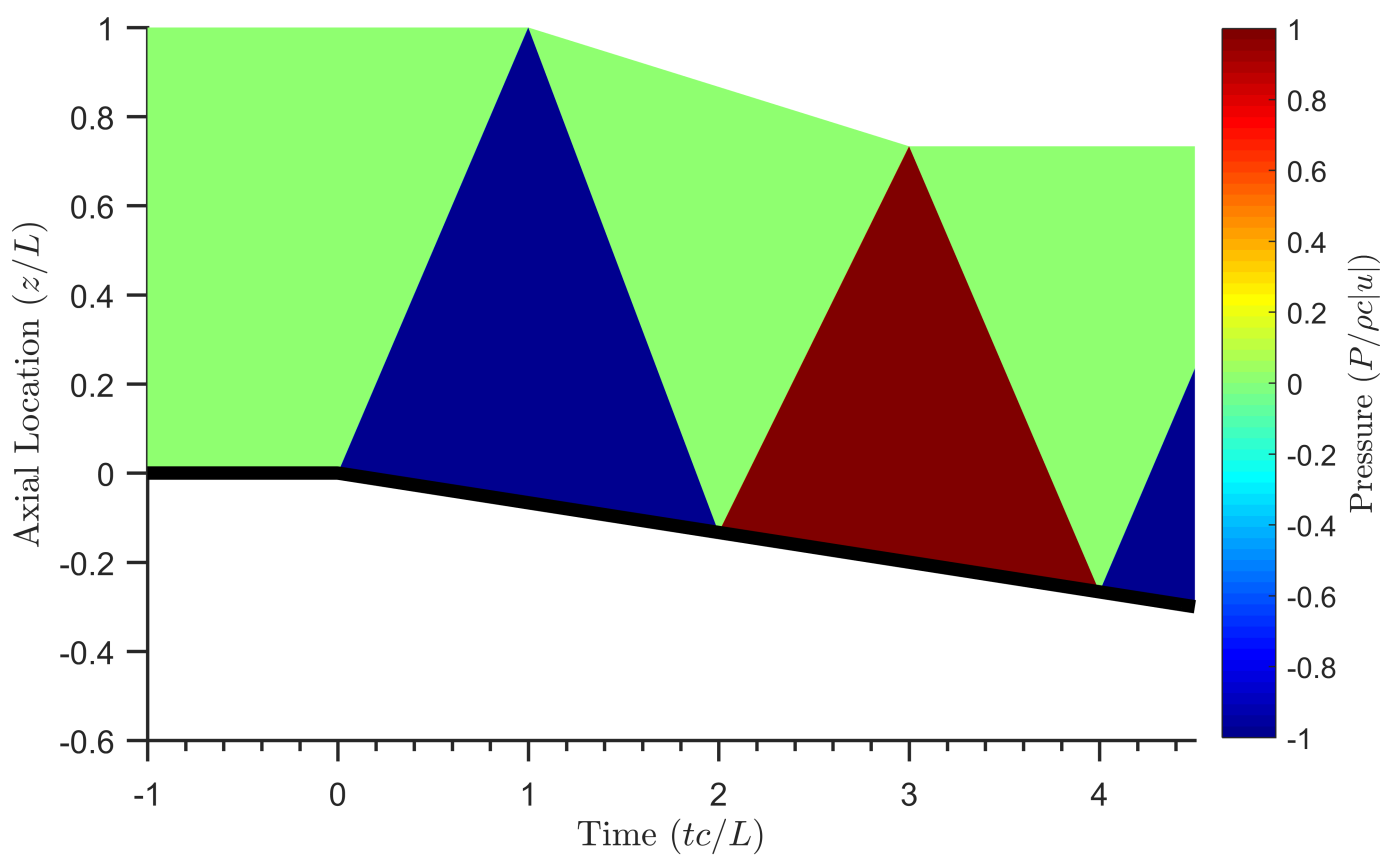

Figure 2.8: Space-time diagram of the pressure transient created by the impulsive acceleration of the syringe. The syringe contains an ideal fluid which can sustain tension. Results obtained with the method-of-characteristics. All results are nondimensional.

The method-of-characteristics is used to predict the wave dynamics for this idealized scenario. The numerical methods described in Appendix A were implemented in Matlab to solve this problem. Figure 2.8 is a space-time diagram of the computed liquid pressure; this is equivalent to Figure 2.7. The results shown are non-dimensional and were obtained with $u / c=-3.33 \times 10^{-3}$, a value which is representative of the magnitude of motion in a SureClick autoinjector device. The reverberations of the wave generated upon the impulsive acceleration of the syringe are again visible. The change of sign of the pressure wave at the free end is also noticeable, resulting in the non-dimensional pressure oscillating between -1 and 1 .

The ideal fluid assumption is now relaxed. From now on the fluid can't sustain tension, and cavitation is possible. The cavitation inception pressure corresponds to $P_{\text {vap. }}$. It has been shown that real liquids can sometimes sustain a significant amount of tension before they begin to cavitate (Franc and Michel, 2005; Trevana, 1987). In other words, bubbles do not necessarily start forming and growing in the liquid as soon as the pressure is reduced below the vapor pressure. A static delay can be caused by the cohesion between the molecules. There can also be a dynamic delay 
if the pressure is reduced much faster than the liquid can evaporate because phase change is not an instantaneous process.

In most engineering applications where the time scales of interest are sufficiently long (milliseconds to seconds) it is typical to assume that cavitation begins as soon as the pressure is reduced at or below the vapor pressure of the liquid. This is the typical approach used, for example, when studying the transient events in piping systems (Wylie and Streeter, 1993). This is because most liquids used in engineering applications contain a sufficiently large number of nuclei for the dynamic and static delays to be much shorter than the characteristic time scale of interest. This is expected to be the case in a syringe, particularly because it is generally possible to observe with the naked eye air bubbles within the liquid drug solution prior to actuation of the autoinjector.

Cavitation occurs if the change in velocity of the syringe due to the impulsive acceleration is large enough, causing the pressure to drop below the vapor pressure. Equation 2.3 is used to estimate how substantial the change in velocity of the syringe needs to be for cavitation to occur for a water column which is accelerated downward $(u<0)$, as previously assumed:

$$
u<\frac{P_{v a p}-P_{0}}{\rho_{l} c_{l}} .
$$

In the syringe of a SureClick, the pressure $P_{0}$ before actuation is approximately ambient pressure, or $101 \mathrm{kPa}$. The vapor pressure of water $P_{\text {vap }}$ is approximately $2.3 \mathrm{kPa}^{2}$ With those values of $P_{0}$ and $P_{\text {vap }}$, one obtains that $u<-0.067 \mathrm{~m} / \mathrm{s}$ is expected to cause cavitation in liquid water. This result indicates there is no need for the change in velocity created by the impulsive acceleration of the syringe to be substantial for cavitation to occur, but only a modest downward motion will suffice. This explains why bare hands can be used to cause cavitation in a beer bottle, resulting in failure of the glass container (Daily et al., 2012).

A space-time diagram which illustrates this physical situation is shown in Figure 2.9. Upon the rapid acceleration of the syringe, a relatively weak tension wave forms in the liquid. No matter what the magnitude of $u$ is, the magnitude of the tension wave is $\Delta P=\left(P_{\text {vap }}-P_{0}\right)$. This is because the pressure at the bottom of the container inside the cavity which forms is maintained at $P_{v a p}$, while the pressure at the free-

\footnotetext{
${ }^{2}$ The vapor pressure of the drug solution is not known, but we anticipate from Raoult's law that the vapor pressure of the solution will be slightly lower than that of the solvent (water) for a dilute solution.
} 


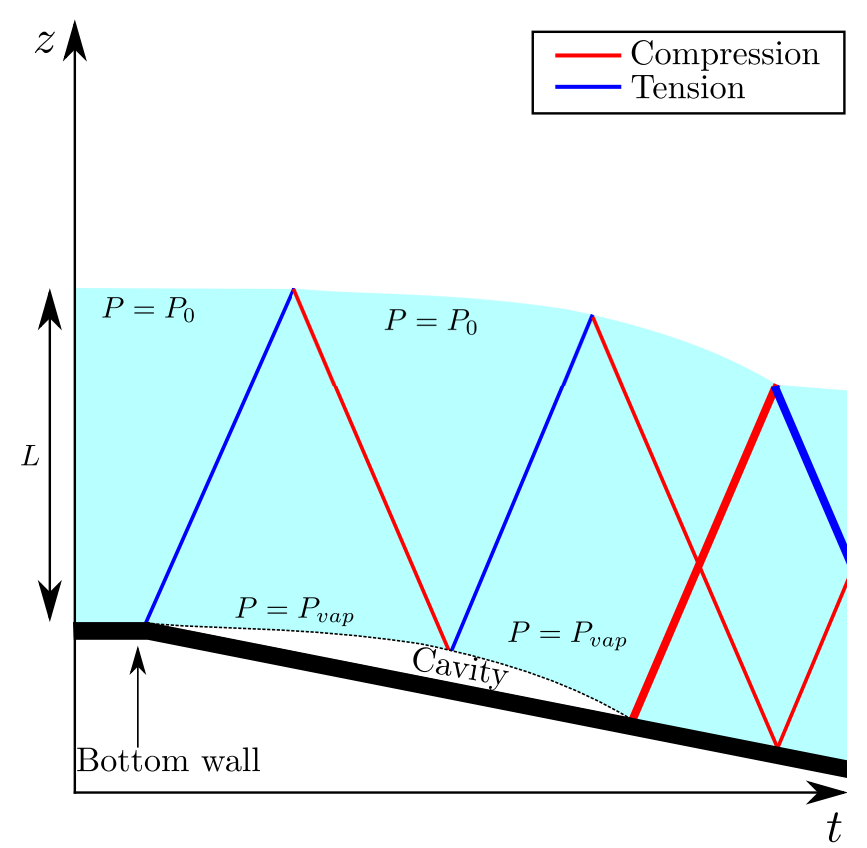

Figure 2.9: Space-time diagram of the pressure transient created by the impulsive acceleration of the syringe. The syringe contains a real fluid which does not sustain tension.

end is maintained at ambient pressure $P_{0}$. This is significantly different from the situation where cavitation is not allowed to occur (see Figures 2.7 and 2.8).

The change in velocity of the liquid due to the passage of the initial tension wave is modest, approximately $0.067 \mathrm{~m} / \mathrm{s}$. This is because the pressure change due to the tension wave is modest. The minimal velocity change each passage of a wave can incur to the fluid explains why the liquid can't keep up with the motion of the container, and there is a loss of contact: a cavity forms. Note that during the existence of the cavity the pressure wave has sufficient time to complete multiple round trips throughout the syringe, but this could not be shown in the simplified schematic. Furthermore, for reasons explained before the reflection of the pressure wave at the free end results in a sign change: the tension wave becomes a compression wave. Also, the pressure waves, upon reaching the bottom end of the container, do not reflect on the rigid wall of the container, but instead reflect on the boundary of the cavity. The pressure inside the cavity is approximately constant and equal to $P_{v a p}$. Therefore, the reflection of the pressure waves at the bottom of the container also results in a sign change: the compression wave becomes a tension wave. 


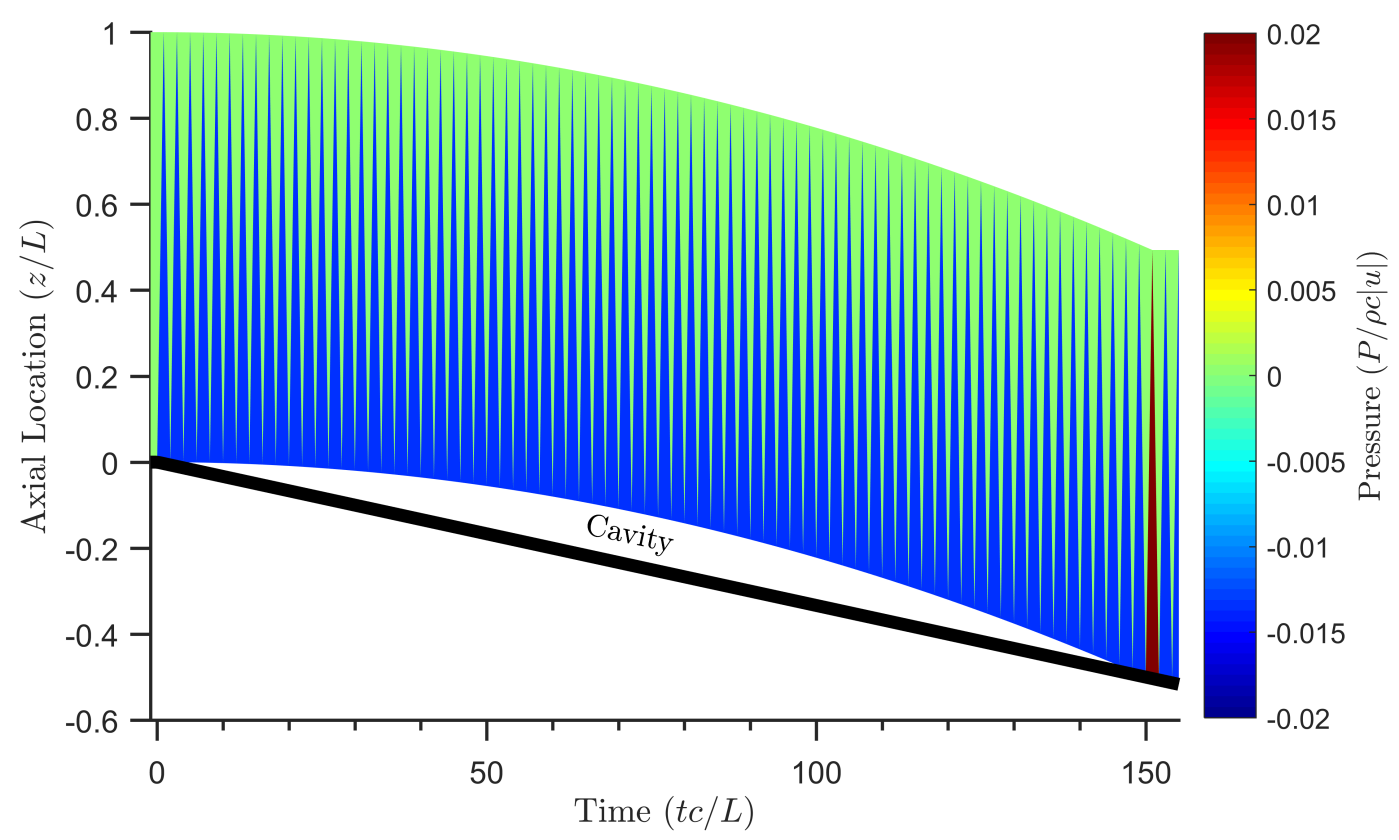

Figure 2.10: Space-time diagram of the pressure transient generated upon the impulsive acceleration of the syringe. The syringe contains a real fluid which does not sustain tension. Results obtained with the method-of-characteristics in combination with a cavitation model. All results are non-dimensional.

After multiple round trips of the pressure wave within the liquid - approximately $|u|\left(\rho_{l} c_{l}\right) /\left(P_{0}-P_{\text {vap }}\right)$ of them ${ }^{3}$ - the liquid has been accelerated forward sufficiently that the cavity starts diminishing in size. After several more round trips of the pressure wave - approximately $|u|\left(\rho_{l} c_{l}\right) /\left(P_{0}-P_{\text {vap }}\right)$ more of them - the cavity collapses. The collapse of the cavity results in the production of a relatively strong compression wave. This is the same pressure increase as the one shown at time "D" in Figure 2.6; this is investigated further in Chapters 3, 4, and 6.

The method-of-characteristics along with a cavitation model is used to predict the wave mechanics for this more realistic situation. The methods discussed in Appendix A were implemented in Matlab to solve this problem, and the results are shown in Figure 2.10. The results shown are for $P_{\text {vap }}=0$ and $u / c=-6.66 \times 10^{-2}$. This value of $u / c$ is not representative of the magnitude of motion in a SureClick autoinjector; it has been exaggerated to make the growth and collapse of the cavity more noticeable. Note that the color scale of the non-dimensional pressure is different from the color scale used in Figure 2.8.

\footnotetext{
${ }^{3}$ In a SureClick device, $|u|$ is approximately $8 \mathrm{~m} / \mathrm{s}$, and the number of round trips is approximately equal to 116
} 
Multiple reverberations of the weak pressure wave within the liquid are observed, along with the slow but continuous acceleration of the liquid content. The cavity forming at the bottom of the container is also visible. A strong compression wave is created upon collapse of the cavity. The color scale is saturated: the non-dimensional pressure due to the compression wave forming upon collapse is of order 1. This result should however be interpreted with great care due to the limitations of the cavitation model used (see Appendix A). In particular, the cavitation model assumes each cavitation bubble occupies the entire cross section of the syringe. In reality, a collection of smaller, almost spherical bubbles with a radius less than the radius of the syringe form to later collapse. The method-of-characteristics coupled with a cavitation model is an adequate tool to predict the overall, average behavior, but it is inadequate to predict the pressures created by the rapid collapse of cavitation bubbles. The collapse of the cavitation bubble along with the peak pressure created by this event is further investigated in Chapter 6.

To summarize, the rapid acceleration of the syringe can create a significant pressure transient. If the change in velocity of a downward accelerated syringe (i.e., $u<0$ ) is small $\left(u>\left(P_{\text {vap }}-P_{0}\right) /\left(\rho_{l} c_{l}\right)\right)$, cavitation does not occur. Conversely, cavitation occurs if the change in velocity of the syringe is large $\left(u<\left(P_{v a p}-P_{0}\right) /\left(\rho_{l} c_{l}\right)\right)$. When cavitation is not present, the maximum liquid pressure is created shortly after acceleration; the peak pressure occurs within one round trip of the pressure wave in the syringe. When cavitation is present, the maximum liquid pressure is delayed and occurs only when the cavity collapses. The rapid collapse of such cavities can create stresses which are sufficiently large to break glass (Daily et al., 2012).

\subsubsection{Event 2: Syringe Deceleration}

Event 1 accelerates the syringe forward to introduce the needle into the patient. The syringe needs to be decelerated upon reaching the right penetration depth for the needle (see frame B in Figure 2.1). The magnitude of syringe deceleration, or event 2 , can be substantial, as large as $10^{3}$ to $10^{4} \mathrm{~m} / \mathrm{s}^{2}$ (see Chapter 3). Large decelerations of a liquid-filled container can create a significant pressure and stress transient. The transient resulting from the deceleration is, however, fundamentally different from the transient resulting from the large acceleration of the syringe (event 1) described above.

The origin of the transient created by the abrupt deceleration of the syringe and the liquid it contains is twofold: 
- a pressure wave is created in the liquid, and this pressure wave creates stresses and strains in the wall of the syringe;

- a stress wave is created directly in the wall of the syringe.

The mechanism for creating the pressure and stress transient is illustrated in Figure 2.11. Panels A and B show the syringe assembly (i.e., the syringe, the needle, the plunger-stopper, and the liquid drug) traveling downward. All components of the assembly are traveling together at the same velocity $u$, and the liquid inside the syringe is at pressure $P_{0}$. In panel $\mathrm{C}$, the needle has reached the right penetration depth and the syringe is rapidly decelerated. The deceleration results from a mechanical contact or impact between the syringe assembly and a feature which is part of the autoinjector's shell. For simplicity, the syringe deceleration is assumed to be impulsive. This is a restrictive assumption which is relaxed later.

Immediately after the impulsive deceleration, the liquid and the solid elements adjacent to the point of contact come to a complete stop. The fluid and the solid elements far above the point of contact, however, are still traveling downward with velocity $u$ since they do not know yet about the deceleration which occurred at the point of contact. This is because the information about the deceleration originating at the point of contact propagates at a finite speed; this information is propagated through acoustic waves traveling within the solid and the liquid at the respective sound speeds $c .^{4}$

Upon deceleration of the syringe, an acoustic compressive pressure wave forms in the liquid and propagates away from the point of contact. For historical reasons, this type of pressure transient which arises from the abrupt deceleration of a liquid column is most often called a water hammer (Bergant, Simpson, and Tijsseling, 2006; Watters, 1984; Wiggert and Tijsseling, 2001; Wylie and Streeter, 1993). Water hammers are a common issue in piping systems. They occur when the flow of a liquid inside a pipe is brought to a stop by the rapid closure of a valve. Water hammer events can sometimes create a significant pressure surge which can cause damage to piping and support systems in both residential plumbing and industrial facilities.

The water hammer effect in the syringe is illustrated using the space-time diagram shown in Figure 2.12 for a case without cavitation (cavitation does not occur if

\footnotetext{
${ }^{4}$ The fluid-structure interaction between the liquid and the solid can alter the speed at which weak disturbances propagate in both the liquid and the solid (Shepherd and Inaba, 2010).
} 


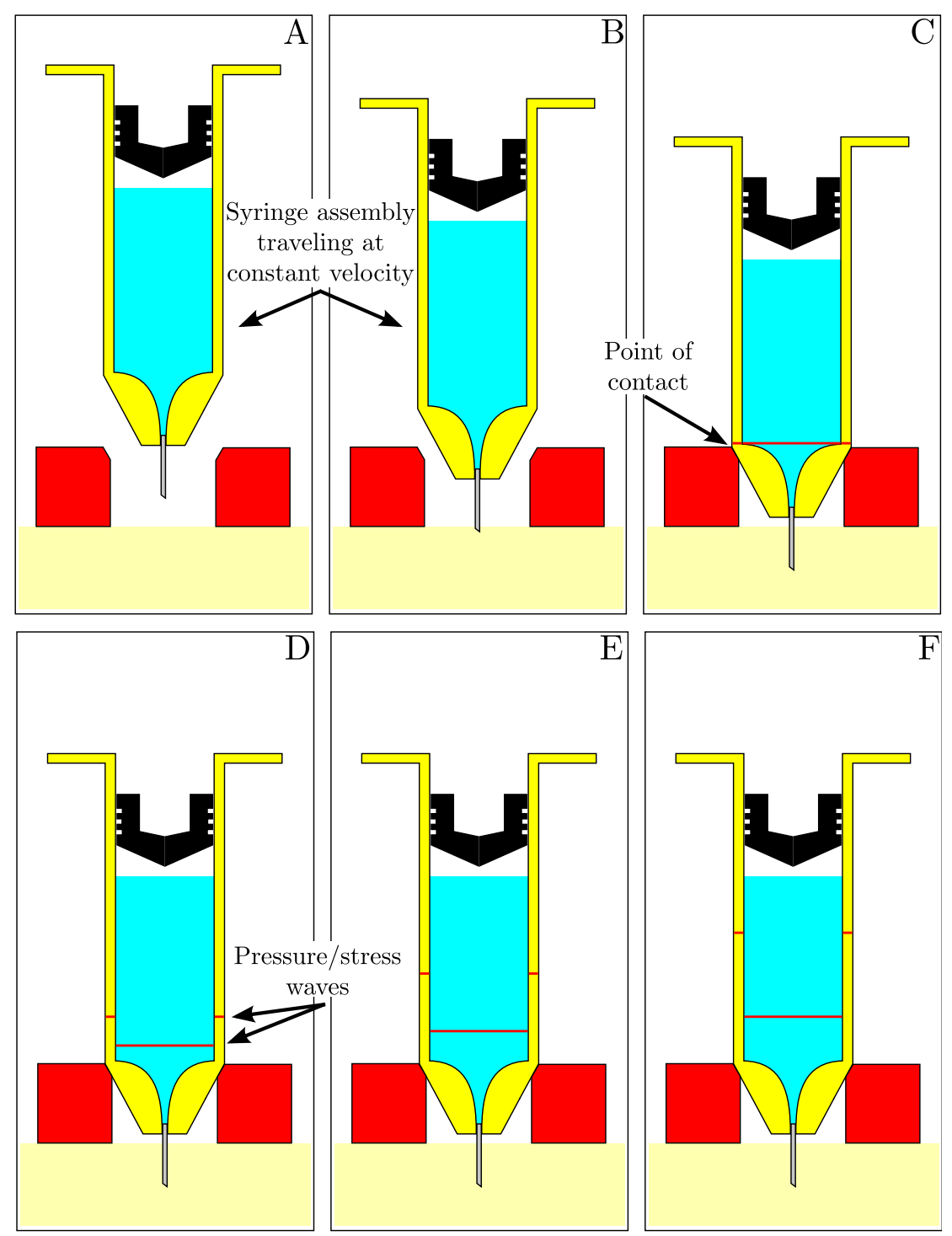

Figure 2.11: Simplified schematic showing the rapid deceleration of the syringe when it reaches its travel limit. The propagation of the pressure and stress waves created by the rapid deceleration is illustrated.

$u>\left(P_{\text {vap }}-P_{0}\right) /\left(\rho_{l} c_{l}\right)$, or if the syringe is assumed to contain an ideal fluid which can sustain tension). The same assumptions as for event 1 are made in order to create the space-time diagram shown in Figure 2.12.

The fluid and the container are initially (region 0 ) traveling at a velocity $u$. Note that $u<0$, which means the syringe and its content are traveling downward. The container is then impulsively decelerated to a complete stop. The liquid adjacent to the bottom wall of the container comes to a stop at the same time as the container, 
sending a compression pulse with magnitude

$$
\Delta P=\rho_{l} c_{l}|u|
$$

in the liquid. The effect of the compression wave is to increase the liquid pressure, and to bring the liquid to a stop (region 1).

The compression wave arrives later at the top surface of the water column, which is assumed to be a free end (i.e., constant pressure boundary condition). There, the compression wave reflects as a tension wave and begins to travel downward in the liquid toward the bottom wall. Upon reaching the rigid, bottom wall the wave reflects back into the liquid. The magnitude of the reflected wave is unchanged, but the reflection of the wave on a rigid boundary results in the doubling of the pressure in the immediate vicinity of the wall. Without accounting for dissipation the pressure wave reverberates indefinitely between the two boundaries.

Another consequence of the impulsive deceleration is that an acoustic compressive axial stress wave forms in the solid wall of the container and propagates away from the point of contact. Because the sound speed in solids is typically larger than the sound speed in liquids, the axial stress wave in the solid is traveling faster than the pressure wave in the liquid. This is depicted in panels $\mathrm{C}$ through $\mathrm{F}$ of Figure 2.11. Because the fluid and solid motion is only weekly coupled (discussed later in this section), the stress wave propagating in the syringe can be treated separately.

It is interesting that only minor modifications to the space-time diagram shown in Figure 2.12 are necessary so it applies to the axial stress $\sigma_{z}$ created in the wall of the container. Indeed, acoustic theory permits the calculation of the axial stress $\sigma_{z}$ in the wall of the container (Davis, 1988; Royer, Morgan, and Dieulesaint, 2000). The water hammer effect, despite its misleading name, can also occur in solids. A modified space-time diagram is shown in Figure 2.13. Note that the stress and velocity in each region are indicated in Table 2.3 due to the lack of space within the figure itself. Note that the following typical sign convention is adopted in this thesis: compression in a liquid is indicated with positive pressures, and compression in a solid is indicated with negative stresses.

The method-of-characteristics discussed in Appendix A was used to generate a space-time diagram equivalent to those shown in Figures 2.12 and 2.13. The nondimensional results shown in Figure 2.14 are valid for both the pressure and the stress transients: this is a consequence of the non-dimensionalization of the results. Note that the results are non-dimensionalized using the density and sound speed of 


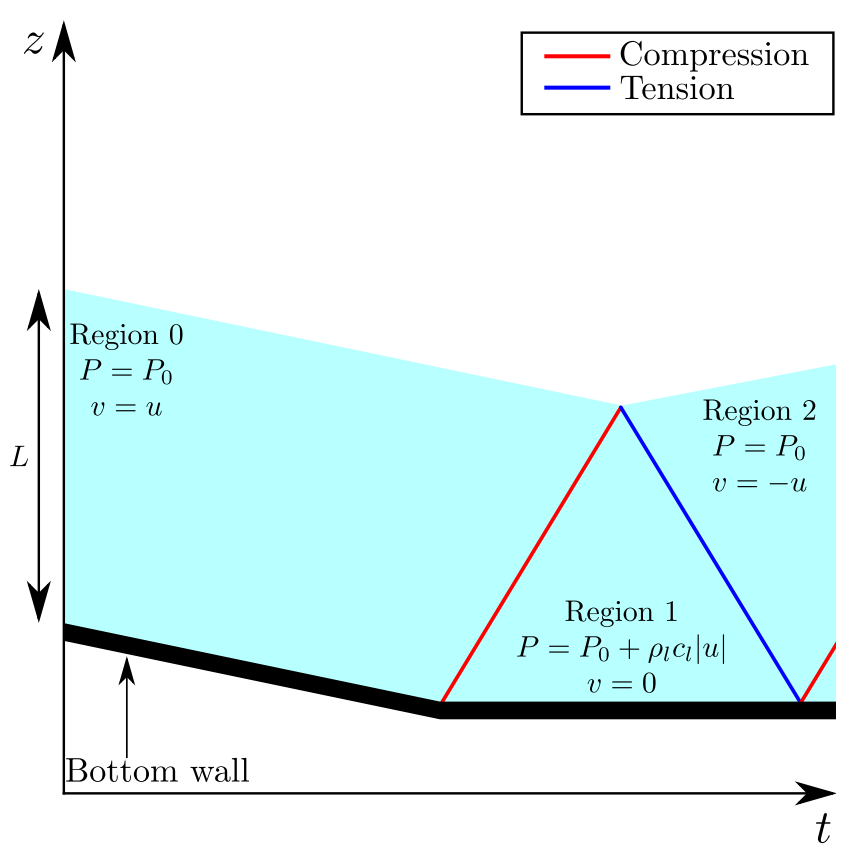

Figure 2.12: Space-time diagram of the pressure transient in the liquid created by the impulsive deceleration of the syringe. The syringe contains an ideal fluid, and cavitation does not occur.

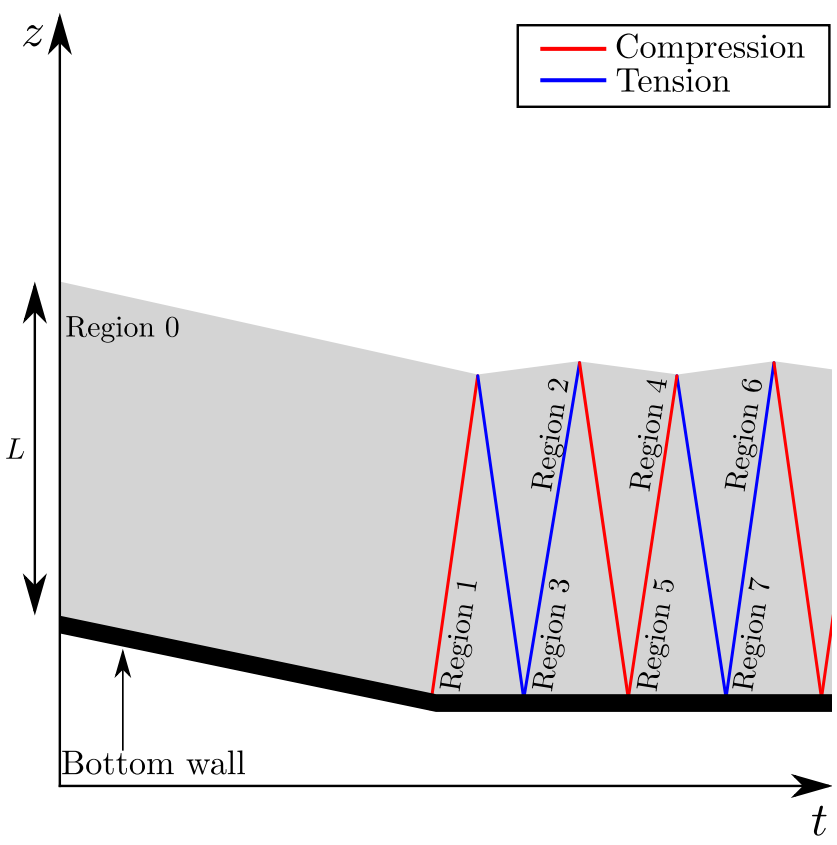

Figure 2.13: Space-time diagram of the stress transient created by the impulsive deceleration of the syringe. The syringe contains an ideal fluid, and cavitation does not occur. The axial stress and velocity of the solid in each region are indicated in Table 2.3. 
Table 2.3: Axial stress and velocity within regions 0 to 7 identified in Figure 2.13.

\begin{tabular}{llc}
\hline Region & Axial stress & Velocity \\
\hline 0 & $\sigma_{z, 0}$ & $u$ \\
1 & $\sigma_{z, 0}-\rho_{s} c_{s}|u|$ & 0 \\
2 & $\sigma_{z, 0}$ & $-u$ \\
3 & $\sigma_{z, 0}+\rho_{s} c_{s}|u|$ & 0 \\
4 & $\sigma_{z, 0}$ & $u$ \\
5 & $\sigma_{z, 0}-\rho_{s} c_{s}|u|$ & 0 \\
6 & $\sigma_{z, 0}$ & $-u$ \\
7 & $\sigma_{z, 0}+\rho_{s} c_{s}|u|$ & 0 \\
\hline
\end{tabular}

the liquid for the pressure transient, and the density and sound speed of the syringe wall for the stress transient. Also, the time axis is non-dimensional, giving the impression the pressure and stress transients occur at the same time scale, but this is of course not the case. The physics predicted with the method-of-characteristics is identical to what was shown and discussed earlier using the simplified diagrams.

For a syringe which is initially traveling downward $(u<0)$, cavitation is expected to occur in the liquid if $u<\left(P_{v a p}-P_{0}\right) /\left(\rho_{l} c_{l}\right)$. The onset of cavitation can substantially modify the wave dynamics within the liquid. The method-of-characteristics with a cavitation model was used to generate a space-time diagram which depicts this situation. The non-dimensional results are shown in Figure 2.15. The compression wave created by the impulsive deceleration of the syringe travels upward, and reflects on the free end. The compression wave becomes a tension wave when this happens. The tension wave and the reflected tension wave together reduce the pressure below vapor pressure, and cavitation occurs. As seen before while studying event 1 , a cavity forms at the bottom of the container, and the cavity later collapses (not shown).

The force applied on the syringe by the autoinjector feature which is responsible for decelerating the syringe creates stresses directly in the wall of the syringe. This remains true even if the syringe is not filled with a liquid. In other words, the stress wave which is directly created in the wall of the syringe does not result from the interaction between the fluid and the solid, but results from the interaction between the syringe and the autoinjector feature which is responsible for decelerating the syringe. Because the syringe contains a liquid and there is two-way interaction 


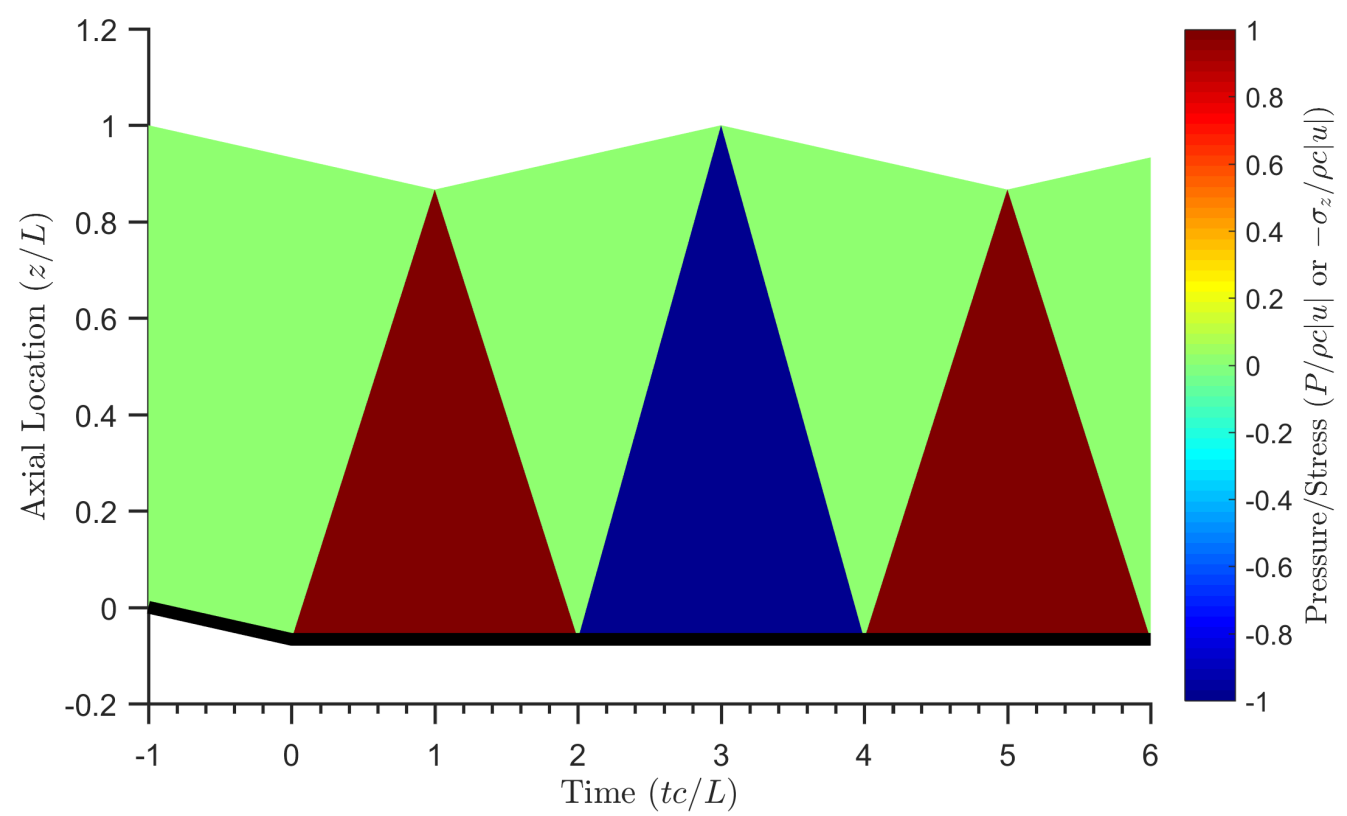

Figure 2.14: Space-time diagram of the pressure transient (without cavitation) or the stress transient created by the impulsive deceleration of the syringe. Results obtained using the method-of-characteristics. All results are non-dimensional.

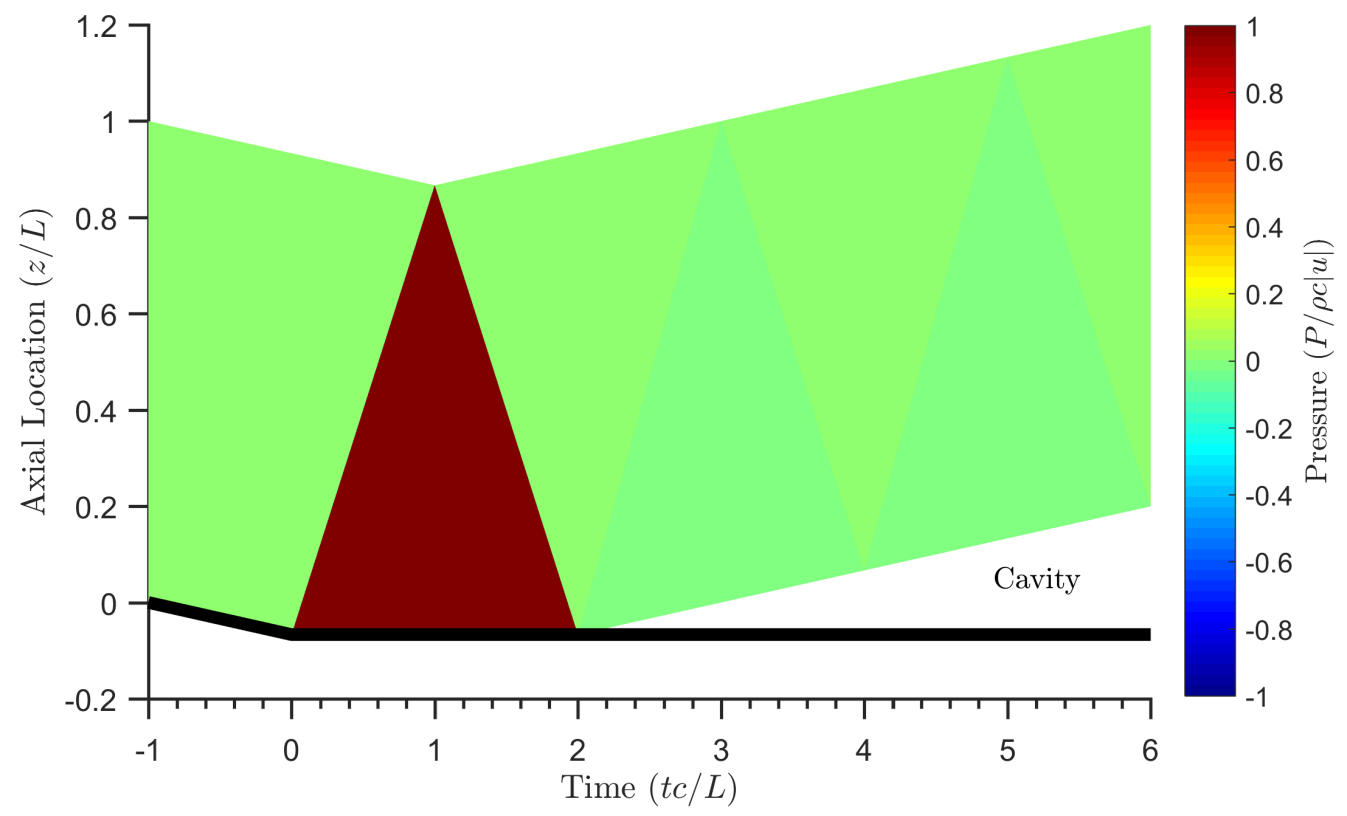

Figure 2.15: Space-time diagram of the pressure transient (with cavitation) created by the impulsive deceleration of the syringe. Results obtained using the method-ofcharacteristics. All results are non-dimensional. 


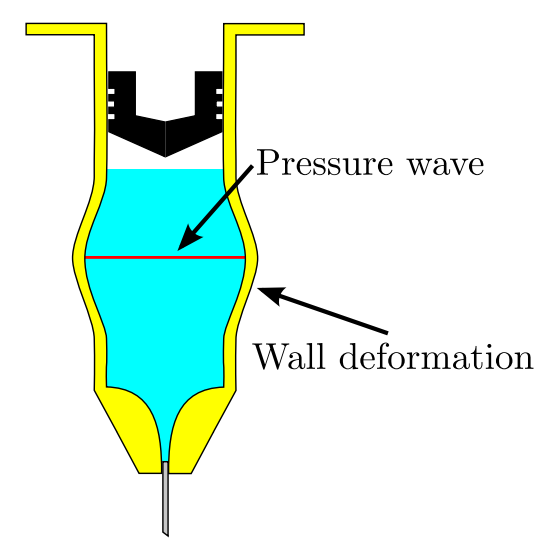

Figure 2.16: Deformation of the syringe wall resulting from its interaction with a pressure wave propagating in the liquid. The drawing is not to scale, and the deformation of the syringe is exaggerated.

between the fluid and the solid, the propagation of the stress wave in the syringe wall creates pressure waves within the liquid. The magnitude of those pressure waves is however relatively small, a consequence of the weak fluid-structure interaction between the fluid and the solid. The intensity of the fluid-structure interaction is often represented using the parameter $\beta$ (Shepherd and Inaba, 2010), introduced and discussed in Chapter 4 (see Equation 4.1). The parameter $\beta$ depends on the acoustic properties of the liquid and solid (i.e., density and sound speed) and the geometry of the container (i.e., radius and thickness of the wall). In a SureClick device using a glass syringe, the parameter $\beta$ is approximately 0.22 , a value which is relatively small as discussed by Shepherd and Inaba (2010).

The weak two-way coupling between the liquid and the solid also means that the pressure wave created and traveling in the liquid creates stresses and strains in the solid. The deformation of the container resulting from its interaction with the pressure wave is qualitatively shown in Figure 2.16. The deformation of the syringe, or strain, is primarily in the circumferential direction, creating a stress which is primarily in the circumferential or hoop direction. This is investigated and discussed further in Chapters 3 and 4.

The analysis presented above assumes the deceleration of the syringe is instantaneous. This assumption is now relaxed, and a constant deceleration which occurs over a finite time $\Delta t$ is assumed instead. The resulting linear velocity profile is shown in Figure 2.17. Of course, the case $\Delta t=0$ corresponds to the instantaneous deceleration previously discussed. The following analysis applies both to the solid 


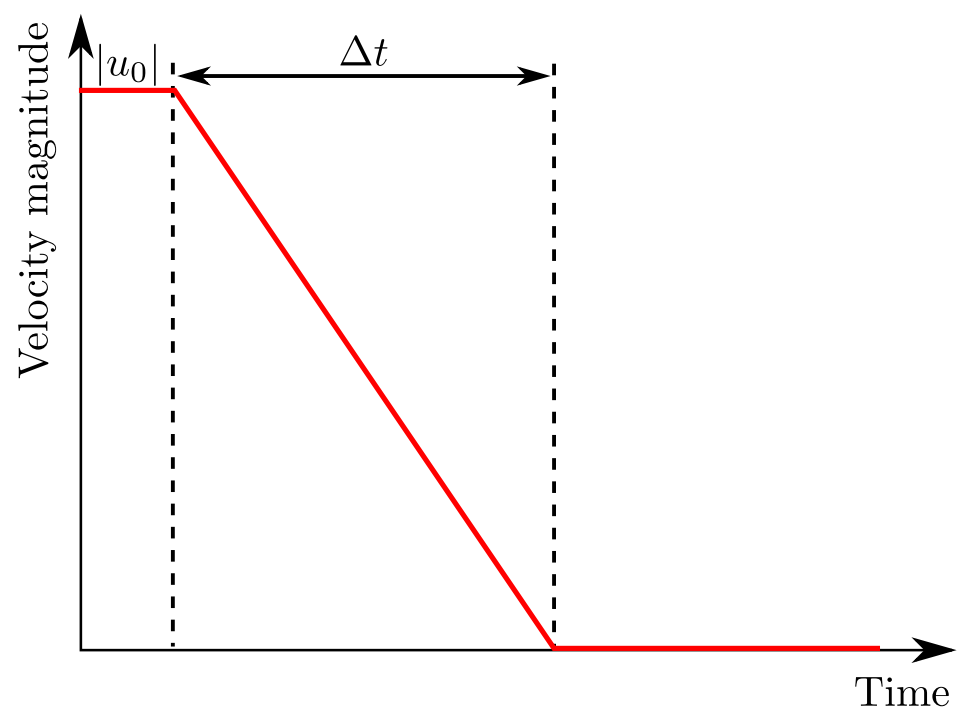

Figure 2.17: Velocity of the syringe during a deceleration of constant magnitude over a time $\Delta t$.

wall of the syringe and the liquid column contained inside the syringe, as long as the appropriate physical properties are used.

There are two deceleration regimes which need to be distinguished:

- regime 1: $\Delta t<\frac{2 L}{c}$;

- regime 2: $\Delta t \geq \frac{2 L}{c}$.

The first regime $(\Delta t<2 L / c)$ corresponds to a deceleration which occurs over a short time $\Delta t$; the deceleration is complete before the waves can complete a round trip within the specimen (i.e., the syringe wall or the liquid column). This corresponds to the "impulsive" deceleration regime, and the results shown above for an instantaneous deceleration are valid estimates. The second regime $(\Delta t \geq 2 L / c)$ corresponds to a deceleration which occurs over a long time $\Delta t$; the waves can complete one or more round trips within the syringe or liquid before the deceleration is complete. In this regime it is inadequate to assume an impulsive deceleration. Note that the deceleration regime in the solid wall and the liquid may be different.

In a SureClick device the deceleration regime of the liquid falls in regime 1 if the syringe deceleration occurs over a time $\Delta t$ which is less than approximately $68 \mu \mathrm{s}$, and the deceleration regime of the syringe wall falls in regime 1 if $\Delta t$ is less than 
approximately $21 \mu \mathrm{s}$. The experimental results from Chapter 3 indicate the syringe deceleration in a SureClick occurs over a time which is longer than $68 \mu \mathrm{s}$ : the deceleration is in regime 2 for both the liquid and the solid.

It is possible to estimate the expected peak pressure or the peak axial stress resulting from a deceleration in regime 2. An analytic solution can be obtained as long as cavitation does not occur. If cavitation occurs, the method-of-characteristics must be utilized. The analytic solution is obtained using stress wave theory and acoustics (Kolsky, 1953; Pierce, 1989). One important assumption is that this is a one-dimensional problem. In a solid, this assumption is reasonable as long as the magnitude of the axial stress remains large compared to the magnitude of the hoop and the radial stresses. In a liquid, this assumption is valid as long as the motion of the fluid elements is mostly in the axial direction.

The analytic solution is obtained by solving the following mathematical problem. At time $t<0$, a specimen of length $L$ is moving at a constant velocity $u_{0}$ in the negative $z$ direction. At times $t>0$ a force $F$ is applied on the bottom end of the specimen located at $z=0$. The force $F$ is oriented in the positive $z$ direction and decelerates the specimen at a rate $a$. The end of the specimen located at $z=L$ corresponds to a stress free or constant pressure end.

The initial velocity $u_{0}$ of the specimen is not important in this problem; it is eliminated by switching to an unaccelerated frame of reference traveling at velocity $u_{0}$. In other words, the pressure and stress waves are created by changes in velocity, not the velocity itself.

The equivalent mathematical problem is formulated using an acoustic potential $\phi(z, t)$ and necessitates solving the wave equation. To simplify the notation, all subscripts used in the following mathematical development indicate derivatives.

$$
\begin{array}{lc}
\phi_{t t}(z, t)=c^{2} \phi_{z z}(z, t) & (0<z<L, 0<t<\infty), \\
\phi(z, 0)=0 \text { and } \phi_{t}(z, 0)=0 & (0<z<L), \\
\phi_{z}(0, t)=a t \text { and } \phi_{t}(L, t)=0 & (0<t<\infty),
\end{array}
$$

where:

$$
\begin{gathered}
\sigma(z, t)=\rho \phi_{t}(z, t), \\
u(z, t)=\phi_{z}(z, t) .
\end{gathered}
$$

Note that $u(z, t)$ is the velocity at time $t$ of an element located at an axial location $z$, and $c$ corresponds to the sound speed in the specimen. Also, $\sigma$ can be replaced 
with $-P$ as the solution is valid for both the liquid and the solid, as long as the appropriate physical properties are used (i.e., $L, \rho$ and $c$ ).

The following linear transformation is performed before solving:

$$
\phi(z, t)=\psi(z, t)-(L-z) a t,
$$

and the mathematical problem becomes:

$$
\begin{array}{lr}
\psi_{t t}(z, t)=c^{2} \psi_{z z}(z, t) & (0<z<L, 0<t<\infty), \\
\psi(z, 0)=0 \text { and } \psi_{t}(z, 0)=(L-z) a & (0<z<L), \\
\psi_{z}(0, t)=0 \text { and } \psi_{t}(L, t)=0 & (0<t<\infty) .
\end{array}
$$

The solution is obtained using standard techniques of separation of variables (Greenberg, 1998). Once the solution $\psi(z, t)$ is obtained, the linear transformation given by Equation 2.11 is used once more to recover $\phi(z, t)$ :

$$
\phi(z, t)=\sum_{n=1,3, \ldots}^{\infty} \frac{16 L^{2} a}{c n^{3} \pi^{3}} \sin \left(\frac{n \pi c}{2 L} t\right) \cos \left(\frac{n \pi}{2 L} z\right)-(L-z) a t
$$

The stress (or pressure) is calculated using Equation 2.9:

$$
\sigma(z, t)=\rho \sum_{n=1,3, \ldots}^{\infty} \frac{8 L a}{n^{2} \pi^{2}} \cos \left(\frac{n \pi c}{2 L} t\right) \cos \left(\frac{n \pi}{2 L} z\right)-\rho(L-z) a .
$$

The result above is general and applies to all axial locations in the specimen. This result can be used to find the location and value of the peak stress or pressure. Because of the $\cos \left(\frac{n \pi}{2 L} z\right)$ term, the maximum stress or pressure clearly occurs at $z=0$, where:

$$
\sigma(0, t)=\rho \sum_{n=1,3, \ldots}^{\infty} \frac{8 L a}{n^{2} \pi^{2}} \cos \left(\frac{n \pi c}{2 L} t\right)-\rho a L .
$$

Using the methods described by Greenberg (1998) it is possible to show that Equation 2.17 corresponds exactly to the half-range cosine expansion of:

$$
\sigma(0, t)= \begin{cases}-\rho a c t, & \text { if } 0 \leq t \geq \frac{2 L}{c}, \\ \rho a(c t-4 L), & \text { if } \frac{2 L}{c} \leq t \geq \frac{4 L}{c},\end{cases}
$$


a result which is easier to interpret than the infinite series from Equation 2.17. Note that Equation 2.18 is the mathematical representation of only the first period of the solution given by Equation 2.17; the solution is periodic with a period equal to $4 L / c$. Thus, the maximum stress or pressure occurs at $t=2 L / c$, and it is equal to:

$$
\begin{gathered}
\sigma(0,2 L / c)=-2 \rho a L . \\
P(0,2 L / c)=2 \rho a L .
\end{gathered}
$$

The non-dimensional results obtained with Equation 2.5 (valid in deceleration regime 1) and Equations 2.19 and 2.20 (valid in deceleration regime 2) are plotted in Figure 2.18. The horizontal axis corresponds to $\Delta t c / L$, where $\Delta t$ is the duration of the deceleration event, as indicated in Figure 2.17. A value of $\Delta t c / L=2$ corresponds to the boundary between deceleration regimes 1 and regime 2: the wave can complete one entire round trip during the deceleration event. Results obtained with the method-of-characteristic are also shown in Figure 2.18 for comparison. The method-of-characteristics results were obtained for the deceleration of a specimen from a velocity $u$ to zero in a time $\Delta t$ ( $\Delta t$ is defined in Figure 2.17) and correspond to the peak magnitude of the pressure or stress inside the specimen. The reader should recall that $u<0$ since the specimen is initially traveling downward. The relation between acceleration, initial velocity and $\Delta t$ is $a=-u / \Delta t$.

The results obtained with the method-of-characteristics are in agreement with the results obtained using Equations 2.5, 2.19 and 2.20. Two regimes are distinguished. For $\Delta t c / L \leq 2$, the peak magnitude of the pressure or stress is unaffected by $\Delta t c / L$; the peak pressure or stress is determined by the change in velocity, not the magnitude of the deceleration. For $\Delta t c / L>2$, the peak magnitude of the pressure or stress decreases as $\Delta t c / L$ increases. This is because the peak magnitude of the pressure or stress is determined by the magnitude of the acceleration, which is controlled by $\Delta t c / L$.

Finally, note that there are also two acceleration regimes. The discussion and estimates developed for the deceleration of the syringe wall or the liquid content also apply when the bottom end of the specimen is accelerated from zero to $u$ at a constant rate (event 1 ). The main difference is that tension waves are initially created instead of compression waves. It is important to recall that the analytic expressions are not valid after the onset of cavitation.

To summarize, the rapid deceleration of the syringe creates a pressure and stress transient which is significant in both the solid and the liquid. The transients are 


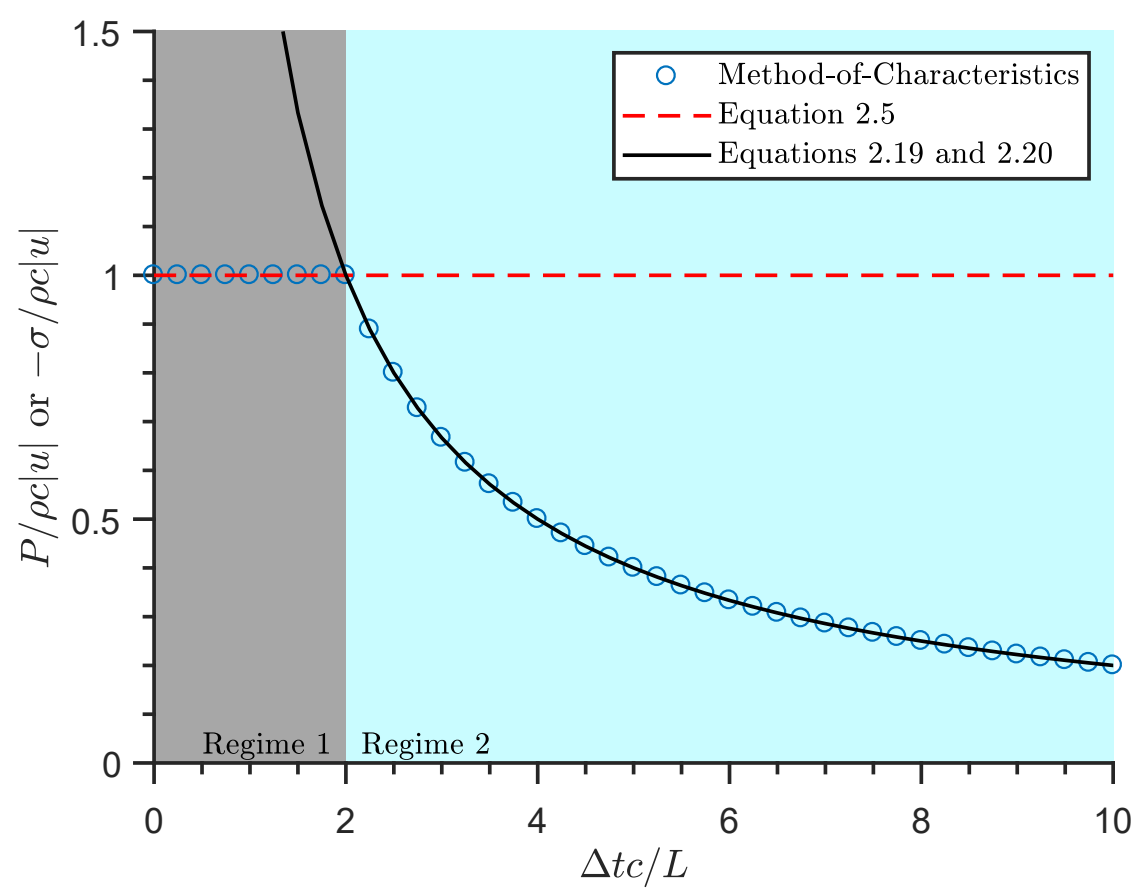

Figure 2.18: Peak pressure or stress created by a constant deceleration of the syringe over a time $\Delta t$. Two regimes are shown (see the text for explanations).

similar to water hammer events in piping systems. The deceleration of the syringe creates pressure waves within the liquid which, in turn, create stresses in the syringe wall due to the two-way coupling between the solid and the liquid. The deceleration of the syringe also creates a compressive axial stress wave in the container's wall. For an acceleration/deceleration which is in regime 2 (i.e., non-impulsive), increasing $\Delta t$, where $\Delta t$ is the time over which the change in velocity occurs, reduces the magnitude of the acceleration/deceleration, and reduces the magnitude of the peak pressure and stress. The acceleration/deceleration of a syringe in a SureClick device is in regime 2 .

\subsubsection{Event 3: Impact of the Driving Rod on the Plunger-Stopper}

The spring-actuated driving rod is used to apply a force of magnitude $F$ on the plunger-stopper. This is illustrated in panels $\mathrm{C}$ and D of Figure 2.1. This force is responsible for the pressurization of the liquid contained inside the syringe. Pressurization of the liquid is necessary to create a pressure gradient through the needle, and force the medicament to be extruded into the patient. As described before, the amount of force $F$ needed to extrude a specific drug volume in a given amount of time is generally estimated using Equation 1.1, and this is commonly known as 
syringeability. Equation 1.1 assumes a steady and fully-developed Poiseuille flow in the needle (Kundu, Cohen, and Dowling, 2012), and no friction between the plunger-stopper and the syringe barrel.

If force $F$ is slowly and gradually applied on the plunger-stopper (i.e., when the force is applied in a quasi-static manner without any impact between the driving rod and the plunger-stopper) the pressure in the syringe slowly increases to reach a maximum quasi-static value $P_{q s}$. The liquid pressure then remains approximately constant in the syringe until the injection is complete. This quasi-static pressure $P_{q s}$ in the syringe is approximately $P_{q s}=F / A_{b, i}$, where $A_{b, i}$ is the inner cross-sectional area of the syringe barrel. In a real situation there is friction between the plungerstopper and the syringe barrel, and therefore the measured value of $P_{q s}$ is expected to be less than $F / A_{b, i}$. In a SureClick autoinjector device, the magnitude of $P_{q s}$ is approximately $1 \mathrm{MPa}$.

The pressure history at a particular axial location along the barrel of the syringe resulting from the quasi-static pressurization of the syringe is qualitatively illustrated in Figure 2.19 (dashed curve). The quasi-static pressurization of the syringe corresponds to the ideal pressurization profile; this is not a transient event, and the stresses within the syringe can be estimated using a simple shell theory or the Lamé solution (Bower, 2009; Jones, 1989). The strains created in a SureClick autoinjector by the quasi-static pressure are estimated and measured experimentally in Chapter 3.

If instead the spring force $F$ is applied on the plunger-stopper in an impulsive manner such as when the driving rod impacts on the plunger-stopper, the above quasi-static analysis does not hold. When this is the case, the dynamics of the event must be accounted for in predicting the pressure history in the syringe since the instantaneous pressure may exceed significantly the quasi-static value $P_{q s}$. The characteristics of this momentary excursion of the liquid pressure above $P_{q s}$ depend greatly on the presence and size of an air gap between the plunger-stopper and the liquid drug solution (see Figure 2.3). The cases with and without an air gap are treated separately.

The simplest impulsive-loading case to analyze and understand is when there is no air gap between the plunger-stopper and the liquid. This can correspond to two different configurations: 1) a syringe without any air gap inside, or 2) a syringe with an air gap inside, but positioned in a vertical, tip-up orientation. A very short time after the impact of the spring-actuated driving rod on the plunger-stopper at a velocity $u$, the plunger-stopper is moving at a velocity $u_{p s, 0}$. This means the plunger- 
stopper is abruptly accelerated to a velocity $u_{p s, 0}$ due to the impact event. Because there is a direct contact between the liquid and the plunger-stopper, the motion of the plunger-stopper forces the liquid immediately adjacent to the plunger-stopper to accelerate impulsively, and to move at the same velocity $u_{p s, 0}$.

The abrupt acceleration of the liquid creates a water hammer type of transient: a compressive pressure wave of magnitude

$$
\Delta P=\rho_{l} c_{l}\left|u_{p s, 0}\right|
$$

is created in the liquid. This pressure wave travels from top to bottom in the syringe (i.e., from below the plunger-stopper toward the tip of the syringe). As before, the propagation of this pressure wave in the liquid creates stresses in the syringe and slightly deforms the walls (see Figure 2.16). The strains created by the propagation of the pressure wave in the syringe are measured and discussed further in Chapter 3. The typical pressure history at any axial location along the barrel of the syringe resulting from this type of transient event is shown in Figure 2.19 (continuous curve). The pressure rises abruptly, almost in a discontinuous fashion. After reaching its peak value, the pressure in the syringe decays to a value equal to the quasi-static pressure $P_{q s}$. This type of transient event has been studied in the past by Shepherd and Inaba (2010) and Inaba and Shepherd (2010).

The transient due to the impulsive-loading of the plunger-stopper resulting from the impact of the driving-rod on the plunger-stopper is more complex when there is an air gap between the plunger-stopper and the liquid. When this is the case, the liquid in the syringe becomes pressurized as the air gap gets compressed. The magnitude of motion of the air inside the gap is small, at most $10 \mathrm{~m} / \mathrm{s}$. As a useful approximation, the compression of the air gap is considered to be isentropic:

$$
\frac{P_{\text {gap }}}{\rho_{\text {gap }}^{\gamma}}=\text { constant },
$$

and the pressure within the air gap is approximately uniform; this approximation is adequate because the pressure waves can reverberate multiple times within the air gap during the compression, as illustrated with the results from Chapter 3. The air gap is effectively acting like a pressure boundary condition for the liquid column, such that a pressure rise within the air gap sends a train of compressive pressure waves in the liquid, the role of which is to equilibrate the liquid pressure, and make it equal to the air gap pressure. 


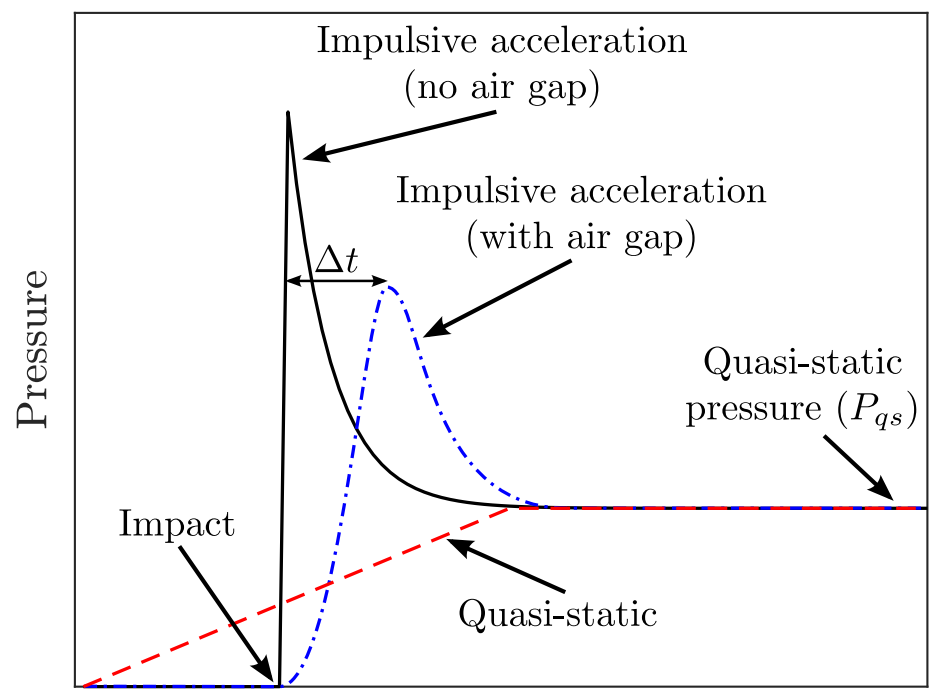

Time

Figure 2.19: Pressure history at a fixed location along the barrel of the syringe upon application of a force $F$ on the plunger-stopper. The dashed curve is for a quasistatic application of force $F$. The continuous curve and the dashed-dot curve are respectively for the impulsive application of force $F$ on the plunger-stopper without and with an air gap below the plunger-stopper.

The pressure history in the air gap or at any axial location along the syringe barrel is shown in Figure 2.19 (dashed-dot curve). Because the liquid is pressurized through the relatively slow compression of the air gap, which happens over a finite amount of time, the pressure rise is not as sharp or abrupt as for the case without an air gap (continuous curve). There is again an excursion of the instantaneous pressure above $P_{q s}$, and the pressure eventually decays to the quasi-static pressure $P_{q s}$.

Generally, the rise time $\Delta t$ of the pressure pulse increases as the initial air gap size $\delta_{0}$ increases, and as $u_{p s, 0}$ decreases $\left(\Delta t \sim \delta_{0} / u_{p s, 0}\right)$. Estimating the maximum air gap compression and the peak pressure in the air gap in this dynamic problem is no simple matter. The complexity arises from the fact that the driving-rod, the plungerstopper, the air gap and the liquid together behave like a damped mass-spring system (see Chapter 4). Furthermore, the pressure in the air gap behaves in a non-linear fashion as the air gap becomes compressed (i.e, the air gap behaves like a non-linear spring). The pressure within the air gap is approximately:

$$
P_{\text {gap }}=P_{\text {gap }, 0}\left(\frac{\delta_{0}}{\delta}\right)^{\gamma},
$$


where $\delta$ is the air gap size, as indicated in Figure 2.3. The air gap compression is discussed further in Chapter 4, where a model which can be used to estimate the peak pressure and the rise time of the pressure pulse is introduced.

In summary, if the force $F$ applied on the plunger-stopper increases slowly to the final value, this results in a slow and gradual increase of the liquid pressure to a value $P_{q s}=F / A_{b, i}$. This is the idealized pressurization profile as there are no excursions of the pressure above $P_{q s}$. On the other hand, when the force $F$ is applied impulsively on the plunger-stopper the peak pressure in the liquid can exceed $P_{q s}$, and this creates stresses and strains in the syringe's walls. As discussed in Chapter 3 , peak magnitudes as large as 4 to 10 times the value of $P_{q s}$ have been measured in a SureClick autoinjector. The pressure waves created in the liquid are very sharp when there is a direct contact between the plunger-stopper and the liquid. Adding and increasing the size of the air gap reduces the sharpness of the pressure waves created in the liquid (the sharpness of the pressure waves is further discussed in Chapters 3 to 5).

To end this section, it is important to recognize that all three transient events are of very short duration (i.e., a few milliseconds at most). The transient events are followed by the extrusion phase, which is relatively long (i.e., a few seconds). The liquid pressure during the extrusion phase is equal to $P_{q s}$. Equation 1.1 can be used successfully to estimate the syringeability of a drug solution, but it can't be used to predict the peak pressure in the liquid or the peak wall stresses in the syringe. Although Equation 1.1 can be used to scale some parameters of the autoinjector device in a quasi-static sense, it does not say anything about the potential for device failure related to the dynamic events.

\subsection{SureClick Autoinjector}

One of the autoinjector devices used by Amgen is commercialized under the SureClick brand. The exterior of a SureClick autoinjector used for the delivery of a drug called Enbrel (etanercept) is shown in Figure 2.20. The SureClick autoinjector is fabricated by SHL Group (commercialized under the DAI and DAI 2 brands). The SureClick autoinjector is discussed extensively in this thesis to study the pressure and stress transients resulting from the three dynamic events previously described. 
Before use

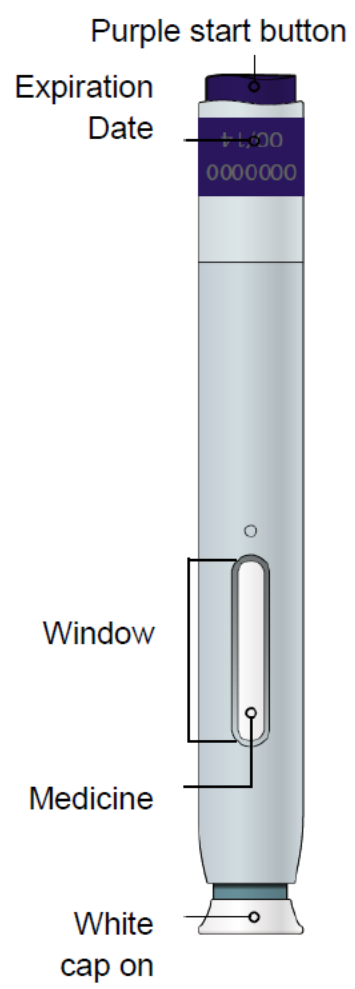

After use

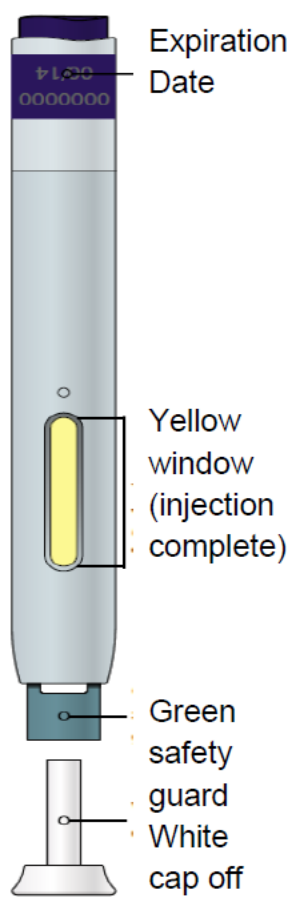

Figure 2.20: SureClick autoinjector device used by Amgen. Reproduced from Amgen (2016).

The external user actions required to operate a SureClick autoinjector are briefly reviewed. The various steps are described in the user manual distributed with the device (Amgen, 2016), and the sequence can be summarized as follows:

1. Remove the SureClick autoinjector from the package.

2. Inspect the SureClick autoinjector.

3. Prepare and clean the injection site. A proper injection site can be a thigh, the abdomen, or the outer area of an upper arm.

4. Pull the needle shield off (i.e., the white cap) when you are ready to inject.

5. Stretch or pinch the injection site to create a firm surface.

6. Place the end of the autoinjector on the skin at a right angle, and firmly push down the autoinjector onto the skin. This unlocks the internal mechanism.

7. When ready to inject, press the actuation button (a "click" is heard). 


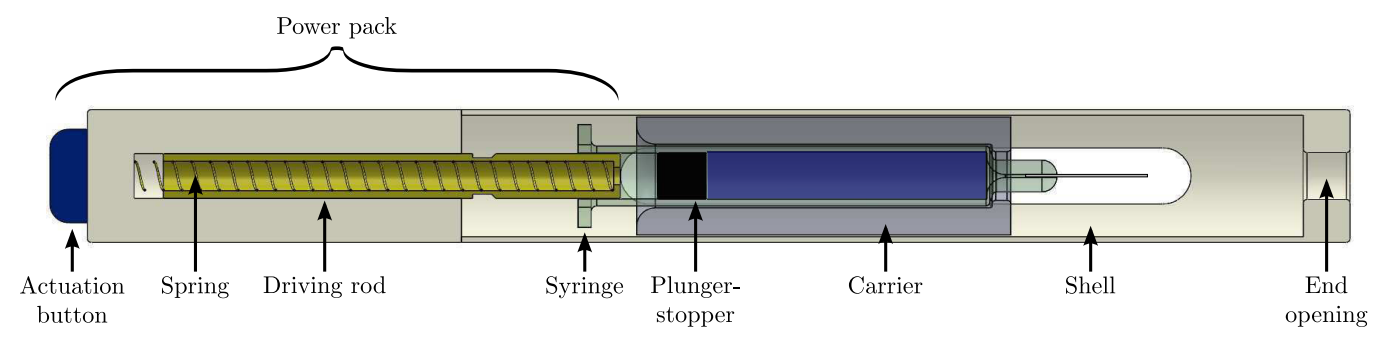

Figure 2.21: Schematic of the key features of a SureClick autoinjector device.

8. The injection is complete when a second "click" is heard, up to 15 seconds after actuation.

9. Remove the autoinjector and dispose.

The transient events (events 1,2, and 3) occur between steps 7 and 8 of the user sequence.

Figure 2.21 is a simplified schematic of the internal components of a SureClick autoinjector. In a SureClick device, the pre-filled syringe is mounted inside a syringe carrier. The syringe carrier is fabricated with plastic, and its primary role is to support the syringe within the device. The syringe carrier also ensures proper alignment of the syringe within the device. The contact point between the syringe and the syringe carrier is located at the shoulder level. Note that a needle is preattached to the syringe before the device is distributed to patients.

The power pack is responsible for actuation of the device. A coiled spring is located inside the driving rod. The spring is pre-compressed before the device is distributed to patients, eliminating the need to "prime" the device before use.

The stiffness of the spring in the SureClick autoinjector considered herein is approximately $500 \mathrm{~N} / \mathrm{m}$. Note that there exist different versions of SureClick with different spring stiffness. The pre-filled syringe has the following approximate dimensions: $6 \mathrm{~mm}$ in inner diameter, $8 \mathrm{~mm}$ in outer diameter, and $64 \mathrm{~mm}$ in length (more precise values are provided in Chapter 3). The diameter of the shell of the autoinjector is approximately $18 \mathrm{~mm}$, and the length of the device is approximately $150 \mathrm{~mm}$.

When the user activates the device by depressing the actuation button located at the top of the power pack, the internal mechanism of the power pack (not shown) releases the spring-actuated driving rod. The driving rod is then accelerated and impacts on the plunger-stopper, setting the syringe, the carrier and the liquid contained inside the syringe into motion (event 1). The impact of the driving-rod on the plunger-stopper 
also initiates the pressurization of the syringe (event 3 ). Due to the friction between the plunger-stopper and the syringe, events 1 and 3 are not entirely decoupled from one another. In fact, no needle insertion mechanism is shown in Figure 2.21. Even if one is physically present in a SureClick, results indicate it does not play a significant role in the actuation sequence. As explained in Chapter 3, the acceleration of the syringe results from the friction between the plunger-stopper and the syringe, and from the pressure increase at the bottom of the syringe.

A few milliseconds after actuation the syringe carrier reaches its travel limit, and both the liquid and the syringe stop moving (event 2). Injection of the drug into the patient then follows over approximately 5-15 seconds. 
Chapter 3

\section{IN SITU MEASUREMENTS}

This chapter is on the in situ experimental study of the pressure and stress transients in a SureClick autoinjector device. The first objective of this chapter is to thoroughly describe the experimental methods developed and used. The second objective is to show and discuss typical results obtained using those novel experimental techniques. The experimental results are used to better understand the pressure and stress transient resulting from events 1,2, and 3 described in Chapter 2. The coupling between the dynamic events is also investigated, and the performances of both glass and plastic syringes are discussed.

\subsection{Methodology and Material}

Quantitative measurements of the pressure and stress transients in a SureClick autoinjector are performed using a combination of digital high-speed cameras, pressure sensors, and strain gauges. Digital high-speed imaging of the moving components makes it possible to verify the sequence of events and timing within the device. Quantitative image analysis enables measurements of the impact velocity between the various components, and the acceleration/deceleration of the components. A pressure transducer mounted inside the syringe makes it possible to measure the liquid pressure throughout actuation. Strain gauges mounted on the outer surface of the syringe make it possible to measure the deformation or strains of the glass in the hoop (i.e., circumferential) and axial directions. The information obtained with the strain gauges is useful because it can be used to infer the wall stresses.

Instrumenting and preparing a syringe for an experiment is a difficult and lengthy task. The difficulties are caused by several factors, such as:

- the small size of the pre-filled syringe;

- the lack of openings or the inconvenient geometry and placement of openings which can be used to route the wires connecting the pressure and strain gauges to the amplifying electronics;

- the possibility for the gauges and wires to interfere with the motion of the internal components of the autoinjector; 
- the possibility for the motion of the internal components of the autoinjector to damage the gauges or the wires.

Despite the difficulties mentioned above it was possible to successfully develop and use in situ experimental techniques. The protocol used to instrument and test an autoinjector is described below in a sequential manner. Note that all the techniques are described in the context of instrumenting and testing a SureClick autoinjector device, but it is relatively straightforward to adapt the methods to instrument and test a different injection device.

\subsubsection{Step 1: Modifying the Shell}

An essential diagnostic in studying an autoinjector device is high-speed imaging of the moving components. Unfortunately, the geometry of the outer shell of a SureClick autoinjector is inadequate for this. The outer shell is opaque, and the position of the window (see Figure 2.20) does not make it possible to observe the motion of the internal components throughout actuation. Note that the purpose of the window built into the device is to make it possible for the user to inspect the drug solution for discoloration and contamination before injection, not to observe the motion of the internal components.

There are two different alternatives which can be used to make high-speed imaging of the internal components of a SureClick possible. The first option is to fabricate a new shell using optically clear material. Optically clear shells can be fabricated using stereolithography. Vapor polishing the final product improves the clarity and translucence of the shell. This first method is preferred because the geometry of the shell is not altered.

The second option is to increase the size of the window which is already present (see Figure 2.20). The size of the window is extended in the axial direction until it becomes possible to observe the tip of the syringe, the tip of the driving rod, the

plunger-stopper and the flange of the syringe throughout actuation. The modifications to the shell can be performed using a rotary cutting tool.

Both approaches were used successfully to obtain the data shown and discussed in this thesis. Also, choosing one option over the other is inconsequential as it does not create a measurable effect on the results. The translucent shells used in this study were fabricated by the company 3D Systems. 


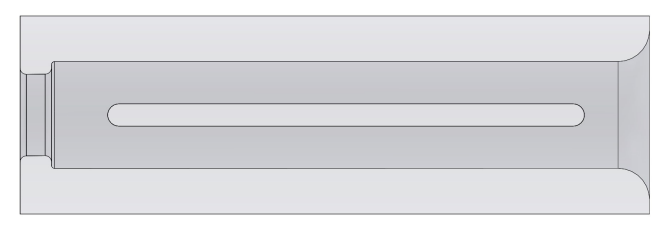

Figure 3.1: Schematic of a syringe carrier with an oblong slit for the leadwires of the strain gauges to be mounted on the syringe.

\subsubsection{Step 2: Modifying the Syringe Carrier}

The plastic syringe carrier of a SureClick device has the shape of a cylindrical shell opened at both ends. There is sufficient space between the outer surface of the syringe and the inner surface of the syringe carrier to accommodate several strain gauges mounted on the syringe barrel. There is, however, no suitable opening in the syringe carrier to route the leadwires of the strain gauges to the outside of the autoinjector device.

This issue is resolved by making an oblong slit in the side wall of the syringe carrier. There is no need for the slit to be very large; a width of approximately $1 \mathrm{~mm}$ is sufficient. The axial length and position of the slit is determined by the intended location of the strain gauges to be installed on the syringe wall. An example of a modified syringe carrier with an oblong slit for routing the leadwires of the strain gauges is shown in Figure 3.1.

\subsubsection{Step 3: Removing the Needle}

The pre-filled syringe used in a SureClick autoinjector comes with a pre-attached needle. The needle must be removed prior to instrumenting the syringe. This is necessary because the leadwires of the pressure transducer will later be routed to the outside of the autoinjector through the opening located in the syringe tip, where the needle is pre-attached. The inner diameter of the needle is too small to accommodate the two leadwires connected to the pressure transducer, but the opening left after the removal of the needle is sufficiently large.

The pre-attached needle on a glass syringes is generally secured using UV cured adhesives (Sacha, Rogers, and Miller, 2015). To remove the needle, heat is applied on the needle and the tip of the syringe using a heat source such as a blow torch. This makes the adhesive degrade, and it becomes possible to pull the needle out without much effort using regular pliers. The needle must be appropriately discarded in a sharps container. 
The use of a heat source is of course not possible when testing plastic syringes. It is often possible to use pliers to pull on the needle and extract it from the tip of the syringe. In some cases the force needed to extract the needle is too large, and this causes the needle to break, leaving a portion of the needle within the syringe. Those syringes need to be discarded, and it might be necessary to communicate with the syringe manufacturer to obtain engineering samples without a pre-attached needle. The plastic syringes used in this study did not pose a significant problem, and gently pulling on the needle using pliers works generally well to remove the needle.

\subsubsection{Step 4: Mounting the Pressure Transducer}

PCB Piezotronics fabricates ICP tourmaline underwater blast pressure sensors. The sensing element of an ICP sensor is typically suspended and sealed in an insulating vinyl tube which is filled with silicone oil. The vinyl tube enclosing the sensing element is, however, too large to fit within the pre-filled syringe used in a SureClick autoinjector; the inner diameter of the syringe is only $6 \mathrm{~mm}$.

A PCB 138M186 tourmaline underwater blast pressure sensor is used in this study. The 138M186 is a modified version of the ICP sensors described above: it comes without an enclosing vinyl tube. A photo of the pressure transducer is shown in Figure 3.2. The sensing element is approximately $4 \mathrm{~mm} \times 4 \mathrm{~mm} \times 1.5 \mathrm{~mm}$. Due to its small size the transducer can be introduced inside the syringe and submerged in the liquid content. The transducer does not need to be attached to the wall of the syringe, but can be left floating freely in the liquid. The sensitivity of the

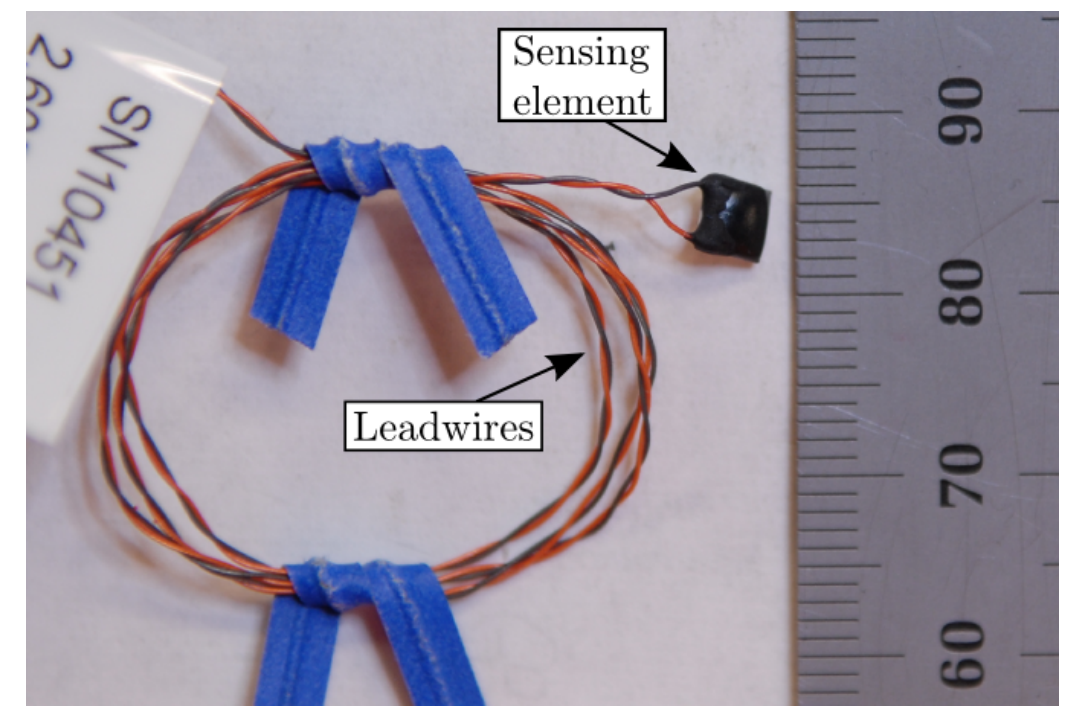

Figure 3.2: PCB 138M186 tourmaline underwater blast pressure sensor. 
pressure transducer is approximately $0.44 \mathrm{~V} / \mathrm{MPa}$, and it can measure pressures up to $13.8 \mathrm{MPa}$.

The first step in mounting the pressure transducer in the syringe is to cut and shorten the pre-attached leadwires, leaving only 1.0 to 1.5 inches of leadwires attached to the transducer. It is important to be very gentle with the leadwires connected to the transducer as they tend to easily break at their root, and it becomes extremely difficult, if at all possible, to repair the transducer when this happens.

The second step is to introduce two foot-long magnet wires inside the syringe through the opening in the tip. Belden magnet wires AWG (American Wire Gauge) \#34 (0.16 $\mathrm{mm}$ in diameter) and \#38 (0.101 $\mathrm{mm}$ in diameter) are used in this study. Whenever possible the larger diameter magnet wire is used, but this is not always possible. Different syringe models have different geometries, and the size of the opening located within the tip of the syringe can even vary between different syringe samples of the same model. This is particularly true for glass syringes.

Note that the conductive core of the magnet wires is coated with a polyester insulator. The insulating coating needs to be removed at both ends of each magnet wire over a distance of approximately one quarter of an inch. This is necessary in order to later reconnect the pressure sensor to the amplifying electronics. Failure to remove the insulating coating results in poor connectivity between the transducer and the amplifying electronics. Removal of the coating is performed by scraping off the polyester with a razor blade, melting the solvent with a heat source such as a lighter, or using an appropriate solvent such as acetone.

Once the magnet wires are routed through the opening in the tip of the syringe, the next and third step is to connect the magnet wires to the leadwires of the pressure transducer. A standard soldering iron and solder are used for this. The fourth step is to apply a thin coating of insulating material on the connections between the magnet wires and the leadwires. The Vishay-Micro-Measurement M-Coat A liquid polyurethane coating designed to protect and insulate strain gauges is used for this purpose. Standard non-conductive epoxy also works well. Failure to properly insulate the connections often results in shorting of the transducer and signal loss during actuation of the autoinjector. It is necessary to allow sufficient time for the protective coating to properly set before moving to the next step.

The fifth and final step is to position the pressure transducer within the syringe. The transducer is introduced in the syringe and positioned at the appropriate axial 


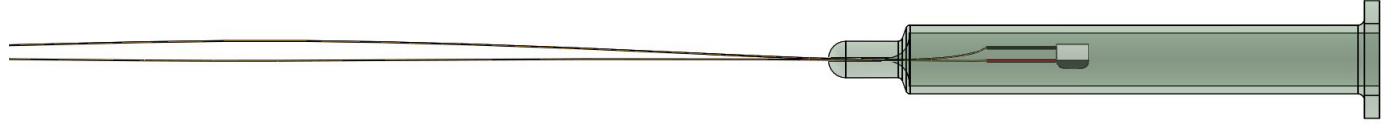

Figure 3.3: Schematic of a PCB 138M186 tourmaline underwater blast pressure sensor mounted inside a syringe. The black and red leadwires of the pressure sensor are connected to magnet wires running through the opening in the syringe tip.

location by pulling gently on the magnet wires from the outside of the syringe. The final result is similar to the instrumented syringe shown in Figure 3.3.

The pressure transducer is tested before moving to the next step. Testing of the transducer is achieved by connecting the magnet wires to the signal conditioner, and powering-on the system. The front display of the signal conditioner is verified for indication of an anomaly with the transducer. An oscilloscope is also used to monitor the output signal and confirm it is stable and free from spurious noise. Experience has shown that anomalies with the pressure transducer generally result from a poor connection between the magnet wires and the leadwires of the pressure transducer. Note that a PCB model 480C02, battery-operated, constant-current signal conditioner is used to power the pressure transducer.

\subsubsection{Step 5: Filling the Syringe}

The syringe is filled with de-ionized water, silicone oil, a placebo, or an actual drug solution. Once the liquid is inside the syringe, the plunger-stopper is positioned in the syringe barrel at the appropriate depth. If necessary, an air gap is introduced within the syringe at this stage of the process. The air gap is introduced by letting some liquid slowly exit the syringe through the tip before positioning the plungerstopper. The motion of the plunger-stopper into the syringe barrel is kept to a minimum to avoid altering the lubrication film between those two parts.

The bottom opening of the syringe is then properly dried and sealed using standard 5-minute epoxy. Sealing of the syringe tip is recommended to prevent the plungerstopper from being pushed down into the syringe barrel during actuation, and extrude the liquid out of the syringe. Pushing the plunger-stopper down into the syringe can result in permanent damage to the pressure transducer. Furthermore, the epoxy prevents the magnet wires from moving, and this fixes the position of the pressure transducer inside the syringe. 
Note that a limited number of experiments were performed without a sealed tip to confirm that sealing the tip does not affect the results. On the time scale of the dynamic events there is no flow through the tip of the syringe, and sealing the tip does not have a measurable effect on the results. Of course, sealing the tip is inadequate if one is interested in making measurements during the relatively long extrusion phase which follows the transient events.

\subsubsection{Step 6: Preparing the Syringe Surface}

Adherence of the strain gauges to the surface of the syringe is improved by locally abrading the syringe surface in the areas where strain gauges are to be installed. Abrading the surface of a glass syringe is achieved with a rotary tool and a grinding stone. A rotary tool can also be used with plastic syringes, but regular sand paper works better.

After properly abrading the syringe, its surface is degreased, conditioned, and neutralized. ${ }^{1}$ The instructions provided by the manufacturer of the strain gauges detail the procedure used in this study (Vishay - Micro-Measurement, 2014). Note that the manufacturer's instructions are different for plastic and glass samples. This is because some of the chemicals used in the degreasing, conditioning, and/or neutralizing process react moderately to strongly with some plastics.

\subsubsection{Step 7: Installing the Strain Gauges}

The syringes used in autoinjector devices are generally relatively small, and this is the case for a SureClick autoinjector. This makes the task of installing strain gauges on the syringe challenging. The strain gauges installed on the exterior wall of the syringe can't interfere with the motion of the components inside the autoinjector device, nor can the motion of the internal components interfere with the functioning of the strain gauges. This creates additional challenges and imposes restrictions on the maximum size of the strain gauges and the wiring between the strain gauges and the signal conditioners.

The in situ experiments reported in this chapter are all performed using miniature strain gauges C2A-06-015LW-120 from Vishay - Micro-Measurements. The size of the matrix is $1.9 \mathrm{~mm} \times 1.37 \mathrm{~mm}$, and the gauge size is $0.38 \mathrm{~mm} \times 0.50 \mathrm{~mm}$. The maximum strain which can be measured is approximately $3 \%(30,000 \mu \epsilon)$, which is adequate for both glass and plastic syringes. The resistance of the grid is $120 \mathrm{Ohms}$.

\footnotetext{
${ }^{1}$ The syringe surface needs to be degreased, conditioned, and neutralized even if the surface is not abraded.
} 
The signal conditioners used with the strain gauges are model $2310 \mathrm{~b}$ from Vishay Micro Measurements in a quarter-bridge configuration.

The procedure used to install the strain gauges matches as closely as possible the instructions provided by the manufacturer (Vishay - Micro-Measurement, 2010b). There are, however, a few deviations from the recommended process due to geometrical constrains.

The C2A-06-015LW-120 strain gauges come with pre-attached leadwires. One deviation from the procedures recommended by Vishay is to cut the leadwires of the strain gauges before bonding them to the syringe surface, leaving approximately 2 inches of leadwires attached to each gauge. The leadwires are very similar to magnet wires. There is an insulating coating protecting the conductive core, and it needs to be removed from the tip of the leadwires. As before, this is done using a razor blade, a heat source, or a solvant.

The strain gauges are then bonded to the surface of the syringe using an M-Bond 200 kit from Vishay - Micro-Measurements (Vishay - Micro-Measurement, 2018). After bonding each gauge, thumb pressure is applied and maintained on the gauge for at least two minutes to maximize adherence to the surface of the syringe. Maintaining a firm pressure on the strain gauge is important due to the small radius of curvature of the syringe.

A protective coating is applied on the strains gauges after they are all properly bonded to the syringe surface. For all experiments reported in this chapter, the strain gauges are coated using M-Coat A from Vishay - Micro-Measurement. MCoat $\mathrm{A}$ is a liquid polyurethane which insulates and protects the strain gauges from the environment.

The leadwires of the strain gauges are bonded to the surface of the syringe using regular adhesive tape. This is necessary to avoid pulling the strain gauges off from the surface of the syringe in the remaining steps. The adhesive tape can be removed before performing the experiment, but this is not necessary as long as there is an adequate strain relief loop (Vishay - Micro-Measurement, 2010b).

Finally, the quality of the strain gauge installation is verified as recommended by the manufacturer (Vishay - Micro-Measurement, 2010a). Figure 3.4 shows a simplified schematic of a syringe instrumented with strain gauges and a pressure transducer. The syringe is ready to be mounted in the autoinjector device. 


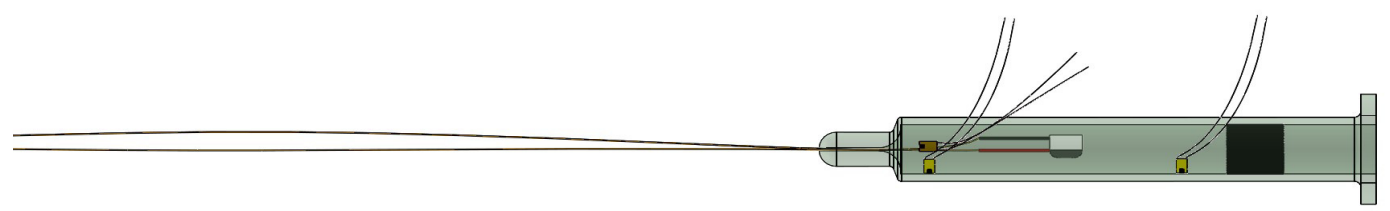

Figure 3.4: Schematic of a syringe instrumented with C2A-06-015LW-120 strain gauges and a PCB 138M186 pressure transducer.

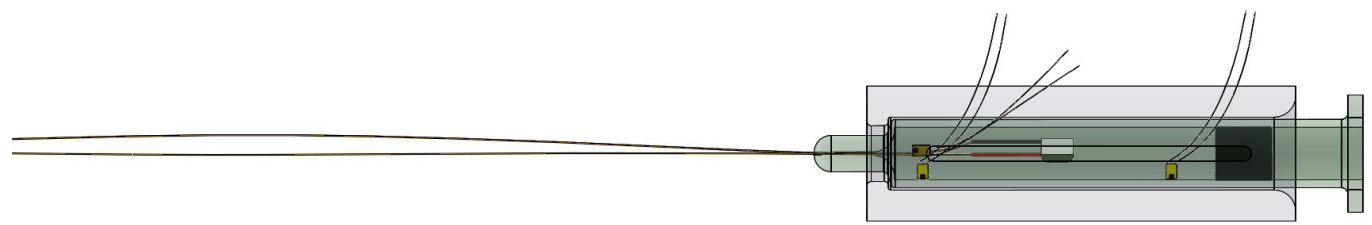

Figure 3.5: Schematic of an instrumented syringe mounted into a syringe carrier.

Note that up to six strain gauges were successfully installed on a pre-filled syringe mounted in a SureClick. However, it is recommended not to install more than three strain gauges on each syringe. Increasing the number of strain gauges increases the failure rate of the in situ experiments.

\subsubsection{Step 8: Assembling the Autoinjector}

The leadwires of the strain gauges attached to the surface of the syringe are wrapped around the syringe prior to mounting the syringe in the carrier. Then, the syringe is introduced and positioned inside the syringe carrier. Fine tweezers are used to pull the leadwires of each strain gauge through the oblong slit that was previously made in the syringe carrier. The result is similar to the simplified schematic shown in Figure 3.5.

Once this is done, the leadwires of the strain gauges are wrapped around the syringe carrier. The syringe carrier and the syringe are then introduced inside the main autoinjector shell. Following this, fine tweezers are used to pull the leadwires of the strain gauges through the window of the shell. Finally, the power pack is installed and secured in place. The final result is similar to what is shown in the simplified schematic of Figure 3.6.

\subsubsection{Step 9: Mounting the Autoinjector on a Support}

The autoinjector is mounted on a special fixture to keep it steady during actuation. The specific design of the fixture used to hold the instrumented device depends upon 


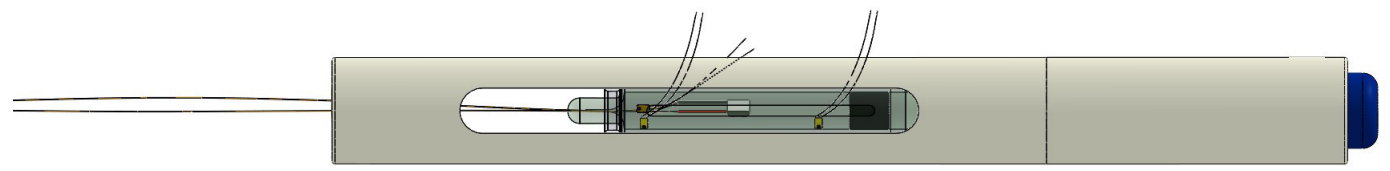

Figure 3.6: Schematic of a spring-actuated autoinjector device instrumented with strain gauges and a pressure transducer.

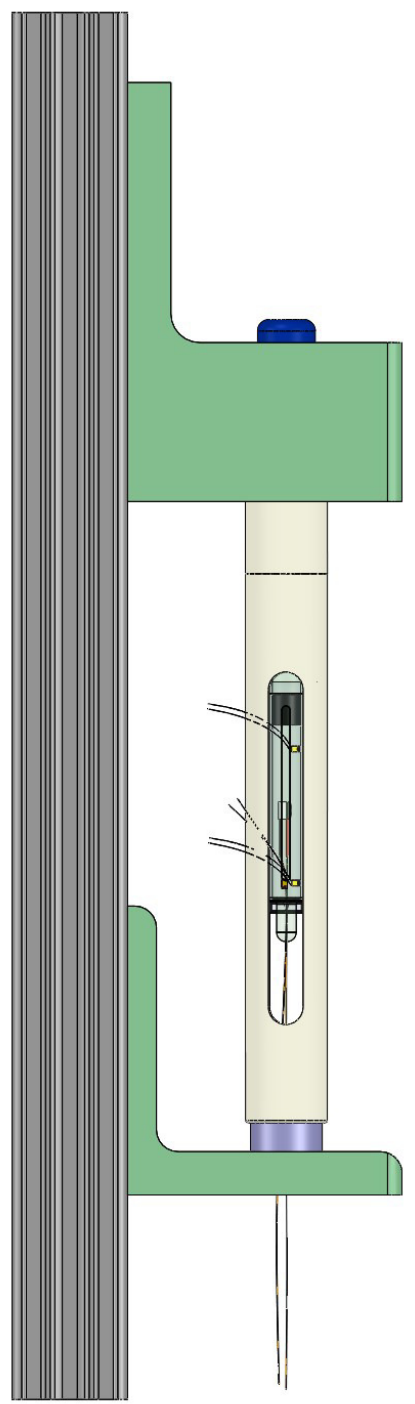

Figure 3.7: Schematic of an autoinjector device instrumented with strain gauges and a pressure transducer. The autoinjector is mounted in aluminum blocks on a T-slot extrusion. 
the geometry of the autoinjector under study. A fixture consisting of two custommade sliding mounts attached to a T-slot extrusion is used to hold the SureClick device. This is shown in Figure 3.7. The bottom sliding mount represents the patient's limb where the injection would take place, and the top sliding mount represents the patient's hand which would be holding the device.

The autoinjector is not rigidly mounted on the fixture. The clamping force is small and does not cause any deformation of the autoinjector's shell, and clamping of the device does not interfere with the motion of the internal components. Furthermore, the autoinjector is mounted on a deformable nylon ring which allows for some vibration of the device during actuation.

\subsubsection{Step 10: Final Preparation}

The pressure transducer and the strain gauges are reconnected to the signal conditioners and the amplifying electronics. All connections are tested for proper electrical connectivity and robustness. A loose connection often results in the cables becoming disconnected during actuation when the syringe is in motion.

A PCB 352A21 accelerometer is mounted on the top sliding mount, next to the power pack. A PCB 482A21 signal conditioner is used to power the accelerometer. The output signal from the accelerometer is used to reliably trigger the oscilloscope and the digital high-speed cameras upon release of the spring-actuated driving rod. A Yokogawa DL850 oscilloscope with a 12-bit vertical resolution and a sampling rate of $100 \mathrm{MHz}$ is used to acquire the experimental data.

In most experiments there are two high speed cameras mounted close to the autoinjector along with multiple bright light sources. The cameras used are a combination of Phantom v7.0g, v711, v1612, and v2011. The first camera is used to image the tip of the driving rod, the plunger-stopper, and the air gap. The second camera is used to image the bottom half of the syringe. The light sources are a combination of Visual Instrumentation Corporation model 201010B and GS Vitec model LT-V9-15. The final configuration is similar to what is schematically shown in Figure 3.8.

The signal-conditioners and amplifiers for the strain gauges need to be adjusted properly to optimize signal quality. Choosing the right excitation voltage for the strain gauges is critical: an excitation voltage which is too small results in a noisy signal, and an excitation voltage which is too large can result in overheating of the strain gauge. This can damage the strain gauge and the surface to which it is mounted. This is particularly true for plastic syringes. The proper excitation 


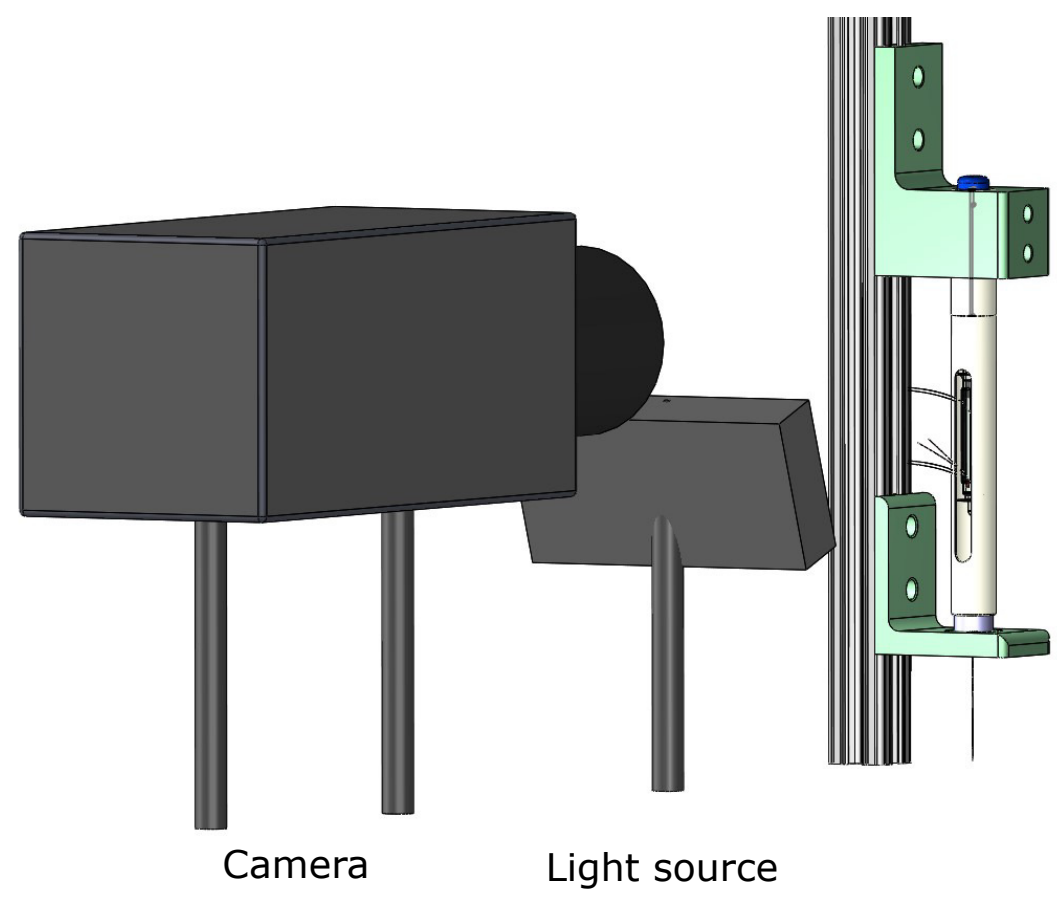

Figure 3.8: Schematic of an autoinjector device instrumented with strain gauges, a pressure transducer, and a high speed camera. The autoinjector is mounted in aluminum blocks on a T-slot extrusion.

voltage can be approximately determined using some charts, or it can be determined more accurately through experiments (Vishay - Micro-Measurement, 2010c). An excitation voltage of $1.0 \mathrm{~V}$ is used for all experiments performed with a glass syringe, and the excitation voltage is reduced to $0.5 \mathrm{~V}$ when a plastic syringe is used. The gain on the signal-conditioners is set to 800 in all cases.

The autoinjector is ready for actuation. Note that each syringe and each plungerstopper are used only once. This is because the inner surface of the syringe and/or the plunger-stopper are lubricated, and the motion of the plunger-stopper within the syringe alters the lubrication film. As a consequence of this, performing more than one experiment with the same syringe and/or plunger-stopper yields dissimilar results from one experiment to the other due to inconsistent friction forces.

\subsubsection{Limitations}

One limitation of the techniques described above is that instrumentation of the syringe is limited to its barrel. Unfortunately, the pressure transducer is too large to be installed within the conical section of the syringe. Also, the outer surface of the syringe in the vicinity of the cone and the tip of the syringe is generally irregular, 
and the radius of curvature is small. This makes it impossible to attach strain gauges to the surface of the syringe in this region.

Another limitation comes from the device supports, which are stiffer than in a real life application where a patient would use his hand to hold the device. It is possible the user won't maintain the device in a perfectly vertical orientation, something which is not accounted for. Furthermore, the user hand is likely to apply a force on the shell of the autoinjector which is not only oriented in the axial direction, and this could create a circumferential deformation of the shell of the autoinjector. In a real application, there would also be a small but detectable jerking motion of the device, creating axial and lateral motion of the shell of the autoinjector during actuation. This can affect the location of the air gap and create deformation of the shell. Also, the insertion of the needle into human tissues is expected to create friction on the needle, and this could reduce the peak magnitude of syringe acceleration. The effects of those elements should be investigated in a future study.

\subsection{Results and Discussion}

Typical results for four different configurations are shown and discussed in this section. The four configurations are:

- configuration 1: glass syringe without an air gap;

- configuration 2: glass syringe with an air gap;

- configuration 3: plastic syringe without an air gap;

- configuration 4: plastic syringe with an air gap.

All results are obtained using a SureClick autoinjector. A BD HyPack $1 \mathrm{~mL}$ glass syringe is used in configurations 1 and 2. A Daikyo Crystal Zenith $1 \mathrm{~mL}$ plastic syringe from West Pharma is used in configurations 3 and 4. The estimated physical properties and the approximate dimensions of both syringes are summarized in Table 3.1. Note that Appendix B contains a list of all in situ tests which have been performed to date.

In all four configurations the autoinjector device is maintained in a vertical, tipdown configuration throughout actuation, as shown in Figure 3.7. When present, the air gap is therefore located immediately below the plunger-stopper, as shown in Figure 2.3. Without an air gap there is a direct contact between the plunger-stopper and the liquid content. 
Table 3.1: Estimated physical and geometrical properties of a BD HyPack and a Daikyo Crystal Zenith pre-filled syringe.

\begin{tabular}{llll}
\hline Property & BD HyPack & Daikyo & Units \\
\hline Material & Borosilicate & Cyclic-olefin polymer & - \\
Density $\left(\rho_{s}\right)$ & 2230 & 1020 & $\mathrm{~kg} / \mathrm{m}^{3}$ \\
Young's modulus $(E)$ & 69 & 2.6 & $\mathrm{GPa}$ \\
Poisson's ratio $(v)$ & 0.3 & 0.3 & - \\
Barrel inner diameter $\left(D_{b, i}\right)$ & 6.35 & 6.25 & $\mathrm{~mm}$ \\
Barrel outer diameter $\left(D_{b, o}\right)$ & 8.15 & 7.95 & $\mathrm{~mm}$ \\
Syringe length $\left(L_{s}\right)$ & 64.0 & 64.0 & $\mathrm{~mm}$ \\
Barrel length $\left(L_{b}\right)$ & 54.4 & 54.6 & $\mathrm{~mm}$ \\
\hline
\end{tabular}

The liquid content of the syringe is either di-ionized water or a drug solution. Even if only four typical cases are reported in this chapter, a large number of tests were performed with water or a drug solution in the syringe. There is no measurable variation between the results obtained with water or a drug solution during the transient events. The extrusion phase, on the other hand, is highly dependent on the viscosity of the liquid. The extrusion phase is not discussed herein.

The viscosity of the de-ionized water is approximately $1 \mathrm{cP}$. The proprietary drug solution has a viscosity which is between 8 to $12 \mathrm{cP}$. The exact value of the viscosity depends primarily on the temperature of the drug solution. The density and sound speed of the drug solution and water are similar - our measurements indicate the acoustic impedance of the drug solution is approximately $10 \%$ larger than the acoustic impedance of water.

The syringes are instrumented with a pressure transducer and three strain gauges using the methods described in Section 3.1. The strain gauges are positioned and oriented as shown in Figure 3.4. There is one strain gauge below the plunger-stopper and one strain gauge above the cone of the syringe to measure the circumferential deformation of the glass (i.e., the hoop strains $\epsilon_{\theta}$ ). In addition, there is a strain gauge above the cone of the syringe to measure the axial deformation of the glass (i.e., the axial strains $\epsilon_{z}$ ). The pressure transducer is located approximately half-way between the plunger-stopper and the cone of the syringe. 


\subsubsection{Configuration 1: Glass Syringe Without an Air Gap}

The first configuration studied is that of a glass syringe without an air gap (see Figure 2.3). A BD HyPack $1 \mathrm{~mL}$ syringe filled with drug solution is used. Multiple experiments were performed in this configuration, but only one representative case is reported.

The position and velocity of the driving rod, the plunger-stopper and the syringe are obtained from careful post-processing of the videos recorded with a digital high-speed camera at a rate of 14,000 frames per second. The built-in feature of the Phantom Camera Control software is used to extract the axial position of the internal components. Matlab is used to differentiate the position to obtain the velocity and acceleration. A Savitzky-Golay filter is applied on the position history prior to performing the numerical differentiation to reduce noise. Note that the driving rod is tracked using its tip, the plunger-stopper is tracked using its top surface (i.e., the surface which is not in contact with the liquid drug solution), and the syringe is tracked using the topmost surface of its flange.

The position and the velocity of each component are shown in Figure 3.9. The system of coordinates shown in Figure 2.3 is used. The reference for the measurement of all axial locations is the syringe tip.

Initially, prior to actuation, all internal components of the autoinjector are at rest. Actuation of the device occurs between $-2 \mathrm{~ms}$ and $-0.2 \mathrm{~ms}$. This is when the user depresses the actuation button. The motion of the actuation button produces some motion of the internal components during that time period, but it is not substantial. This minimal motion of the internal components is necessary before the internal mechanism of the power pack can release the spring-actuated driving rod. This is also what determines the minimal initial separation between the driving rod and the top surface of the plunger-stopper.

The spring-actuated driving rod is released at approximately $-0.2 \mathrm{~ms}$. Following its release, the driving rod accelerates rapidly toward the plunger-stopper. At $0 \mathrm{~ms}$ the driving rod impacts on the top surface of the plunger-stopper at a velocity of approximately $6.5 \mathrm{~m} / \mathrm{s}$. The initial average acceleration of the plunger-stopper and the syringe following this impact event is approximately $15,000 \mathrm{~m} / \mathrm{s}^{2}$ in magnitude, or 1,500 times the gravitational acceleration. The acceleration of the syringe occurs nearly simultaneously with the acceleration of the plunger-stopper (within $0.1 \mathrm{~ms}$ ). 


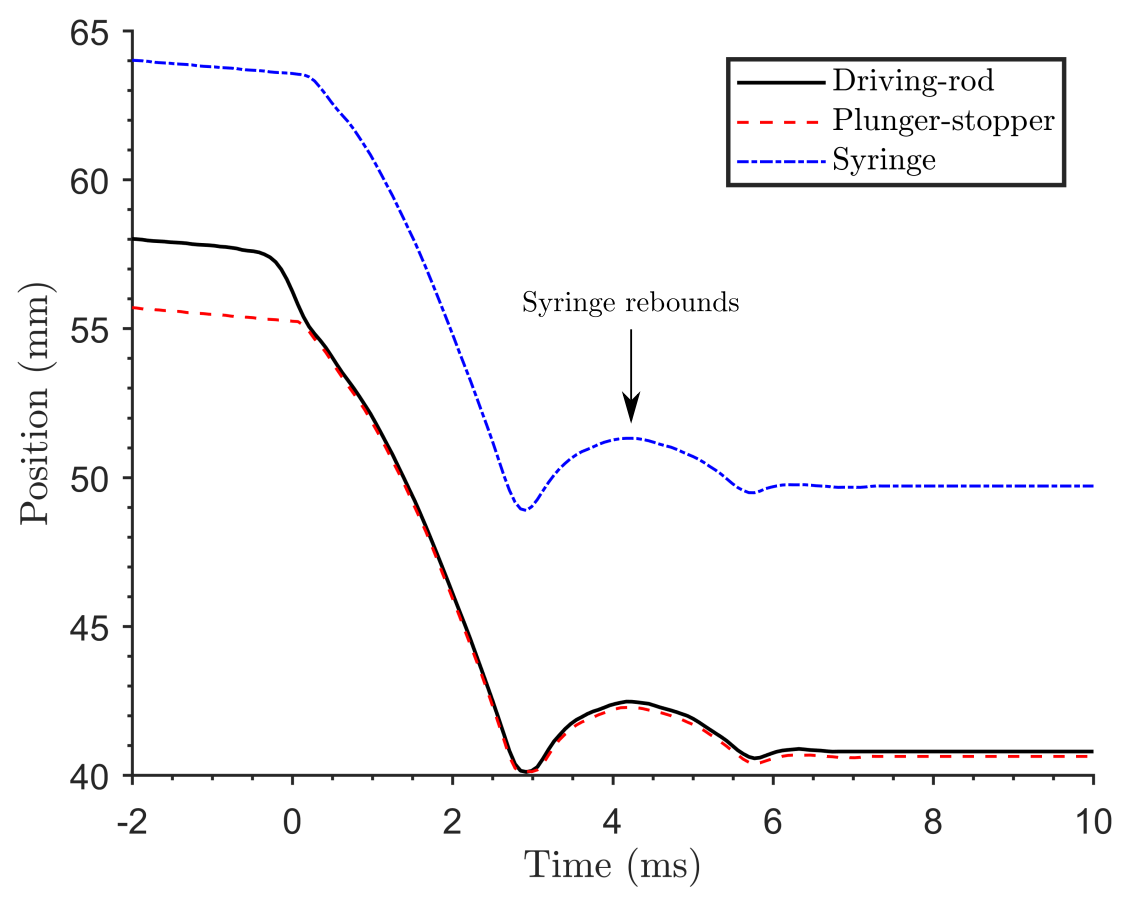

(a) Position

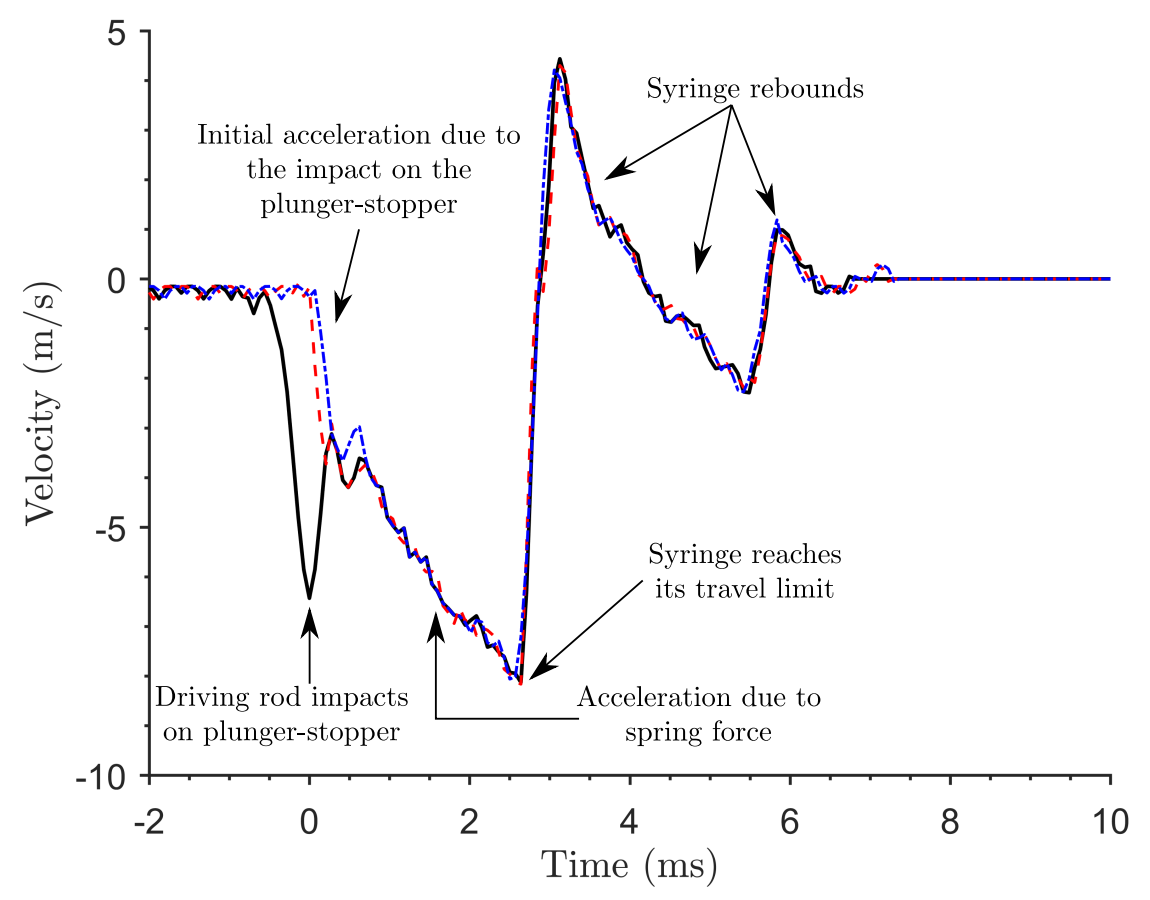

(b) Velocity

Figure 3.9: Position and velocity of the moving components in a SureClick autoinjector - glass syringe without an air gap (test SC-033). 


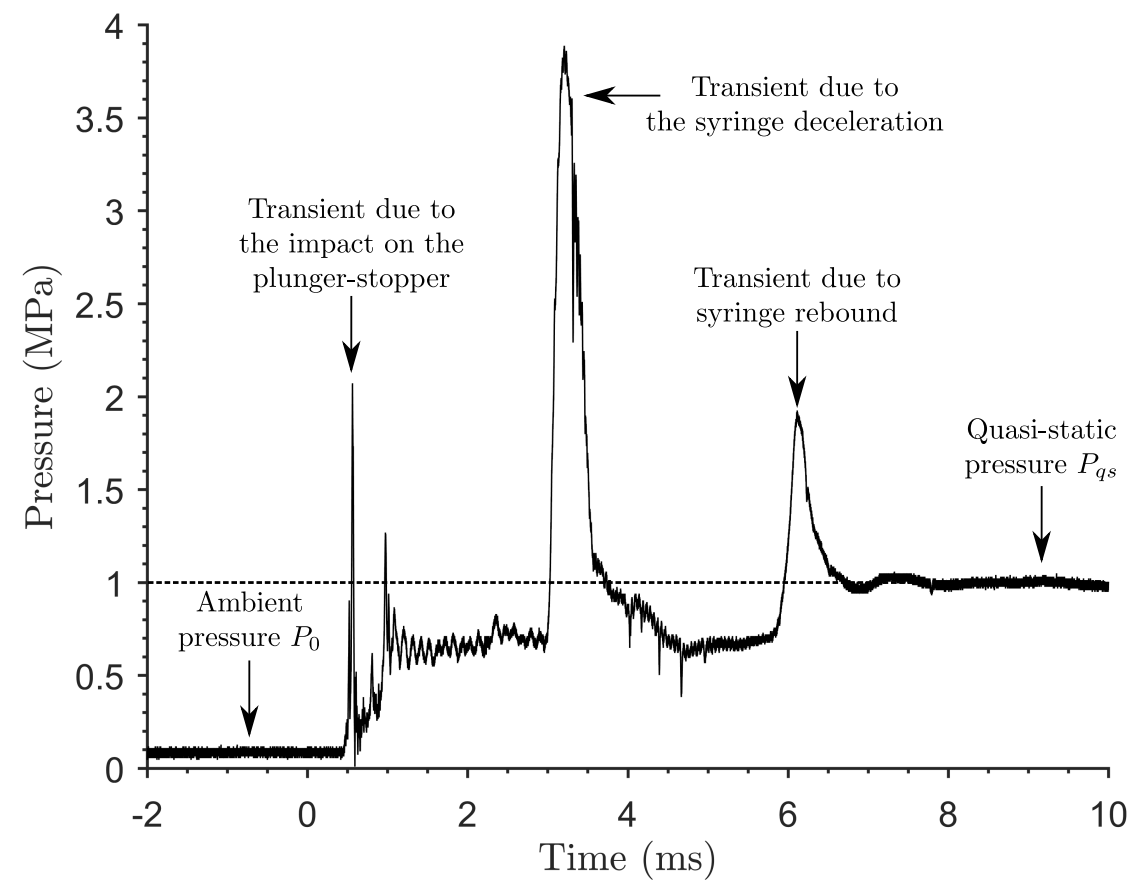

Figure 3.10: Liquid pressure in a SureClick autoinjector - glass syringe without an air gap (test SC-033).
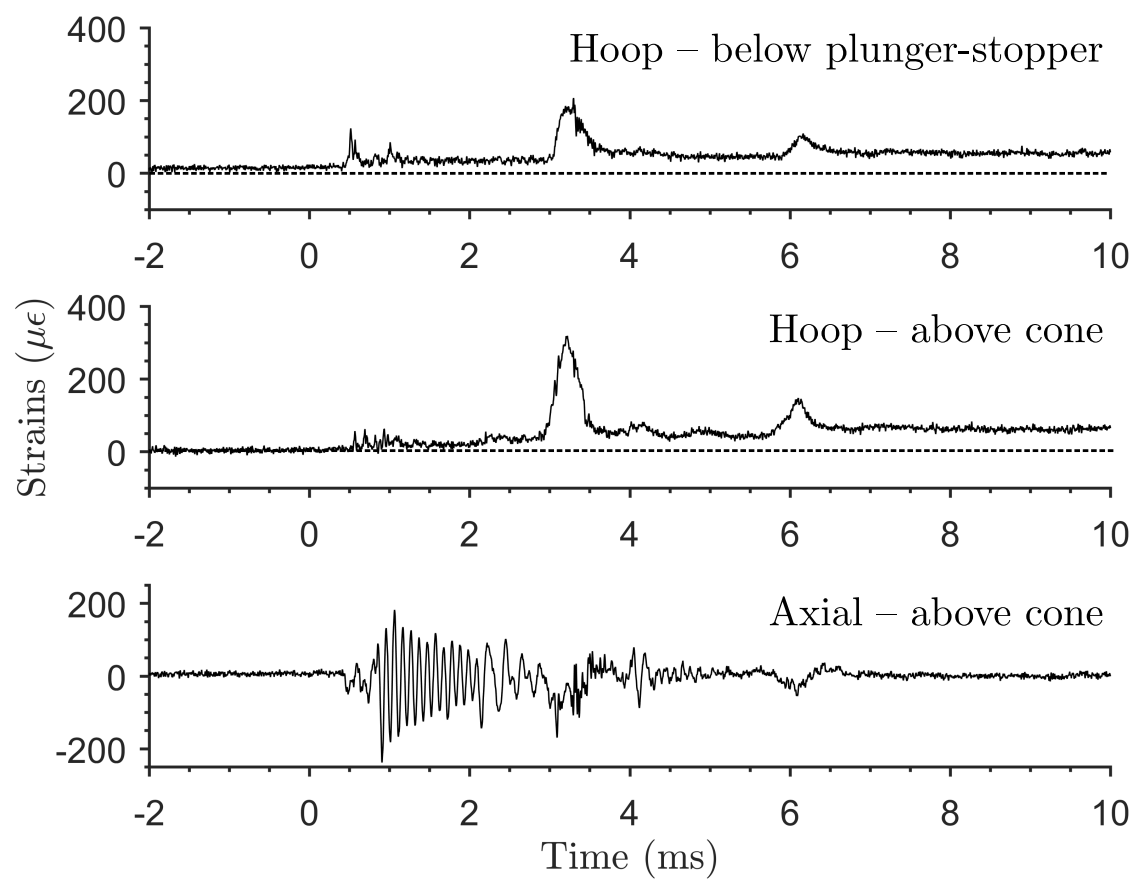

Figure 3.11: Hoop and axial strains on the barrel of the syringe in a SureClick autoinjector - glass syringe without an air gap (test SC-033). 
The initial acceleration of the syringe is created by two mechanisms. First, there is friction between the plunger-stopper and the syringe. Second, the liquid pressure on the bottom wall of the syringe accelerates the syringe downward. When there is a direct contact between the plunger-stopper and the liquid, the liquid pressure rises rapidly after the impact event, promptly initiating acceleration of the syringe. The peak internal liquid pressure during this acceleration event is approximately $2 \mathrm{MPa}$ (see Figure 3.10). This results in a downward force of $56 \mathrm{~N}$ on the syringe and the syringe carrier. The combined mass of the syringe and the syringe carrier is approximately $8 \mathrm{~g}$, and Newton's Second Law of motion implies that the magnitude of the acceleration due to the liquid pressure applied on the syringe is about $7,000 \mathrm{~m} / \mathrm{s}^{2}$. This result suggests the magnitude of the acceleration due to friction between the plunger-stopper and the syringe is approximately $8,000 \mathrm{~m} / \mathrm{s}^{2}$. This analysis indicates that friction between the syringe and the plunger-stopper as well as pressurization of the liquid are equally important in creating the initial acceleration of the syringe.

The initial, rapid acceleration of the syringe is followed by a much smaller acceleration of constant magnitude. This takes place between $0.75 \mathrm{~ms}$ and $2.5 \mathrm{~ms}$, and the magnitude of the acceleration is approximately $2,000 \mathrm{~m} / \mathrm{s}^{2}$. During that time period there is no relative motion between the driving rod, the plunger-stopper and the syringe; all components are moving together. This constant acceleration results from the steady spring force of approximately $28 \mathrm{~N}$ applied on the plunger-stopper. The total mass accelerated by the spring is approximately $12.5 \mathrm{~g}$. Newton's Second Law implies the expected acceleration is $2,240 \mathrm{~m} / \mathrm{s}^{2}$, comparable to the measured acceleration of $2,000 \mathrm{~m} / \mathrm{s}^{2}$.

Approximately $2.5 \mathrm{~ms}$ after the impact of the driving rod on the plunger-stopper, the syringe, the plunger-stopper and the driving rod are all traveling downward at a velocity of approximately $8 \mathrm{~m} / \mathrm{s}$ when the syringe suddenly reaches its travel limit. All components are then rapidly decelerated. The average magnitude of the deceleration is approximately $36,000 \mathrm{~m} / \mathrm{s}^{2}$. This means the shell of the autoinjector applies a force of $450 \mathrm{~N}$ on the assembly formed by the syringe and its content, the syringe carrier, the plunger-stopper, the driving rod and the spring. The assembly does not immediately come to rest after reaching its travel limit: it rebounds once between $3 \mathrm{~ms}$ and $6 \mathrm{~ms}$. The rebound of the assembly is primarily due to the compliance and elasticity of the syringe carrier and the shell of the autoinjector.

The transient events end approximately $8 \mathrm{~ms}$ after the impact of the driving rod on the plunger-stopper. When the syringe comes to rest, the plunger-stopper and 
the driving rod are pushed into the barrel of the syringe to extrude the liquid drug. The motion of the plunger-stopper and the driving rod during this quasi-static phase of actuation is very small however $(\approx-0.004 \mathrm{~m} / \mathrm{s})$, and it can't be observed in Figure 3.9 because of the vertical scale. The velocity of the moving components is approximately three orders of magnitude larger during the transient events than it is during the quasi-static extrusion phase.

The liquid pressure history is shown in Figure 3.10. Note that pressures are indicated as absolute pressure in this chapter, not relative or gauge pressure. Prior to actuation of the autoinjector the liquid is at atmospheric pressure $P_{0}$. After the transient events are over (i.e., after $8 \mathrm{~ms}$ ), the pressure in the syringe is approximately constant and equal to $1 \mathrm{MPa}$, or 10 times atmospheric pressure. This corresponds to the extrusion pressure $\left(P_{q s}+P_{0}\right)$, where $P_{q s}$ is the quasi-static pressure created by the spring force applied on the plunger-stopper:

$$
P_{q s}=\frac{F}{A_{b, i}},
$$

where $A_{b, i}$ is the inner cross-sectional area of the syringe barrel.

In the first $8 \mathrm{~ms}$ following the release of the driving rod there are three transient events with significant pressure excursions above the extrusion pressure of $1 \mathrm{MPa}$. The first excursion of the pressure above $1 \mathrm{MPa}$ occurs at around $0.5 \mathrm{~ms}$ when the pressure rapidly jumps to approximately $2 \mathrm{MPa}$. This pressure increase results from the impulsive acceleration of the plunger-stopper into the liquid as described in Section 2.2 (event 3). Equation 2.21 is used to estimate the pressure rise $\Delta P$ due to the impulsive acceleration of the plunger-stopper. The experimental results indicate the velocity of the plunger-stopper after the impact of the driving rod is $3.7 \mathrm{~m} / \mathrm{s}$, and one obtains that $\Delta P=5.5 \mathrm{MPa}$. This is substantially more than what is measured experimentally because Equation 2.21 assumes the creation of pressure waves in the liquid is decoupled from the acceleration of the syringe, but this is not the case. The coupling between both events results from significant friction between the plunger-stopper and the syringe. For this reason Equation 2.21 represents an upper bound on the expected peak magnitude of the pressure pulse.

Following the impulsive acceleration of the plunger-stopper, the syringe is rapidly accelerated (event 1 of Section 2.2), creating tension waves within the liquid. This results in the pressure dropping immediately after the driving rod impact event, another factor in the reduction of the peak pressure from the ideal value predicted by Equation 2.21. In the present case of no air gap, the liquid is pressurized at the 
same time as the syringe is accelerated. This has the important consequence that the tension waves created through syringe acceleration are not sufficient to reduce the pressure to or below the vapor pressure $P_{v a p}$ of the liquid content. The cavity formation shown in Figure 2.5 is suppressed, and the pressure transient shown in Figure 2.6 is eliminated: cavitation does not occur.

The second excursion of the pressure occurs between 2.5 and $3.5 \mathrm{~ms}$, resulting from the rapid deceleration of the syringe (event 2 of Section 2.2). During the deceleration of the syringe the liquid pressure reaches $3.9 \mathrm{MPa}$, or close to 40 times atmospheric pressure. This is a pressure which is almost four times larger than the extrusion pressure. The peak liquid pressure upon deceleration of the syringe can't be estimated using Equation 2.5. This is because the deceleration of the syringe is not impulsive and falls within regime 2, as explained in Section 2.2 (event 2). The deceleration of the syringe occurs over $0.5 \mathrm{~ms}$, and this leaves enough time for the pressure waves to complete approximately 10 round trips within the liquid throughout the deceleration. Equation 2.20 is used to estimate the magnitude of the pressure increase due to the syringe deceleration: $\Delta P=2.9 \mathrm{MPa}$. However, because the liquid pressure before the deceleration begins is already $0.65 \mathrm{MPa}^{2}$, the expected peak pressure at the bottom wall of the syringe during the rapid deceleration is the sum, or $(2.9+0.65) \mathrm{MPa}=3.55 \mathrm{MPa}$. This compares relatively well with the measured value of $3.9 \mathrm{MPa}$.

The third excursion of the pressure occurs between 6 and $7 \mathrm{~ms}$, and results from the rebound of the syringe. The origin of this transient event is identical to that of the transient taking place between 2.5 and $3.5 \mathrm{~ms}$ : it is due to the deceleration of the syringe after the rebound. The maximum pressure due to this second deceleration of the syringe is approximately $2 \mathrm{MPa}$. This is two times less than the peak pressure observed during the first deceleration because the deceleration is of lesser magnitude.

The strain signals are shown in Figure 3.11. A strain gauge measures the ratio of total deformation to the initial dimension of the material to which it is attached $(\epsilon=\Delta L / L)$ (Hannah and Reed, 1992). The strains are indicated in microstrains $(\mu \epsilon)$, and $1 \mu \epsilon$ corresponds to a deformation of $1 \times 10^{-4} \%$. A positive strain indicates the material is being stretched, or is under tension, and a negative strain indicates the material is being compressed.

\footnotetext{
${ }^{2}$ The pressure in the syringe is larger than $P_{0}$ before the deceleration occurs due to the spring force applied on the plunger-stopper.
} 
Hoop strain signals exhibit the same features as the pressure signal because the circumferential deformation of the syringe is primarily caused by the liquid pressure. The circumferential deformation is initially zero because the liquid pressure is initially the same as atmospheric pressure. After the transient events (i.e., after $8 \mathrm{~ms}$ ) there is a residual, positive hoop strain of approximately $50-60 \mu \epsilon$. The residual hoop strain is due to the extrusion pressure of $1 \mathrm{MPa}$ that remains in the syringe. This can be confirmed using a static shell theory to relate hoop strains and internal pressure (Jones, 1989):

$$
\epsilon_{\theta}=\frac{\left(P-P_{0}\right)}{2}\left(\frac{D_{b, o}+D_{b, i}}{D_{b, o}-D_{b, i}}\right) \frac{\left(1-v^{2}\right)}{E},
$$

where $E$ and $v$ are respectively the Young's modulus and the Poisson's ratio of the syringe material. Using the values shown in Table 3.1, Equation 3.2 predicts a hoop strain of $52 \mu \epsilon$ for an internal pressure of $1 \mathrm{MPa}$. This strain is in reasonable accord with the observed values.

The transient events in the syringe result in much larger hoop strains, up to $320 \mu \epsilon$, or over 6 times the strains observed during the extrusion phase of operation. This is larger than the factor of 4 previously measured between the peak pressure and the extrusion pressure, indicating the strains are created by the combined effect of the internal liquid pressure and the stress waves created directly in the syringe wall. The largest peak value of the measured hoop strains occurs when the syringe is rapidly decelerated, and the liquid pressure is maximum. The peak hoop strain is larger at the bottom of the syringe, immediately above the syringe shoulder, where the point of contact between the syringe and the shell of the autoinjector is located. The liquid pressure is expected to be larger in this region due to the reflection of pressure waves at the bottom of the syringe.

The expansion of the syringe in the circumferential direction due to the internal pressure causes the glass to contract in the axial direction. This is known as the Poisson effect (Hibbeler, 2010). Under a uniaxial stress, when only one stress component is non-zero, there is a simple relation between the axial and the hoop strains (Hibbeler, 2010):

$$
\epsilon_{z}=-v \epsilon_{\theta} .
$$

It is possible to use the results from Figure 3.11 to show that Equation 3.3 is reasonably well verified before $2.5 \mathrm{~ms}$ and after $8 \mathrm{~ms}$, which suggests the hoop stress is the dominant stress component during those time periods, and that stress is primarily uniaxial. 
The high frequency oscillations in the axial strains observed between $0.75 \mathrm{~ms}$ and $2.5 \mathrm{~ms}$ have not been explained yet. The peak magnitude of the high frequency signal is approximately $200 \mu \epsilon$. The $56 \mathrm{~N}$ pressure force applied on the syringe tip is expected to create a reverberating axial stress wave, but the predicted magnitude of the strains resulting from this wave is $40 \mu \epsilon$, inconsistent with the magnitude of the high frequency oscillations. The high frequency oscillations could result from the excitation of a natural frequency of the coupled system formed by the syringe carrier, the syringe, and the liquid it contains. This could also be the result of an interaction between the syringe and the syringe carrier or the shell into which it is sliding.

The axial strains resulting from the rapid deceleration of the syringe at around $2.5 \mathrm{~ms}$ are more complex. A part of the axial strains again results from the Poisson effect: the large internal pressure creates a circumferential deformation which, in turn, creates an axial deformation. There is however an additional component to the axial strains which comes from the stress wave created within the glass itself as the syringe is decelerated. This stress wave originates from the $450 \mathrm{~N}$ force generated by the contact between the syringe assembly and the shell of the autoinjector.

It is possible to estimate the peak axial stress $\sigma_{z}$ in the glass upon the rapid deceleration of the syringe. The syringe deceleration falls in regime 2 (see event 2 of Section 2.2). Equation 2.19 predicts a peak axial stress of $-8.7 \mathrm{MPa}$ for an acceleration of $36,000 \mathrm{~m} / \mathrm{s}^{2}$ and a glass syringe barrel length of $54 \mathrm{~mm}$.

The stresses in the syringe are inferred from the measured strains shown in Figure 3.11. The calculation uses Hooke's law assuming plane stress (Bower, 2009; Young and Budynas, 2001). Results discussed earlier have shown the stresses are primarily uniaxial before $2.5 \mathrm{~ms}$ and after $8 \mathrm{~ms}$, but this is not necessarily the case between $2.5 \mathrm{~ms}$ and $8 \mathrm{~ms}$. For the plane stress assumption to be valid requires the radial stress to be negligible relative to the axial and hoop stresses. This is the case on the outer surface of the syringe barrel where the measurements are performed. The syringe carrier does not apply any significant radial force on the exterior wall of the syringe barrel. The hoop $\left(\sigma_{\theta}\right)$ and axial $\left(\sigma_{z}\right)$ stresses are:

$$
\begin{gathered}
\sigma_{\theta}=\frac{E}{1-v^{2}}\left(\epsilon_{\theta}+v \epsilon_{z}\right), \\
\sigma_{z}=\frac{E}{1-v^{2}}\left(\epsilon_{z}+v \epsilon_{\theta}\right) .
\end{gathered}
$$


The maximum hoop stress is $\sigma_{\theta} \approx 22 \mathrm{MPa}$, and the maximum axial stress is $\sigma_{z} \approx-8.3 \mathrm{MPa}$. The maximum axial stress is in reasonable agreement with the value obtained using Equation 2.19.

The failure of glass, a brittle material, is often predicted using the maximum principal stress theory (Bower, 2009; Hibbeler, 2010). The theory states that failure occurs when the maximum principal stress $\sigma_{1}$ exceeds the uniaxial tensile strength of the material. The principal stresses $\sigma_{1}>\sigma_{2}>\sigma_{3}$ are obtained through diagonalization of the stress tensor (Bower, 2009).

The principal stresses are not directly measured experimentally but inferred from hoop and axial strain measurements or simulations. From both finite-element simulations and the Lamé solution for a long cylinder under pressure (Bower, 2009) we know that the shear stresses are negligible along the barrel of the syringe. This is demonstrated later in Chapter 5. Therefore, $\sigma_{1} \approx \sigma_{\theta}$, and we infer the peak value of the maximum principal stress on the outer surface of the syringe is $22 \mathrm{MPa}$. The stresses on the inner surface of the barrel in the vicinity of the cone, where stress concentrations occurs, or where the shear stresses are non-negligible, can be significantly larger than $22 \mathrm{MPa}$. This is discussed further in Chapter 5 with the help of simulation results obtained through finite-element methods.

The magnitude of the stress is one of several factors to consider in evaluating the potential for syringe failure. A complete assessment of the probability of glass failure is challenging (Callister and Rethwisch, 2014; McLellan and Shand., 1984). Glass is a brittle material which fails in tension, and its failure is mainly governed by the presence of microscopic flaws where stress concentration occurs (Callister and Rethwisch, 2014). There exists a large gap between the theoretical strength and the practical strength of glass. There is also great variability in the permissible working stress (i.e., the permissible maximum value of $\sigma_{1}$ ), the value of which is influenced by factors such as the type of glass, the condition of the glass and its surface, and the heat or chemical treatment applied during manufacturing (McLellan and Shand., 1984). Typical values of the working stress of glass can vary somewhere between 6 MPa and $90 \mathrm{MPa}$ (McLellan and Shand., 1984). This suggests the transient events taking place within the syringe when there is no air gap produce tensile stresses which are capable of causing failure of the glass syringe at a low but detectable occurrence rate.

To summarize, pressure and strain measurements were performed along the barrel of a glass syringe without an air gap between the plunger-stopper and the liquid. 
The results demonstrate that the transient events taking place within the first $8 \mathrm{~ms}$ of device actuation can result in significant accelerations and decelerations of the components, and in substantial impact velocities. As a consequence, the instantaneous pressure reaches values which are up to four times higher than the pressure required for drug extrusion. These transient internal pressures create measurable strains within the syringe, and we infer that significant stresses are created within the glass. The impact of the syringe on the shell of the autoinjector also creates strains and associated stresses within the syringe.

Note that the results obtained in configuration 1 are also representative of the case of a syringe which has an air gap, but is positioned in a vertical, tip-up configuration. In this configuration, the air gap is located in the tip of the syringe, and there is a direct contact between the plunger-stopper and the liquid.

\subsubsection{Configuration 2: Glass Syringe With an Air Gap}

The second configuration studied is that of a glass syringe with an air gap between the plunger-stopper and the liquid (see Figure 2.3). A BD HyPack $1 \mathrm{~mL}$ syringe filled with the same drug solution as in configuration 1 is used. Multiple experiments were performed in this configuration with various air gap sizes, but only one representative case with an initial air gap size $\delta_{0}$ of $3 \mathrm{~mm}$ is reported and discussed. The autoinjector is maintained in a vertical, tip-down configuration, meaning the air gap is located below the plunger-stopper.

The position and velocity of the driving rod, the plunger-stopper and the syringe are shown in Figure 3.12. There is a gap in the syringe's motion data between $1.6 \mathrm{~ms}$ and $2.2 \mathrm{~ms}$ because the motion of the plunger-stopper and the driving rod is not accessible throughout the entire test. Data from other tests has shown that the plunger-stopper, the driving rod, and the syringe all move together 1.25 to $1.5 \mathrm{~ms}$ after the impact of the driving rod on the plunger-stopper.

The impact of the driving rod on the plunger-stopper occurs at $0 \mathrm{~ms}$. There are noticeable differences between Figure 3.9 (no air gap) and Figure 3.12 (with an air gap). One difference is the change in timing between plunger-stopper and syringe acceleration. Without an air gap the syringe and the plunger-stopper are accelerated together at the same rate, but this is not the case when an air gap is present.

Just after impact of the driving rod on the plunger-stopper the average acceleration of the plunger-stopper is approximately $27,000 \mathrm{~m} / \mathrm{s}^{2}$, and the average acceleration of the syringe is approximately $7,000 \mathrm{~m} / \mathrm{s}^{2}$. The acceleration of the plunger-stopper 


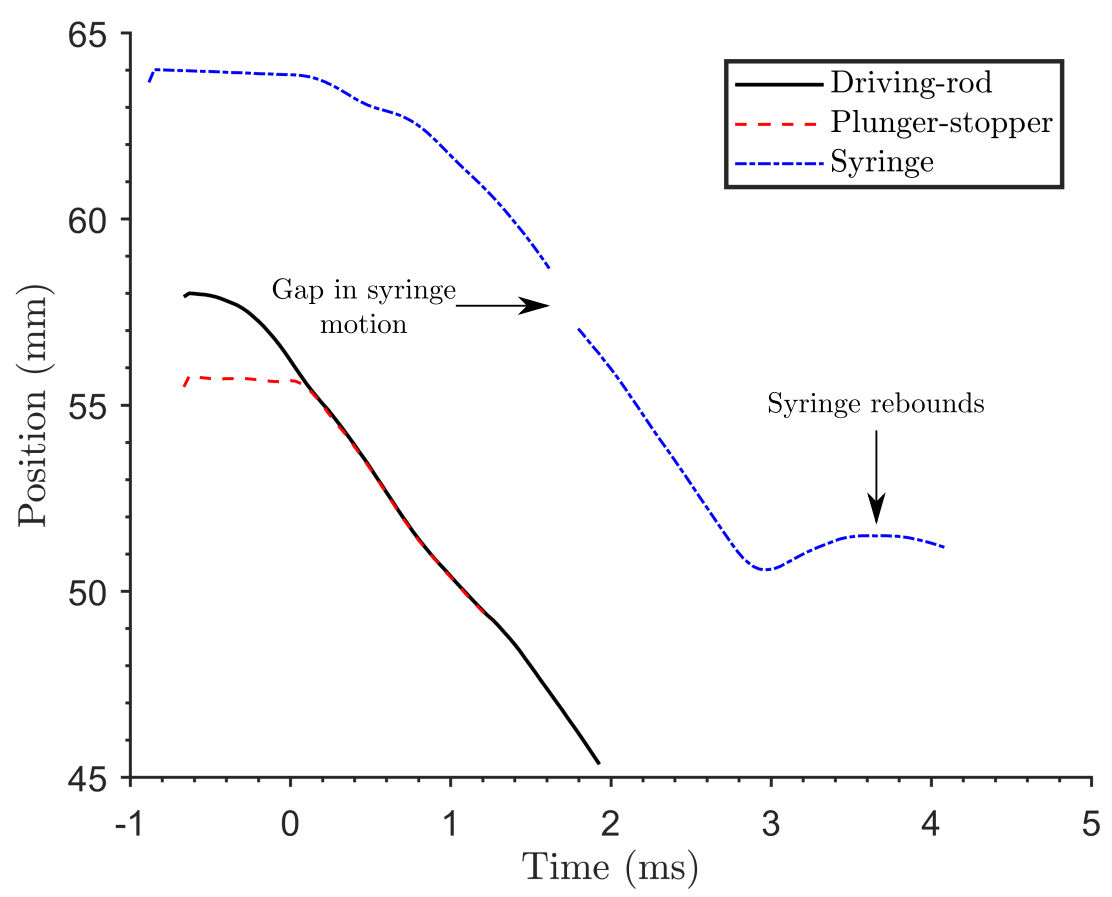

(a) Position

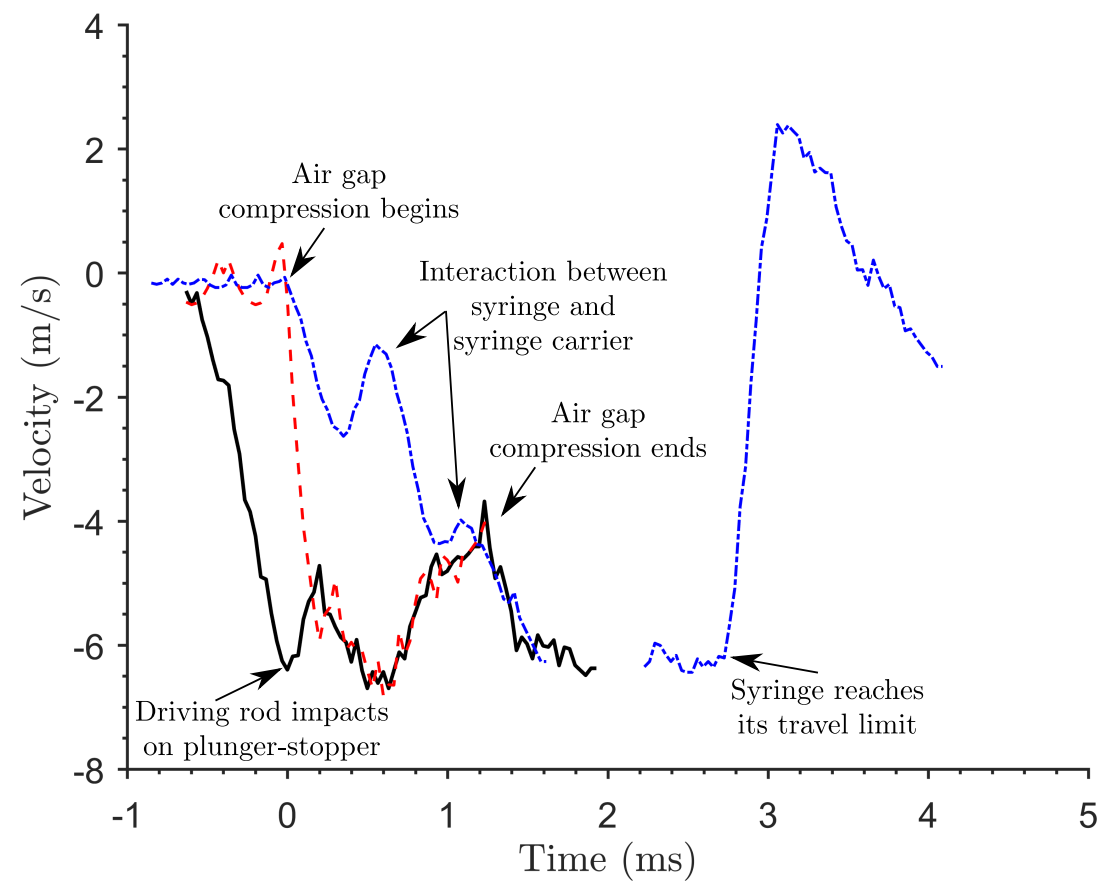

(b) Velocity

Figure 3.12: Position and velocity of the moving components in a SureClick autoinjector - glass syringe with an air gap (test SC-178). 


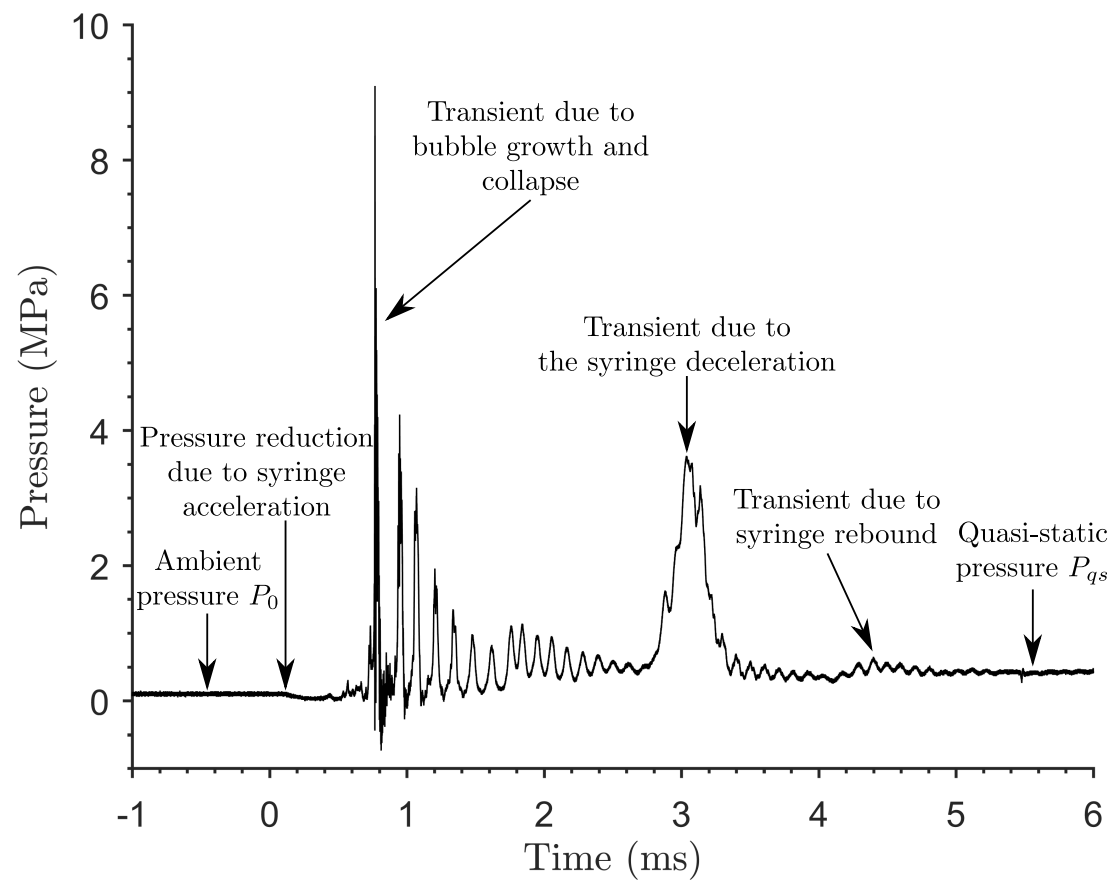

Figure 3.13: Liquid pressure in a SureClick autoinjector - glass syringe with an air gap (test SC-178).
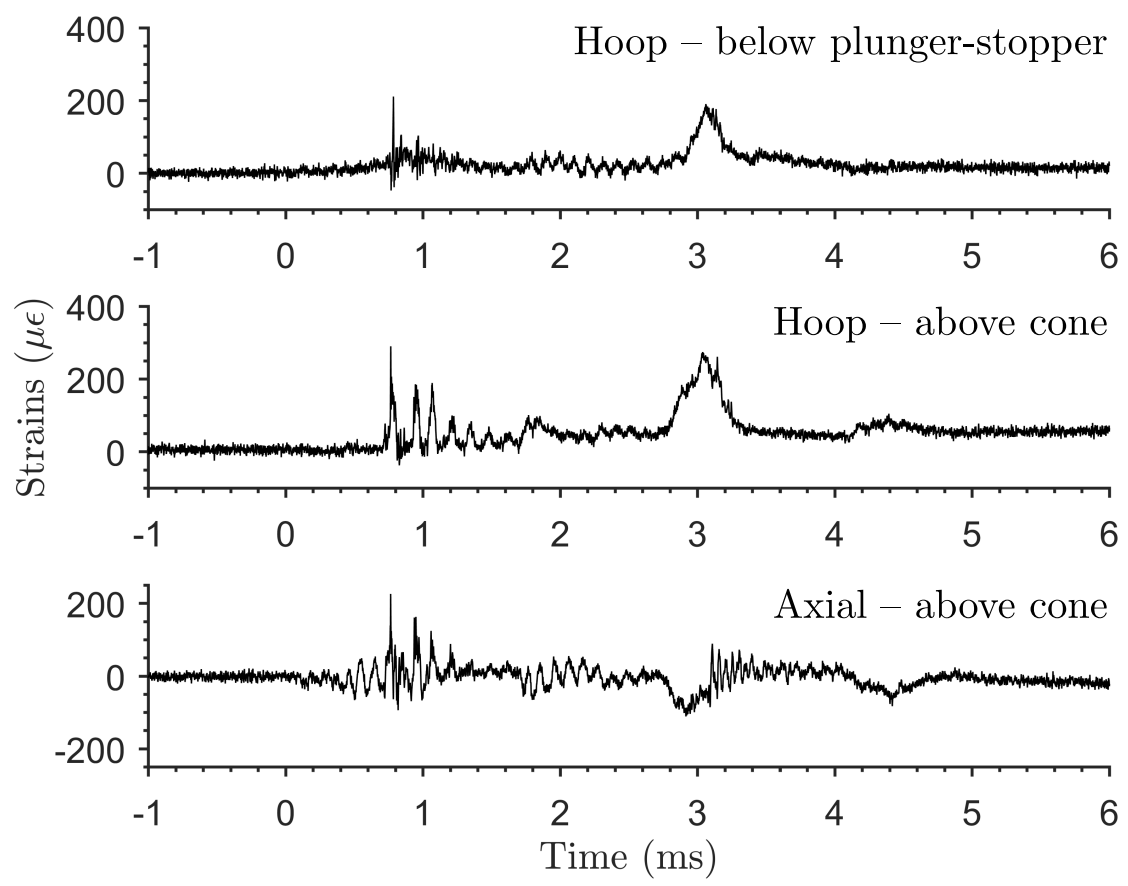

Figure 3.14: Hoop and axial strains on the barrel of the syringe in a SureClick autoinjector - glass syringe with an air gap (test SC-178). 
is almost twice as large as for the no-air-gap case due to the slower response of the air gap to compression in comparison to the liquid; the pressure within the air gap takes a relatively long time to increase. Without an air gap the pressure below the plunger-stopper increases abruptly after impact, immediately accelerating the liquid and the syringe.

The acceleration of the syringe with an air gap is approximately 50\% smaller than the acceleration of a syringe without an air gap. Because the air gap pressure and the liquid pressure slowly increase when there is an air gap, acceleration of the syringe results almost entirely from the friction between the syringe and the plunger-stopper. With an air gap the liquid pressure applied at the bottom of the syringe only contributes minimally to the acceleration of the syringe.

Deceleration of the syringe is observed between $0.4 \mathrm{~ms}$ and $1 \mathrm{~ms}$ after the impact event. The magnitude of the deceleration is approximately $8,000 \mathrm{~m} / \mathrm{s}^{2}$. The motion of the syringe carrier (not shown) was studied in separate tests, and it was found that the deceleration results from an elastic interaction between the syringe and the carrier. This interaction results from the compliance of the plastic carrier. The average deceleration of the syringe upon reaching its travel limit at approximately $2.8 \mathrm{~ms}$ is $26,000 \mathrm{~m} / \mathrm{s}^{2}$. The characteristics of this deceleration are almost identical to the no-air-gap case, but it is of lesser magnitude due to test-to-test variations.

The liquid pressure is shown in Figure 3.13. The events taking place after $2.5 \mathrm{~ms}$ (i.e., the transients due to syringe deceleration and syringe rebound) are very similar to what was observed and discussed without an air gap (configuration 1). This, along with the results from other experiments, suggests the deceleration of the syringe and the other moving components is relatively insensitive to the presence of an air gap between the plunger-stopper and the liquid. In this test, the baseline of the pressure transducer signal shifted during actuation of the autoinjector. As a consequence, the extrusion pressure is incorrectly registered as $0.4 \mathrm{MPa}$, which is inconsistent with the hoop strains in the syringe (see Figure 3.14). Using the hoop strains and Equation 3.2 we infer the extrusion pressure to be around $1 \mathrm{MPa}$, as expected.

The transient events taking place within the first $2.5 \mathrm{~ms}$ following impact of the driving rod on the plunger-stopper are different from what was observed without an air gap. The pressure does not increase immediately after the impact of the driving rod on the plunger-stopper, but instead decreases. This pressure decrease is caused by the syringe acceleration which creates tension waves, as discussed in Section 2.2 (event 1). 
With an air gap, pressurization of the liquid does not occur before the syringe is substantially accelerated. Without pre-pressurization of the liquid, the tension waves are sufficient to reduce the liquid pressure to sub-atmospheric values, which results in transient cavitation: bubbles form, grow, and collapse. One such rapid bubble collapse occurs at $0.8 \mathrm{~ms}$. This results in the production of a sharp and substantial pressure wave with a magnitude close to $10 \mathrm{MPa}$, or 100 times atmospheric pressure. This is 10 times the pressure needed for drug extrusion. The oscillatory pressure signal indicates that the bubbles grow and collapse multiple times, resulting in successive sharp peaks of decaying amplitude. It has been confirmed using high speed imaging that cavitation is indeed responsible for these features of the pressure trace. This is also confirmed in Chapter 4 using a large-scale model autoinjector.

To obtain clearer images of the bubble dynamics due to cavitation, an experiment was performed with a non-instrumented syringe. A sequence of images showing the growth and collapse of a cavitation bubble within the cone of the syringe is shown in Figure 3.15. Bubble growth begins at $0 \mu$ s when acceleration of the syringe begins. The cavity grows for approximately $360 \mu \mathrm{s}$. The collapse of the cavity is rapid and occurs over approximately $60 \mu \mathrm{s}$. It is possible to see the bubble rebound and collapse a second time from 450 to $510 \mu \mathrm{s}$.

When an air gap is present the large acceleration of the syringe consistently causes bubbles or cavities to form and collapse in the cone of the syringe. Larger air gaps tend to increase the time lag between pressurization of the liquid and acceleration of the syringe, resulting in more severe cavitation events. Cavitation bubbles can also form away from the cone. An example of this is shown in Figure 3.16. Two large bubbles form, grow and collapse along the barrel of the syringe. As expected, the exact location of bubble growth and collapse varies between experiments. The collapse of the two bubbles is non-symmetric, and the collapse of bubbles close to a wall can generate significant wall stresses (Brennen, 1995).

The hoop and axial strains are shown in Figure 3.14. The distinctive signature of the collapsing bubbles is visible on the hoop strains measured above the cone of the syringe between 0.8 and $1.6 \mathrm{~ms}$. One would however expect the peak hoop strains to be larger than $300 \mu \epsilon$ in order to be consistent with the recorded peak pressure of $10 \mathrm{MPa}$. We recall that the peak hoop strains were approximately $320 \mu \epsilon$ for a peak pressure of $4 \mathrm{MPa}$ when there was no air gap. This inconsistency can be explained by the location of the collapse relative to the strain gauge. The collapsing bubble which produces the large pressure signal of $10 \mathrm{MPa}$ is very close to the 


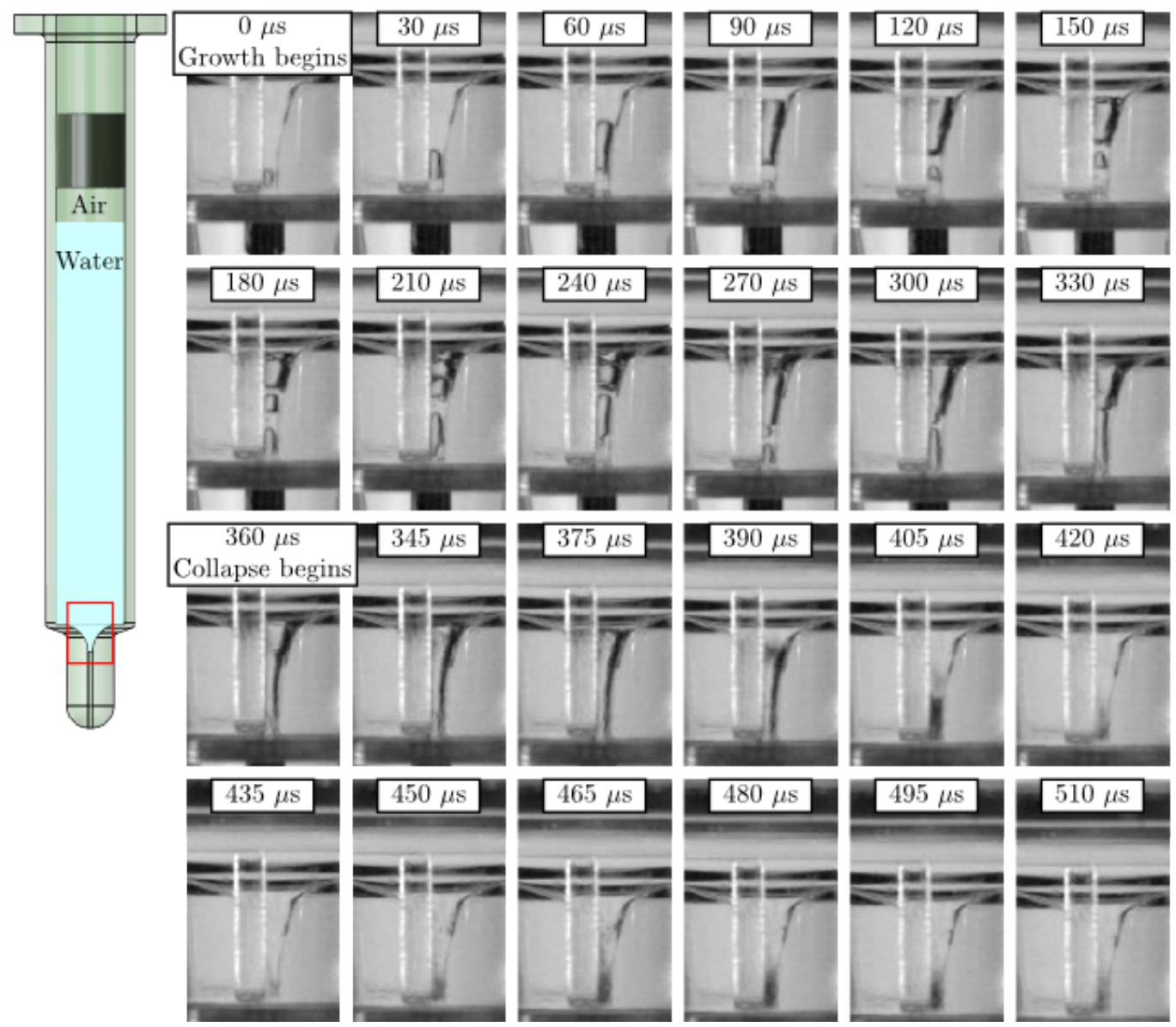

Figure 3.15: Growth and collapse of a bubble in the cone area of a glass syringe when an air gap is present (test SC-0137).

pressure sensor, but relatively far from the strain gauges. Following the collapse and rebound of a cavitation bubble, a shock wave forms and propagates away from the bubble. The magnitude of the wave decays rapidly as it travels away from the bubble (Brennen, 1995). Neglecting the effect of the walls, the decay is expected to be in $1 / r$, where $r$ is the distance from the center of the collapsing bubble.

The strain gauge located below the plunger-stopper does not exhibit the distinctive signature of cavitation in the form of multiple pressure peaks. This is because that strain gauge is essentially responding to the air gap pressure which is isolated from the weakened cavitation pressure pulses arriving at this location.

In summary, the presence of an air gap between the plunger-stopper and the liquid modifies the timing between pressurization and acceleration of the syringe. With an air gap the acceleration takes places before the liquid pressure increases, and 


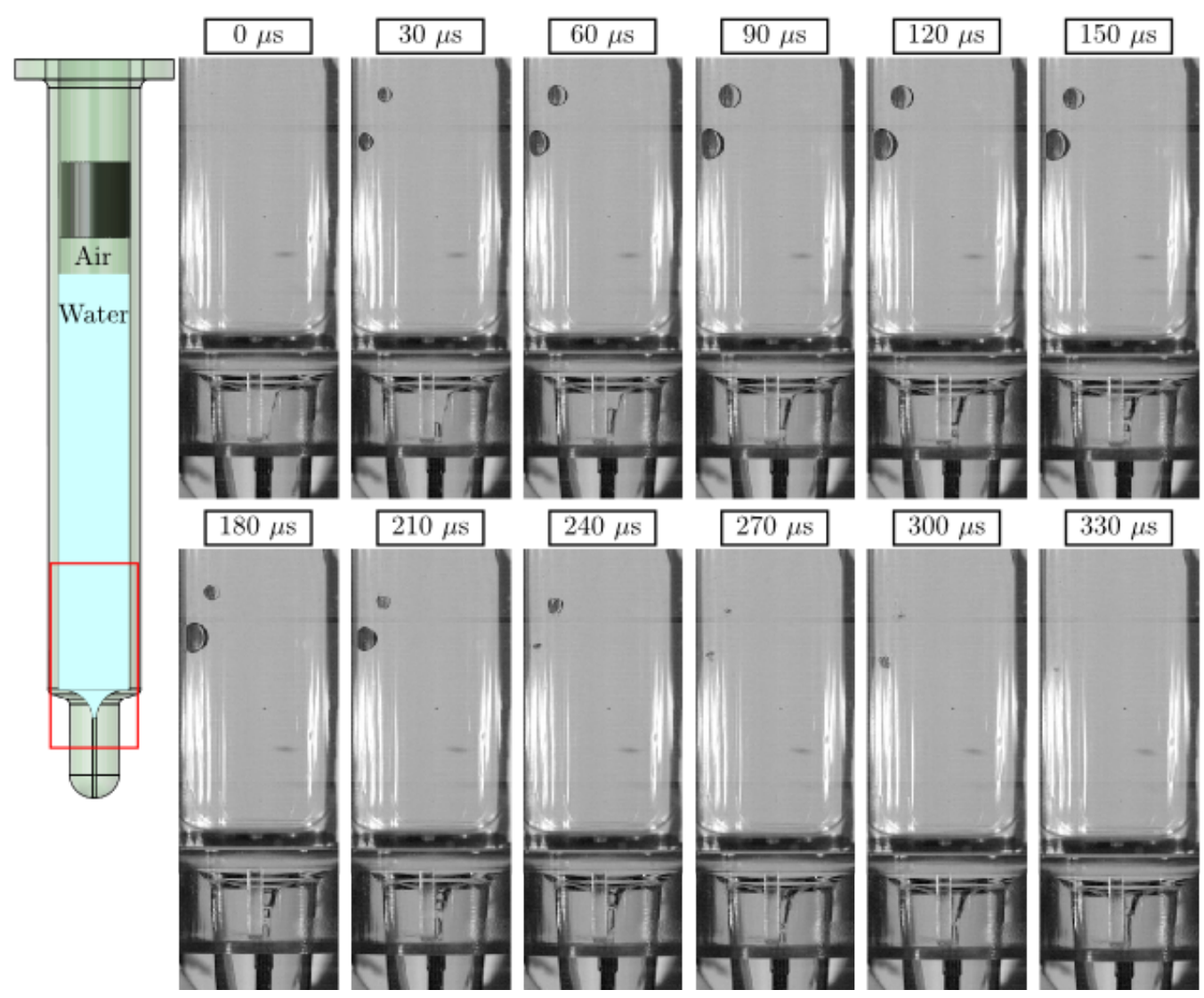

Figure 3.16: Growth and collapse of bubbles along the barrel of a glass syringe when an air gap is present (test SC-0137).

cavitation occurs. The collapse of the cavitation bubbles can create significant, but highly localized, pressures within the liquid. The collapse of a bubble close to a wall can result in substantial wall stresses, likely capable of causing failure of the syringe. The pressure and stress transients generated upon deceleration of the moving components occurring when the syringe reaches its travel limit are not significantly affected by the presence of an air gap.

\subsubsection{Configuration 3: Plastic Syringe Without an Air Gap}

The third configuration studied is that of a plastic syringe without an air gap. The plastic syringe is a Daikyo Crystal Zenith $1 \mathrm{~mL}$ syringe from West Pharma. Multiple experiments were performed in this configuration, but only one representative case is reported and discussed. The emphasis of the discussion is on the differences between the results obtained with a glass (i.e., configuration 1) and a plastic syringes. 
Note that the syringe material is not the only difference between the BD HyPack and the Daikyo Crystal Zenith syringes. The lubrication of the syringes and the plunger-stoppers is also different. The plunger-stopper and the inner surface of the BD HyPack syringe are coated with a thin layer of silicone oil. As mentioned in Chapter 2, siliconization of the components is the typical approach used to lubricate pre-filled syringes and plunger-stoppers. In the contrary, the inner surface of the Daikyo syringe and the plunger-stopper are not siliconized. In fact, no lubrication at all is applied on the syringe, and the plunger-stopper is coated with the Daikyo Flurotec film (West Pharmaceutical Services, n.d.), a dry lubricant. The break-loose force and the dynamic friction are therefore expected to be significantly different between the two syringe systems. In particular, the relation between friction and relative velocity of the plunger-stopper into the syringe is expected to be different.

Results obtained with the Daikyo plastic syringe are shown in Figures 3.17 to 3.19. The motion of the internal components, the liquid pressure and the strains are not qualitatively different from what was recorded using a glass syringe without an air gap (i.e., configuration 1) during the time period which immediately follows the impact of the driving rod on the plunger-stopper (i.e., $0 \mathrm{~ms}$ to $1 \mathrm{~ms}$ ). The impact of the driving rod on the plunger-stopper is again responsible for the pressurization and the acceleration of the syringe. Quantitatively, the acceleration of the syringe immediately after the impact event is approximately $22,000 \mathrm{~m} / \mathrm{s}^{2}$, a value which is significantly larger than the $15,000 \mathrm{~m} / \mathrm{s}^{2}$ recorded in configuration 1 with a glass syringe. The substantial difference is explained by the smaller mass of the plastic syringe compared to the glass syringe. The total mass of the liquid content, syringe, and syringe carrier is $5.2 \mathrm{~g}$ for the plastic syringe, compared to $7.0 \mathrm{~g}$ for the glass syringe.

The reader is reminded about the absence of an air gap in the present configuration. As such, the pressure rises rapidly in the syringe following the impact of the driving rod on the plunger-stopper. The results indicate the acceleration and the pressurization of the syringe occur almost simultaneously, and no cavitation occurs in the syringe. This behavior is identical to the one observed in configuration 1 with a glass syringe.

The deceleration of the syringe upon reaching its travel limit occurs between $2.5 \mathrm{~ms}$ and $4.0 \mathrm{~ms}$. The magnitude of the deceleration is $34,000 \mathrm{~m} / \mathrm{s}^{2}$, comparable to the $36,000 \mathrm{~m} / \mathrm{s}^{2}$ recorded in configuration 1 . There is however a significant difference between the behavior of the plastic and the glass syringe near the end of the rapid 


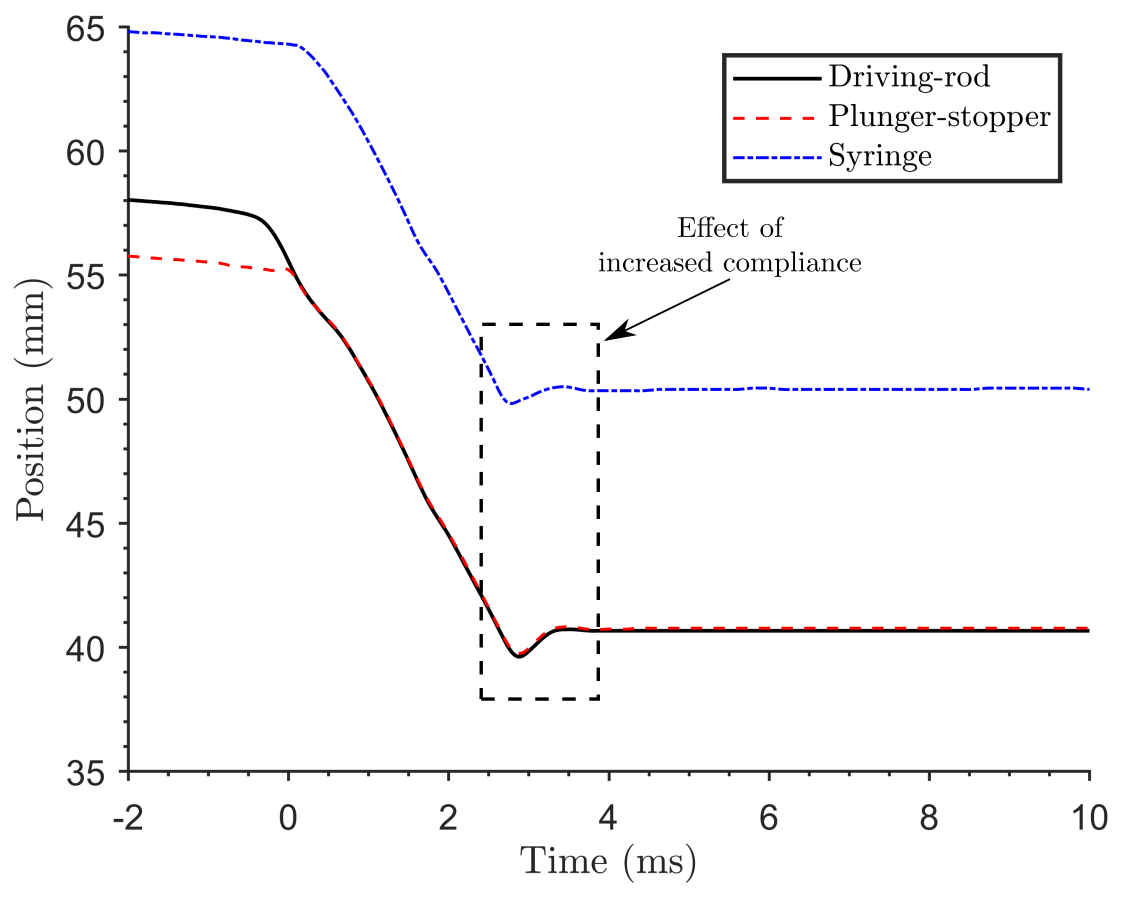

(a) Position

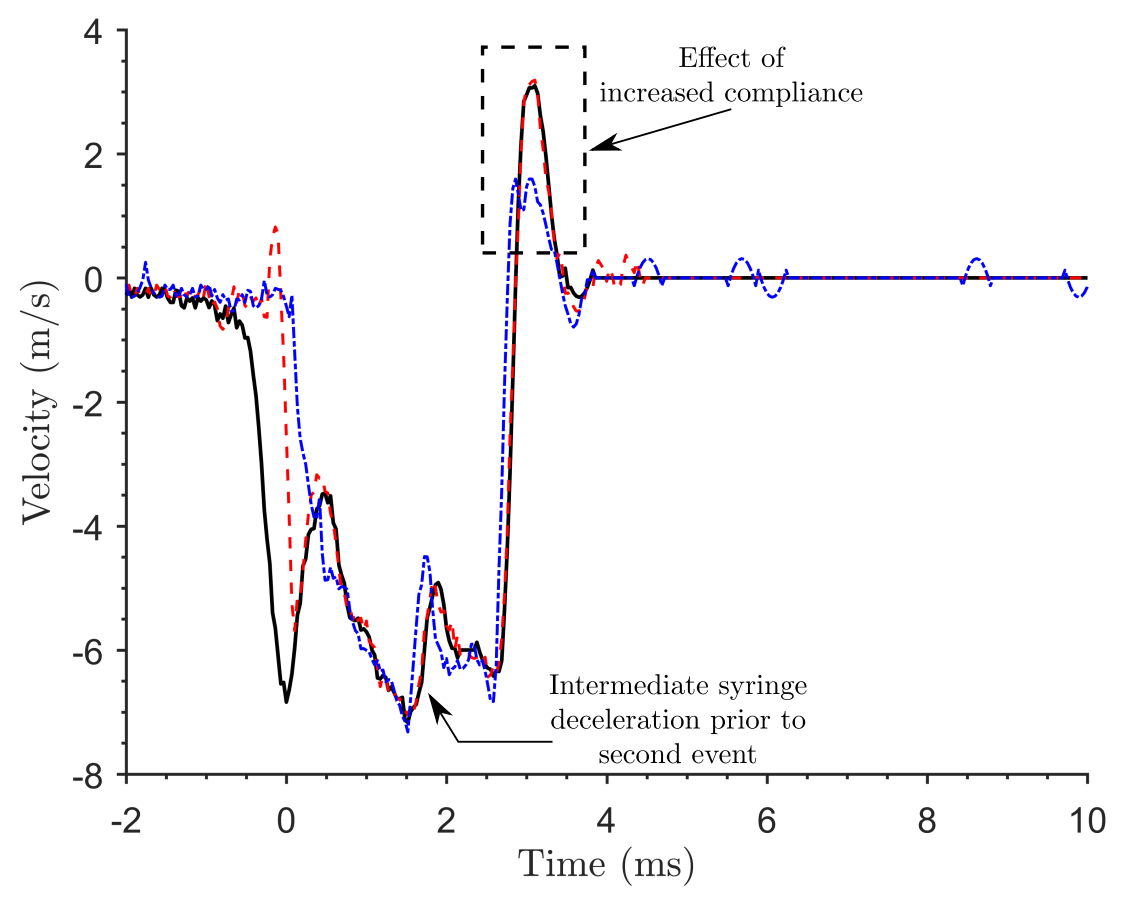

(b) Velocity

Figure 3.17: Position and velocity of the moving components in a SureClick autoinjector - plastic syringe without an air gap (test SC-200). 


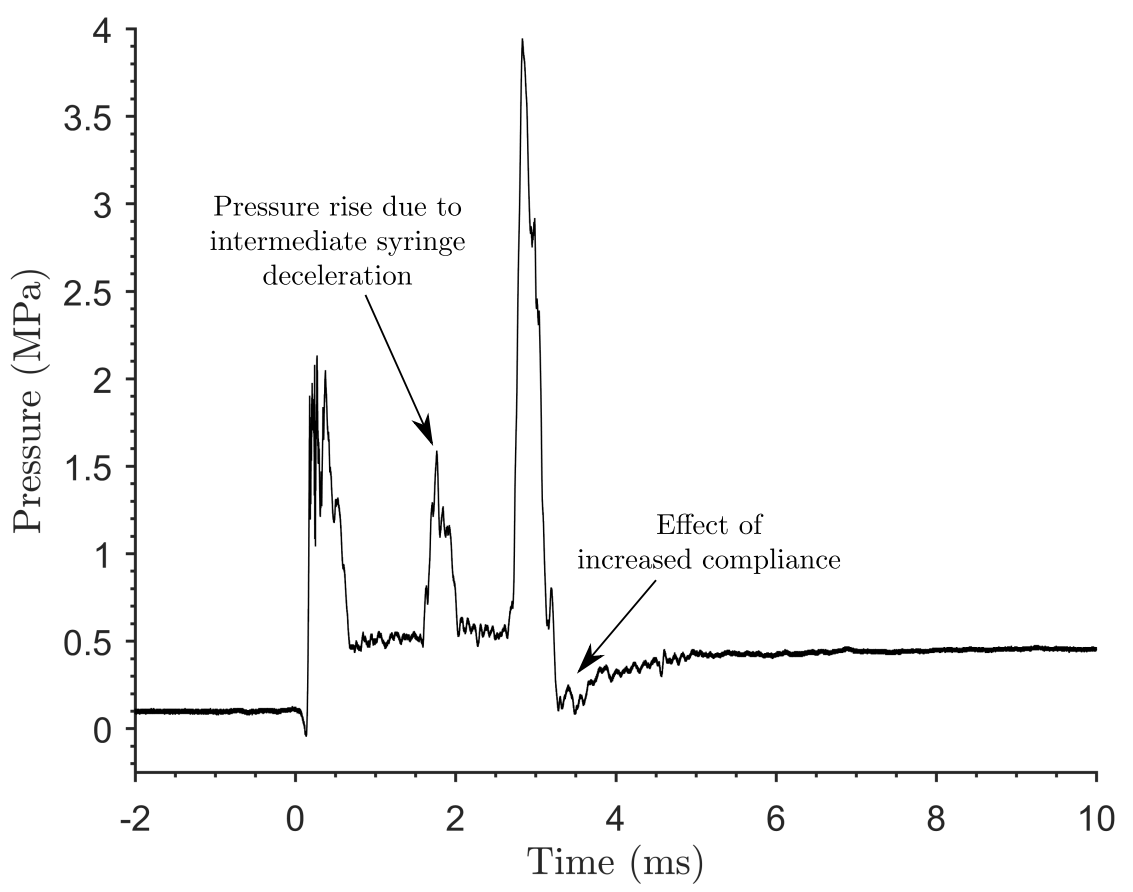

Figure 3.18: Liquid pressure in a SureClick autoinjector - plastic syringe without an air gap (test SC-200).
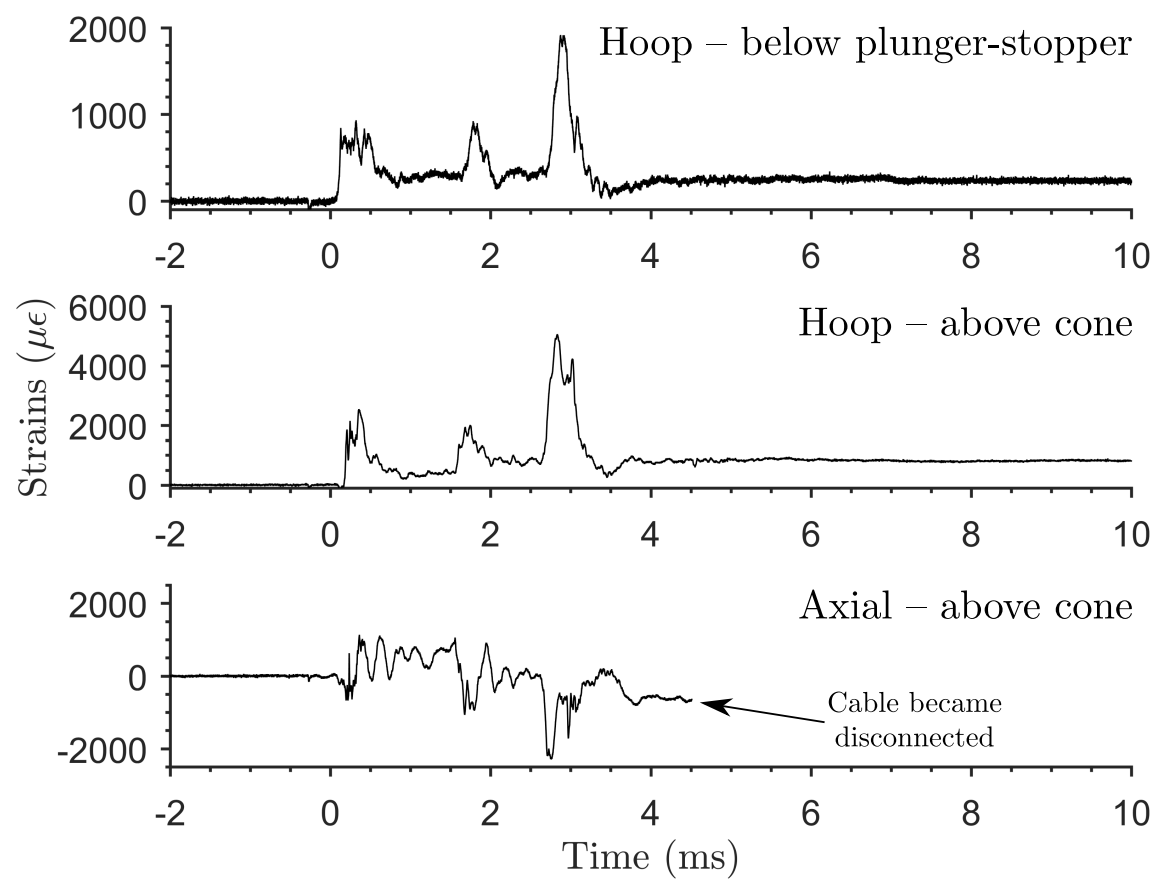

Figure 3.19: Hoop and axial strains on the barrel of the syringe in a SureClick autoinjector - plastic syringe without an air gap (test SC-200). 
deceleration. There is more compliance in the system when a plastic syringe is used, and the increased compliance has a measurable effect on the motion of the internal components (see Figure 3.17a). There is a noticeable overshoot in the displacement of the components past the equilibrium, or extrusion position, as the syringe reaches its travel limit. Furthermore, the rebound of the syringe is not as important.

The current hypothesis is that the overshoot is caused by a substantial deformation of the syringe and/or the plastic syringe-carrier in the vicinity of the point-of-contact between those two components. This could not be confirmed visually as the pointof-contact is virtually impossible to observe. The effect of this increased compliance is also visible in Figure 3.17b: the syringe does not travel at the same velocity as the plunger-stopper and the driving rod during the rebound, which indicates there is a momentary decrease in the force applied on the top surface of the plunger-stopper. This, in turn, causes a momentary decrease in liquid pressure during the syringe rebound (see Figure 3.18).

Another difference between the results obtained in configurations 1 and 3 is the weak, momentarily deceleration of the plastic syringe at around $2.0 \mathrm{~ms}$. This occurs between the impact of the driving rod on the plunger-stopper and the syringe deceleration resulting from the syringe reaching its travel limit. This is not a difference which is believed to result from using a plastic syringe, but instead results from test-to-test variation in the results.

The weak, momentarily deceleration of the plastic syringe at around $2.0 \mathrm{~ms}$ is noticeable on the velocity plot (see Figure 3.17b), and it also causes a pressure increase (see Figure 3.18). This deceleration of the syringe could be caused by an interaction between some of the components inside the autoinjector. This hypothesis is supported visually using the high-speed video for this test. It is possible to observe the shell of the device deform in the circumferential direction, and this appears to apply a force on the syringe carrier in the radial direction. This pushes the syringe and the syringe carrier sideways. The root cause of this interaction between the components has not been identified.

The hoop and axial strains measured with a plastic syringe (see Figure 3.19) are qualitatively similar to the strains measured with a glass syringe (see Figure 3.11). Quantitatively, the strains measured with a plastic syringe are one order of magnitude larger than the strains measured with a glass syringe. This is explained by the different Young's modulus of elasticity of the materials, as indicated in Table 3.1. 
The larger strains measured with a plastic syringe do not necessarily indicate that the maximum principal stress $\sigma_{1}$ is larger with a plastic syringe than with a glass syringe. Equations 3.4 and 3.5 are used to estimate the wall stresses. The maximum principal stress $\sigma_{1} \approx \sigma_{\theta}$ along the barrel is estimated to be $14 \mathrm{MPa}$, a value which is less than the $22 \mathrm{MPa}$ previously obtained with a glass syringe. The peak compressive axial stress in the plastic syringe is estimated to be $-4.4 \mathrm{MPa}$, a magnitude which is less than the -8.3 MPa previously obtained with a glass syringe. This difference is explained using Equation 2.19, which suggests the peak axial stress depends linearly on the density of the syringe material.

To summarize, the results for a glass and a plastic syringe without an air gap are qualitatively similar. In both cases, pressurization of the liquid content and acceleration of the syringe occur nearly simultaneously. As a result, cavitation is suppressed. The system formed by the plastic syringe and the syringe carrier is more compliant than the system formed by the glass syringe and the syringe carrier. The increased compliance alters the behavior of the system during the final stage of the rebound. The increased compliance of the plastic syringe also results in larger strains, not to be mistakenly associated with larger stresses. Because the Young's modulus of glass is approximately 26.5 times larger than the Young's modulus of plastic (see Table 3.1), the strains are expected to be approximately 26.5 times larger in the plastic syringe than in the glass syringe for the same stress $\sigma$ applied on the syringe wall $(\epsilon \sim \sigma / E)$.

\subsubsection{Configuration 4: Plastic Syringe With an Air Gap}

The fourth and last configuration studied is that of a plastic syringe with an air gap of initial size $\delta_{0}=3 \mathrm{~mm}$. The plastic syringe used is again a Daikyo Crystal Zenith $1 \mathrm{~mL}$ syringe from West Pharma. Multiple experiments were performed in this configuration with various initial air gap sizes, but only one representative case is reported and discussed.

Typical results obtained with a Daikyo plastic syringe are shown in Figures 3.20 to 3.22. Similar to configuration 3 , the results indicate the plastic syringe and the syringe carrier form a more compliant system than the glass syringe and the syringe carrier.

Overall, the results obtained with the plastic syringe that initially has an air gap (configuration 4) are very similar to the results obtained with the plastic syringe that does not initially have an air gap (configuration 3). This behavior is unexpected. 


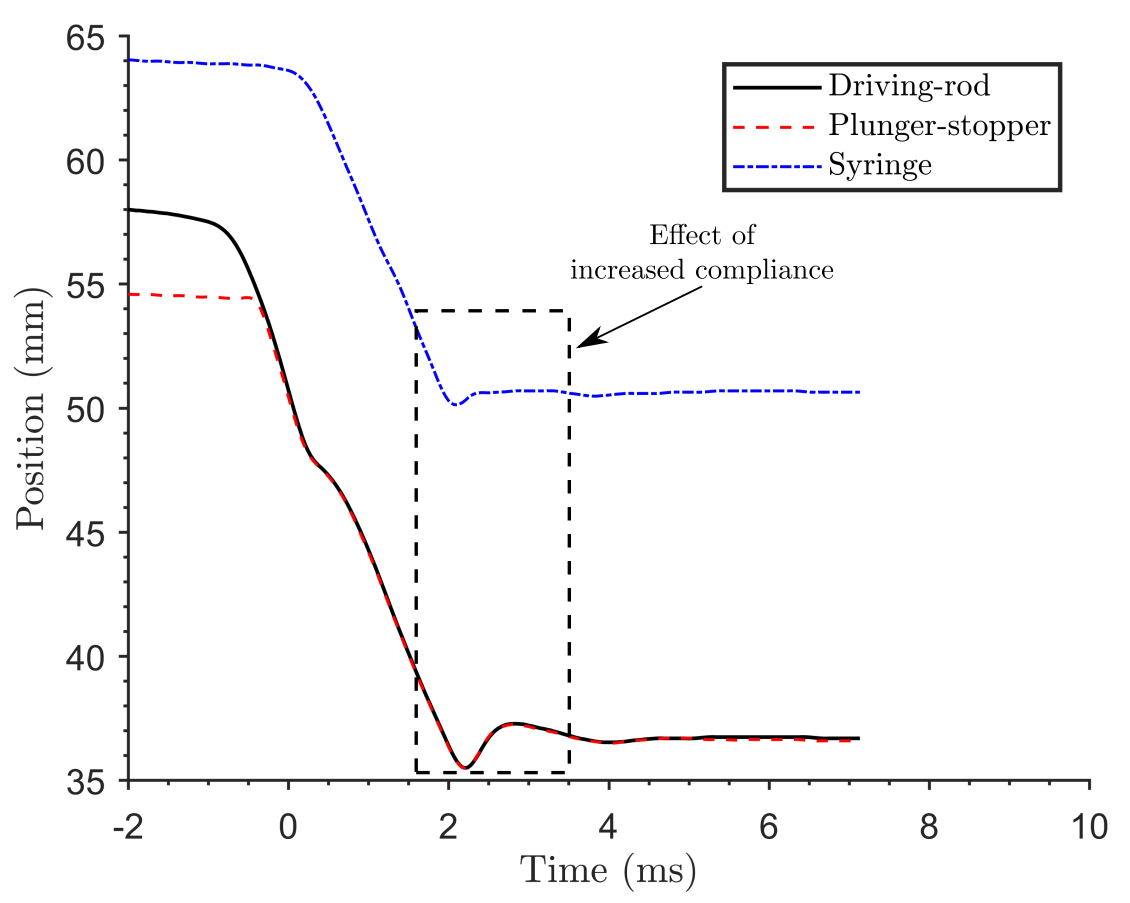

(a) Position

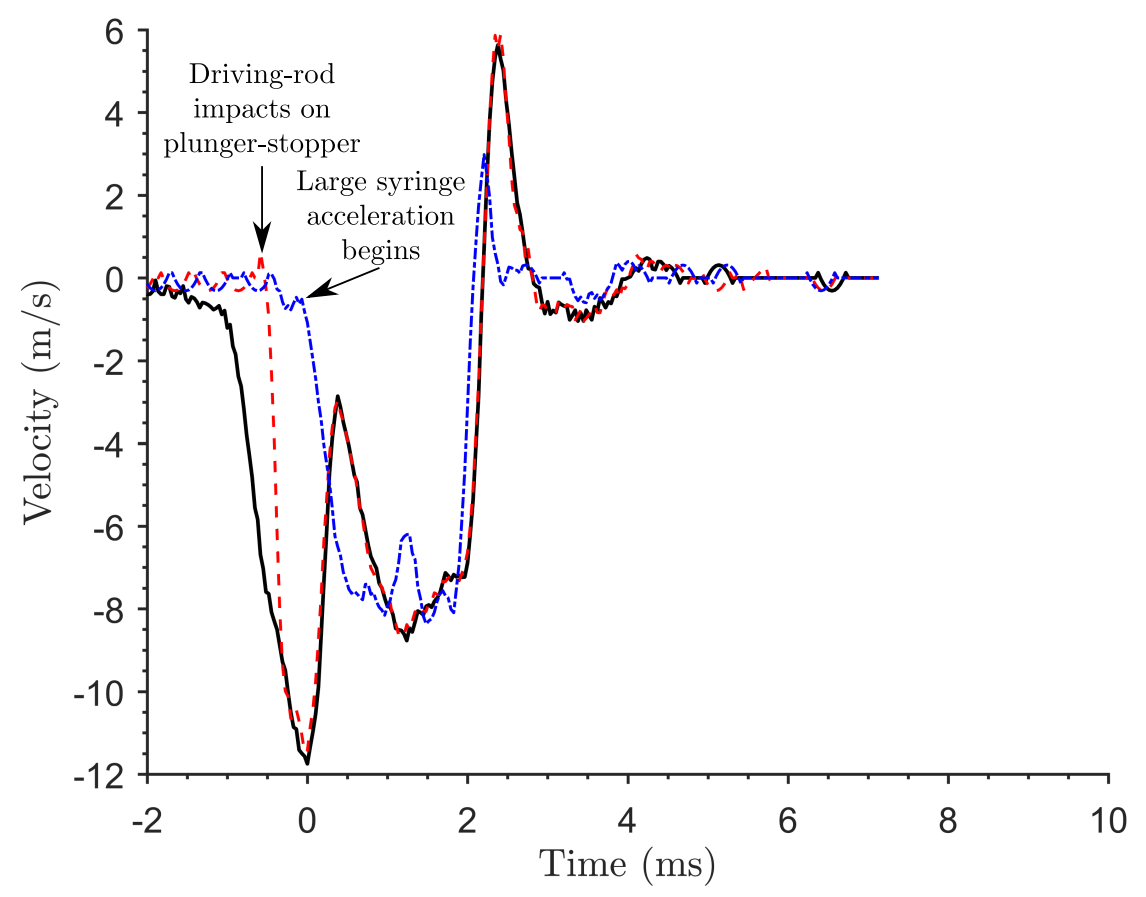

(b) Velocity

Figure 3.20: Position and velocity of the moving components in a SureClick autoinjector - plastic syringe with an air gap (test SC-201). 


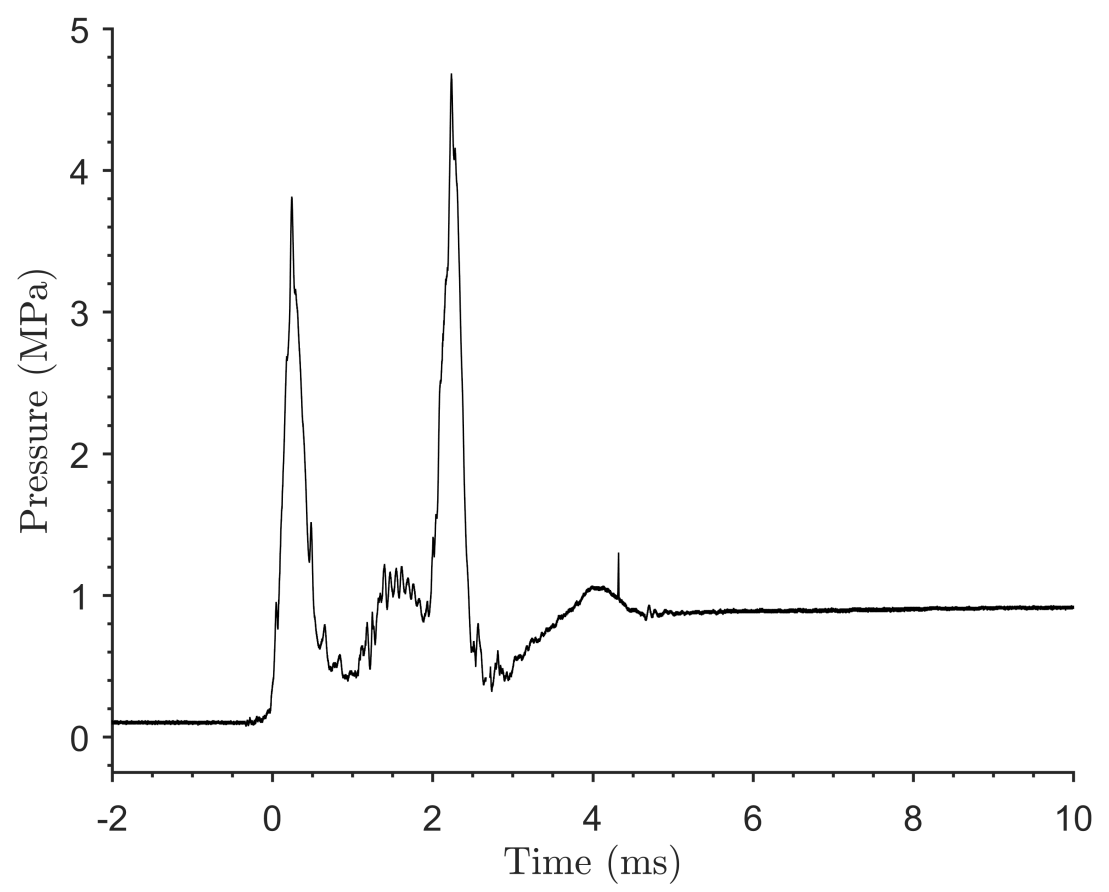

Figure 3.21: Liquid pressure in a SureClick autoinjector - plastic syringe with an air gap (test SC-201).
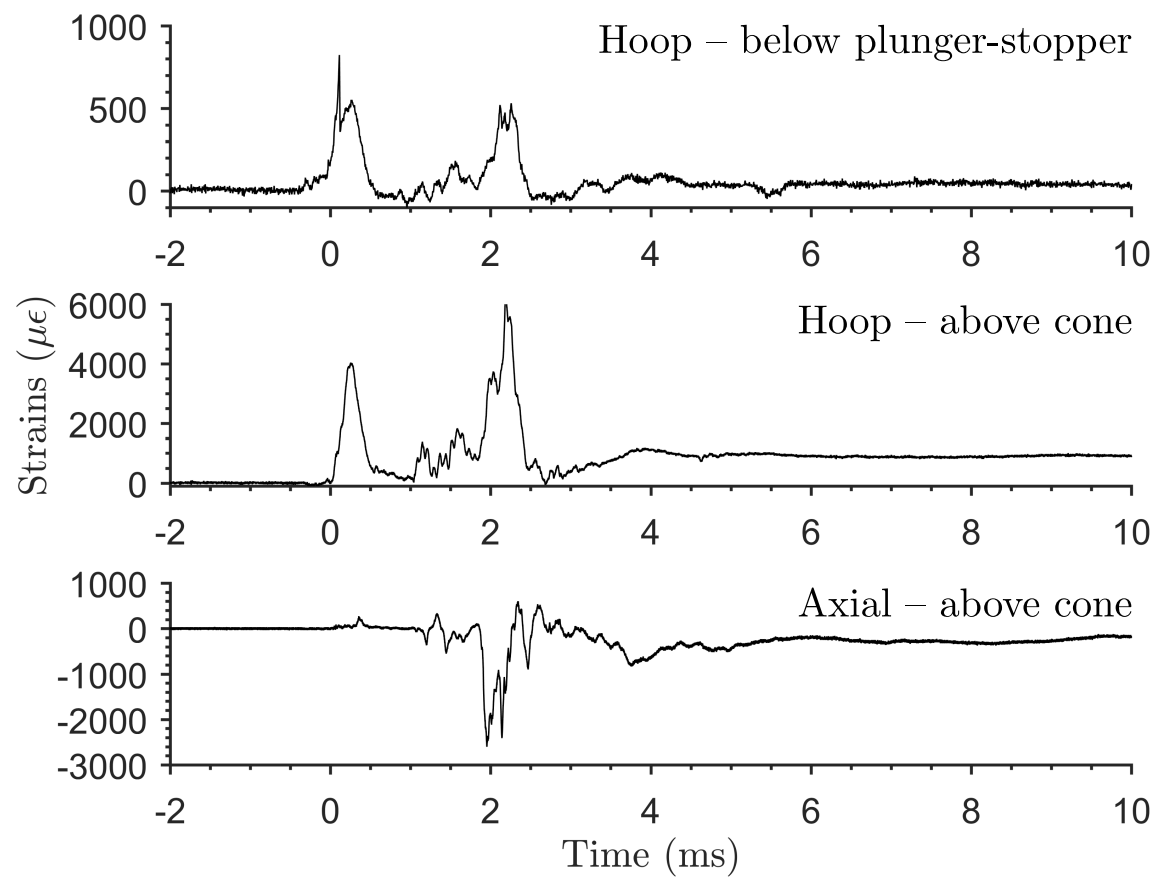

Figure 3.22: Hoop and axial strains on the barrel of the syringe in a SureClick autoinjector - plastic syringe with an air gap (test SC-201). 
Earlier results obtained with a glass syringe have shown that adding an air gap in the syringe below the plunger-stopper affects the results substantially by creating cavitation, and the same is expected when adding an air gap in a plastic syringe. The fact that this is not the case indicates there are fundamental differences in the performance of the plastic and the glass syringes in the SureClick autoinjector.

The most significant difference between the glass and the plastic syringes is in the timing between pressurization of the liquid content and acceleration of the syringe. With the plastic syringe the large acceleration of the syringe begins $0.5 \mathrm{~ms}$ after the impact of the driving rod on the plunger-stopper (see Figure 3.20b). This leaves sufficient time for the air gap to become substantially compressed, and for the liquid pressure to increase before the syringe is rapidly accelerated. Pressurization of the syringe prior to acceleration suppresses cavitation. The situation is the opposite when a glass syringe is used: the acceleration of the plunger-stopper and the syringe occur almost simultaneously (see Figure 3.12b), not leaving sufficient time for the air gap to be compressed and the liquid pressure to increase prior to syringe acceleration.

Several experiments were performed with non-instrumented Daikyo syringes with and without an air gap. The results confirm that cavitation is generally not observed. In the few cases where cavitation is observed, the cavitation event is very mild; the cavities or bubbles remain very small, and they do not collapse as violently as in the case of a glass syringe.

An interesting question arises in light of those results: what could explain the substantially different relative timing between acceleration and pressurization of the syringes? Figure 3.23 is used to answer this important question. It is a sequence of images showing the motion of the driving rod, the plunger-stopper, and the syringe. The sequence at the top of Figure 3.23 is for a glass syringe, and the sequence at the bottom is for a plastic syringe. The syringes used in those experiments are empty of liquid, which means the motion of the syringes results almost entirely from the friction between the plunger-stopper and the syringe barrel. All frames are separated by $0.2 \mathrm{~ms}$. The black horizontal lines visible in each frame are on the syringe outer surface. The motion of the syringe is indicated with the red, continuous curve, and the motion of the top surface of the plunger-stopper is indicated with the blue, dashed curve.

Figure 3.23 indicates the motion of the syringe and the plunger-stopper are significantly different for a plastic and a glass syringe. The plunger-stopper appears to stick to the barrel of the glass syringe. The glass syringe and the plunger-stopper, soon 


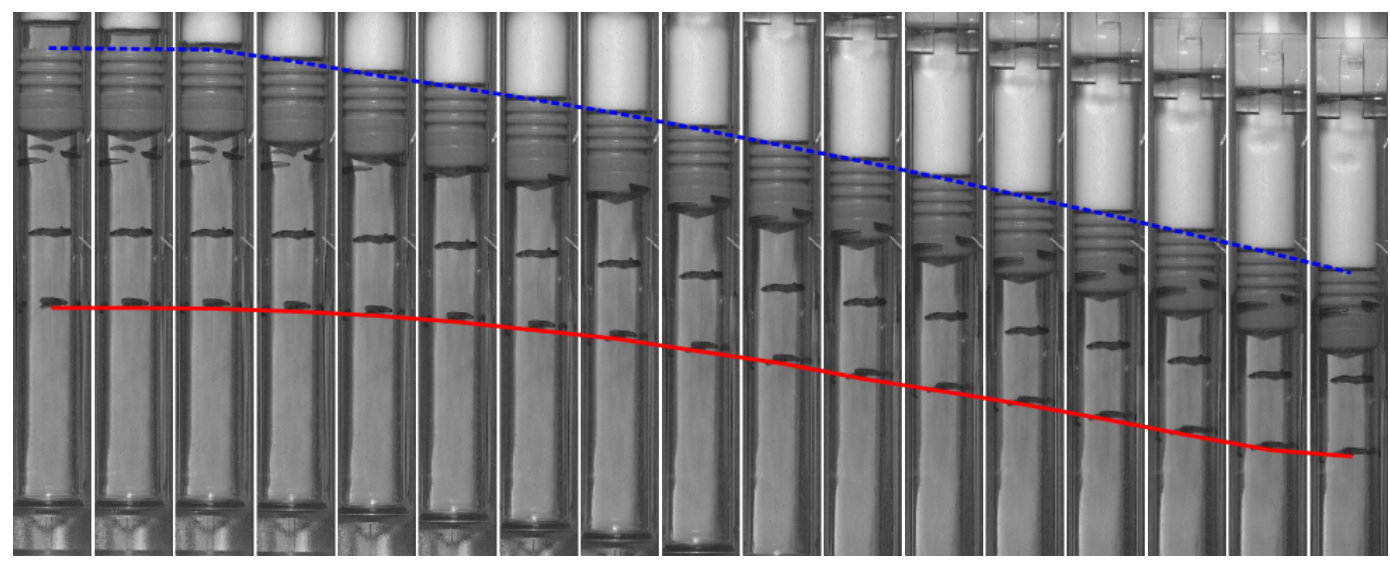

(a) Glass syringe (test SC-215)

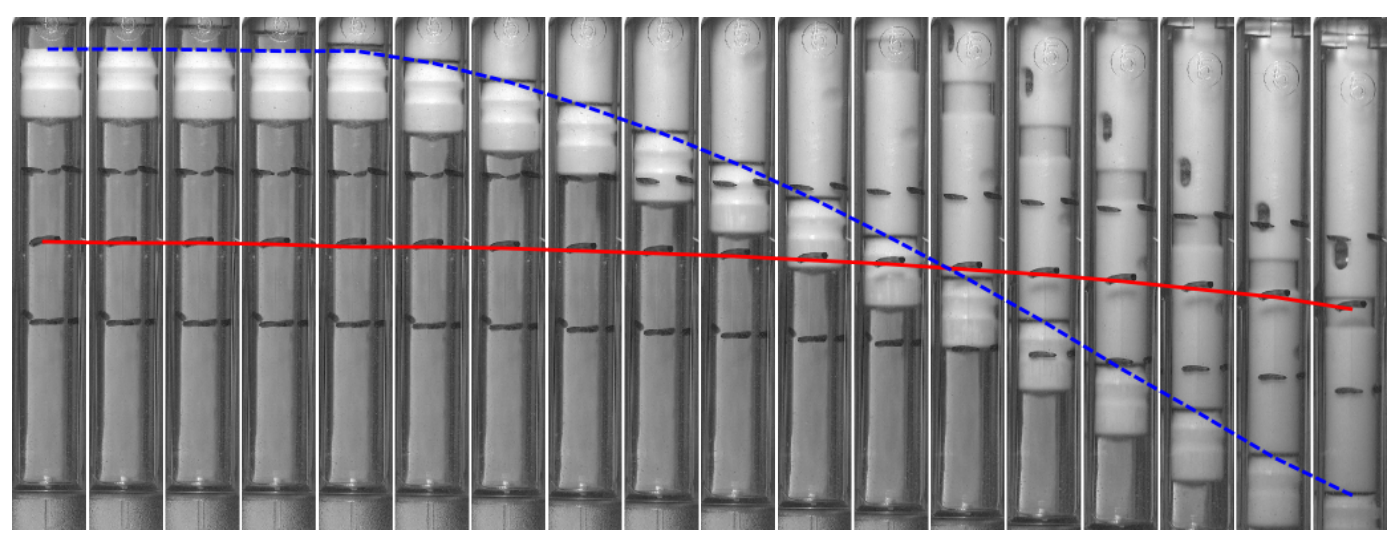

(b) Plastic syringe (test SC-214)

Figure 3.23: Sequence of images showing the motion of the driving rod, the plungerstopper and the syringe ( $0.2 \mathrm{~ms}$ between successive frames). The red, continuous curves indicate the position of the syringe. The blue, dashed curves indicate the position of the plunger-stopper.

after the impact event, travel at a similar velocity; there is minimal relative motion of the plunger-stopper into the barrel. In the contrary, the plunger-stopper does not stick to the barrel of the plastic syringe. The friction is minimal, and the relative motion of the plunger-stopper into the syringe is large. Those results indicate the coefficient of friction between the plunger-stopper and the syringe is much lower for the Daikyo than the BD HyPack syringes in the highly dynamic regime under consideration. ${ }^{3}$

The reduced friction force between the plunger-stopper and the plastic syringe barrel accounts for the absence of cavitation. The reduced friction results in a substantial air

\footnotetext{
${ }^{3}$ Note that the results could be different in a quasi-static regime more representative of the extrusion phase.
} 
gap compression before the syringe is accelerated. This is because the acceleration of the syringe primarily results from the liquid pressure applied on the bottom wall of the syringe rather than the friction between the plunger-stopper and the syringe, even when an air gap is present. Because the syringe is pressurized prior to being accelerated, the tension waves created upon acceleration are not sufficiently large to reduce the pressure to sub-atmospheric values, and cavitation is inhibited.

To summarize, the friction force between the plunger-stopper and the syringe barrel during the transient events is much smaller with the plastic syringe than with the glass syringe in a SureClick device. This results in the syringe pressurization occurring before the syringe acceleration, even when an air gap is present. This has the effect of suppressing cavitation. The results suggest the syringe material (i.e., glass vs plastic) is not the likely cause for the different friction forces. The different lubrication film between the plunger-stopper and the syringe barrel likely explain the differences, but this needs to be confirmed experimentally. One possibility would be to test plastic syringes lubricated with silicone oil and to compare the results with those discussed in this chapter. Note that the results could be different when using the same syringe models in combination with a different autoinjector device.

\subsubsection{Friction Estimates}

The results obtained with glass and plastic syringes indicate the friction force between the plunger-stopper and the syringe is a key design parameter which affects whether or not cavitation occurs inside the syringe. Actuation of the autoinjector device is a highly dynamic event which involves impacts and large accelerations of the components. The friction force between the plunger-stopper and the syringe needs to be evaluated in a similar highly dynamic regime representative of the autoinjector under study. This is important because the friction between the plunger-stopper and the syringe during the transient phase can be substantially different from the friction force measured during the quasi-static extrusion phase. The reader is reminded that the relative velocity of the plunger-stopper into the syringe barrel is only $0.004 \mathrm{~m} / \mathrm{s}$ during the extrusion phase.

The velocity of the plunger-stopper and the syringe along with the relative velocity between both are shown in Figure 3.24. The data supports the earlier conclusion: there is more friction between the plunger-stopper and the syringe when the glass syringe is used. 


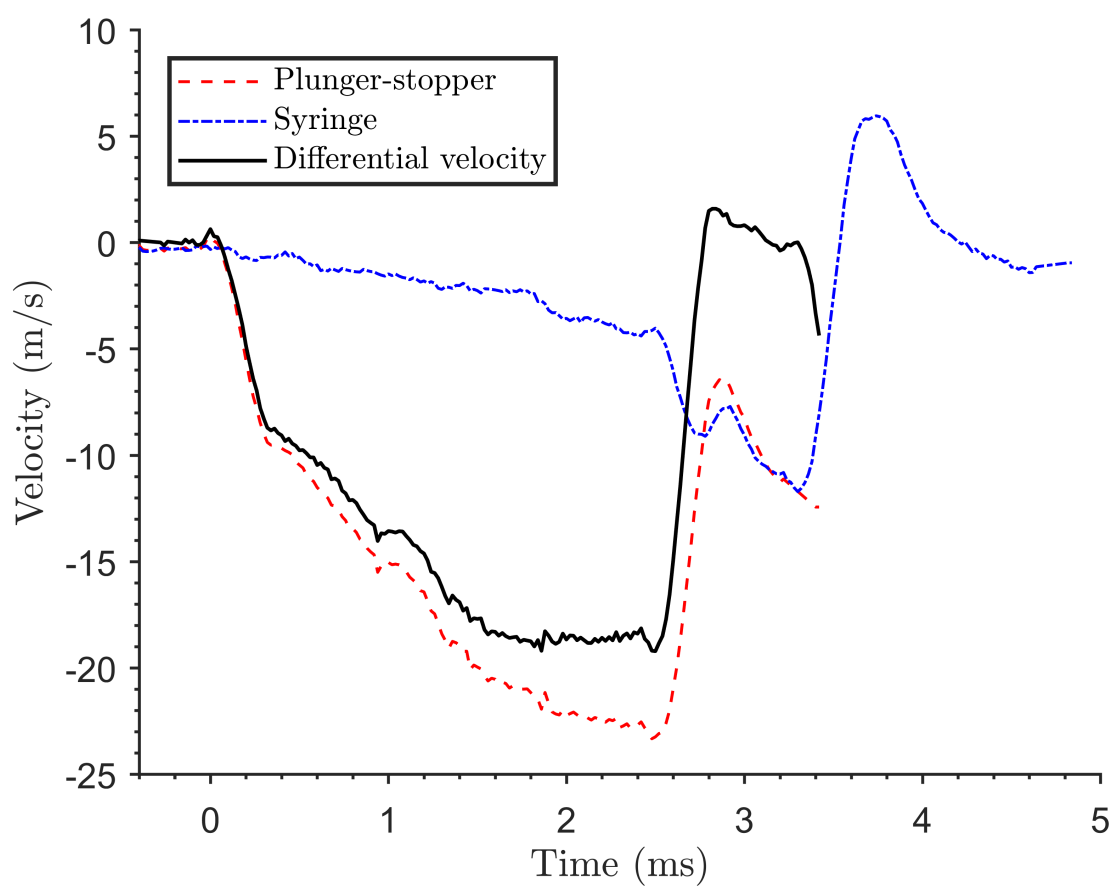

(a) Plastic syringe

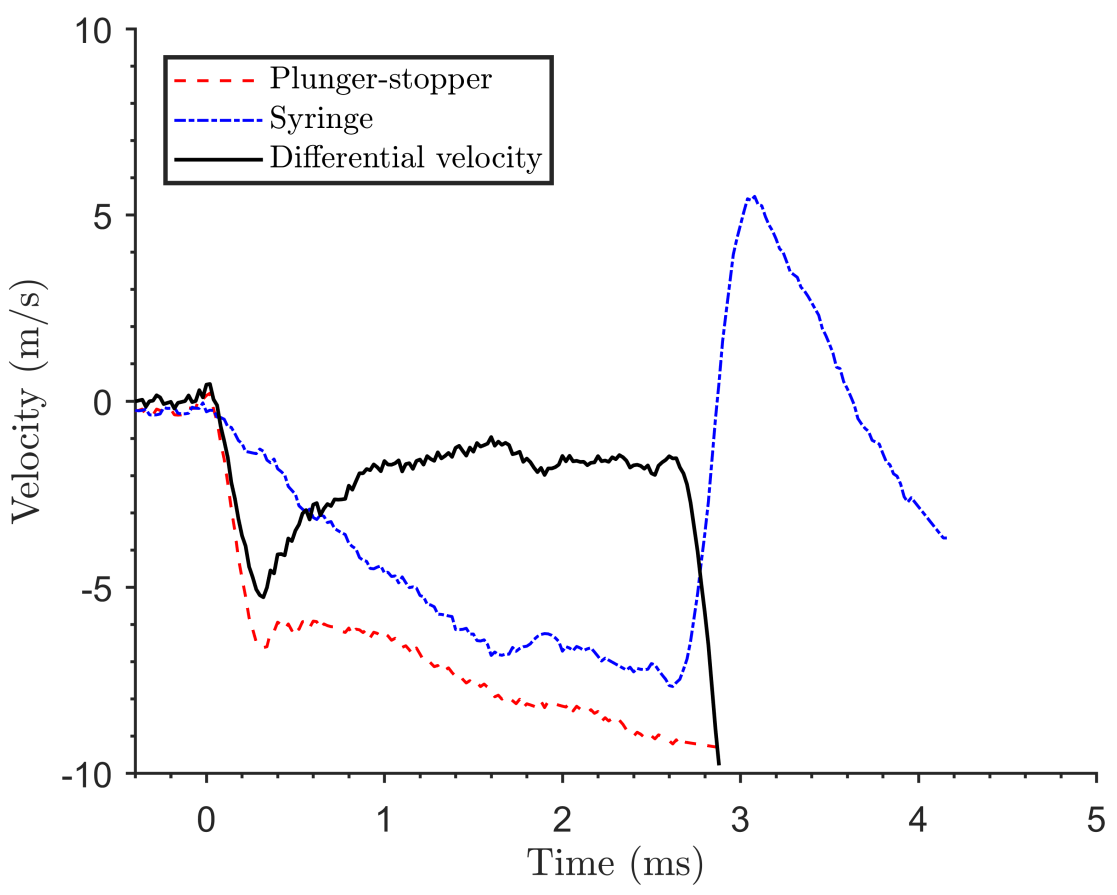

(b) Glass syringe

Figure 3.24: Position and velocity of the moving components in a SureClick autoinjector with an empty syringe. 
Quantitative friction estimates are obtained using the data from Figure 3.24. The acceleration of the syringe immediately after the impact of the driving rod on the plunger-stopper is computed. Because we know the mass of the syringe and the syringe-carrier, Newton's second law can then be used to indirectly estimate the friction force.

The initial acceleration of the empty BD glass syringe and the syringe carrier upon impact of the driving rod on the plunger-stopper is approximately $4,000 \mathrm{~m} / \mathrm{s}^{2}$. The mass of the syringe and the syringe carrier is $6.0 \mathrm{~g}$. Newton's second law indicates the friction between the plunger-stopper and the syringe is approximately $24 \mathrm{~N}$. The initial acceleration of the empty Daykio plastic syringe and the syringe carrier upon impact of the driving rod on the plunger-stopper is approximately $1,500 \mathrm{~m} / \mathrm{s}^{2}$. The mass of the syringe and the syringe carrier is $4.2 \mathrm{~g}$. Newton's second law indicates the friction force between the plunger-stopper and the syringe is approximately $6.2 \mathrm{~N}$, or four times less than the friction measured with the glass syringe. 


\section{Chapter 4}

\section{LARGE SCALE MODEL AUTOINJECTOR}

The results and analysis from Chapters 2 and 3 suggest the transient events in the syringe of an autoinjector device share similarities with those observed in fluid-filled pipelines during water hammer events (Bergant, Simpson, and Tijsseling, 2006; Watters, 1984; Wiggert and Tijsseling, 2001; Wylie and Streeter, 1993). Inaba and Shepherd (2010) and Shepherd and Inaba (2010) examined pressure transients which are closely related to the present work. There are four main differences between these previous studies and the syringe situation:

1. the mechanism of initiating the transient events;

2. the presence of an air gap in the syringe;

3. the converging section terminating the syringe;

4. the translational motion of the syringe.

The primary objective of this chapter is to use experimental measurements on a scaled-up model autoinjector to investigate and explain the effect of these features on measured pressure and strains. Using a scaled-up model autoinjector is particularly useful because it becomes easier to instrument the test setup and perform the measurements, especially within the cone area of the syringe. This, in turn, makes it easier to study and understand the physics at play.

The secondary objective of this chapter is to use numerical simulations and modeling to predict the pressure in the liquid, and the strains/stresses in the syringe wall. LSDYNA is used for this purpose. The comparison between the experiments and the numerical simulations is particularly useful to validate the ability of LS-DYNA at predicting accurately the transient events considered in this thesis.

The first section of this chapter focuses on items 1 to 3 of the list above: the syringe model is static. The effect of the translational motion is important, and it is discussed separately in the second section of this chapter. 


\subsection{Static Large-Scale Model Autoinjector}

The experiments and numerical simulations performed using the static, large-scale model autoinjector test setup are discussed in this section. Five test cases which are representative of the physics at play are discussed in detail.

\subsubsection{Experimental Setup}

Figure 4.1 is a schematic of the experimental test setup. Note that the $z$-axis, or longitudinal axis, is defined downward positive, and all distances are measured relative to the top end of the aluminum tube. This convention is different from the one used earlier in Chapters 2 and 3, but it is a more convenient convention for the discussions included in this chapter. The experimental apparatus consists of three main components: the guide tube, the projectile, and the test specimen.

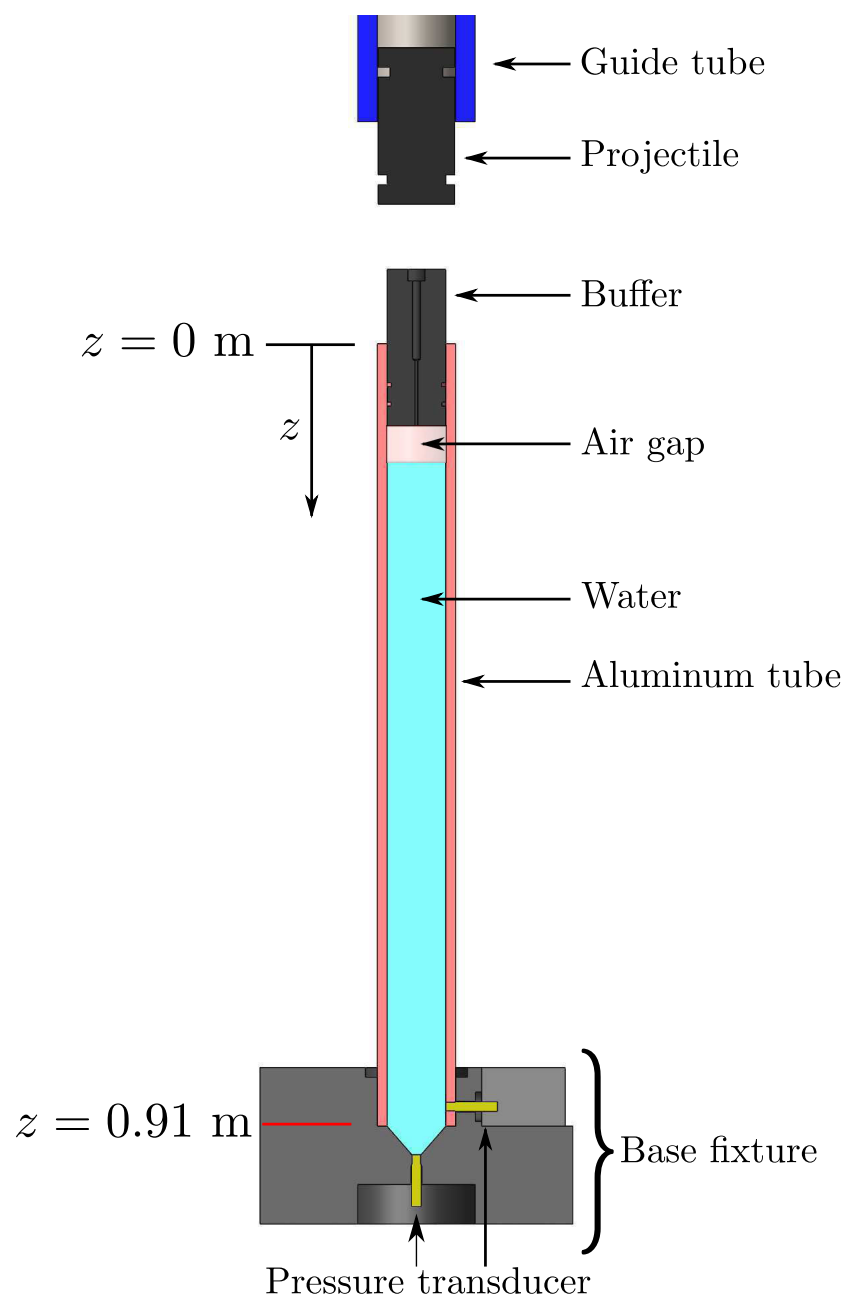

Figure 4.1: Schematic of the static, large-scale model autoinjector experimental setup. 
The guide tube - inner diameter of $50.8 \mathrm{~mm}$ and length of approximately $2.1 \mathrm{~m}$ - is only partially shown in Figure 4.1. The purpose of this tube is to guide the projectile while it is vertically accelerated to velocities up to $6.4 \mathrm{~m} / \mathrm{s}$ using gravity alone. The projectile consists of a $0.5 \mathrm{~kg}$ aluminum cylinder $-50.7 \mathrm{~mm}$ in diameter and $102 \mathrm{~mm}$ in length - which can slide freely within the guide tube.

The test specimen consists of a thick-wall aluminum tube with a length of $0.91 \mathrm{~m}$, an outer diameter of $50.8 \mathrm{~mm}$, and an inner diameter of $38.1 \mathrm{~mm}$. The tube is filled with de-ionized water. Note that several experiments were performed with degassed, de-ionized water (a list of all tests performed is available in Appendix B). Using degassed water does not create measurable differences, and the results are not reported. The aluminum tube is mounted into a cylindrical base fixture which is bolted to heavy plates resting on the ground (not shown). The overall mass of the test specimen, including the base fixture and the plates, is over $50 \mathrm{~kg}$.

The three base fixtures shown in Figure 4.2 are used. The first two base fixtures (Figures $4.2 \mathrm{a}$ and $4.2 \mathrm{~b}$ ) are fabricated with aluminum. The aluminum tube is positioned into the base fixture as shown in Figure 4.1, and it is secured in place using a shrink fit. In the first geometry (Figure 4.2a), the bottom of the aluminum tube is terminated with a flat end perpendicular to the $z$-axis. In the second geometry (Figure 4.2b), the aluminum tube is terminated with a conical section similar to that of a syringe. The half-angle of the cone is $41^{\circ} .{ }^{1}$ In both geometries there are two ports for mounting piezoelectric pressure transducers.

The third base fixture (Figure 4.2c) is fabricated using optically-clear polycarbonate. The polycarbonate was vapor polished after machining to ensure optical clarity of the final product. This fixture is taller because it contains a $76 \mathrm{~mm}$ long straight section of tube terminated with a $41^{\circ}$ cone. The aluminum tube is positioned into the base fixture as shown in Figure 4.2c, and it is secured in place using epoxy. The aluminum tube used with the polycarbonate base fixture is $76 \mathrm{~mm}$ shorter to make sure the overall distance between the top end of the tube and the entrance of the cone is $0.91 \mathrm{~m}$, the same as for the other two base fixtures. The third base fixture makes it possible to observe the water and cavitation within the cone and the last $76 \mathrm{~mm}$ of straight tube.

The pressure transducers mounted into the base fixtures are also shown in Figure 4.1. The precise locations of the transducers are indicated in Table 4.1. For the

\footnotetext{
${ }^{1} \mathrm{~A} 41^{\circ}$ half-angle was chosen based on the standard tools available for the fabrication of the conical tip.
} 


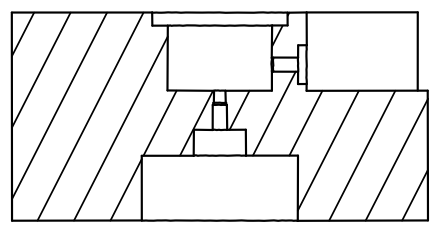

(a) Cross-section view of the aluminum base fixture without a cone.

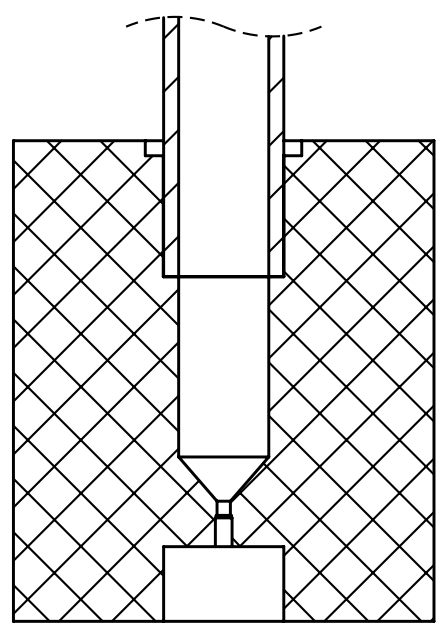

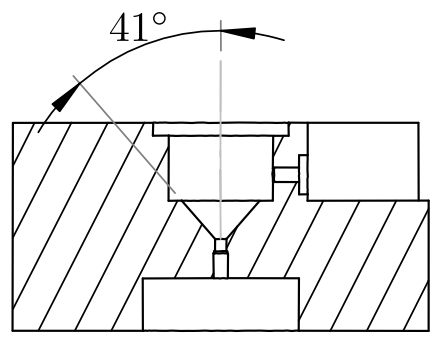

(b) Cross-section view of the aluminum base fixture with a cone.

(c) Cross-section view (left) and isometric view (right) of the polycarbonate base fixture with a partial view of the aluminum tube.

Figure 4.2: Schematic of the base fixtures - single hatch for aluminum, and double hatch for polycarbonate - used with the static, large-scale model autoinjector.

aluminum base fixture which has a conical section, one transducer is located above the converging section, and the other one is positioned at the apex of the conical section. The polycarbonate base fixture only has one port for mounting a pressure transducer, and it is located at the apex of the cone.

The test specimen is sealed at its top end using a $104 \mathrm{~mm}$ long polycarbonate cylinder used as a buffer between the projectile and fluid. The mass of the buffer is $134 \mathrm{~g}$. There are two O-rings between the buffer and the aluminum tube for sealing. There is a small hole along the longitudinal axis of the buffer which is closed using a socket screw before an experiment. This opening allows for the introduction of an air gap of controlled size between the bottom end of the buffer and the water contained in 
Table 4.1: Axial location of the pressure transducers in the static, large-scale model autoinjector.

\begin{tabular}{ccc}
\hline Transducer & Without the cone & With the cone \\
\hline P1 & $895 \mathrm{~mm}$ & $895 \mathrm{~mm}$ \\
P2 & $910 \mathrm{~mm}$ & $927 \mathrm{~mm}$ \\
\hline
\end{tabular}

Table 4.2: Axial location of the strain gauges in the static, large-scale model autoinjector.

\begin{tabular}{c|ccccccc}
\hline Station & S1 & S2 & S3 & S4 & S5 & S6 & S7 \\
$z(\mathrm{~mm})$ & 127 & 254 & 381 & 508 & 635 & 762 & 857 \\
\hline
\end{tabular}

the tube, as shown in Figure 4.1. For all cases reported in this section, the bottom end of the buffer is located at $z=\left(51-\delta_{0}\right) \mathrm{mm}$, where $\delta_{0}$ is the initial air gap size. In addition to the pressure sensors (PCB 113A23 or PCB 113B23) there are 14 strain gauges to measure the hoop and axial strains at 7 axial locations on the outer surface of the aluminum tube. The strain gauges are a combination of Vishay CEA06-125UN-350/P2 and HBM K-LY4-3-05-350-3-2. The location of each gauge is indicated in Table 4.2. Note that no strain gauge is installed at station S7 with the polycarbonate base fixture.

The pressure sensors are powered using a PCB model 483A constant-current signal conditioner. The strain gauges are powered using Vishay model 2310B signal conditioners in a quarter-bridge configuration. The excitation voltage is $10 \mathrm{~V}$, and the gain is set to 500. The data acquisition is performed using two National Instruments model PCI-6133 data acquisition systems (14 bit vertical resolution, $2 \mathrm{MHz}$ sampling rate), or a Yokogawa DL850E oscilloscope (12 bit vertical resolution, $10 \mathrm{MHz}$ sampling rate).

A high-speed video camera (Vision Research Phantom V7.0G) is used to visualize the contact between the projectile and the buffer, making it possible to track the projectile and the buffer to study their interaction, and to measure the impact velocity. When using the polycarbonate base fixture, a second high-speed video camera (Vision Research Phantom V1612) is used to visualize the cavitation events within the visible section of the tube and cone. 
Aluminum

projectile

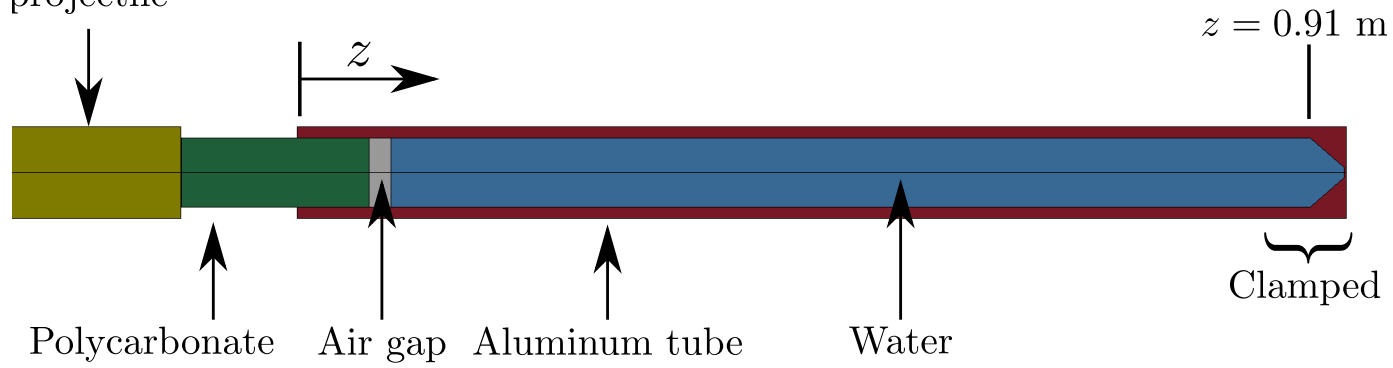
buffer

Figure 4.3: LS-DYNA model of the static, large-scale model autoinjector. The example shown is for the base fixture with a converging section. The schematic is not to scale.

The analogy between the test setup and an actual autoinjector device is as follows: the projectile corresponds to the spring-actuated driving rod, the buffer corresponds to the plunger-stopper, the aluminum tube corresponds to the syringe, and the water corresponds to the drug solution.

\subsubsection{LS-DYNA Numerical Model}

Numerical simulations were performed using LS-DYNA (Hallquist, 2016), a generalpurpose finite element code which can model fluid-structure interaction. The geometry of the LS-DYNA model is shown in Figure 4.3. The model is 2D axisymmetric, and the mesh is constructed using Lagrangian shell elements. All components are meshed using a structured grid, except for the conical section. The elements are approximately $0.5 \mathrm{~mm} \times 0.5 \mathrm{~mm}$ in size unless otherwise indicated, and this yields a total of $\approx 110,000$ elements. Refinement of the computational grid by a factor of 4 does not affect the results significantly: the peak pressures, stresses and strains vary by at most $8 \%$. The refined grid is not used to perform the numerical simulations due to the increased computational cost and the relatively small effect on the simulation results. The Courant number is 0.5 in all simulations.

The projectile, buffer, air gap, water, and wall are all modeled as separate material regions or parts. The base fixture is not modeled and is approximately taken into account through a boundary condition; the nodes of the wall which would be in contact with the base fixture are all rigidly clamped. The elements forming the air gap are also constrained to avoid getting a highly distorted mesh; they can only deform axially. 
Table 4.3: Parameters used in the equations of state used in the LS-DYNA model.

\begin{tabular}{ll|llll}
\hline Property & Units & Aluminum & Polycarbonate & Water & Air \\
\hline$E$ & $\mathrm{GPa}$ & 69.6 & 2.6 & & \\
$\rho_{0}$ & $\mathrm{~kg} / \mathrm{m}^{3}$ & 2700 & 1200 & 1000 & 1.23 \\
$v$ & - & 0.33 & 0.37 & & \\
$c$ & $\mathrm{~m} / \mathrm{s}$ & & & 1500 & \\
$\gamma$ & - & & & & 1.4 \\
\hline
\end{tabular}

The nodes at the buffer-air gap interface are shared by the two components. The same is true about the nodes at the air gap-water interface. For the cases where no air gap is present, the nodes at the buffer-water interface are shared by both parts. Sharing the nodes between two parts eliminates the need for a contact model.

At the projectile-buffer interface and at the water-wall interface, the LS-DYNA built-in surface-to-surface contact model is used (Hallquist, 2016). This contact model can only account for compression between the two surfaces; tensile forces are not transmitted between the two surfaces, such that the cavitation inception pressure corresponds to zero absolute pressure. In this model, whenever the liquid experiences tension, there is a loss of contact between the water and the wall, mimicking cavitation. The formation of the voids forces the pressure to remain at or above zero absolute pressure. The growth and the collapse of those voids locally mimic the effect of the bubbles during cavitation.

A linear-elastic constitutive model (Bower, 2009) is used for all solid parts. A Mie-Grüneisen equation of state (Wu, Zong, and Sun, 2014) is used for the water. The gas in the air gap is modeled as an isentropically compressed perfect gas (see Equation 2.22). The parameters used in each equation of state are shown in Table 4.3. Note that $S_{1}=S_{2}=S_{3}=0$ in the Mie-Grüneisen equation of state used to model water. With this, the Mie-Grüneisen equation of state reduces to an acoustic formulation where $\Delta P=\rho_{0} c \Delta u$.

Initially, all components are at rest except for the projectile that is traveling at the impact velocity $V_{0}$. The projectile and the buffer are initially a small distance apart $(0.1 \mathrm{~mm})$. Gravity is not accounted for in the simulations. Finally, all simulations are terminated shortly after the onset of cavitation due to the absence of an explicit cavitation model in the numerical simulations. 


\subsubsection{Results}

Five representative cases are reported to illustrate the effect of an air gap and a converging section:

- case 1: no converging section, no air gap;

- case 2: with a converging section, no air gap;

- case 3: no converging section, $3.5 \mathrm{~mm}$ air gap;

- case 4: no converging section, $12 \mathrm{~mm}$ air gap;

- case 5: with a converging section, $12 \mathrm{~mm}$ air gap.

\subsubsection{Case 1}

The first case considered is the simplest configuration with no air gap and no cone. The water column is pressurized through a direct contact between the buffer and the liquid; the liquid at the interface is forced to move with the buffer. The measured impact velocity of the projectile on the buffer is $5.7 \mathrm{~m} / \mathrm{s}$.

This configuration was examined previously by Inaba and Shepherd (2010), but without the base fixture used in the present study. Another difference is that Inaba and Shepherd used a polycarbonate tube instead of an aluminum tube. As a result, the coupling between the liquid and the structure was substantially more important than in the present study. Despite the differences, the wave dynamics described in detail by Inaba and Shepherd is essentially the same as in the present study.

Because there are many reverberations of the stress waves within the projectile and the buffer during the slowing of the buffer, the projectile and buffer can be treated as rigid bodies. The transit time of the stress waves is $36 \mu s$ in the projectile, and $44 \mu s$ in the buffer; this is shorter than the rise time of the pressure, approximately 75-100 $\mu$ s. The idea that the motion of the projectile and the buffer is governed by rigid body mechanics has been validated through numerical simulations; making the buffer and the projectile rigid does not change the results other than producing a small increase of the peak pressures. This is of course a simplifying assumption, and the reader should see Kojima et al. (2017) for a more detailed treatment of stress wave dynamics in the projectile and buffer.

The maximum pressure in the liquid below the buffer can be estimated using acoustic theory (see Chapter 2 and Appendix A). Assuming the initial velocity of the buffer is 
the impact velocity $V_{0}$, the compression wave generated upon impact has a magnitude $\Delta P \approx \rho c V_{0}$, resulting in a peak relative pressure of $8.55 \mathrm{MPa}$. As discussed by Shepherd and Inaba (2010), this pressure increase is expected to be followed by an exponential decay since the buffer begins slowing down immediately after impact, thus creating expansion waves which follow the initial compression wave.

Pressure transducers P1 and P2 are located very close to the bottom wall where the wave reflects. As a consequence, the measured peak pressure is expected to be larger than 8.55 MPa. When the wave reaches the bottom wall, it is partly transmitted through the base fixture and partly reflected into the water, as explained in Chapter 2 (see Figure 2.4, Equations 2.1 and 2.2, and Table 2.1).

For the present experiment, medium 1 is water and medium 2 is aluminum. Table 2.1 indicates $\Delta P_{r} \approx 0.81 \Delta P_{i}$. When the incident wave reflects at the bottom wall, the pressure increase there is the sum of the incident and the reflected waves, that is $1.81 \Delta P_{i}$. In the present case, the expected pressure is therefore $\approx 15.5 \mathrm{MPa}$. Note that when the polycarbonate base fixture is used, the sum of the incident and the reflected wave is smaller; it is $1.30 \Delta P_{i}$. This is a consequence of the lower acoustic impedance of polycarbonate compared to aluminum.

Pressures P1 and P2 are shown in Figure 4.4. Both P1 and P2 are very similar in trend and magnitude; this is because there is no converging section, and both pressure transducers are located only $50 \mathrm{~mm}$ apart. The peak pressure measured experimentally is $16.1 \mathrm{MPa}$, within $4 \%$ of the $15.5 \mathrm{MPa}$ predicted using acoustic theory. The peak pressure predicted with LS-DYNA is within $10 \%$ of the experimental value. The first pressure wave is followed by a second wave of smaller amplitude (reaching the bottom at $\sim 1.4 \mathrm{~ms}$ ), and this is immediately followed by a first cavitation event. The cavitation event approximately spans from $2 \mathrm{~ms}$ to $31 \mathrm{~ms}$ (note that the time-axis in Figure 4.4 is discontinuous). This is followed by a few more cavitation events of decreasing duration and intensity, which are not shown and discussed; see Inaba and Shepherd (2010) for more information on this.

The wave dynamics in the test specimen is further explained using Figure 4.5. Upon impact of the projectile on the buffer (label 1), a pressure wave forms in the liquid. This wave travels down the tube, partially reflects off the bottom wall (label 2 ), and then travels upward. After one round trip in the tube, the pressure wave partially reflects on the buffer (label 3). The reflection of the wave on the buffer creates a second pressure wave that later reaches the bottom of the tube (label 4). The reflection of the pressure wave on the buffer (label 3) also initiates an upward 


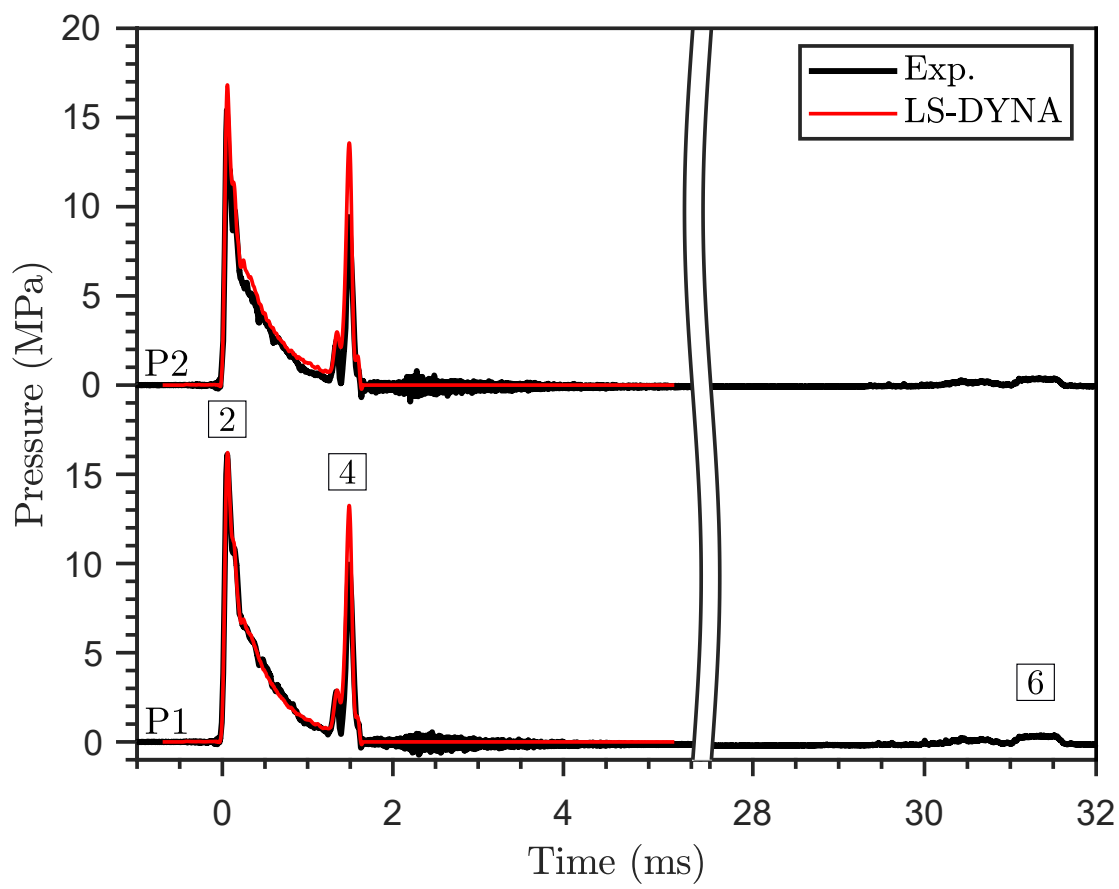

Figure 4.4: Pressure at the bottom end for case 1 of the static, large-scale model autoinjector experiments - the time axis is discontinuous (test GG-0030).

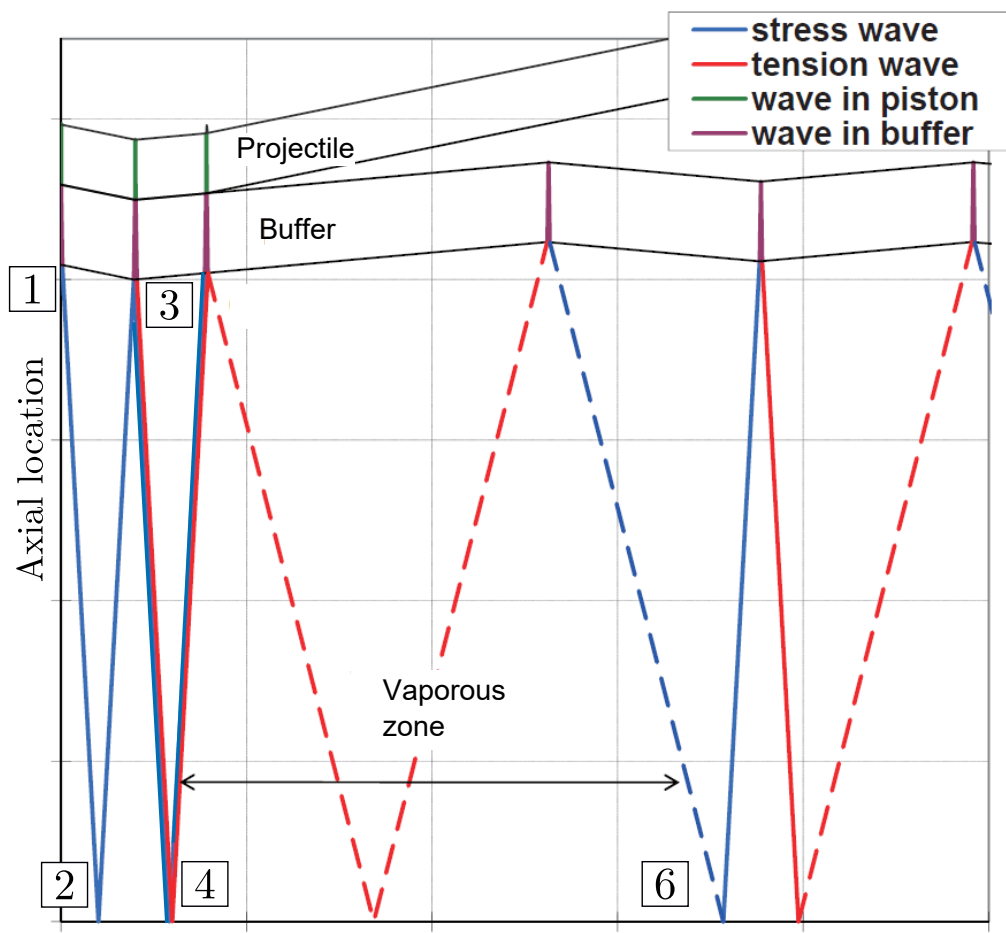

Time

Figure 4.5: Wave dynamics in the static, large-scale model autoinjector test setup (adapted from Inaba and Shepherd (2010) with permission). 
motion of the buffer. The upward motion of the buffer produces tension waves which immediately follow the second pressure wave. The tension waves result in distributed cavitation in the water column. Visual confirmation of this is provided in case 2. This cavitation event ends after the direction of motion of the buffer is once more reversed; the downward motion of the buffer sends a compression wave that collapses the bubbles as it propagates from top to bottom. The arrival of this compression wave at the bottom of the tube is detected by the pressure transducers (label 6).

This is followed by several cycles of cavitation of decaying duration and intensity, but this occurs over much longer times than shown in the figures. This is similar to what was observed and reported by Inaba and Shepherd (2010) and Shepherd and Inaba (2010). Note that the main focus of this thesis is on the events which take place shortly after the impact of the projectile on the buffer (i.e., labels 1 through 5).

The hoop $\left(\epsilon_{\theta}\right)$ and axial $\left(\epsilon_{z}\right)$ strains from the experiment and the simulations are shown in Figure 4.6 for the first $5 \mathrm{~ms}$ after impact. The bottommost trace corresponds to location S1, and the topmost trace corresponds to location S7, as summarized in Table 4.2. The scale for the strains is shown to the right of the plot.

The oblique lines shown in Figure Figure 4.6 have a slope which corresponds to the Korteweg speed $c_{K}$. The Korteweg speed is the expected velocity of the pressure waves in the liquid for the fluid-structure coupled problem in the absence of cavitation. It can be evaluated as follows:

$$
c_{K}=\frac{c_{l}}{\sqrt{1+\beta}}, \quad \beta=\frac{K}{E}\left(\frac{D_{o}+D_{i}}{D_{o}-D_{i}}\right),
$$

where $c_{l}$ is the sound speed in water, and $\beta$ is the FSI coupling parameter with $K$ the bulk modulus of water, $D_{i}$ and $D_{o}$ the inner and outer diameter of the aluminum tube, and $E$ the Young's modulus of the tube (Shepherd and Inaba, 2010). Larger values of the coupling parameter $\beta$ indicate that the pressure waves propagating in the liquid tend to create larger strains in the wall of the tube. Equivalently, it also indicates that stress waves propagating in the wall of the tube tend to create pressure waves of larger magnitude in the liquid. In the present case, $\beta$ is equal to 0.22 and $c_{K}=1350 \mathrm{~m} / \mathrm{s}$, only $10 \%$ lower than $c_{l}$. This indicates the intensity of coupling is relatively small. Note that the value of $\beta$ is also equal to 0.22 in a pre-filled glass syringe, as indicated in Section 2.2.3. The experimental results indicate that both pressure and strains waves appear to propagate with the Korteweg speed. 


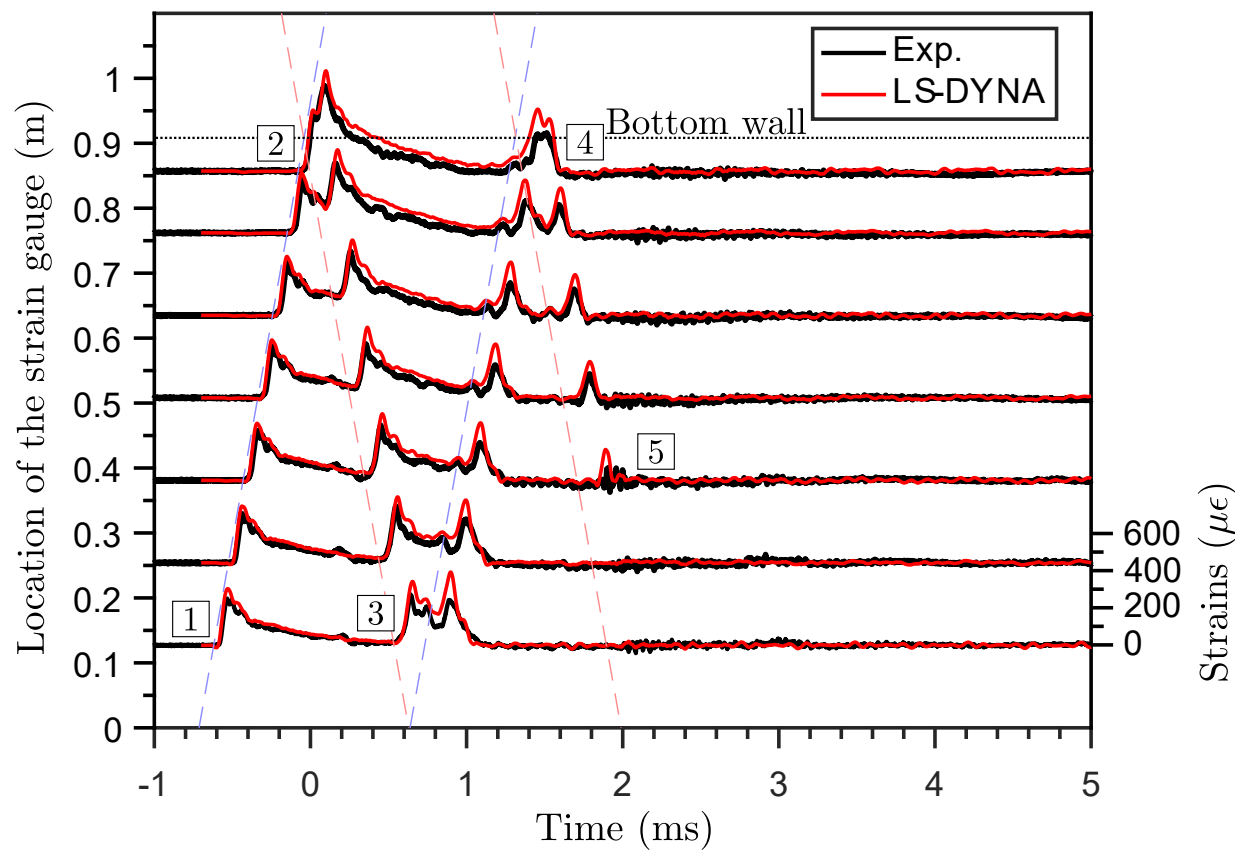

(a) Hoop strains

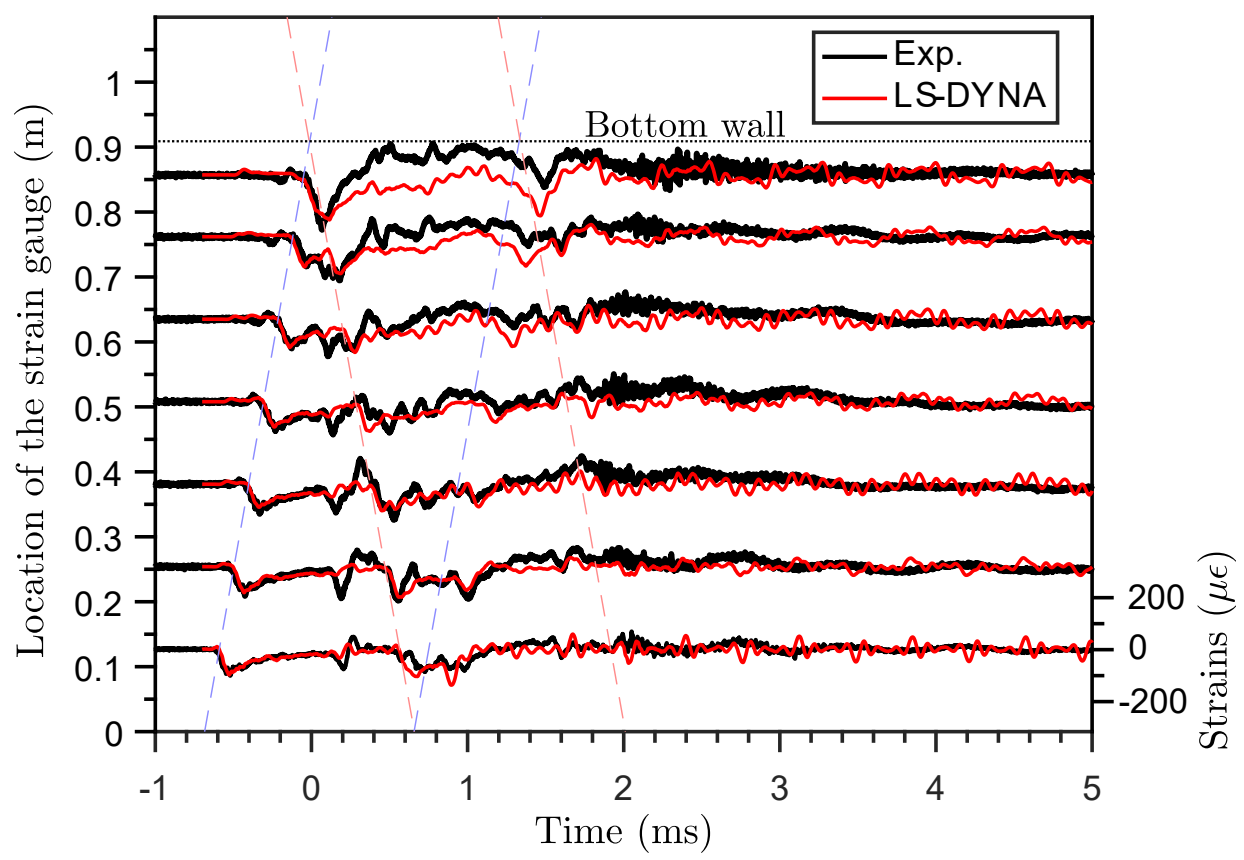

(b) Axial strains

Figure 4.6: Hoop and axial strains for case 1 of the static, large-scale model autoinjector experiments (test GG-0030). 

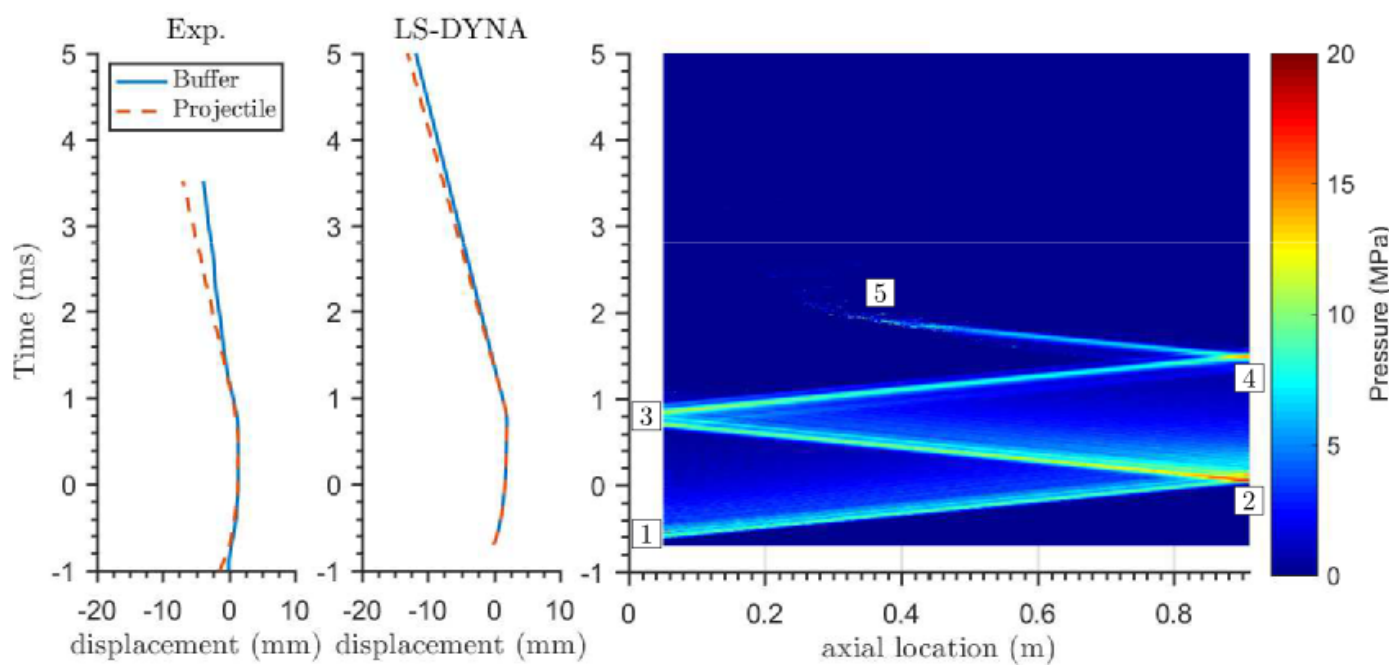

Figure 4.7: Motion of the buffer and the projectile with a space-time pressure plot (LS-DYNA) for case 1 of the static, large-scale model autoinjector experiments (test GG-0030).

Returning to Figure 4.6, there is reasonable agreement between the experiment and the simulations, especially for the hoop strains. The axial strains predicted by LSDYNA close to the bottom of the test specimen are not in good agreement with the experiment, which could be due to the sensitivity of the axial strains to the boundary conditions. The base fixture and the plates to which it is bolted are not modeled in detail, but the tube end is treated as fixed in the simulations. On the contrary, the boundary conditions applied on the top end of the tube are modeled realistically, allowing motion in both radial and axial directions. There, the agreement between the experiment and the simulation is much better for axial strains.

Figure 4.7 is a space-time plot of the pressure, along with the motion of the projectile and the buffer. The measured and simulated motions of the projectile and buffer are in good agreement up to $2 \mathrm{~ms}$. The space-time plot of the pressure from the simulation illustrates the propagation of the pressure wave along the axis of symmetry of the tube.

The dynamics of the transient behavior is now examined using Figures 4.6a and 4.7. At approximately $t=-0.6 \mathrm{~ms}$ (label 1 ), the projectile impacts on the buffer. This pushes the buffer down and creates a pressure wave within the liquid, as described above. This pressure wave, the first incident wave, propagates downward into the liquid at the Korteweg speed of $1350 \mathrm{~m} / \mathrm{s}$ (i.e., parallel to the characteristic lines shown in Figure 4.6a). When the wave reaches the bottom of the tube at 
approximately $t=0 \mathrm{~ms}$ (label 2), reflection occurs, creating a wave traveling upward which will be called the first reflected wave.

The hoop strains associated with the first incident and reflected pressure waves are approximately $300 \mu \epsilon$, except close to the bottom wall where the hoop strains are close to $600 \mu \epsilon$ due to the pressure increase associated with reflection.

When the first reflected wave reaches the bottom end of the buffer, it reflects (label 3 ), creating the second incident wave traveling downward into the liquid. This wave, however, is immediately followed by tension waves due to the motion of the buffer. This is because upon reflection of the first reflected wave on the buffer (label 3), the resulting liquid pressure accelerates the buffer upward, and this upward motion results in the production of tension waves just behind the second incident wave (see Section 4.2.3 for more details on the reflection of pressure waves on an accelerated boundary). Those tension waves also explain why the second reflected wave (the one created during event 4) is eventually annihilated (label 5).

Regarding the axial strains, assuming zero axial stress and using shell theory yields $\epsilon_{z}=-v \epsilon_{\theta}$ (Bitter and Shepherd, 2013). This assumption is only valid close to the top end of the tube, and it implies the axial strains are produced through the Poisson effect. Using the experimental results it is possible to verify that the relation above is approximately satisfied; the magnitude of the axial strains is approximately one-third that of the hoop strains.

\subsubsection{Case 2}

Case 2 is identical to case 1, except the test is performed using the aluminum base fixture which has a cone. The impact velocity of the projectile on the buffer is $5.6 \mathrm{~m} / \mathrm{s}$. Acoustic theory, as introduced earlier, predicts a peak pressure of $15.2 \mathrm{MPa}$ at the bottom of the test specimen. However, shock focusing could occur within the cone (Apazidis and Eliasson, 2019; Sturtevant and Kulkarny, 1976), and this could result in a larger peak pressure at the tip (P2). Shock focusing in the cone is further investigated in Chapter 5.

Pressures $\mathrm{P} 1$ and $\mathrm{P} 2$ are shown in Figure 4.8. We recall that transducer P1 is mounted above the cone, and transducer $\mathrm{P} 2$ is mounted at the apex of the cone. The maximum value of P1 and P2 within the first $5 \mathrm{~ms}$ is $14.8 \mathrm{MPa}$, which is in reasonable accord with the predicted value of $15.2 \mathrm{MPa}$. The fact that the peak pressures recorded 


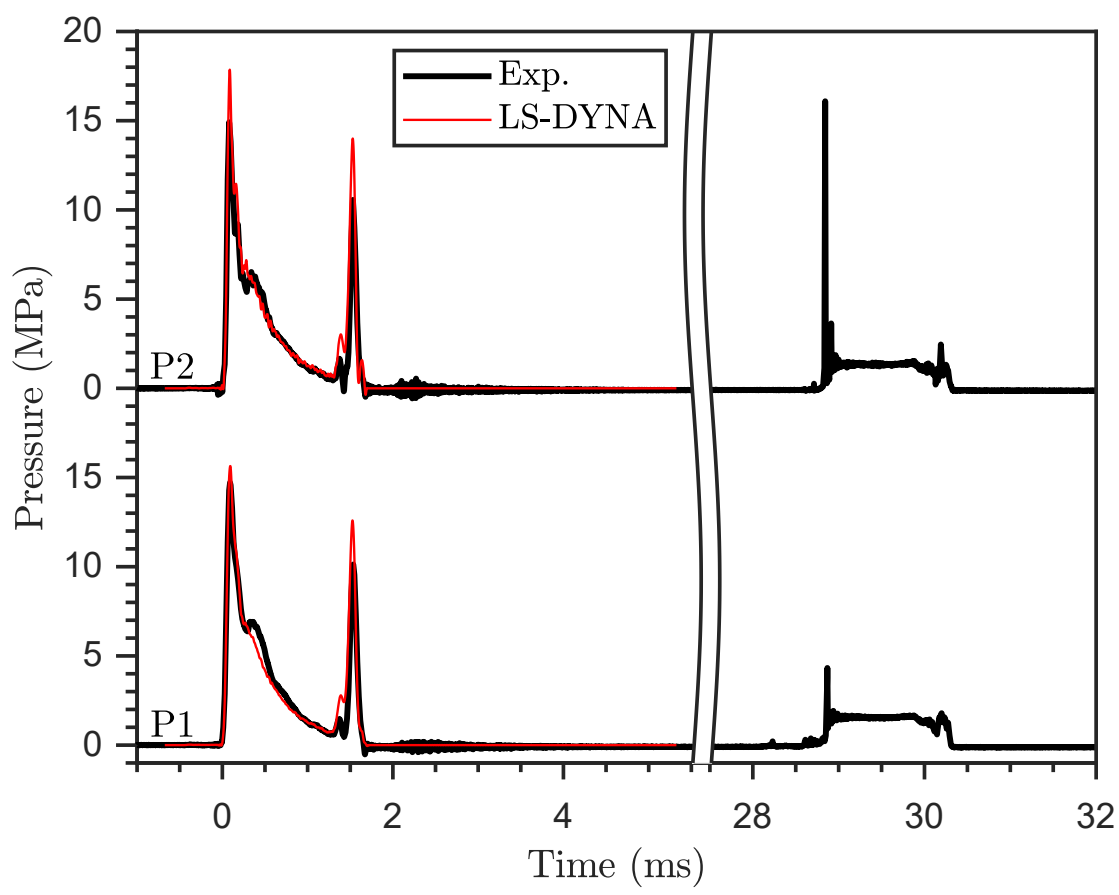

Figure 4.8: Pressure at the bottom end for case 2 of the static, large-scale model autoinjector experiments - the time axis is discontinuous (test GG-0240).

above the cone (P1) and at the tip of the cone (P2) are similar suggests that shock focusing does not occur.

Shock focusing does not occur in this test because the transit time $D_{b, i} / c_{l}$ for pressure waves within the converging section is $\sim 25 \mu$ s, smaller than the rise time of the first incident pressure wave of approximately $100 \mu \mathrm{s}$. The pressure has time to equilibrate throughout the cone during pressurization, and shock focusing does not occur.

Pressures P1 and P2 due to the collapse of the cavitation bubbles, $29 \mathrm{~ms}$ after impact, are substantially different. The peak pressure above the cone (P1) is $4.3 \mathrm{MPa}$, and the peak pressure at the tip of the cone $(\mathrm{P} 2)$ is $16.1 \mathrm{MPa}$. In repeat tests, the peak pressure measured at the tip of the cone was consistently higher than the peak pressure measured above the cone upon bubble collapse.

The explanation for the larger pressure at the tip of the cone is twofold. First, shock focusing can occur (Apazidis and Eliasson, 2019; Sturtevant and Kulkarny, 1976). The rise time of the pressure wave is approximately $10 \mu \mathrm{s}$, and this is less than the acoustic transit time of the waves within the cone. The pressure is not uniform throughout the cone during pressurization, and amplification of the 


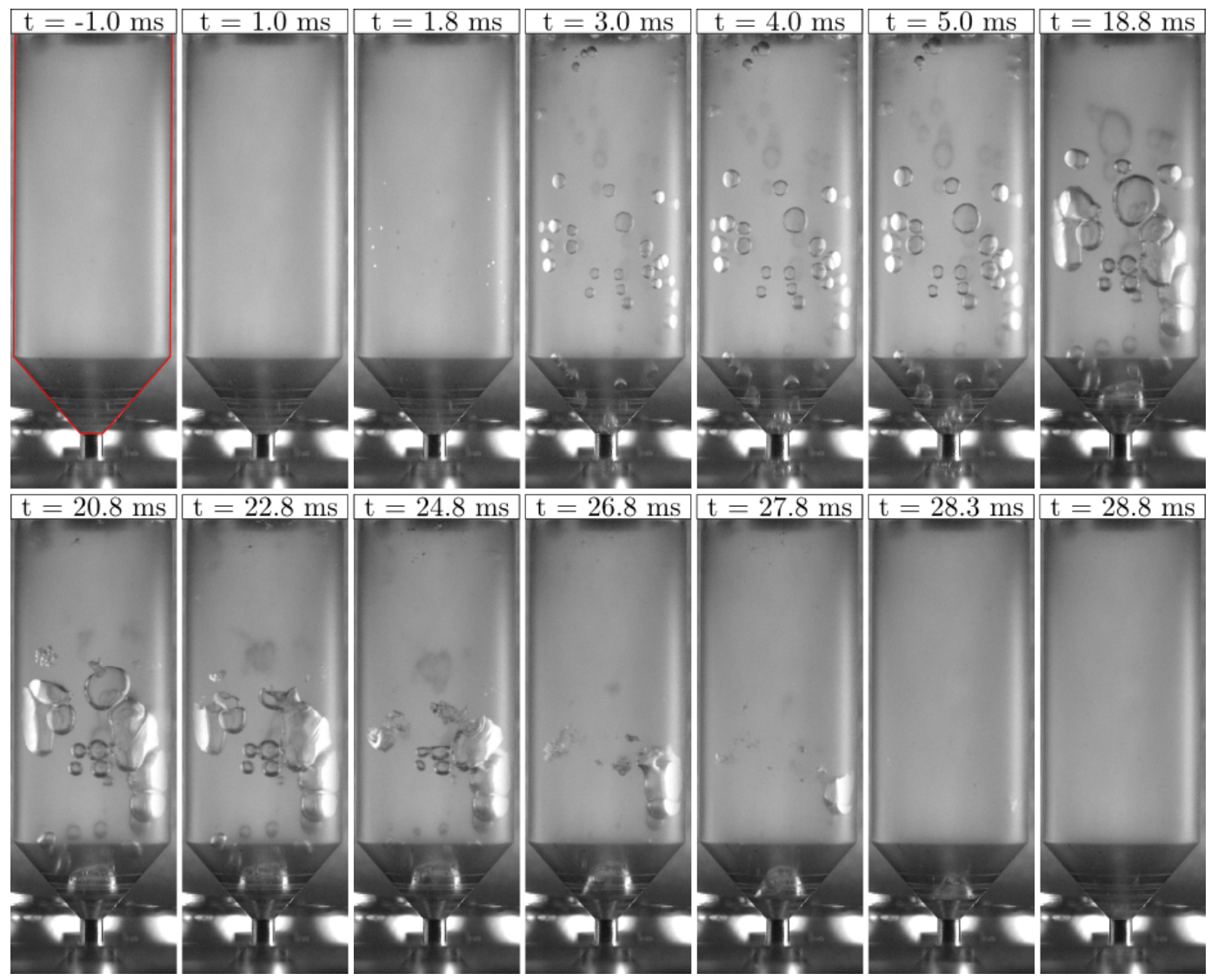

Figure 4.9: Sequence of images showing distributed cavitation for case $2 b$ of the static, large-scale model autoinjector experiments (test GG-0215).

pressure is possible (see Chapter 5). Second, the collapse of bubbles within the cone can be enhanced by the geometry; focusing of the pressure waves on the axis of symmetry can accelerate the collapse of bubbles. The collapse of bubbles in a cone is investigated and discussed in Chapter 6.

The strains for case 2 are not shown; they are very similar to the strains shown for case 1. This is because the wave dynamics within the test specimen is the same as for case 1, except locally near the cone. The measured strains are insensitive, however, to the local effect of the cone due to the placement of the gauges away from this region.

Case 2 was repeated a number of times with the polycarbonate base fixture in order to observe bubble dynamics in the cone (tests GG-0200 to GG-0215). The timing of the events and the magnitude of the peak pressures are similar to those obtained with the aluminum base fixture. A sequence of frames obtained with the polycarbonate base fixture is shown in Figure 4.9; this test is identified as case 2b. The time stamp 
shown at the top of each frame can be used to approximately locate each frame on the pressure history shown in Figure 4.8 (the reader is however reminded that Figure 4.8 was obtained with an aluminum base fixture). The edges of the straight tube and the cone are identified in the first frame of Figure 4.9.

The first two frames $(t=-1.0 \mathrm{~ms}$ and $t=1.0 \mathrm{~ms})$ show no sign of cavitation. This is expected since no tension waves have reached the bottom end of the tube yet, and this region is under compression (i.e., $P>0 \mathrm{MPa}$ ). The first tension waves reach the bottom of the tube at $t=1.8 \mathrm{~ms}$, and nucleation of several bubbles distributed throughout the visible portion of the tube is observed. The polycarbonate base fixture introduces optical distortions in the images, and it is only possible to clearly distinguish the bubbles forming on the front of the tube. The bubbles forming away from the front are visible, but out of focus.

The growth of multiple bubbles is observed from $t=3.0 \mathrm{~ms}$ to $t=18.8 \mathrm{~ms}$. In particular, a few bubbles forming and growing in the cone coalesce to create a larger bubble approximately centered on the axis of symmetry, and located near the bottom of the cone. The collapse of the bubbles takes place from $t=20.8 \mathrm{~ms}$ to $t=28.8 \mathrm{~ms}$. The bubbles successively collapse from top to bottom due to the slow progression of a compression wave in the bubbly mixture. The collapse of the bubbles is asymmetric, as expected, due to the proximity of the walls and the shock-induced origin of the collapses (Brennen, 1995).

\subsubsection{Case 3}

Case 3 is identical to case 1, except there is a $3.5 \mathrm{~mm}$, air gap between the bottom of the buffer and the water surface. The impact velocity of the projectile on the buffer is $5.5 \mathrm{~m} / \mathrm{s}$. The air gap drastically affects both the interaction of the projectile and buffer, as well as the transmission of pressure waves into the liquid column.

Pressures P1 and P2 are shown in Figure 4.10 for the first $5 \mathrm{~ms}$ after impact. The dynamics taking place after $5 \mathrm{~ms}$ is very similar to what was observed and described for cases 1 and 2. The signals recorded using P1 and P2 are very similar in trend and magnitude. The pressure history is however more complicated than it was in cases 1 and 2; there are now multiple pressure peaks. The measured peak pressure is approximately $11.0 \mathrm{MPa}, 30 \%$ lower than the peak pressures measured for case 1 . This is because the water column is now pressurized through the isentropic compression of the air gap. LS-DYNA does not predict all the fine details of the 


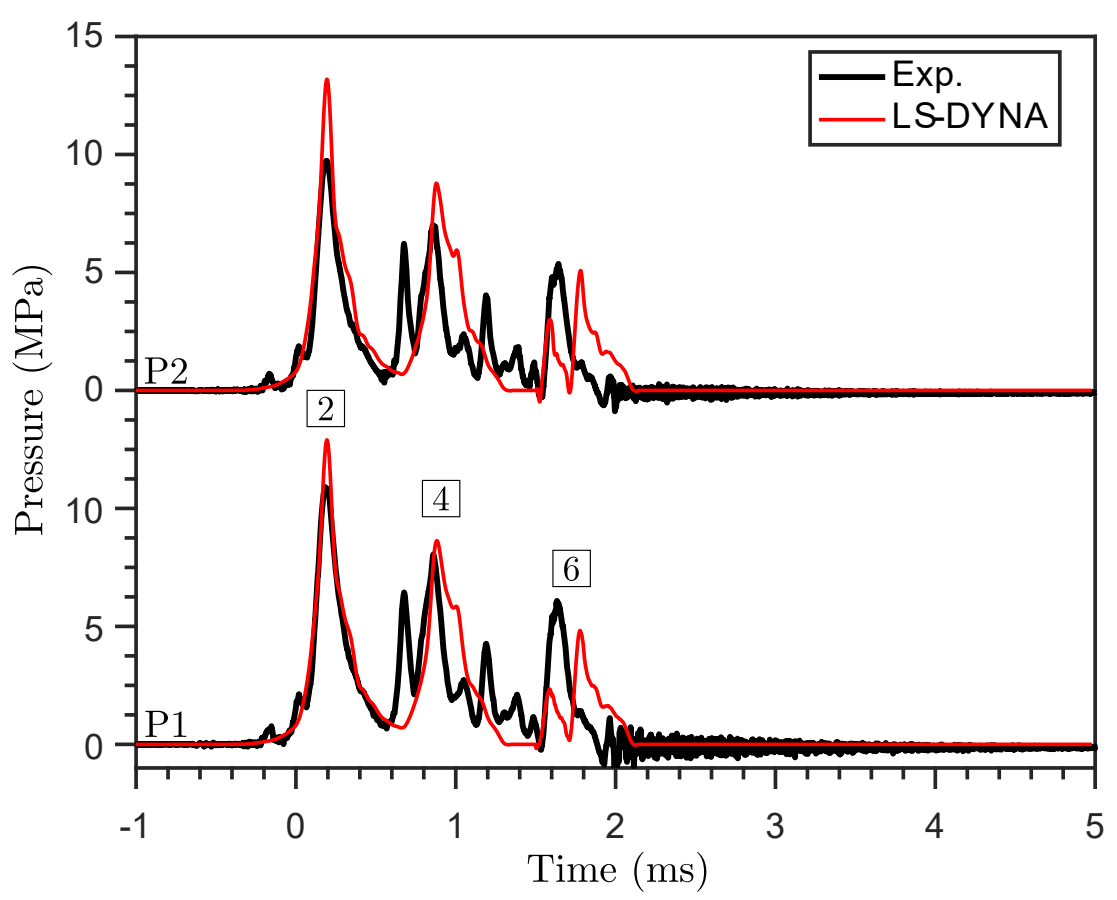

Figure 4.10: Pressure at the bottom end for case 3 of the static, large-scale model autoinjector experiments (test GG-0033).

experimental pressure traces, but it does predict the presence of multiple pressure peaks.

The motion of the projectile and the buffer along with a space-time plot of the pressure is shown in Figure 4.11. The projectile rebounds from the buffer repeatedly, resulting in multiple impacts between the projectile and the buffer, which is different from cases 1 and 2 where only one impact was observed. When there is an air gap, the projectile, the buffer and the air gap form a spring-mass system with the air gap being equivalent to a non-linear spring. Although the results are not shown here, using rigid body mechanics (i.e., conservation of momentum and energy) with a non-linear spring for the air gap enables reasonable quantitative predictions of the interactions between the projectile, the buffer, and the air gap. It is possible to approximate the pressure within the air gap as uniform since the waves transit sufficiently rapidly $(10 \mu \mathrm{s})$ within the air gap that there are multiple reverberations during the compression or the expansion of the gap.

In Figure 4.11 there are now 3 distinguishable impacts between the projectile and the buffer (labels 1, 3, and 5), each of which results in the production of a pressure wave. Each of these waves reflect at the bottom wall (labels 2, 4, and 6), and 


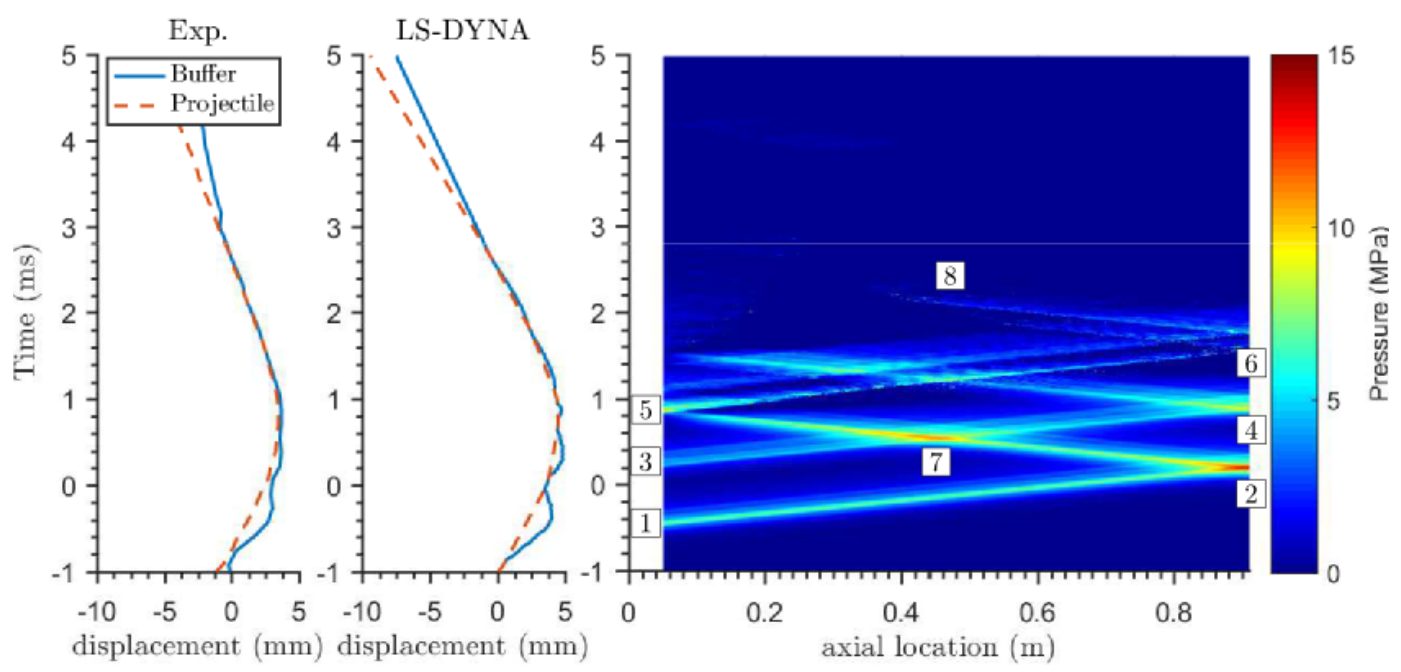

Figure 4.11: Motion of the buffer and the projectile with a space-time pressure plot (LS-DYNA) for case 3 of the static, large-scale model autoinjector experiments (test GG-0033).

propagate upward toward the buffer. However, only the wave due to the first impact has enough time to reach the top of the liquid column. This is because the reflection of the first compression wave (label 5) happens at a free surface, and this creates a tension wave. This can be understood using Equations 2.1 and 2.2 along with the data shown in Table 2.1, which indicate that $\Delta P_{r} \approx-\Delta P_{i}$ : the sign of the pressure wave changes, and a compression wave becomes a tension wave upon reflection.

As in case 1, the buffer starts moving upward after reflection of the first wave occurs (label 5), and this creates relatively strong tension waves. The tension waves forming at the top end of the tube propagate throughout the tube and interfere destructively with the second and third waves before they reach the buffer. At $t=2.5 \mathrm{~ms}$ (label 8), the entire water column is under the influence of the tension waves, and distributed cavitation occurs. This is identical to what happens in cases 1 and 2, and this was also observed by Inaba and Shepherd (2010). Images of the distributed cavitation are not included since the cavitation event is similar to the one reported for case 2 (see Figure 4.9).

Another interesting feature is the possibility of having some constructive interference between the multiple waves propagating within the tube. This is observed at label 7 , where the incident wave due to the second impact interacts constructively with the reflected wave due to the first impact. The constructive interference can result in peak pressures and strains in locations away from the bottom of the tube. 


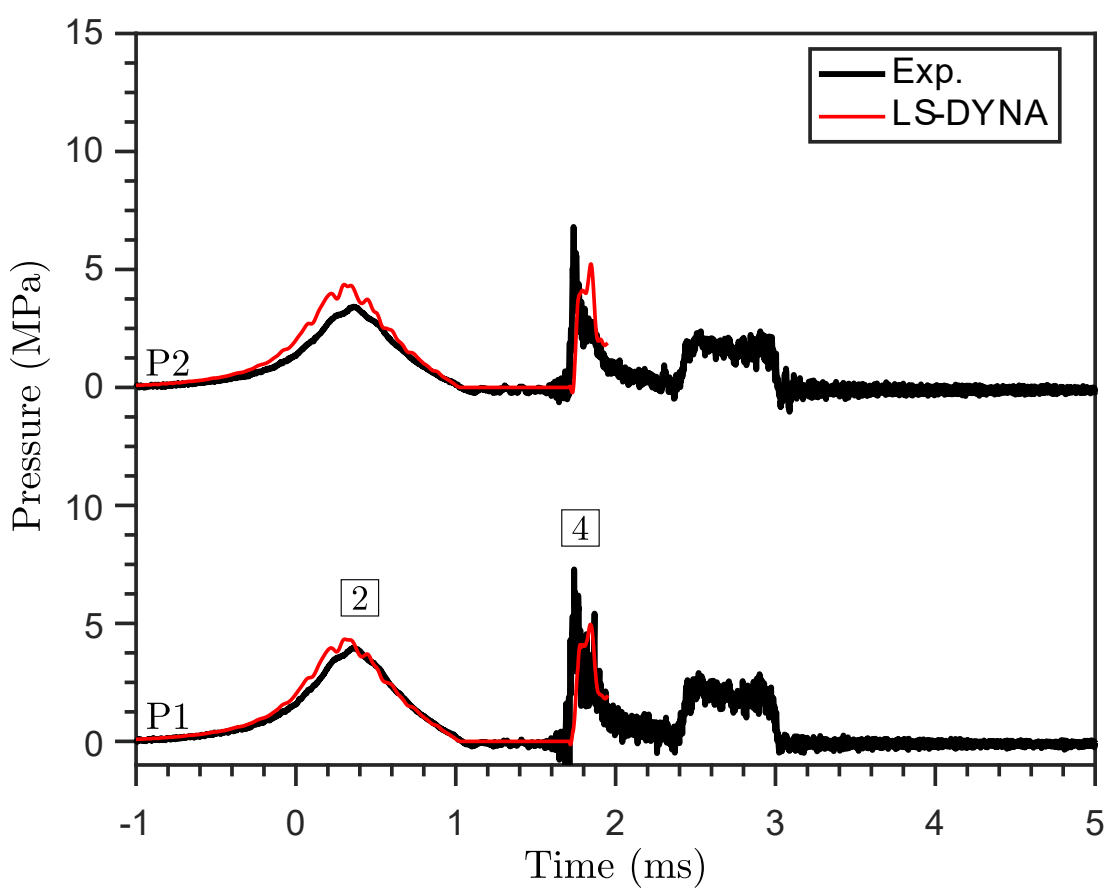

Figure 4.12: Pressure at the bottom end for case 4 of the static, large-scale model autoinjector experiments (test GG-0034).

\subsubsection{Case 4}

Case 4 is identical to case 3 , except there is initially a $12.0 \mathrm{~mm}$ air gap between the buffer and the water surface. The impact velocity of the projectile on the buffer is $5.6 \mathrm{~m} / \mathrm{s}$. The size of the air gap drastically affects the timing of the multiple impacts between the projectile and the buffer.

Pressures P1 and P2 are shown in Figure 4.12 up to $5 \mathrm{~ms}$ after impact. The two pressure traces are close in trend and magnitude. The match between the experiment and the simulation is also good. It is now possible to distinguish two main pressure waves. The first one reaches a peak pressure of approximately $3.9 \mathrm{MPa}$ at $t=0.3 \mathrm{~ms}$, and the pressurization happens slowly in comparison to the wave transit times through either gas or liquid; the pressure takes $\sim 1 \mathrm{~ms}$ to reach its peak value. The second pressure wave is sharp and reaches a peak value of $7.2 \mathrm{MPa}$ at $t=1.75 \mathrm{~ms}$. The rise time associated with this pressure wave is approximately $30 \mu \mathrm{s}$. Between the first and the second pressure waves ( $t$ between 1.0 and $1.6 \mathrm{~ms}$ ), the liquid is under the influence of tension waves, and cavitation occurs. Unlike cases 1 and 2, the second pressure wave is not due to the reflection of the first pressure wave, but instead results from a second impact of the projectile on the buffer. 


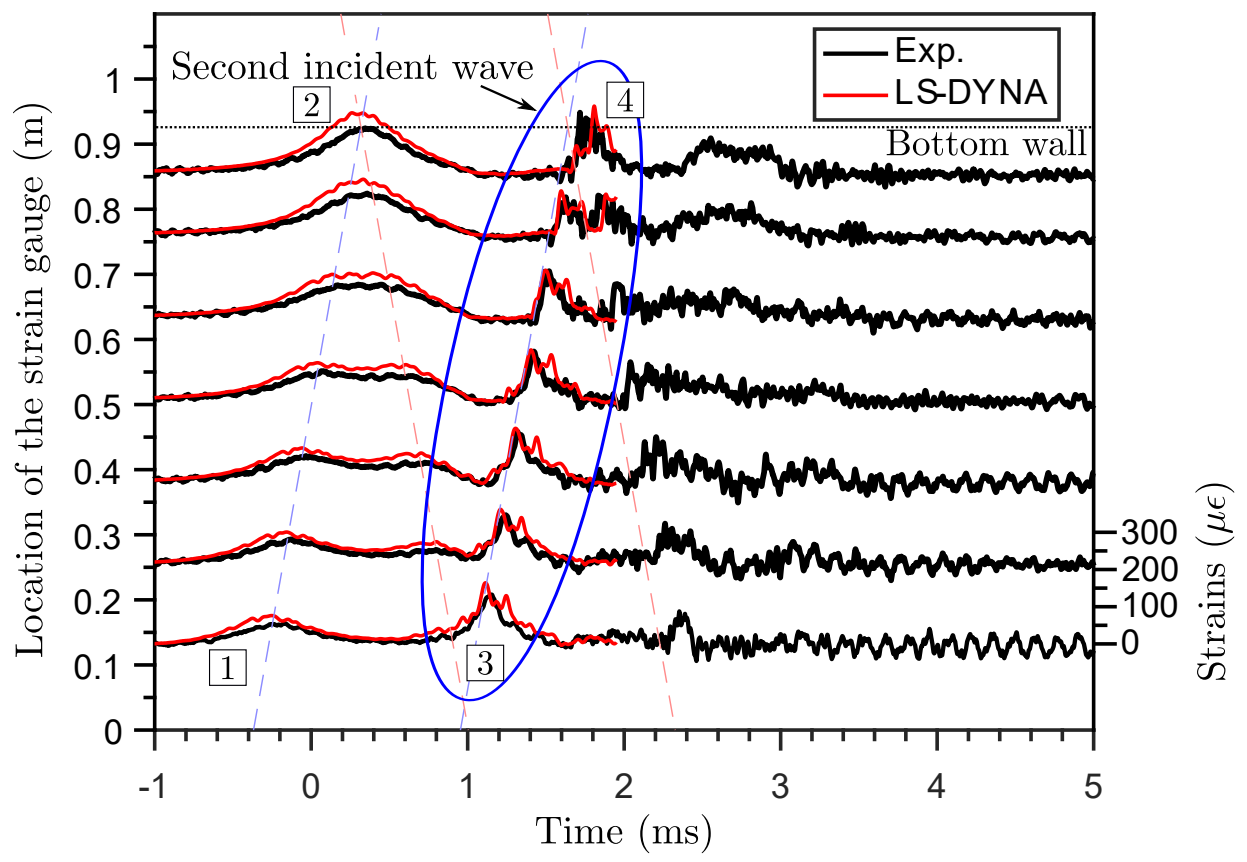

Figure 4.13: Hoop strains for case 4 of the static, large-scale model autoinjector experiments (test GG-0034).

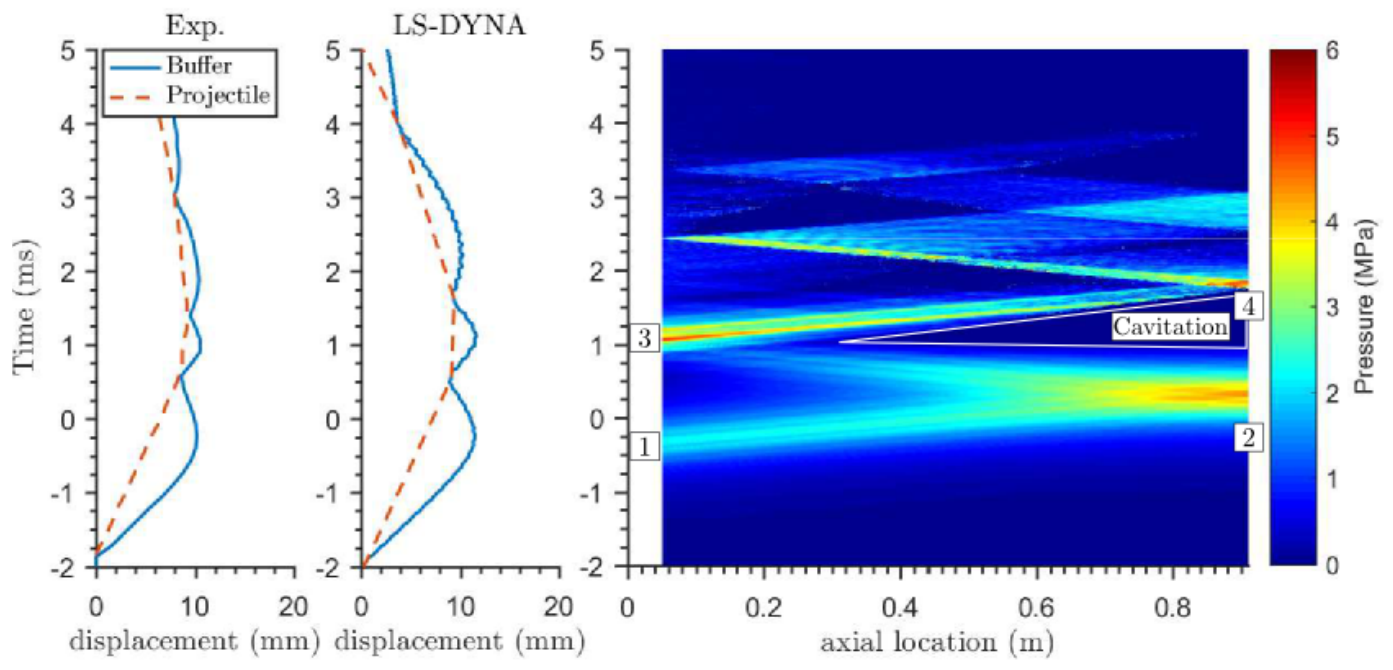

Figure 4.14: Motion of the buffer and the projectile with a space-time pressure plot (LS-DYNA) for case 4 of the static, large-scale model autoinjector experiments (test GG-0034). 
Table 4.4: Rise time of the second incident wave for case 4 of the static, large-scale model autoinjector experiments (test GG-0034).

\begin{tabular}{r|ccccccccc}
\hline Station & $\mathrm{S} 1$ & $\mathrm{~S} 2$ & $\mathrm{~S} 3$ & $\mathrm{~S} 4$ & $\mathrm{~S} 5$ & $\mathrm{~S} 6$ & $\mathrm{~S} 7$ & $\mathrm{P} 1$ & $\mathrm{P} 2$ \\
$\Delta T(\mu \mathrm{s})$ & 360 & 170 & 160 & 130 & 70 & 70 & 50 & 30 & 30 \\
\hline
\end{tabular}

The projectile and buffer's motion along with a space-time plot of the pressure are shown in Figure 4.14. There are multiple impacts between the projectile and the buffer. The first one (label 1) is responsible for the slow pressurization of the water column through compression of the air gap. This corresponds to the first pressure pulse visible on both P1 and P2. When this first wave reflects off the bottom wall (label 2), a compression wave traveling upward is created. When this wave reaches the top of the tube and reflects off the free surface between the air and the liquid, it becomes a tension wave for the same reason as in case 3, and causes distributed cavitation. This tension wave is followed by the second incident wave, which is caused by the second impact of the projectile on the buffer (label 3). This second incident wave is propagating into a bubbly mixture created by the cavitation event caused by the tension wave.

The collapse of the cavities under pressure reduces the void fraction, increases the wave speed, and results in wave steepening. Wave steepening occurs because the head of the wave travels faster than the tail: the head travels in a bubbly liquid which has a larger void fraction than the void fraction at the tail of the wave. This is similar to the wave steepening leading to shock wave observed with simplified models such as the traffic flow equation and Burger's equation (Whitham, 1999). This results in the second incident pressure wave becoming sharp before reaching the bottom end of the tube. This explains the very short rise time of the second pressure wave (see Table 4.4).

The steepening of the second pressure wave is also observed on the hoop strains shown in Figure 4.13. For stations S3 to S7, careful reading of the plot shows that the second incident wave is preceded by a negative hoop strain, which indicates the liquid is locally at a sub-atmospheric pressure. Visually, the steepening of the wave is observed between locations S3 to S7. The rise times of the hoop strains and pressures associated with the second incident wave are summarized in Table 4.4.

LS-DYNA appears to simulate the steepening of the pressure wave despite the absence of an explicit cavitation model. As mentioned previously, this is because 


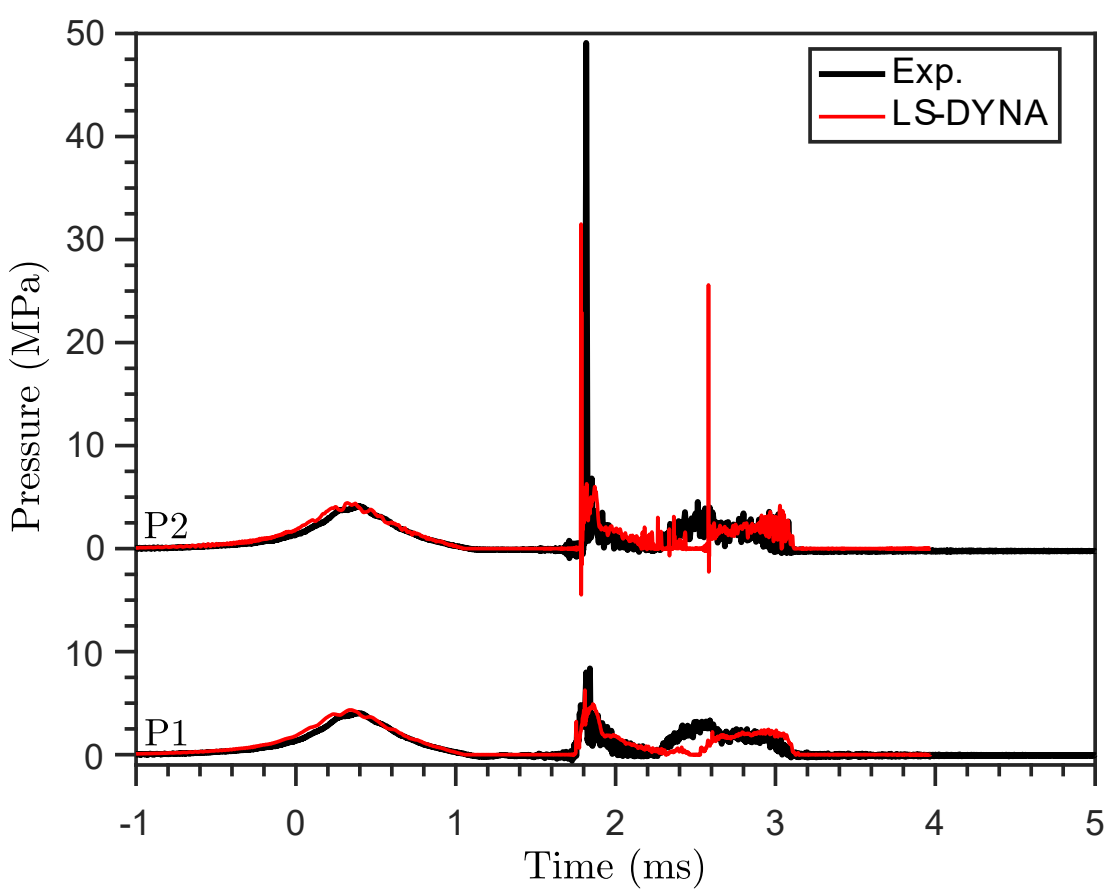

Figure 4.15: Pressure at the bottom end for case 5 of the static, large-scale model autoinjector experiments (test GG-0099).

of the boundary condition between the aluminum tube and the water which mimics the effect of cavitation. A separate simulation was performed using a boundary condition which allows for tensile forces between the two surfaces. This eliminates the pseudo-cavitation model and suppresses wave steepening.

\subsubsection{Case 5}

Case 5 is identical to case 4, except the test specimen is terminated with a converging section. The impact velocity of the projectile on the buffer is $6.4 \mathrm{~m} / \mathrm{s}$. The dynamics of cases 4 and 5 are identical; only the pressure at the bottom of the test specimen differs between these cases.

Both P1 and P2 are shown in Figure 4.15. As in case 4, there are two main pressure waves created by the two impacts between the projectile and the buffer. The first wave which has a rise time of order $\sim 1 \mathrm{~ms}$ is considered first. The peak pressure is approximately 4.3 MPa, and it is well predicted by LS-DYNA. The peak pressure due to the first wave is the same above the cone (P1) and at the tip of the cone (P2); there is no amplification of the pressure due to the converging section. There is no shock focusing because the transit time of the acoustic waves in the cone (25 $\mu \mathrm{s})$ 
is much smaller than the $1 \mathrm{~ms}$ rise time of the first pressure wave. The rise time of the second pressure wave is approximately $16 \mu s$ (measured using P2). The peak pressure measured at the apex of the converging section $(\mathrm{P} 2)$ is approximately $50 \mathrm{MPa}$, and the peak pressure measured above the converging section (P1) is about 8.4 MPa, or 6 times lower than P2.

Identical to case 2, the explanation for the larger peak pressure at the tip of the cone is twofold. First, there is shock focusing within the cone (Apazidis and Eliasson, 2019; Sturtevant and Kulkarny, 1976). Shock focusing is possible because the rise time of the pressure wave $(16 \mu \mathrm{s})$ is less than the acoustic transit time of the waves within the cone $(25 \mu \mathrm{s})$. The strain signals for case 5 are not shown because they are similar to those shown for case 4 . Wave steepening is observed and responsible for the short rise time of the second pressure wave. Second, there is a rapid bubble collapse within the cone in the vicinity of the tip where the pressure transducer is mounted. Visual confirmation of this is provided below.

Repeated tests (referred as test cases 5b) were performed with the same test conditions, but the aluminum base fixture was replaced with the polycarbonate base fixture, making it possible to visualize the cavitation events in the cone. The timing of the events and the magnitude of the peak pressures with the polycarbonate base fixture is similar to what was obtained with the aluminum base fixture. There is however one significant difference between cases 5 and $5 \mathrm{~b}$ : wave steepening does not occur when the polycarbonate base fixture is used.

The absence of wave steepening results from a milder distributed cavitation event in the tube when the polycarbonate fixture is used. The steepening of the second incident wave, as explained earlier using case 4 , is caused by the propagation of the second incident wave in a bubbly mixture due to cavitation. If the cavitation event is milder, there are less bubbles and/or they remain smaller, resulting in less or no steepening at all of the second incident wave as it propagates down the tube.

The milder distributed cavitation event in the tube terminated with the polycarbonate fixture is due to the lower acoustic impedance of polycarbonate $(\approx 3 \mathrm{MPa} \cdot \mathrm{s} / \mathrm{m})$ compared to aluminum ( $\approx 15 \mathrm{MPa} \cdot \mathrm{s} / \mathrm{m})$. With the aluminum base fixture, $81 \%$ of the first incident wave is reflected at the bottom wall, compared to $30 \%$ with the polycarbonate base fixture. We recall that the reflected part of the first incident wave, upon reaching the interface between the liquid and the air gap, becomes a tension wave, and this is what creates the tension wave responsible for the cavitation event. The reduction in the magnitude of the reflected wave indicates that the magnitude of 

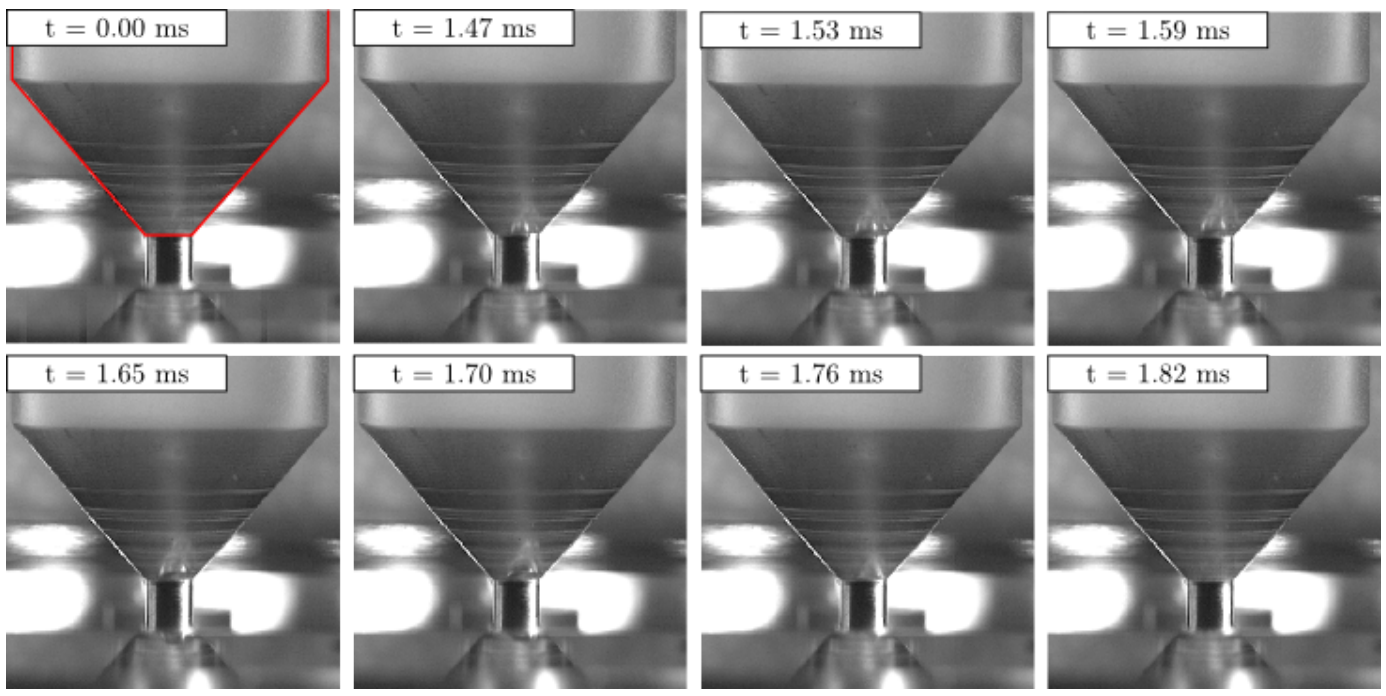

Figure 4.16: Sequence of images showing cavitation at the tip of the cone for tests performed with a large air gap (test GG-0235).

the tension wave is smaller in case 5 b than case 5 , thus causing a milder cavitation event.

Figure 4.16 is a sequence of images of the cavitation event in the cone for case $5 \mathrm{~b}$. The polycarbonate base fixture was used to obtain this sequence of images. The time stamps shown at the top of each frame can be used to approximately position each frame on the pressure history shown in Figure 4.15 (the reader is reminded that Figure 4.15 was obtained with an aluminum base fixture).

The first frame (i.e., $t=0.00 \mathrm{~ms}$ ) is taken before the arrival of the tension wave at the bottom of the tube, and there is no cavitation. The frames from $t=1.47 \mathrm{~ms}$ to $t=1.59 \mathrm{~ms}$ show the growth of a cavitation bubble. The bubble appears to nucleate in the vicinity of the tip of the cone, where the pressure transducer is located. The collapse of the bubble is shown with the frames $t=1.65 \mathrm{~ms}$ to $t=1.82 \mathrm{~ms}$. The collapsing bubble remains close to the pressure transducer, which partially explains the larger peak pressure recorded at the tip of the cone compared to the peak pressure recorded above the cone.

\subsubsection{Conclusion on Cases 1 to 5}

The impulsively-generated pressure and strain transients inside a static, cylindrical, fluid-filled tube were studied experimentally and numerically. The effect of an air gap and a converging section were explained using five different cases. 
Case 1, the simplest case, has no air gap and no converging section. It was found that the upward motion of the buffer upon reflection of the pressure wave on the buffer creates tension waves, and this causes distributed cavitation to occur throughout the tube. The reflection of pressure waves on an accelerated boundary is revisited and discussed in more details in the next section.

Case 2 is identical to case 1 , except there is a converging section at the bottom end of the tube. No shock focusing of the primary pressure wave generated upon the impact of the projectile on the buffer was observed. The peak pressure recorded upon collapse of the cavitation bubbles was found to be much larger at the tip of the cone than above the cone. The amplification is due to a combination of shock focusing and the effect of the cone on the collapsing bubbles. Shock focusing is discussed further in Chapter 5, and collapsing bubbles are discussed further in Chapter 6.

Case 3 has the same geometry as case 1, but there is a small $(3.5 \mathrm{~mm})$ air gap between the buffer and the water. The presence of an air gap drastically affects the dynamics of the projectile and buffer; there are now multiple collisions between the projectile and the buffer, resulting in multiple pressure waves within the tube. Constructive interference between the waves may occur.

Case 4 is identical to case 3, except the air gap is larger than in case $3(12 \mathrm{~mm})$. The size of the air gap drastically affects the timing of the multiple impacts between the projectile and the buffer. As a result, the wave generated through the second impact propagates in a cavitating liquid, and wave steepening leading to shock waves is possible.

Case 5 is identical to case 4 , except there is a converging section at the bottom end of the tube. The pressure measured at the tip of the cone is substantially larger than the pressure measured above the cone. The amplification of the pressure is due to a combination of shock focusing and the rapid collapse of a bubble in the immediate vicinity of the pressure transducer.

The material used to fabricate the base fixture terminating the tube affects the wave dynamics in the liquid. Materials of lower acoustic impedance, such as polycarbonate, result in reflected waves of lesser magnitude. In some cases this can result in milder cavitation events in the liquid, reducing the effect of wave steepening. The results suggest that combining an air gap and a converging section can create high pressures, as much as $50 \mathrm{MPa}$, in the cone area of a syringe geometry. The 
translational motion of the syringe was not taken into account in this section. The effect of the syringe acceleration and deceleration is important, and this is the subject of the next section.

\subsection{Dynamic Large-Scale Model Autoinjector}

The results from Chapter 3 indicate the acceleration and the deceleration of the syringe can be large, 15,000 to $36,000 \mathrm{~m} / \mathrm{s}^{2}$. The syringe motion can largely affect the transient events. This section is an extension of the work presented in the previous section to include the translational motion of the syringe. The syringe motion is primarily investigated using experimental work, but a limited number of LS-DYNA numerical simulations are also discussed.

\subsubsection{Experimental Setup}

A schematic of the experimental setup is shown in Figure 4.17. Figure 4.17a is a view of the complete test setup, and Figure $4.17 \mathrm{~b}$ is a zoomed-in view of the projectiles and the syringe. Note that the $\zeta$-axis in Figure $4.17 \mathrm{a}$ is in a stationary frame, it is defined downward positive, and it is referenced from the top end of the syringe guide tube. The $z$-axis in Figure $4.17 \mathrm{~b}$ is in an accelerated frame, it is defined downward positive, and it is referenced from the top end of the syringe barrel. The experimental apparatus consists of five main components: the projectile guide tube, the projectile assembly, the test specimen or syringe, the syringe guide tube, and the decelerator.

The projectile guide tube is only partially shown in Figure 4.17a. This is the same guide tube as the one used in the static, large-scale model autoinjector experiments reported in Section 4.1. The purpose of this tube is to guide the projectiles while they are vertically accelerated to velocities up to $6.4 \mathrm{~m} / \mathrm{s}$ using gravity alone.

The projectile assembly consists of two concentric projectiles: the inner and the outer projectiles. The inner projectile is a $0.33 \mathrm{~kg}$ aluminum cylinder diameter $=39.2 \mathrm{~mm}$ and length $=102 \mathrm{~mm}-$ which can slide freely within the outer projectile. The outer projectile is a $0.44 \mathrm{~kg}$ aluminum cylinder - inner diameter $=38.5 \mathrm{~mm}$, outer diameter $=50.1 \mathrm{~mm}$, and length $=204 \mathrm{~mm}-$ which can slide freely within the guide tube. A \#00 nylon screw (about $1.2 \mathrm{~mm}$ diameter) is used to fix the inner projectile inside the outer projectile at an adjustable position $\lambda_{0}$ prior to a test (see Figure 4.17b). The nylon screw breaks as soon as one of the two projectiles impacts on the syringe barrel and/or the buffer, resulting in both projectiles traveling independently thereafter. 


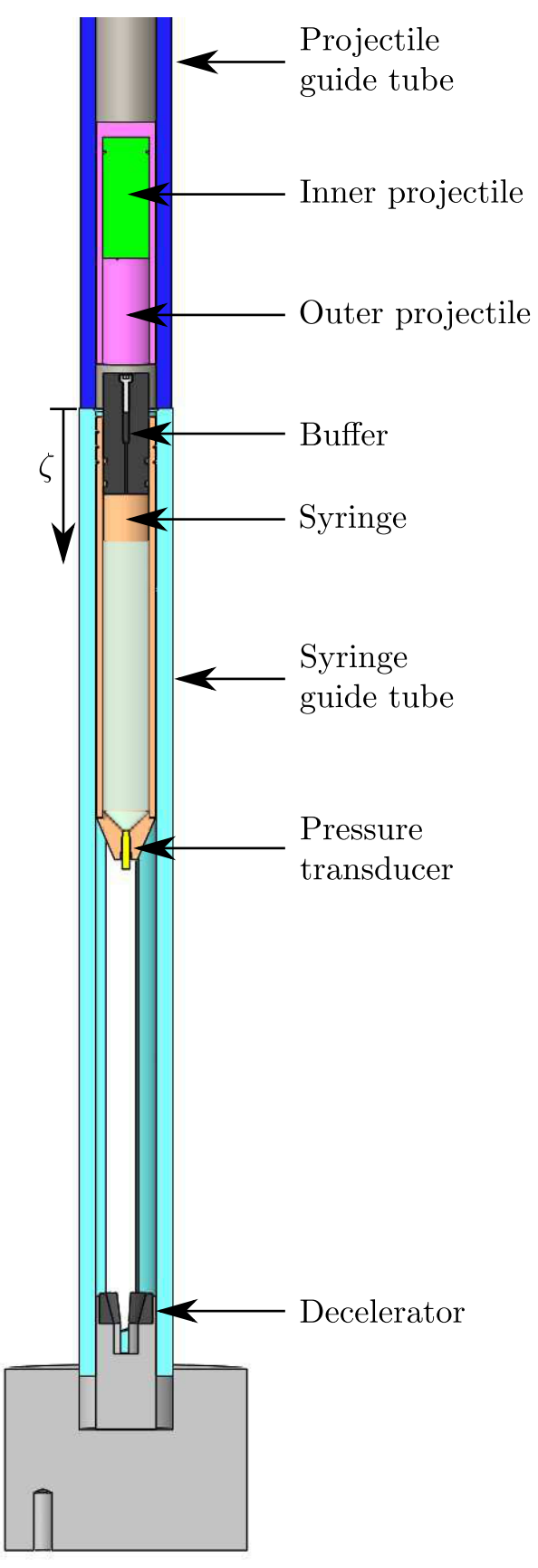

(a) View of the entire test setup

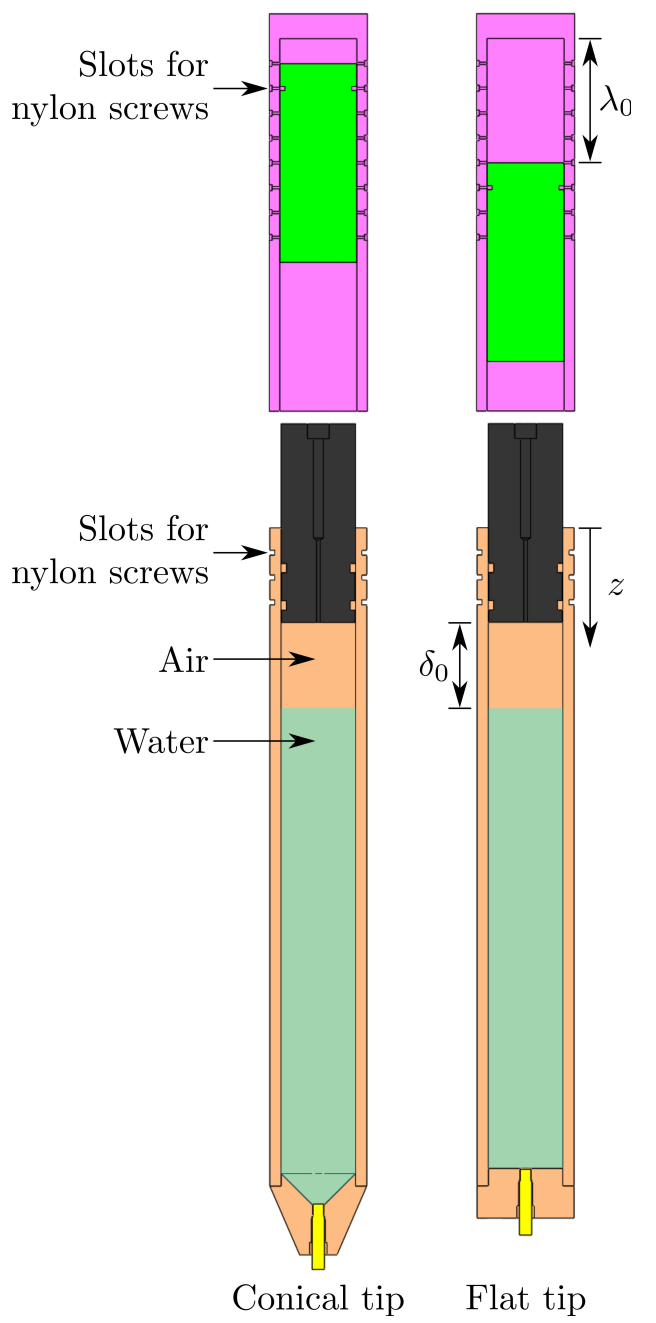

(b) Zoomed-in view of the syringe, the buffer and the projectile assembly

Figure 4.17: Schematic of the dynamic, large-scale model autoinjector experimental setup. 
The test specimen consists of a thick-wall aluminum or polycarbonate tube with an outer diameter of $49.5 \mathrm{~mm}$ and an inner diameter of $38.1 \mathrm{~mm}$. The polycarbonate tube is clear and vapor polished, making it possible to observe the inside of the syringe. As shown in Figure $4.17 \mathrm{~b}$, the tube is either terminated with a flat wall or a cone. The half-angle of the cone is $41^{\circ}$, identical to the half-angle of the cone used in Section 4.1. The tip, which contains the flat wall or the cone, is a separate part that is glued to the bottom end of the barrel. The tip with a flat wall is fabricated using aluminum. Two conical tips were fabricated: one with aluminum, and another one with clear, vapor polished polycarbonate. The length of the barrel is $337 \mathrm{~mm}$. The overall syringe length, including the tip, is $353 \mathrm{~mm}$ for the syringe terminated with a flat wall, and $372 \mathrm{~mm}$ for the syringe terminated with a cone. The mass of the aluminum and polycarbonate syringes are respectively $0.81 \mathrm{~kg}$ and $0.36 \mathrm{~kg}$.

The liquid inside the syringe during an experiment is de-ionized water. Degassed, de-ionized water was also tested, but this did not create significant differences in the measured pressure or strains as compared to tests without degassing (see Appendix B for a list of all tests performed). Apparently, there are sufficient nucleation sites in both the water and the wall of the sample that the cavitation behavior is not prohibited by degassing. The results obtained with degassed water are not reported.

The syringe guide tube is an aluminum cylinder with a length of $813 \mathrm{~mm}$, an inner diameter of $50.5 \mathrm{~mm}$, and an outer diameter of $76.1 \mathrm{~mm}$. The guide tube ensures proper alignment and motion of the different components during a test. A \#00 nylon screw (about $1.2 \mathrm{~mm}$ diameter) is used to hold the syringe inside the guide tube, well above the decelerator, prior to an experiment. As soon as one of the two projectiles impacts on the syringe and/or buffer, the nylon screw breaks, and the syringe is accelerated downward. The syringe guide tube is mounted into an aluminum base fixture which is bolted to heavy stainless steal plates that are bolted and epoxied to the floor. A tight fit is used between the guide tube and the base fixture, and no adhesive is used to secure it in place. There are two oblong axial slots 180 degrees apart on the syringe guide tube used for syringe visualization and routing of the wires connected to the pressure and strain gauges.

The top end of the syringe barrel is initially located at $\zeta=203 \mathrm{~mm}$. After completion of a test, the top end of the syringe is located at $\zeta=343 \mathrm{~mm}$. The travel distance of the syringe is therefore $140 \mathrm{~mm}$.

A decelerator is located at the bottom end of the guide tube to stop the syringe motion upon reaching its travel limit. It is a piece of urethane (Shore 60A hardness) 


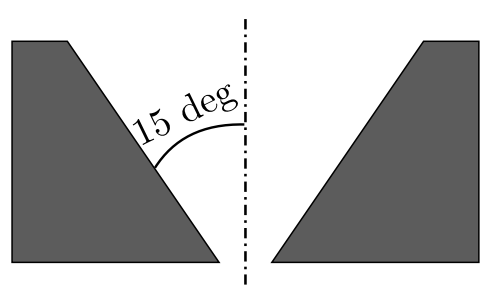

Conical tip

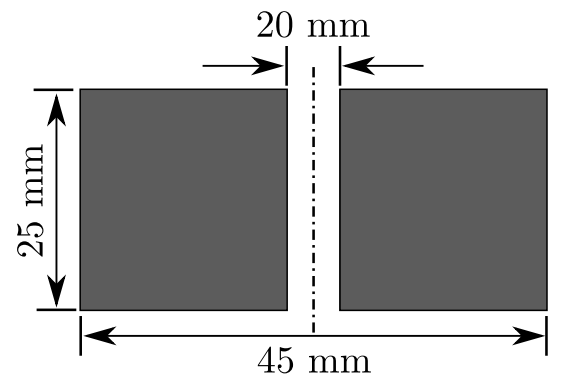

Flat tip

Figure 4.18: Schematic of the decelerators used to stop the syringe in the dynamic, large-scale model autoinjector experimental setup.

prepared using a home-made mold. The elastomer is from Frosch Polymer Corp (URS-5160). The decelerator is supported with an aluminum mount. The shape of the decelerator is different depending on the tip geometry: the two different shapes and the dimensions of the decelerators are shown in Figure 4.18.

The test specimen is sealed at its top end using a $104 \mathrm{~mm}$ long polycarbonate cylinder used as a buffer between the projectile and fluid. This is the same $134 \mathrm{~g}$ buffer as the one used in Section 4.1. There is a small hole along the longitudinal axis of the buffer which is closed using a socket screw before a test. This opening allows for the introduction of an air gap of controlled size $\delta_{0}$ between the bottom end of the buffer and the water contained in the tube, as shown in Figure 4.17. For all cases reported in this section, the bottom end of the buffer is located at $z=53 \mathrm{~mm}$.

The syringe is instrumented with a piezoelectric pressure transducer PCB 113B23 mounted into the tip. The outer surface of the aluminum syringe is instrumented with 12 to 14 strain gauges to measure the hoop and axial strains. The strain gauges are a combination of Vishay CEA-06-125UN-350/P2 and HBM K-LY4-3-05-3503-2. The nominal location of each strain gauge is indicated in Table 4.5. The exact location of the strain gauges is reported on the plots that contain the strain signals. The same electronics as in Section 4.1 is used to power the sensors and acquire the data.

Two high-speed video cameras - a combination of Vision Research Phantom V7.0G, V711, and/or V1612 - are used to visualize the projectiles, the buffer and the syringe, making it possible to track the components. Quantitative image analysis makes it possible to obtain the velocity and acceleration of each component. When a clear, polycarbonate syringe is used, it is possible to visualize the cavitation events. 
Table 4.5: Nominal axial location of the strain gauges mounted on the outer surface of the syringe used in the dynamic, large-scale model autoinjector experimental setup.

\begin{tabular}{c|ccccccc}
\hline Station & S1 & S2 & S3 & S4 & S5 & S6 & S7 \\
$z(\mathrm{~mm})$ & 73 & 121 & 175 & 232 & 279 & 327 & 345 \\
\hline
\end{tabular}

The analogy between the test setup and an actual autoinjector is as follows: the projectile assembly corresponds to the spring-actuated power pack, the buffer corresponds to the plunger-stopper, the aluminum/polycarbonate tube corresponds to the syringe, and the water corresponds to the drug solution. The decelerator corresponds to the autoinjector feature which is responsible for decelerating the syringe upon reaching the right penetration depth for the needle.

In the tests reported herein, only the outer projectile is used. The outer projectile can be oriented as shown in Figure 4.17 to impact on the syringe wall. The orientation of the outer projectile can also be reversed to impact only on the buffer. This approach was used for simplicity, making it possible to use the same projectile for all tests.

Another possibility (not reported in this thesis) is to have the outer projectile impact on the syringe wall, causing the syringe to accelerate (event 1 in Section 2.2), and the inner projectile impact on the syringe buffer, pressurizing the air gap and the liquid inside the syringe (event 3 in Section 2.2). The timing between the impact on the buffer and the syringe wall is controlled by varying distance $\lambda_{0}$ (see Figure $4.17 \mathrm{~b}$ ). A large value of $\lambda_{0}$ results in the impact of the inner projectile on the buffer occurring before the outer projectile impacts on the syringe wall, causing pressurization of the liquid prior to acceleration of the syringe. Figure 4.17b shows two different initial configuration of the projectiles: small $\lambda_{0}$ on the left-hand side, and large $\lambda_{0}$ on the right-hand side.

Note that damping material in the form of two stacked O-rings is introduced between the syringe wall and the outer projectile. This prevents the creation of large axial stress waves in the syringe due to the impact event. A metal-to-metal impact creates large amplitude stress waves which take a long time to dissipate, and this pollutes the measurements. The presence of damping material reduces the magnitude of the syringe acceleration, but the acceleration remains large enough to create substantial 


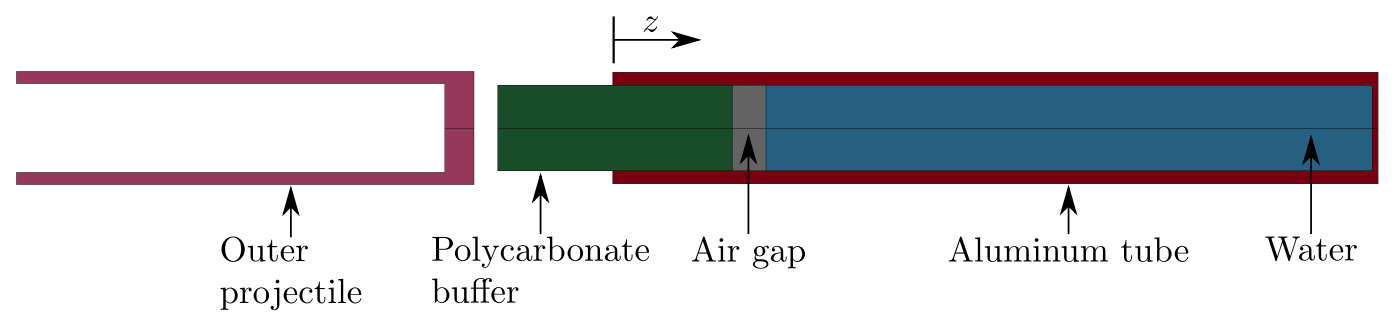

Figure 4.19: LS-DYNA model used to simulate the transient events created by the impact of the reversed outer projectile on the buffer of the syringe in the dynamic, large-scale model autoinjector.

pressure and stress transients. No damping material is used when the reversed outer projectile is used to impact on the buffer.

The travel distance of the syringe is relatively large, resulting in a temporal separation of 50-60 ms between the syringe acceleration and deceleration. This is sufficient time for the transient events created by the impact of the outer projectile on the syringe wall or buffer to dissipate before the syringe is decelerated. This means that events 1 and 3 (i.e., pressurization and acceleration) are decoupled from event 2 (i.e., deceleration). For this reason, events 1 and 3 are studied separately from event 2 , both experimentally and numerically.

\subsubsection{LS-DYNA Numerical Model}

Numerical simulations were performed using LS-DYNA (Hallquist, 2016). The model is constructed using the same methodology as in Section 4.1. The syringe acceleration and pressurization are modeled separately from the syringe deceleration. This shortens the simulation time by avoiding to simulate the translation of the syringe between the acceleration and deceleration events. No pressure and stress transients occur during this uninteresting time period.

The geometry of the LS-DYNA model used to simulate the pressure and stress waves created by the impact of the reversed outer projectile on the buffer is shown in Figure 4.19. The simulation is initialized with all parts at rest, except for the projectile which is traveling toward the buffer at $6.0 \mathrm{~m} / \mathrm{s}$. The syringe is free to accelerate as a result of the pressure force applied on the wall of the tip. There is no friction between the buffer and the syringe in this model. The grid is constructed with approximately 150,000 shell elements.

The geometry of the LS-DYNA model used to simulate the pressure and stress waves created by the syringe deceleration is shown in Figure 4.20. No projectiles 


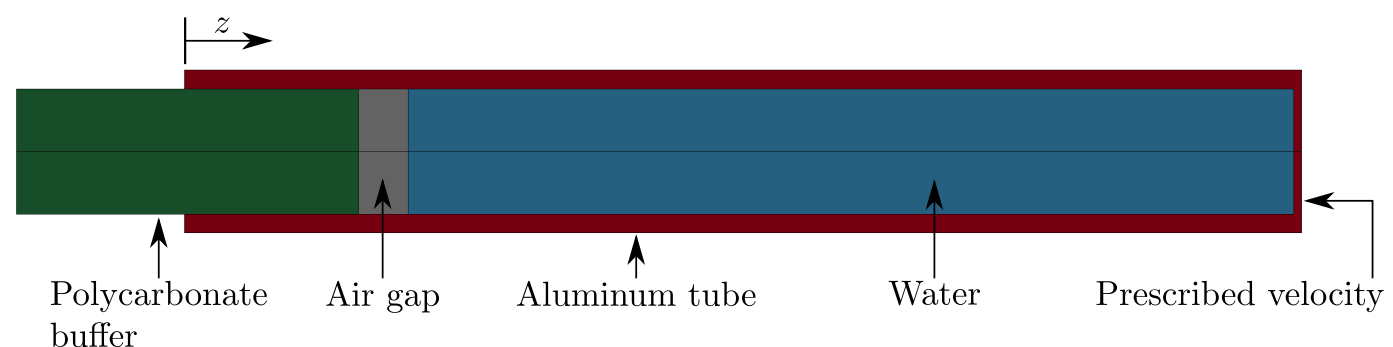

Figure 4.20: LS-DYNA model used to simulate the transient events created by the deceleration of the syringe in the dynamic, large-scale model autoinjector.

or decelerator are present in this model. The simulation is initialized with all parts traveling at a uniform and constant velocity $u_{0}$. Shortly after initialization, the bottom wall of the syringe is rapidly decelerated to a velocity $u_{1}$ by prescribing the velocity on the boundary identified in Figure 4.20 as "Prescribed velocity". A constant deceleration resulting in a linear velocity profile is used for simplicity. There are approximately 110,000 shell elements in this model.

Refinement of the computational grid by a factor of 4 does not affect the results significantly. The peak pressures, stresses and strains vary by no more than $7 \%$ when using the refined grid. Due to the relatively small effect on the results and the increase in computational time, the refined grid was not utilized to perform the numerical simulations.

LS-DYNA numerical simulations of the impact of the outer projectile on the syringe wall were performed, but the agreement between the numerical results and the experiments is not satisfactory. For this reason, the numerical model and results are not reported. The discrepancy between the simulations and the experiments is due to the absence of an explicit cavitation model in the simulations. The impact of the outer projectile on the syringe wall is immediately followed by a cavitation event, and the absence of a cavitation model makes it nearly impossible to simulate with fidelity the transient events created by the syringe acceleration resulting from the impact of the outer projectile on the syringe wall.

\subsubsection{Results: Syringe Acceleration and Pressurization}

Several cases are discussed in this subsection to study the acceleration and pressurization of the syringe. Cases where the reversed outer projectile impacts on the buffer only are discussed first. This is followed by a discussion of cases where the outer projectile impacts on the syringe wall. The list of test cases discussed herein is: 
- Impact on the buffer only:

- case 6: syringe terminated with a flat wall, no air gap;

- case 7: syringe terminated with a flat wall, $5.1 \mathrm{~mm}$ air gap;

- case 8: syringe terminated with a cone, $5.1 \mathrm{~mm}$ air gap;

- case 9: syringe terminated with a flat wall, $15.2 \mathrm{~mm}$ air gap.

- Impact on the syringe wall only:

- case 10: syringe terminated with a flat wall, no buffer;

- case 11: syringe terminated with a flat wall, no air gap;

- case 12: syringe terminated with a cone, no buffer;

\subsubsection{Case 6}

Several tests where the reversed outer projectile impacts on the buffer were performed. The physics and results in this configuration are analogous to what was presented and discussed for the static, large-scale model autoinjector in Section 4.1. The emphasis of the present discussion is on the differences created by the syringe motion.

Case 6 is for a syringe terminated with a flat wall, and no air gap between the buffer and the liquid. Case 6 is the equivalent of case 1 from Section 4.1. The syringe and buffer velocity is shown in Figure 4.21. The impact of the projectile on the buffer occurs at $-0.8 \mathrm{~ms}$, resulting in the acceleration of the buffer into the syringe. Because there is no air gap between the buffer and the syringe liquid content, a sharp pressure wave is immediately created in the liquid (this will be discussed later using the pressure trace).

The explanation for the syringe acceleration is twofold. First, the impact of the projectile on the buffer creates relative motion between the buffer and the syringe. After impact of the projectile on the buffer, friction between the syringe and the buffer applies a force oriented in the positive $z$ direction on the syringe. The friction force has not been measured experimentally, but the results indicate the friction force $f$ is small (i.e., $f / m_{s}$ is small); the friction force does not create a measurable acceleration of the syringe, as shown in Figure 4.21. The syringe acceleration begins $0.5 \mathrm{~ms}$ after the impact of the projectile on the buffer. 


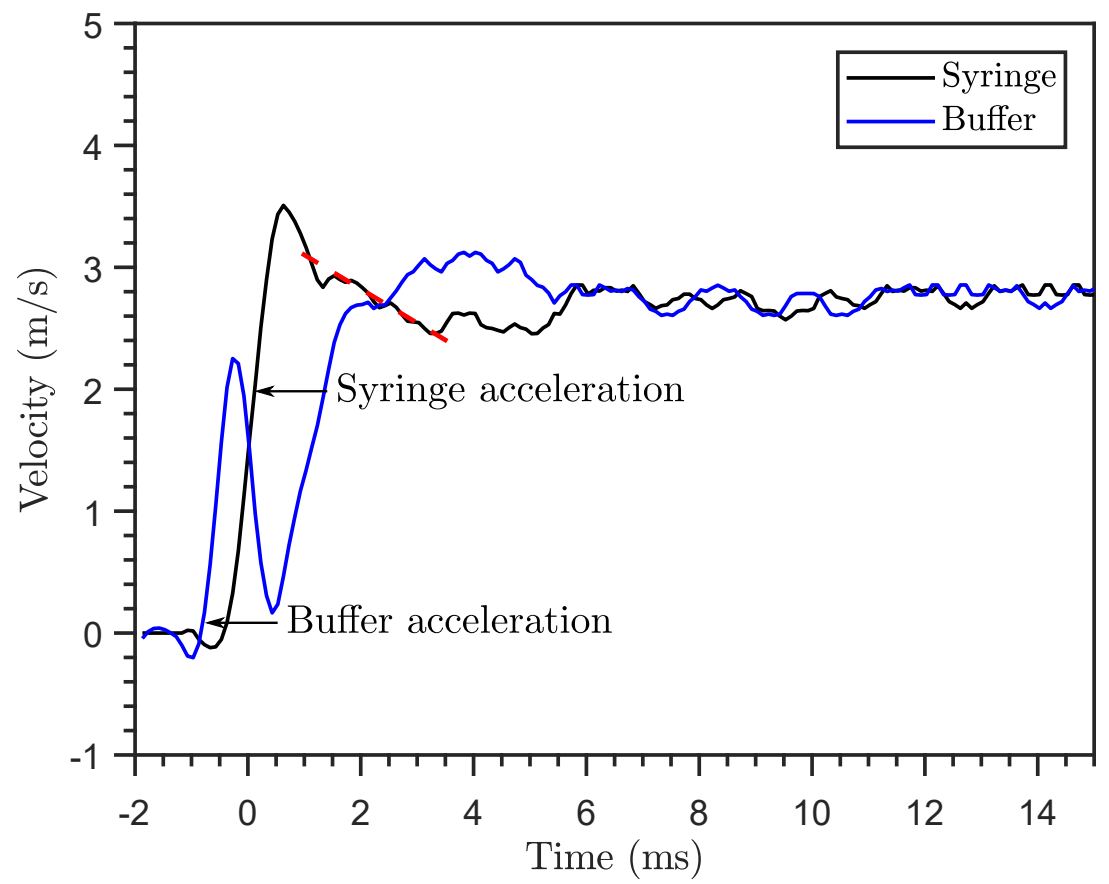

Figure 4.21: Velocity of the syringe and the buffer for case 6 of the dynamic, large-scale model autoinjector experiments (test LS-0353).

The second mechanism which is responsible for the syringe acceleration is the pressure pulse created in the syringe liquid content by the motion of the buffer into the syringe. The pressure applied on the syringe tip creates a net force oriented in the positive $z$ direction, resulting in a positive acceleration. The pressure pulse created by the acceleration of the buffer into the syringe travels from the buffer bottom surface to the syringe tip in approximately $0.2 \mathrm{~ms}$, indicating that one would expect the syringe acceleration to begin no more than $0.2 \mathrm{~ms}$ after the impact of the projectile on the buffer. As indicated before, the measured delay is $0.5 \mathrm{~ms}$ (note that the uncertainty on this delay is approximately $0.2 \mathrm{~ms}$ due to the frame rate of the camera). A possible explanation for the longer than expected delay is the presence of the nylon screw that maintains the syringe in position prior to a test: as the pressure force applied on the syringe tip increases, the nylon screw begins to deform, and the shear force has to increase sufficiently for the screw to break. The average magnitude of the syringe acceleration is $\approx 5,000 \mathrm{~m} / \mathrm{s}^{2}$.

The rapid acceleration of the syringe is followed by a weak deceleration, identified with a red-dashed curve in Figure 4.21. The weak deceleration is the result of a cavitation event inside the syringe (to be discussed further using the pressure 


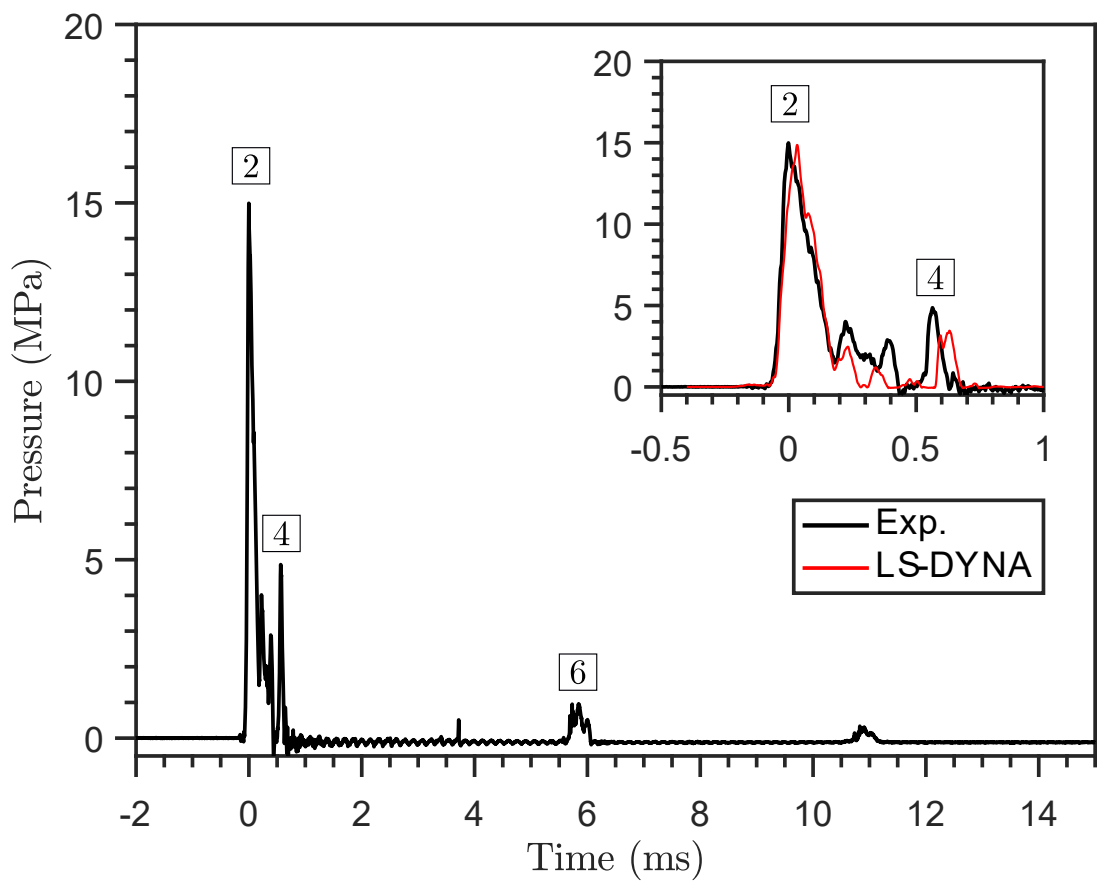

Figure 4.22: Pressure at the bottom wall for case 6 of the dynamic, large-scale model autoinjector experiments (test LS-0353).

trace). During a cavitation event, the absolute pressure applied on the interior wall of the syringe tip is approximately vapor pressure, or $2.3 \mathrm{kPa}$ (this corresponds approximately to a $-0.1 \mathrm{MPa}$ relative pressure). The absolute pressure applied on the exterior wall of the tip is always ambient pressure. The pressure difference creates a net force on the syringe tip, oriented in the negative $z$ direction. The magnitude of this net force ${ }^{2}$ is approximately $114 \mathrm{~N}$. Newton's second law of motion $(F=m a)$ indicates this should create a deceleration with a magnitude of $141 \mathrm{~m} / \mathrm{s}^{2}$. The results shown in Figure 4.21 indicate the magnitude of the deceleration is approximately $200 \mathrm{~m} / \mathrm{s}^{2}$, reasonably close to the estimated value given the simplicity of the approximation.

Figure 4.22 is a plot of the pressure measured with the transducer mounted into the syringe tip. This pressure trace is to be compared with the pressure signal obtained for case 1 in Section 4.1. The labels in Figures 4.4, 4.5 and 4.22 identify the same features. Note that the timescales in both tests are different due to the different syringe lengths, but the results are otherwise similar.

\footnotetext{
${ }^{2}$ The force corresponds to the pressure difference across the tip multiplied by the inner crosssectional area of the tip.
} 


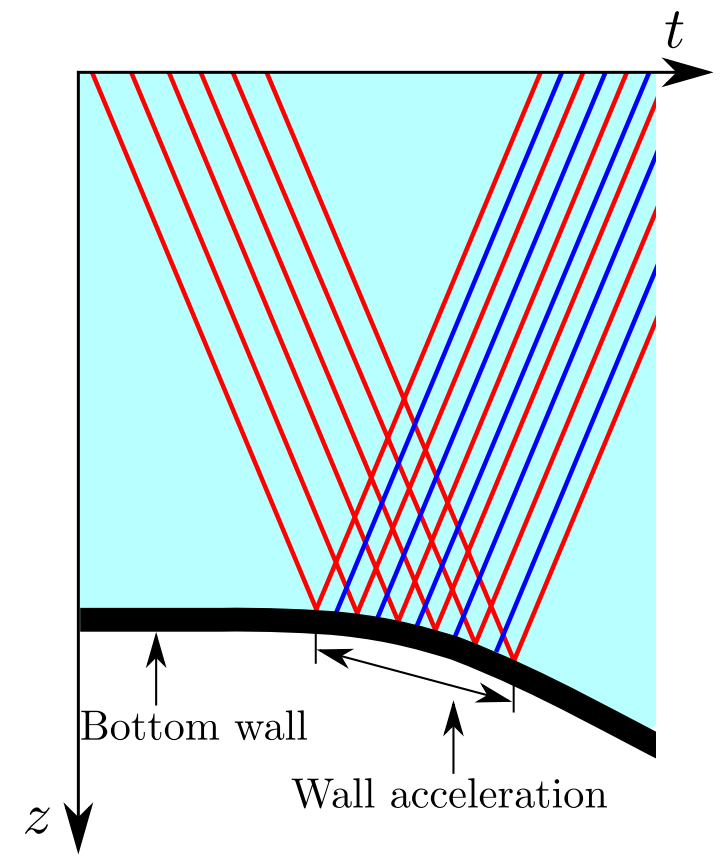

Figure 4.23: Reflection of a pressure pulse on an accelerated, rigid wall.

The peak magnitude of the pressure pulse is approximately 15.0 MPa with the syringe motion, comparable to the $16.1 \mathrm{MPa}$ obtained without the syringe motion. The peak magnitude of the reflected wave, identified with label 4 , is significantly weaker with the syringe motion: $4.7 \mathrm{MPa}$ vs 10.0 MPa. The lower magnitude of the reflected wave is a consequence of the syringe acceleration, effectively acting as a pressure relief mechanism. The acceleration of the syringe bottom wall results in the production of tension waves which reduce the magnitude of the pressure. This process is similar to what happens when a compression wave reflects on the bottom surface of the buffer, forcing the buffer to accelerate and creating tension waves. In other terms, the reflection of pressure waves on a wall which is free to accelerate is "softer" than the reflection of pressure waves on a wall which is fixed in space.

This concept is illustrated using the space-time diagram in Figure 4.23. The spacetime diagram corresponds to a zoomed-in view of the wall: the top of the liquid column and the mechanism creating the pressure pulse are not represented. The wall is assumed to be rigid, but it has a finite inertia. A pressure pulse, represented with the red lines, is created in the liquid and propagates toward the wall. The pressure pulse is a continuum of compression and tension waves which increases the pressure from $P_{0}$ to a peak value $\Delta P_{i}$, and then reduces the pressure to its original 
value $P_{0}$. The pressure pulse, upon reaching the bottom wall, increases the pressure in the vicinity of the wall. This creates a downward-oriented force on the wall, and forces the boundary to accelerate down. The continuum of pressure waves forming the pulse reflects on the rigid wall and then propagates upward in the liquid. The resulting acceleration of the bottom wall results in the formation of tensile waves (indicated with blue lines) which interfere with the incident pressure pulse. The effect of the tensile waves is to reduce the magnitude of the reflected pressure pulse. The monograph of Cole (1948) on underwater explosions introduces a mathematical model for the reflection of a pressure pulse on an accelerated boundary. In the context of acoustic and under the assumption of 1D motion, the wall pressure is:

$$
P(t)=2 P_{i}(t)-\rho_{0} c u_{w}(t)
$$

where $P_{i}(t)$ is the instantaneous pressure of the incident pulse, and $u_{w}(t)$ is the velocity of the wall (it is important to recall that $u_{w}(t)>0$ due to the orientation of the $z$ axis). Note that this result is valid whether or not the acceleration of the wall is created by the pressure pulse or not; the change in velocity could be caused by an external actuator.

The peak wall pressure is obtained by evaluating Equation 4.2 at $t_{\max }$, where $t_{\max }$ corresponds to the instant when $P_{i}(t)$ is equal to its peak value $\Delta P_{i}$ :

$$
P_{\text {max }}=2 \Delta P_{i}-\rho_{0} c u_{w}\left(t=t_{\text {max }}\right) .
$$

This result indicates the reduction in peak magnitude of the pressure pulse is determined by the velocity of the boundary when $P_{i}(t)$ is maximum rather than the terminal velocity of the boundary after the pulse has completely reflected on the wall. Also, Equation 4.3 indicates the peak wall pressure is equal to $2 \Delta P_{i}$ if the wall is stationary $\left(u_{w}=0\right)$. This is the expected result for the reflection of acoustic waves on a fixed, rigid wall (see Chapter 2).

The case of a syringe is more complicated than the simplified situation described above. This is because more than one pressure pulse are created in the liquid, and each pulse creates some acceleration of the syringe. The multiple pulses can interfere, indicating that the relative timing of the events creating the pressure pulses is important.

Returning to case 6 , the velocity of the boundary when the wall pressure reaches its first maximum (label 2) is approximately $1.1 \mathrm{~m} / \mathrm{s}$. Equation 4.3 indicates the 
wall motion is expected to decrease the peak wall pressure by 1.7 MPa. When the pressure reaches its second maximum (label 4), the velocity of the syringe is approximately $3.5 \mathrm{~m} / \mathrm{s}$, expected to create a pressure reduction of approximately 5.3 MPa. As noted before, the magnitude of the peak wall pressure created by the reflected wave is $4.7 \mathrm{MPa}$ with the motion (case 6), and 10.0 MPa without (case 1). The difference is $5.3 \mathrm{MPa}$, equal to the predicted value. Note that the buffer motion also creates a further pressure decrease when the pressure pulse reflects on the bottom wall of the buffer, and this has not been accounted for. This explains why the difference in peak wall pressure created by the incident pulse (label 2) and the reflected pulse (label 4) is larger, 10.3 MPa for case 6.

As for case 1 in Section 4.1, the return of the reflected wave - a tension wave - at the bottom of the syringe (label 4) is followed by a cavitation event. The cavitation event ends a few milliseconds later (label 6), creating a small but detectable pressure increase. There are a few more detectable cycles of cavitation within the syringe, not all shown in Figure 4.22.

The hoop and axial strains are shown in Figure 4.24. These can be compared with the strains in Figure 4.6 obtained without the syringe motion. Apart from the different timescales, the hoop strains with and without the syringe motion are analogous, and the minor differences are explained by the pressure decrease created by the acceleration of the syringe tip.

The axial strains with and without the syringe motion differ more substantially. This is mostly because the boundary condition applied at the bottom end of the test specimen is different for both situations. Without the syringe motion, the stress waves at the bottom end of the specimen are almost entirely transmitted to the heavy base fixture supporting the syringe. With the syringe motion, the bottom end approximately corresponds to a stress free end. There, the axial stress waves are reflected into the syringe wall, but the sign of the waves changes upon each reflection. This is the same boundary condition as applied at the top end of the syringe wall. The stress free boundary conditions applied at both extremities of the test specimen result in a large number of reverberations of the stress waves in the syringe wall. The decay in magnitude is slow because there is only a small amount of dissipation in the system.

The peak magnitude of the hoop strains is approximately $400 \mu \epsilon$ with the syringe motion. This is lower than the $600 \mu \epsilon$ measured without the syringe motion. Because the hoop strains primarily result from the liquid pressure applied on the 


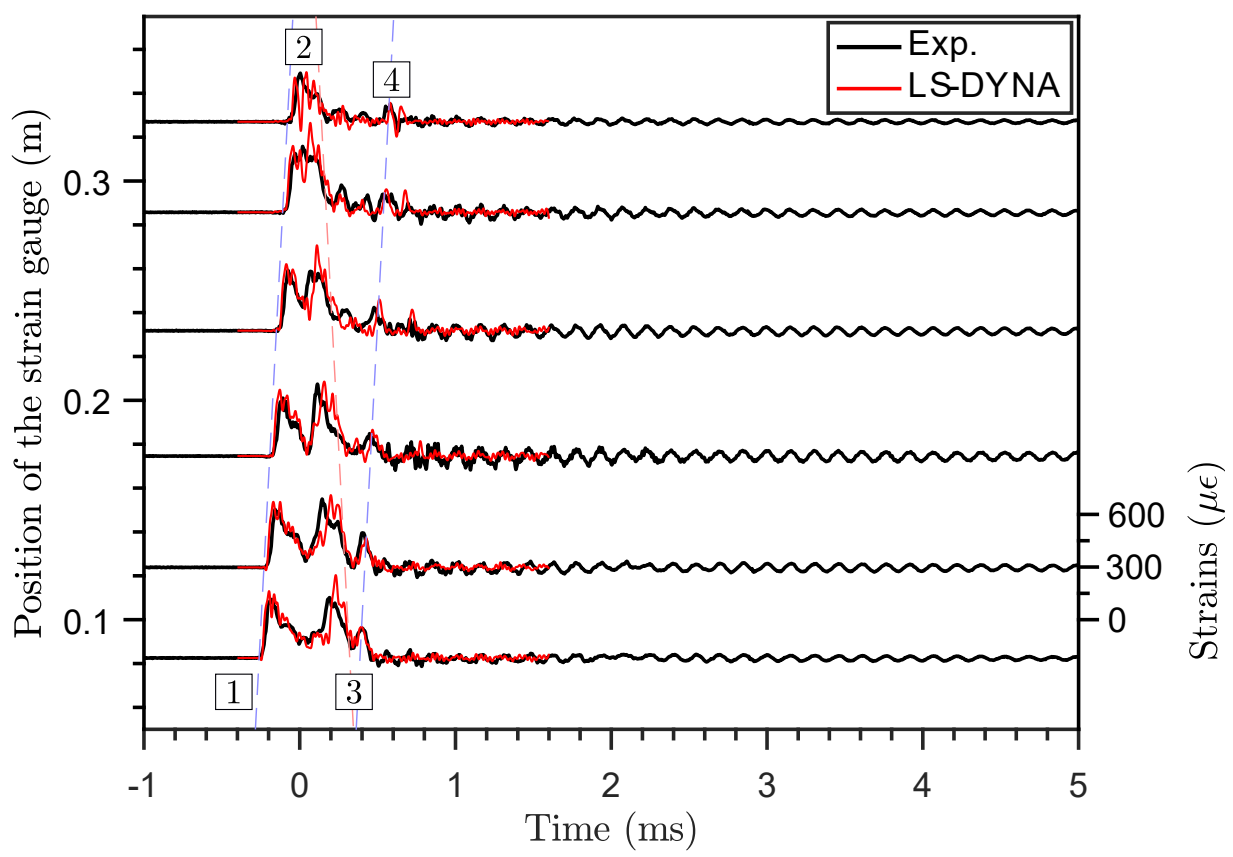

(a) Hoop strains

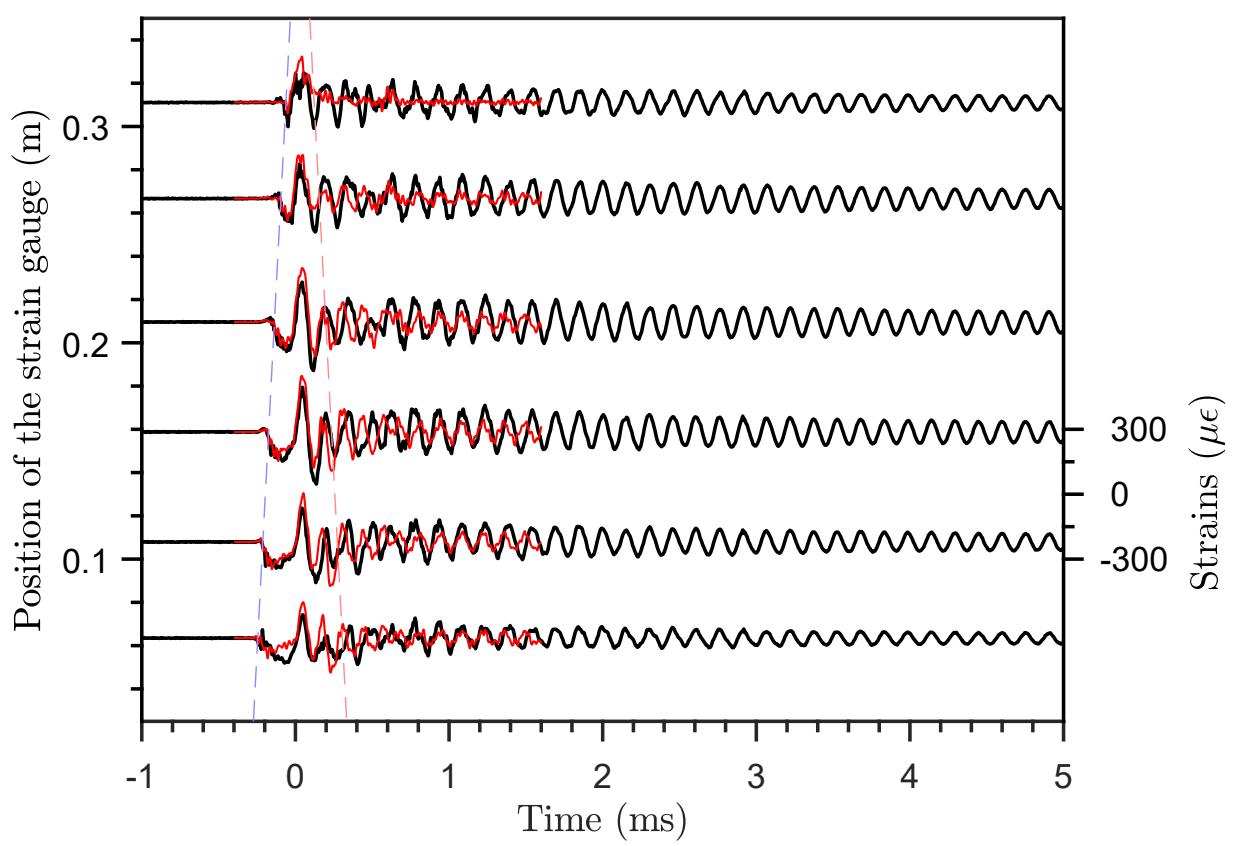

(b) Axial strains

Figure 4.24: Hoop and axial strains for case 6 of the dynamic, large-scale model autoinjector experiments (test LS-0353). 
inner syringe wall, this difference is consistent with the pressure decrease caused by the syringe acceleration which has been reported above.

The peak magnitude of the axial strains is approximately $-240 \mu \epsilon$ in compression, and $200 \mu \epsilon$ in tension. Without the syringe motion, the axial strains are created by the Poisson effect (i.e., the magnitude is approximately $\epsilon_{z}=-v \epsilon_{\theta}$ ). With the syringe motion, the strains are too large to be accounted for entirely by the Poisson effect. One factor explaining this is the multiple axial reverberations of the stress waves in the syringe walls, resulting in complex interactions and interference between the various stress waves in the system. As indicated before, the axial reverberations observed when the syringe motion is included are a result of the stress free boundary condition applied at the bottom of the syringe, and this is different from the boundary condition enforced when the syringe is static.

Numerical results obtained with LS-DYNA are shown in Figures 4.22 and 4.24. The numerical simulations are purposely stopped shortly after the onset of cavitation due to the absence of an explicit cavitation model. The pressure predicted by LS-DYNA is in reasonable accord with the experimental results, both in trend and in magnitude. The same is true about the hoop and axial strains, except for the magnitude of the reverberating axial stress waves, which is under predicted. The results suggest that LS-DYNA is able to predict with reasonable fidelity the pressure and strains immediately after the transient events are initiated.

The results obtained with the static, large-scale model autoinjector indicate that introducing an air gap between the buffer and the liquid changes the results substantially. This is also true for the experiments performed with the syringe motion. Introducing an air gap between the buffer and the liquid modifies the dynamics of the interactions between the projectile and the buffer, and it decrease the peak magnitude of the pressure and strains. Figure 4.25 is a plot of the peak pressure recorded in the syringe tip as a function of initial air gap size $\delta_{0}$. Data obtained with a flat tip and a conical tip are both shown, along with the LS-DYNA numerical predictions for a flat tip. There are five experimental data points for each combination of tip and initial air gap size.

The tests performed with a flat tip exhibit a clear trend toward a decrease in peak pressure as the initial air gap size increases. This is due to the damping effect of the air gap, as indicated in Section 4.1. For the same impact velocity of the projectile on the buffer, larger air gaps result in a smaller peak pressure inside the gap and, 


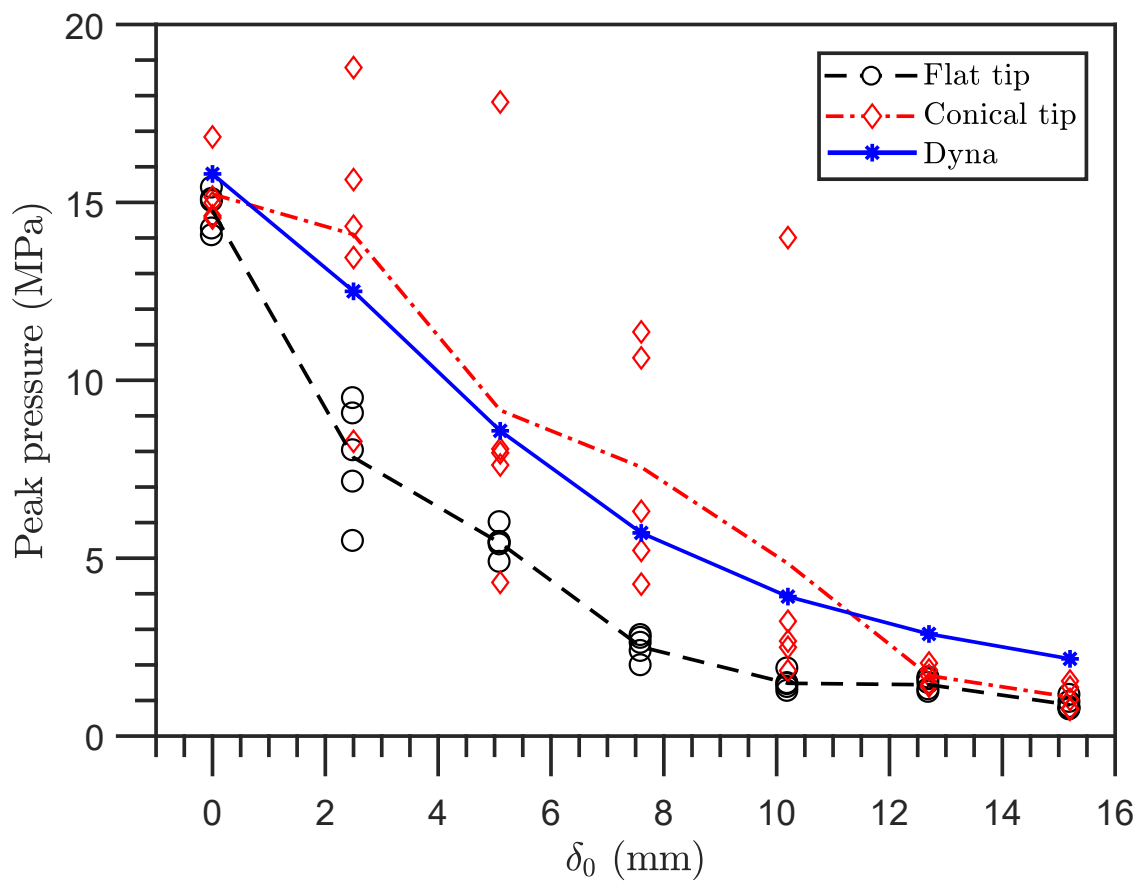

Figure 4.25: Peak pressure in the syringe tip as a function of initial air gap size. The outer projectile impacts on the buffer of the syringe in the dynamic, large-scale model autoinjector.

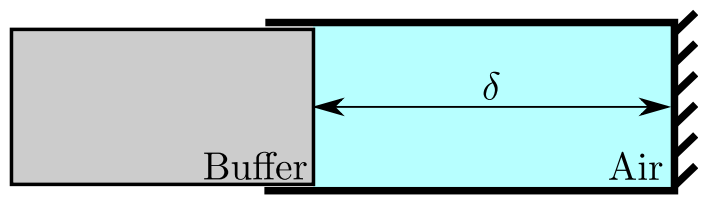

Figure 4.26: Schematic of the simplified model of the isentropic compression of an air gap.

in turn, this has the effect of creating pressure waves in the liquid with a weaker amplitude.

A simplified model, schematically represented in Figure 4.26, was developed to illustrate this effect. The model consists of a buffer, initially traveling at a velocity $u_{0}$, which compresses an air gap of initial size $\delta_{0}$. The coupling between the air gap and the liquid inside the syringe is neglected. The model assumes the buffer isentropically compresses the air gap:

$$
P_{\text {gap }}(t)=P_{0}\left(\frac{\delta_{0}}{\delta(t)}\right)^{\gamma} .
$$




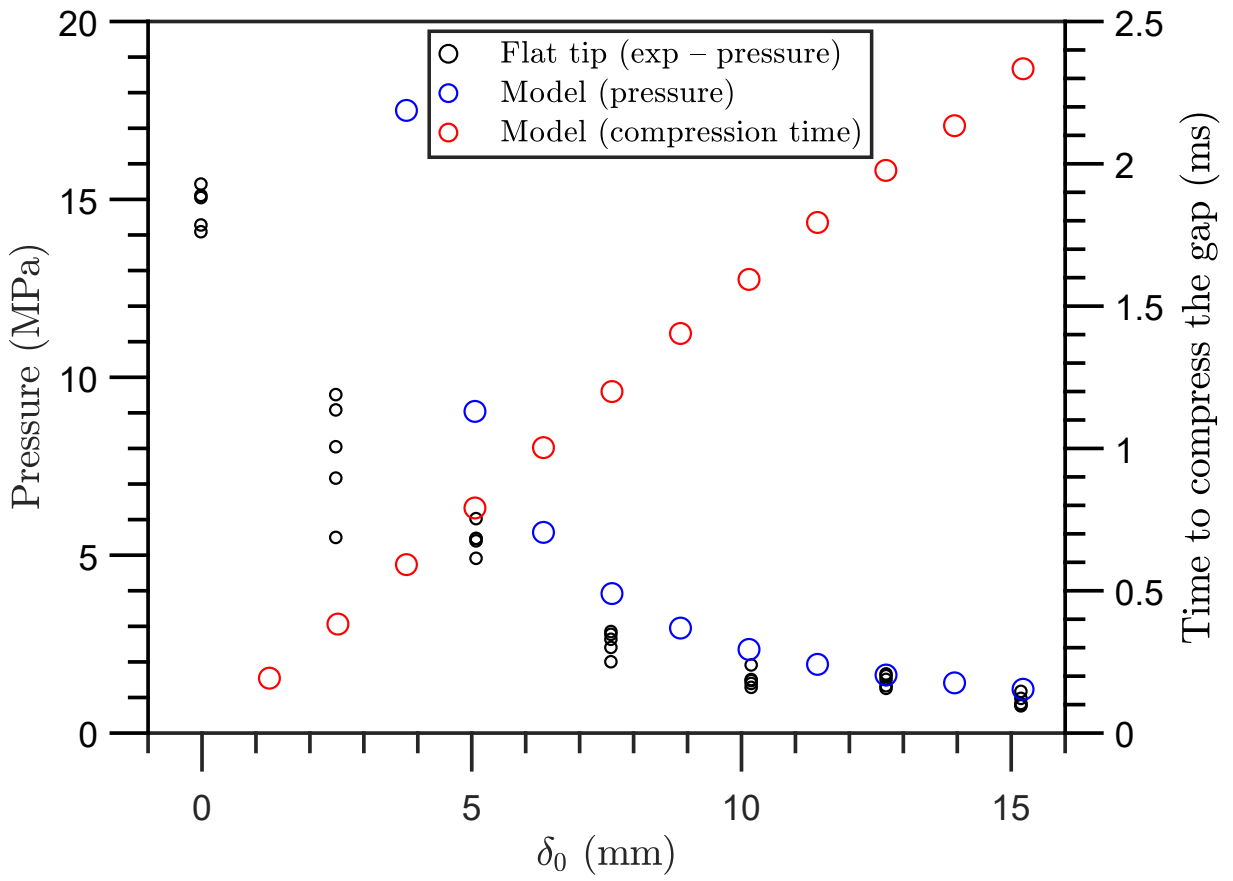

Figure 4.27: Peak air gap pressure and air gap compression time obtained with the simplified model. The peak wall pressure measured with a flat tip (see Figure 4.25) are plotted for comparison.

With this, the equation of motion of the buffer is:

$$
m_{b f} \frac{\mathrm{d}^{2} \delta(t)}{\mathrm{d} t^{2}}=P_{0} A\left[\left(\frac{\delta_{0}}{\delta}\right)^{\gamma}-1\right] .
$$

Numerical integration of the differential equation provides a time history of $\delta$ and the pressure inside the air gap. The peak pressure in the air gap and the time needed to reach the minimum air gap height, or the rise time of the pressure pulse, as a function of initial air gap height are shown in Figure 4.27. The calculation assumes $P_{0}=101 \mathrm{kPa}$ (ambient pressure), $\gamma=1.4, m_{b f}=0.134 \mathrm{~kg}, A=1.14 \times 10^{-3} \mathrm{~m}^{3}$, and an initial buffer velocity of $7 \mathrm{~m} / \mathrm{s}$ to the right.

The model results indicate the peak pressure in the air gap is expected to decrease as the initial air gap size increases. The rise time of the pressure pulse created through air gap compression increases approximately linearly with air gap size. The model predicts unrealistically large peak pressures for air gaps which are less than $5 \mathrm{~mm}$ in size. This is because for small air gaps the pressure increases more rapidly, and the motion of the right boundary of the air gap (see Figure 4.26) needs to be accounted for. The pressure increase in the gap sends compression waves in the liquid which accelerate the right-hand boundary of the gap to the right, increasing the value of $\delta$. 
The trend predicted by the model is in reasonable agreement with the experiments for $\delta>5 \mathrm{~mm}$.

Returning to Figure 4.25, the vertical scatter in the data is relatively small for the no-air-gap cases and for air gaps larger than $6 \mathrm{~mm}$. The vertical scatter is more significant for the cases with an initial air gap size of $2.5 \mathrm{~mm}$ and $5.1 \mathrm{~mm}$. Identical to what was observed with the static test setup, adding an air gap results in multiple impacts between the projectile and the buffer. Because the air gap behaves like a non-linear spring, the dynamics of the interactions between the buffer and the projectile is very sensitive to the precise air gap size and projectile velocity at impact, especially when the air gap is relatively small. The dependence of the results on air gap size is a significant factor which partially explains the test-to-test variation of the results.

Except for the no-air-gap cases and the tests performed with a 12.7-15.2 mm air gap, replacing the flat tip with a conical tip results in significantly larger peak pressures, as much as 6 times larger. The vertical scatter in the peak pressures recorded with the conical tip is substantial. The effect of the cone is investigated further using test cases 7,8 , and 9 .

Figure 4.25 indicates that without an air gap the cone does not result in any amplification of the peak pressure. This is surprising at first, but there is a valid explanation for this behavior. Test case 6 was repeated 5 times: repeat tests have shown that cavitation consistently occurs in the syringe when there is no air gap below the buffer. Identical to case 2 from Section 4.1, the pressure increase at the end of the cavitation event (label 6) is amplified when a cone is present. The incident pressure pulse (label 2), however, is not amplified in the cone due to its relatively long rise time.

Despite the amplification inside the cone, the magnitude of the pressure measured at the end of the cavitation event remains smaller than the magnitude of the incident pressure pulse. Without an air gap the peak pressure in the syringe is due to the incident pressure pulse (unaffected by the cone) rather than the collapse of bubbles at the end of the cavitation event (affected by the cone). The fact the peak pressure results from the incident pressure pulse rather than the cavitation event explains the limited vertical scatter in the data obtained without an air gap. As the air gap size increases, the magnitude of the incident pressure pulse decreases, and the peak pressure in the system becomes due to the collapse of cavitation bubbles, creating more variability in the results. 


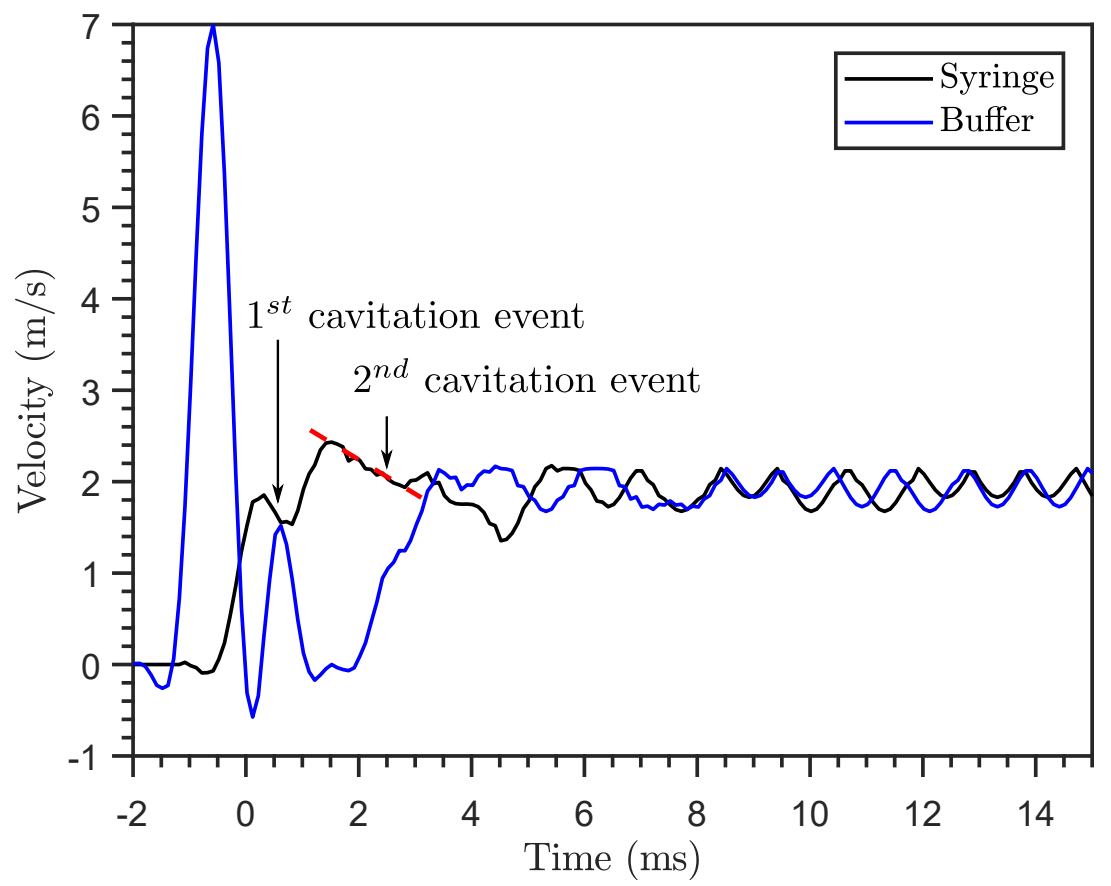

Figure 4.28: Velocity of the syringe and the buffer for case 7 of the dynamic, large-scale model autoinjector experiments (test LS-0345).

Figure 4.25 indicates that LS-DYNA predicts the correct order of magnitude for the peak pressure in the syringe, but it has a tendency to overestimate the peak pressure. The agreement between the experiments and the simulations is best without an air gap. The discrepancies observed when an air gap is present are likely caused by the absence of friction between the buffer and syringe in the numerical model. Several attempts were made to include friction without modeling the O-rings, but satisfactory results were not obtained.

\subsubsection{Case 7}

Case 7 is identical to case 6 , except there is a $5.1 \mathrm{~mm}$ air gap initially located between the buffer and the water. The results shown are representative of all tests performed with a 2.5 to $10.2 \mathrm{~mm}$ air gap. Case 7 is analogous to case 4 without the syringe motion.

The velocity of the syringe and the buffer are shown in Figure 4.28. The projectile impacts on the buffer at approximately $-1.3 \mathrm{~ms}$, accelerating the buffer into the syringe. The peak velocity of the buffer is $7 \mathrm{~m} / \mathrm{s}$, larger than the $2 \mathrm{~m} / \mathrm{s}$ measured for case 6. This is because without an air gap (case 6), the direct contact between 


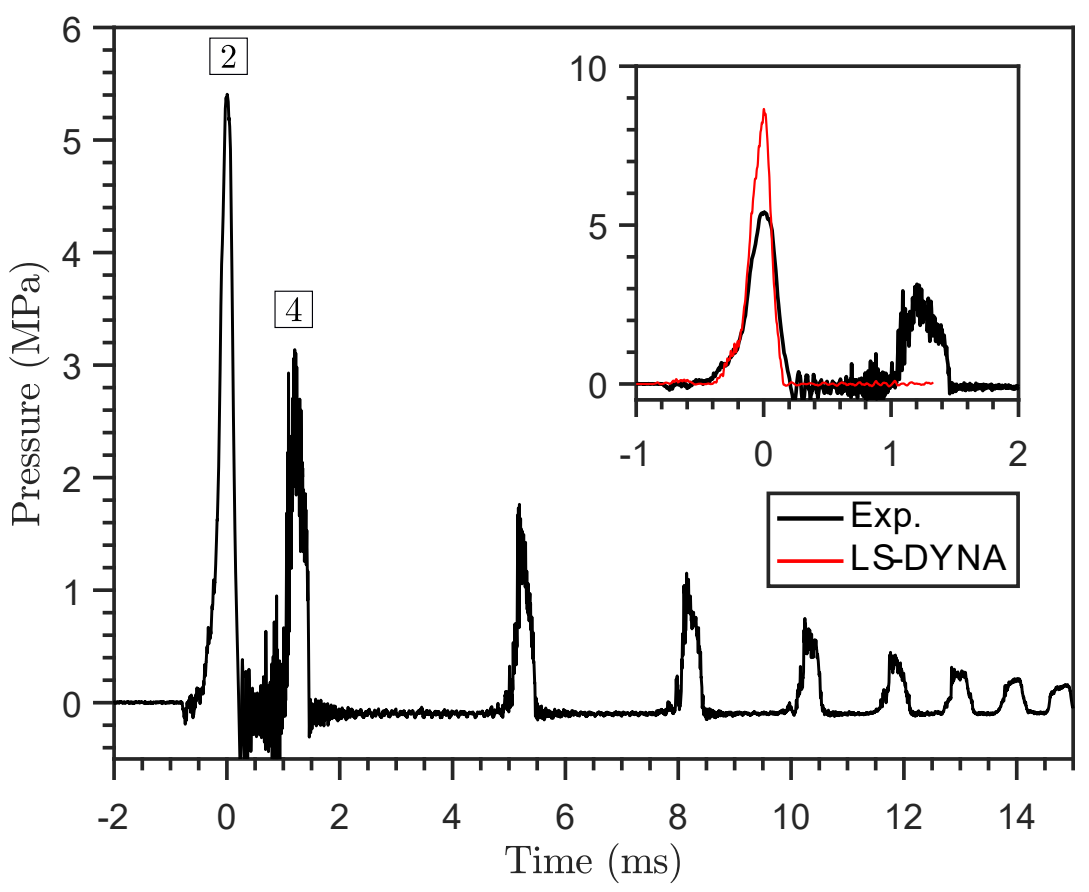

Figure 4.29: Pressure at the bottom wall for case 7 of the dynamic, large-scale model autoinjector experiments (test LS-0345).

the buffer and the liquid rapidly increases the liquid pressure, preventing the buffer from being substantially accelerated. With an air gap, the pressure below the buffer increases more slowly, and this allows the buffer to be accelerated to a larger velocity.

The syringe acceleration begins $0.8 \mathrm{~ms}$ after the acceleration of the buffer begins. This confirms the friction between the buffer and the syringe does not play a significant role in accelerating the syringe; it is the pressure increase in the tip which accounts for the acceleration. The $0.8 \mathrm{~ms}$ delay is longer than the $0.5 \mathrm{~ms}$ delay measured without an air gap (case 6). This is again due to the slower rise time of the pressure pulse.

The syringe is accelerated until $0.3 \mathrm{~ms}$, experiences a deceleration labeled as "1st cavitation event", is again accelerated, and again decelerated due to a second cavitation event. The motion of the syringe is consistent with the evolution of the pressure in the tip, discussed below.

Figure 4.29 is a plot of the pressure in the syringe. Labels 2 and 4 identify the same features as those identified in Figure 4.12 due to the similar physics between cases 4 and 7. The first incident pressure pulse (label 2) is due to the compression wave created by the first impact of the projectile on the buffer, which compresses and 
pressurizes the air gap. The second pressure pulse (label 4) is caused by the second impact of the projectile on the buffer, not a reflected wave.

Identical to case 4 of Section 4.1, the pressure pulse created by the second impact of the projectile on the buffer propagates in a cavitating liquid, and cavitation bubbles are forced to collapse as the pressure pulse propagates in the bubbly liquid. This is visible on the hoop and axial strains shown in Figure 4.30. When the second impact of the projectile on the buffer occurs (label 3), the bottom half of the liquid column is already experiencing cavitation. The arrival of the second incident wave at the bottom of the syringe is indicated with label 4.

The agreement between the LS-DYNA simulations and the experiments is reasonably good. LS-DYNA over predicts the magnitude of the pressure pulse created at the first impact, an effect which is likely due to the absence of friction in the numerical model. LS-DYNA also predicts the pressure pulse created by the second impact is entirely annihilated before reaching the bottom wall, but this is not in agreement with the experimental measurements. This is particularly noticeable on the hoop strains shown in Figure 4.30a.

The axial strains predicted by LS-DYNA are not in agreement with the measurements. Significant axial strains are created and measured immediately after the impact of the projectile on the buffer, something which is not predicted by LSDYNA. Those features could result from the breakage of the nylon screw holding the syringe prior to a test, friction between the buffer and the syringe, and misalignment of the syringe in the guide tube resulting in lateral impacts between those components.

\subsubsection{Case 8}

Case 8 is identical to case 7 , except the syringe is terminated with a straight cone. Case 8 is equivalent to case 5 from Section 4.1, except the motion of the syringe is now included. Figure 4.31 is a plot of the pressure measured at the apex of the syringe tip. The peak magnitude of the pressure pulse created by the first impact of the projectile on the buffer (label 2) does not differ significantly when a flat tip or a conical tip is used: $5.4 \mathrm{MPa}$ for case 7 , and 5.7 $\mathrm{MPa}$ for case 8. This is because the rise time of the incident pressure pulse is approximately $720 \mu \mathrm{s}$, much longer than the $25 \mu$ s acoustic transit time of the waves in the cone, and no amplification occurs. 


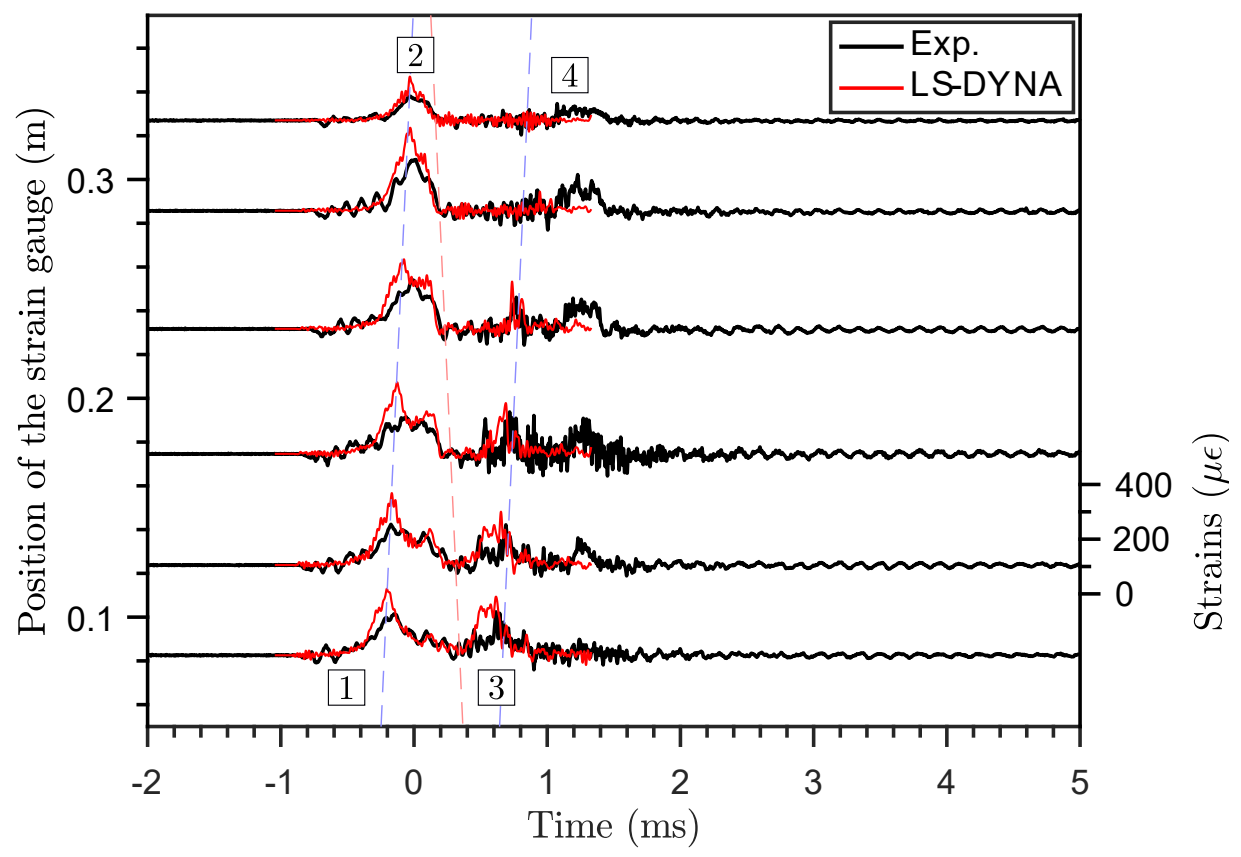

(a) Hoop strains

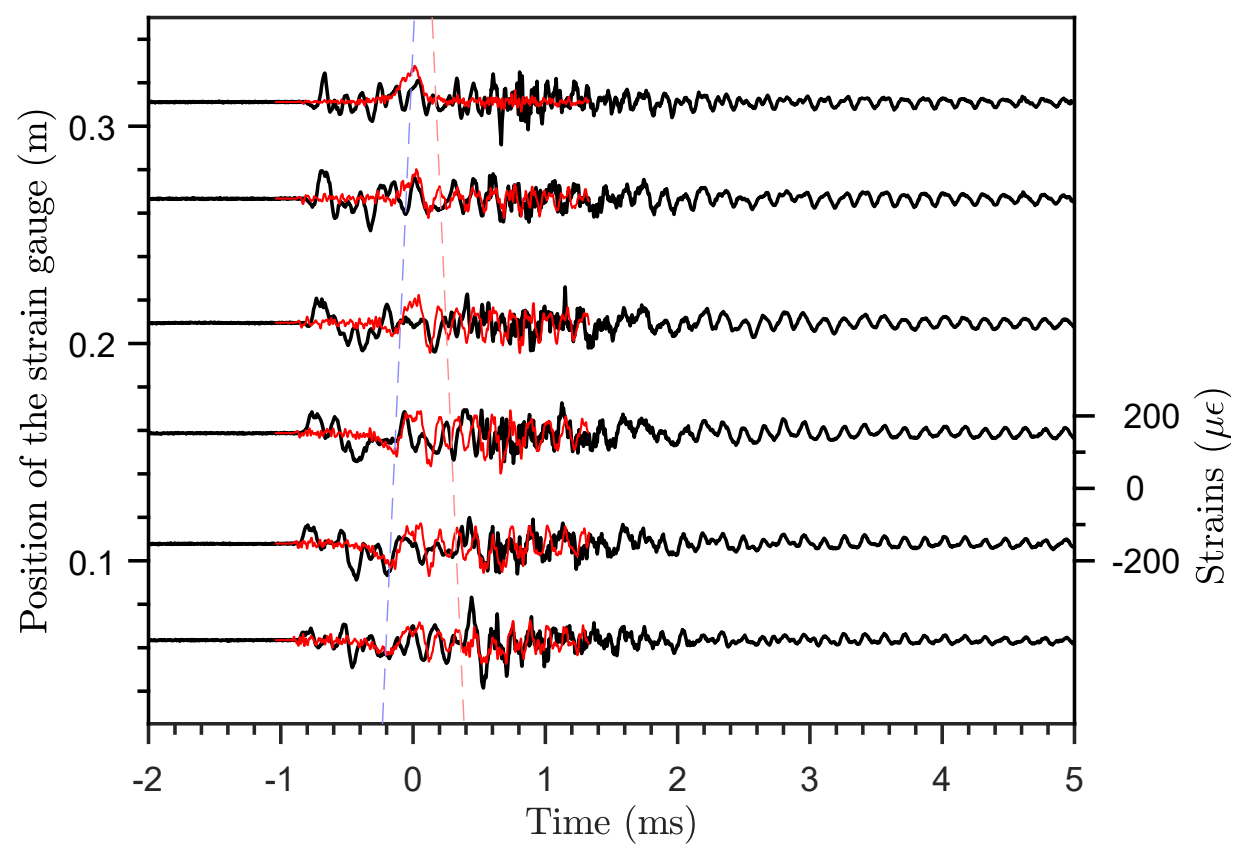

(b) Axial strains

Figure 4.30: Hoop and axial strains for case 7 of the dynamic, large-scale model autoinjector experiments (test LS-0345). 


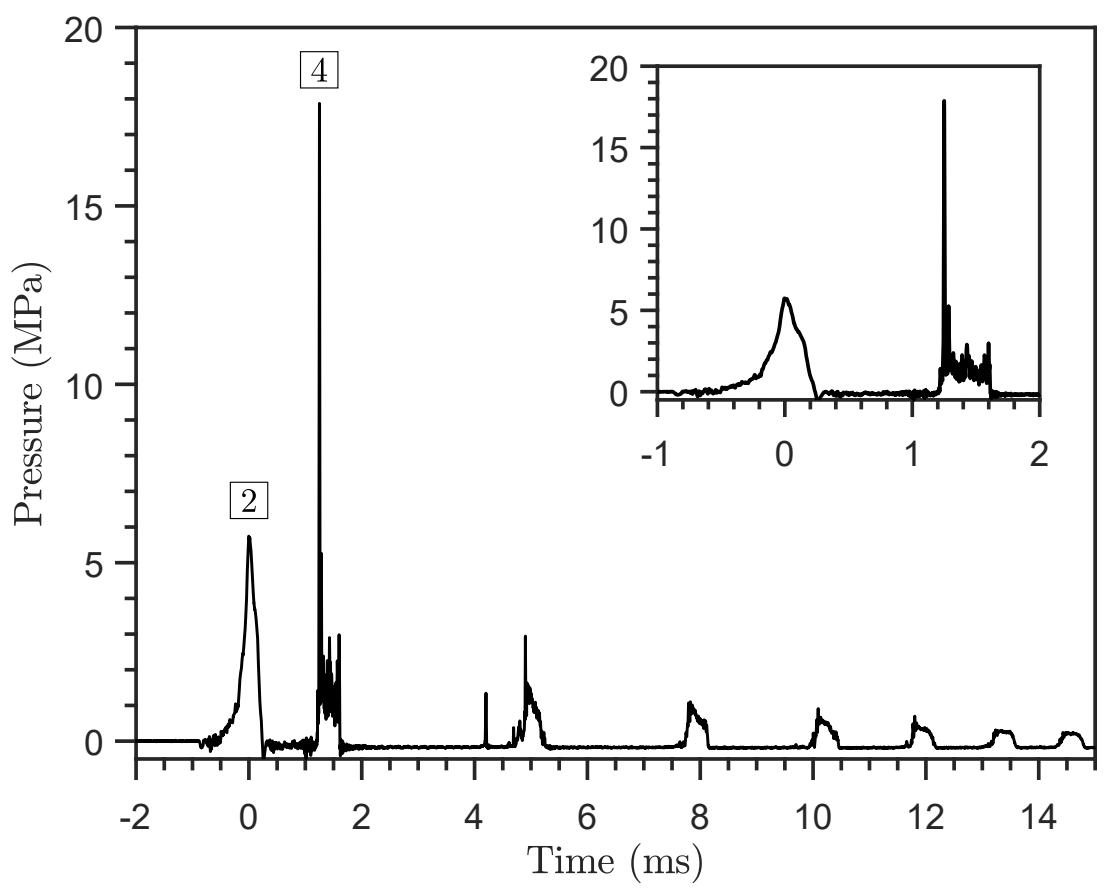

Figure 4.31: Pressure at the bottom wall for case 8 of the dynamic, large-scale model autoinjector experiments (test LS-0208).

The peak magnitude of the pressure pulse resulting from the second impact of the projectile on the buffer (label 4) is substantially affected by the conical tip. A peak magnitude of 3.0 MPa is measured with the flat wall, compared to $17.9 \mathrm{MPa}$ with the cone. The explanation for the larger pressure, as before, is twofold. First, there is the potential for shock focusing inside the cone due to the sharpness of the second pressure pulse. The second pressure pulse becomes sharp as it propagates in a bubbly liquid (see Section 4.1). Second, the collapse of cavitation bubbles in the tip can be enhanced by the conical geometry. This is investigated further in Chapter 6 .

The hoop and axial strains are not shown because they are very similar to the strains measured with a flat tip (case 7). This is because the effect of the cone is local, and the strain gauges are positioned away from the cone.

\subsubsection{Case 9}

Case 9 is identical to case 6, except there is a $15.2 \mathrm{~mm}$ air gap between the buffer and the water. The motion of the syringe and the buffer is shown in Figure 4.32. The projectile impacts on the buffer at approximately $-2.5 \mathrm{~ms}$, accelerating the buffer into the syringe up to a velocity of $8 \mathrm{~m} / \mathrm{s}$. The syringe acceleration begins approximately 


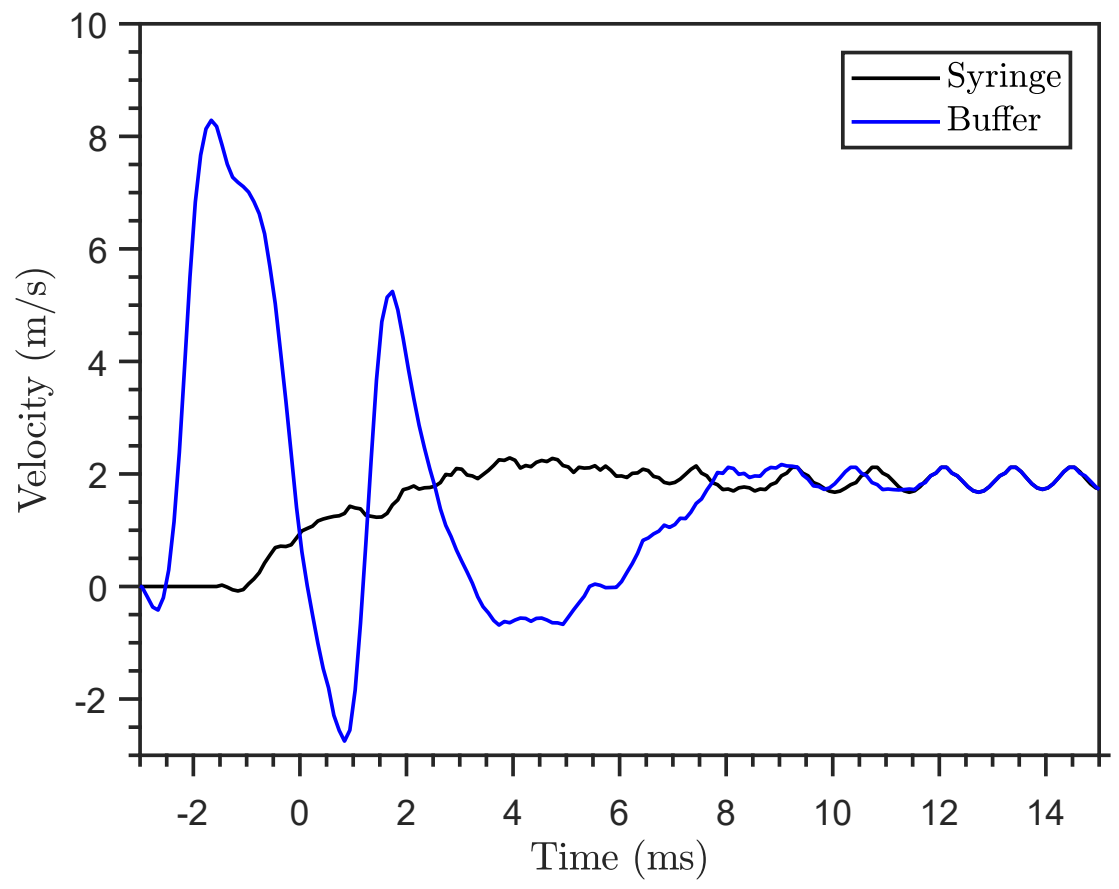

Figure 4.32: Velocity of the syringe and the buffer for case 9 of the dynamic, large-scale model autoinjector experiments (test LS-0329).

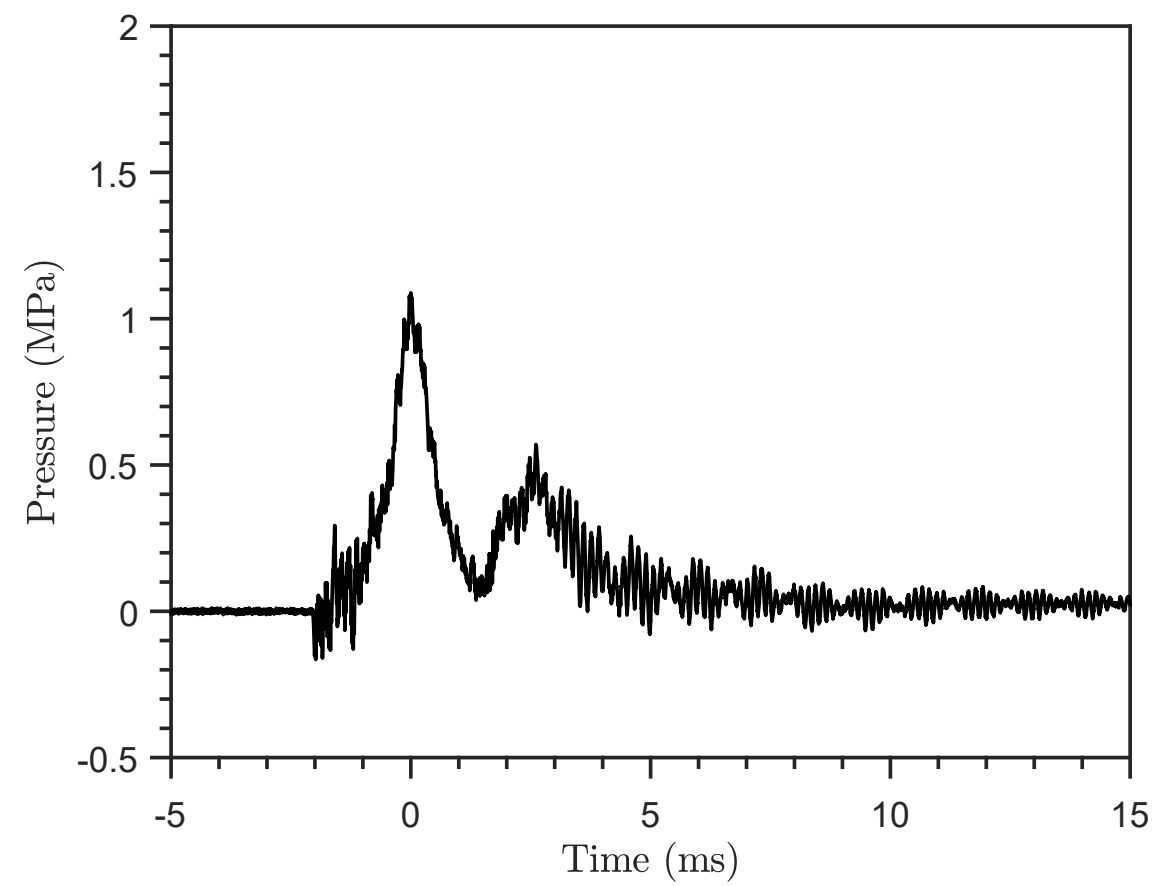

Figure 4.33: Pressure at the bottom wall for case 9 of the dynamic, large-scale model autoinjector experiments (test LS-0329). 
$1.5 \mathrm{~ms}$ after the impact of the projectile on the buffer. This relatively long delay is due to the slow rise time of the pressure in the air gap and, consequentially, in the tip of the syringe. The force applied on the syringe tip needs to increase sufficiently for the nylon screw holding the syringe to break. The acceleration of the syringe occurs over approximately $4 \mathrm{~ms}$, and the maximum syringe velocity is $2 \mathrm{~m} / \mathrm{s}$. The pressure in the tip is discussed below.

Figure 4.25 indicates the results obtained with the cone and the flat tip are similar for such a large air gap, and no amplification occurs within the cone. This is because the impact of the projectile on the buffer creates a pressure pulse which is weak due to the damping effect of the air gap; the peak pressure at the bottom wall is only 1.1 MPa, and the magnitude of the pressure pulse is too small to result in cavitation. This is confirmed with the pressure plot shown in Figure 4.33.

Note that without the syringe motion, a $15.2 \mathrm{~mm}$ air gap results in cavitation in the liquid (see cases 4 and 5 in Section 4.1). Without the syringe motion, the magnitude of the incident pressure wave created by the impact of the projectile on the buffer is approximately 4.0 MPa, substantially larger than the $1.1 \mathrm{MPa}$ measured with the syringe motion. The reduction in peak pressure and the absence of cavitation is another confirmation that the syringe motion effectively acts as a pressure relief mechanism.

\subsubsection{Case 10}

Test cases 10 to 15 are performed with the reversed outer projectile: the impact of the projectile occurs on the syringe wall rather than on the buffer. Several tests were performed in this configuration. As indicated at the beginning of this section, damping material in the form of two stacked O-rings is introduced between the projectile and the syringe wall. The impact of the projectile on the damping material accelerates the syringe. The acceleration, in turn, creates a pressure and stress transient.

Case 10 is for a syringe terminated with a flat wall, filled with water, but with no buffer sealing the syringe. This is equivalent to having an air gap which is infinite in size. Although it may not be obvious at first, this configuration without a buffer is the simplest configuration possible for this type of transient event. The effect of adding the buffer and reducing the air gap size is discussed later. 


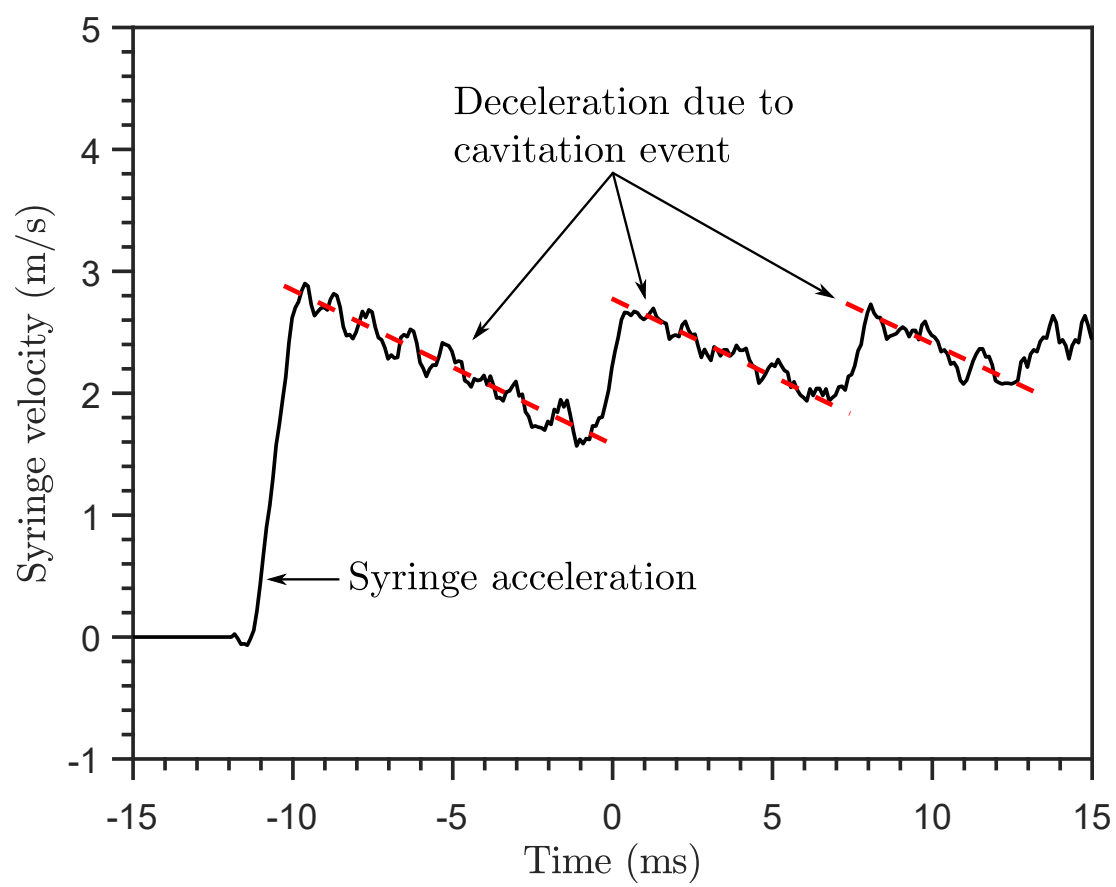

Figure 4.34: Velocity of the syringe for case 10 of the dynamic, large-scale model autoinjector experiments (test LS-0322).

The velocity of the syringe is shown in Figure 4.34. The impact of the projectile on the syringe wall occurs at $-11.5 \mathrm{~ms}$, promptly accelerating the syringe. The magnitude of the syringe acceleration is approximately $3,000 \mathrm{~m} / \mathrm{s}^{2}$. Note that the projectile rebounds on the O-rings, and it does not impact a second time on the syringe wall in the time period of interest.

The large syringe acceleration is followed by a weak deceleration, indicated with a red-dashed curve in Figure 4.34. This is again the result of a cavitation event in the syringe. The magnitude of the deceleration is approximately $121 \mathrm{~m} / \mathrm{s}^{2}$, close to the expected magnitude of $141 \mathrm{~m} / \mathrm{s}^{2}$. The positive acceleration of the syringe around $0 \mathrm{~ms}$ is the result of a substantial pressure increase at the bottom of the syringe, creating a large force on the tip of the syringe. This pressure increase occurs when the first cavitation event ends, and this is what triggers the data acquisition system. This cycle is repeated a few times.

Figure 4.35 is a plot of the pressure measured at the bottom of the syringe. The pressure history supports the observations reported above. The syringe acceleration begins at $-11.5 \mathrm{~ms}$ (label 1), resulting in a pressure decrease. As explained in Section 2.2.2, the acceleration of the syringe creates tension waves which reduce 


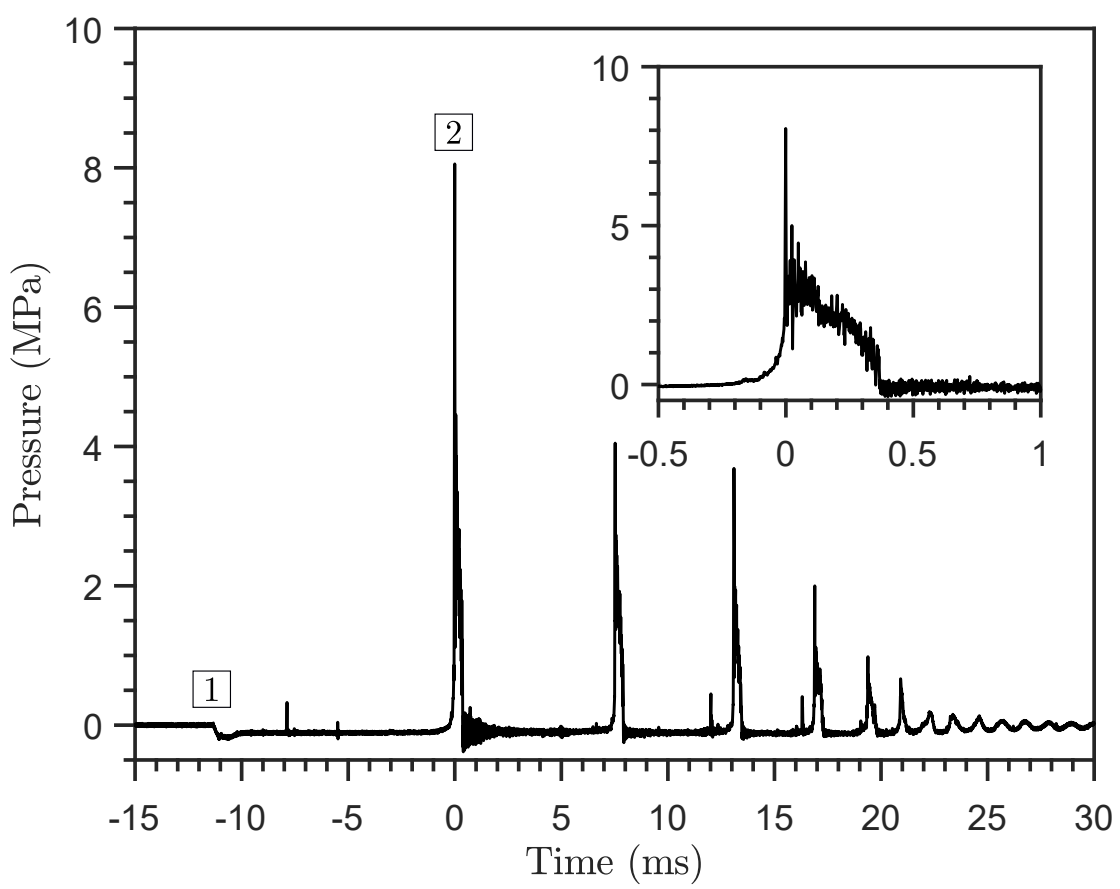

Figure 4.35: Pressure at the bottom wall for case 10 of the dynamic, large-scale model autoinjector experiments (test LS-0322).

the liquid pressure to vapor pressure, and cavitation occurs. The tension waves originate in the vicinity of the tip, and propagate toward the free surface located at the top end of the syringe. There, the tension waves reflect as compression waves. The role of the tension and compression waves is to accelerate the liquid forward.

The first cavitation event lasts until $0 \mathrm{~ms}$, ending with a substantial pressure increase (label 1) up to 8.0 $\mathrm{MPa}$. The pressure increase is created by the collapse of a large cavity or bubble in the syringe. This is confirmed later with images obtained from tests performed with a clear syringe.

Figure 4.35 indicates this cycle (i.e., cavitation event followed by a pressure increase) is repeated several times. The peak pressure created at the end of each cavitation event decreases in amplitude from cycle to cycle due to dissipation in the system. The duration of each cavitation event (i.e., the time lapse between successive peaks) also becomes shorter, indicating a faster propagation of pressure waves in the bubbly liquid during the cavitation event. This, in turn, indicates the average void fraction decreases from one cycle to another (Brennen, 1995).

The pressure signals from Figures 4.35 can be compared with the pressure signal from Figure 3.13 ( 0 to $2.5 \mathrm{~ms}$ ) obtained with the in situ methods. Both signals 
exhibit similar features. In both cases the transient events begin with a pressure decrease from ambient conditions to vapor pressure. This is followed by multiple pressure peaks created by cycles of cavitation. The peak magnitude of pressure is similar ( 8 to $9 \mathrm{MPa}$ ), and the amplitude of the pressure peaks caused by the successive collapses of cavities decreases monotonically from cycle to cycle. The time scales for both tests are different, however, because the syringes used in both setups have different size; the syringe used in the large-scale model autoinjector is approximately 6 times larger. The similarities noted suggest the physics at play is similar in the autoinjector device and the dynamic, large-scale model autoinjector.

Returning to the results for test case 10, the duration of the first cavitation event is $11.5 \mathrm{~ms}$. A simple model which provides an estimate for the expected duration of this cavitation event was developed. The model assumes the syringe and the liquid column inside the syringe move independently as two rigid bodies. This approximation is justified because the pressure waves and stress waves can complete multiple axial round trips in $11.5 \mathrm{~ms}$. The model assumes the syringe is impulsively accelerated to a velocity $u_{0}$, which corresponds to the velocity of the syringe after the impact event. A cavity immediately starts forming between the liquid column and the syringe: the syringe is moving, but the liquid column is still stationary.

The pressure inside the cavity is vapor pressure, creating a net force oriented in the negative $z$ direction applied on the syringe. The equation of motion for the syringe is:

$$
u_{s}(t)=u_{0}-\frac{\left(P_{0}-P_{\text {vap }}\right) A_{i} t}{m_{s}} .
$$

This equation indicates the syringe experiences a linear deceleration as a result of the low pressure which subsists inside the cavity forming between the syringe and the liquid water. Equivalently, the low pressure in the cavity creates a net force oriented in the positive $z$ direction applied on the water column. The equation of motion for the liquid column is:

$$
u_{l}(t)=\frac{\left(P_{0}-P_{v a p}\right) t}{\rho_{l} L_{l}}
$$

The difference in velocity $\Delta u(t)$ between the syringe and the liquid column dictates the growth and collapse of the cavity. The velocity difference is:

$$
\Delta u(t)=u_{s}(t)-u_{l}(t)=u_{0}-\left(P_{0}-P_{v a p}\right) t\left(\frac{A_{i}}{m_{s}}+\frac{1}{\rho_{l} L_{l}}\right),
$$


and the volume of the cavity is:

$$
V_{\text {cav }}(t)=A_{i} \int_{0}^{t} \Delta u\left(t^{\prime}\right) \mathrm{d} t^{\prime}=u_{0} t A_{i}-\frac{A_{i}\left(P_{0}-P_{v a p}\right) t^{2}}{2}\left(\frac{A_{i}}{m_{s}}+\frac{1}{\rho_{l} L_{l}}\right) .
$$

The collapse time is obtained when $V_{c a v}=0$. The nontrivial solution of $V_{c a v}=0$ from Equation 4.9 is:

$$
t_{\text {collapse }}=\frac{2 u_{0} m_{s} \rho_{l} L_{l}}{\left(P_{0}-P_{\text {vap }}\right)\left(A_{i} L_{l} \rho_{l}+m_{s}\right)} .
$$

Evaluating with numbers that are representative of the experiments $\left(u_{0}=3 \mathrm{~m} / \mathrm{s}\right.$, $\rho_{l}=1,000 \mathrm{~kg} / \mathrm{m}^{3}, L_{l}=0.286 \mathrm{~m}, P_{0}=101,325 \mathrm{~Pa}, P_{v a p}=2,300 \mathrm{~Pa}$, and $A_{i}=1.14 \times$ $10^{-3} \mathrm{~m}^{3}$ ) yields $t_{\text {collapse }}=12.1 \mathrm{~ms}$, in very reasonable agreement with the measured value of $11.5 \mathrm{~ms}$.

The hoop and axial strains for case 10 are shown in Figure 4.36. The hoop strains confirm the pressure pulse forms at the bottom wall (label 2) and propagates upward at the Korteweg speed, toward the free surface. A precursor wave also forms in the syringe wall, visible from the detectable hoop deformation occurring prior to the arrival of the pressure pulse (i.e., strains to the left of the oblique line indicating the Korteweg speed). The pressure pulse reflects on the free surface (label 3) as a tension wave. The reflected tension wave, upon reflection on the bottom wall, initiates the second cavitation event (label 4).

The peak magnitude of the hoop strains is approximately $212 \mu \epsilon$. The quasi-static, thin shell theory (Equation 3.2) is used to estimate the magnitude of the pressure pulse needed to create a $212 \mu \epsilon$ hoop strain. A value of $2.1 \mathrm{MPa}$ is obtained, and this is not in agreement with the measured peak pressure of 8.0 MPa. In other words, an 8.0 MPa pressure pulse is expected to create hoop strains that are significantly larger than $212 \mu \epsilon$. The zoomed-in view of the pressure pulse shown in the top-right corner of Figure 4.35 provides an explanation for this. Although the pressure does reach a peak value of $8.0 \mathrm{MPa}$, the pressure does not remain this large for an extended period of time: the pressure is greater than $2.5 \mathrm{MPa}$ for only $6 \mu \mathrm{s}$.

There are three reasons why the short pulse is not visible in the hoop strains: 1) the frequency response of the strain gauges and signal conditioners is not large enough to capture such short pulses; 2) the sharp pressure pulse attenuates as it travels away from the end of the tube; 3 ) inertial effects in the syringe wall are important, and the wall of the tube can't respond this fast to a pressure pulse. The transit time of the waves for a round trip through the thickness of the tube is $4.2 \mu \mathrm{s}$, comparable to 


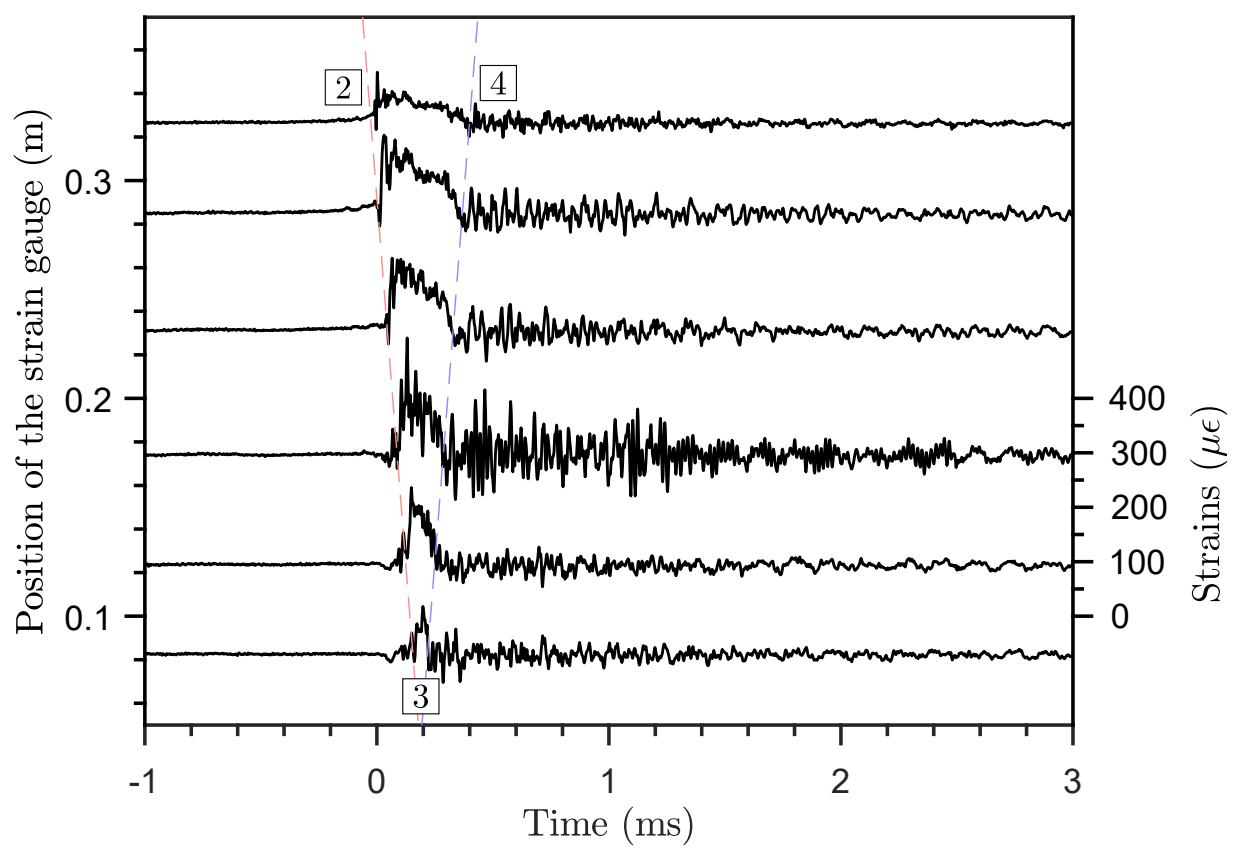

(a) Hoop strains

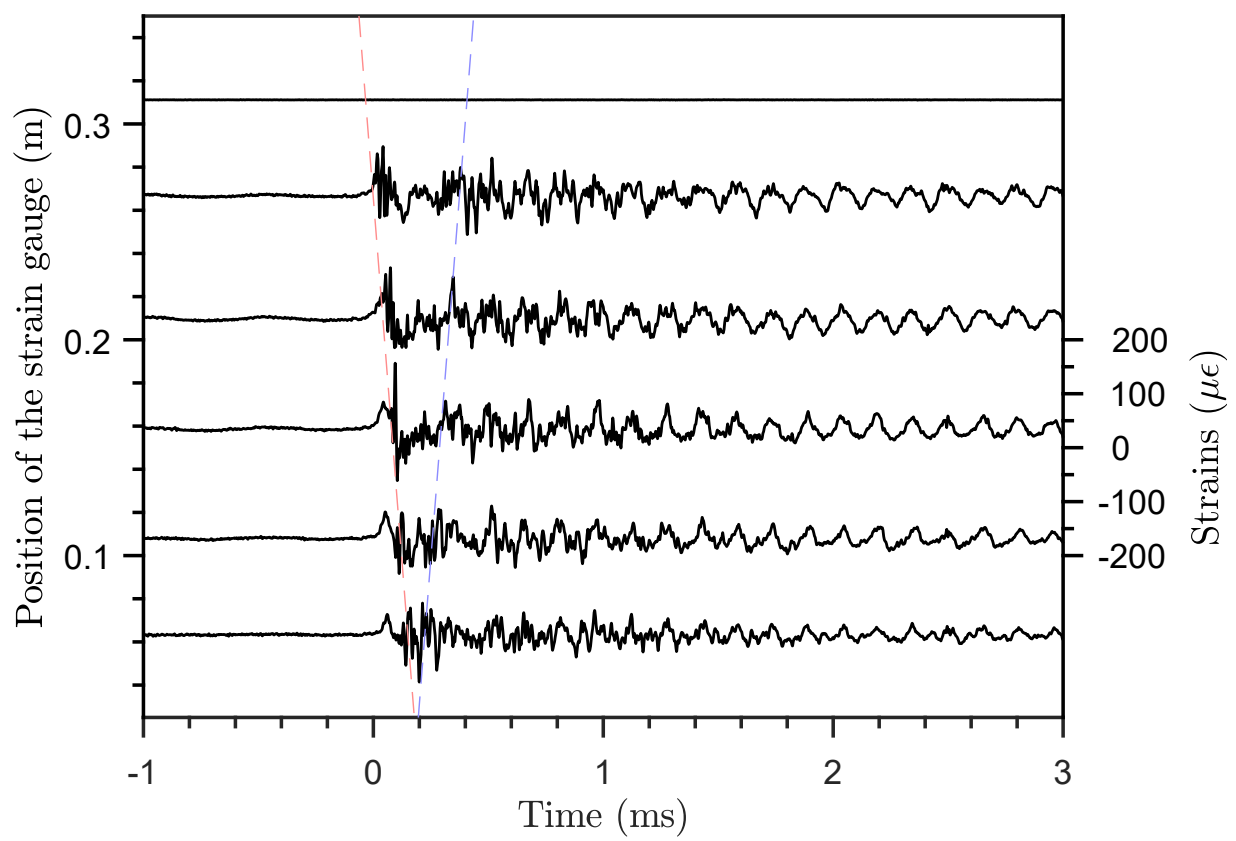

(b) Axial strains

Figure 4.36: Hoop and axial strains for case 10 of the dynamic, large-scale model autoinjector experiments (test LS-0322). 
the $6 \mu$ s duration of the pressure pulse, and the natural period of motion in the hoop direction is $75 \mu$ s (see Equation 4.12 discussed below), longer than the duration of the pulse. The pressure however remains around 2.0 MPa for an extended period of time, close to $0.5 \mathrm{~ms}$, creating the measurable $212 \mu \epsilon$.

The peak magnitude of the axial strains is $121 \mu \epsilon$ in tension, and $-96 \mu \epsilon$ in compression. The axial strains result from both the Poisson effect and the pressure force applied on the tip. The pressure force applied on the tip tends to create a tensile axial stress in the syringe walls, while the Poisson effect tends to create a compressive stress. The two effects are competing, resulting in complex interactions. The axial strains confirm there is a detectable precursor wave traveling in the syringe wall, ahead of the pressure pulse and to the left of the oblique line indicating the Korteweg speed. The precursor wave creates a tensile axial strain of $49 \mu \epsilon$.

A quasi-static thin shell approximation is used to estimate the magnitude of the tensile stress wave created by the pressure force applied in the tip, and, in turn, the magnitude of the precursor axial strain:

$$
\epsilon_{z}=\frac{P R_{i}^{2}}{\left(R_{o}^{2}-R_{i}^{2}\right) E} .
$$

This quasi-static estimate is adequate as long as the rise time of the pressure $P$ is small compared to the natural period of oscillation $T$ of the tube in the hoop direction (Shepherd and Inaba, 2010):

$$
T=\pi\left(R_{i}+R_{0}\right) \sqrt{\frac{\rho_{s}\left(1-v^{2}\right)}{E}},
$$

equal to approximately $75 \mu \mathrm{s}$ for the aluminum syringe. The expected value of $\epsilon_{z}$ for a $2 \mathrm{MPa}$ pressure applied on the tip is $44 \mu \epsilon$, close to the measured magnitude of $49 \mu \epsilon$. Note that the stress wave in the syringe wall reverberates multiple times, creating oscillatory axial strain signals over a long period of time. Note that the axial transit time for a round trip of the stress wave in the axial direction is approximately $125 \mu \mathrm{s}$.

A repeat test was performed with a clear, polycarbonate syringe, referenced as test case $10 \mathrm{~b}$. The pressure in the syringe is shown in Figure 4.37, and it is similar to what was obtained for case 10. Figure 4.38 is a sequence of images of the cavitation event inside the syringe, in the vicinity of the tip. The time stamp above each frame can be used to position the frames on the pressure plot from Figure 4.37. 


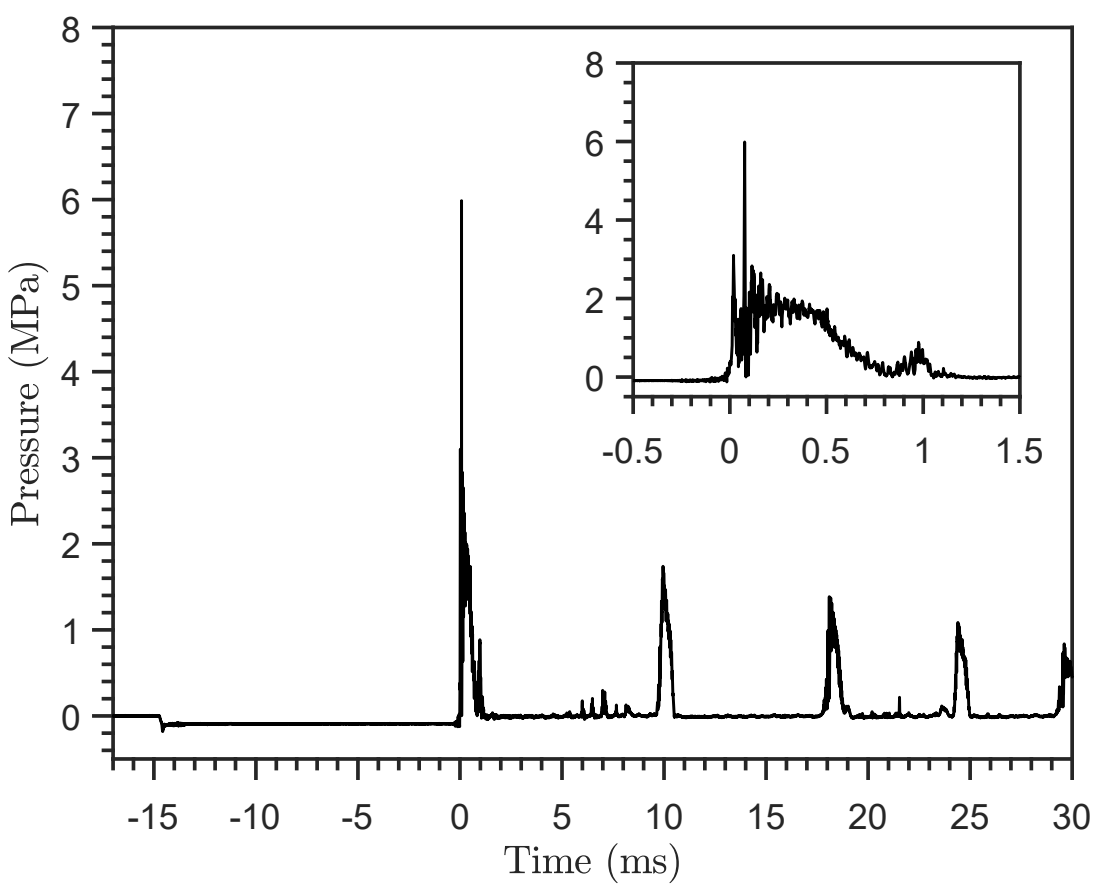

Figure 4.37: Pressure at the bottom wall for case 10b of the dynamic, large-scale model autoinjector experiments.

Initially $(-16.0 \mathrm{~ms})$, no cavitation is observed. The syringe is stationary, and the pressure inside corresponds to ambient pressure. The impact of the projectile on the syringe wall occurs at around $-15.0 \mathrm{~ms}$, causing the syringe to rapidly accelerate. The tension waves created by the acceleration of the wall and the tip reduce the pressure to vapor pressure, promptly initiating cavitation. A distributed cavitation event occurs away from the tip, where multiple relatively small bubbles form and collapse from $-14.9 \mathrm{~ms}$ to $-11.7 \mathrm{~ms}$. There is a column separation event at the very bottom of the syringe.

The column separation event begins with the formation of multiple small bubbles attached to the flat wall at $-14.9 \mathrm{~ms}$. From $-13.8 \mathrm{~ms}$ to $-11.7 \mathrm{~ms}$, the bubbles grow and coalesce to form a single cavity which occupies the entire cross section of the syringe. This is followed by the vertical growth of the cavity until $-7.3 \mathrm{~ms}$, and the collapse of the cavity. Complete collapse occurs at $0.0 \mathrm{~ms}$, an event which creates a substantial pressure increase in the tip. This is followed by a second cavitation event, the beginning of which is visible in the last two frames, from $1.3 \mathrm{~ms}$ to $2.1 \mathrm{~ms}$. The duration of the first cavitation event for test $10 \mathrm{~b}$ is $15.0 \mathrm{~ms}$, longer than the $11.5 \mathrm{~ms}$ duration measured for test 10 with the aluminum syringe. The difference 


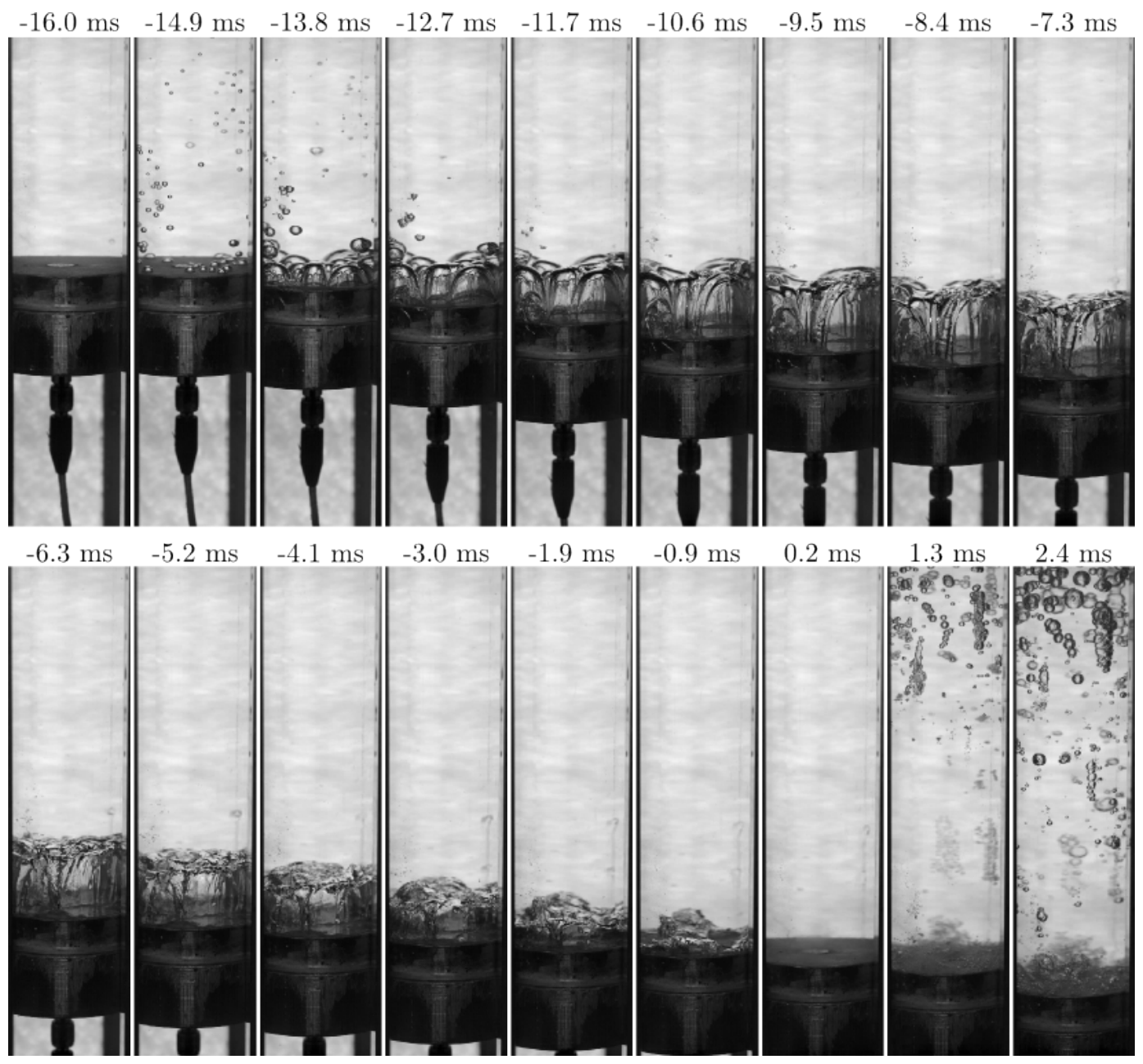

Figure 4.38: Sequence of images showing the cavitation event at the bottom of the syringe for test $10 \mathrm{~b}$ of the dynamic, large-scale model autoinjector experiments (test LS-0382).

is explained by the lower mass of the polycarbonate syringe $-0.36 \mathrm{~kg}$ for the polycarbonate syringe and $0.81 \mathrm{~kg}$ for the aluminum syringe - which results in the syringe being accelerated to a larger velocity after the impact of the projectile on the syringe wall. The velocity of the syringe after impact is $4.3 \mathrm{~m} / \mathrm{s}$ for case $10 \mathrm{~b}$, compared to $3.3 \mathrm{~m} / \mathrm{s}$ for case 10 .

Adding a buffer at the top of the syringe affects the results significantly. Qualitatively, the measured signals all look alike, but the peak magnitude of the pressure and strains are changed. Figure 4.39 is a plot of the peak pressure as a function of initial air gap size. In general, increasing the air gap size results in a larger peak pressure. This trend is investigated further using case 11. 


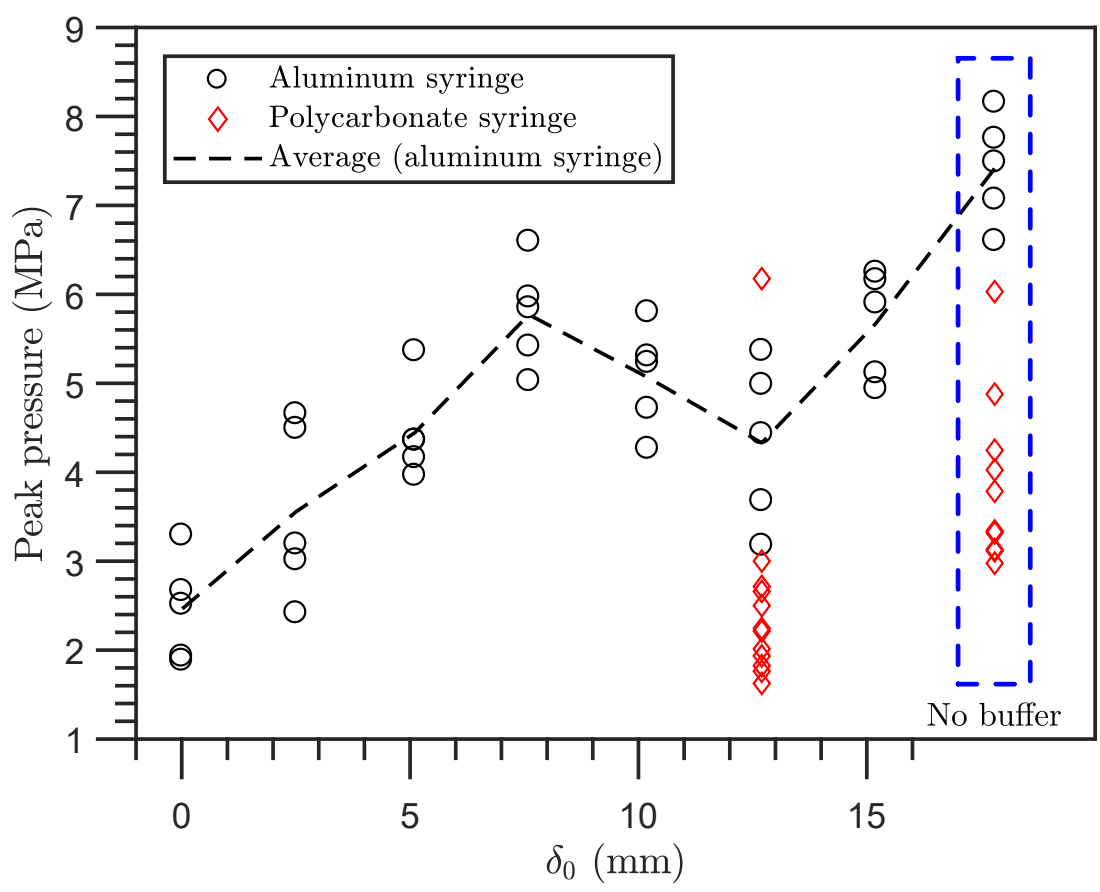

Figure 4.39: Peak pressure in the syringe tip as a function of initial air gap size. The outer projectile impacts on the syringe wall. This is for the dynamic, large-scale model autoinjector test setup with a flat tip.

Figure 4.39 also indicates there is substantial vertical scatter in the measured peak pressure, both for the aluminum and the polycarbonate syringes. The polycarbonate syringe is used to investigate this further. Results suggest that variability in the position of the bubble with respect to the pressure transducer in the final stage of the collapse is the key factor in determining the peak magnitude of the pressure pulse. A bubble that collapses close to the transducer creates a larger measured pressure than a bubble collapsing far away from the transducer. This is because the magnitude of the pressure waves forming upon bubble collapse decays rapidly. Neglecting the effect of the wall, the decay is expected to be in $1 / r$, where $r$ is the distance from the center of the collapsing bubble (Brennen, 1995; Franc and Michel, 2005).

Figure 4.40 contains sequences of images of the final stage of bubble collapse for two different test cases. Figure 4.40a is for test case 10b, discussed above. Figure 4.40b is for test case 10c, which is another repeat test with the same experimental conditions. Figure 4.40a indicates that the final bubble collapse occurs right above the pressure transducer in test case $10 \mathrm{~b}$. This results in a peak pressure of 6.0 MPa. It is even possible to observe two successive collapses of the bubble, in agreement with the 

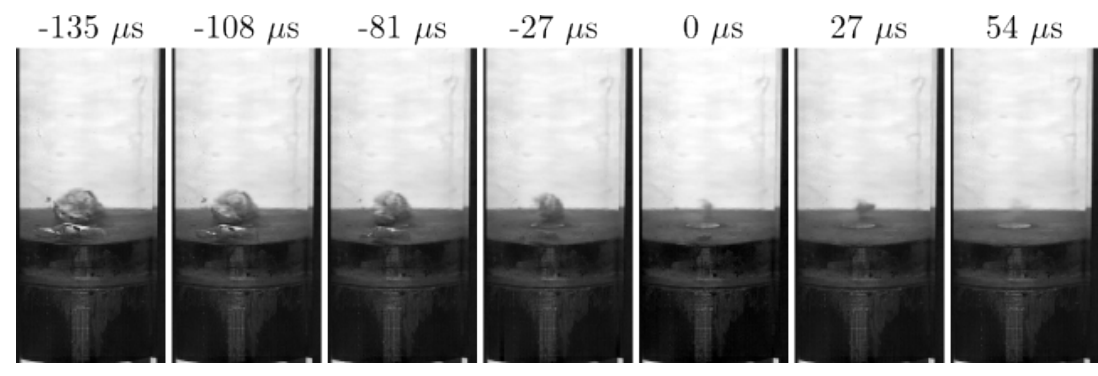

$81 \mu \mathrm{s}$

(a) Test case $10 \mathrm{~b}$ (test LS-0382)
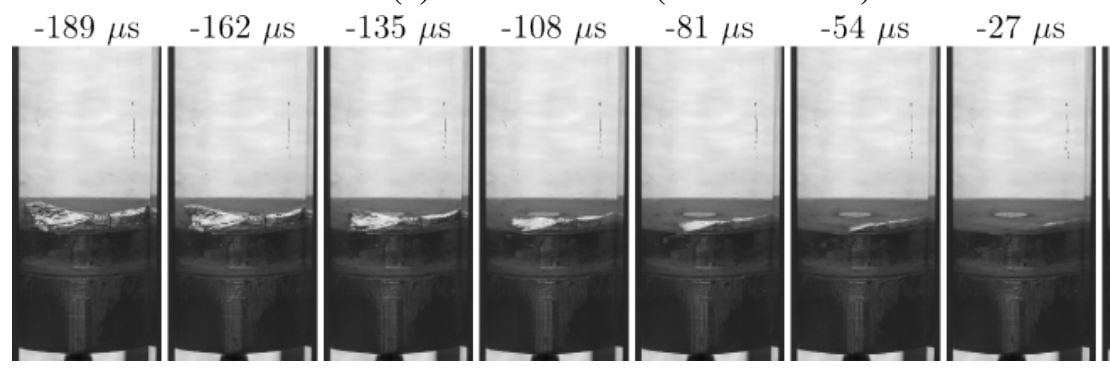

$0 \mu \mathrm{s}$

(b) Test case 10c (test LS-0388)

Figure 4.40: Final stage of bubble collapse for tests cases $10 \mathrm{~b}$ and 10c performed without a buffer. This is for the dynamic, large-scale model autoinjector.

pressure trace shown in Figure 4.37. The first collapse occurs at $0.0 \mu$ s, resulting in a peak pressure of $3.1 \mathrm{MPa}$. The bubble rebounds, and collapses again $54 \mu$ s later. The second collapse results in a peak pressure of 6.0 MPa.

Figure $4.40 \mathrm{~b}$ indicates that the final stage of bubble collapse is different for test 10c. The collapse occurs in the front of the tube, away from the tip of the transducer, and no bubble rebound is observed. The pressure history for this test case is not shown, but the peak pressure is only 3.0 MPa, half the peak pressure measured for test $10 \mathrm{~b}$.

\subsubsection{Case 11}

Case 11 is identical to case 10, except the buffer is present and there is no air gap in the syringe. The velocity of the syringe is not shown because it is similar to what is shown for case 10. Figure 4.41 is a plot of the pressure at the bottom of the syringe. The impact of the projectile on the syringe wall results in a pressure decrease and promptly initiates cavitation. The duration of the cavitation event is shorter than without a buffer, only $7.6 \mathrm{~ms}$ in duration. The shorter growth and collapse of the cavity indicates the cavity does not grow as large as for cases without the buffer. This was confirmed visually using the polycarbonate syringe. The peak pressure upon collapse of the smaller cavity is approximately $2.4 \mathrm{MPa}$, and this is not followed by a subsequent cavitation event. 


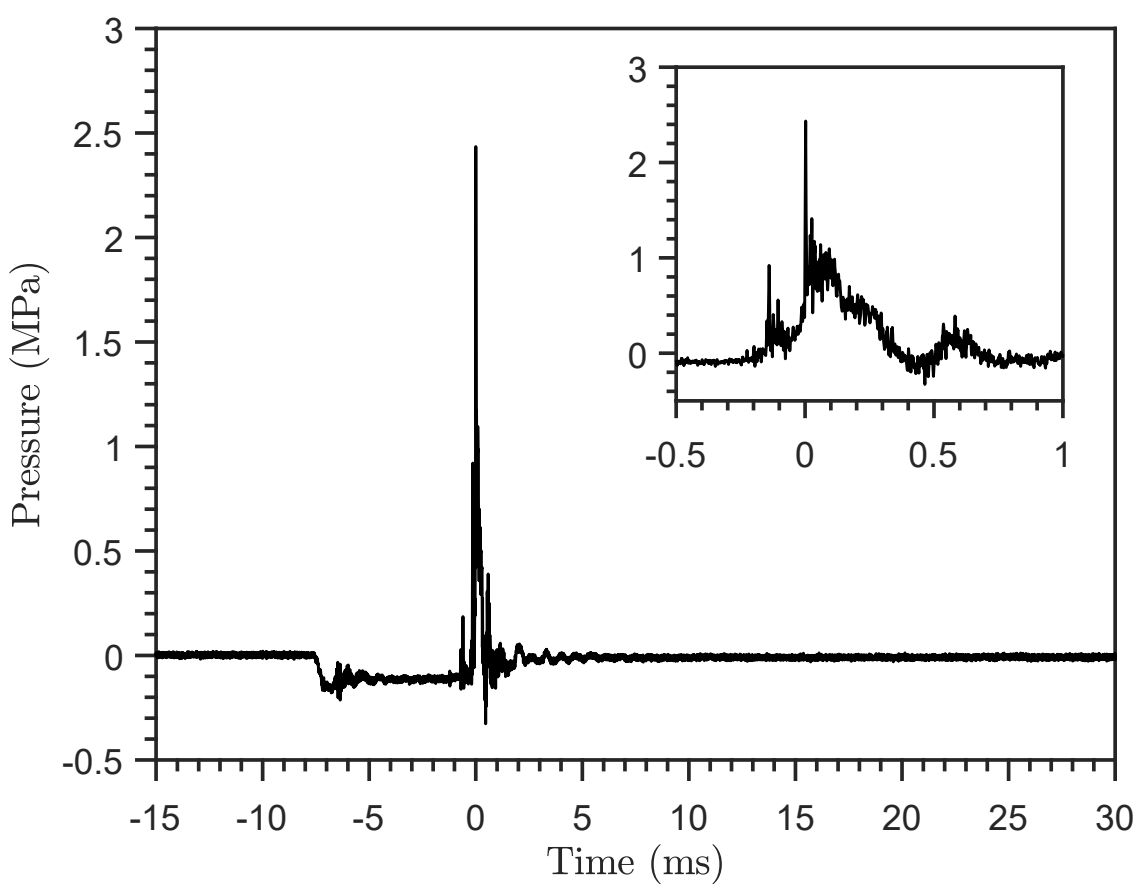

Figure 4.41: Pressure at the bottom wall for case 11 of the dynamic, large-scale model autoinjector experiments (test LS-0318).

The absence of an air gap results in a strong coupling between the motion of the liquid and the buffer. This explains the absence of a second cavitation event. The friction between the buffer and the syringe wall creates dissipation, resulting in a more rapid decay of the pressure waves in the liquid.

There is a relatively simple explanation for the shorter duration of the cavitation event, and the smaller magnitude of the peak pressure. Without a buffer, the acceleration of the liquid column results entirely from the tension waves created in the syringe tip. When a buffer is present and when there is no air gap, the friction between the buffer and the syringe results in the production of compression waves originating from below the buffer. This is because the acceleration of the syringe results in the downward acceleration of the buffer due to friction. The motion of the buffer contributes at creating the motion of the liquid content. In other words, the tension waves created in the tip are not the only mechanism for accelerating the liquid forward. The compression waves originating from below the buffer are what limits the growth of the cavity forming in the tip.

The presence of a buffer also affects the wave mechanics: the top boundary condition on the water column is not a true constant pressure boundary condition. The 
reflection of the waves occurs on the buffer surface rather than a free surface. This type of soft reflection on an accelerated wall was discussed earlier.

Introducing and increasing the size of the air gap between the buffer and the liquid results in a weaker coupling between the buffer and the liquid motion. As the air gap size is increased, the boundary condition applied on the top end of the water column becomes more similar to a constant pressure boundary condition. The end result is that increasing the air gap size increases the duration of the cavitation event, leaving enough time for the cavity to grow larger, thus creating larger pressures upon collapse.

\subsubsection{Case 12}

Replacing the flat tip with a straight cone results in larger measured peak pressures. Figure 4.42 is a plot of the peak pressure as a function of initial air gap size with the conical tip. A comparison of Figures 4.39 and 4.42 indicates the peak pressure measured with the cone is up to 6 times larger than the peak pressure measured with the flat tip. Figure 4.42 indicates there is again substantial variability in the results, visible in the form of vertical scatter. The test-to-test variability is again explained by the precise location of the bubble with respect to the pressure transducer during the final collapse.

Case 12 is identical to case 10 , but uses a conical tip instead of a flat tip. This test case is used to investigate the creation of larger peak pressures with the conical tip. The syringe velocity is shown in Figure 4.43, and it is similar to the motion of the syringe observed with the flat tip (see Figure 4.34). This is expected since the motion of the syringe primarily depends on the inertia of the syringe, a parameter which is virtually unchanged when switching from one tip to the other.

Figure 4.44 is a plot of the pressure in the syringe tip. Qualitatively, the pressure trace is similar to that of case 10 , but the vertical scale is substantially different. With the cone, the peak pressure resulting from the first bubble collapse is approximately 43.0 $\mathrm{MPa}$. The rise time of the numerous pressure peaks is short, ranging between 1 to $10 \mu \mathrm{s}$. This is shorter than the acoustic transit time of the pressure waves within the cone (approximately $25 \mu \mathrm{s}$ ), indicating that shock focusing is likely to occur.

The peak pressure created at the end of each cavitation event does not decrease monotonically. The fourth collapse, for example, creates a peak pressure which is larger than the peak pressure created by the third collapse. The most plausible 


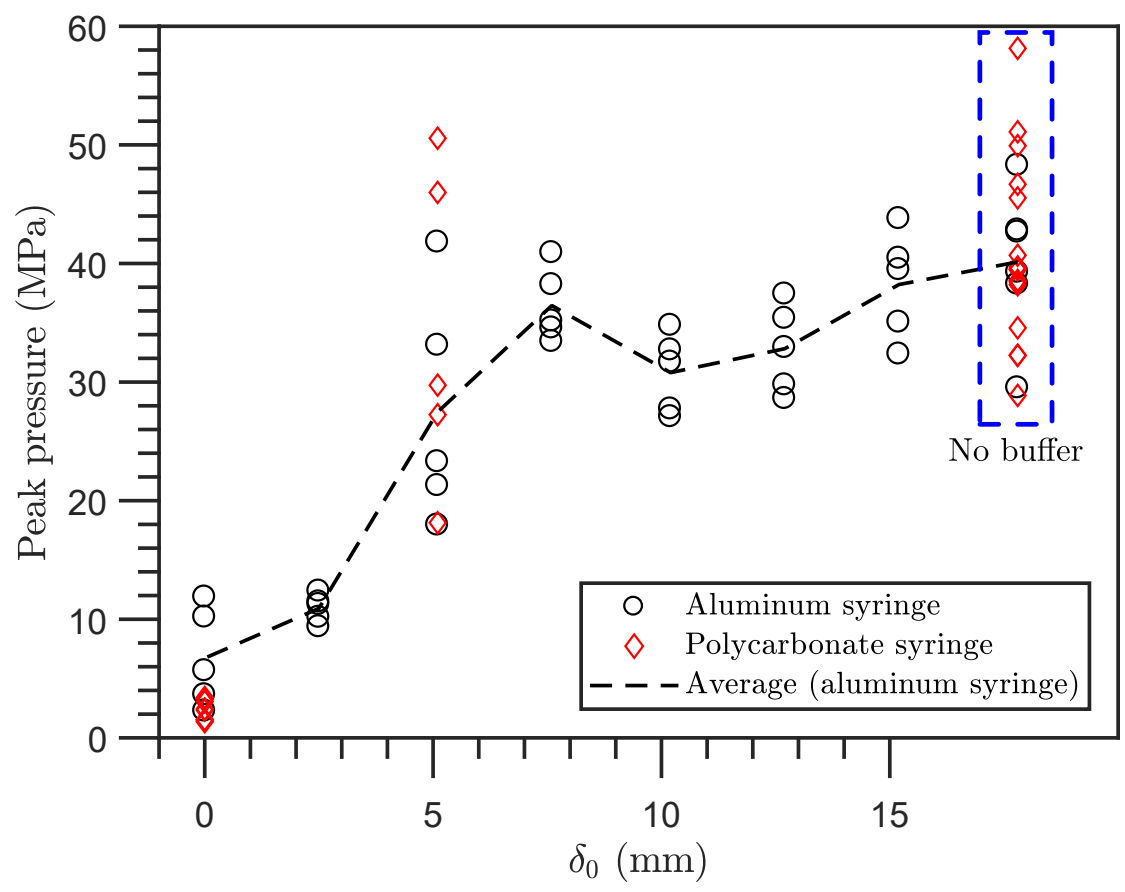

Figure 4.42: Peak pressure in the syringe tip as a function of initial air gap size. The outer projectile impacts on the syringe wall. This is for the dynamic, large-scale model autoinjector test setup with a conical tip.

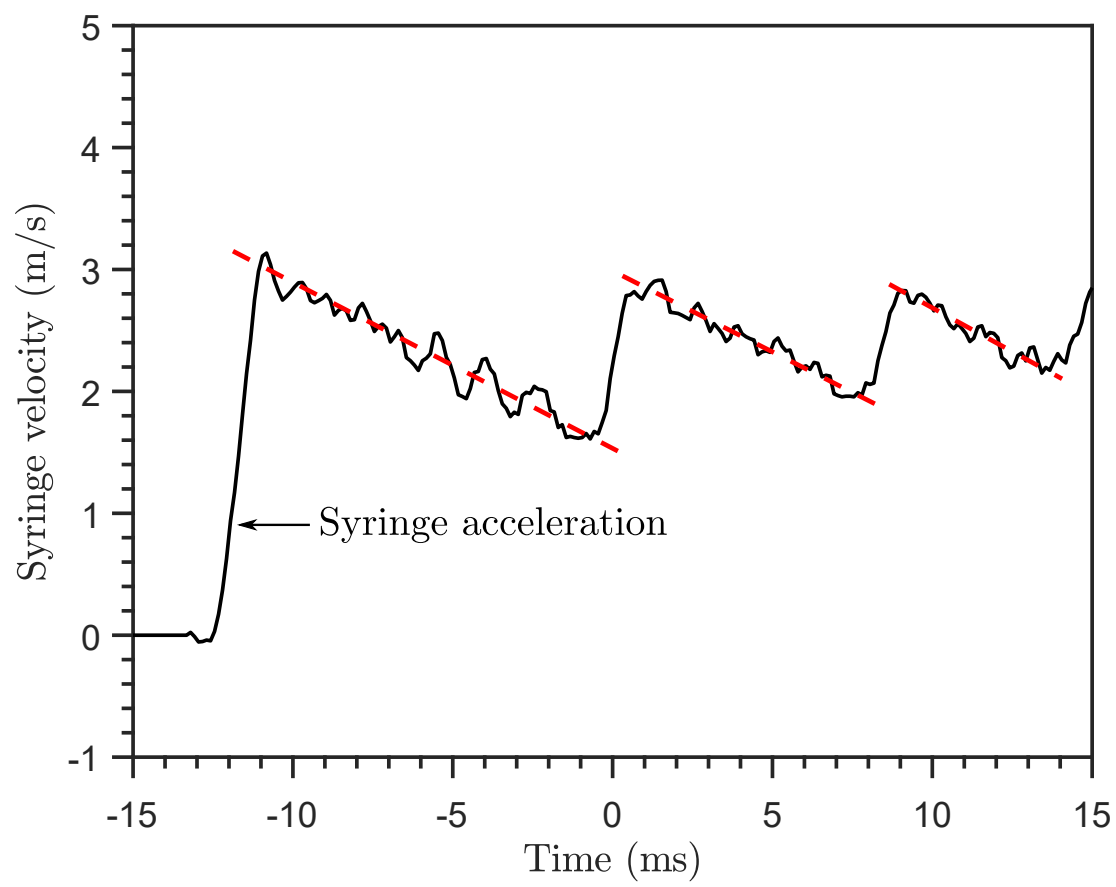

Figure 4.43: Velocity of the syringe for case 12 of the dynamic, large-scale model autoinjector experiments (test LS-0263). 


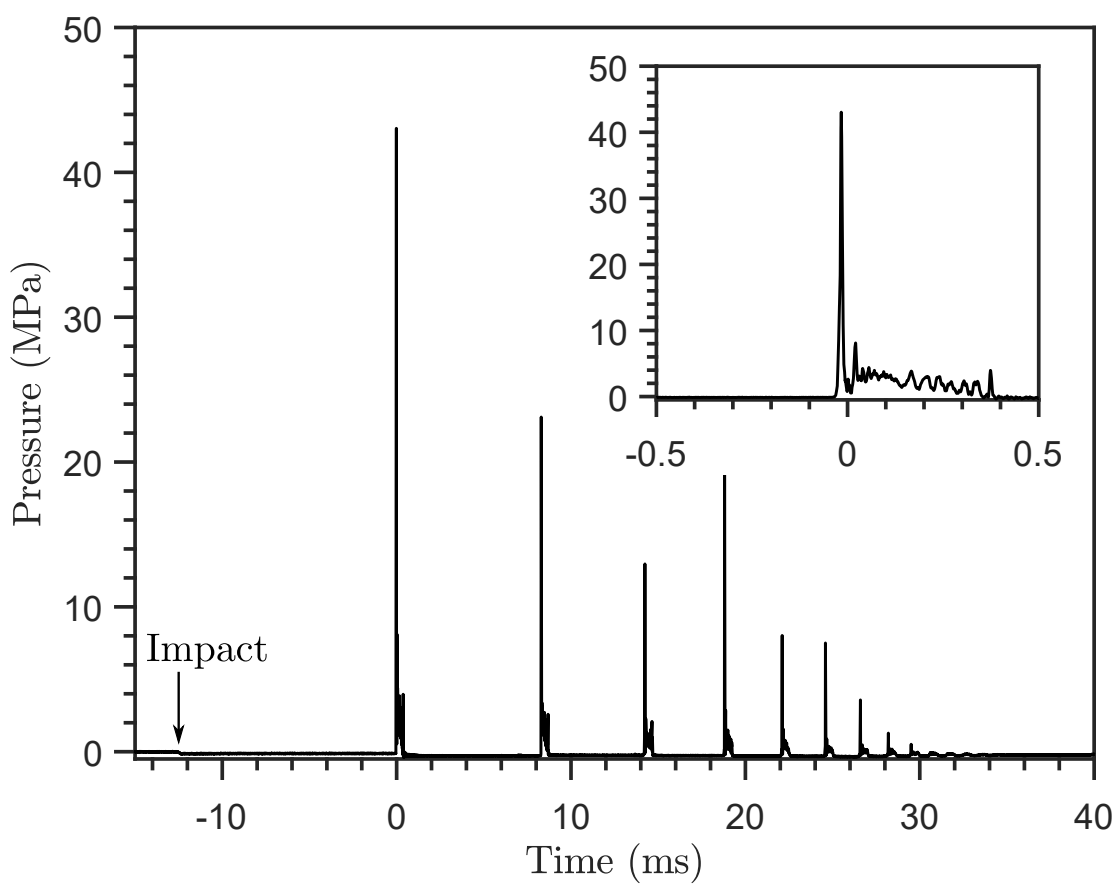

Figure 4.44: Pressure at the bottom wall for case 12 of the dynamic, large-scale model autoinjector experiments (test LS-0263).

explanation for this behavior is the movement of the bubbles inside the syringe tip, resulting in some collapses occurring closer to the transducer than others. It was not possible to confirm this visually using the clear, polycarbonate syringe. This is because the wave mechanics is weakly modified by the increased compliance of the syringe wall, suppressing this interesting behavior. Instead, experiments were performed with the aluminum syringe in combination with the clear tip. The same behavior is observed, and the hypothesis was visually confirmed.

The hoop and axial strains are shown in Figure 4.45. The strains are not different in trend and magnitude from the strains shown for case 10. This indicates the effect of the cone is local, and the peak magnitude of the pressure waves propagating outside the cone is unaffected by the geometry of the tip.

A repeat test was performed with a clear, polycarbonate syringe. It is referenced as test case $12 \mathrm{~b}$. Figure 4.46 is a plot of the pressure in the tip of the cone. The first collapse is similar to the first collapse in test case 12 , but the subsequent collapses are different. This is because the transmission, reflection, and propagation of waves between the water and the syringe wall is affected by the change in material. 


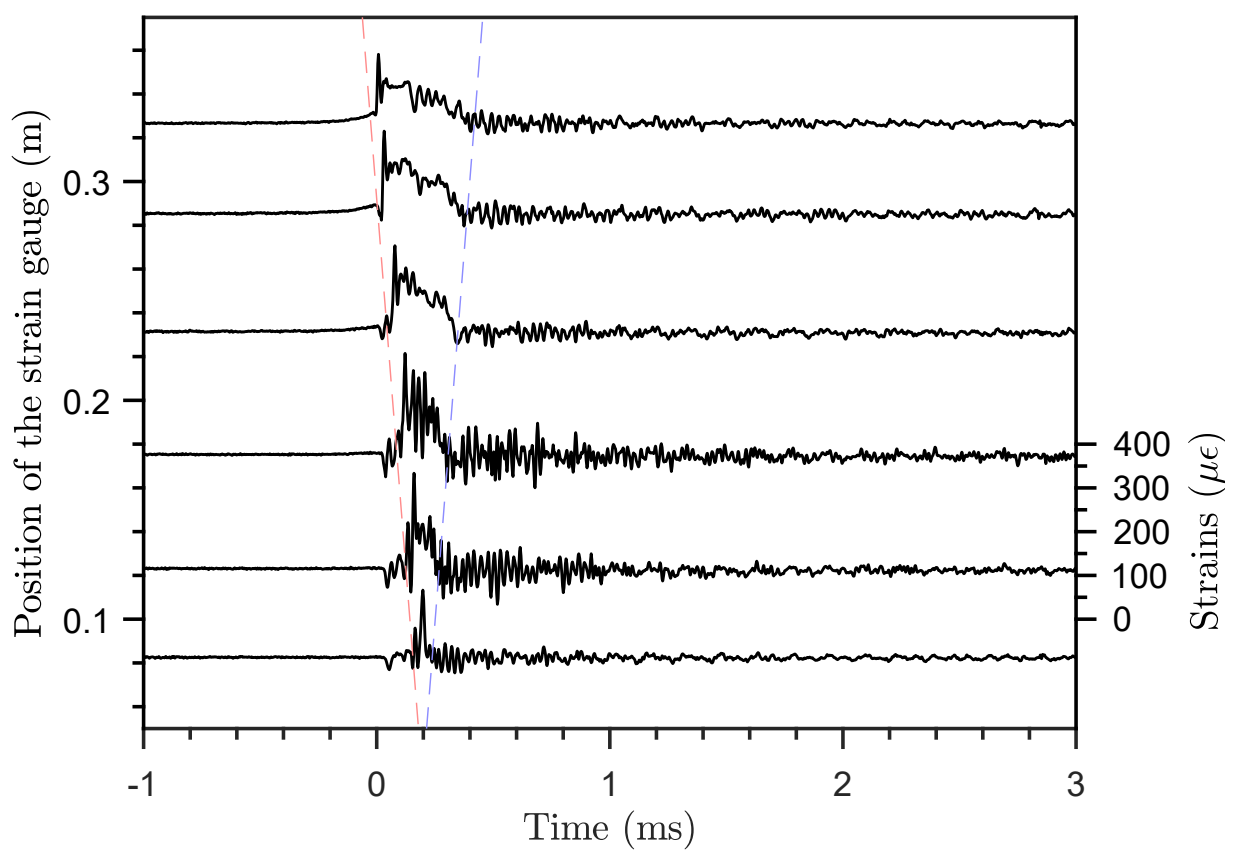

(a) Hoop strains

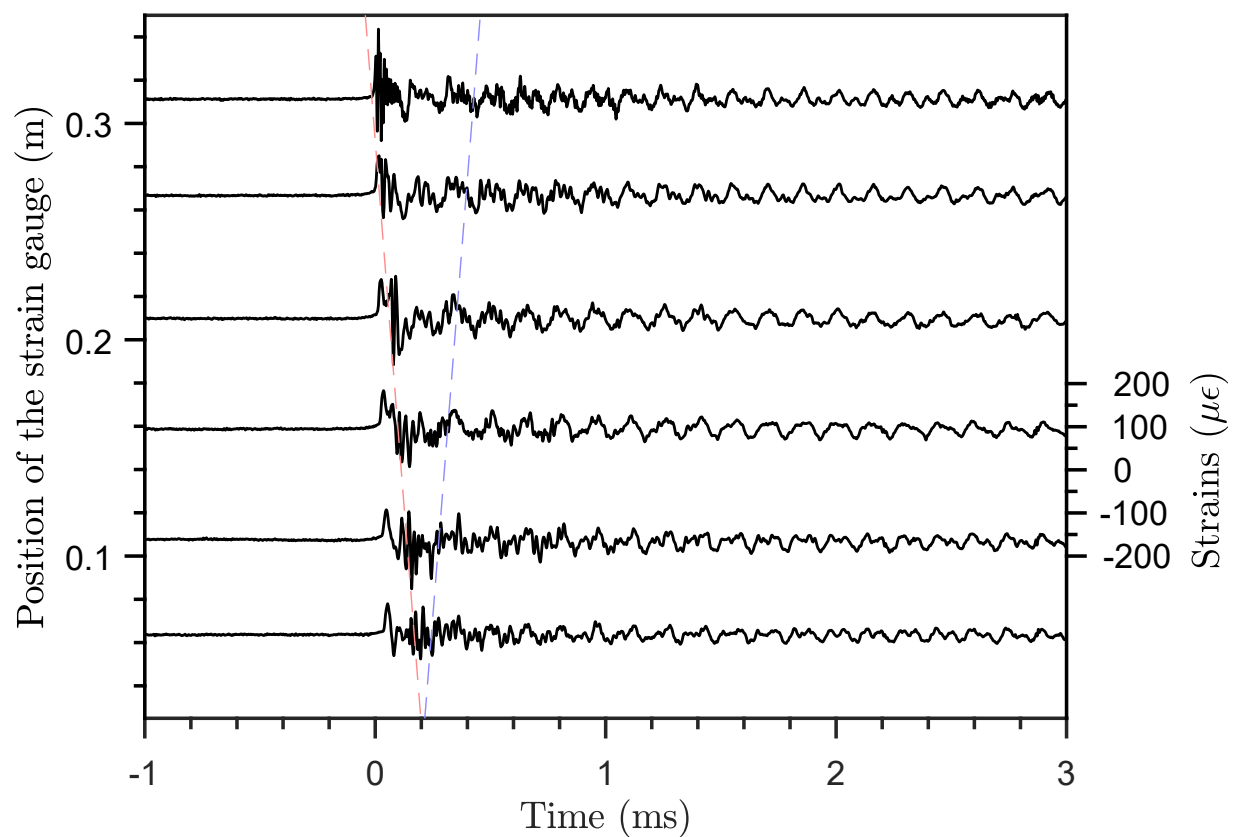

(b) Axial strains

Figure 4.45: Hoop and axial strains for case 12 of the dynamic, large-scale model autoinjector experiments (test LS-0263). 


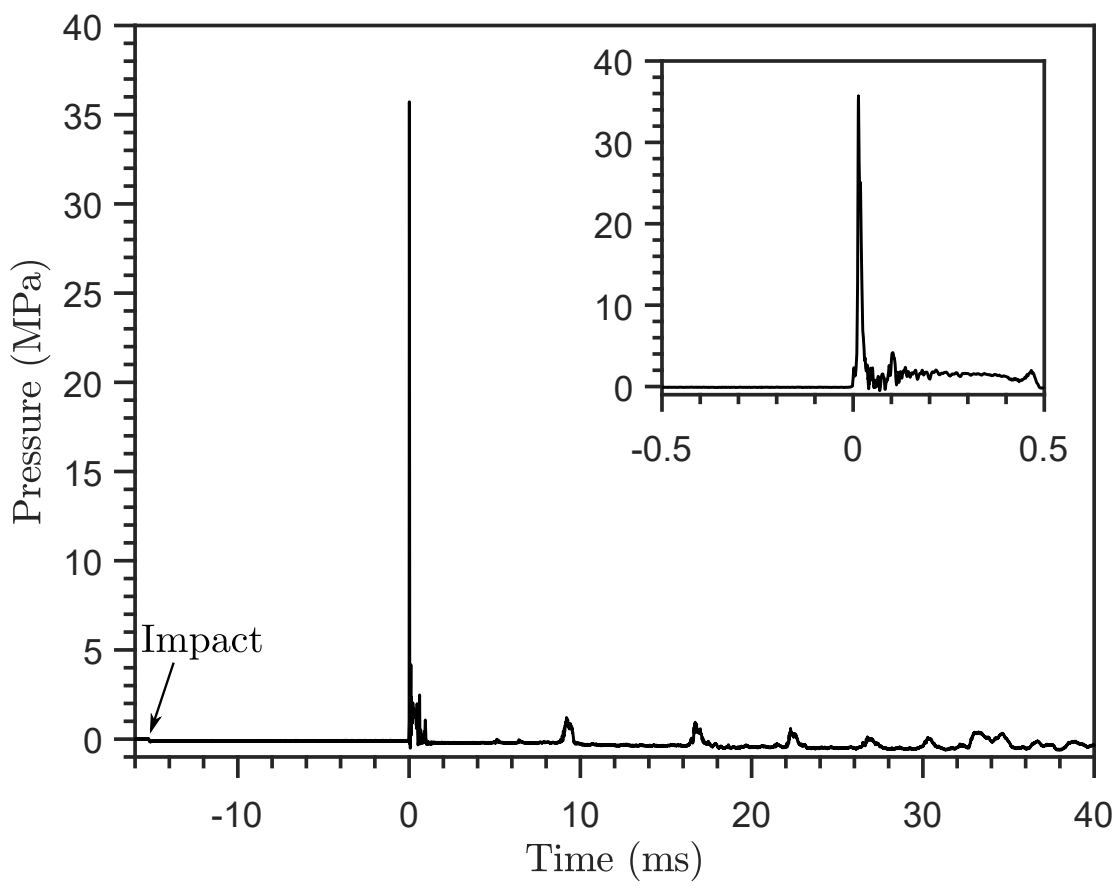

Figure 4.46: Pressure at the bottom wall for case $12 \mathrm{~b}$ of the dynamic, large-scale model autoinjector experiments (test LS-0402).

Figures 4.47 and 4.48 are two sequences of images of the first cavitation event. Figure 4.47 shows the growth and collapse of a large cavity in the cone. The joint between the tip and the barrel partially obscures the view. Nevertheless, it is possible to distinguish the growth and collapse of the cavity, and the results are analogous to what was observed and reported for case 10 with a flat tip. Multiple bubbles nucleate, grow, and coalesce to eventually form a single cavity that occupies the entire cross section of the tube. The single cavity later collapses, and this is followed by a second cavitation event.

Figure 4.48 is a sequence of images of the final stage of bubble collapse. It is possible to see the cavity is very close to the tip of the pressure transducer when it collapses. This behavior is representative of all cases performed with a clear conical tip. It appears the geometry of the cone forces the bubbles to collapse close to the tip of the cone.

\subsubsection{Summary of Cases 6 to 12}

The impact of the outer projectile on the buffer to pressurize the syringe was studied using experiments and numerical simulations. The results indicate that smaller air 

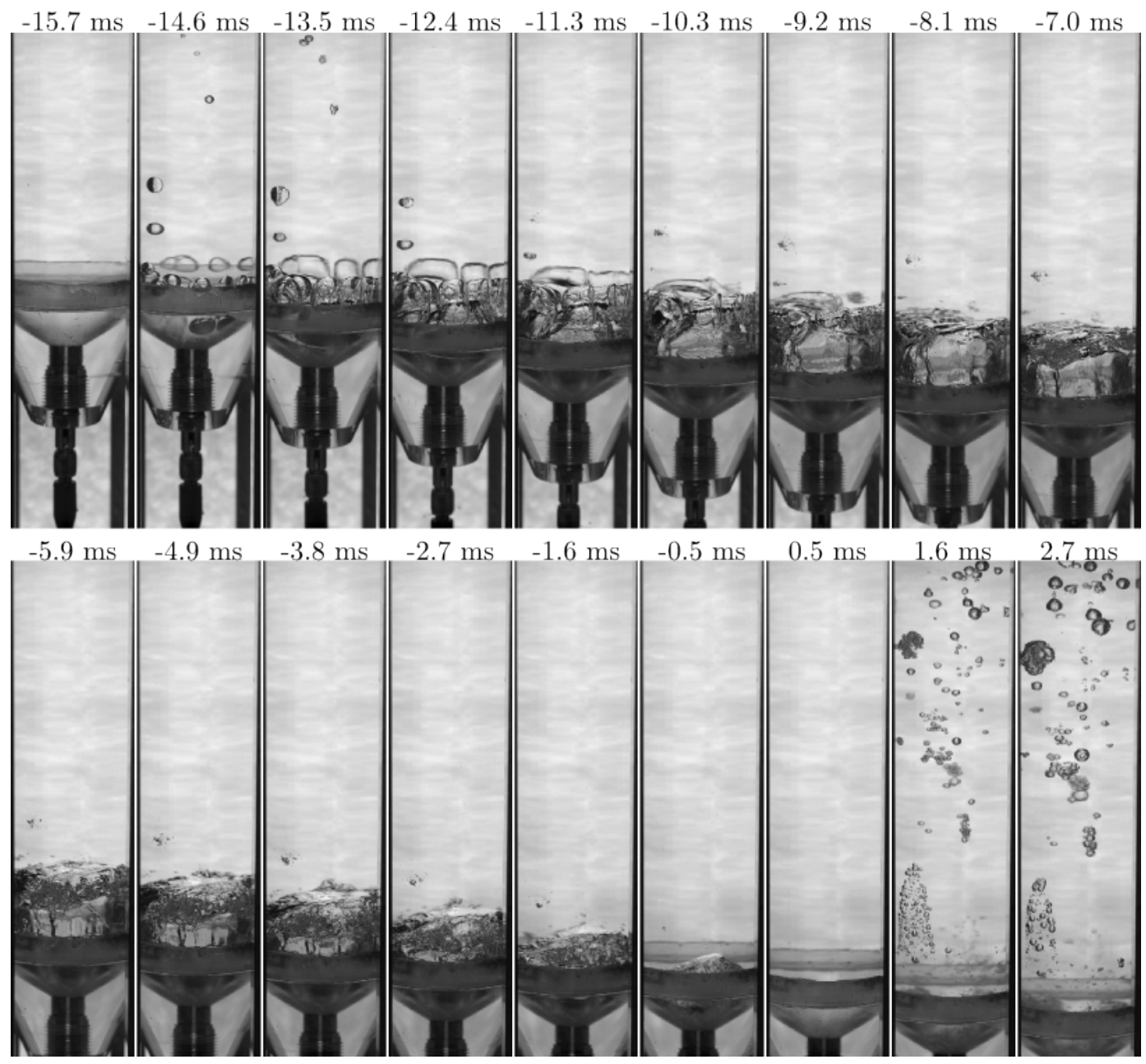

Figure 4.47: Sequence of images showing the cavitation event at the bottom of the syringe for test $12 \mathrm{~b}$ of the dynamic, large-scale model autoinjector experiments (test LS-0402).

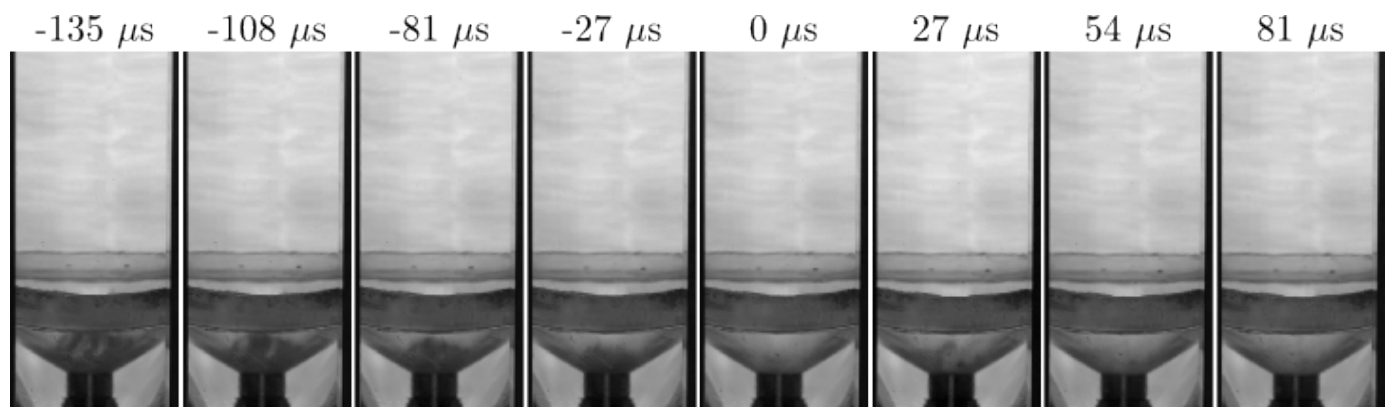

Figure 4.48: Final stage of bubble collapse for test $12 \mathrm{~b}$ of the dynamic, large-scale model autoinjector experiments (test LS-0402). 
gaps tend to create larger peak pressures at the bottom of the tube. This is because for the same impact velocity of the projectile on the buffer, large air gaps do not become as compressed as small air gaps due to the larger mass of air to be compressed. Because the liquid is pressurized through pressure waves originating from the air gap, a smaller air gap pressure results in a smaller peak pressure at the bottom of the tube. For example, with a flat tip and an air gap size of $2.5 \mathrm{~mm}$, a peak pressure of 5 to $10 \mathrm{MPa}$ is measured at the wall. With an air gap size of $10.2 \mathrm{~mm}$, the peak pressure is reduced to approximately $1.5 \mathrm{MPa}$.

The impact of the projectile on the buffer results in cavitation within the syringe, except when a large air gap is used. This is because the magnitude of the pressure waves is damped by the large air gap, and the pulses are too weak to reduce the pressure at or below vapor pressure. For test cases where cavitation occurs, there is substantial variability in the measured peak pressure, explained by variability in the location of the bubble upon collapse. Bubbles collapsing very close to the transducer result in larger measured pressures.

Replacing the flat tip with a straight cone results in substantial amplification of the peak pressure, except when cavitation does not occur. Without cavitation, all the pressure waves have a rise time which is substantially more than the acoustic transit time of the pressure waves within the cone. The peak pressures measured with the conical tip are as much as 6 times larger than the peak pressures measured with a flat tip.

The transient events created by the impact of the outer projectile on the syringe wall are substantially different from the transient events created by the impact of the reversed outer projectile on the buffer. First and foremost, the transient begins with a cavitation event due to tensile waves, not a pressure increase due to compression waves.

The peak pressures are created by the collapse of a large cavity which forms in the tip of the syringe. Larger air gaps tend to create larger pressures because the cavity grows to a larger size before collapse. Larger cavities result in larger velocities of the bubble wall at collapse and create larger peak pressures. There is substantial variability in the measured peak pressure, and this has to do with the location of the bubble in the final stage of collapse with respect to the pressure transducer.

Replacing the flat tip with a straight cone results in consistent amplification of the peak pressures, regardless of the size or presence of an air gap. The pressure with 
the conical tip is as much as 6 times larger than the pressure with a flat tip. Visual observations suggest the geometry of the conical tip forces the bubble to collapse very close to the tip of the cone, where the transducer is located.

\subsubsection{Results and Discussion: Syringe Deceleration}

The syringe deceleration is studied by using the outer projectile to impact on the syringe wall. The reversed outer projectile can also be used to impact on the buffer, but preliminary testing revealed that the transient events created upon the syringe deceleration are insensitive to how the syringe is accelerated. This is because the transient events created by the syringe deceleration occur sufficiently later that they are decoupled from the transient events created by the impact of the projectile on the syringe wall or buffer.

Three cases are discussed to illustrate the physics at play during the deceleration of the syringe:

- case 13: syringe terminated with a flat wall, large air gap;

- case 14: syringe terminated with a flat wall, no air gap;

- case 15: syringe terminated with a cone, large air gap.

\subsubsection{Case 13}

The case of an aluminum syringe terminated with a flat tip and with a large, $15 \mathrm{~mm}$ air gap between the buffer and water is discussed first. Figure 4.49 is a plot of the syringe velocity moments before and after the deceleration. Note that the projectile does not make contact with the syringe or buffer between the acceleration and the deceleration events; the projectile is further up in the guide tube, rebounding from the impact event which occurred around $0 \mathrm{~ms}$. This is illustrated in the sequence of images shown in Figure 4.50, where the motion of the projectile and the syringe is shown from before the impact of the projectile on the syringe until after the syringe deceleration.

The deceleration of the syringe occurs $60 \mathrm{~ms}$ after the trigger event. The same trigger event as in the previous subsections is used, making it simpler to locate the syringe deceleration in time with respect to the other transient events. The syringe velocity is approximately $2.2 \mathrm{~m} / \mathrm{s}$ before the impact of the syringe on the decelerator, and $-0.7 \mathrm{~m} / \mathrm{s}$ after the impact. This indicates the syringe does not come to a stop, but 


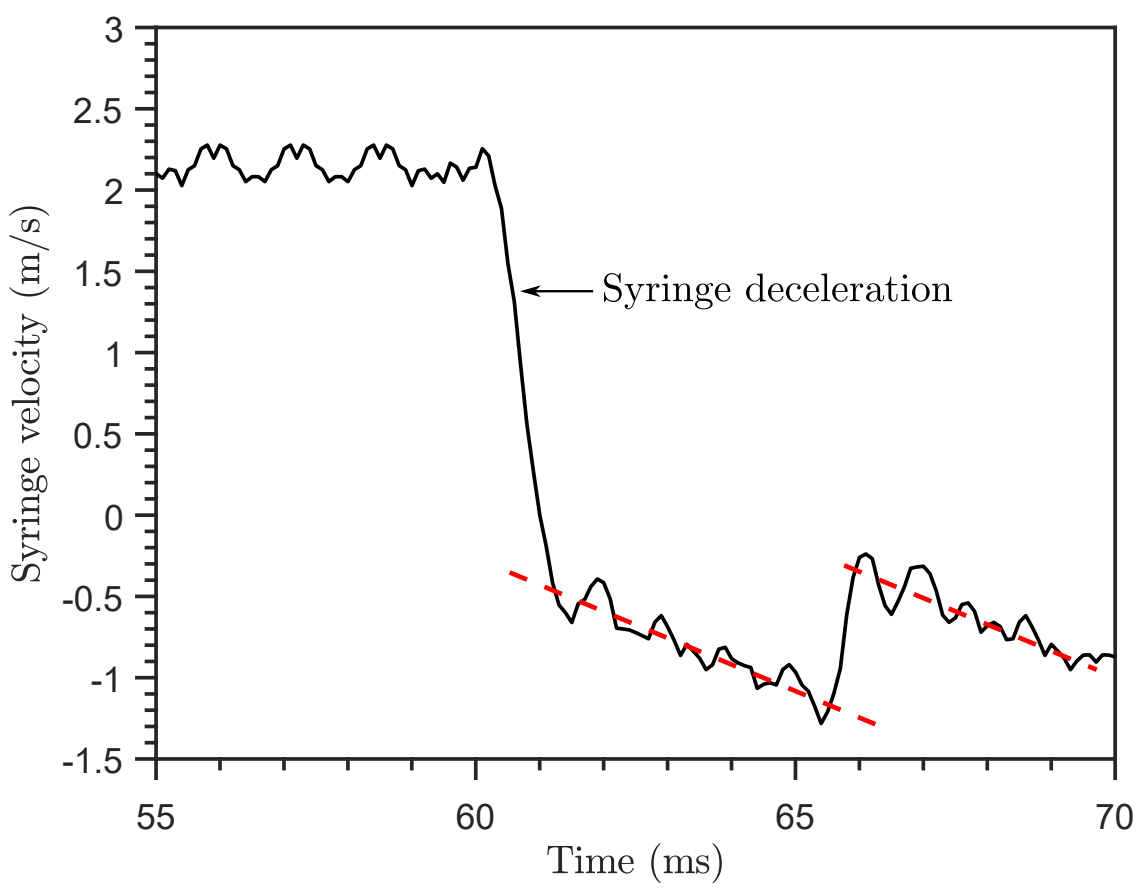

Figure 4.49: Velocity of the syringe for case 13 of the dynamic, large-scale model autoinjector experiments (test LS-0296).

rebounds on the decelerator. The magnitude of the deceleration is approximately $10,000 \mathrm{~m} / \mathrm{s}^{2}$. The liquid contained inside the syringe experiences a regime 1 or impulsive type of deceleration (see Chapter 2), and Equation 2.5 is used to estimate the peak pressure. The change in velocity $\Delta u$ of the syringe is $2.9 \mathrm{~m} / \mathrm{s}$, and this is expected to create a pressure pulse with a peak magnitude of $4.4 \mathrm{MPa}$.

The rapid deceleration of the syringe is followed by a deceleration of much lesser magnitude, indicated with a red-dashed line in Figure 4.49. This occurs between 61 and $65 \mathrm{~ms}$, and again between 66 and $70 \mathrm{~ms}$. The magnitude of the deceleration is $140 \mathrm{~m} / \mathrm{s}^{2}$. As before, the cavitation events are responsible for those weak decelerations. We recall that the expected magnitude of the deceleration is $141 \mathrm{~m} / \mathrm{s}^{2}$, close to the measured value of $140 \mathrm{~m} / \mathrm{s}^{2}$.

Figure 4.51 is a plot of the pressure in the syringe tip. A few labels are positioned on the plot to identify key features. Label 1 identifies the beginning of the syringe deceleration, creating a compressive pressure pulse in the liquid. The peak pressure resulting from the deceleration is $4.4 \mathrm{MPa}$, equal to the value predicted using acoustic theory. The pressure pulse predicted using LS-DYNA is also shown, and it is in reasonable accord with the measurements if the acceleration and velocity change 


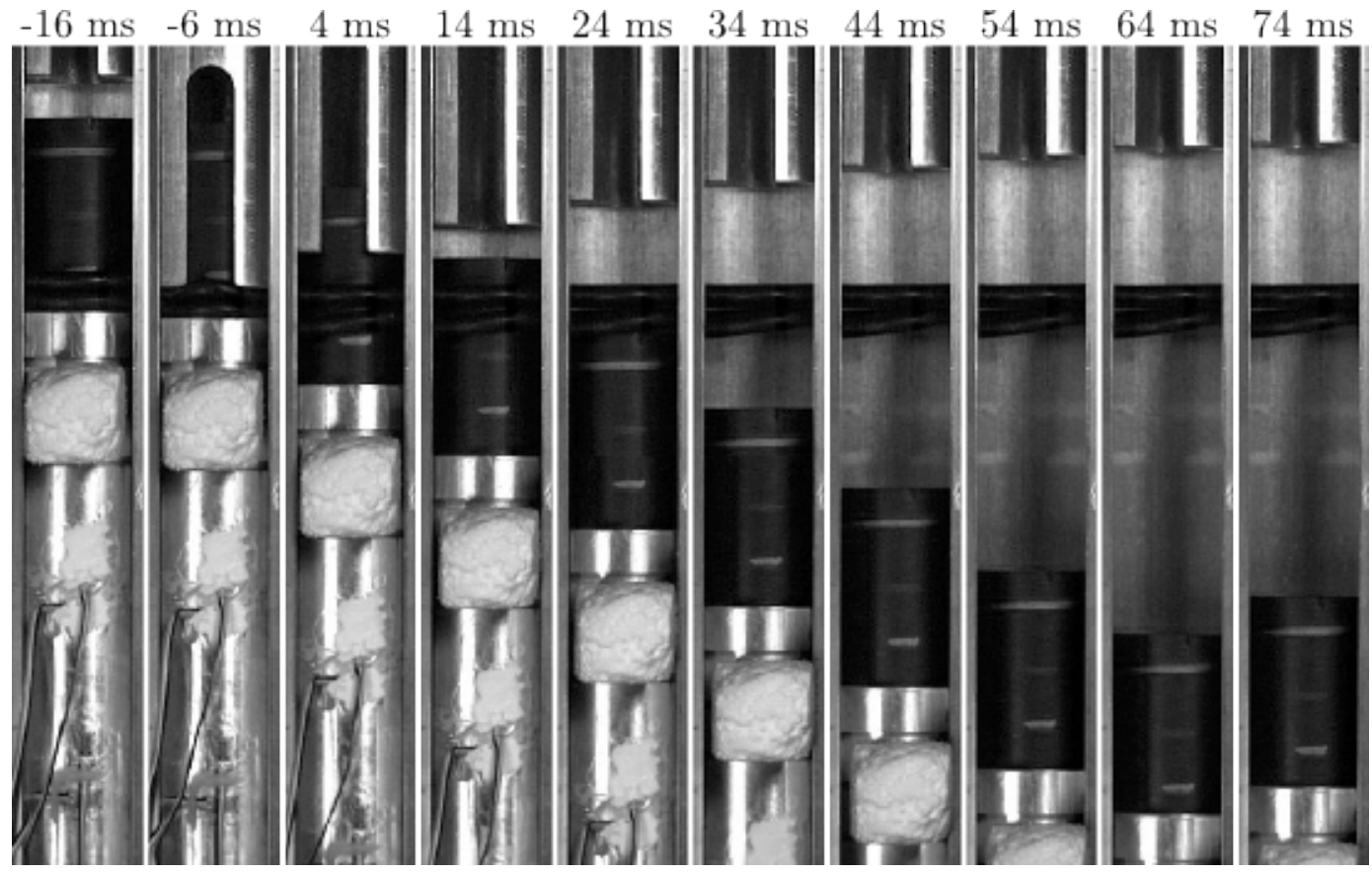

Figure 4.50: Sequence of images showing the motion of the projectile, the buffer and the syringe for case 13 of the dynamic, large-scale model autoinjector experiments (test LS-0296).

are selected to be approximately the same as in the experiments. All the simulation results shown in this subsection are for a $12,000 \mathrm{~m} / \mathrm{s}^{2}$ deceleration, and a change in velocity of $3 \mathrm{~m} / \mathrm{s}$.

The compression wave forms in the tip of the syringe and propagates in the liquid toward the buffer. The wave reflects as a tensile wave on the air-water interface located below the buffer. The reflected wave propagates toward the syringe tip and reflects as a tensile wave on the flat wall, promptly initiating distributed cavitation. Label 3 identifies the beginning of the first distributed cavitation event. The pressure is constant and approximately equal to vapor pressure throughout the cavitation event. The tensile wave continues to propagate in the water, reflects at the top end of the syringe on the air-water interface as a compression wave, and propagates toward the bottom of the syringe. Label 4 identifies the end of the first cavitation event, marked by a rapid increase in pressure due to the return of the compression wave and the collapse of cavitation bubbles.

This cycle is repeated several times, but the magnitude of the pressure pulse and the severity of the cavitation event decreases between each cycle. The decrease in severity of the cavitation event is inferred from the shortening of the time interval 


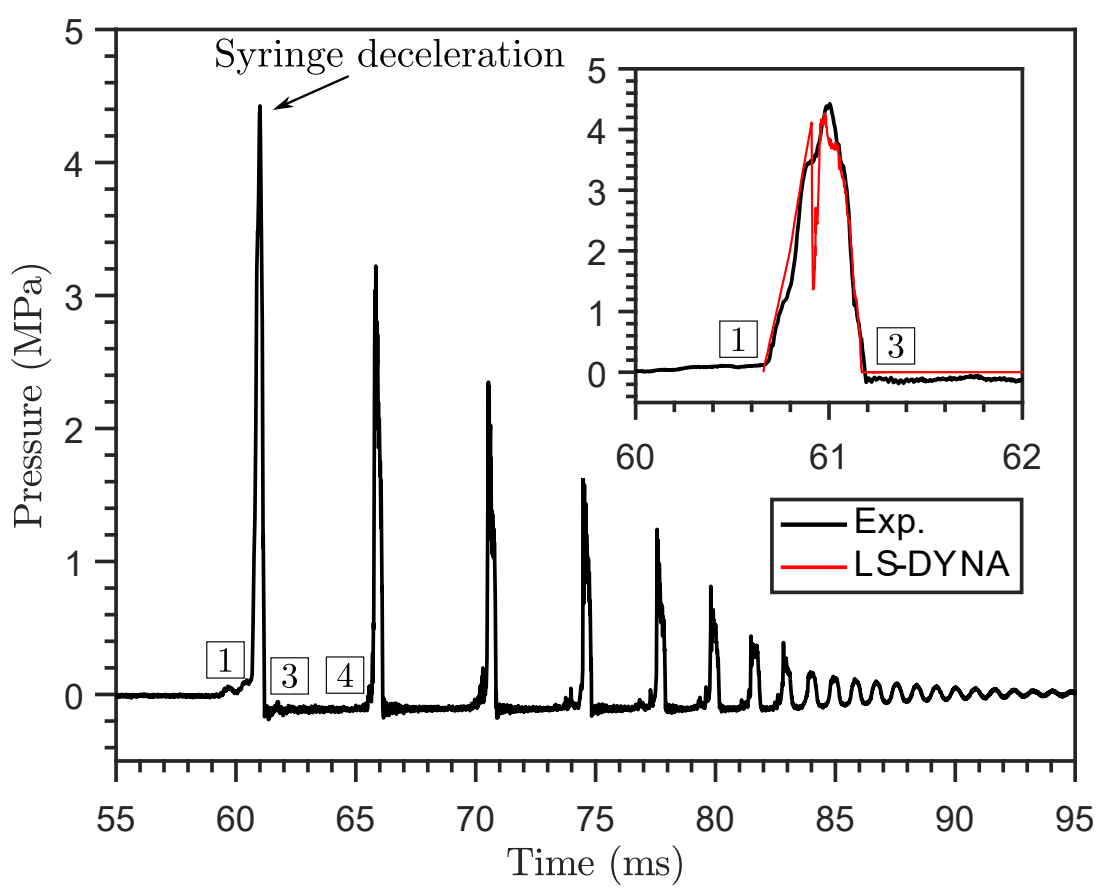

Figure 4.51: Pressure at the bottom wall for case 13 of the dynamic, large-scale model autoinjector experiments (test LS-0296).

between two successive pressure peaks, indicating an increase in the average wave speed. This, in turn, is directly correlated with a decrease of the average void fraction within the liquid column during the cavitation event (Brennen, 1995).

The hoop and axial strains on the outer surface of the syringe barrel are shown in Figure 4.52. The oblique, dashed lines in Figure 4.52 have a slope which corresponds to the Korteweg speed. The strains result from the combined effect of the hoop stress created by the pressure pulse traveling in the liquid, and the axial stress wave traveling in the syringe wall, created by the contact force between the syringe and the decelerator. The pressure and stress waves travel at different speeds and repeatedly reflect from the ends to create the rather complex strain distributions shown in Figure Figure 4.52. The boundary conditions on the water column are also different from the boundary conditions on the syringe wall. To obtain some insight as to the wave mechanics, a simplified model of the fixture and boundary conditions is considered.

The simplified schematic from Figure 4.53 illustrates the wave mechanics in the water and the syringe walls under the simplifying assumption of no radial stress waves. The schematic is in a moving frame attached to the syringe. The boundary 


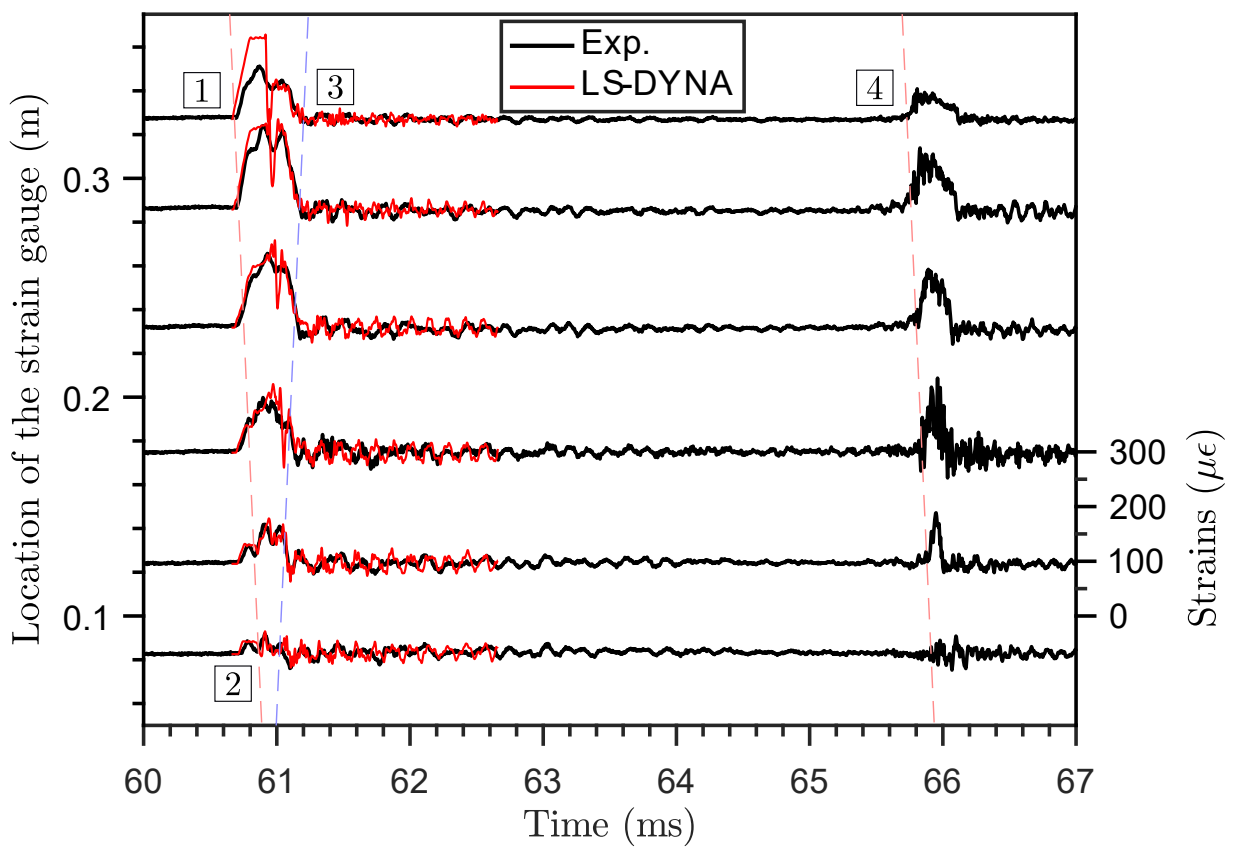

(a) Hoop strains

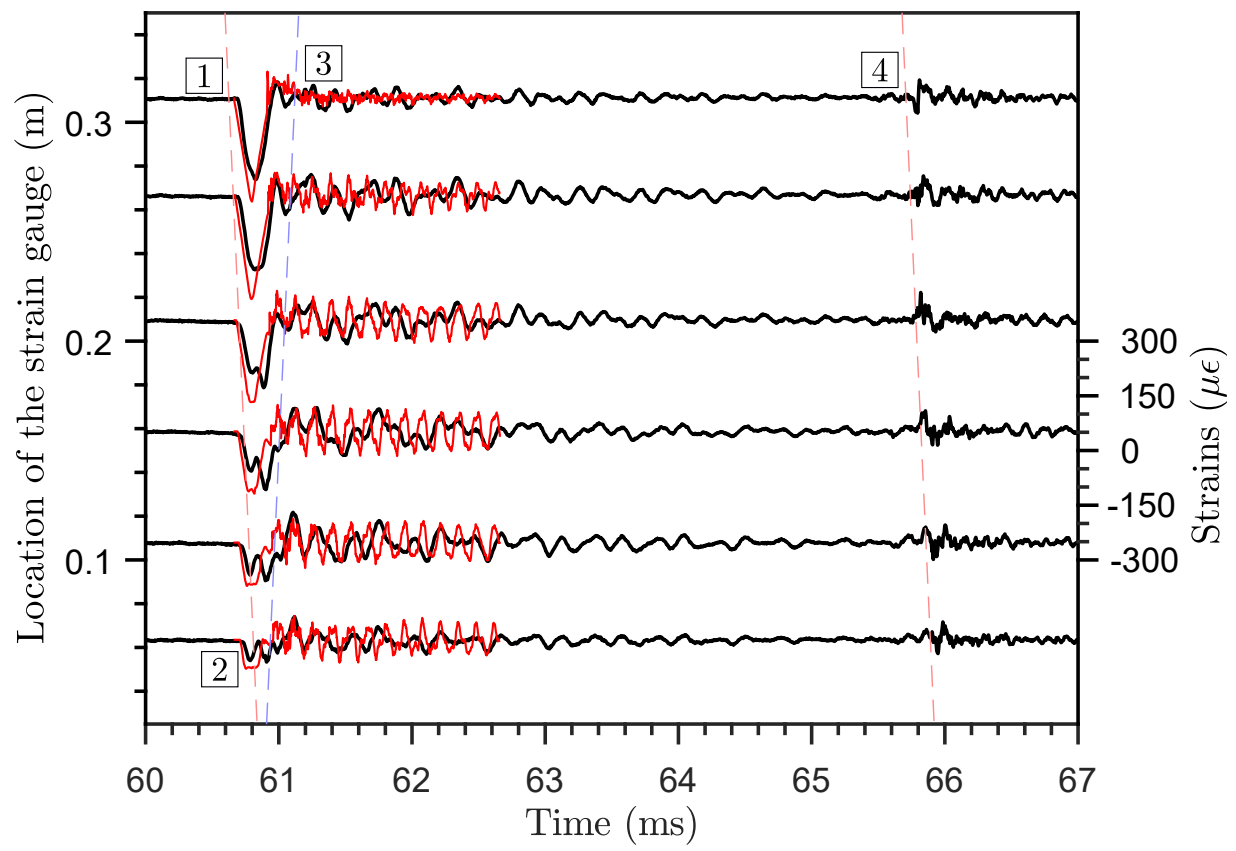

(b) Axial strains

Figure 4.52: Hoop and axial strains for case 13 of the dynamic, large-scale model autoinjector experiments (test LS-0296). 


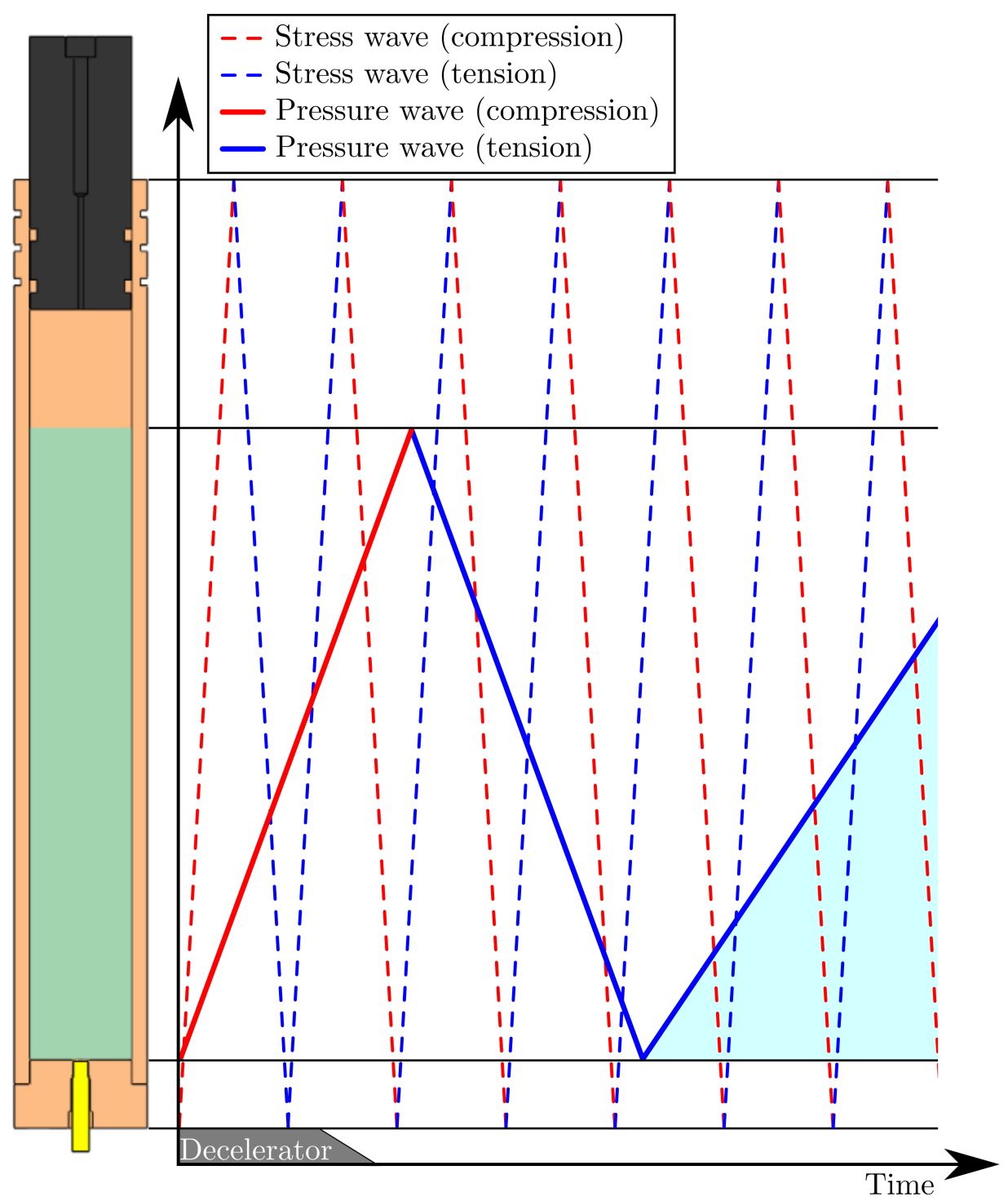

Figure 4.53: Schematic of the wave mechanics in the syringe wall and the liquid column during and after the syringe deceleration for case 13.

condition at the bottom of the syringe barrel changes over time. During the syringe deceleration, there is a contact between the decelerator and the syringe wall, resulting in the partial transmission and reflection of stress waves upon reaching the interface. The first reflection of the stress wave at the bottom wall results in no sign change of the axial stress wave. Later on there is a loss of contact between the syringe and the decelerator, and the boundary becomes stress free. The subsequent reflections of the axial stress wave on that boundary result in a sign change. Note that the boundary condition on the top end of the syringe barrel is always a stress free end, and the stress waves always changes sign upon reflection there. 
The wave mechanics in the liquid is similar to what is explained in Section 4.1. The pressure pulse does not change sign upon reflection in the tip. The pressure pulse changes sign when it reflects on the interface between the air gap and the liquid. When no air gap is present, the reflection of the pressure pulse occurs on the moving surface of the buffer; this type of reflection on a moving boundary has been explained in Section 4.2.3.

The pressure and stress waves reverberate multiple times in the system. There is constructive and destructive interference between the multiple waves. The shaded area corresponds to a region where cavitation occurs.

The hoop strains (see Figure 4.52a) primarily result from the hoop stress created by the pressure pulse forming during the rapid deceleration of the syringe (label 1). The hoop strain wave primarily travels at the Korteweg speed, but there is also a precursor wave traveling faster. Close to the top end of the tube, positive hoop strains are observed to the left of the oblique line that indicates the Korteweg speed. This is because the compressive axial stress wave, which travels in the syringe wall at approximately $5,500 \mathrm{~m} / \mathrm{s}$, creates hoop strains through the Poisson effect.

The largest hoop strains are observed close to the bottom end of the syringe. There the maximum hoop strains are approximately $150 \mu \epsilon$. The hoop strains at the top end of the tube are close to zero, a consequence of the stress free boundary condition. Label 3 indicates the beginning of a cavitation event, visible in the form of a plateau in the hoop strains. When the cavitation event ends (label 4), the pressure pulse propagating in the liquid becomes visible again, creating a positive hoop strain signal which travels at the Korteweg speed.

The LS-DYNA simulation predicts the hoop strains with reasonable accuracy. It is also possible to estimate the expected maximum hoop strains using a quasi-static thin shell theory (Equation 3.2). A 4.4 MPa pressure wave is expected to create a $194 \mu \epsilon$ hoop strain. The predicted value is of the same order as the measured value $(150 \mu \epsilon)$.

Figure $4.52 \mathrm{~b}$ is a plot of the axial strains. The axial strains are primarily created by the axial stress wave forming during the rapid deceleration of the syringe (label 1). The peak magnitude of the axial strains is approximately $-220 \mu \epsilon$, in compression, close to the tip the syringe. The axial strains close to the top end are negligible, a consequence of the stress free boundary condition. The axial strain wave is observed to travel faster than the Korteweg speed, as expected. 
The LS-DYNA simulation predicts the axial strains with reasonable accuracy. It is also possible to estimate the magnitude of the axial stress wave using Equation 2.19. Assuming a deceleration of $10,000 \mathrm{~m} / \mathrm{s}^{2}$, the axial stress wave is $\sigma_{z}=-16 \mathrm{MPa}$. Under a uniaxial stress assumption, this is expected to create $-235 \mu \epsilon$ of axial strain, close to the measured value of $-220 \mu \epsilon$.

A repeat test, referenced as test $13 \mathrm{~b}$, was performed with the clear, polycarbonate syringe. A sequence of images is shown in Figure 4.54. The buffer is shown at the top, and the flat wall of the syringe tip is shown at the bottom. Before the deceleration, the air gap and the interface between the air and the water are not well defined. Before a test the interface between the water and air is sharp and horizontal, but the transient events created by the acceleration and pressurization of the syringe result in a mixture region. ${ }^{3}$ This has not been taken into account in the numerical simulations, where a well defined air gap is assumed. This also indicates that assuming the deceleration event is entirely decoupled from the acceleration and the pressurization events is, in fact, a simplifying assumption.

The wave speed in the mixture region can be significantly different than the wave speed in pure liquid water or air. The relatively good match between the experimental and numerical results however suggests that this has negligible effects on the results.

Figure 4.54 indicates the cavitation event can be classified as distributed cavitation. Cavitation is confined to the bottom half of the syringe. The collapse of the cavitation bubbles occurs from top to bottom, resulting from the slow propagation of the compressive pressure pulse in the bubbly liquid.

\subsubsection{Case 14}

Case 14 is identical to case 13, except there is no air gap between the buffer and the liquid. The velocity of the syringe is shown in Figure 4.55, and it is not significantly different from the velocity of the syringe measured in case 13.

Figure 4.56 is a plot of the pressure in the tip of the syringe. There are significant differences between the results of cases 13 and 14. One difference is in the magnitude of the pressure pulse resulting from the large syringe deceleration, between labels 1 and 3. Without an air gap, the peak pressure is 5.7 MPa, larger than the $4.4 \mathrm{MPa}$ measured with an air gap. The explanation for the larger pressure is twofold. First,

\footnotetext{
${ }^{3}$ The results obtained with a clear syringe suggest there are Rayleigh-Taylor instabilities at the interface.
} 


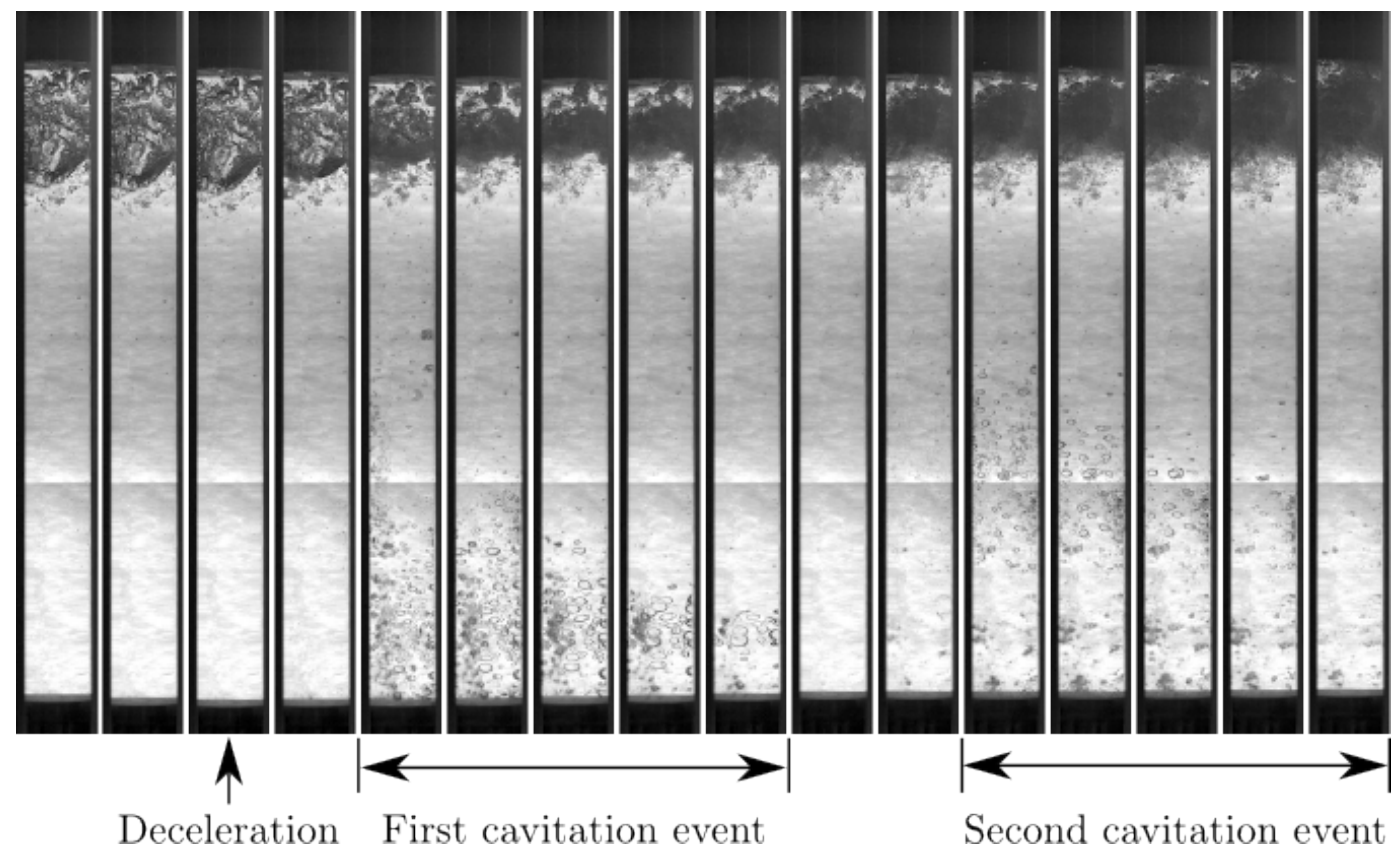

Figure 4.54: Sequence of images of the cavitation events for case $13 \mathrm{~b}$ of the dynamic, large-scale model autoinjector experiments (test LS-0417). There is $62.5 \mu$ s between successive frames.

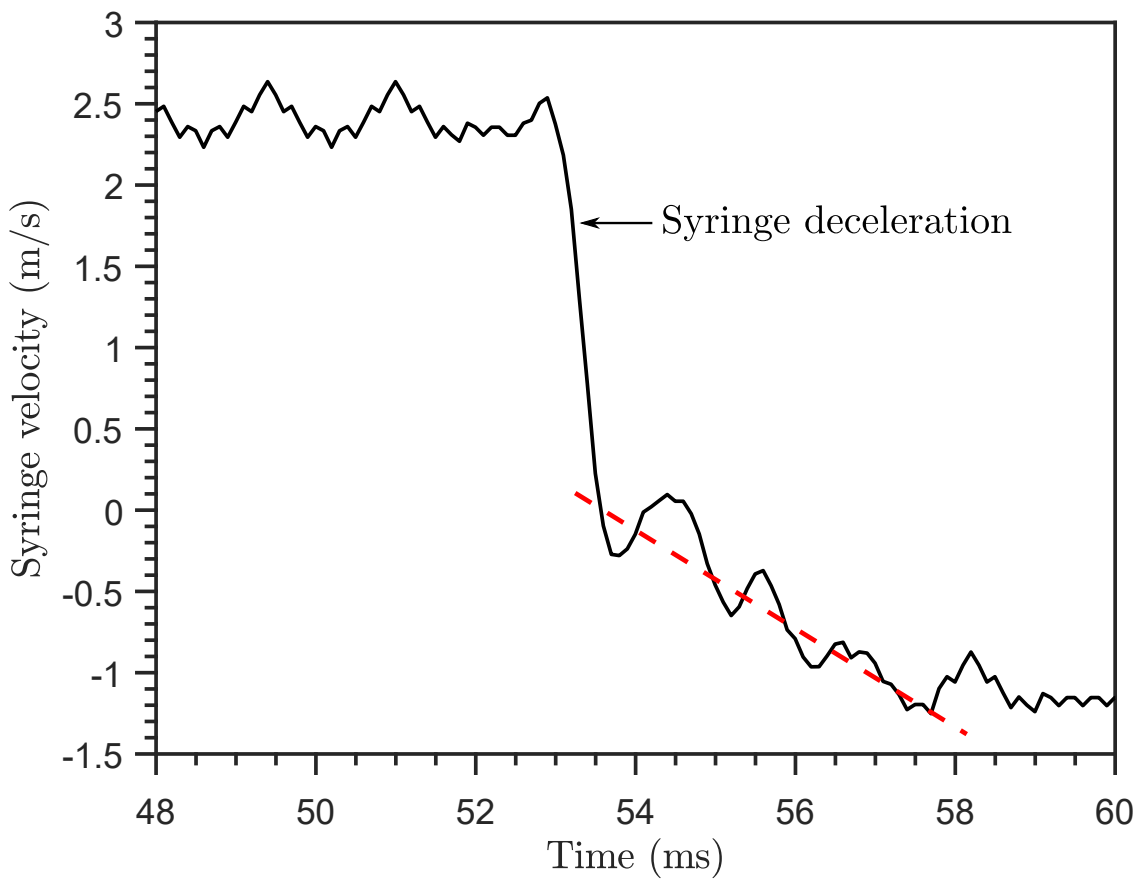

Figure 4.55: Velocity of the syringe for case 14 of the dynamic, large-scale model autoinjector experiments (test LS-0319). 


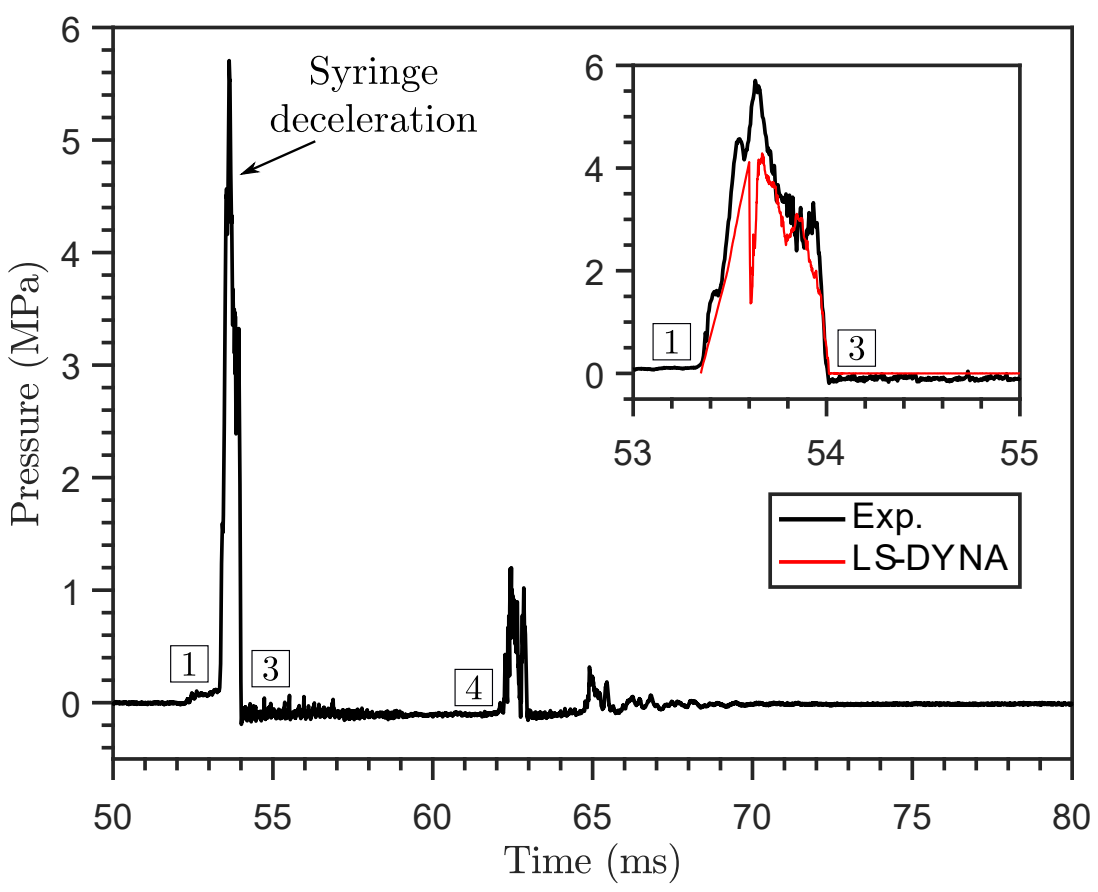

Figure 4.56: Pressure at the bottom wall for case 14 of the dynamic, large-scale model autoinjector experiments (test LS-0319).

the reflection of the pressure wave upon reaching the top of the water column occurs on the buffer rather than on a free surface. The implications of this were described earlier. Second, the direct contact between the buffer and the liquid results in the production of compression waves due to the inertia of the buffer. This is also the case when there is an air gap, but the damping effect of the air gap reduces the magnitude of those compression waves.

Several tests were performed with and without an air gap to investigate the effect of air gap size on peak pressure. A box plot of the peak pressure as a function of air gap size is shown in Figure 4.57. When an air gap is present, the peak pressure is relatively insensitive to the air gap size. This is because the pressure waves creating the peak magnitude of pressure originate from the syringe tip rather than the end of the tube closed with the buffer, and the peak pressure is created before the pressure waves can complete one round trip between the bottom and the top end of the syringe. The peak pressure without an air gap is consistently larger, however, than the peak pressure with an air gap due to the compression waves created as a result of the inertia of the buffer, as explained above. The variability in the results 


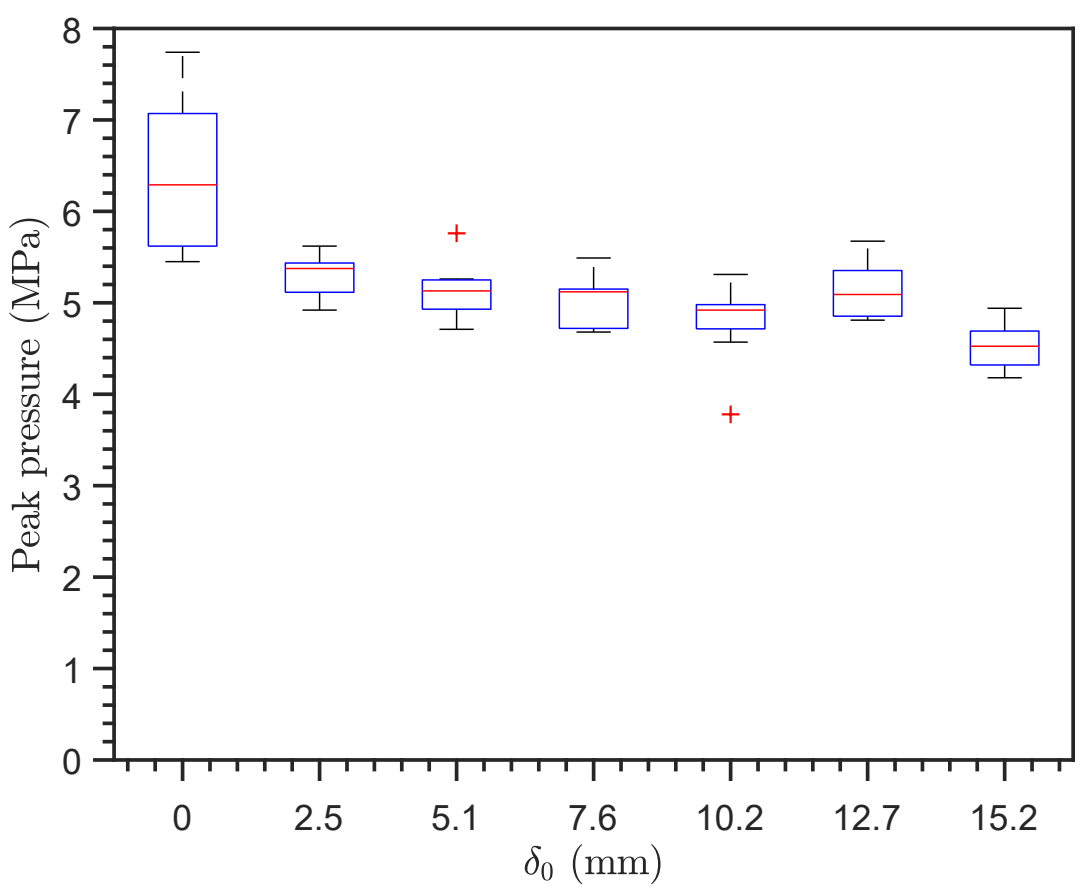

Figure 4.57: Box plot of the peak pressure at the flat wall of the tip during the rapid deceleration of the syringe in the dynamic, large-scale model autoinjector experiments.

is created by variations in the deceleration profile of the syringe, and variability in the buffer motion.

Another difference between cases 13 and 14 is in the number of repeated cavitation events. Only 1 long and 1 short cavitation events are observed when no air gap is present. Without an air gap, the motion of the liquid column is strongly coupled to the motion of the buffer; the motion of the liquid necessarily results in the motion of the buffer. This stronger coupling creates more dissipation due to friction between the buffer and the syringe. This is not the case when there is an air gap because there is room for the liquid to move without creating significant motion of the buffer.

The hoop and axial strains are not shown. This is because both the hoop and axial strains are similar to what was shown for case 13. The LS-DYNA results (not shown) are in accord with the measurements.

\subsubsection{Case 15}

Case 15 is identical to case 13 , except the syringe is terminated with a conical tip instead of a flat wall. The results of cases 13 and 15 differ substantially. We 


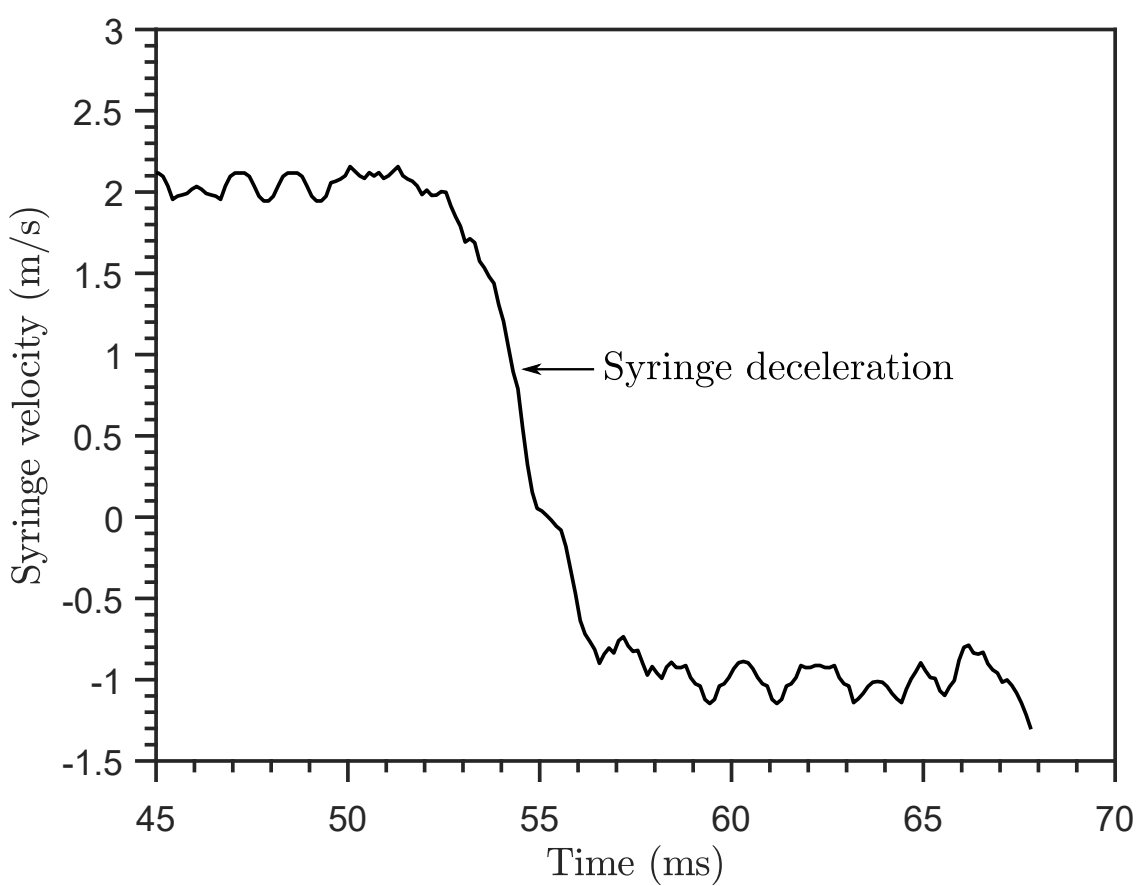

Figure 4.58: Velocity of the syringe for case 15 of the dynamic, large-scale model autoinjector experiments (test LS-0227).

recall that the geometry of the decelerator used with a flat tip is different from the geometry of the decelerator used with a conical tip (see Figure 4.18). The different geometry of the decelerator accounts for the differences between cases 13 and 15 . The deceleration profile of the syringe is different for both cases, and this makes it difficult to identify the effect of the cone itself on the transient events.

The velocity of the syringe is shown in Figure 4.58. The magnitude of the deceleration is only $1,000 \mathrm{~m} / \mathrm{s}^{2}$, one order of magnitude less than for cases 13 and 14 . This is a deceleration which falls in regime 2 (see Chapter 2) for both the liquid column and the syringe wall. With a flat tip, the deceleration of the liquid column was in regime 1 .

Equation 2.20 predicts a peak pressure of $0.6 \mathrm{MPa}$ for a deceleration of $1,000 \mathrm{~m} / \mathrm{s}^{2}$. The expected peak pressure is one order of magnitude smaller than the peak pressure measured with the flat tip. The measured pressure is shown in Figure 4.59. The peak magnitude is approximately $0.5 \mathrm{MPa}$, close to the predicted value. The pressure trace indicates there is no cavitation in the liquid. This is because the magnitude of the tensile pressure waves created by the reflection of this weak transient event 


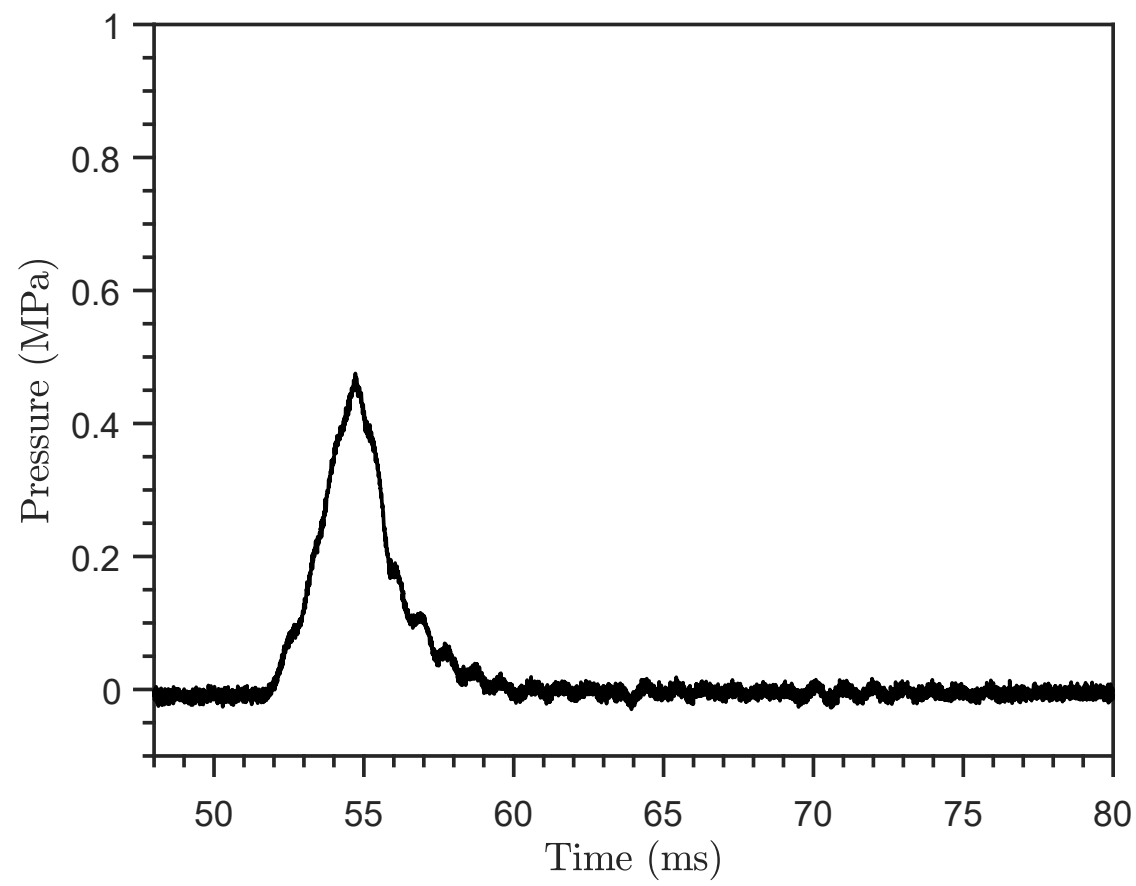

Figure 4.59: Pressure at the bottom wall for case 15 of the dynamic, large-scale model autoinjector experiments (test LS-0227).

from the free surface is not sufficiently large to reduce the liquid pressure to vapor pressure.

Similar to the pressure and deceleration, the magnitude of the hoop and axial strains is one order of magnitude less than the strains measured for cases 13 and 14. The peak magnitude of the strains is 25-30 $\mu \epsilon$. The hoop and axial strains are not shown because it is not possible to learn anything new about the transient events from those signals.

The peak pressure measured in the cone is close to the value predicted using Equation 2.20. This indicates that shock focusing does not occur in the cone. This is expected because the rise time of the pressure pulse is over $2 \mathrm{~ms}$, significantly larger than the $25 \mu \mathrm{s}$ acoustic transit time of the pressure waves within the cone.

\subsubsection{Summary of Cases 13 to 15}

The pressure and stress transients inside a water-filled syringe which is rapidly decelerated were studied experimentally and numerically. Three cases were reported and analyzed. 
Case 13 has a large air gap between the buffer and the liquid, and it uses a syringe terminated with a flat tip. The deceleration of the syringe is large, approximately $10,000 \mathrm{~m} / \mathrm{s}^{2}$. The strains resulting from this transient event are complex due to the interaction of an axial stress wave traveling in the syringe wall at $5,500 \mathrm{~m} / \mathrm{s}$ with a pressure pulse traveling in the liquid at $1,350 \mathrm{~m} / \mathrm{s}$.

Case 14 is identical to case 13, except there is no air gap between the buffer and the liquid. This results in a stronger coupling between the liquid and the buffer motion. The peak magnitude of the pressure pulse in the liquid is larger than for case 13, but it is also attenuated more rapidly.

Case 15 is identical to case 13 , except that a syringe terminated with a conical tip is used. Unfortunately, the magnitude of the deceleration created by the decelerator is one order of magnitude less than for cases 13 and 14. The deceleration is not impulsive, and the transient events are weak. Shock focusing can't occur due to the long rise time of the pressure wave.

LS-DYNA was successful at predicting the magnitude of the pressure and strains in the system as long as the appropriate magnitude of velocity and deceleration is prescribed. Furthermore, the simple estimates developed in Chapter 2 proved to predict reasonably well the peak pressure and strains.

Designing a second version of this experiment should be considered in the future. Investigating the effect of the cone on the pressure and stress transients requires the magnitude of the deceleration to be approximately the same across all cases. This necessitates a revision of the design of the decelerators and/or the outside geometry of the syringe tip. 


\author{
Chapter 5
}

\title{
NUMERICAL SIMULATIONS OF SHOCK FOCUSING AND SYRINGE STRESSES
}

Numerical simulations are used in this chapter to examine the details of shock focusing and the stresses created in the syringe wall during the transient events which can occur in an autoinjector device. The purpose of the first section on shock focusing is to determine under what conditions shock focusing can occur in a syringe, and to quantify the magnitude of pressure wave amplification inside the cone. The purpose of the second section on the syringe wall stresses is to determine which transient event(s), among events 1,2, and 3 from Chapter 2, can account for the consistent cone failures observed by Amgen during clinical trials (see Chapter 1).

\subsection{Shock Focusing}

Results from Chapters 3 and 4 have shown that sharp pressure waves can form inside the syringe of an autoinjector device. On multiple occasions it was mentioned that sharp pressure waves can be amplified within the cone of the syringe as a result of shock focusing. The justification of this statement is the subject of this section. A sharp pressure wave, so far, was assumed to have a rise time which is less than or approximately equal to the acoustic transit time of the pressure waves within the cone. The acoustic transit time is defined as the time needed for pressure waves to travel inside the cone $\left(D_{b, i} / c_{l}\right)$.

The physical mechanism resulting in the amplification of pressure waves within the cone is reviewed. Then, the effect of three key parameters on the magnitude of shock focusing is investigated:

1. the half-angle of the cone terminating the syringe;

2. the rise time or "sharpness" of the incident pressure waves entering the cone;

3. the acoustic impedance of the syringe wall.

The last subsection is on shock focusing in a real syringe geometry. In most pre-filled syringes used with autoinjector devices the half-angle of the cone is not constant; 


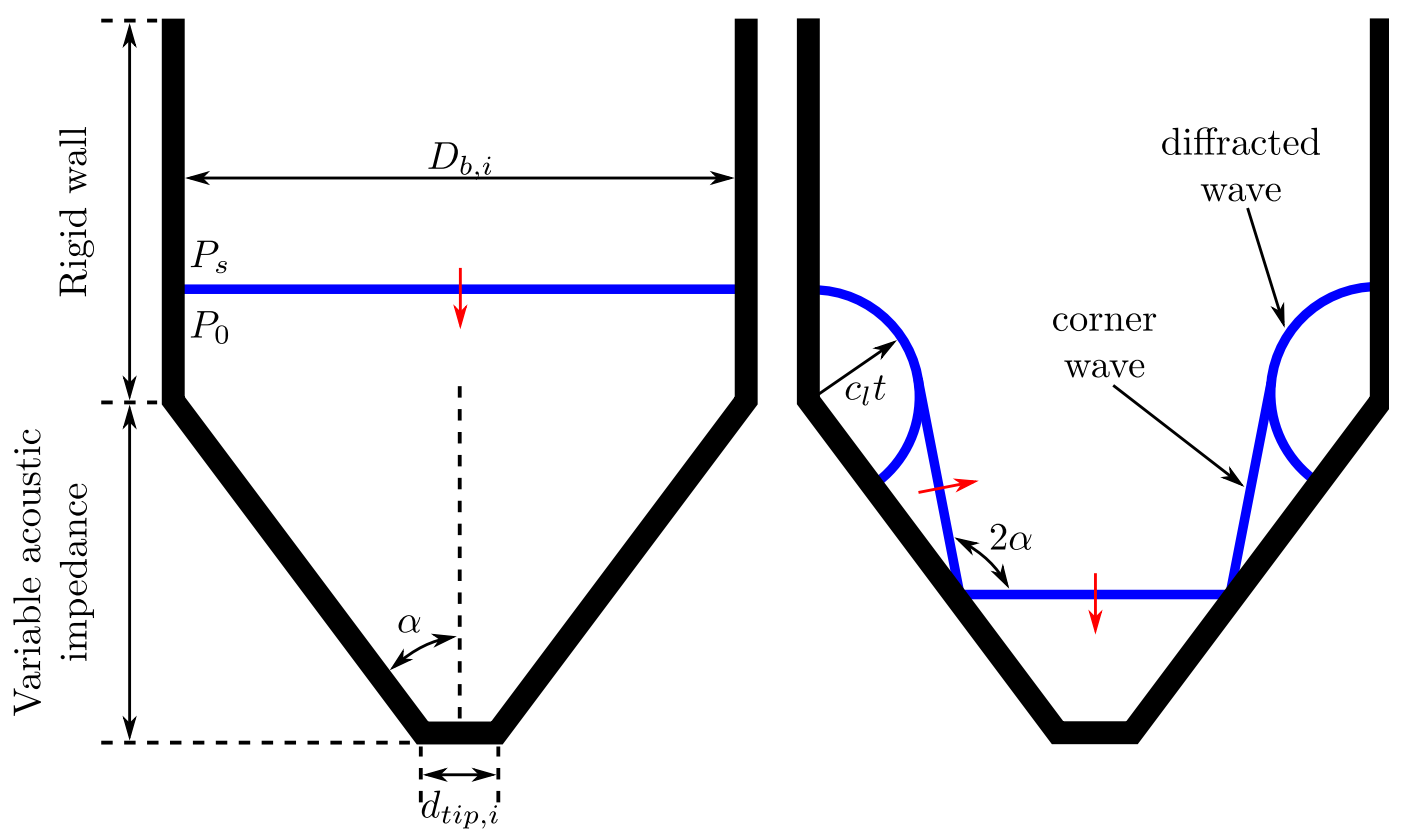

Figure 5.1: Cross-sectional view of the geometry used in the numerical simulations on shock focusing in a straight cone. The left-hand side is the initial configuration, and the right-hand side depicts the situation after the pressure wave has entered the cone.

it is a function of axial location. This can have an effect on the magnitude of the amplification occurring inside the cone.

\subsubsection{Numerical Model}

Figure 5.1 is a cross-sectional view of the geometry used in LS-DYNA (Hallquist, 2016) to predict the amplification of pressure waves in a straight cone. The left-hand side of Figure 5.1 represents the initial configuration: a pressure wave is traveling downward in a syringe-like geometry filled with water. For simplicity, the syringe barrel is terminated with a truncated straight cone. The diameter of the barrel is $D_{b, i}$, the diameter of the tip is $d_{t i p, i}$, and the half-angle of the cone is $\alpha$. The pressure ahead and behind the incident pressure wave is respectively $P_{0}$ and $P_{s}$. The pressure wave has a sufficiently low amplitude (i.e., $\left.\left(P_{s}-P_{0}\right) \ll \rho_{l} c_{l}^{2}\right)$ that it can be considered an acoustic disturbance traveling at sound speed $c_{l}$.

The wall of the syringe barrel and cone has a finite thickness in the numerical model. A non-reflecting boundary condition is applied on the outer boundary of the wall, effectively making the wall infinitely thick. For this reason, no wall thickness is indicated in Figure 5.1. Although this geometry does not replicate the finite wall 
thickness of the syringe, it avoids the complication associated with wave mechanics in the shell and enables concentrating on the focusing event alone.

The wall of the syringe barrel is assumed to be rigid (i.e., it has an infinite acoustic impedance). This is to avoid fluid-structure interaction between the pressure wave and the barrel. Making the barrel rigid results in the pressure wave propagating unaltered inside the barrel, making it easier to isolate the effect of the cone on the pressure field. The wall of the cone terminating the syringe is modeled as rigid in Sections 5.1.2 to 5.1.4, and modeled using a linear-elastic constitutive model in Section 5.1.5. The Poisson's ratio is $v=0.3$, and the acoustic impedance of the cone is defined as $Z_{s}=\rho_{s} \sqrt{E / \rho_{s}}$. This definition of the acoustic impedance uses the 1D definition of sound speed in solids. The acoustic impedance of the cone varies parametrically in the numerical simulations of Section 5.1.5, and values of $Z_{s}$ are indicated for each case discussed. Note that $\rho_{s}$ is constant and equal to $2230 \mathrm{~kg} / \mathrm{m}^{3}$, and the acoustic impedance is modified by changing the value of $E$.

An acoustic formulation is used to model the liquid water. The same parameters as in Chapter 4 are used $\left(\rho_{l}=1000 \mathrm{~kg} / \mathrm{m}^{3}\right.$, and $\left.c_{l}=1500 \mathrm{~m} / \mathrm{s}\right)$, resulting in an acoustic impedance $Z_{l}=1.5 \mathrm{MPa} \cdot \mathrm{s} / \mathrm{m}$. A surface-to-surface boundary condition is used between the liquid water and the solid wall. This boundary condition allows sliding between the water and the solid wall.

All simulations are performed in 2D axisymmetric mode, and the flow solver is inviscid. The contraction ratio $D_{b, i} / d_{t i p, i}$ is equal to 9 across all simulations (the physical dimension of $D_{b, i}$ is $40 \mathrm{~cm}$, similar to the $38.1 \mathrm{~cm}$ inner diameter of the large scale syringes used in Chapter 4 ). The computational grid is constructed using 750,000 to 2,250,000 shell elements. The number of shell elements depends on the half-angle, which affects the length of the cone. Note that further refinement of the numerical grid does not affect the results significantly: a grid that contains 4 times as many computational elements results in variations of approximately $10 \%$ of the peak pressure in the tip.

\subsubsection{Shock Focusing in a Straight Cone}

The focusing of shock waves in a straight cone or a wedge has been thoroughly studied in the context of gasdynamics; some important work in this area has been performed here at Caltech, including the work of Bond et al. (2009); Russel (1967); Setchell, Storm, and Sturtevant (1972). The monograph of Apazidis and Eliasson (2019) should be consulted for a thorough introduction to shock focusing and past 
research in this field. In most experimental studies, a shock tube (i.e., the working fluid is a gas) was used to create a shock wave upstream of a straight cone or a wedge. The Mach number of the incident pressure wave was typically greater than 1.5, and the shock focusing effect resulted in the shock becoming moderate to strong. Nonlinear effects are important in those studies, and real gas effects were often observed. Most studies have investigated only one half-angle and have focused on understanding the process through which the shock wave is amplified as it propagates toward the apex of the cone rather than performing parametric studies. One important finding confirmed by past studies is that the amplification mechanism of the pressure on the axis of the cone or wedge is not a continuous process: the shock is alternately strengthened by the repeated crossing of the Mach reflections across the diameter of the cone or wedge, a concept which is clearly discussed by Setchell, Storm, and Sturtevant (1972).

This process is partially illustrated in the right-hand side of Figure 5.1 for acoustic waves. The schematic illustrates the physical situation after the incident wave has entered the straight cone: a corner wave and a diffracted wave have formed. The corner wave results from the reflection of the incident wave on the oblique wall of the cone. Because this is an acoustic problem, the reflection of the incident wave on the wall is regular (Apazidis and Eliasson, 2019; Courant and Friedrichs, 1976): the angle between the wall and the incident wave is the same as the angle between the wall and the corner wave. The diffracted wave forms as a result of the wave turning the corner between the syringe barrel and the cone.

The corner and the diffracted waves converge toward the axis of symmetry of the syringe. Upon reaching the axis of symmetry they reflect and propagate toward the wall of the cone. This cycle is repeated and creates stepwise changes in pressure on the axis of symmetry. The convergence of the waves toward the axis means that some of the energy transported by the waves is focused on the axis of symmetry; the energy is concentrated into a volume of decreasing size. Although it is typical to think of pressure as a force per unit area $\left(\mathrm{N} / \mathrm{m}^{2}\right)$, it is also possible to think of pressure as an energy density $\left(\mathrm{J} / \mathrm{m}^{3}\right)$. It then becomes easier to understand the amplification mechanism: the focusing of the energy transported by the pressure waves on the axis of symmetry increases the energy density. This, in turn, translates into a substantial increase in liquid pressure.

There are important differences between the present and previous studies on shock focusing. In the present work, the pressure waves entering the cone are acoustic 
(the Mach number is approximately 1) rather than moderate to strong shocks, and the waves propagate in liquid water rather than a gas. The study aims at quantifying the magnitude of pressure wave amplification at the tip of the cone as a function of the half-angle of the cone, the rise time of the pressure pulse, and the acoustic impedance of the cone.

The rise time of the pressure pulse is a parameter which could influence the magnitude of amplification within the cone. Pressure pulses with a slow rise time are expected to experience only a small amount of amplification within the cone. The preliminary study of Johnson et al. (2014) on the focusing of stress waves in a cone has shown that stress waves with an increasing ramp tend to be less amplified than top-hat types of stress waves. However, only one slope for the ramp was investigated. In the context of autoinjector devices, it is important to quantify the effect of the rise time of the pressure pulse on the focusing effect because the rise time of pulses created in syringes is largely affected by the size of the air gap located between the plunger-stopper and the liquid, as described in Chapters 3 and 4.

Eliasson et al. (2010) and Wang and Eliasson (2012) performed an experimental study on the focusing of shock waves inside structures with varying acoustic impedance. The geometries, a double wedge and a logarithmic spiral, were filled with water. Both studies indicate the focusing effect is affected by the acoustic impedance of the cone or spiral, but the effect of the acoustic impedance on the peak pressure was not quantified. The acoustic impedance of the confining geometry affects the proportion of the incident wave which is reflected in the liquid and transmitted in the solid. Physical intuition suggests that cones with rigid walls rather than soft walls could create more amplification of the pressure. In the context of autoinjector devices, this has important implication for glass and plastic syringes, two materials with dissimilar acoustic impedances. The acoustic impedance of glass syringes is approximately 4.7 times larger than the acoustic impedance of plastic syringes, suggesting the focusing effect could be significantly different in glass and plastic syringes.

The shock focusing process in a straight cone filled with water is illustrated in Figure 5.2, a sequence of images obtained using LS-DYNA. The results shown are for a half-angle of 30 degrees, an infinitely sharp pressure wave, and a rigid cone. The left-hand side is a pseudo-schlieren which makes it possible to track the pressure waves in the cone. The right-hand side is the pressure field. Note that the incident pressure wave, which is initially infinitely sharp, does not appear to be infinitely 


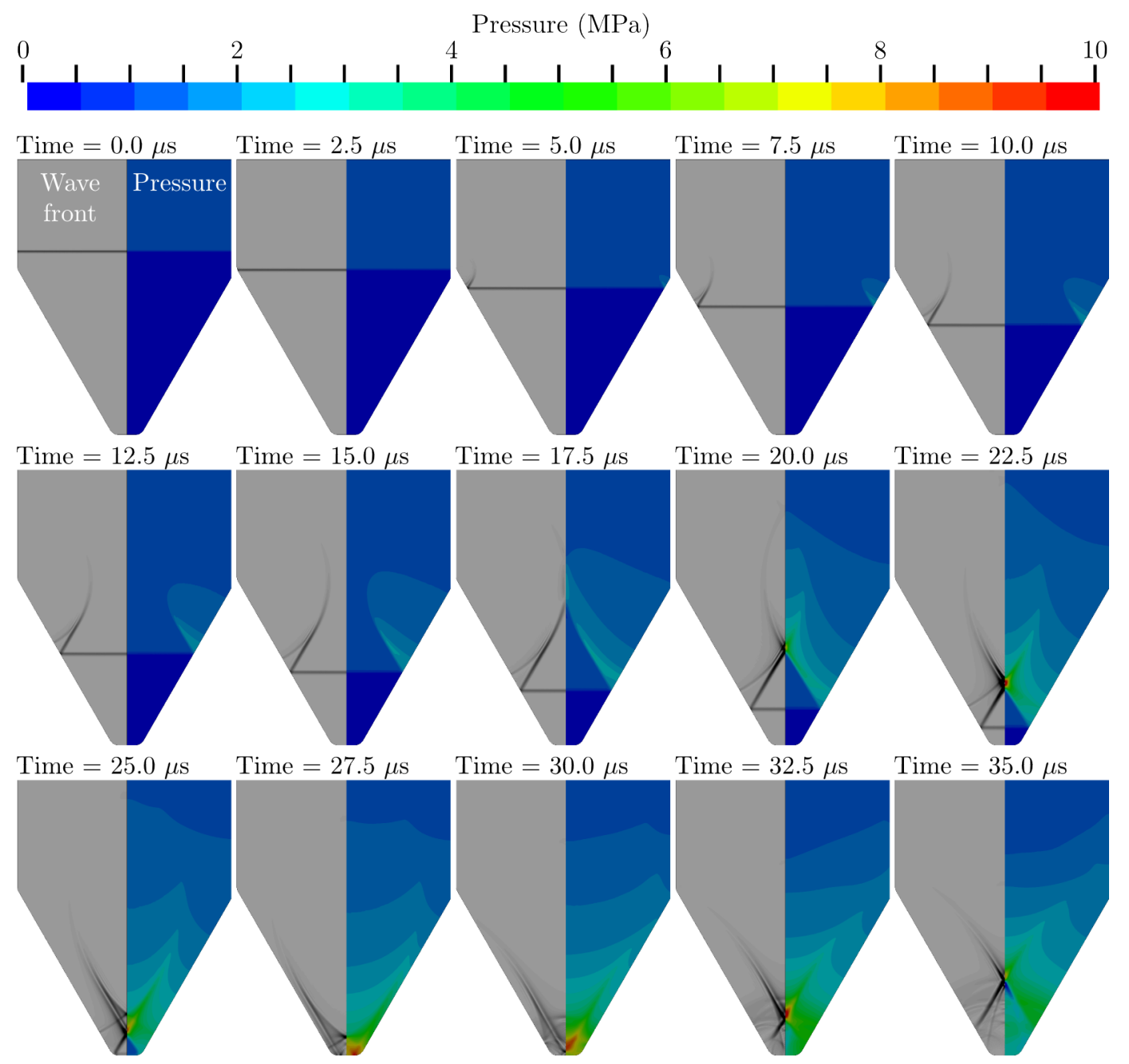

Figure 5.2: Amplification of a sharp pressure wave entering a straight, rigid cone with a half-angle of 30 degrees. The left-hand side of each frame is a pseudoschlieren, and the right-hand side is the pressure field.

sharp in Figure 5.2, but instead has a finite thickness and rise time. This results from numerical dissipation due to the low order of the numerical methods in LS-DYNA. Initially, the pressure wave is above the corner located at the junction between the syringe barrel and the cone. The pressure ahead of the pressure wave is $0 \mathrm{MPa}$, and the pressure behind is $1 \mathrm{MPa}$. After $5.0 \mu \mathrm{s}$, the incident pressure wave enters the cone, and a corner wave and a diffracted wave start forming. The propagation of those waves is not purely in the axial direction. The radial component of motion makes the corner wave and the diffracted wave reverberate in the radial direction within the cone. 


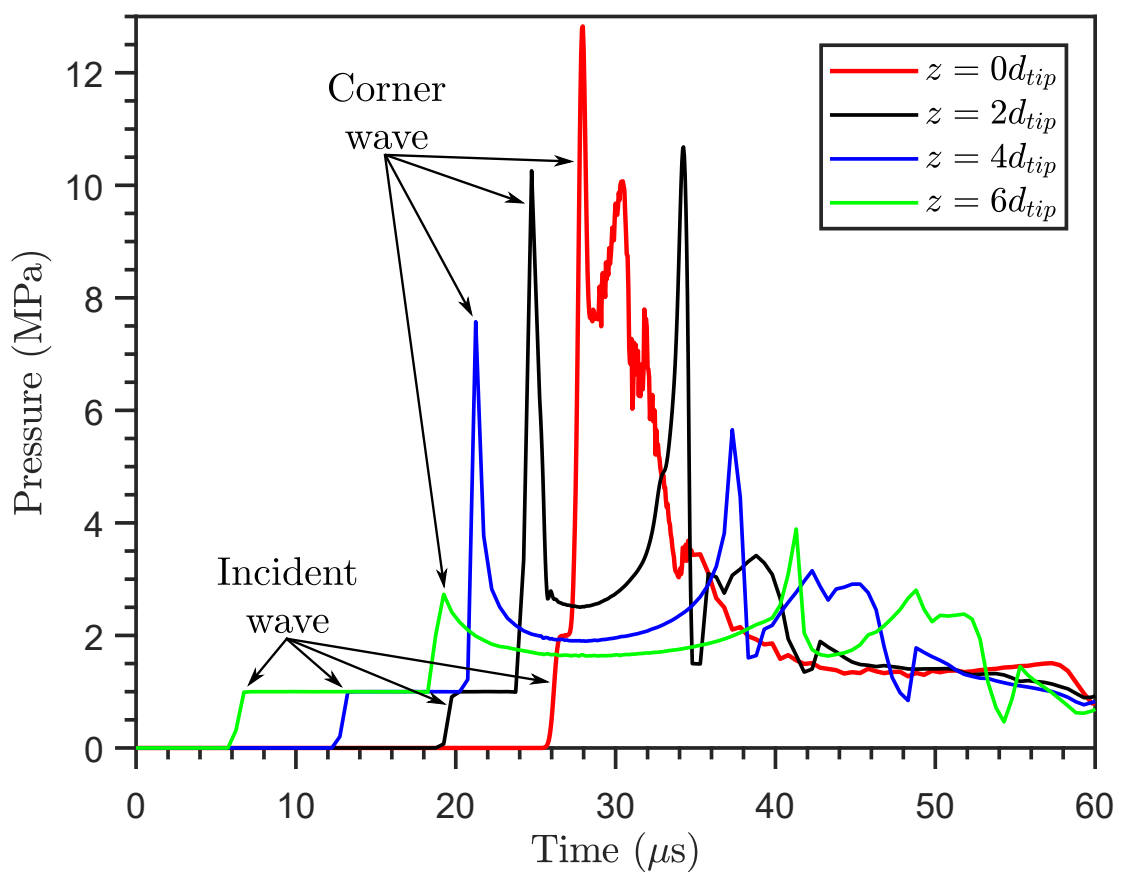

Figure 5.3: Pressure history on the cone axis and the tip wall for a sharp pressure wave entering a straight, rigid cone with a half-angle of 30 degrees.

From $5.0 \mu$ s to $15.0 \mu$ s the corner wave and the diffracted wave converge on the axis of symmetry. The magnitude of those waves increases as this happens, resulting in a larger pressure increase upon their passage. After $17.5 \mu$ s the corner wave and the diffracted wave reach the centerline of the cone and reflect. The corner wave then consists of two approximately straight segments. The radial component of motion of those two segments is in opposite directions. The cone has the effect of "folding" the incident wave on itself within the cone.

The axial progression of the incident and the corner waves toward the tip of the cone is uninterrupted. The pressure waves are focused into the tip between $27.5 \mu \mathrm{s}$ and $30 \mu \mathrm{s}$, resulting in a peak pressure in the tip that is approximately $12.5 \mathrm{MPa}$, or 12.5 times the initial magnitude of the incident wave. Following this, the pressure waves still exhibit a radial motion, but the axial direction of travel is reversed. The pressure waves propagate away from the tip, toward the top of the syringe, and the pressure in the tip decays.

The evolution of pressure at 4 stations located on the axis of symmetry is shown in Figure 5.3. The stations are positioned at $z=0 d_{t i p}$ (at the tip wall) and upstream of the tip wall at $z=2,4$ and 6 tip diameters. The results indicate the pressure increase 
on the axis of symmetry is not continuous, as expected: a first pressure increase occurs upon arrival of the incident wave, and a second pressure increase occurs when the corner wave converges on the axis of symmetry. This is the same behavior which was observed in previous research studies (Bond et al., 2009; Setchell, Storm, and Sturtevant, 1972). The pressure history also indicates the pressure in the vicinity of the tip does not remain large for a very long time: the pressure is greater than $5 \mathrm{MPa}$ for approximately $5.4 \mu \mathrm{s}$, and above $1 \mathrm{MPa}$, the magnitude of the incident wave, for approximately $33 \mu \mathrm{s}$.

\subsubsection{Effect of the Half-Angle}

The example discussed above demonstrates that substantial amplification of the incident pressure wave is possible within the cone. The magnitude of the amplification is expected to depend on the half-angle of the cone. This dependence is investigated in this subsection. All the numerical simulations are initialized with an infinitely sharp pressure wave, and the cone is rigid.

$\hat{P}_{t i p}$ is defined as the spatial average of the pressure applied on the flat wall of the tip which has a diameter $d_{t i p, i}$ (see Figure 5.1). The maximum value of $\hat{P}_{t i p}$ throughout an entire numerical simulation is used to define an amplification factor $\lambda$ :

$$
\lambda=\frac{\max \left\{\hat{P}_{t i p}\right\}}{P_{s}} .
$$

The amplification factor $\lambda$ quantifies the amplifying effect of the cone on the magnitude of the incident pressure wave.

The results for a few numerical simulations are shown in Figure 5.4. Note that a logarithmic scale is used for the vertical axis. The case $\alpha=90$ degrees corresponds to the situation where no cone is present, and the syringe is terminated with a flat wall perpendicular to the axis of symmetry of the syringe. Acoustic theory (see Appendix A) predicts the amplification factor should be $\lambda=2$ for a half-angle of 90 degrees. This is because no corner wave or diffracted wave form, and the incident wave is entirely reflected by the flat rigid wall, resulting in the doubling of the pressure in the vicinity of the wall upon reflection. LS-DYNA correctly predicts $\lambda=2$ for a half-angle of 90 degrees.

The numerical simulations indicate the amplification factor increases as the halfangle of the cone is reduced. This means that a deeper cone which converges at a shallower angle tends to focus more energy at its tip. The exponential relation between $\lambda$ and $\alpha$ is expected to depend on the contraction ratio $D_{b, i} / d_{t i p, i}$. Physical 


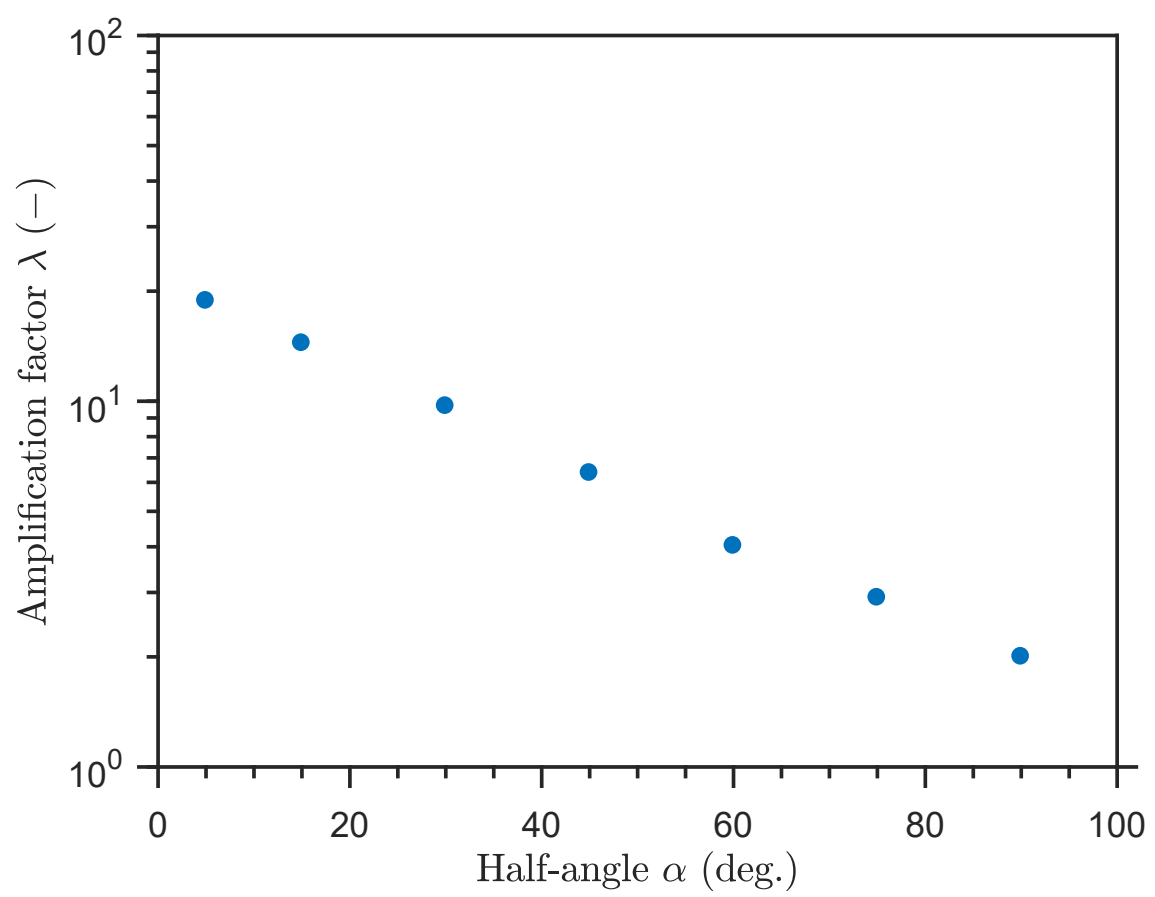

Figure 5.4: Evolution of the amplification factor as a function of the half-angle $\alpha$. The results are for a rigid cone and an initially sharp pressure wave.

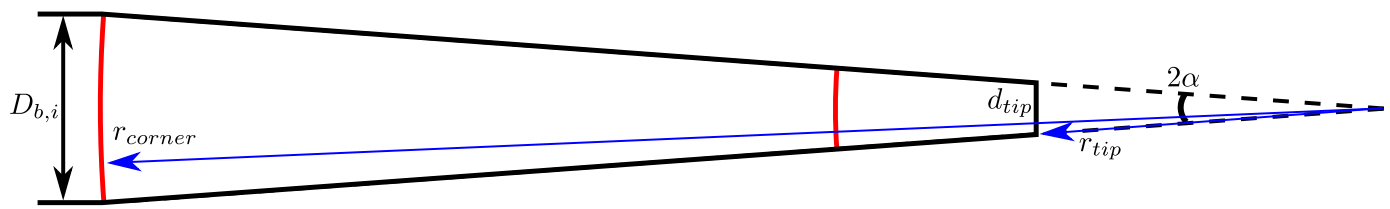

Figure 5.5: Spherical convergence of an acoustic wave.

intuition suggests that larger contraction ratios should result in a larger amplification of the pressure waves. This has been confirmed through numerical simulations, but the results are not reported.

It is also possible to investigate the limit of $\alpha$ going to 0 . Because the ratio $D_{b, i} / d_{t i p, i}$ is constant across all simulations, decreasing $\alpha$ results in the cone becoming deeper. For a very elongated cone, the propagation of the incident wave into the cone is similar to the spherical convergence of an acoustic wave. This is illustrated in Figure 5.5, where the convergence of a segment of a spherical wave inside a cone is represented. The curvature of the wave is negligible if $r_{c o r n e r} / D_{b, i}$ and $r_{t i p} / d_{t i p_{i}}$ are small, which is the case in the limit of $\alpha$ which tends to zero. This explains why the current problem, where the wave front is initially straight, can be approximated by a spherical wave. 
The magnitude of a spherically converging acoustic wave is inversely proportional to the radius of the wave (Pierce, 1989; Whitham, 1999): $\Delta P \sim 1 / r$. In the present case:

$$
\frac{r_{\text {corner }}}{r_{\text {tip }}}=\frac{D_{b, i}}{d_{\text {tip }, i}}=9 .
$$

Therefore the magnitude of the spherically converging wave is expected to increase by a factor of 9 between the corner of the cone and the tip of the cone. Doubling of the spherically converging wave occurs upon reflection on the wall of the tip; the peak pressure at the wall is therefore expected to be 18 times larger than the magnitude of the incident pressure wave. This is in good agreement with the amplification factor for a half-angle of 5 degrees, which is equal to 18.8. This result also indicates that increasing the ratio $D_{b, i} / d_{t i p, i}$ is expected to result in larger amplification factors.

It is important to recall that the code used to perform the simulations reported herein is inviscid, and the results are expected to depend on the size of the elements forming the mesh; smaller elements are expected to result in larger peak pressures. This is particularly true for the pressure waves converging toward the axis of symmetry. For a perfect fluid, the cylindrical or spherical convergence of acoustic waves results in unbounded pressures. In reality, real processes such as viscous dissipation regularize the problem, resulting in finite pressures. In the present case, the pressure on the axis is obviously bounded. This is because the solution is regularized by the numerical dissipation in the code, and the amount of dissipation is intrinsically related to the size of the grid elements. This caveat is important and suggests the peak magnitude of pressure should be interpreted with great care, and it is preferable to focus on the trends and relative variations.

\subsubsection{Effect of the Rise Time}

The numerical results obtained in the previous subsection demonstrate that a straight cone can substantially amplify sharp pressure waves, especially when the half-angle is relatively small. One would expect that increasing the rise time of the incident pressure wave, or making it "less sharp", has the effect of mitigating the amplification of pressure waves within the cone. This is investigated in this subsection. Figure 5.6 is a plot of the amplification factor as a function of the rise time of the incident pressure wave. The rise time of the incident wave is normalized by the acoustic transit time of pressure waves within the cone: $D_{b, i} / c_{l}$. The results shown in Figure 5.6 are for a rigid cone with a half-angle of 30 degrees. 


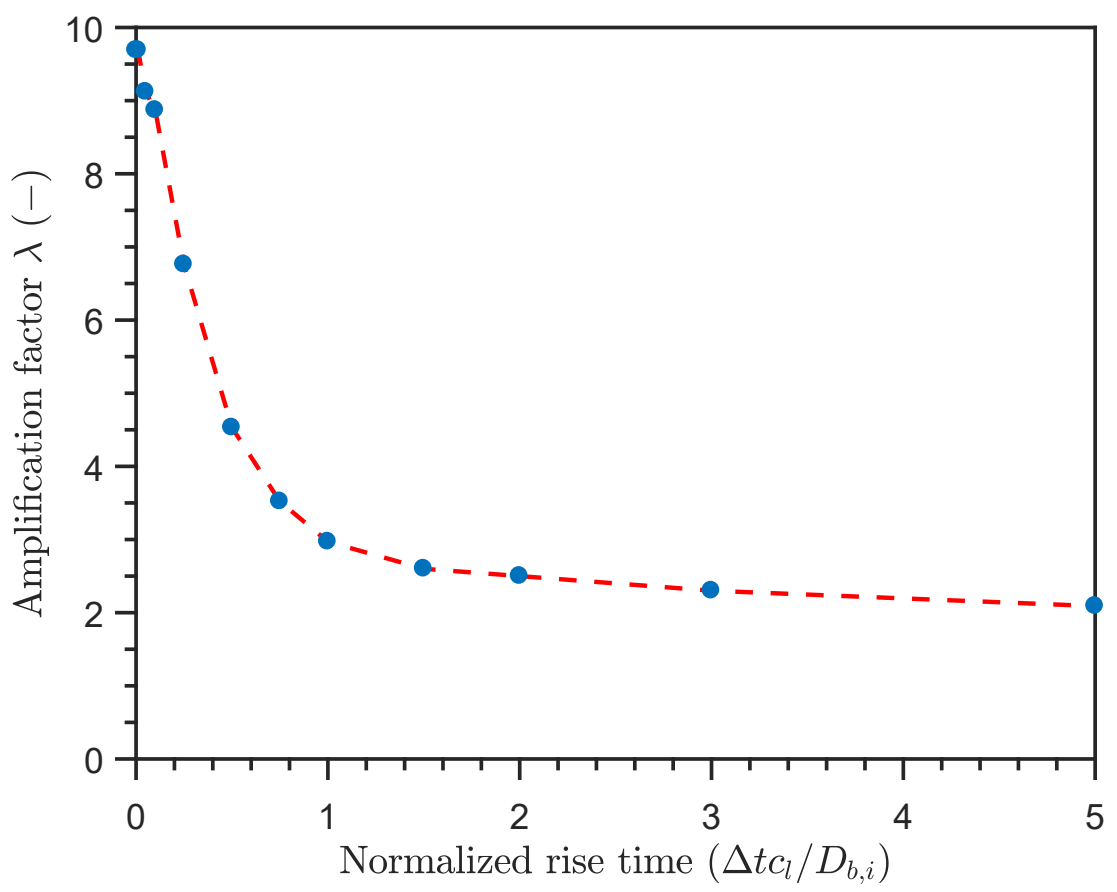

Figure 5.6: Evolution of the amplification factor as a function of the normalized rise time of the incident pressure wave. The results are for a rigid cone with a half-angle of 30 degrees.

The results suggest that pressure waves with a rise time which is less than 0.5 acoustic transit time are substantially amplified within the cone. There is moderate amplification of pressure waves with a rise time between 0.5 and 1 acoustic transit time. The amplification factor for pressure waves with a rise time that is more than 1 acoustic transit time is approximately 2, the same as when the syringe is terminated with a flat wall perpendicular to the axis of symmetry (i.e., a half-angle of 90 degrees). This indicates the pressure waves do not feel the presence of the cone when the rise time is more than 1 acoustic transit time, and the pressure is essentially uniform in the radial direction.

One assumption frequently made in the previous chapters of this thesis is that shock focusing can only occur when the rise time of pressure waves is less than the acoustic transit time of the pressure waves within the cone. The numerical results reported in this subsection suggest this assumption is adequate.

The evolution of the average tip pressure is shown in Figure 5.7 for a cone with a half-angle of 30 degrees and multiple rise time of the incident wave. The results indicate that increasing the rise time of the incident wave not only has the effect 


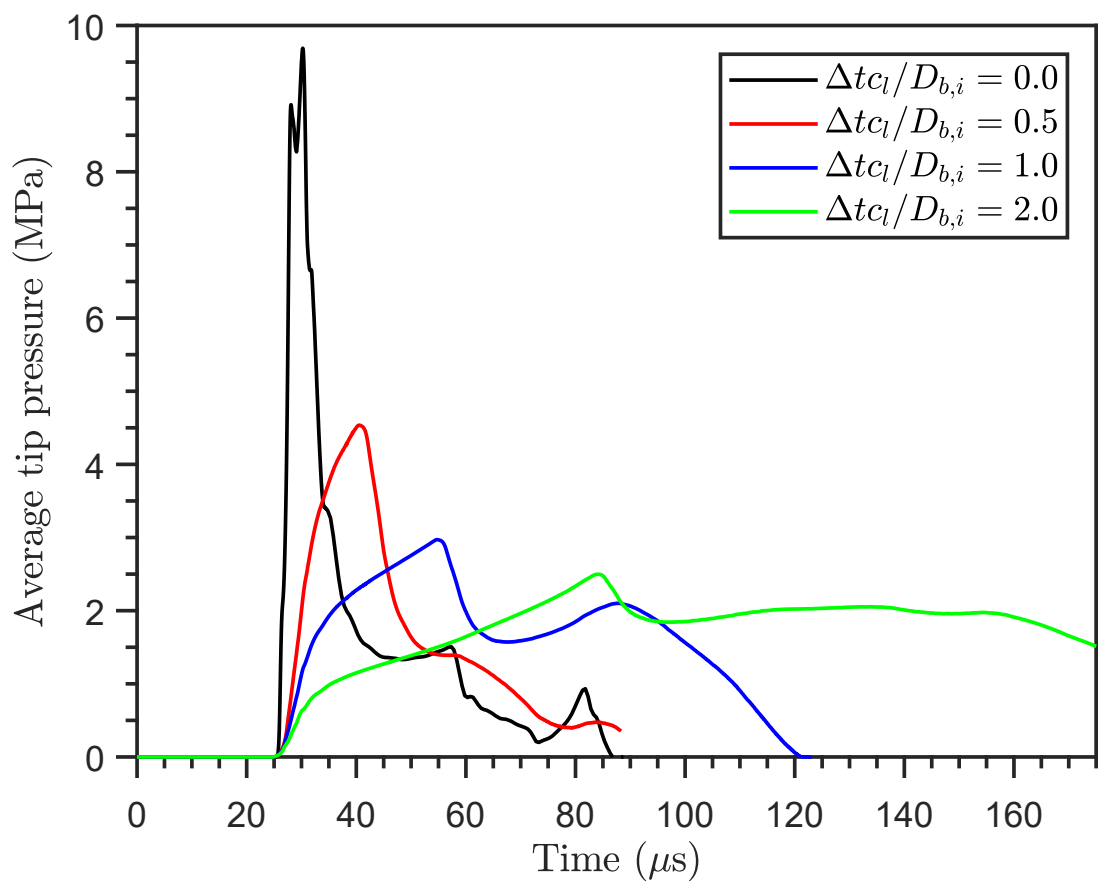

Figure 5.7: History of the average tip pressure for a pressure wave entering a straight, rigid cone with a half-angle of 30 degrees. The average tip pressure is shown for different rise times of the incident wave.

of reducing the peak pressure in the tip, but also changes the characteristic time scale or width of the pressure pulse. For a rise time $\Delta t c_{l} / D_{b, i}=0$, the tip pressure remains above $1 \mathrm{MPa}$, the magnitude of the incident wave, for $33 \mu \mathrm{s}$. When the rise time is increased to $\Delta t c_{l} / D_{b, i}=1.0$, the pressure remains above $1 \mathrm{MPa}$ for $79 \mu \mathrm{s}$. As discussed in Shepherd and Pintgen (2007), the response of the structure depends on the duration of the pressure pulse. Long pulses are approximately equivalent to a quasi-static loading of the structure, whereas short pulses are equivalent to an impulsive loading. In addition to temporal duration, the spatial extent of the pressure pulse is significant: highly localized pulses result in smaller peak deformations and stresses than a spatially-extended pulse of the same peak pressure.

\subsubsection{Effect of the Acoustic Impedance}

The acoustic impedance of the wall forming the cone is yet another factor which can influence the magnitude of shock focusing. The corner wave, as indicated before, is created by the reflection of the incident wave on the oblique wall of the cone. Another part of the incident wave is reflected by the flat wall of the tip. The reflection of waves at the interface between two media is intrinsically dependent 
on the acoustic impedance of the two media in contact, as discussed in Chapter 2. Equation 2.1 indicates that reducing the acoustic impedance of the cone should result in a weaker corner wave, and, in turn, less amplification of the pressure waves inside the cone. In other words, as the acoustic impedance of the cone is reduced, more energy is transmitted into the wall instead of being reflected and focused into the liquid.

Figure 5.8 is a plot of the amplification factor as a function of the reflection coefficient at the boundary between the liquid and the solid. The results for a few half-angles are shown. Note that the results for a half-angle of 90 degrees (i.e., reflection on a flat wall) are obtained analytically using acoustic theory (see Chapter 2 and Appendix A). The amplification factor for a half-angle of 90 degrees is equal to:

$$
\lambda_{90}=\left(\frac{Z_{s}-Z_{l}}{Z_{s}+Z_{l}}\right)+1 .
$$

Shock focusing occurs for data points which are above the red-dashed line. A data point which is close or directly on the red-dashed line indicates the incident pressure wave does not feel the effect of the cone, and the pressure in the tip is close or identical to what would be obtained for a syringe terminated with a flat wall.

For a rigid wall (i.e., reflection coefficient equal to 1), the effect of the half-angle is substantial, and smaller half-angles result in a larger amplification factor. The effect of the half-angle of the cone becomes less significant as the reflection coefficient is reduced. Furthermore, for reflection coefficients which are not unity it is not possible to predict which half-angle results in the largest amplification factor. For example, for a reflection coefficient equal to 0.5 a half-angle of 60 degrees results in an amplification factor which is larger than the amplification factor obtained with a half-angle of 15 degrees. This unexpected effect has to do with the number of radial reverberations of the corner wave inside the cone. Each reflection of the corner wave on the wall of the cone results in a part of the wave being transmitted into the wall of the cone, reducing the amount of energy which can be focused into the liquid. Smaller half-angles result in more reverberations of the corner wave because the cone is deeper.

A reflection coefficient which is equal to 0 corresponds to the situation where the acoustic impedances of the liquid and the solid are matched. Effectively, the incident pressure wave does not feel the boundary between the liquid and the solid, and no reflection occurs. The amplification factor is therefore expected to be 1 for all half-angles, and this is in accord with the numerical results. 


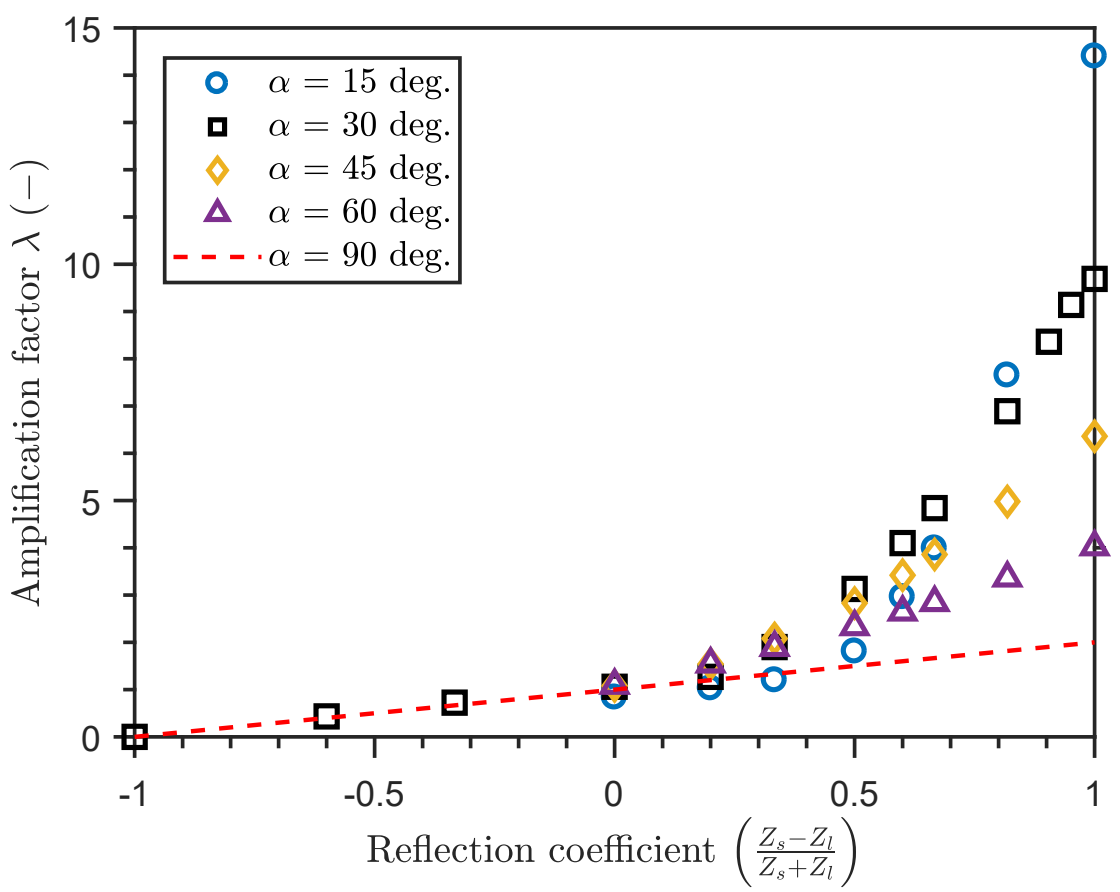

Figure 5.8: Evolution of the amplification factor of the cone as a function of the reflection coefficient.

Reflection coefficients which are less than 0 result in the production of a tensile corner wave. This explains why the amplification factor is less than 1. A reflection coefficient which is equal to -1 corresponds to the situation where there is no wall. The outer edge of the cone is a constant pressure boundary condition, and the amplification factor is expected to be 0 , in accord with the numerical simulations. This result is independent of the half-angle of the cone.

The results demonstrate that shock focusing is largely dependent on the acoustic impedance of the wall. Reflection coefficients larger than 0.5 result in substantial amplification of the incident pressure wave, and the effect of the half-angle of the cone is important. Reflection coefficients less than 0.5 result in only a small amount of amplification, and the effect of the half-angle is negligible.

For illustration purposes, a rigid cone with a half-angle of 30 degree results in an amplification factor of 9.7. If the same cone is made with glass and polycarbonate, the amplification factors are respectively 6.5 and 1.5 . This suggests that substantial shock focusing is possible in glass syringes, but only a small amount of amplification is expected in plastic syringes. 


\subsubsection{Focusing in the Cone of a Syringe}

The straight cone geometry used in the simulations reported above is similar but not identical to the geometry of an actual pre-filled syringe. In most pre-filled syringes the half-angle is not constant, but instead varies with axial location. Because the amplification factor is a strong function of the the half-angle, the specific shape of the cone has an effect on the amplification factor. Furthermore, a real syringe geometry is not terminated by a flat wall, but is instead terminated with a narrow channel leading to the needle.

Shock focusing in a BD HyFlow $1 \mathrm{~mL}$ pre-filled syringe is studied in this subsection. The precise geometry of the cone was measured by engineers at Amgen using a Nikon VMA-2520 microscope (private communication). A sequence of images showing the evolution of the pressure and the propagation of pressure waves is shown in Figure 5.9. The magnitude of the incident, sharp pressure wave is $1 \mathrm{MPa}$. The syringe wall, assumed to be rigid, is not shown. A constant pressure boundary condition is applied on the bottom end of the channel located below the cone. The left-hand side of each panel is a colored pseudo-schlieren which illustrates the propagation of the incident, the corner, and the diffracted waves. The right-hand side of each panel is a contour plot of the pressure field.

Between 2.75 and $4.75 \mu \mathrm{s}$, the diffracted wave and the corner wave form and converge toward the axis of symmetry. While this occurs, the pressure becomes non-uniform in the radial direction; the pressure is larger in the vicinity of the axis of symmetry. The corner wave is not straight but curved due to the non-constant half-angle of the cone. Between 5.75 and $6.75 \mu$ s a region of substantial pressure develops in the cone of the syringe, where the cross-sectional area is minimum. The pressure there is approximately $5 \mathrm{MPa}$, or 5 times larger than the initial magnitude of the incident pressure wave. The pressure history in the narrower section of the cone is shown in Figure 5.10. Although the pressure becomes large, the pulse has a short duration: the pressure is larger than $2 \mathrm{MPa}$ for only $2.75 \mu \mathrm{s}$.

The results demonstrate that substantial amplification of pressure waves can occur in the cone of a syringe despite the half-angle not being constant. Note that the cone was assumed to be rigid. A cone with a finite acoustic impedance, as demonstrated earlier, results in a reduction of the amplification factor. Results for a non-rigid wall with a finite thickness are shown in Section 5.2. 


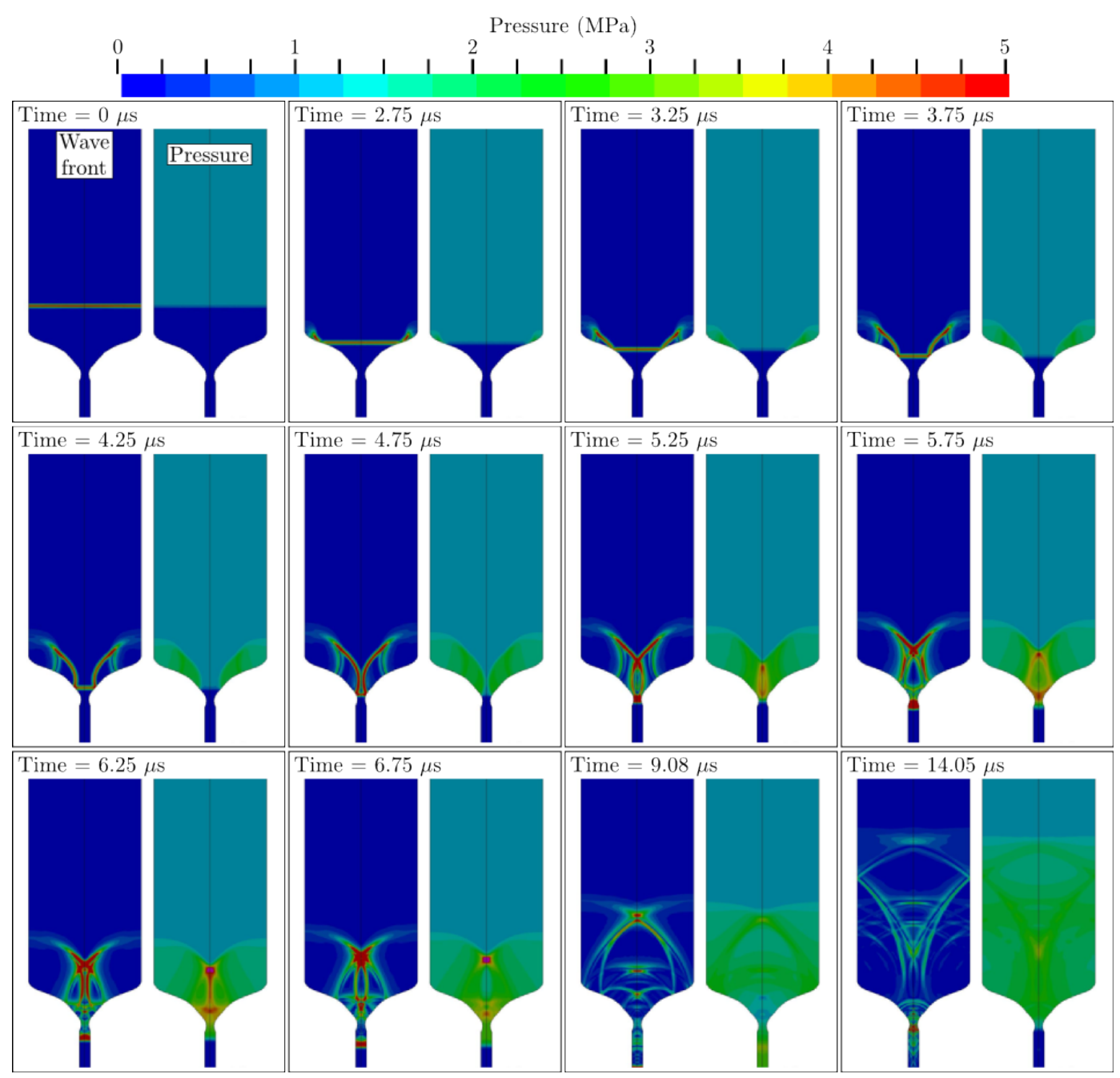

Figure 5.9: Amplification of a sharp pressure wave entering the cone of a BD Hyfloe $1 \mathrm{~mL}$ pre-filled syringe. The left-hand side of each frame is a colored pseudo-schlieren, and the right-hand side is the pressure field.

\subsubsection{Summary of Shock Wave Focusing}

Numerical simulations confirmed it is possible for acoustic pressure pulses to be amplified inside the cone of a syringe. For a truncated cone with a convergence ratio of 9 , amplification factors as large as 18.8 were predicted. The amplification results from the convergence of corner waves toward the axis of symmetry of the cone.

The magnitude of the amplification depends on the half-angle of the cone. Deeper cones with smaller half-angles result in a larger amplification than cones with a large half-angle. The amplification factor is equal to 2 for half-angles of 90 degrees, and twice the contraction ratio for small half-angles approaching zero. 


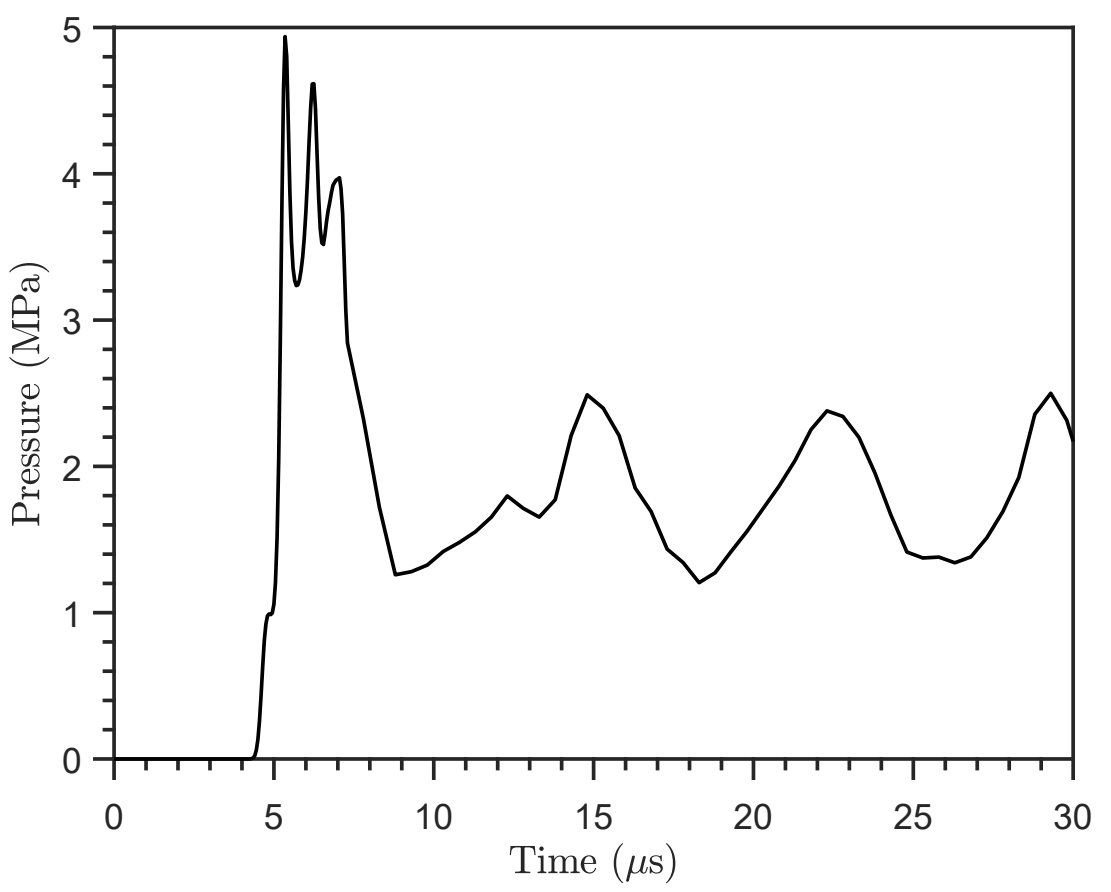

Figure 5.10: Pressure history in the narrower section of the syringe cone.

The acoustic impedance of the cone is another factor which affects the magnitude of pressure wave amplification. Amplification of the incident wave is possible for reflection coefficients greater than 0.5 . The rise time of the incident pressure wave also affects the magnitude of the amplification in the cone. A rise time of more than 1 acoustic transit time does not create much amplification of the pressure wave (the amplification factor is approximately equal to 2 ).

Simulations performed with an actual BD syringe geometry demonstrate that substantial amplification is possible within the cone of pre-filled syringes. The peak pressure is predicted to occur where the cross-sectional area of the cone is minimum. An amplification factor of 5 is predicted for a rigid cone, the worst case scenario. The amplification factor is expected to be less than 5 for cones with a finite acoustic impedance and wall thickness.

The numerical results have also shown that the characteristic time scale of the pressure pulses created in the tip of a cone or the cone of a syringe is short, of order 1-100 $\mu \mathrm{s}$. The duration of the pulse is influenced by the rise time of the incident wave. The response of the material is expected to be influenced not only by the peak magnitude of the pulse, but also by the duration of the pulse. Strain rate effects 
and inertial effects could be significant and need to be accounted for (Jones, 1989; Shepherd and Pintgen, 2007; Smith and Hetherington, 1994).

\subsection{Wall Stresses}

Chapter 1 indicates that Amgen recorded the failure of several SureClick autoinjector devices during clinical trials at a low, but detectable rate of 30 parts-per-million. Forensic analysis of the broken glass revealed the failures originated on the inner surface of the cone. The forensic study concluded that an excessive hoop stress resulting from a large liquid pressure is believed to be the root cause of syringe failures, but the origin of the excessive hoop stress was not determined.

Based on forensic evidence, the transient events which create large stresses away from the cone area can't explain the consistent failures originating from within the cone. Chapters 2, 3, and 4 have shown that actuation of a SureClick autoinjector can create deleterious pressure and stress transients inside the device, but the study was mostly focused on the syringe barrel due to experimental constrains. An alternative to study the stresses in the cone region is to use numerical simulations.

In this section, LS-DYNA is used to predict the magnitude and location of relatively large stresses resulting from events 1, 2, and 3 (see Chapter 2). The numerical simulations are performed on an actual pre-filled syringe geometry. A process of elimination is used to determine, as best as possible, which dynamic event(s) can explain the consistent failures originating from within the cone.

\subsubsection{Numerical Model}

The numerical simulations reported in this section are performed using LS-DYNA (Hallquist, 2016). The inviscid simulations are performed in 2D axisymmetric mode. The model, shown in Figure 5.11, is constructed using approximately 1,200,000 Lagrangian shell elements, unless otherwise indicated, and consists of two parts: the liquid (blue) and the solid (red). Further refinement of the computational grid by a factor of 4 results in variations of the peak pressures, stresses and strains that are $7 \%$ or less. The refined grid is not utilized due to the increased computational time and the relatively small effect on the quantities of interest.

The liquid contained inside the syringe is modeled as water using the same acoustic formulation as in Chapter 4 with $\rho=1000 \mathrm{~kg} / \mathrm{m}^{3}$ and $c_{l}=1500 \mathrm{~m} / \mathrm{s}$. The syringe wall is modeled as a borosilicate glass using a linear elastic constitutive relation (Bower, 2009) with $\rho_{s}=2230 \mathrm{~kg} / \mathrm{m}^{3}, E=65.0 \mathrm{GPa}$, and $v=0.3$. No failure model 


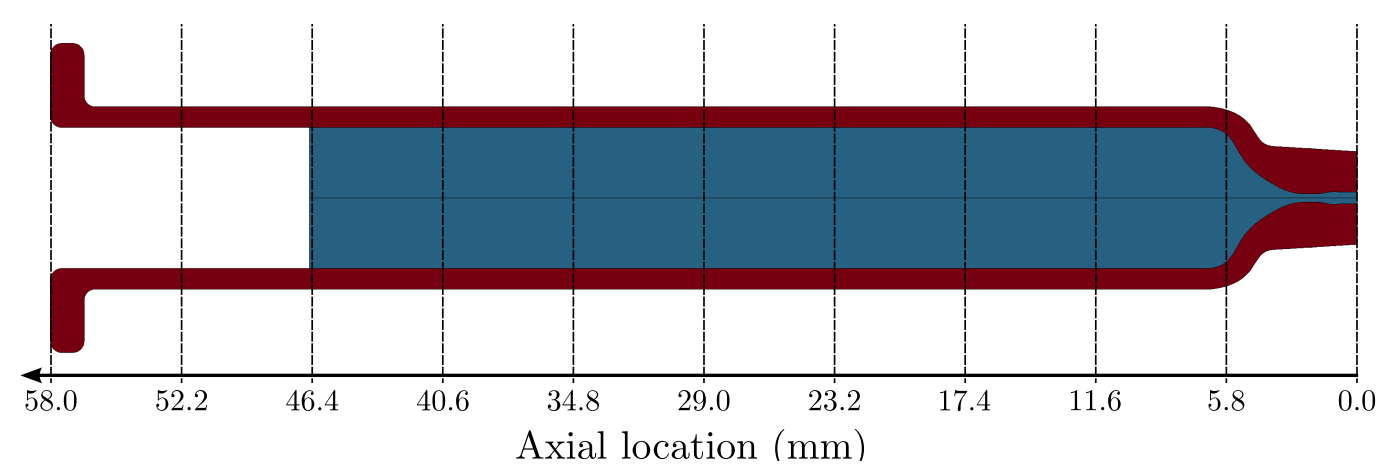

Figure 5.11: LS-DYNA syringe model of a $1 \mathrm{ml} \mathrm{BD} \mathrm{Hypack} \mathrm{syringe.}$

is used because the objective is only to determine the magnitude and location of the peak stresses in the syringe wall. The contact between the glass and water is modeled using a surface-to-surface two-way contact.

The geometry of the syringe tip and cone corresponds to the geometry of a $1 \mathrm{~mL}$ BD Hypack. The precise geometry of the outer and the inner surfaces in the cone region was measured by Amgen (private communication) using a Nikon VMA-2520 microscope. The geometry of the barrel and the flange is approximate.

Several components of the SureClick autoinjector device, such as the plungerstopper, the driving rod, the needle and the syringe carrier, are not modeled. Modeling those components would significantly increase the complexity of the numerical model, and this would add unnecessary uncertainty on the results. Instead, the effect of those components is approximately taken into account through appropriate boundary conditions.

A distributed load is applied on the top boundary of the liquid column to create the internal liquid pressure. This is illustrated in Figure 5.12a. The distributed load can be a function of time. Two different syringe support mechanisms representative of autoinjector devices are considered: 1) a simple support located on the flange (see Figure 5.12b); 2) a simple support located on the shoulder (see Figure 5.12c). Note that only one of the two simple supports is used at a time, and the simple supports only constrain the motion in the axial direction. Furthermore, the simple supports have a finite size: the force applied is distributed over a region which is approximately $0.7 \mathrm{~mm}$ in length. The results obtained with each simple support are discussed separately. Away from the simple support, a stress free boundary condition is applied on the outer surface of the syringe. 


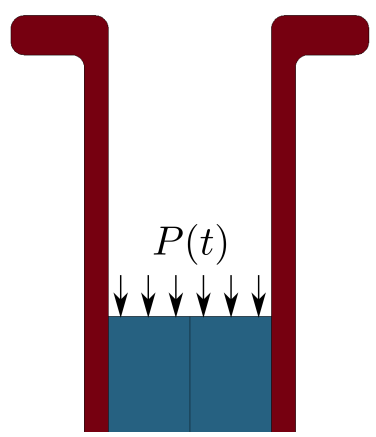

(a) Pressure

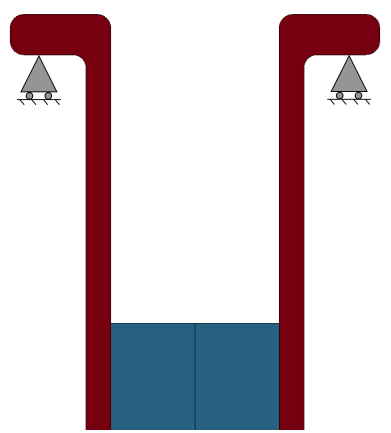

(b) Simply supported shoulder

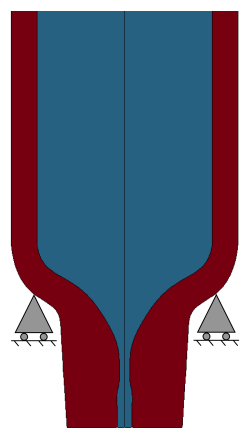

(c) Simply supported flange

Figure 5.12: Schematic representation of the boundary conditions used in the LSDYNA numerical model.

\subsubsection{Static Internal Loading}

The syringe wall stresses under static internal loading are studied in this subsection. The static load is created by a constant and uniform liquid pressure applied on the syringe inner surface. This static loading situation is not representative of the transient events that occur inside an autoinjector during actuation. Studying this situation is nevertheless relevant for two reasons: 1) it is important to understand the static case before studying the more complicated dynamic or transient situation; 2) the liquid pressure is approximately constant and uniform during the extrusion phase of autoinjector actuation, when the transient events are terminated. Note that all static simulations are performed with an implicit solver to minimize simulation time.

\subsubsection{Simply Supported Flange}

The first static case that is discussed is for a syringe supported using the flange. The stresses are normalized by the magnitude $P$ of the constant internal pressure. Figure 5.13 is a contour plot of the normalized maximum principal stress $\sigma_{1} / P$. All regions under compression $\left(\sigma_{1} / P<0\right)$ are colored in gray, and all normalized stresses exceeding 4 are colored in magenta.

There are three regions where $\sigma_{1} / P$ is relatively large:

- the junction between the flange and the barrel;

- the inner surface of the barrel;

- the inner surface of the syringe shoulder. 


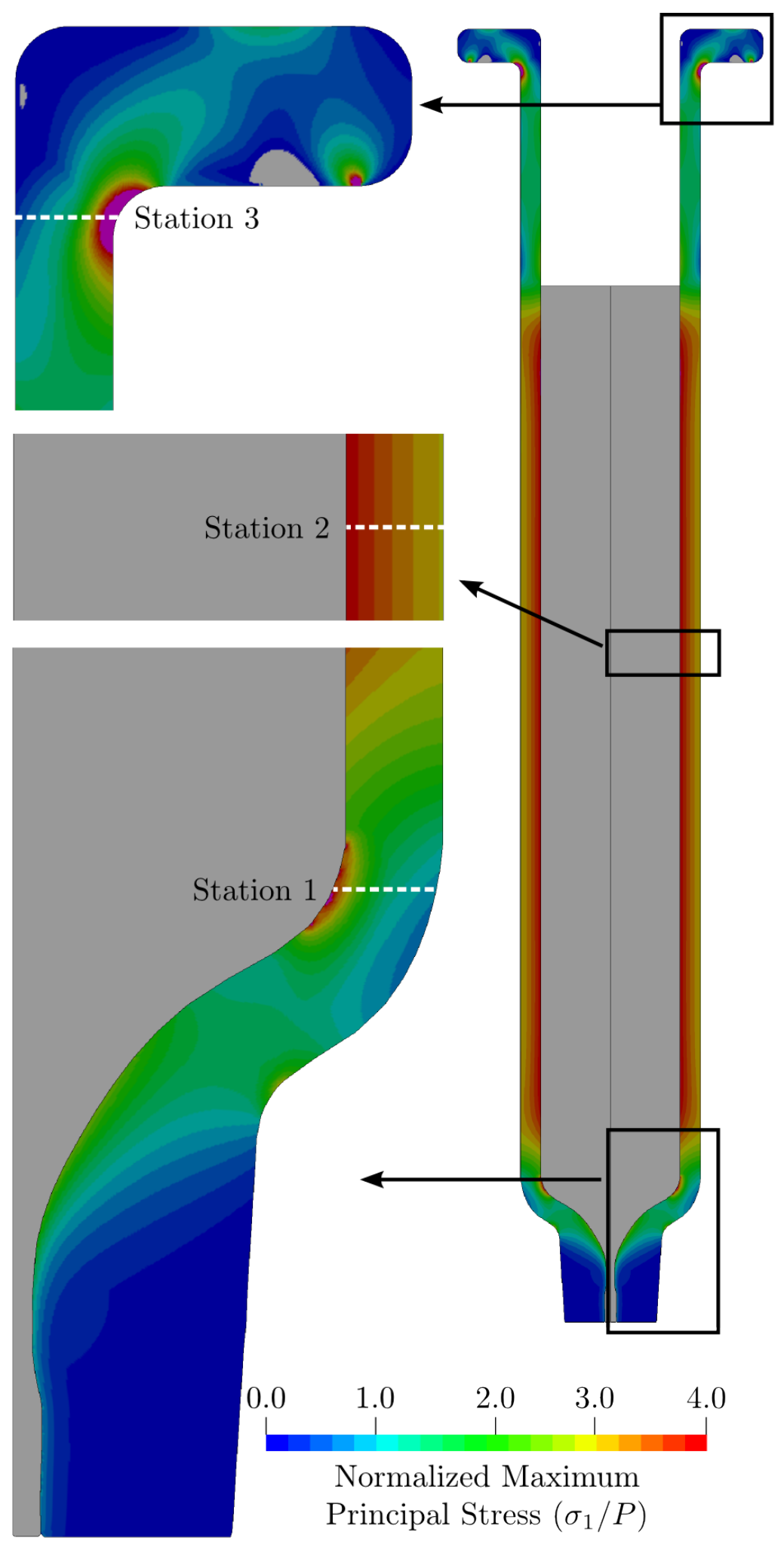

Figure 5.13: Maximum principal stress in the syringe wall for a constant and uniform liquid pressure of $1 \mathrm{MPa}$. The syringe is supported at the flange. 


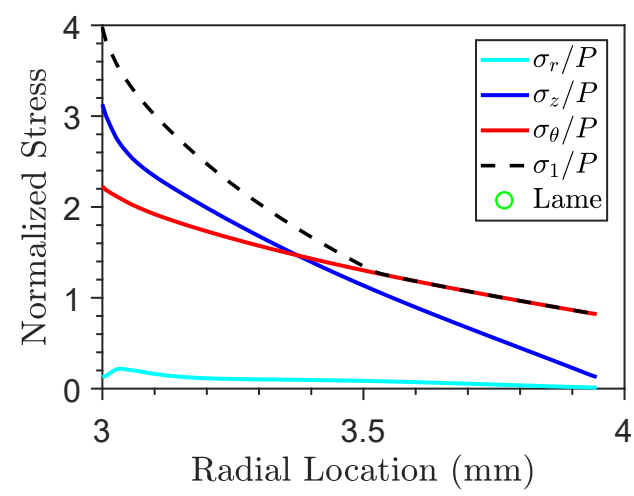

(a) Station 1 (shoulder)

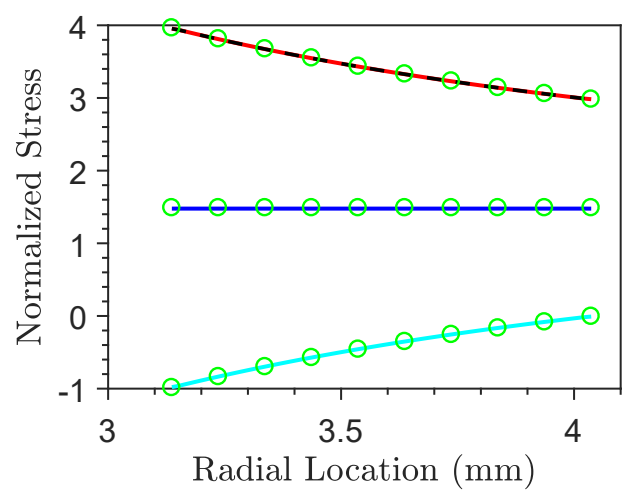

(b) Station 2 (barrel)

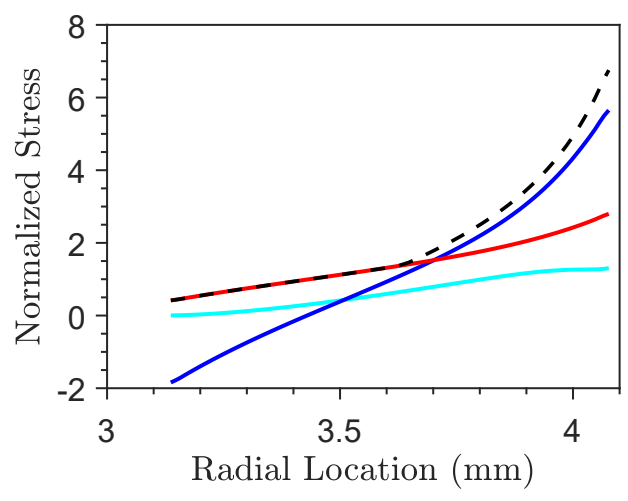

(c) Station 3 (flange)

Figure 5.14: Radial, axial, hoop, and maximum principal stress distribution at three different stations in the syringe's wall for a constant and uniform liquid pressure. The syringe is supported at the flange. Stations 1 to 3 are identified in Figure 5.13.

A zoomed-in view of the normalized maximum principal stress in those regions is shown on the left-hand side of Figure 5.13. Failure is most likely to occur in those regions due to the relatively large magnitude of the tensile stresses. Three stations are indicated with white-dashed lines in Figure 5.13. Figure 5.14 is a plot of the normalized radial, axial, hoop, and maximum principal components of stress along those lines as a function of radial location measured from the centerline of the syringe.

The stresses at station 1 (shoulder) are shown in Figure 5.14a. The stresses are larger on the interior surface, and the dominant stress components are in the hoop and the axial directions. The peak value of the normalized maximum principal stress is approximately 4 on the inner surface.

The stresses at station 2 (barrel) are shown in Figure 5.14a. The dominant stress component is in the hoop direction. The stresses predicted using the Lamé solution 
(Bower, 2009) are also plotted in Figure 5.14a; the LS-DYNA results are within $1 \%$ of the Lamé solution. The stresses predicted with LS-DYNA are validated by the good agreement with the Lamé solution. The peak value of the normalized maximum principal stress is approximately 4 on the inner surface of the barrel.

The stresses at station 3 (flange) are shown in Figure 5.14a. The stresses are larger on the outer surface of the syringe, where the dominant stress component is in the axial direction. The peak value of the normalized maximum principal stress is approximately 7 .

The results shown in Figure 5.14 suggest that under static conditions, the normalized maximum principal stress is well approximated by:

$$
\frac{\sigma_{1}(r)}{P}=\max \left\{\frac{\sigma_{\theta}(r)}{P}, \frac{\sigma_{z}(r)}{P}\right\} \text {. }
$$

This approximation is generally adequate because the shear stresses remain small in the syringe wall, except in the shoulder region.

Figure 5.15 is a plot of the normalized maximum principal, hoop, and axial components of stress along the inner and the outer surface of the syringe. The data shown in Figure 5.15 confirms that Equation 5.4 is generally an adequate estimate for the maximum principal stress, except near the syringe shoulder. This is important because the maximum principal stress was not measured using the in situ techniques from Chapter 3. Instead, the strains were measured along the barrel of the syringe, and the axial and hoop components of stress were inferred using Hooke's law. The approximation of Equation 5.4 makes it possible, in turn, to infer the maximum principal stress.

The results from Figure 5.15 confirm there are three regions where the normalized maximum principal stress is relatively large: the junction between the flange and the barrel, the inner surface of the barrel, and the inner surface of the syringe shoulder. Among those, the junction between the flange and the barrel experiences the largest stresses. This is the location where failure is most likely to occur when the syringe is supported by the flange and statically loaded.

\subsubsection{Simply Supported Shoulder}

The second case that is considered is identical to the first one, except the syringe is supported at the shoulder. The stresses are again normalized by the magnitude $P$ of the constant and uniform internal pressure. Figure 5.16 is a contour plot 


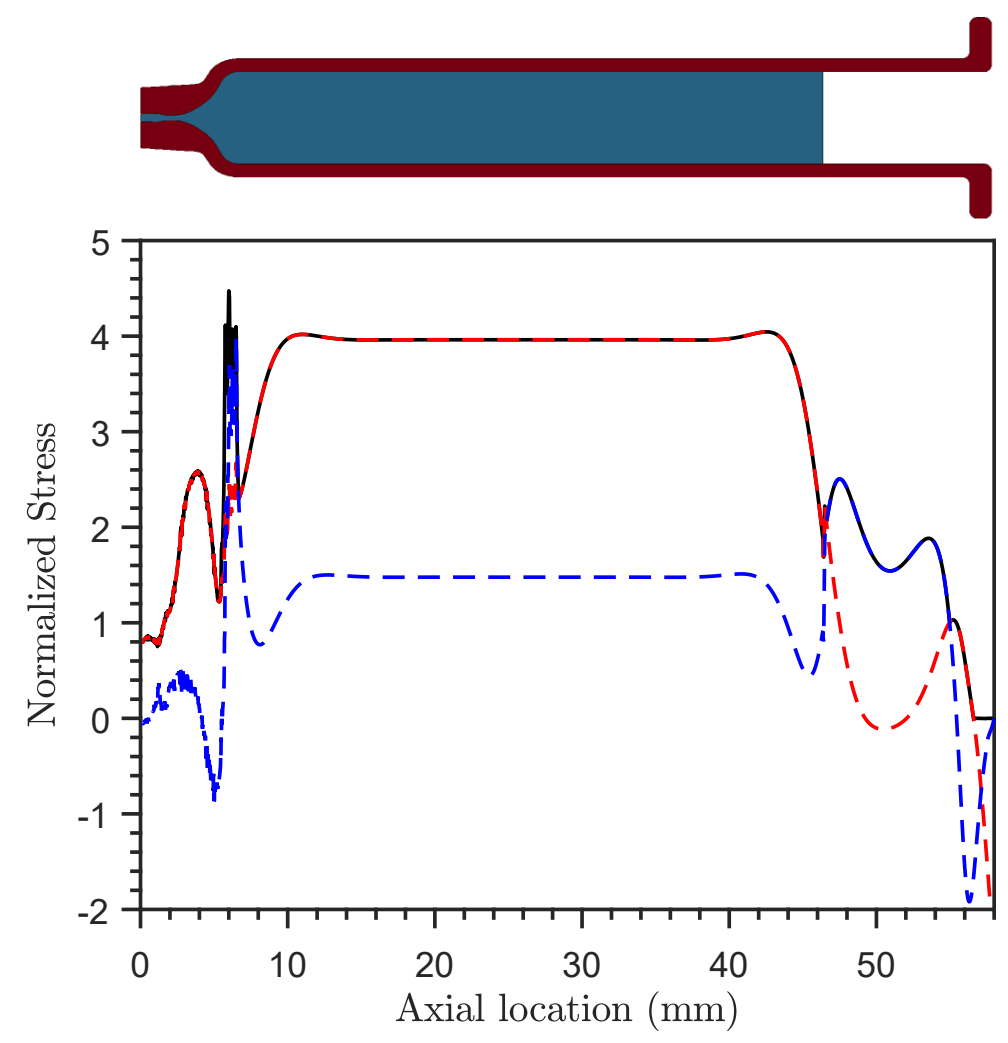

(a) Stresses along the inner surface

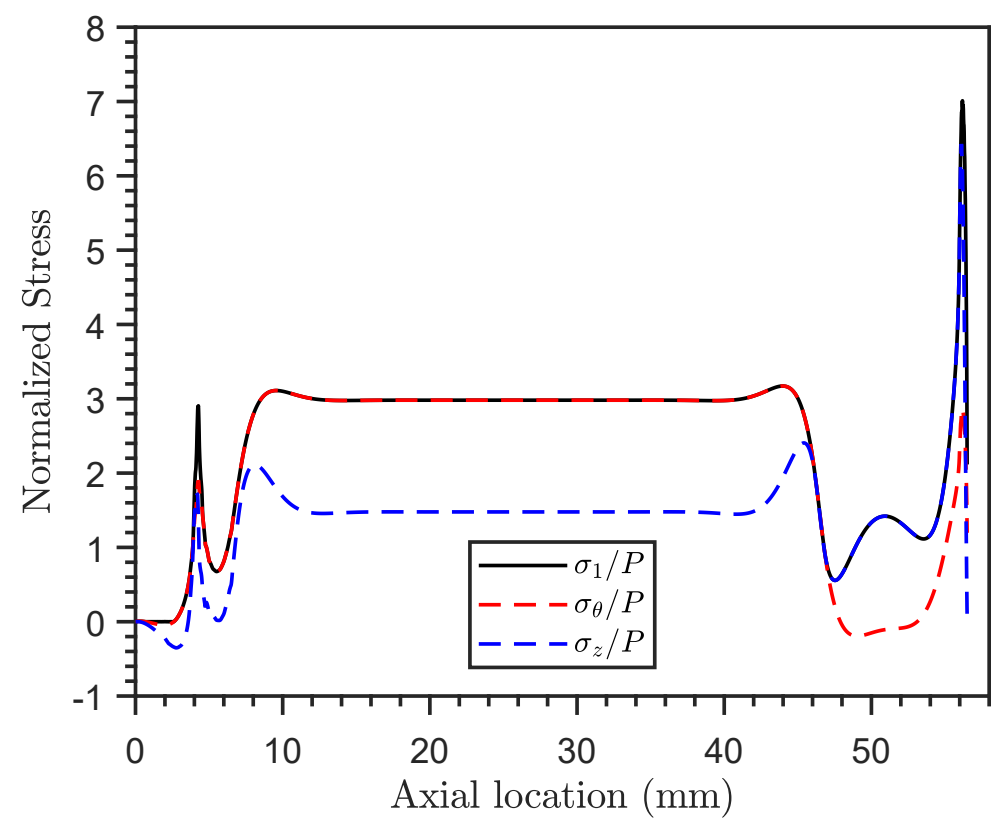

(b) Stresses along the outer surface

Figure 5.15: Maximum principal, hoop and axial stresses along the inner and the outer surface of the syringe for a constant and uniform liquid pressure. The syringe is supported at the flange. 
of the normalized maximum principal stress $\sigma_{1} / P$. Regions under compression $\left(\sigma_{1} / P<0\right)$ are colored in gray, and all normalized stresses exceeding 4 are colored in magenta.

The results in Figure 5.15 indicate that moving the support from the flange to the shoulder reduces the stresses in the flange, as expected. There are now two regions where $\sigma_{1} / P$ is relatively large:

- the inner surface of the barrel;

- the inner surface of the syringe shoulder.

A zoomed-in view of the normalized maximum principal stress in those regions is shown on the left-hand side of Figure 5.16. Failure is most likely to occur in one of those regions. Three stations are indicated with white-dashed lines in Figure 5.16. Figure 5.17 is a plot of the normalized radial, axial, hoop, and maximum principal components of stress along those lines as a function of radial location measured from the centerline of the syringe.

The stresses at station 1 (bottom shoulder) are shown in Figure 5.17a. Note that station 1 corresponds to the location of the simple support. The simple support creates substantial compressive stresses on the outer portion of the syringe wall. Compressive stresses are not discussed because the compressive strength of glass is significantly larger than its tensile strength. The normalized stresses at station 1 do not exceed a value of approximately 2 , which occurs on the inner surface of the syringe.

The stresses at station 2 (upper shoulder) are shown in Figure 5.17b. There are substantial tensile stresses. The normalized maximum principal stress is equal to 4 on the inner surface of the syringe. The results indicate that the shear stresses are relatively large in this region and Equation 5.4 does not provide an accurate estimate for the normalized maximum principal stress.

The stresses at station 3 (barrel) are shown in Figure 5.17c. The Lamé solution (Bower, 2009) is also plotted for comparison. The numerical predictions of LSDYNA are validated by the Lamé solution, as in the previous case. The stresses are larger on the inner surface of the barrel, where the normalized maximum principal stress is equal to 4 .

Figure 5.18 is a plot of the normalized maximum principal, hoop and axial components of stress along the inner and the outer surfaces of the syringe. The data shown 


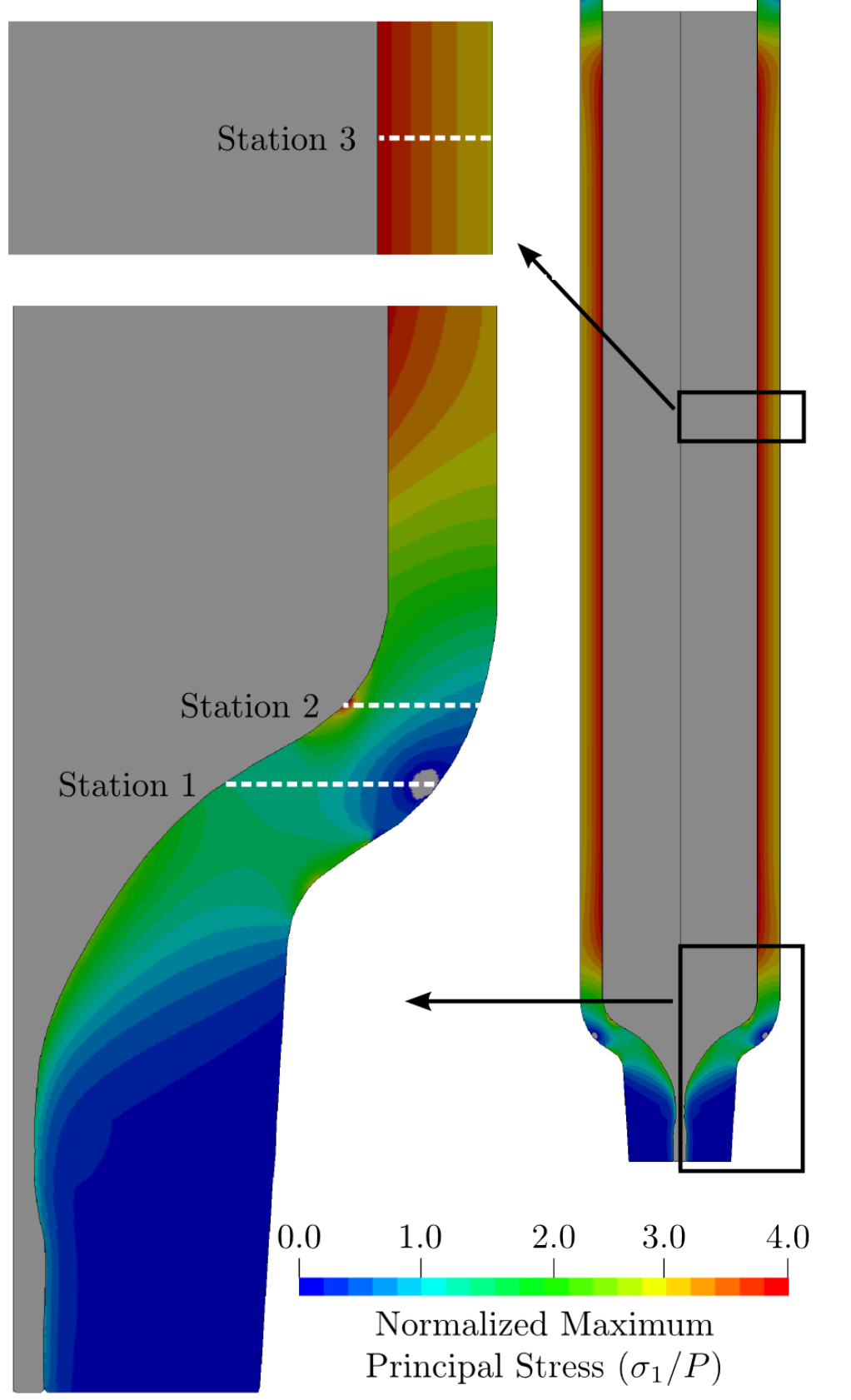

Figure 5.16: Maximum principal stress in the syringe wall for a constant and uniform liquid pressure of $1 \mathrm{MPa}$. The syringe is supported at the shoulder. 


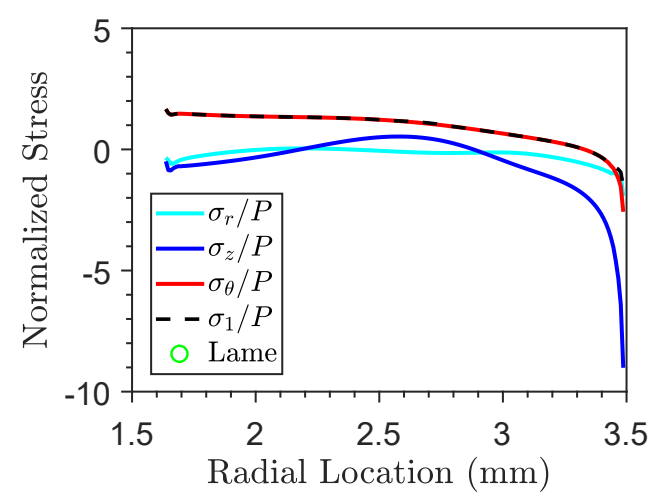

(a) Station 1 (bottom shoulder)

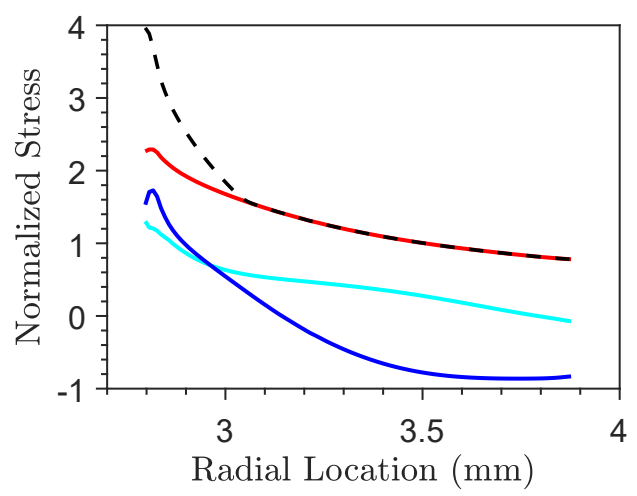

(b) Station 2 (upper shoulder)

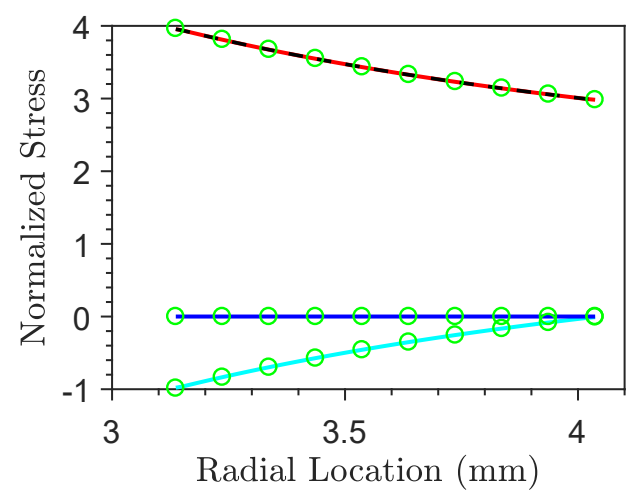

(c) Station 3 (barrel)

Figure 5.17: Radial, axial, hoop, and maximum principal stress distribution at three different stations in the syringe's wall for a constant and uniform liquid pressure. The syringe is supported at the shoulder. Stations 1 to 3 are identified in Fig. 5.16.

in Figure 5.18 is another confirmation that Equation 5.4 is an adequate estimate for the maximum principal stress, except in the vicinity of the syringe shoulder. The results also confirm that the largest maximum principal stresses, as indicated before, occur on the inner surface of the syringe shoulder, and on the inner surface of the syringe barrel. Note that the normalized maximum principal stress is at most 4, compared to 7 for a syringe which is supported using the shoulder. This indicates that supporting the syringe using the shoulder reduces the peak stresses in the syringe wall, suggesting it is preferable to support the syringe using the shoulder rather than the flange.

\subsubsection{Summary of Static Stresses}

The syringe wall stresses under static loading conditions were studied in this subsection. The stresses are relatively large in the following regions: 


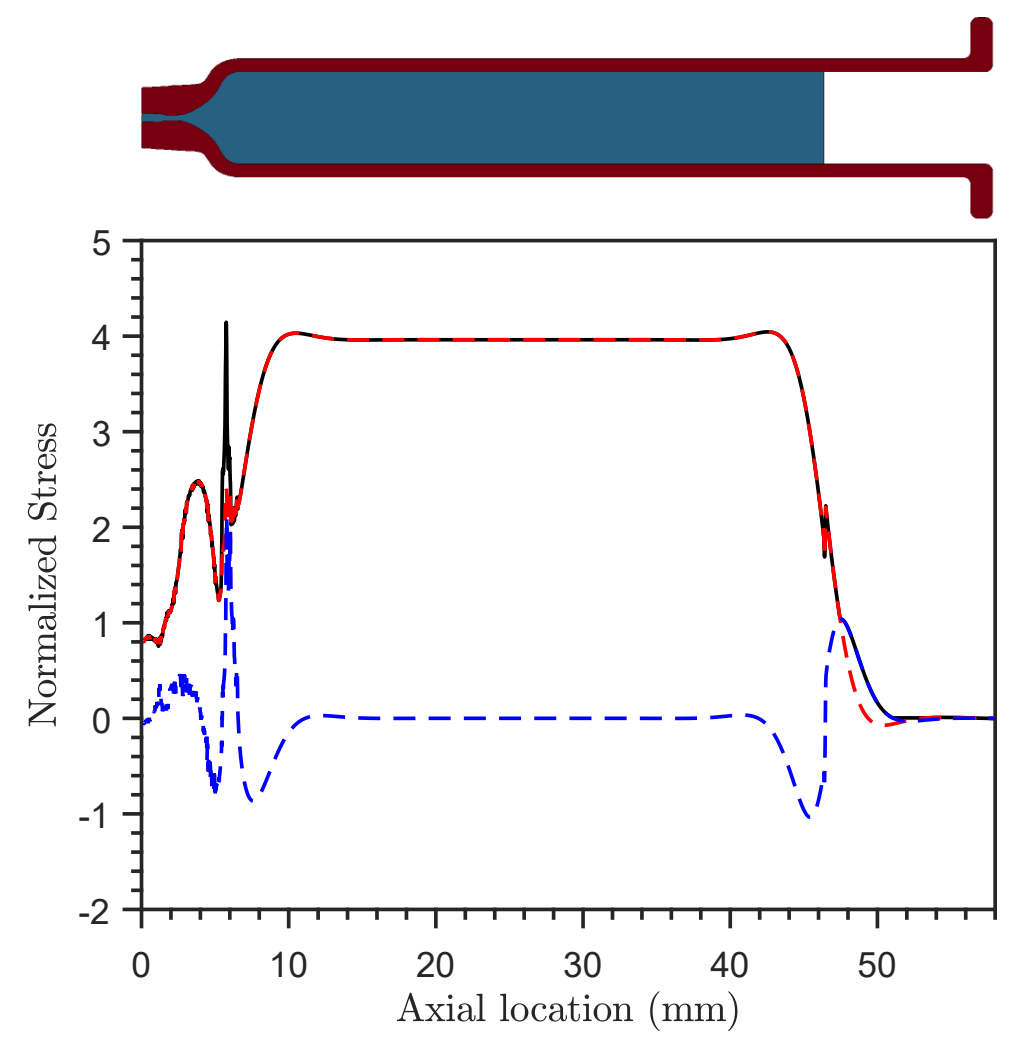

(a) Stresses along the inner surface

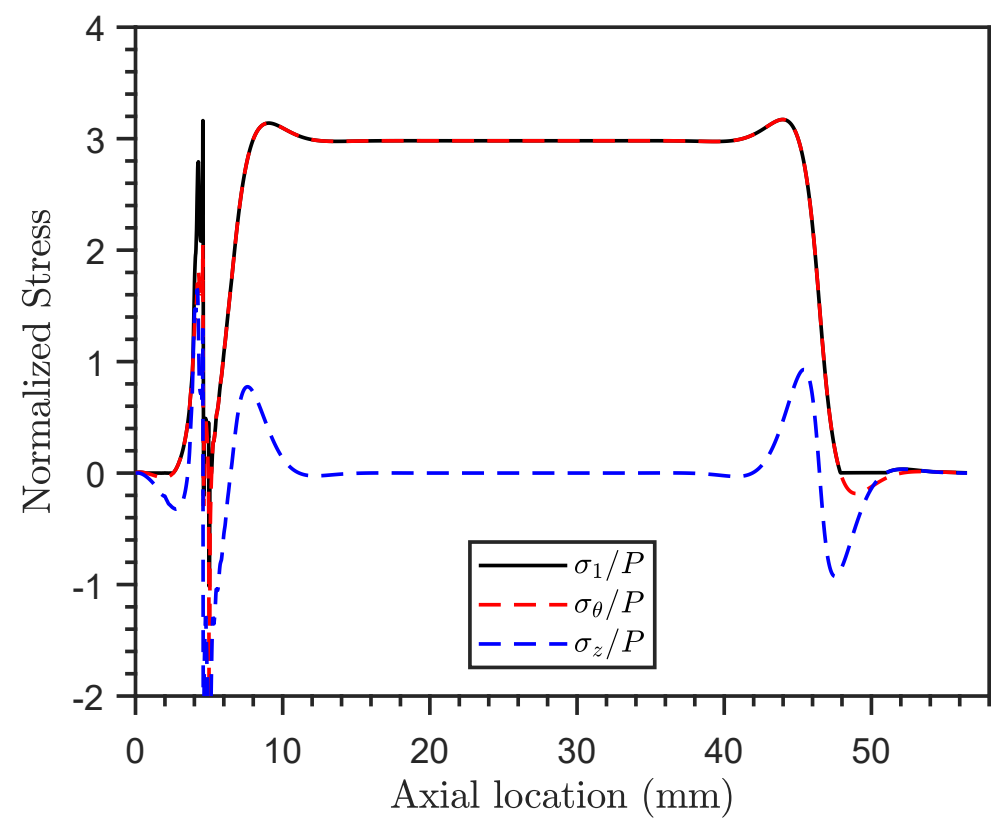

(b) Stresses along the outer surface

Figure 5.18: Maximum principal, hoop and axial stresses along the inner and the outer surface of the syringe for a constant and uniform liquid pressure. The syringe is supported at the shoulder. 
- the junction between the flange and the barrel (if the syringe is supported at the flange);

- the barrel of the syringe;

- the inner \& the outer surfaces of the shoulder;

indicating this is where failure is more likely to occur.

The results indicate that supporting the syringe using the shoulder instead of using the flange has three consequences: 1) it reduces substantially the stresses in the flange region; 2) it reduces the axial component of stress in the barrel to zero; 3 ) it creates a region under compression in the vicinity of the shoulder. Overall, the peak stresses are almost reduced by a factor of two when the syringe is supported using the shoulder rather than using the flange.

Statically loading the syringe inner surface through a constant and uniform liquid pressure does not create relatively large tensile stresses within the cone of the syringe. This result is important because it suggests that consistent failures originating within the cone region can't be attributed to the static loading of the syringe during the extrusion phase.

\subsubsection{Dynamic Internal Loading}

The pressurization of the syringe resulting from the impact of the driving rod on the plunger-stopper is dynamic rather than static. This is because the motion of the plunger-stopper creates pressure waves in the liquid and stress waves in the syringe glass. As demonstrated in the previous section, pressure waves can be amplified within the cone region, and this means the stresses in the cone could be larger than what was predicted for the static case. This is particularly true when the pressure waves are sharp.

Numerical simulations are performed using the explicit solver of LS-DYNA, the approach which is most suitable for wave propagation problems. The same mesh as in the static cases is used. The same boundary conditions as before (see Figure 5.12) are used, except for a few changes. First, $P(t)$ is not a constant anymore. Instead, the top end of the water column is impulsively pressurized:

$$
\frac{P(t)}{P_{\text {max }}}= \begin{cases}0 & \text { if } t<0, \\ 1 & \text { if } t \geq 0 .\end{cases}
$$


The impulsive loading of the top surface of the water column results in the creation of a sharp pressure wave propagating toward the cone area of the syringe. This corresponds to the worst case scenario because sharp pressure waves result in the largest shock focusing effect within the cone.

Second, the channel located below the syringe cone is sealed to prevent liquid from flowing outside the syringe. Fluid elements tend to flow through the cone and the tip of the syringe, resulting in a highly distorted mesh. Sealing the tip of the syringe resolves this issue, but this also modifies the boundary condition applied on the water column. The result is that it creates liquid pressures inside the syringe, in the vicinity of the tip, which are larger than if a free end allowing a flow through the tip was used. The simulated conditions therefore correspond to the worst case scenario for estimating stresses.

The pressure and stresses are a function of both space and time due to the dynamic nature of this problem. All quantities of interest (i.e., pressure and stress components) are plotted along the inner and the outer surfaces of the syringe for each case discussed, similarly to what is shown in Figures 5.15 and 5.18. The time dependence of each quantity of interest $\xi(z, t)$ is eliminated by keeping only the maximum value for each axial location:

$$
\tilde{\xi}(z)=\max _{t>0} \xi(z, t)
$$

\subsubsection{Simply Supported Flange}

The case of a syringe supported at the flange is considered first. The normalized pressure along the inner surface of the syringe is shown in Figure 5.19. Contrary to the static case where the pressure is uniform, the pressure varies largely throughout the syringe. The largest pressure is observed within the cone area of the syringe, as expected. Amplification of the incident pressure wave results in a normalized pressure which is close to 4.5 in the cone. The pressure in the syringe barrel is also larger than 1 due to the reflection of the incident pressure wave in the cone. The pressure at the top end of the liquid column is 1 because this is a constant pressure boundary condition.

Figure 5.20 is a plot of the normalized maximum principal, hoop and axial components of stress along the inner and the outer surfaces of the syringe. The peak magnitude of the normalized maximum principal stress along the inner surface (see Figure 5.20a) is approximately 14 , and it occurs in the syringe shoulder. There, 


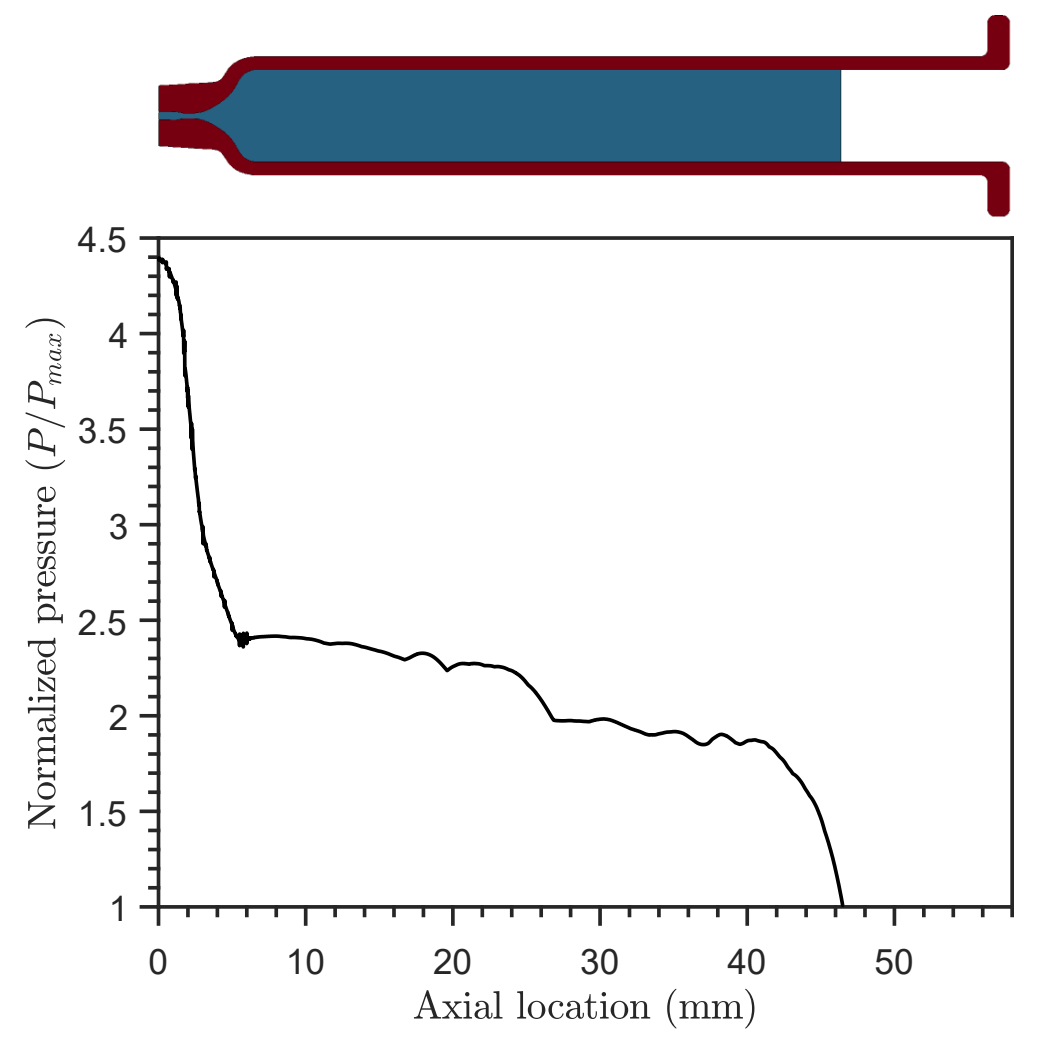

Figure 5.19: Maximum pressure along the inner surface of the syringe. The syringe is supported at the flange, and dynamically pressurized with a sharp pressure wave.

the dominant stress component is in the axial direction. The inner shoulder is the location where failure would most likely occur along the inner surface of the syringe. The peak amplitude of the normalized maximum principal stress is approximately 27 on the outer surface of the syringe (see Figure 5.20b), and it occurs at the junction between the flange and the barrel. There, the dominant stress component is in the axial direction. This is the location where failure would most likely occur along the outer surface of the syringe. The peak magnitude of the normalized maximum principal stress along the outer surface is twice as large as the peak magnitude of the normalized maximum principal stress on the inner surface. The results also indicate the normalized maximum principal stress on the inner and the outer surfaces of the syringe is as much as 4 times larger when the syringe is dynamically pressurized compared to when it is statically pressurized. 


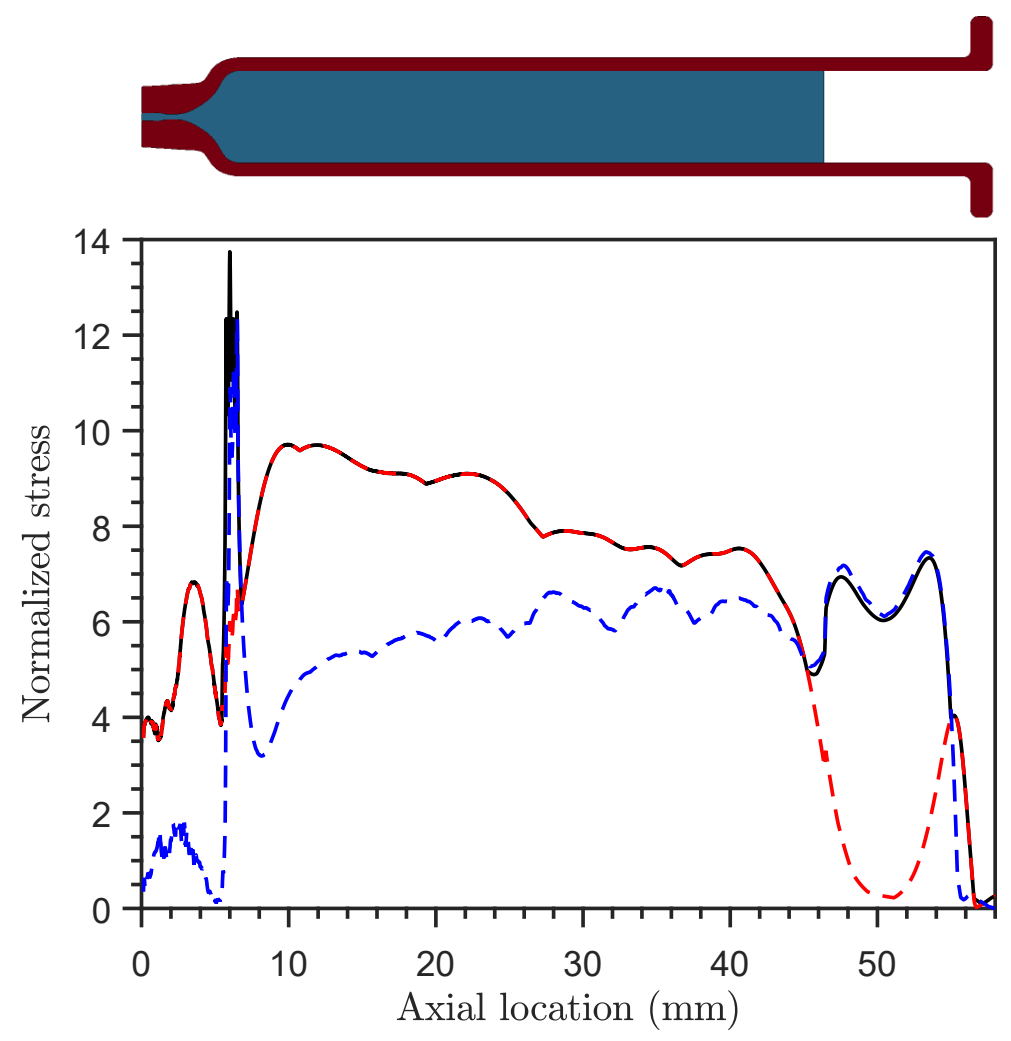

(a) Stresses along the inner surface

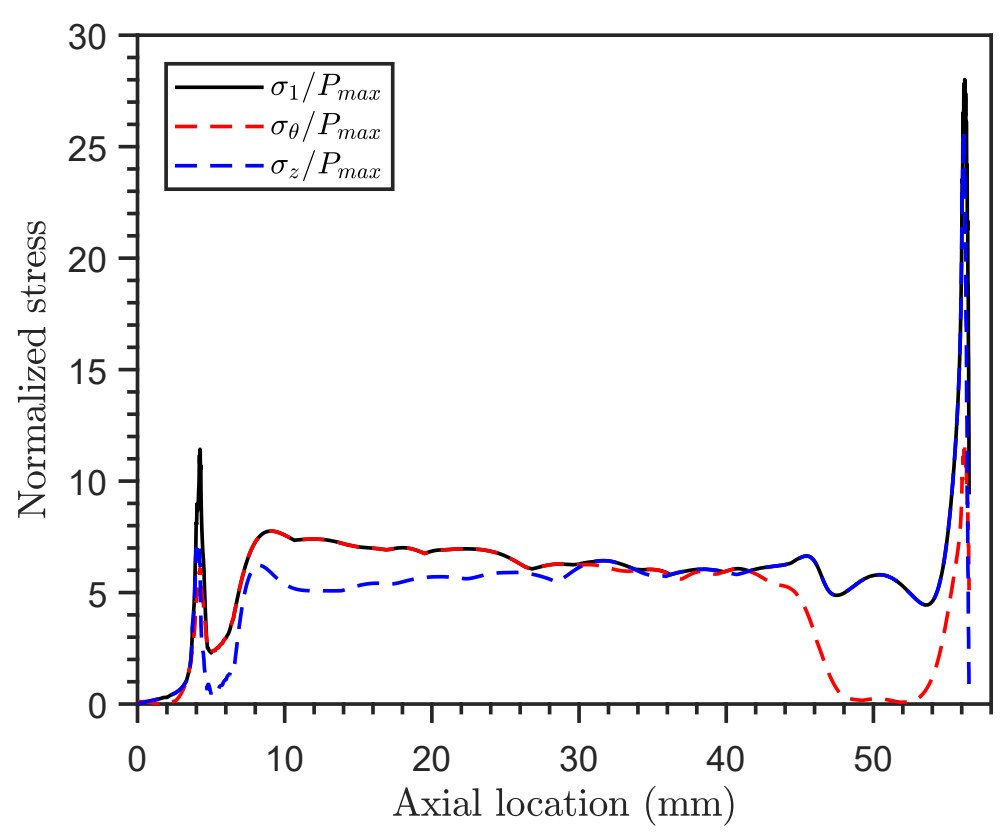

(b) Stresses along the outer surface

Figure 5.20: Maximum principal, hoop and axial stresses along the inner and the outer surface of the syringe. The syringe is supported at the flange, and dynamically pressurized with a sharp pressure wave. 


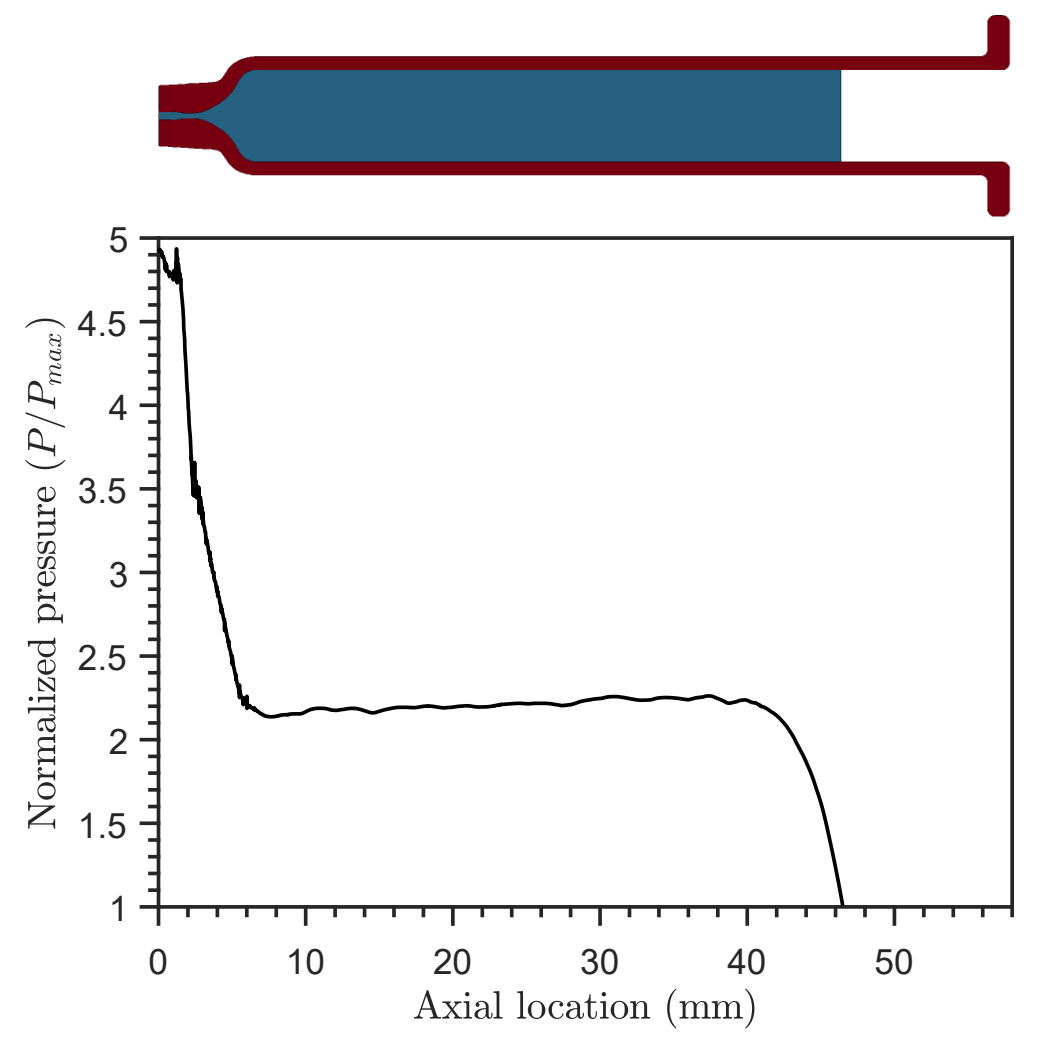

Figure 5.21: Maximum pressure along the inner surface of the syringe. The syringe is supported at the shoulder, and dynamically pressurized with a sharp pressure wave.

\subsubsection{Simply Supported Shoulder}

The case of a syringe supported at the shoulder is now considered. The normalized pressure along the inner surface of the syringe is shown in Figure 5.21. The normalized peak pressure in the cone is approximately 5 , slightly higher than in the previous case. It is interesting, but not unexpected, that a larger peak pressure is predicted when the syringe is supported at the shoulder rather than the flange (5.0 vs 4.5). This is because the boundary condition in the vicinity of the shoulder is effectively stiffer when the syringe is supported at the shoulder. The presence of a simple support near the cone influences the wave dynamics both within the solid and the liquid. Locally, the boundary condition on the outer surface of the syringe is not a stress free boundary, and this has the consequence of further increasing the maximum pressure inside the syringe cone.

Figure 5.22 is a plot of the normalized maximum principal, hoop and axial components of stress along the inner and the outer surfaces of the syringe. The peak 
magnitude of the normalized maximum principal stress on the inner surface (see Figure 5.22a) is approximately 12, and it occurs in the vicinity of the shoulder. There, the dominant stress component is in the axial direction. This is where failure would most likely occur on the inner syringe surface. The maximum principal stress on the inner surface is up to 3 times larger than the maximum principal stress predicted for a statically pressurized syringe.

The peak magnitude of the normalized maximum principal stress on the outer syringe surface (see Figure 5.22b) is approximately 12, identical to the peak magnitude predicted on the inner surface. The peak normalized maximum principal stress occurs in the vicinity of the shoulder, and this is where failure would most likely occur on the outer surface. There, the axial and the hoop components of stress are of similar magnitude. The normalized maximum principal stress is up to 3.7 times larger than for a statically pressurized syringe.

Comparing Figures 5.20 and 5.22 suggests that supporting the syringe at the shoulder instead of supporting it at the flange does not significantly affect the stresses on the inner and the outer surfaces, except in the vicinity of the junction between the flange and the barrel. Supporting the syringe at the flange rather than the shoulder increases the peak magnitude of the normalized maximum principal stress by as much as $100 \%$. This suggests that supporting the syringe at the shoulder is preferable to minimize the potential for syringe failure.

\subsubsection{Summary of Dynamic Loading Stresses}

Numerical simulations were performed to predict the pressure and stresses in a syringe which is dynamically pressurized though a sharp pressure wave. This situation is analog to event 3 in a SureClick autoinjector device (see Chapter 2). The results again demonstrate that amplification of sharp pressure waves is possible within the cone region: amplification factors as large as 4.5 to 5.0 are predicted. The peak magnitude of the normalized maximum principal stress resulting from this type of transient event is consistently 3 to 4 times larger than for a syringe which is statically pressurized.

Despite the presence of shock focusing in the cone, the peak magnitude of the normalized maximum principal stress is predicted to occur in the vicinity of the shoulder and/or the flange, not in the cone. This observation is important, indicating that even when the liquid is pressurized through sharp pressure waves, the syringe will only be likely to fail in the vicinity of the shoulder and/or the flange. This 


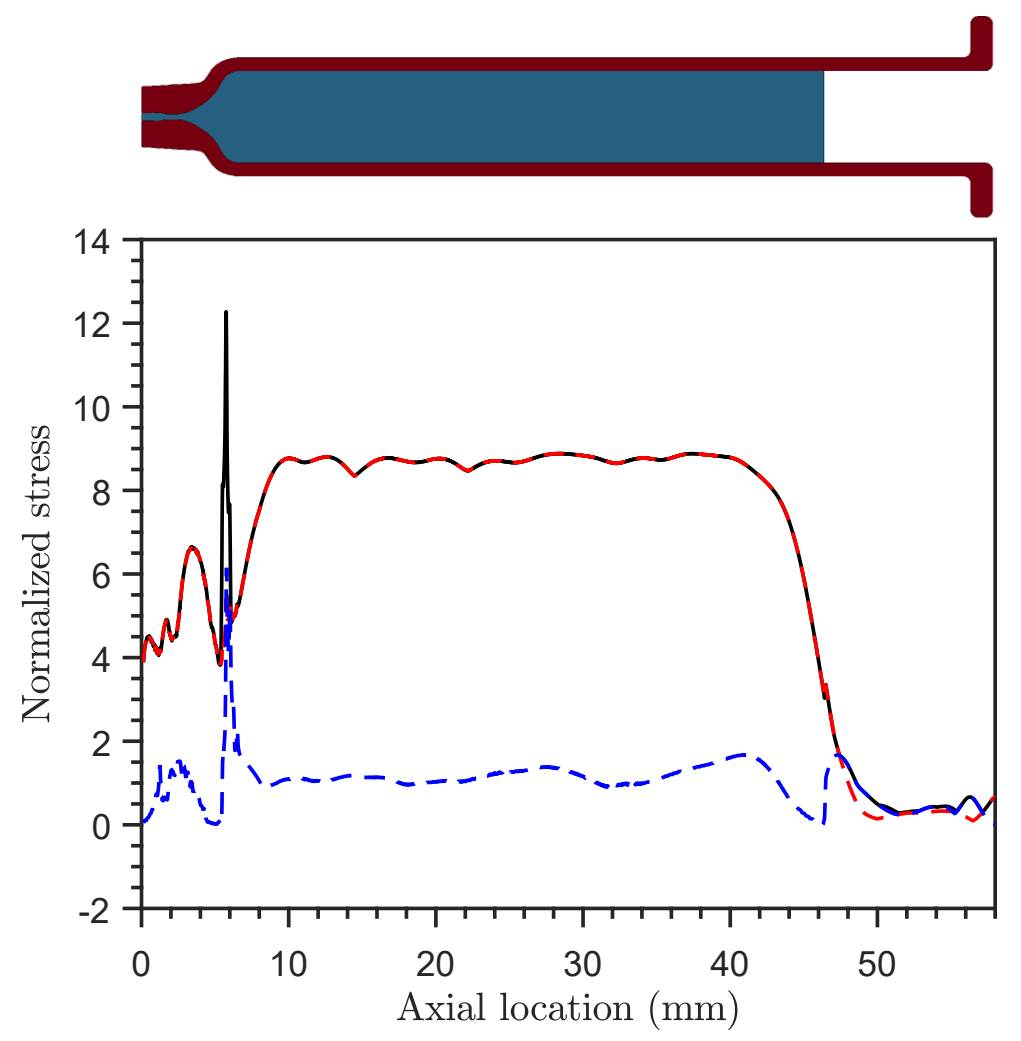

(a) Stresses along the inner surface

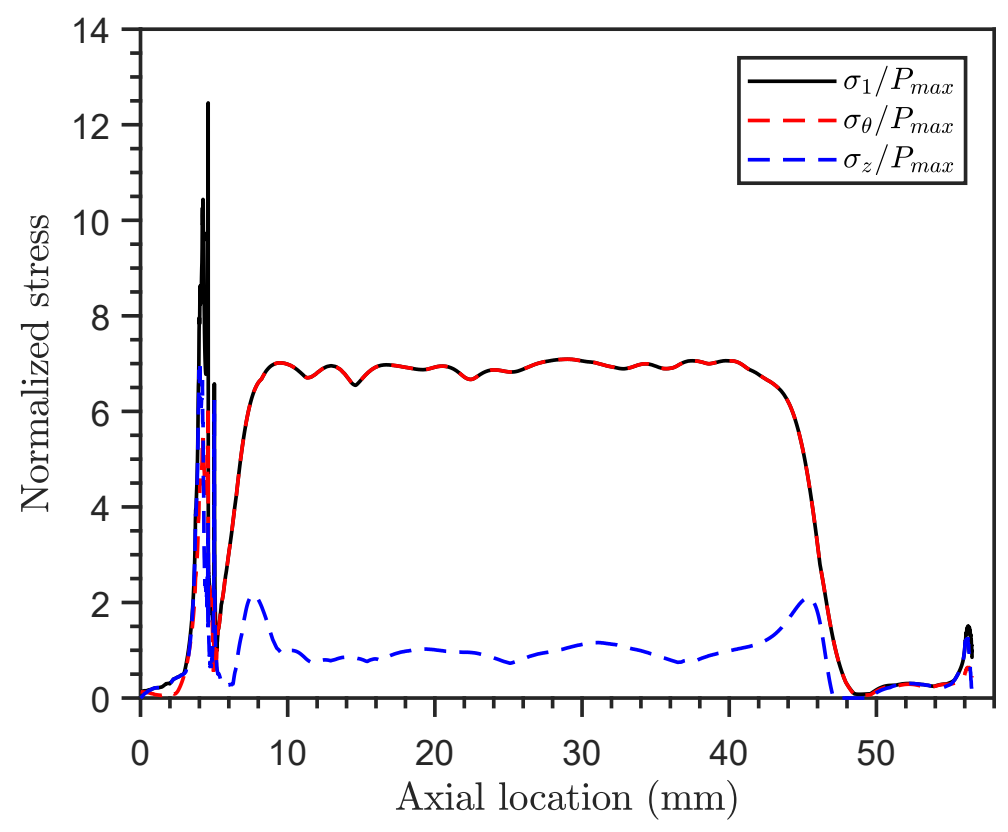

(b) Stresses along the outer surface

Figure 5.22: Maximum principal, hoop and axial stresses along the inner and the outer surface of the syringe. The syringe is supported at the shoulder, and dynamically pressurized with a sharp pressure wave. 
indicates that shock focusing of pressure waves inside the cone is not sufficient to create stresses in the cone that are larger than the stresses created in the shoulder and/or the flange. The dynamic pressurization of the syringe (event 3 ) is not the likely cause for the consistent cone failures observed by Amgen during clinical trials.

\subsubsection{Syringe Deceleration}

The rapid deceleration of the syringe creates pressure waves in the liquid and stress waves in the syringe wall. The magnitude of the pressure and stresses is predicted using the LS-DYNA model and deceleration histories representative of autoinjector operation. The syringe and the liquid content are initially traveling downward at a velocity of $8 \mathrm{~m} / \mathrm{s}$. The syringe is then rapidly decelerated to a complete stop at a rate of $36,000 \mathrm{~m} / \mathrm{s}^{2}$ (i.e., the velocity profile is linear). The deceleration is controlled using a support which is either located at the flange or the shoulder (see Figures $5.12 \mathrm{c}$ and $5.12 \mathrm{~b}$ ).

This problem was studied by Veryst Engineering at Amgen's request in 2014 and 2015 (private communication). The numerical study was performed using Comsol. The primary conclusion of the study was that the peak magnitude of the maximum principal stress occurs away from the cone. The numerical model developed by Veryst Engineering was complex because all components of the autoinjector device were modeled. The model also suffered from numerical convergence issues. Those issues motivate performing the study a second time using the simpler and more robust LS-DYNA numerical model.

The explicit solver of LS-DYNA is used throughout this subsection. Because it is necessary to simulate a relatively long physical time, the grid resolution is reduced to shorten the computational time. The grid used to simulate the syringe deceleration has approximately 600,000 Lagrangian shell elements, compared to $1,200,000$ elements for the grid used to simulate the static and dynamic pressurization of the syringe.

The pressure boundary condition applied on the liquid column is $P=0 \mathrm{MPa}$, which corresponds to a free end. The "simple support" is a region where a linear velocity profile is prescribed. Note that no constraint is applied in the radial direction at the location of the simple support.

Fluid elements tend to flow through the cone and the tip of the syringe during the deceleration event, resulting in a highly distorted mesh. This issue is resolved by merging the nodes between the liquid and syringe parts instead of using a two-way 
surface-to-surface contact. The channel located below the syringe cone is also sealed to prevent liquid from flowing outside of the syringe. This modifies the boundary condition applied on the liquid column, and this has the consequence of modifying the pressure wave dynamics. The result is that it creates liquid pressures in the syringe which are larger than if a free end allowing a flow through the tip was used. The simulated conditions correspond to the worst case for estimating stresses.

Another approach can be used to circumnavigate the mesh distortion issues. The liquid contained in the syringe can be modeled using Eulerian shell elements instead of Lagrangian shell elements. The coupling between the liquid and the solid is modeled using a penalty coupling. This approach is not ideal because it often results in nonphysical leakage of the liquid into the solid, creating spurious stress signals. For this reason, the results obtained using this second approach were not used as part of the present study.

\subsubsection{Simply Supported Flange}

The case of a syringe supported at the flange is considered first. Figure 5.23 is a plot of the pressure along the inner surface of the syringe. The results are kept dimensional in this subsection. ${ }^{1}$ The maximum pressure is approximately $4 \mathrm{MPa}$ in the tip of the syringe, in accord with the peak magnitude of the pressure measured in a SureClick autoinjector device during the rapid deceleration of the syringe (see Chapter 3). The pressure at the top end of the liquid column is $0 \mathrm{MPa}$ due to the constant pressure boundary condition.

Figure 5.24 is a plot of the maximum principal, hoop and axial components of stress along the inner and the outer surfaces of the syringe. The maximum principal stress along the inner surface (see Figure 5.24a) is relatively large at two locations: the shoulder (17 MPa), and the junction between the flange and the barrel (16 MPa). This is where failure would most likely occur along the inner syringe wall. The dominant stress component at those two locations is in the axial direction.

The maximum principal stress along the outer surface (see Figure 5.24b) is relatively large in the vicinity of the junction between the flange and the shoulder. The peak magnitude of the maximum principal stress is $64 \mathrm{MPa}$, and this is where failure would most likely occur. This is not unexpected considering the deceleration is controlled using a support which is located at the flange, and substantial stress

\footnotetext{
${ }^{1}$ The appropriate reference pressure to non-dimensionalize the pressure would be $2 \rho_{l} L_{l} a$. The appropriate reference stress to non-dimensionalize the stresses would be $2 \rho_{s} L_{b} a$
} 


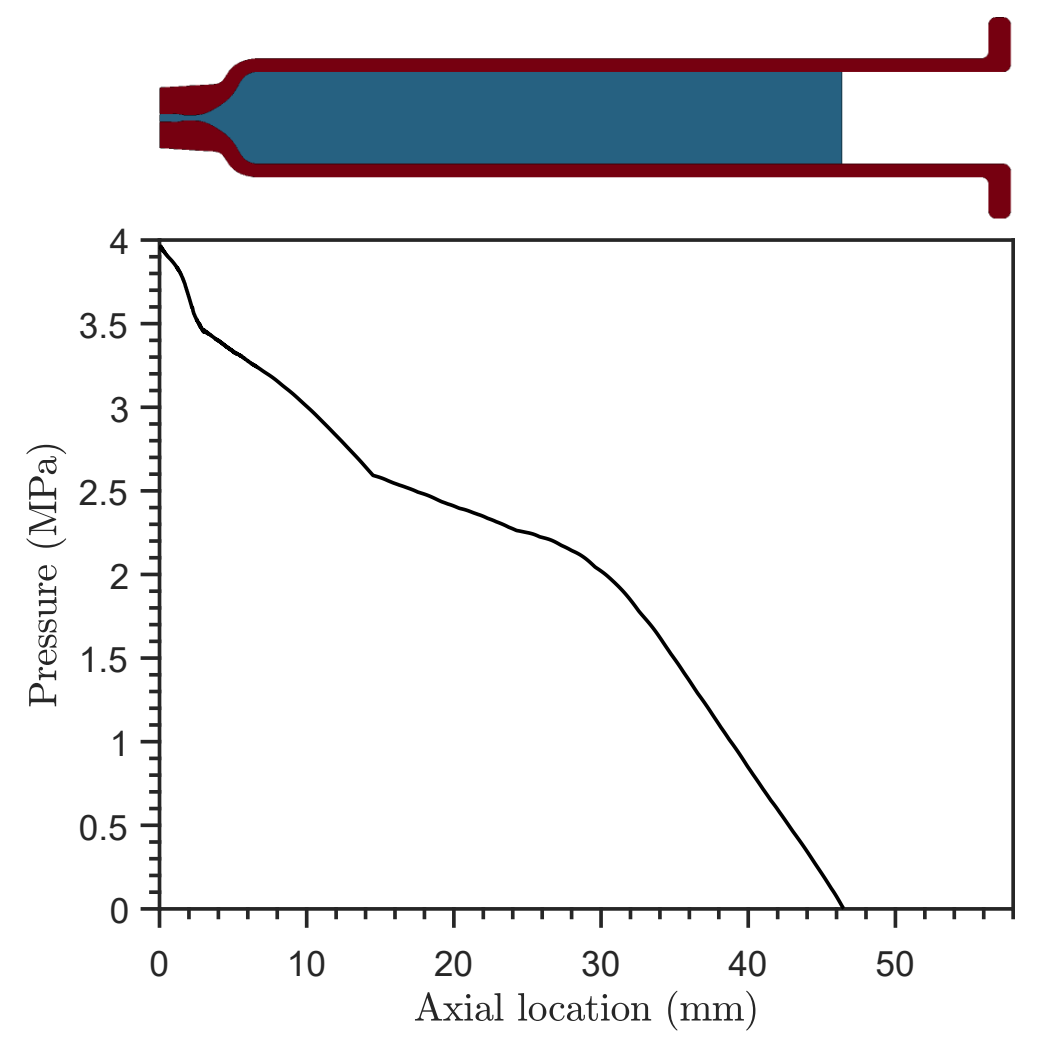

Figure 5.23: Maximum pressure along the inner surface of the syringe. The syringe and its liquid content are initially traveling downward, and the syringe is rapidly decelerated using a support at the flange.

concentration is expected to occur in the vicinity of the flange. There, the dominant stress component is again in the axial direction.

\subsubsection{Simply Supported Shoulder}

The case of a syringe supported at the shoulder is considered next. The maximum pressure along the inner surface of the syringe is shown in Figure 5.25. The results do not differ substantially from what was obtained for a syringe supported at the flange. The peak magnitude of the maximum pressure in the tip is $3.7 \mathrm{MPa}$, and the maximum pressure varies almost linearly to $0 \mathrm{MPa}$ moving toward the free end at the top of the liquid column.

Figure 5.26 is a plot of the maximum principal, hoop and axial components of stress along the inner and the outer surface of the syringe. The peak magnitude of the maximum principal stress on the inner surface (see Figure 5.26a) occurs in the vicinity of the shoulder. The peak magnitude is approximately $15 \mathrm{MPa}$, and 


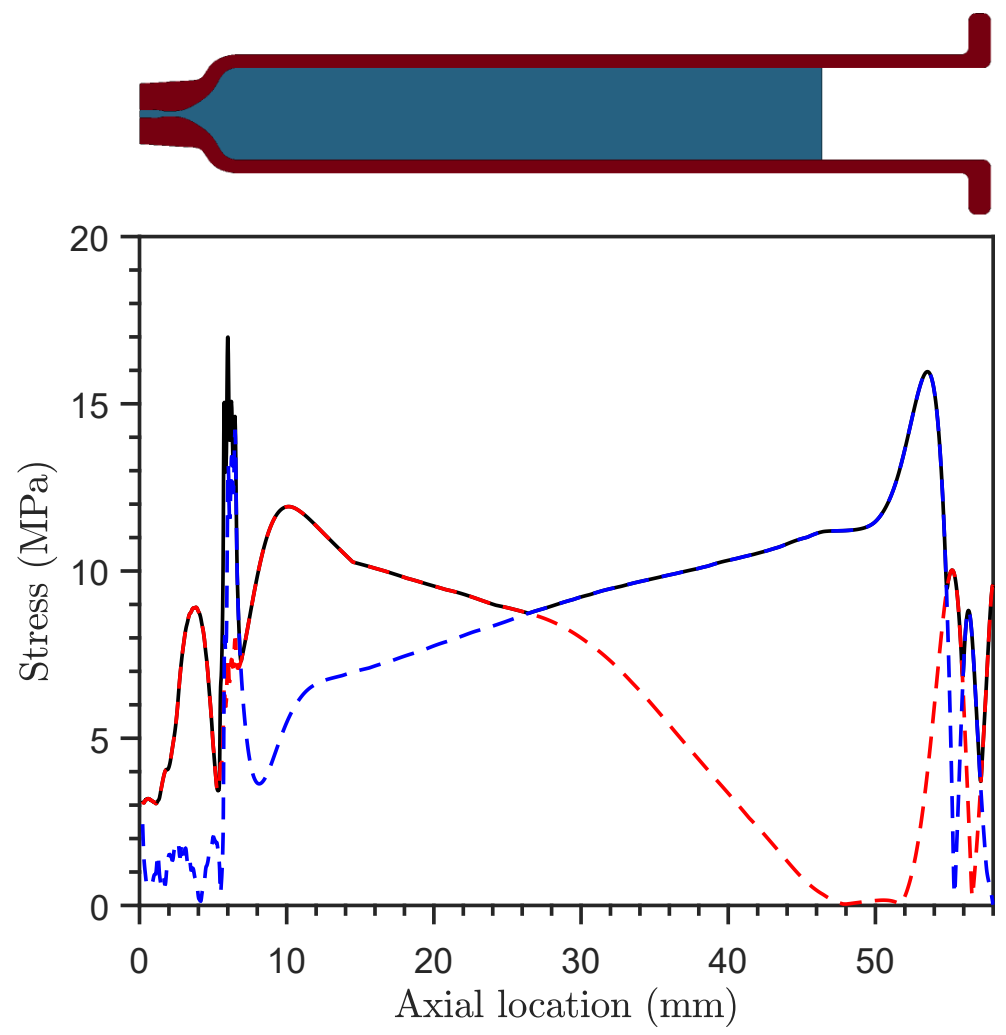

(a) Stresses along the inner surface

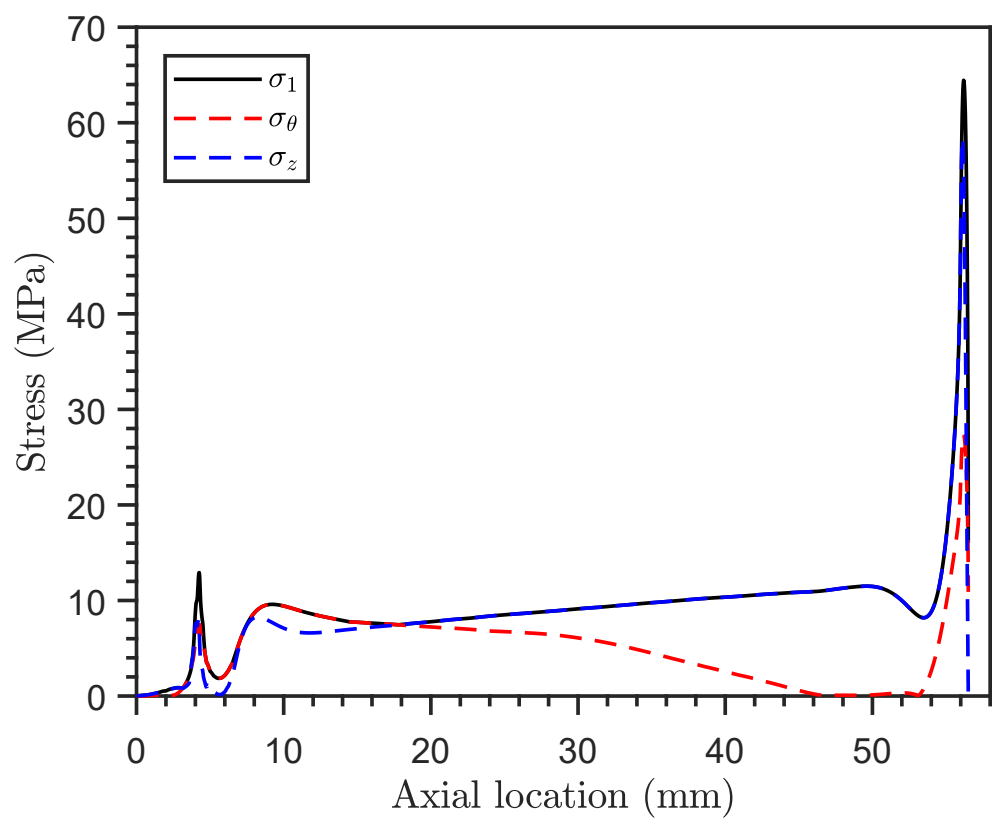

(b) Stresses along the outer surface

Figure 5.24: Maximum principal, hoop and axial stresses along the inner and the outer surface of the syringe. The syringe and its liquid content are initially traveling downward, and the syringe is rapidly decelerated using a support at the flange. 


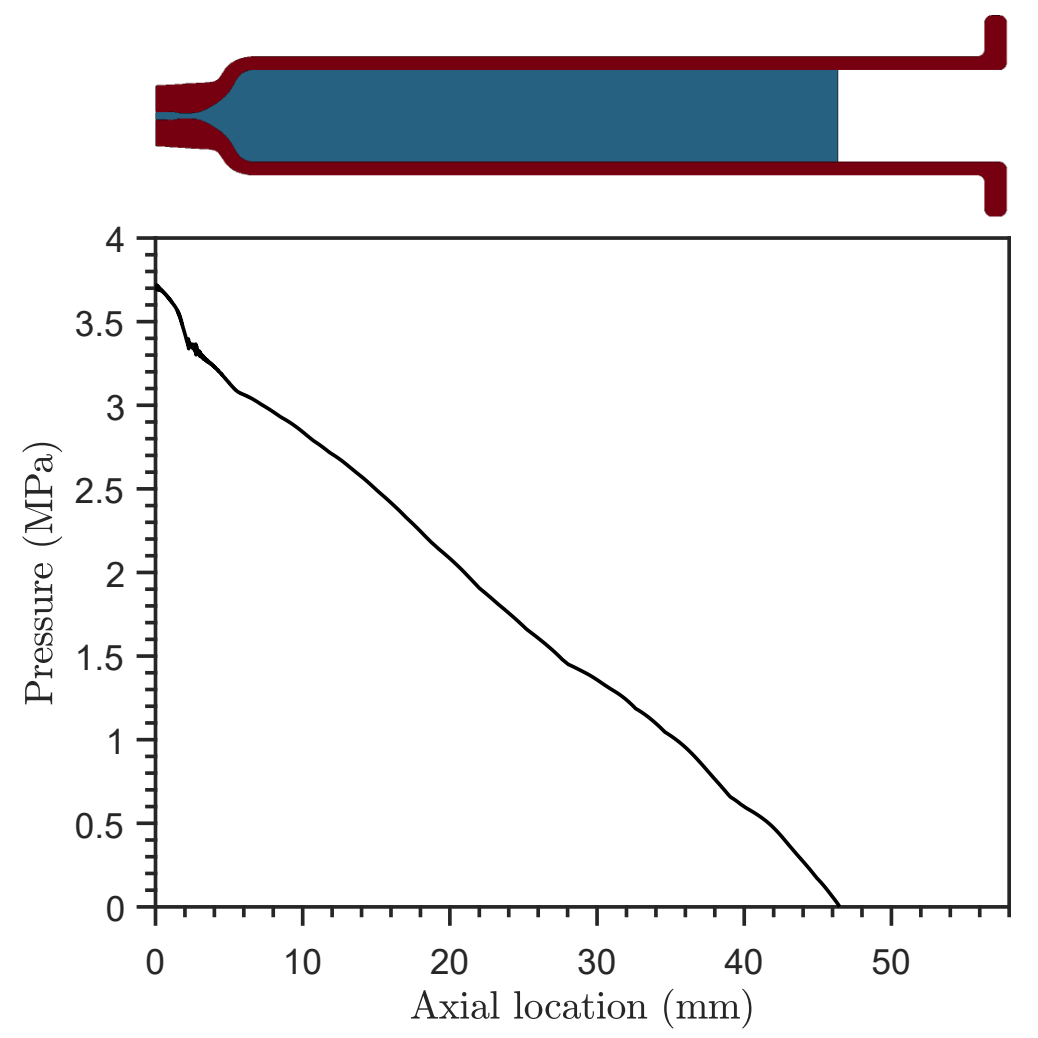

Figure 5.25: Maximum pressure along the inner surface of the syringe. The syringe and its liquid content are initially traveling downward, and the syringe is rapidly decelerated using a support at the shoulder.

both the hoop and axial stress components are of similar magnitude. The stresses are also relatively large along the bottom half of the syringe barrel, between 8 and $20 \mathrm{~mm}$. There, the hoop component of stress is dominant, indicating the stresses likely result from the pressure applied on the inner surface. The magnitude of the axial stress component is substantially less for the syringe supported at the shoulder than for the syringe supported at the flange.

The peak magnitude of the maximum principal stress (see Figure 5.26) is approximately $30 \mathrm{MPa}$ on the outer surface, in the vicinity of the shoulder. The dominant stress component is in the axial direction. This location corresponds to the position of the simple support. The outer shoulder of the syringe is where failure would most likely occur along the outer surface of the syringe.

A comparison of Figures 5.24 and 5.26 indicates that the peak magnitude of the maximum principal stress is twice as large for the syringe supported at the flange than for the syringe supported at the shoulder. This indicates that supporting the syringe 


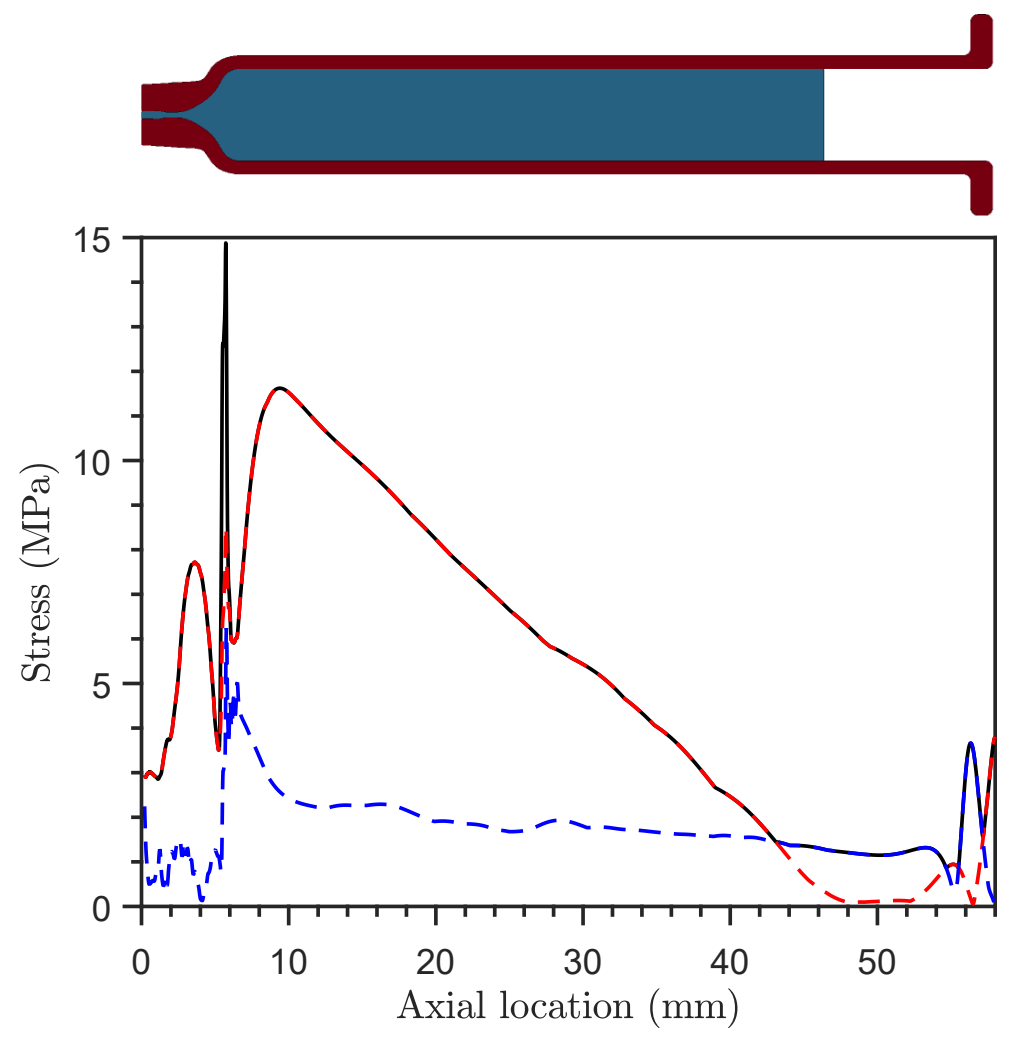

(a) Stresses along the inner surface

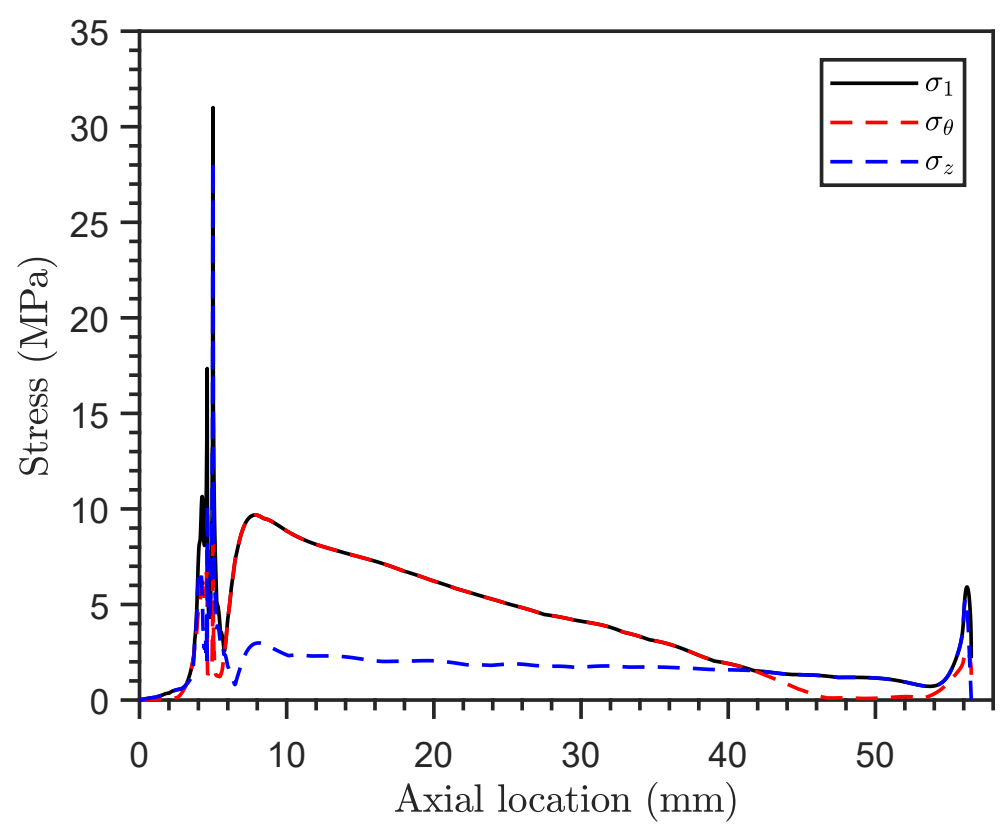

(b) Stresses along the outer surface

Figure 5.26: Maximum principal, hoop and axial stresses along the inner and the outer surface of the syringe. The syringe and its liquid content are initially traveling downward, and the syringe is rapidly decelerated using a support at the shoulder. 
at the shoulder during the deceleration is more appropriate because it reduces the probability for syringe failure.

\subsubsection{Summary of Stresses Created by Syringe Deceleration}

Numerical simulations were performed to predict the pressure and stresses in the syringe during the rapid deceleration event. This corresponds to event 2 of Chapter 2. When the syringe is supported at the flange, failure would most likely occur in the shoulder region, or near the junction between the flange and the barrel. When the syringe is supported at the shoulder, failure is not likely to occur in the vicinity of the flange. Instead, failure is expected to occur close to the shoulder region or the bottom half of the barrel.

The numerical results indicate that the peak stresses created by the rapid syringe deceleration occur away from the cone region. This suggests the rapid syringe deceleration can't explain the consistent cone failures observed by Amgen during clinical trials. This conclusion is consistent with that reached by the engineers at Veryst Engineering in 2014/2015 using a different modeling approach.

\subsubsection{Weibull Curves}

In engineering, the probability of failure of glass is conventionally studied using Weibull distributions (Weibull, 1951). Amgen has prepared Weibull curves for various commonly used pre-filled syringes (private communication). The data used to create the Weibull curves was obtained experimentally using the Ram-Rod testing facility at Amgen. In the Ram-Rod facility, a syringe with or without the tip is supported using the shoulder. The syringe, which is filled with water, is sealed with a plunger-stopper. A rod impacts on the plunger-stopper at a known velocity, creating pressure waves in the syringe. The impact velocity of the rod on the plunger-stopper is varied over a relatively large range of values. The result of each test is binary: failure (1) or no-failure (0).

For each syringe model tested, the first step is to calibrate the testing facility. The calibration step consists of measuring the peak magnitude of pressure inside the syringe as a function of the impact velocity of the rod on the plunger-stopper. A schematic of the configuration used during the calibration phase is shown in Figure 5.27a. Note that the tip of the syringe is removed to perform this calibration. Removal of the tip is necessary in order to position a piezoelectric pressure transducer 


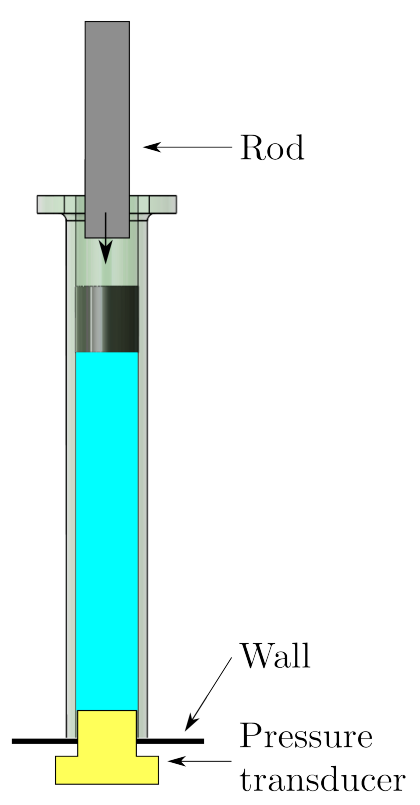

(a) Calibration

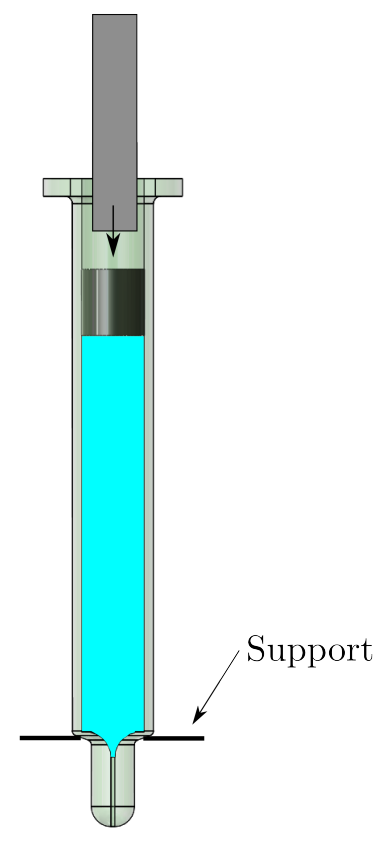

(b) Failure test

Figure 5.27: Schematic of the Ram-Rod testing facility used to construct Weibull curves of various pre-filled syringes.

at the bottom end of the syringe. There is no air gap between the plunger-stopper and the liquid.

The impulsive acceleration of the plunger-stopper into the syringe barrel results in the formation of a pressure wave in the liquid. The pressure wave propagates from top to bottom. The wave, upon reaching the bottom end of the syringe, reflects on the surface of the pressure transducer, resulting in the doubling of the pressure: the peak pressure in the vicinity of the pressure transducer is approximately twice as large as the magnitude of the pressure wave created immediately below the plunger-stopper. The second step is to test a large number of syringes, and to vary the impact velocity of the rod on the plunger-stopper. The tests are performed in the configuration shown in Figure 5.27b: the tip of the syringe is present. For each test the binary result failure (1) or no-failure (0) is recorded. The third and last step is to fit a Weibull curve to the data obtained.

The experiments performed with the Ram-Rod machine are very similar to the numerical experiments reported in Section 5.2.3.2: the syringe is supported the shoulder, and the liquid is impulsively pressurized. Testing with the Ram-Rod 


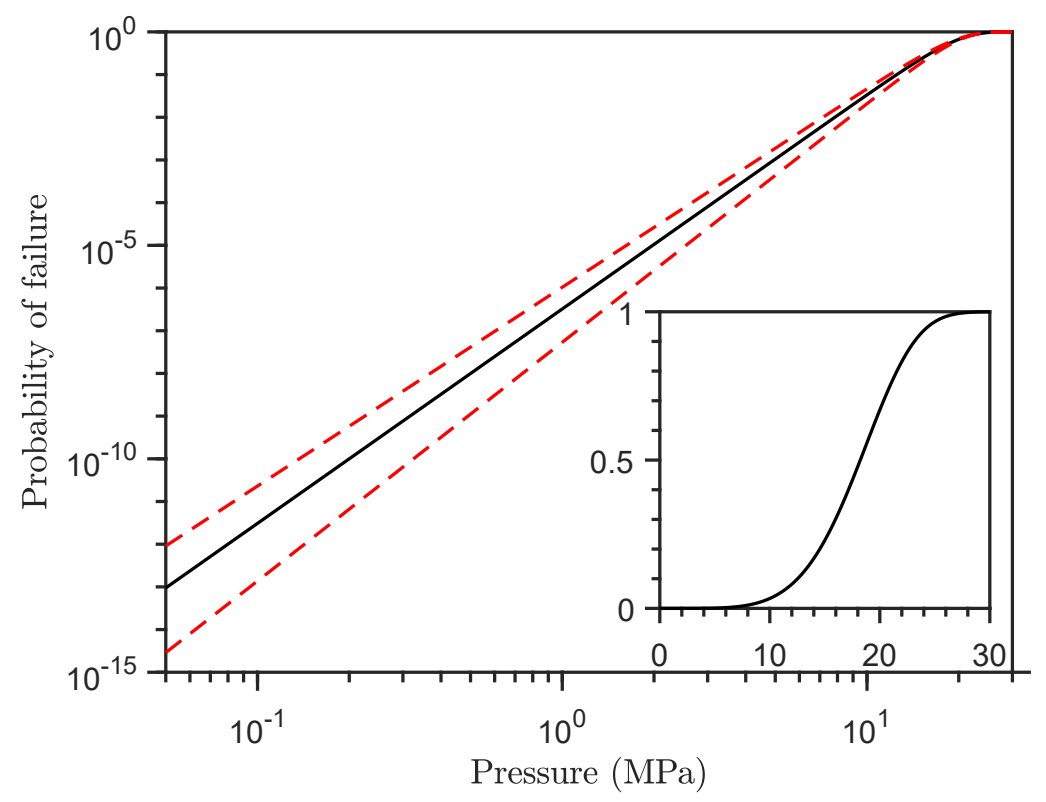

Figure 5.28: Probability of failure as a function of the peak magnitude of the pressure wave created under the plunger-stopper (Weibull curve) for a $1 \mathrm{~mL} \mathrm{BD}$ Hypack pre-filled syringe. The main plot uses double logarithmic coordinates so that the regime of small failure probability is visible. The inset graph shows the more conventional linear representation.

facility has shown that failure, when it occurs, originates in the shoulder region. This observation is consistent with the results reported in Section 5.2.3.2.

Figure 5.28 is a plot of the Weibull curve obtained by Amgen for a $1 \mathrm{ml}$ BD Hypack pre-filled syringe. The red-dashed curves correspond to the $95 \%$ confidence interval. The probability of failure is indicated as a function of the magnitude of the incident pressure wave created under the plunger-stopper. However, in order to apply this to evaluating the potential of glass breaking in an autoinjector the dependent variable needs to be converted to the maximum principal stress.

It is possible to approximately convert from pressure to maximum principal stress using the numerical results from Section 5.2.3.2: the peak magnitude of the normalized maximum principal stress in the shoulder is approximately 12 . Using this simple scaling, the Weibull curve shown in Figure 5.29 is obtained.

The failure rate observed in the field during Amgen's clinical trials was approximately 30 parts-per-million $\left(3 \times 10^{-5}\right)$. The Weibull curve indicates this failure rate could result from a maximum principal stress ranging between 24.6 and $37.2 \mathrm{MPa}$ (the most probable value is $29.4 \mathrm{MPa}$ ). This value and the approach taken in ana- 


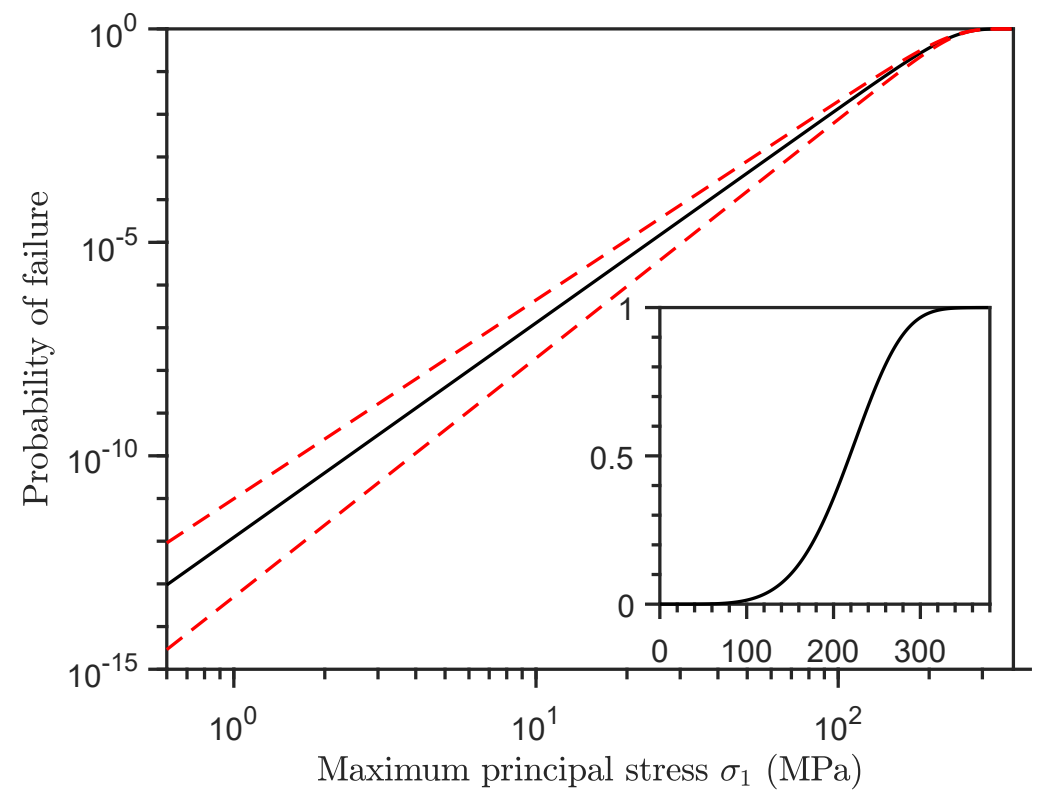

Figure 5.29: Probability of failure as a function of the peak magnitude of the maximum principal stress in the syringe shoulder (Weibull curve) for a $1 \mathrm{~mL} \mathrm{BD}$ Hypack pre-filled syringe. The main plot uses double logarithmic coordinates so that the regime of small failure probability is visible. The inset graph shows the more conventional linear representation.

lyzing the Ram-Rod test results assumes the failure mechanism is independent from the strain rate, the impulse of the pressure wave, or the geometry of the region where failure occurs. All of these factors are known to play a role in determining failure threshold and mechanisms, and the present approach is quite simplistic. While understanding the failure mechanism deserves further attention, this is outside the scope of this thesis.

\subsubsection{Bubble Collapse}

The numerical results obtained so far indicate the impulsive pressurization and the rapid deceleration of the syringe can't explain the consistent failures originating in the cone. The only dynamic event which remains to be investigated is the rapid syringe acceleration. Experiments reported in Chapters 3 and 4 have shown that cavitation bubbles form and collapse in the cone as a result of the syringe acceleration (event 1 of Chapter 2). This type of event is known to locally create large pressures and stresses (Brennen, 1995; Franc and Michel, 2005).

The generation of pressure waves due to bubble growth and collapse within the cone of the syringe is not directly modeled in LS-DYNA because validation testing 


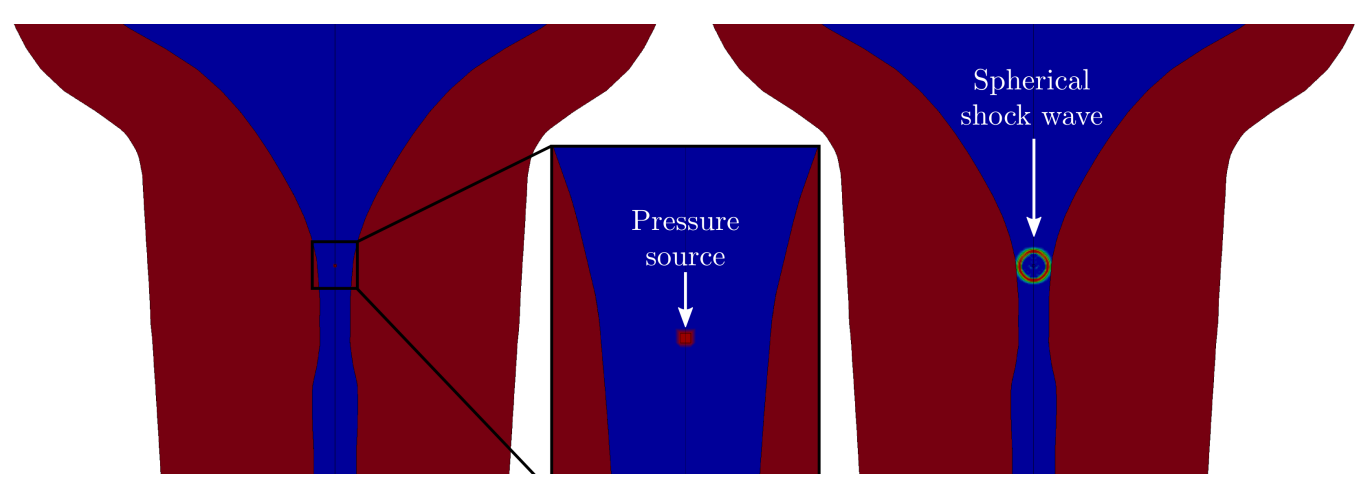

Figure 5.30: Initial pressure source (left \& center) and spherical shock wave (right) used in the LS-DYNA simulations to predict wall stresses.

indicated that the software is poorly suited for this purpose. Instead, the bubble dynamics inside the cone is modeled using more advanced numerical tools. This work is shown and discussed in Chapter 6.

In order to estimate the wall stresses which could be created by the collapse of a bubble inside the cone of a $1 \mathrm{~mL}$ BD Hypack glass syringe, LS-DYNA was used to compute pressure waves from a localized energy source to mimic the effect of the collapsing bubble (see Figure 5.30). The pressure waves are generated by a small region, the size of two grid elements, where the pressure is initially larger than the pressure in the surrounding grid elements by 0.15 to $3.0 \mathrm{GPa}$. This results in the production of a spherical pressure wave propagating away from the source, toward the wall of the syringe. The magnitude of the pressure created on the wall is 2 to $40 \mathrm{MPa}$. The spherical pressure wave is analogous to the pressure wave which would be created by the spherical collapse of a bubble inside the cone of the syringe. The reflection of the spherical pressure wave on the syringe inner surface creates stresses and strains in the cone. This approach is approximate and does not account for the loss of symmetry of the bubble and the formation of a re-entrant jet during collapse, resulting in the production of a wave which is not perfectly spherically symmetric.

The peak magnitude of the maximum principal stress on the inner surface of the cone occurs shortly after the pressure wave reflects on the wall; the maximum stress occurs before the stress waves created in the syringe wall can travel through the thickness of the wall. For this reason, the maximum stress on the inner surface does not depend on the location of the simple support.

Figure 5.31 is a plot of the peak magnitude of the normalized pressure and stress components on the inner surface of the cone. The results are normalized using $P_{\max }$, 
which corresponds to the peak magnitude of the pressure created by the pressure wave on the wall. The peak pressure occurs at $z=2.67 \mathrm{~mm}$, which corresponds to the axial location of the pressure source. The pressure decays on either side of $z=2.67 \mathrm{~mm}$. There are three peaks of the maximum principal stress:

- 0.53 at $z=2.35 \mathrm{~mm}$ (dominated by the axial component of stress)

- 0.47 at $z=2.67 \mathrm{~mm}$ (dominated by the hoop component of stress)

- 0.50 at $z=2.94 \mathrm{~mm}$ (dominated by the axial component of stress)

The results indicate that the peak value of the normalized maximum principal stress on the inner surface is approximately 0.5 .

The dimensional magnitude of the stresses created in the wall of the syringe can be evaluated once the peak wall pressure created by the collapse of a cavitation bubble is known. Estimating the peak pressure due to bubble collapse is a difficult task. The work reported in Chapter 6 suggests the collapse of a bubble inside the cone of a syringe could easily create peak wall pressures in the range of $20-100 \mathrm{MPa}$, indicating that the peak magnitude of the maximum principal stress could range between 10-50 MPa. Based on the Weibull curve shown in Figure 5.29, bubbles collapsing in the cone could possibly account for the 30 part-per-million failure rate observed during clinical trials.

In summary, estimates for the maximum principal stress created in the cone as a result of spherical pressure wave simulating bubble collapse were obtained. This model process indicates that a bubble collapsing inside the cone could potentially create a maximum principal stress with a peak magnitude between $10 \mathrm{MPa}$ and $50 \mathrm{MPa}$. The very localized nature of the bubble collapse combined with the rapid decay in $1 / r$ of the pressure waves created upon collapse result in the rapid decay of the peak wall stresses moving away from the axial location of the bubble. This indicates that the probability of failure due to bubble collapse is significant only in the vicinity of the bubble: a bubble collapsing in the cone can only cause failure in the cone region.

These results suggest that bubbles collapsing inside the cone could explain the consistent cone failures observed during clinical trials. The Weibull curve obtained earlier indicates that a maximum principal stresses with a peak magnitude of 10$50 \mathrm{MPa}$ can create a small but detectable failure rate of the glass syringes based 


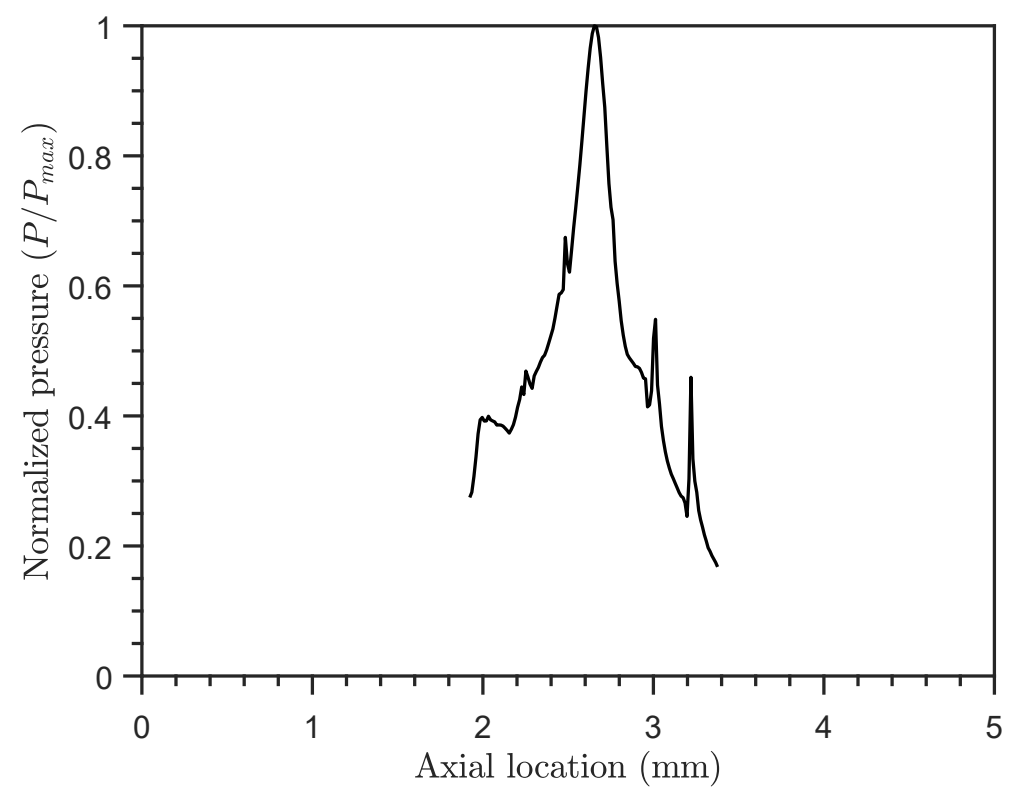

(a) Stresses along the inner surface

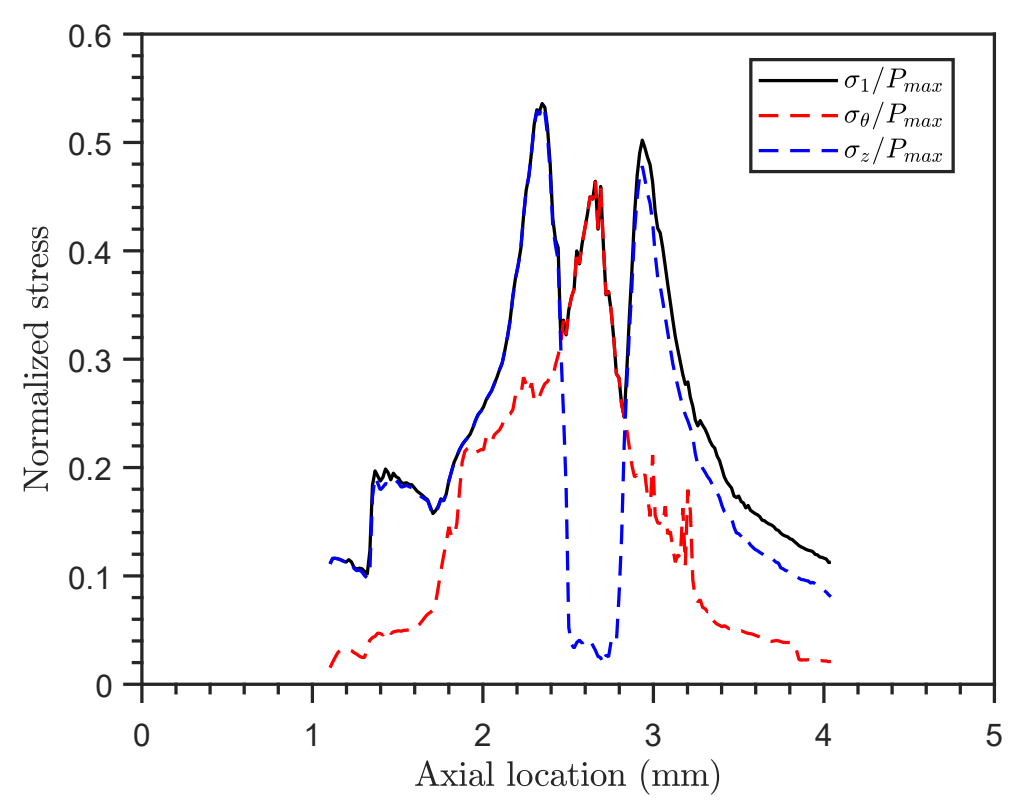

(b) Stresses along the outer surface

Figure 5.31: Pressure, maximum principal stress, hoop stress and axial stress along the inner surface of the syringe. The pressure and stresses are created by a spherical pressure wave similar to the wave created by a bubble collapsing in the cone. 
on the observed $30 \mathrm{ppm}$ failure rate and the interpretation of the Ram-Rod tests in terms of a 24.6 to 37.2 MPa maximum principal stress (see Figure 5.29). 


\section{Chapter 6}

\section{BUBBLE DYNAMICS IN A CONE}

Chapters 2, 3, and 4 have shown that cavitation can occur in the syringe of an autoinjector device during actuation. The results from Chapters 3 and 4 demonstrate that large cavitation bubbles can form, grow, and collapse inside the cone of a syringe, and this can create large pressures and stresses. Peak pressures as large as $50 \mathrm{MPa}$ were measured at the apex of the cone in the large-scale model autoinjector test setup. The results from Chapter 5 obtained with LS-DYNA suggest the collapse of a cavitation bubble is the likely root cause of the syringe failures observed by Amgen during clinical trials.

In this chapter, the shock-induced collapse of a bubble in a straight cone is studied using numerical simulations. The effect of bubble placement within the cone and the effect of the half-angle of the cone are both investigated. The collapse of a bubble in a geometry that resembles a pre-filled syringe is also simulated. The work presented herein is a preliminary study, and more work is needed to develop a better understanding of the rich physics at play. This chapter is not a comprehensive review of cavitation and bubble dynamics. The interested reader should see the monographs of Brennen (1995) and Franc and Michel (2005) for a thorough introduction to the subject.

This chapter has two objectives. The first one is to estimate the peak pressure created on the walls of the cone terminating the syringe upon bubble collapse. The second objective is to determine whether or not the cone has an effect on the collapse of a cavitation bubble.

\subsection{Collapse of a Cavitation Bubble}

It is well known that the collapse of cavitation bubbles can create large pressures and temperatures. Solutions to the Rayleigh-Plesset equation ${ }^{1}$ suggest the peak pressures and temperatures created by the spherical collapse of a bubble could be as large as $10^{9} \mathrm{MPa}$ and $10^{7} \mathrm{~K}$ (Brennen, 1995). Real effects such as diffusion and

\footnotetext{
${ }^{1}$ The Rayleigh-Plesset equation assumes the bubble remains spherical during the growth and the collapse, and compressibility effects are not included in either the gas or surrounding liquid, in addition, mass and energy transfer between the gas and liquid are neglected.
} 
compressibility however prevent the pressure and temperature from reaching such large values.

The nonspherical collapse of a bubble is complex and results in the creation of a re-entrant jet (Plesset and Chapman, 1971). Non-spherical collapses occur when the collapse of the bubble results from the propagation of a shock wave and/or when the bubble collapses in the vicinity of a wall or free surface. The non-spherical collapse of a bubble is known to create more than one shock wave; the structure of the shocks which are emitted depends on the degree of asymmetry during bubble collapse (Supponen et al., 2017).

Fujikawa and Akamatsu (1980) have measured peak pressures as large as $100 \mathrm{MPa}$ upon bubble collapse. More recently, Supponen et al. (2017) reported peak pressures of order $2 \mathrm{GPa}$. The pressure was not measured at the center of the collapsing bubble; it was measured with a transducer located some distance away from the center of the bubble. The pressure pulse created by the collapse of a bubble decays approximately in $1 / r$, where $r$ is the distance measured from the center of the bubble (Brennen, 1995; Franc and Michel, 2005). This indicates the distance between the pressure transducer and the bubble can affect largely the measured pressure.

It is possible to estimate the magnitude of the pressure pulse radiated away from a collapsing bubble with the following rule of thumb:

$$
\Delta P \approx 100 P_{\infty}\left(\frac{R_{m}}{r}\right)
$$

where $R_{m}$ is the maximum bubble radius, and $P_{\infty}$ is the undisturbed pressure far from the bubble (Brennen, 1995). According to this rule of thumb, a bubble collapsing in a liquid where $P_{\infty}$ is approximately atmospheric pressure, such as in a syringe, could create a pressure pulse with magnitude $10 \mathrm{MPa}$ at $r=R_{m}$.

The reflection of the radiated pressure pulse on a wall located in the vicinity of the bubble can create a further pressure increase. A rigid wall, for example, results in the doubling of the pressure upon reflection of the pulse. A wall positioned close to the bubble, however, has an effect on the bubble collapse itself: there is a loss of symmetry during the collapse, and a re-entrant jet or microjet forms, as indicated earlier. The velocity of the microjet can be over $100 \mathrm{~m} / \mathrm{s}$ (Lauterborn and Bolle, 1975), and it is directed toward the wall. The microjet is capable of creating substantial pressure pulses with a magnitude of order $150 \mathrm{MPa}$ (Franc and Michel, 2005). The collapse of a bubble due to the passage of a shock wave results in a 
similar loss of symmetry and the formation of a re-entrant jet, even when no wall is present (Bourne and Field, 1992; Haas and Sturtevant, 1987).

The study of cavitation damage and erosion is still an active research subject receiving great attention. To the author's knowledge, the shock-induced collapse of cavitation bubbles inside a straight cone has not been studied to date. This configuration is potentially interesting for several reasons:

- the cone walls are expected to result in the formation of a microjet oriented toward the apex of the cone;

- the bubble is expected to be processed by the incident pressure wave, followed by the corner wave forming when the incident wave enters the cone (see Chapter 5);

- shock focusing could occur inside the cone (see Chapter 5).

\subsection{Numerical Setup}

Except for the results shown in Section 6.5, the numerical simulations reported in this chapter are performed with an interface-capturing method for compressible multi-component flows that employs an Eulerian finite-volume, weighted essentially non-oscillatory (WENO) scheme. The details about the numerical methods and the verification $\&$ validation of the code are presented elsewhere (Coralic and Colonius, 2014; Meng, 2016).

A first step in understanding the collapse of a bubble in the cone of a syringe is to study the shock-induced collapse of a gas bubble in an otherwise quiescent media. The geometry is depicted in Figure 6.1, where a single bubble is located on the axis of symmetry to make the problem two-dimensional. This configuration avoids having to address the randomness in nucleation events. Initially an air bubble of radius $R_{0}$ is located at a distance $H_{0}$ from the apex of a straight, water-filled cone of half-angle $\alpha$ and depth $L_{c}$. The bubble is initially at equilibrium: $d R / d t=0$ at $t=0$.

An incident weak shock is traveling down the syringe barrel and enters the cone. The pressure and density ahead of the shock are respectively $P_{0}$ and $\rho_{0}$. The pressure behind the shock is $P_{s}$. Other properties are calculated with the shockjump conditions (Courant and Friedrichs, 1976). The initial pressure and density inside the air bubble are respectively $P_{b, 0}$ and $\rho_{b, 0}$. At a later time, the shock has 

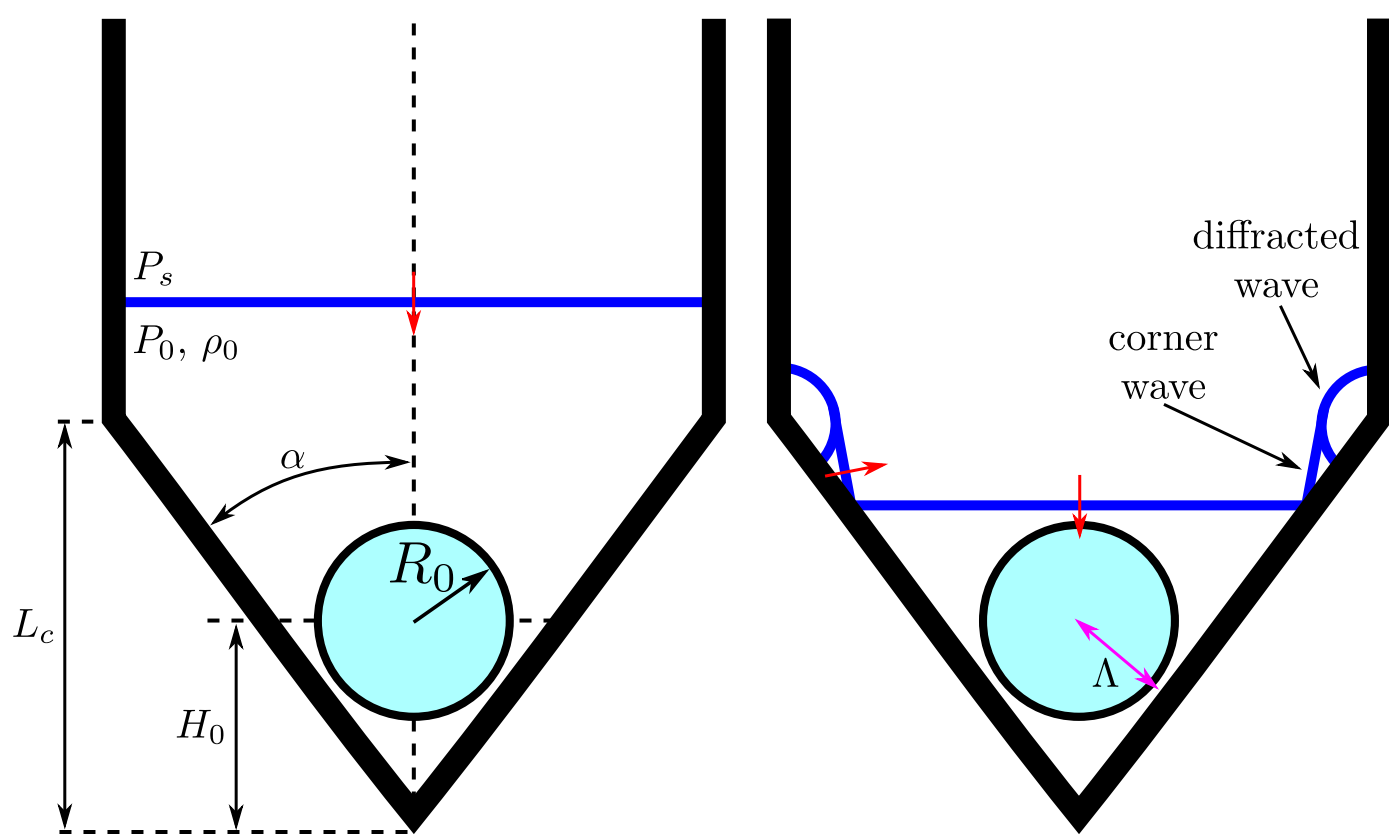

Figure 6.1: Geometry used in the numerical simulations of a bubble collapse inside a straight cone.

entered the cone, depicted on the right-hand side of Figure 6.1. A corner wave and a diffracted wave form, as explained in Chapter 5. The pressure increases in the cone, and this makes the bubble collapse.

All numerical simulations are performed in 2D axisymmetric coordinates. A uniform, structured grid is used in the shock-bubble interaction region, with a resolution of 108 cells per original bubble diameter. The grid further away from the bubble is stretched in both the axial and radial directions to reduce the computational time.

The grid resolution was selected after comparing the results obtained on a benchmark problem using the various grid resolutions reported in Table 6.1. The benchmark problem corresponds to the shock-induced collapse of an initially spherical bubble located in the vicinity of a flat wall (i.e., $\alpha=90$ degrees). The initial offset distance of the bubble from the wall is $\Lambda_{0} / R_{0}=2$, and the magnitude of the incident shock wave is $P_{s} / P_{0}=353$ (same parameters as Johnsen and Colonius (2009)). The effect of the grid on the evolution of bubble volume and the peak pressure on the wall is shown in Figure 6.2. A resolution of 108 cells per original bubble diameter represents a good compromise between computational cost and accuracy of the results. 


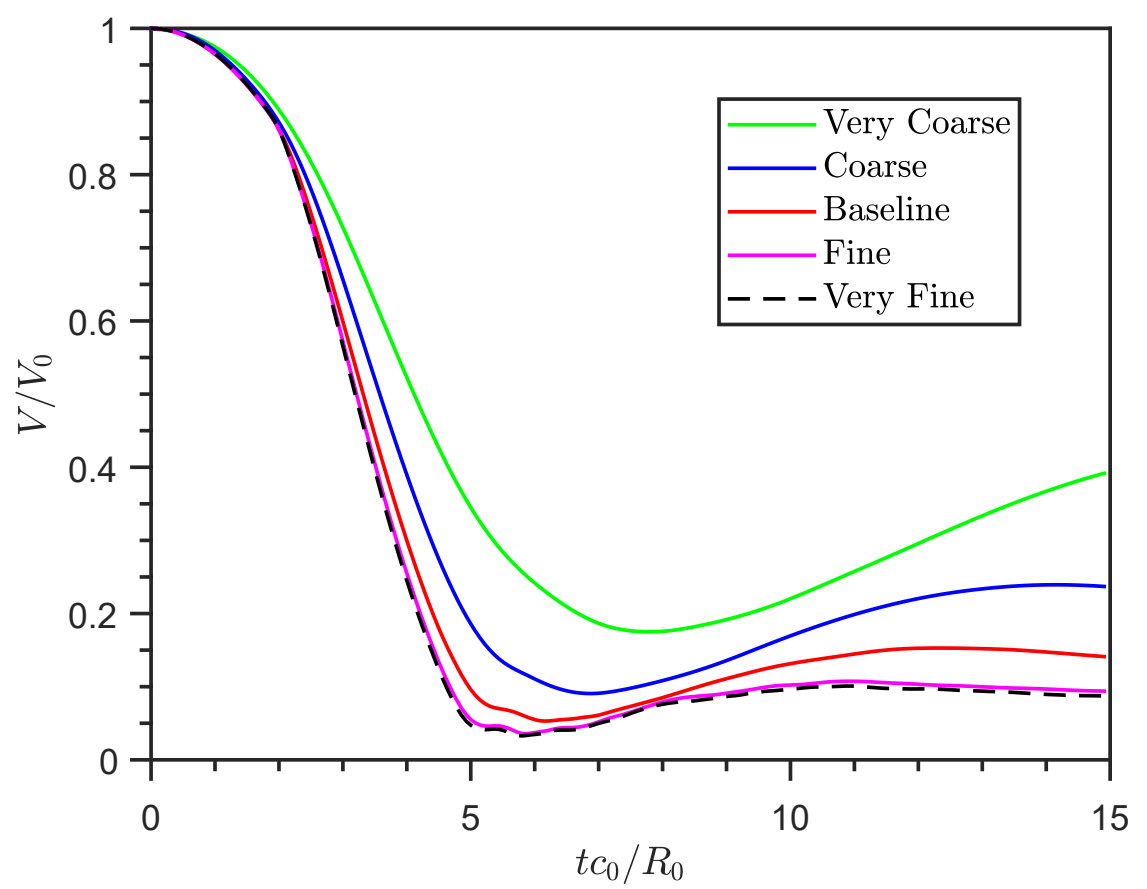

(a) Bubble volume

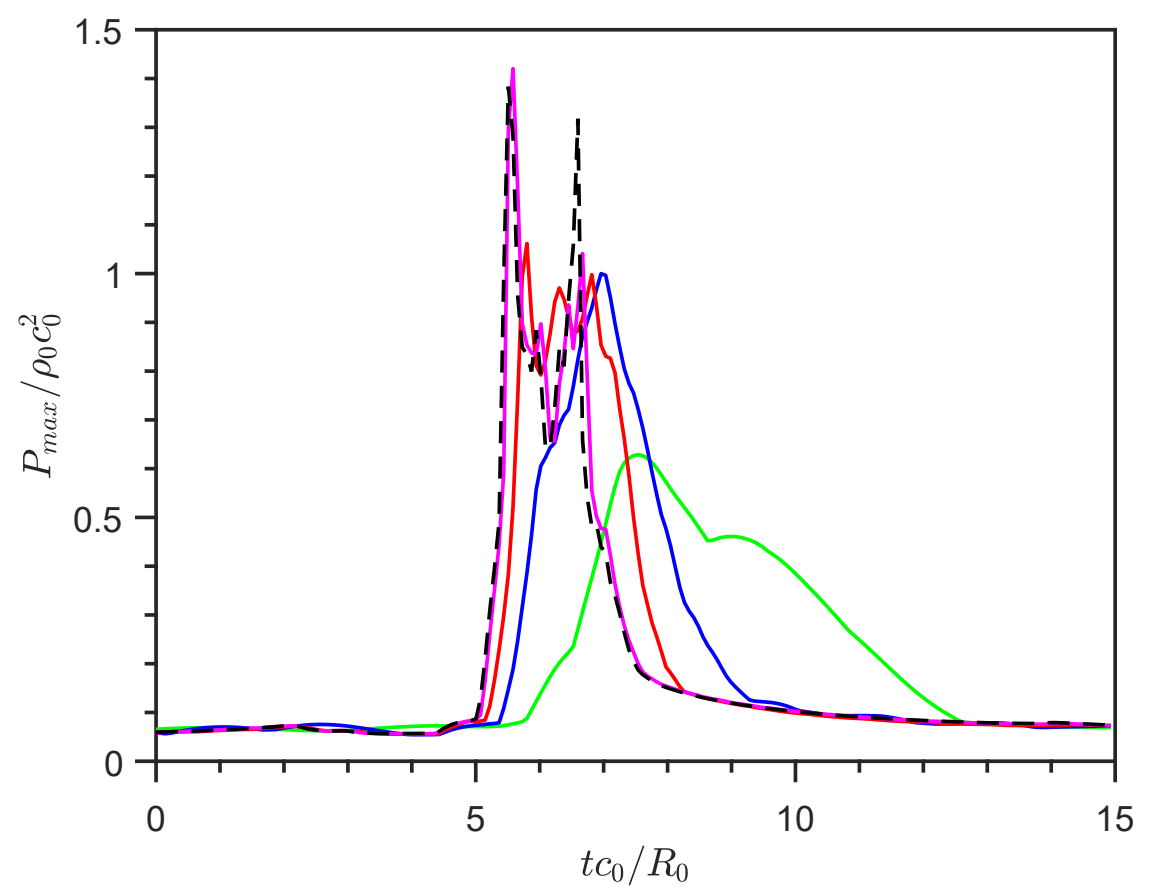

(b) Peak pressure on the wall

Figure 6.2: Effect of the grid resolution on the evolution of bubble volume and peak pressure on the wall. The grid resolutions are reported in Table 6.1. 
Table 6.1: Grid resolutions used to investigate the effect of the mesh on the shockinduced collapse of a bubble in the vicinity of a flat wall.

\begin{tabular}{r|cc}
\hline Case & $\begin{array}{c}\text { Elements per } \\
\text { bubble diameter }\end{array}$ & $\begin{array}{c}\text { Total number } \\
\text { of elements }\end{array}$ \\
\hline Very Coarse & 27 & 11,000 \\
Coarse & 54 & 44,000 \\
Baseline & 108 & 176,000 \\
Fine & 216 & 700,000 \\
Very Fine & 432 & $2,800,000$ \\
\hline
\end{tabular}

All quantities are normalized using $R_{0}, \rho_{0}$ and $c_{0}$, unless otherwise indicated. A stiffened gas equation of state (Harlow and Amsden, 1971) is used for both fluids:

$$
P=(\gamma-1) \rho e-\gamma \pi_{\infty}
$$

where $P$ is the pressure, $e$ is the internal energy, and $\rho$ is the density. The heat capacity ratio $\gamma$ and the normalized stiffness $\pi_{\infty}$ are respectively 1.4 and 0 for air, and 6.68 and 195.25 for water. Setting $\pi_{\infty}=0$ in the equation of state for air reduces Equation 6.2 to the perfect gas relation:

$$
P=(\gamma-1) \rho e .
$$

The shock-induced collapses are simulated with $P_{s} / P_{0}=500, \rho_{b, 0} / \rho_{0}=1.2 \times 10^{-3}$, $L_{c} / R_{0}=10$, and $P_{b, 0}=P_{0}$. For a cavitation bubble in water where the pressure $P_{0}$ inside the bubble is vapor pressure $(2.3 \mathrm{kPa})$, the corresponding value of $P_{S}$ is $1.15 \mathrm{MPa}$. The results from Chapter 3 have shown that pressure waves with a magnitude of 1 to $2 \mathrm{MPa}$ can be created by the impact of the driving-rod on the plunger stopper. The numerical simulations are performed with $\alpha$ ranging from 30 to 90 degrees.

The minimum distance between the center of the bubble and the oblique wall of the cone is defined as $\Lambda$ (see the right-hand side of Figure 6.1). $\Lambda$ is related to the half-angle of the cone and the initial position of the bubble:

$$
\frac{\Lambda}{R_{0}}=\frac{H_{0}}{R_{0}} \sin \alpha .
$$




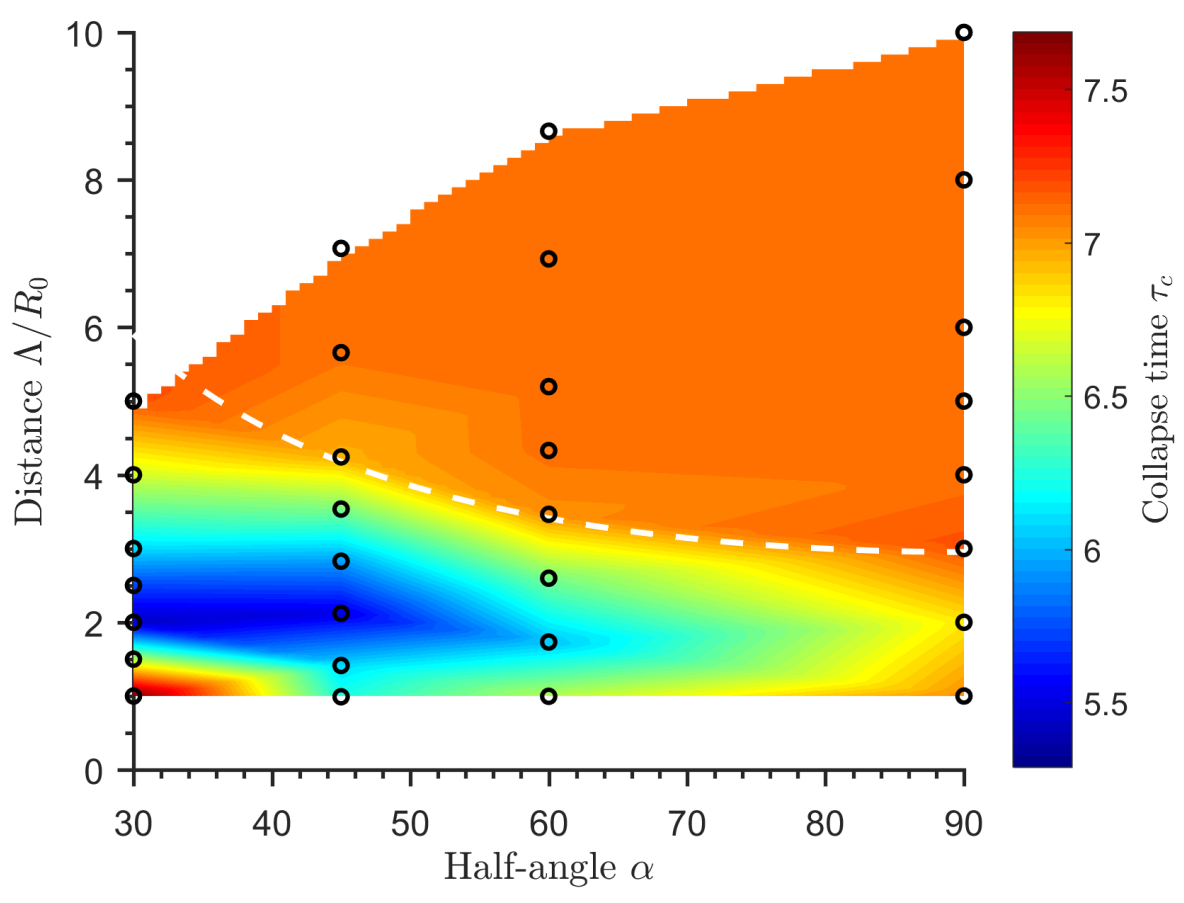

Figure 6.3: Collapse time as a function of the initial location of the bubble and the half-angle of the cone.

Note that $\Lambda=H_{0}$ for $\alpha=90$ degrees. A half-angle of 90 degrees, as in Chapter 5, corresponds to a syringe terminated with a flat wall perpendicular to the axis of symmetry of the syringe barrel: no cone is present.

\subsection{Bubble Collapse Time}

Simulation results of the bubble collapse time are discussed in this section, and a simple model which can approximately predict whether or not the collapse of a bubble is expected to be affect by the cone wall is introduced. Figure 6.3 is a contour plot of the collapse time $\tau_{c}$ (non-dimensional time is indicated with variable $\tau$ ). Each data point, indicated with an open circle, corresponds to a separate numerical simulation. The collapse time is linearly interpolated between the data points to obtain a continuous mapping of the collapse time as a function of the initial bubble location $\Lambda / R_{0}$ and the half-angle of the cone $\alpha$.

The initial bubble location is indicated using distance $\Lambda / R_{0}$ rather than $H_{0} / R_{0}$. Several numerical simulations were performed with a truncated cone identical to the one shown in Figure 5.1. The results from those simulations are not shown and discussed in this thesis. This is because the results obtained with a sharp and a truncated cone approximately collapse when plotted against $\Lambda / R_{0}$. Using $H_{0} / R_{0}$ as 
the dependent variable does not collapse the data, indicating that the key parameter is the distance between the oblique wall of the cone and the bubble, not the distance between the apex of the cone and the bubble. A physical justification for this is provided later.

The data point located in the upper-right corner of Figure 6.3 is for a bubble which is initially located relatively far from the bottom end of a syringe terminated by a flat wall. The collapse time is 7.1, identical to the shock-induced collapse time of a bubble in free space. Because the bubble is so far from the tip, it collapses before the incident wave can complete a round trip between the bubble and the bottom wall. The bubble does not feel the presence of the wall until after it has collapsed.

The bubble collapse time is less than 7.1 in the region located below the white-dashed curve (the significance of the white-dashed curve is discussed later), indicating a more rapid bubble collapse due to the cone. For constant values of $\Lambda / R_{0}$ (i.e., horizontal lines in Figure 6.3), reducing the half-angle reduces the collapse time, indicating that cones with a shallow half-angle can enhance the collapse more substantially than cones with a large half-angle. For constant values of $\alpha$ (i.e., vertical lines in Figure 6.3), the collapse time is minimum at approximately $\Lambda / R_{0}=2$. The collapse of a bubble which is far from the cone is only weakly enhanced or not enhanced at all by the cone. Additionally, the collapse of a bubble located very close to the oblique wall of the cone (i.e., $\Lambda / R_{0} \approx 1$ ) is not enhanced as much as the collapse of a bubble which is initially located at $\Lambda / R_{0} \approx 2$.

Figure 6.4 is a plot of the bubble volume history as a function of time. The bubble volume is normalized with the initial bubble volume $V_{0}$. The simulation results shown in Figure 6.4 are for a half-angle of 45 degree, and the bubble volume history for different values of $\Lambda / R_{0}$ are shown. The bubble volume history for the shockinduced collapse of a bubble in free space is also indicated. Note that time $\tau=0$ corresponds to the arrival of the incident wave on the proximal side of the bubble.

The first portion of bubble collapse is independent from the initial location of the bubble: the volume history of all bubbles follows closely the volume history for the bubble collapsing in free space. For example, with $\Lambda / R_{0}=1.4$ the evolution of bubble volume matches the results obtained for a collapse in free space until $\tau=2.1$. The instant when deviation from the free space collapse occurs is referenced as the "critical time", or $\tau_{\text {crit }}$. After the critical time, the rate at which the bubble collapses is noticeably increased. The critical time appears to be a function of the initial 


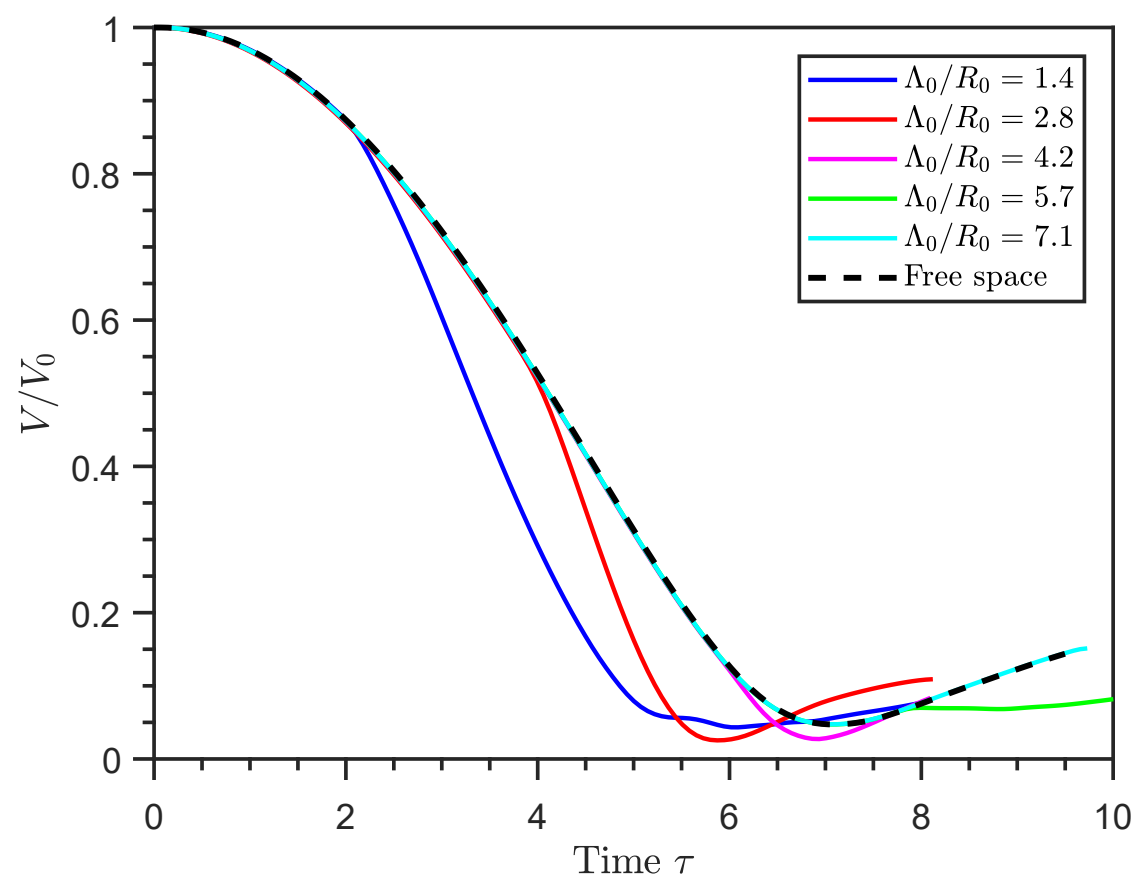

Figure 6.4: Evolution of the bubble volume during collapse in a 45-degree cone.

bubble location: $\tau_{\text {crit }}$ increases with $\Lambda / R_{0}$. For relatively large values of $\Lambda / R_{0}$, the critical time occurs after the bubble reaches its minimum volume $\left(\tau_{c r i t}>\tau_{c}\right)$.

The bubble collapse rate enhancement which occurs around the critical time is demonstrated using Figure 6.5, a sequence of images of the collapse of a bubble in a 45-degree cone with $\Lambda / R_{0}=2.8$. The objective is to identify the mechanism responsible for increasing the collapse rate. The top of each frame is a contour plot of the pressure field. Note that a logarithmic scale is used to color the pressure levels. The bottom of each frame is a numerical schlieren. The bubble volume history for this specific case is plotted at the bottom of Figure 6.5, and the time which corresponds to each frame is indicated with red-dashed lines.

Frame $1(\tau=0)$ is when the incident wave arrives on the distal side of the bubble, and begins to process the bubble. The oblique wall of the cone, the boundary of the bubble, the incident wave and the corner wave are all identified. The incident wave diffracts around the bubble and increases the pressure on the proximal and the distal sides, thus initiating the bubble collapse.

Frame $2\left(\tau=\tau_{c r i t}\right)$ is when the corner wave first begins to process the bubble. This is also when the rate of collapse of the bubble is noticeably increased. Frames 2, 3 and 4 indicate that the corner wave further increases the pressure in the vicinity 


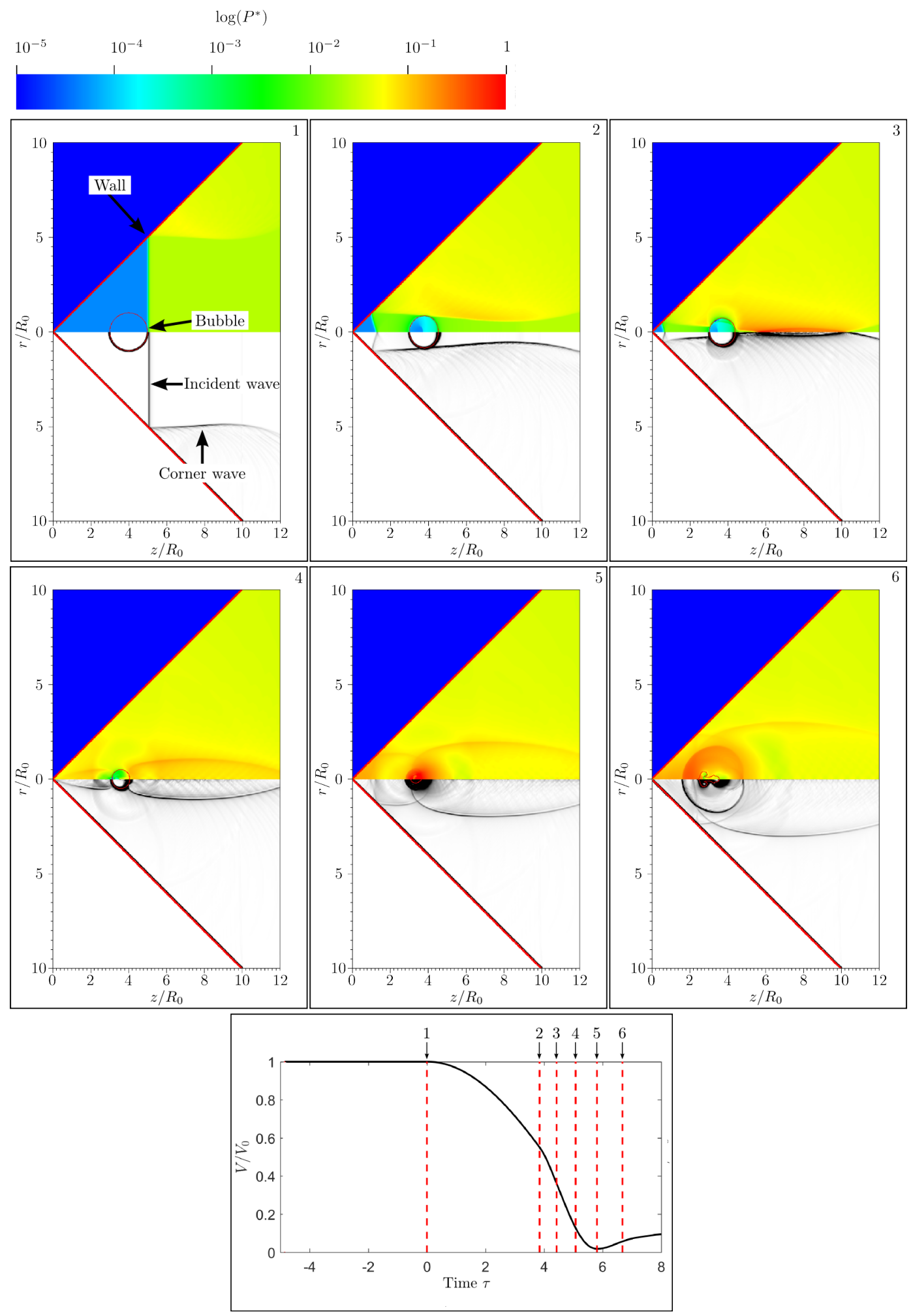

Figure 6.5: Shock-induced collapse of a bubble initially located at $\Lambda / R_{0}=2.8$ in a 45-degree cone. 
of the bubble, which explains the increased rate of collapse. The corner wave, as explained in Chapter 5, is due to the reflection of the incident wave on the oblique wall, and it converges on the axis of symmetry of the cone. The magnitude of the corner wave increases as it converges toward the axis of symmetry. The collapse and rebound of the bubble along with the formation of a shock wave is illustrated in frames 5 and 6 . Note that the shock wave which forms upon bubble collapse and propagates toward the wall is responsible for creating stresses and strains in the wall. The spherical shock wave used in the LS-DYNA simulations of Section 5.2.6 aims at mimicking the effect of this shock wave.

The corner wave is responsible for increasing the rate at which the bubble collapses. Knowing this, it is possible to develop a simple model to predict whether or not a combination of $\Lambda / R_{0}, P_{S} / P_{0}$ and $\alpha$ can result in the enhancement of bubble collapse. Rayleigh (1917) was able to obtain an estimate for the collapse time of a bubble, known as the Rayleigh collapse time:

$$
\tau_{c}=0.915 \sqrt{\frac{1}{P_{s}-P_{b, 0}}} .
$$

Rayleigh obtained this estimate by solving the Rayleigh-Plesset equation. It corresponds to the time needed for a void to collapse completely, from $R=R_{0}$ to $R=0$. This estimate is for a Rayleigh or natural collapse, not a shock-induced collapse. The problem considered herein does not correspond to the complete, natural collapse of a void. Nevertheless, this estimate is useful in scaling numerical results or obtaining an approximate value for the collapse time when analyzing bubble collapse.

The Rayleigh collapse time estimated using Equation 6.5 is approximately 6.1, one time unit shorter than the free-field shock-induced collapse time of 7.1 predicted through numerical simulations. The shock induced collapse time of a bubble in free space is indeed expected to be larger than the Rayleigh collapse time, as explained by Johnsen and Colonius (2009). This is because the shock wave in a shock-induced collapse requires a finite time to travel from the proximal to the distal side of the bubble; the pressure increase around the bubble occurs over a finite time. On the contrary, in a Rayleigh collapse the pressure increase uniformly and instantaneously all around the bubble. Johnsen and Colonius (2009) indicate the difference between the Rayleigh collapse time and the shock-induced collapse time is approximately one time unit. The numbers reported above (7.1 vs 6.1$)$ are in agreement with this estimate. 
Because the incident and the corner waves are weak, they can be considered acoustic disturbances traveling at sound speed. Using this information in combination with geometry, it is useful to estimate the critical time $\tau_{c r i t}$ :

$$
\tau_{\text {crit }} \approx 2\left(\frac{\Lambda}{R_{0}}\right) \sin \alpha .
$$

One limitation of this equation is that the bubble radius is assumed to be equal to $R_{0}$ at $\tau_{\text {crit }}$. This is obviously not true, as shown in Figure 6.4, because the bubble begins to collapse at $\tau=0$ rather than $\tau_{\text {crit }}$. The actual value of $\tau_{\text {crit }}$ is larger than the value obtained using the approximation of Equation 6.6. This is because the corner wave needs to travel a greater distance than assumed before it can reach the boundary of the bubble.

There are two cases to be considered. The first case is when $\tau_{c}<\tau_{c r i t}$ : the corner wave processes the bubble only after the bubble has fully collapsed, and the collapse of the bubble is not expected to be affected by the cone. The second case is when $\tau_{c}>\tau_{c r i t}$ : the corner wave processes the bubble before it fully collapses, and the bubble collapse is expected to be affected by the cone.

Equations 6.5 and 6.6 are combined to obtain the following inequality for determining if the corner wave reaches the bubble before the bubble collapse:

$$
\frac{\Lambda}{R_{0}}<\frac{0.915}{2 \sin \alpha} \sqrt{\frac{1}{P_{s}-P_{b, 0}}} .
$$

If the inequality is satisfied the corner wave is expected to process the bubble prior to bubble collapse, and the cone is expected to enhance the collapse. This inequality is satisfied in the region located below the white-dashed curve shown in Figure 6.3. The agreement between the model and the simulation results suggest the model is reasonable. The collapse time above the white-dashed curve in Figure 6.3 is approximately constant and equal to the shock-induced collapse time of a bubble in free space $\left(\tau_{c}=7.1\right)$, and the collapse time below the white-dashed curve is less than 7.1 .

Equation 6.7 indicates that increasing $P_{s} / P_{b, 0}$ reduces the effect of the cone. For a fixed $\alpha$, increasing $P_{s} / P_{b, 0}$ increases the numerical value of the right-hand side of Equation 6.7, indicating that the bubble must be positioned deeper into the cone for the cone to affect the collapse.

As mentioned earlier, the results from Figure 6.3 indicate that the collapse of bubbles initially located very close to the wall $\left(\Lambda / R_{0} \sim 1\right)$ is not hastened as much as bubbles which are at $\Lambda / R_{0} \approx 2$. This is surprising at first, and it is worth being investigated. 


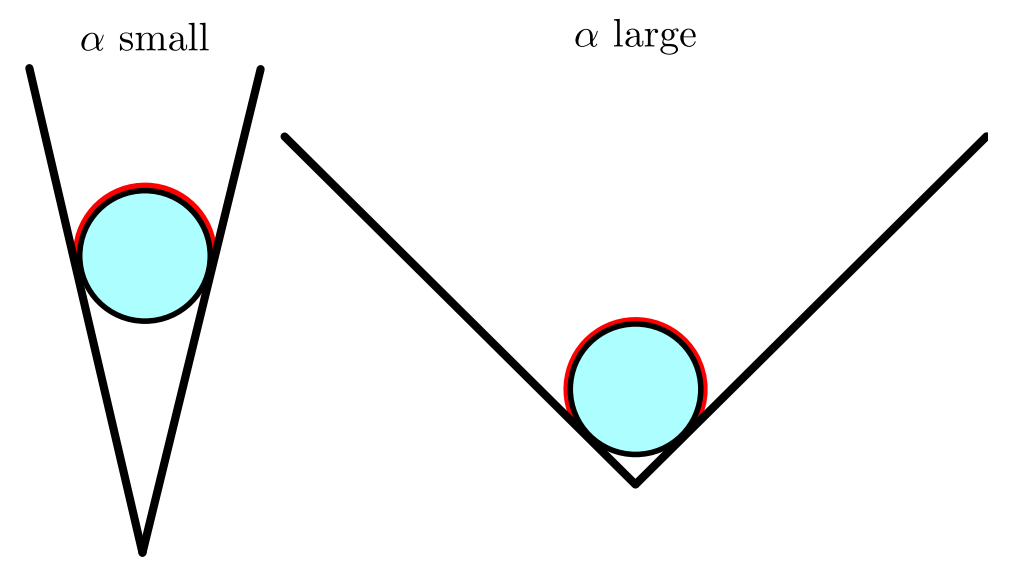

Figure 6.6: Placement of the bubble in a cone with $\Lambda / R_{0}=1$ for a small and a large half-angle.

The sequence of images in Figure 6.5 is for $\Lambda / R_{0}=2.8$, and shows the incident wave diffracts around the bubble, increasing the pressure all around the bubble. When $\Lambda / R_{0}=1.0$, the bubble is initially in contact with the oblique wall of the cone, see Figure 6.6. In this situation, the incident wave can't diffract around the bubble to reach the distal side, and the pressure increases only on the proximal side. Therefore, the bubble collapse is only driven by the pressure increase on the bubble surface area which is highlighted in red in Figure 6.6. The potential for the incident wave to diffract around the bubble to reach the distal side is intrinsically related to the distance between the bubble and the wall. This explains why $\Lambda / R_{0}$ is a more relevant parameter than $H_{0} / R_{0}$.

The proximity of the bubble to the oblique wall also means the portion of the corner wave processing the bubble forms only shortly before processing the bubble. The consequence of this is that the section of the corner wave processing the bubble has a magnitude which is almost identical to the magnitude of the incident wave: substantial focusing has not occurred yet, and the pressure increase due to the corner wave is modest.

\subsection{Pressure in the Tip}

The results from the previous section indicate that the cone can hasten the collapse of a bubble. The purpose of this section is to investigate the effect of the collapsing bubble on the peak pressure created on the wall of the cone.

The peak pressure in the immediate vicinity of the bubble is not investigated in this thesis. Because the shock waves created upon bubble collapse decay approximately in $1 / r$, the peak pressure in the immediate vicinity of the collapsing bubble can differ 


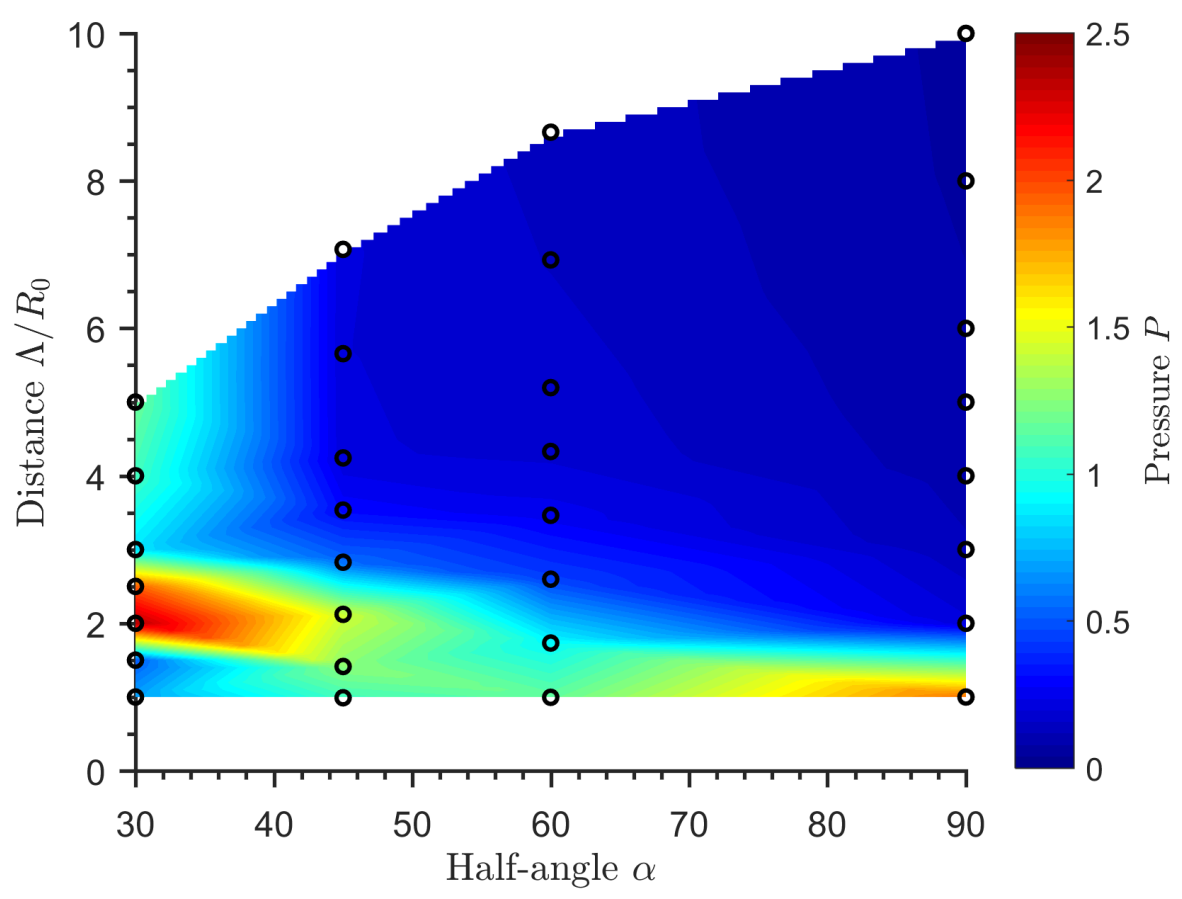

Figure 6.7: Peak wall pressure as a function of the initial location of the bubble and the half-angle of the cone.

greatly from the peak pressure on the wall of the cone. A future study should focus on the peak pressure created by the collapsing bubble in a region which is close to the initial location of the bubble rather than in the tip of the cone. High resolution numerical simulations should also be performed to predict with more accuracy the exact structure of the shock waves created during collapse. The structure of the shock waves should be compared with results available in the literature, such as those of Supponen et al. (2017).

Figure 6.7 is a contour plot of the peak pressure on the oblique wall of the cone. Figure 6.7 indicates that bubbles initially located close to the oblique wall of the cone (i.e., $\Lambda / R_{0}<4$ ) can create peak pressures ranging between 2 and 2.5 (in water this corresponds approximately to pressures ranging between 4.5 and 5.6 GPa). There are two regions of interest where the peak pressure is large: one for $\alpha \approx 90$ degrees and $\Lambda / R_{0} \approx 1$, and another one for $\alpha \approx 30$ degrees and $\Lambda / R_{0} \approx 2$.

Note that for $\alpha=90$ degrees and $\Lambda / R_{0}=10$, the collapsing bubble is located relatively far away from the wall of the cone. Although the collapsing bubble can create large pressures in the vicinity of the bubble, the shock waves reaching the wall of the cone have been considerably weakened: the magnitude of the waves created by bubble 
collapse decay approximately in $1 / r$. For a bubble which tends to be infinitely far from a flat wall (i.e., moving toward the top right corner of Figure 6.7), the peak wall pressure is expected to tend toward a value which is twice the magnitude of the incident wave, 0.045 in the present case. The numerical results indicate the peak wall pressure is approximately 0.06 for $\alpha=90$ degrees and $\Lambda / R_{0}=10$.

For a shock-induced bubble collapse, the contour plot in Figure 6.7 combines the effect of bubble collapse pressure loading and shock focusing on the peak pressure created on the wall. Shock focusing of the incident pressure wave alone can create substantial peak pressures on the cone wall, especially near the tip of the cone as indicated in Chapter 5. To examine the additive effect of the bubble collapse on the peak pressure it is helpful to define an amplification factor $\lambda$ :

$$
\lambda=\frac{\text { Peak wall pressure with a bubble }}{\text { Peak wall pressure without a bubble }} \text {. }
$$

The denominator corresponds to the peak wall pressure when no bubble is present inside the cone: the peak pressure results entirely from shock focusing of the incident pressure wave. The numerator is the peak wall pressure when a bubble is initially located inside the cone.

The peak wall pressure resulting from shock focusing of the incident wave is shown in Figure 6.8 for different half angles; the values shown in Figure 6.8 were obtained without a bubble inside the cone. The peak pressures shown in Figure 6.8 are larger than the peak pressures shown in Figure 5.4. If the working fluid in the cone is water, the results from Figure 6.8 indicate the peak tip pressure is predicted to be 3.6 GPa for a half angle of 30 degrees. For the same half-angle, the results from Figure 5.4 indicate the expected peak pressure is approximately 9.4 MPa. There are two differences between the simulations from this chapter and the simulations from Section 5.1 which explain the different peak pressures: 1) the cone is truncated in Section 5.1, but this is not the case in this chapter; 2) the grid used in this chapter is more refined. Note that the numerical results from Figure 6.8 correspond to the values used in the denominator of Equation 6.8 to compute the amplification factor.

Figure 6.9 is a contour plot of the amplification factor as a function of the initial location of the bubble and the half-angle of the cone. The top plot (Figure 6.9a) uses a color scale which spans the entire range of values of the amplification factor. The bottom plot (Figure 6.9b) is identical to the top plot (Figure 6.9a), except the color scale covers values of $\lambda$ between 0 and 10; all values of $\lambda>10$ are indicated in 


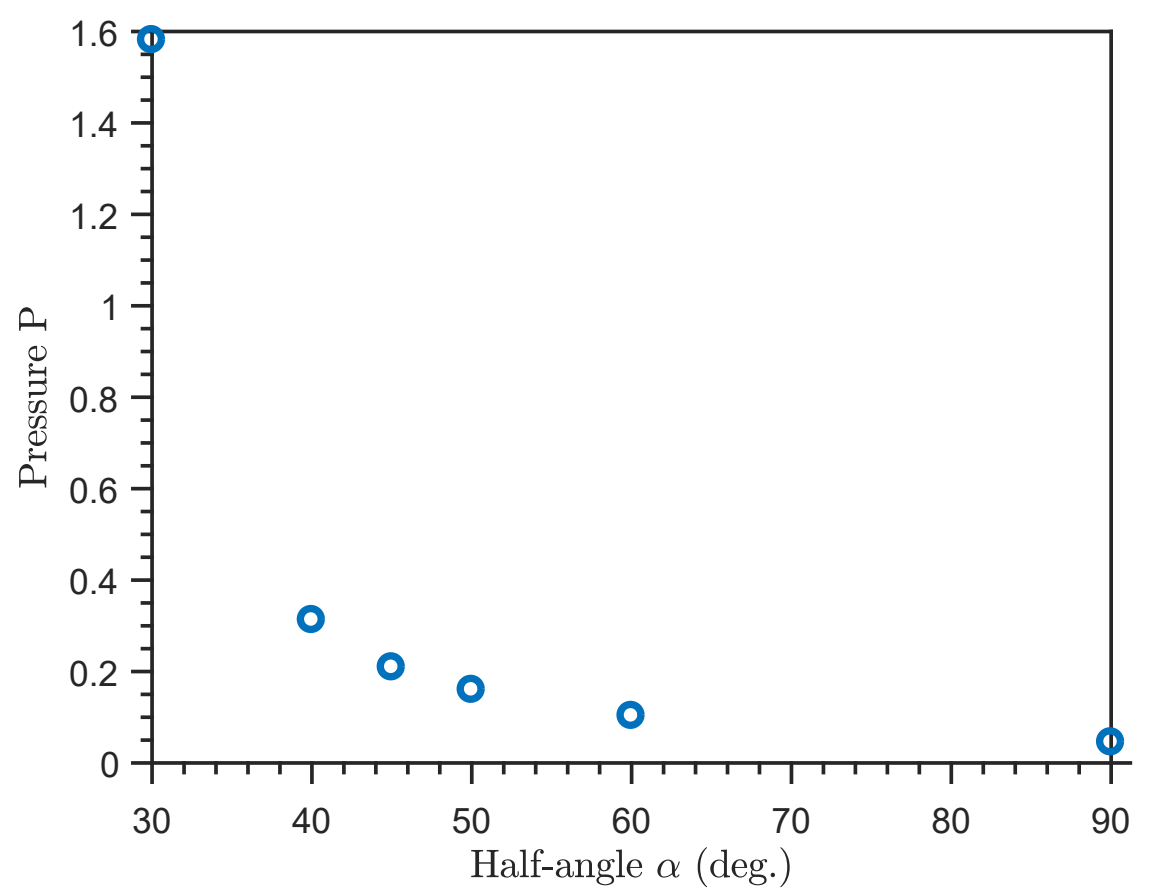

Figure 6.8: Peak wall pressure when no bubble is present in the cone (i.e., effect of shock focusing alone).

dark red. An amplification factor greater than 1 indicates the presence of a bubble increases the peak wall pressure, and vice versa.

The results indicate the amplifying effect of the bubble is most significant (i.e., values of $\lambda$ are the largest) for large half-angles and bubbles which are initially located very close to the wall of the cone $\left(\Lambda / R_{0} \sim 1\right)$. The bubble has only a small effect on the peak wall pressure when the half-angle is small ( $\alpha \approx 30-40$ degrees). This is because for small half-angles the peak wall pressure mostly results from the shock focusing effect, not the bubble collapse itself.

The results indicate there are two regimes to be distinguished. The first regime is for large half-angles. The shock focusing effect is minimal, and the peak pressure on the wall is mostly created by the bubble collapse rather than the focusing of the incident wave. In this regime, the peak pressure created on the wall when a bubble is initially in the cone is large (see Figure 6.7), the peak wall pressure resulting from shock focusing alone is small (see Figure 6.8), and the amplification factor (see Figure 6.9) is large.

The second regime is for small half-angles. The peak pressure on the wall is mostly created by the shock focusing effect rather than the collapsing bubble itself. In this 


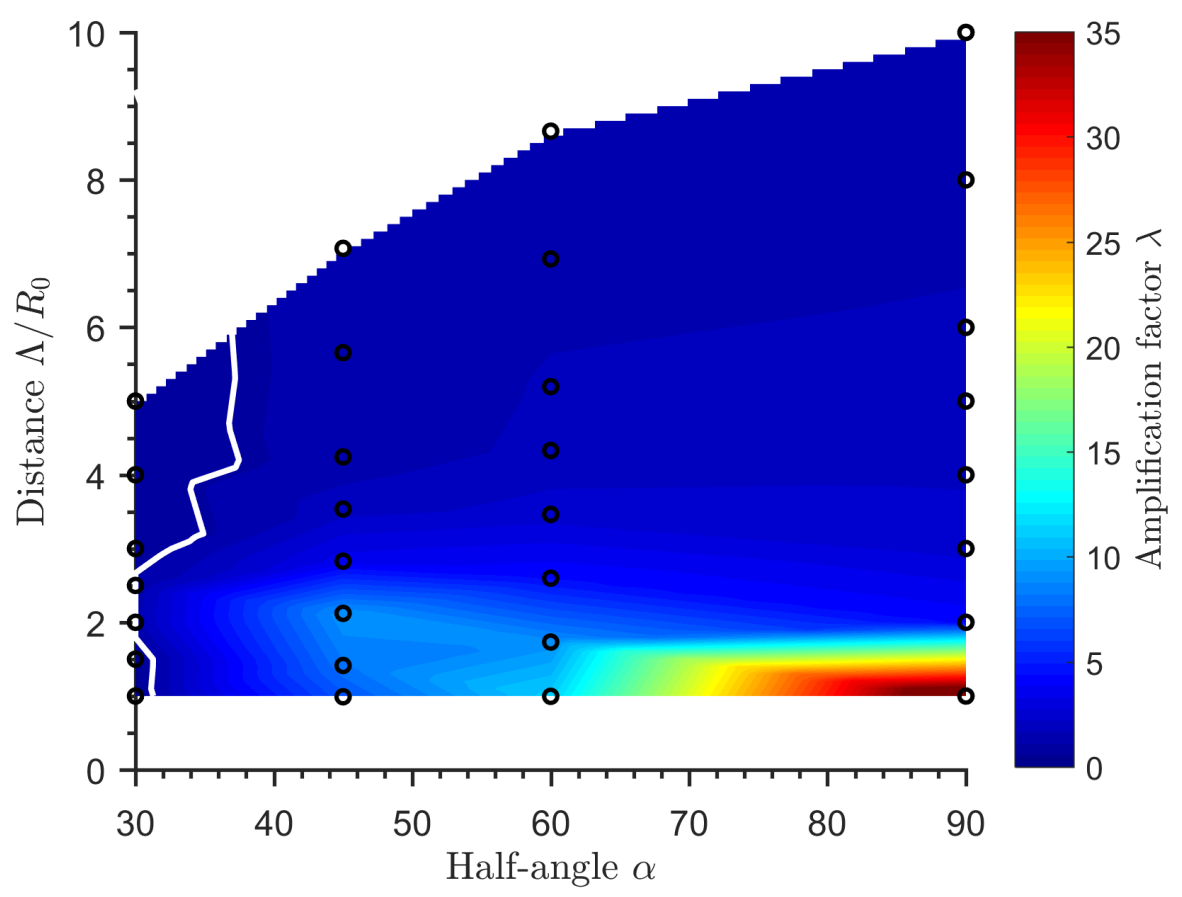

(a) Color scale covers the complete range of $\lambda$.

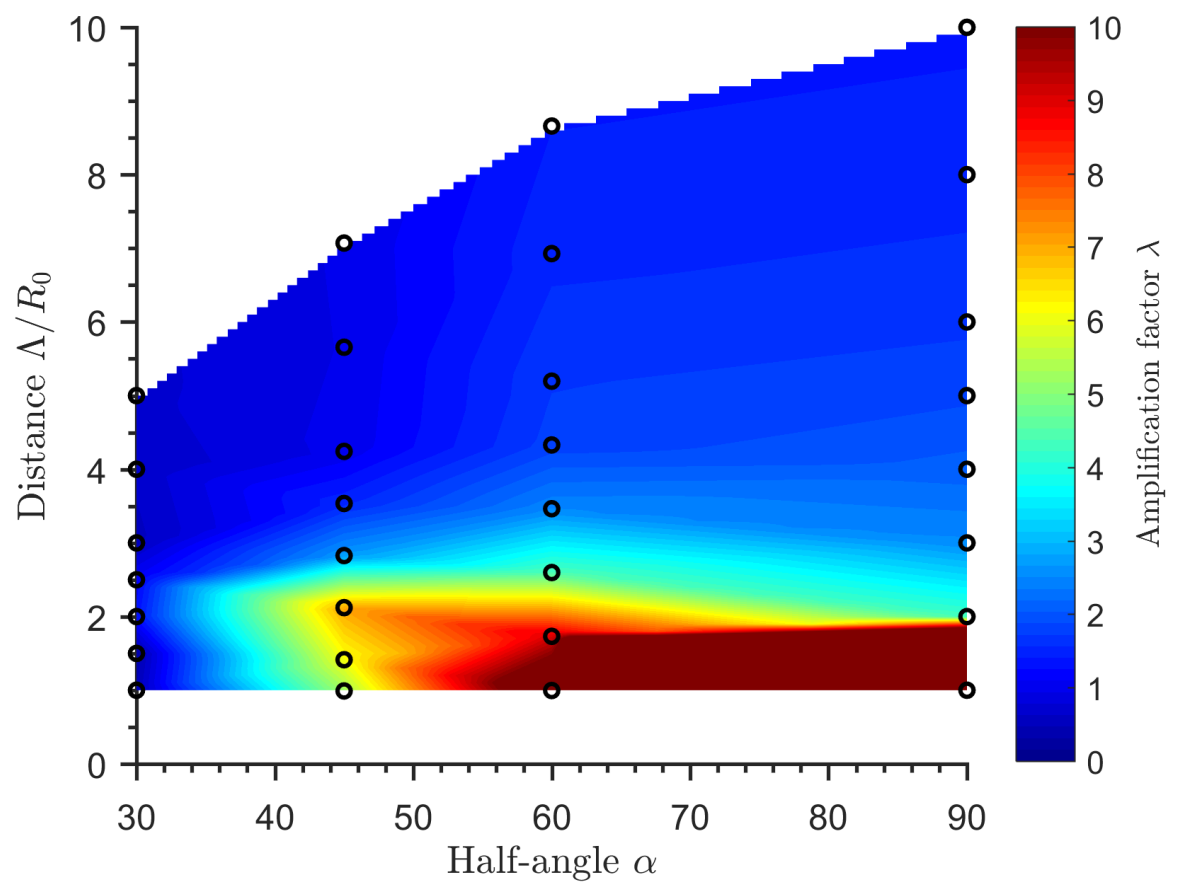

(b) Color covers $\lambda=0$ to 10: values above $\lambda=10$ are colored in dark red.

Figure 6.9: Amplification of the peak wall pressure due to the collapse of a bubble inside the cone. 
regime the peak pressure created on the wall when a bubble is initially in the cone is large (see Figure 6.7), the peak wall pressure resulting from shock focusing alone is large (see Figure 6.8), and the amplification factor (see Figure 6.9) is small.

The white line in Figure 6.9a identifies the isocontour $\lambda=1$ : the amplification factor to the left of that line is less than 1. This indicates that for small half angles, the bubble can in fact reduce the peak pressure on the wall compared to the case without a bubble inside the cone. This is because the bubble is shielding the tip of the cone from the incident and the corner waves, therefore mitigating the shock focusing effect in the vicinity of the tip.

\subsection{Pressure in a Syringe Geometry}

The simplified straight cone geometry used in the previous sections was useful to understand the effect of a cone on a collapsing bubble, and to understand the effect of the bubble on the peak pressure created on the wall. The geometry of a pre-filled syringe can differ substantially from that of a straight cone. A small set of numerical simulations were performed to predict the peak pressure created on the wall of a simplified, syringe-like geometry. Note that the results shown in this section are dimensional.

The geometry and initial configuration of the problem is shown in Figure 6.10. The bubble (black) is located inside the cone of the syringe. The bubble is surrounded by water at pressure $P_{0}$ (blue). Further to the right is a shock wave traveling from right to left. The pressure behind the shock wave (red) is $P_{s}$. The geometry of the cone (brown) is constructed using a stiff fluid: a fluid with an acoustic impedance 1000 times larger than the acoustic impedance of water. Pressure waves are reflected at the boundary between the liquid and the stiff fluid. The magnitude of the reflected waves is $99.8 \%$ the magnitude of the incident waves, a close approximation to a rigid wall. The side walls (gray) correspond to perfectly rigid walls.

The geometry of the syringe is approximately modeled using a few straight segments. The coordinates of the vertices defining the syringe are indicated in Table 6.2. The cone is terminated with a channel (between vertices 1 and 2), the diameter of which is $0.44 \mathrm{~mm}$. The cone is formed with two straight segments: one segment is at 36.7 degrees from the horizontal (between vertices 2 and 3), and the second segment is at 56.8 degrees from the horizontal (between vertices 3 and 4). This geometry is an approximation of a BD Hyflow $1 \mathrm{~mL}$ pre-filled syringe. 

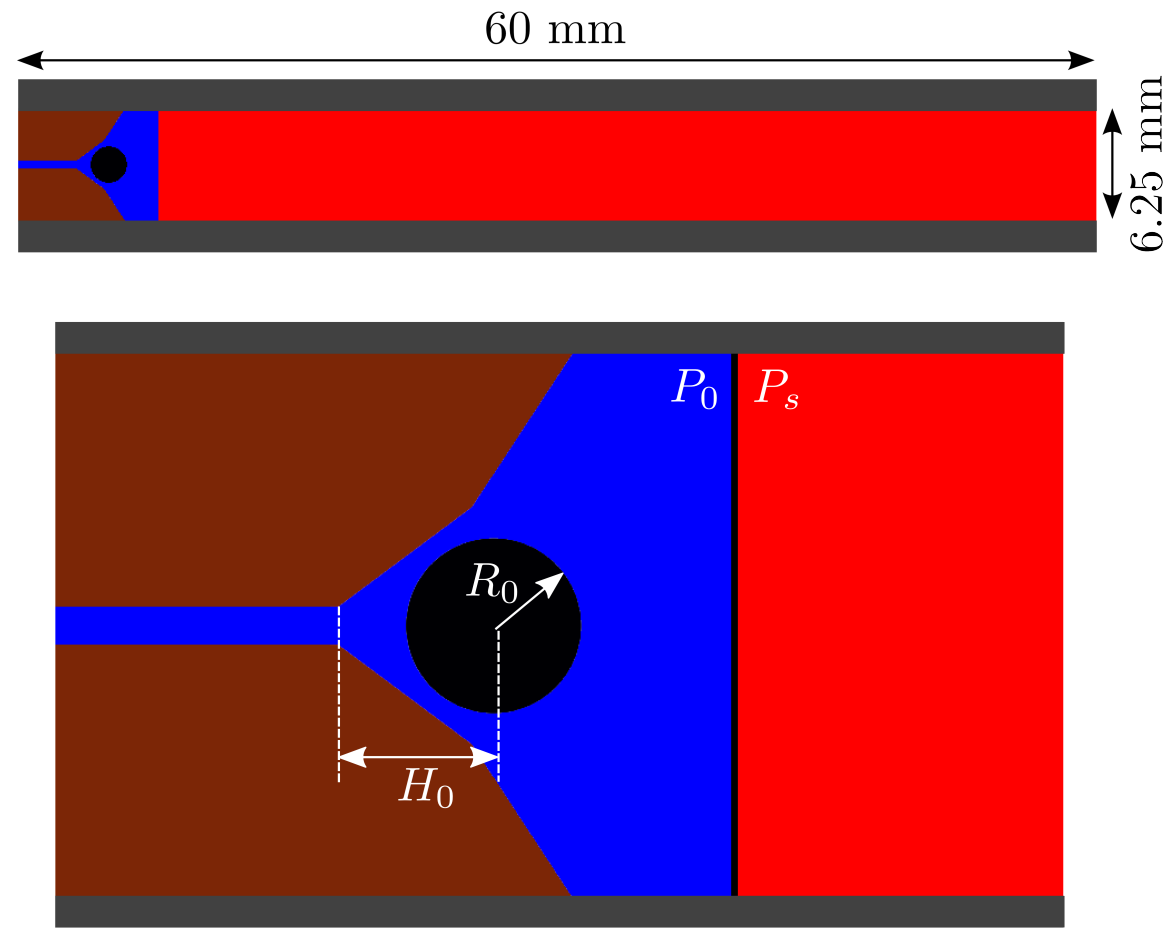

Figure 6.10: Initial configuration used in the numerical simulations performed with MFC and ECOGEN. The bottom view is a zoomed-in view on the cone area.

Table 6.2: Axial and radial location of the vertices in the simplified syringe geometry used in ECOGEN and MFC.

\begin{tabular}{c|ccccc}
\hline Vertex & 1 & 2 & 3 & 4 & 5 \\
\hline$z(\mathrm{~mm})$ & 0.00 & 5.00 & 6.52 & 7.69 & 60.00 \\
$r(\mathrm{~mm})$ & 0.22 & 0.22 & 1.35 & 3.13 & 3.13 \\
\hline
\end{tabular}


The initial radius $R_{0}$ of the bubble is $1 \mathrm{~mm}$, and the initial location $H_{0}$ of the bubble is $1.75 \mathrm{~mm}$. The bubble is located on the axis of symmetry of the syringe. Pressure $P_{0}$ is approximately equal to the vapor pressure of water (i.e., $2500 \mathrm{~Pa}$ ). Selecting pressure $P_{s}$ behind the shock wave is more challenging. An educated guess justified by experimental observations had to be made: a value of $P_{s}=2.5 \mathrm{MPa}$ results in a collapse time which is similar to the collapse time observed in a SureClick autoinjector. The $2.5 \mathrm{MPa}$ figure was obtained using Equation 6.5. This pressure is also in agreement with the magnitude of the pressure waves measured in a real device (see Chapter 3).

Numerical simulations were performed with the same solver as the one used in the previous sections (MFC). Another solver, ECOGEN (Schmidmayer, Marty, et al., 2018; Schmidmayer, Petitpas, and Daniel, n.d.), was also used to make some predictions of the peak pressure created on the wall of the syringe. The same geometry, boundary conditions and initial conditions are used to run the simulations with ECOGEN. One difference is that adaptive mesh refinement (AMR) is available and used in ECOGEN. The structured, non-stretched grid respectively has 20 and 320 cells per initial bubble diameter at the coarsest and finest levels.

A sequence of frames showing the collapse of a bubble in the cone of a syringe is shown in Figure 6.11. The corresponding bubble volume history is shown in Figure 6.12. The results shown were obtained with ECOGEN. Note that both codes predict similar features, except the peak pressures predicted by ECOGEN are larger than the peak pressures predicted by MFC.

At $0 \mu$ s the incident wave arrives on the distal side of the bubble. The incident wave diffracts around the bubble, increases the pressure inside the cone, and initiates the bubble collapse. The reflection of the incident wave on the oblique wall of the cone results in the formation of a corner wave, visible at $1.2 \mu \mathrm{s}$. The corner wave converges toward the axis of symmetry and the bubble, further increasing the pressure surrounding the bubble. The corner wave and the incident wave reflect and propagate away from the bubble thereafter.

At $17.6 \mu \mathrm{s}$, the proximal side of the bubble is moving to the left, reducing the bubble volume. The decrease in bubble volume is almost linear in time, and the collapse is not symmetric. A re-entrant jet forms, visible from $26.6 \mu$ s to $38.1 \mu \mathrm{s}$. Approximately $38.1 \mu \mathrm{s}$ after the beginning of collapse, the re-entrant jet reaches the distal side and divides the bubble into two annular lobes: the proximal and the distal lobes. At $42.5 \mu$ s the proximal lobe collapses, creating an annular shock wave. Part 
Pressure (MPa)
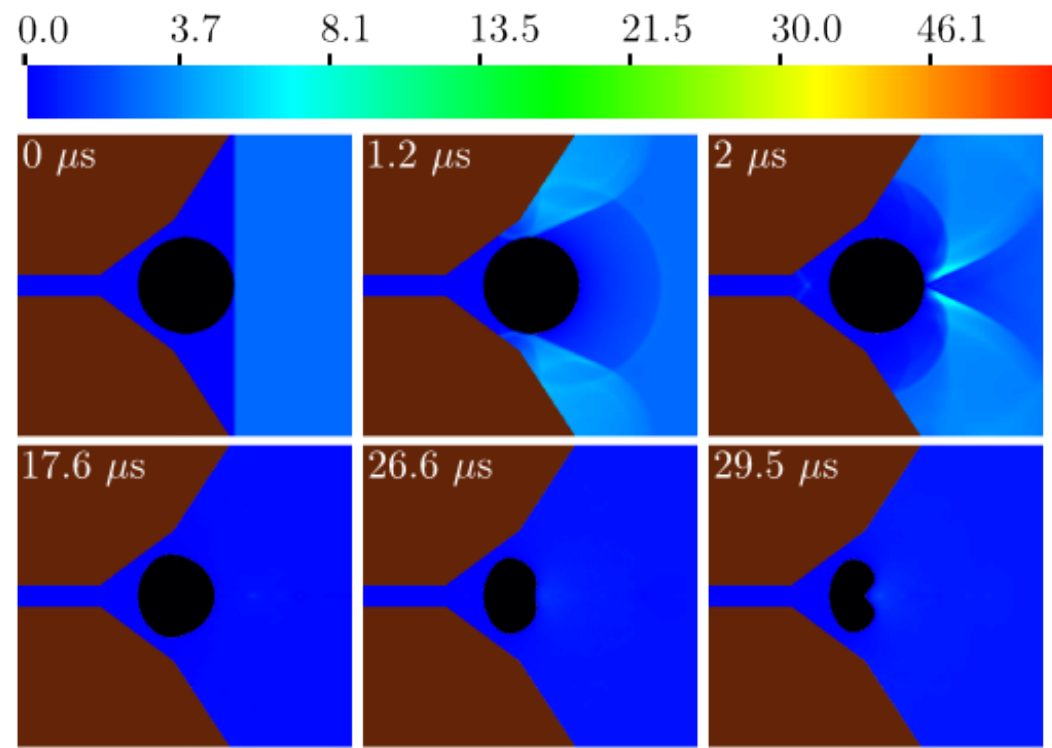

112.5
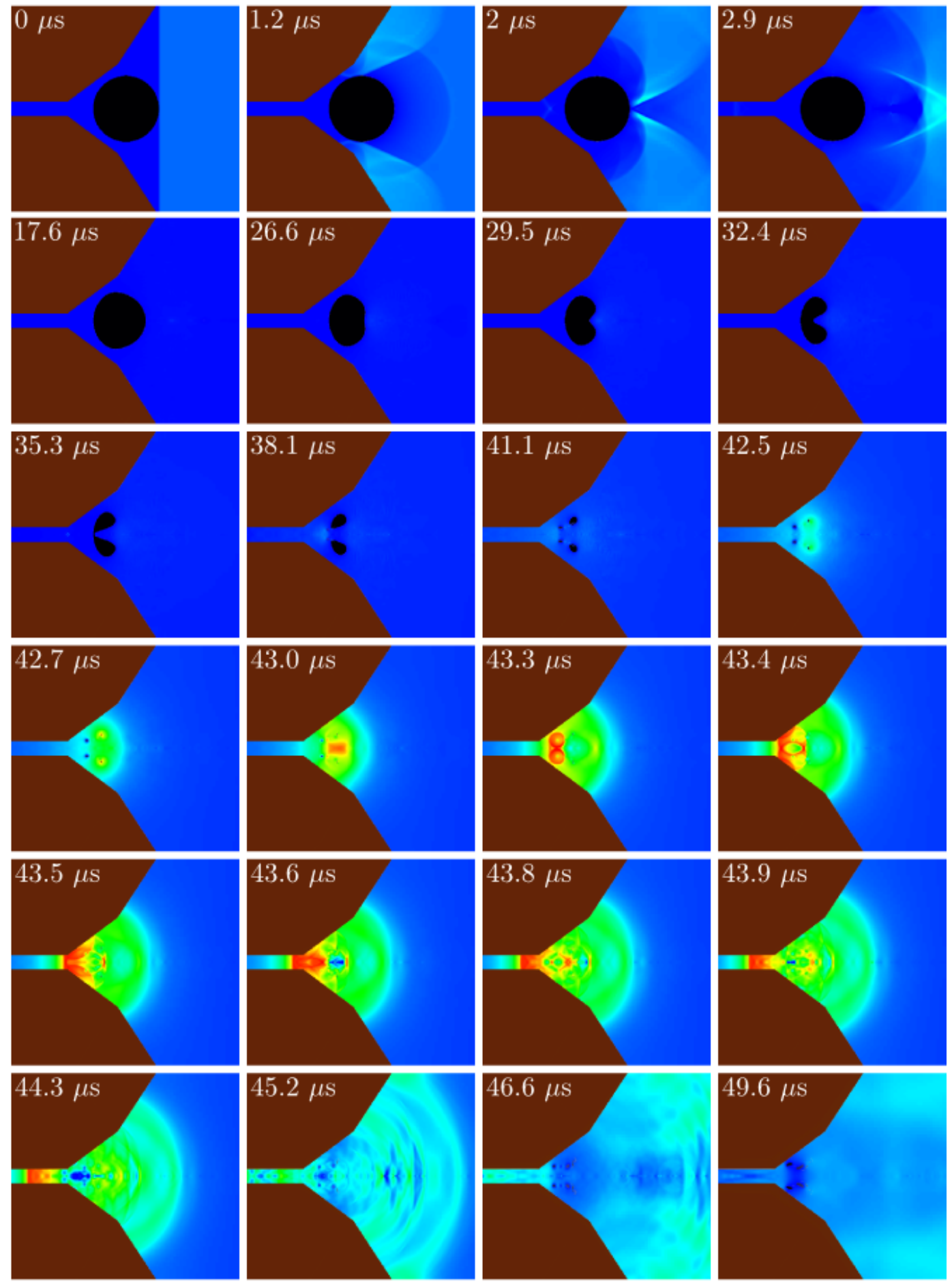

Figure 6.11: Collapse of a bubble inside the cone of a pre-filled syringe. Results obtained using ECOGEN. 


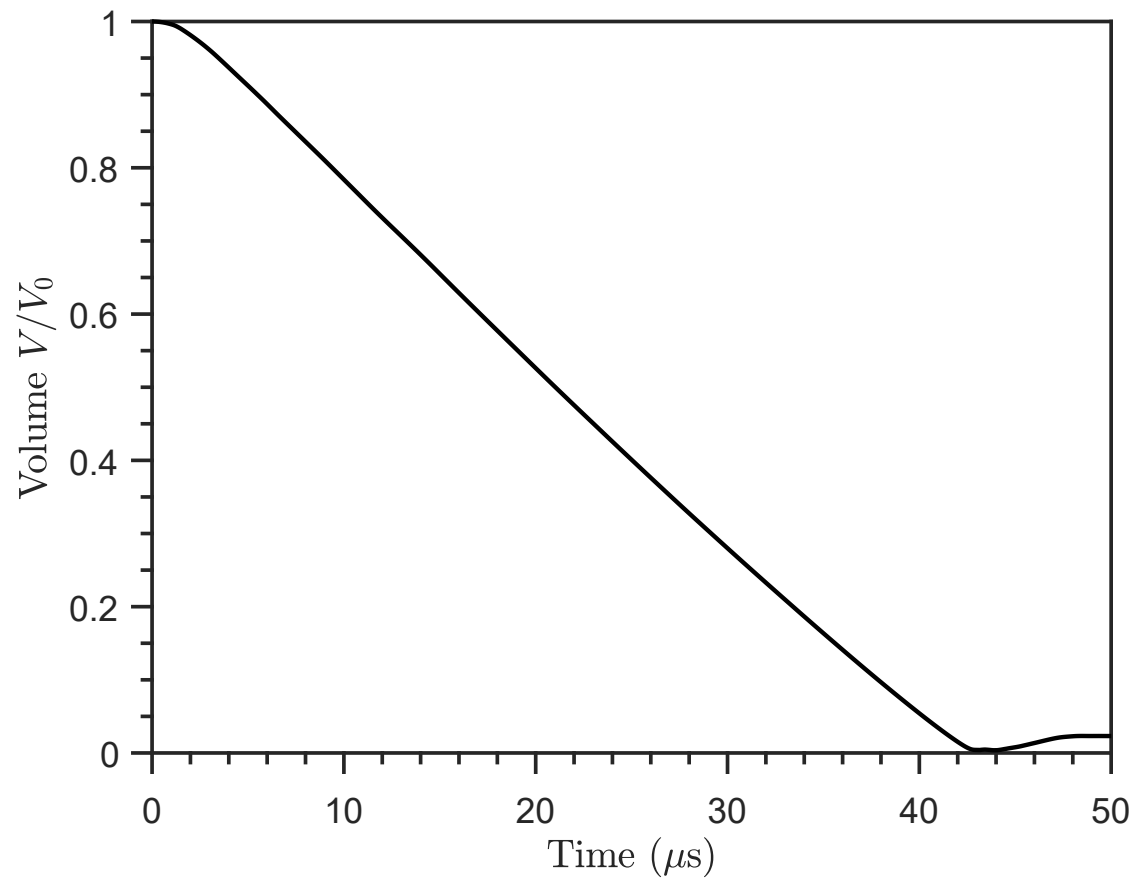

Figure 6.12: Evolution of the bubble volume during collapse in the cone of a syringe. Results obtained using ECOGEN.

of the shock wave converges toward the axis of symmetry, resulting in a substantial pressure increase on the axis of symmetry of the syringe, visible at $43.0 \mu \mathrm{s}$. At $43.3 \mu$ s the distal lobe collapses, creating another annular shock wave which creates large pressures on the oblique wall of the cone and near the entrance of the channel. This occurs from $43.4 \mu$ s to $43.8 \mu$ s. This is followed by a slow decay of the pressure inside the cone.

The peak pressure on each straight segment defining the syringe geometry is plotted in Figure 6.13. Large pressures are created shortly after the bubble reaches its minimum volume and rebounds. The largest pressures are predicted to occur on the segment of the oblique wall which is at 36.7 degrees from the horizontal, and on the channel wall. The peak pressure on the syringe wall is approximately $100 \mathrm{MPa}$. This result was used to calibrate the magnitude of the energy source term in the numerical simulations of Section 5.2.6: the magnitude of the source term was increased until the peak wall pressure created by the spherical shock wave was approximately $100 \mathrm{MPa}$.

MFC predicts a peak pressure of 22.5 MPa on the wall of the cone, substantially less than the peak pressure predicted by ECOGEN. One possible explanation for the 


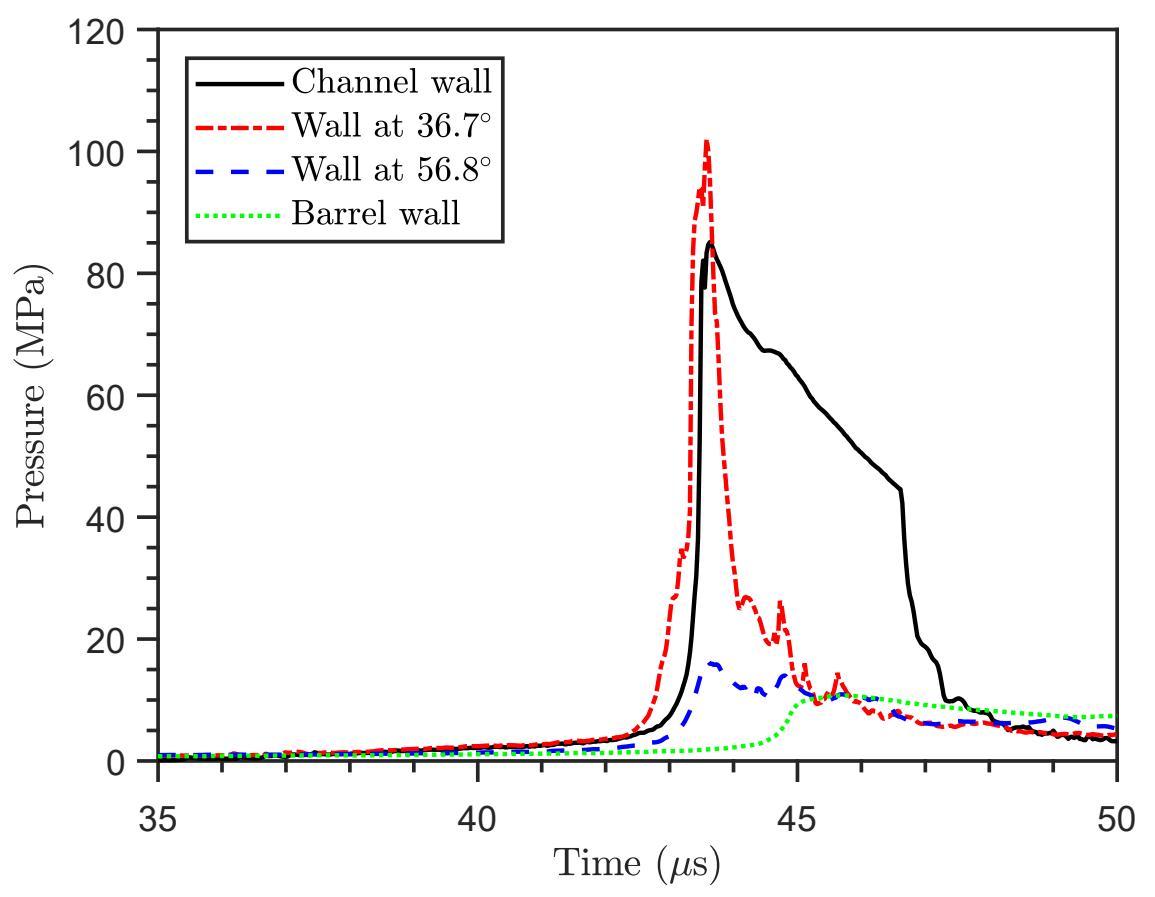

Figure 6.13: Evolution of the peak pressure during collapse in the cone of a syringe. Results obtained using ECOGEN.

different peak pressures is the different resolution of the numerical grids used with MFC and ECOGEN. The numerical grid used with ECOGEN has 320 elements per initial bubble diameter at the most refined level, compared to 108 elements per initial bubble diameter for the grid used with MFC. Another possible explanation for the different peak pressures is the different approaches used in both codes to model the mixture region between the bubble and the water. The effect of the different models on the results is currently being investigated by the developers of ECOGEN and MFC (private communication).

\subsection{Summary}

Numerical simulations were used to study the collapse of bubbles in a straight cone and in a syringe-like geometry. Numerical results have shown that a cone can reduce the collapse time of a bubble. The corner wave is responsible for increasing the rate at which the bubble collapses. A simple model which can predict whether or not the rate at which a bubble collapses can be increased by the cone was developed and validated.

The peak pressure at the apex of a straight cone can be substantial, both with and without a bubble. The large pressures are created by a combination of shock focusing 
of the incident wave, and the shock waves created by the collapsing bubble. For large-half angles, the creation of large pressures is dominated by the shock waves forming upon bubble collapse. For small half-angles, the large pressures created are dominated by the shock focusing effect.

An initially spherical bubble collapsing in a syringe-like geometry can create large pressures on the wall of the syringe. Peak wall pressures as large as $100 \mathrm{MPa}$ were predicted in a pre-filled syringe for a bubble size and a shock wave magnitude representative of what has been observed in the in situ experiments reported in Chapter 3. The results from Chapter 5 indicate that a peak pressure of $100 \mathrm{MPa}$ can create maximum principal stresses of order $50 \mathrm{MPa}$ in the syringe wall. 


\section{Chapter 7}

\section{MITIGATION OF THE PRESSURE AND STRESS TRANSIENTS}

The magnitude of the pressure and stress transients in autoinjector devices can be mitigated through appropriate design modifications. The findings discussed in the previous chapters of this thesis suggest there are multiple ways of reducing the magnitude of the transient events, and directly reduce the probability for device failure. Some key ideas to reduce the magnitude of the transient events are:

1. to reduce the impact velocity of the driving-rod on the plunger-stopper;

2. to reduce the maximum velocity of the syringe prior to deceleration;

3. to reduce the magnitude of syringe acceleration/deceleration;

4. to eliminate cavitation in the syringe.

Although this is not entirely related to the transient events occurring in the device, it is worth to recall that the results from Chapter 5 have shown that supporting the syringe using the shoulder rather than the flange reduces the peak magnitude of the maximum principal stress. The junction between the flange and the syringe barrel has a small radius of curvature, and stress concentration effects are significant there. If a syringe is supported using the flange, it is advisable to use syringes with a thick flange, and a relatively large radius of curvature at the junction between the flange and the barrel.

Reducing the impact velocity, maximum velocity and acceleration/deceleration of the components inside the autoinjector is key to mitigating the pressure and stress transients. This can be done in many ways. For example, torsional springs rather than linear springs can be used to actuate the device. When doing this, a mechanism becomes necessary in order to transform the rotational motion to linear motion, but this type of actuator could provide more control over the velocity of the driving rod and the syringe throughout actuation. Electric motors are another alternative to have better control over the motion of the internal components, but this option is often not viable due to the increased production cost. This is a solution which is more suitable for reusable autoinjector devices. 
Reducing the initial separation between the driving-rod and the plunger-stopper is an effective, low-cost solution to reduce the impact velocity of the driving-rod on the plunger-stopper. A simple mechanical model of a mass accelerated by a spring obeying Hooke's law indicates the velocity of the driving rod upon impact on the plunger-stopper is proportional to $\delta^{1 / 2}$, where $\delta$ is the initial separation between those two components. The SureClick is an example of autoinjector device where the separation $\delta$ can not be zero: there must be a gap of sufficient size for the internal mechanism to release the spring and the driving rod. Efforts should be made during the design process to keep this minimum initial separation as small as possible or, even better, eliminate this constraint such that the driving rod and the plunger-stopper can initially be in contact.

Results from previous chapters indicate it is advisable to eliminate cavitation in the syringe. Pressurizing the syringe prior to accelerating the syringe is one possibility to preclude cavitation. This, however, needs to be considered carefully: pressurizing the syringe too early before the needle penetrates under the patient's skin means that some drug solution can be lost. For most devices, the time delay between actuation and penetration of the needle under the skin is very short - a few milliseconds at most-indicating that if the syringe is pressurized at the same time as it is accelerated, the amount of drug solution lost is minimal. One possibility to synchronize syringe acceleration and syringe pressurization is to eliminate the air gap or head space. This may be difficult to achieve in practice.

When an air gap is present, the syringe acceleration and pressurization can be synchronized by reducing the friction between the plunger-stopper and the syringe. This also has the added benefit of reducing the peak magnitude of syringe acceleration. This idea was discussed in Chapter 3 (see the results obtained with plastic syringes). The use of damping material positioned judiciously between key components inside the device can also be considered to reduce the peak velocities and acceleration/deceleration. This idea is investigated experimentally in the first section of this chapter. This is also reported in the US patent application of Veilleux and Shepherd (2017). Cavitation can be eliminated by reducing the peak magnitude of syringe acceleration. The maximum syringe acceleration allowable while preventing cavitation from occurring in the syringe is investigated theoretically and experimentally in the second section of this chapter. 


\subsection{Dampers for Autoinjector Devices}

The injection and needle motion is controlled primarily by the quasi-static spring force rather than peak pressures or peak forces due to transient events. The large peak pressures and strains reported in Chapter 3 for an undamped SureClick device are therefore unnecessary, and they can be eliminated without affecting the injection function of the device. This can be achieved by adding damping material within the device to absorb a substantial fraction of the mechanical energy, and therefore reduce significantly the impact velocities and the magnitude of the acceleration/deceleration of the moving components.

The dampers must not affect the primary function of the device. The following requirements must be met:

1. the quasi-static force applied on the plunger-stopper should be unaffected: there needs to be enough force for the needle to penetrate under the skin, and the injection time should be virtually unchanged;

2. the placement of damping material should not reduce the penetration depth of the needle under the skin.

One way to satisfy these requirements is to use viscous dampers (shock absorbers) between the moving parts. Introducing viscous dampers inside the device requires substantial changes or additional components in the autoinjector. This is conceivable when designing a new device, but challenging to modify an existing device to include viscous dampers.

Another option is to use a low-resilience foam to damp the impact events inside the autoinjector. A low-resilience foam, such as "memory foam", tends to remain in the deformed configuration over a relatively long time, even after the stress creating the deformation is removed.

To test this idea in the SureClick device, low-resilience polyurethane foam (also know as LRPU or viscoelastic polyurethane foam) is introduced between the driving rod and the plunger-stopper, and between the syringe tip and the shell of the autoinjector. The locations are shown schematically in Figure 7.1. The foam used is from Moldex Pura-Fit 6800 ear protection devices.

The piece of foam introduced between the driving rod and the plunger-stopper has the shape of a disk. The diameter of the disk is approximately $6.8 \mathrm{~mm}$, which is 


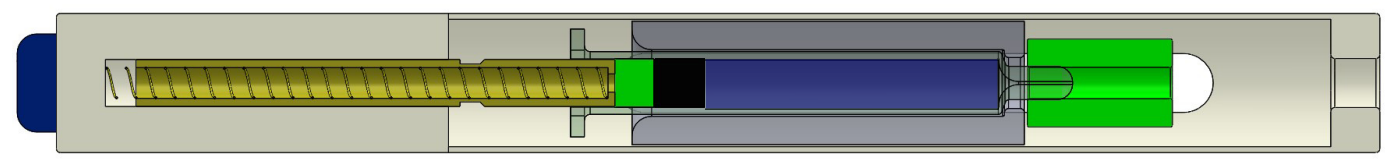

Figure 7.1: Schematic of a SureClick autoinjector with damping material (green) between the driving rod and the plunger-stopper, and between the syringe tip and the shell of the device.

only slightly larger than the inner diameter of the syringe. The height of the disk is approximately $2.5 \mathrm{~mm}$, which corresponds to the initial separation between the driving rod and the plunger-stopper. The uncompressed foam disk entirely fills the gap which is initially present between the driving-rod and the plunger-stopper before actuation.

The piece of foam introduced between the syringe tip and the shell of the autoinjector device has the shape of a hollow cylinder. The length of the cylinder is $17 \mathrm{~mm}$, and the diameter is $11 \mathrm{~mm}$. There is an opening $3 \mathrm{~mm}$ in diameter centered on the axis of symmetry of the cylinder. This is to accommodate the tip of the syringe and the needle.

Multiple experiments were performed to assess the effectiveness of those simple dampers. Only one representative test case performed with a water-filled BD Hyflow $1 \mathrm{~mL}$ glass syringe without an air gap is reported. Except for the placement of damping material, the experimental methods used to obtain the data reported in this section are identical to the methods reported in Chapter 3.

Figure 7.2 is a plot of the velocity of the driving rod, the plunger-stopper, and the syringe. This figure is equivalent to Figure $3.9 \mathrm{~b}$ obtained without damping material. The velocity of the syringe is not available before $2 \mathrm{~ms}$, but results from Chapter 3 indicate the syringe and the plunger-stopper move together with the same velocity when no air gap is present.

The driving rod begins to compress the foam located between the driving rod and the plunger-stopper at around $0 \mathrm{~ms}$. The compression of the foam initiates the acceleration and pressurization of the syringe. The peak magnitude of syringe acceleration is approximately $8500 \mathrm{~m} / \mathrm{s}^{2}, 43 \%$ less than the $15,000 \mathrm{~m} / \mathrm{s}^{2}$ measured without damping material. The syringe begins to compress the damping material located between the tip and the shell of the autoinjector around $2 \mathrm{~ms}$, initiating the syringe deceleration. The peak magnitude of the syringe deceleration is approximately $12,000 \mathrm{~m} / \mathrm{s}^{2}, 67 \%$ 


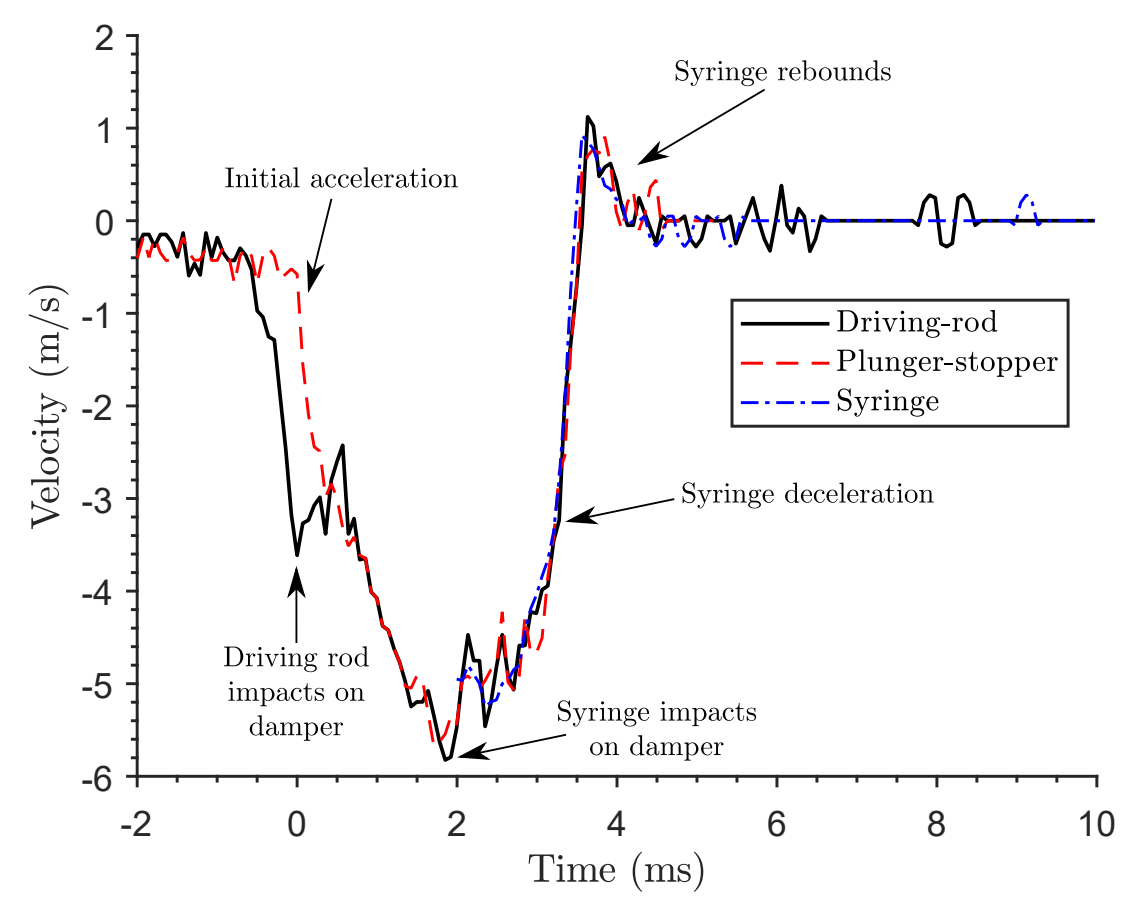

Figure 7.2: Velocity of the moving components in a SureClick autoinjector with damping material.

less than the $36,000 \mathrm{~m} / \mathrm{s}^{2}$ measured without damping material. As an added benefit, the damping material also mitigates the syringe rebound.

These results demonstrate that damping material introduced in the autoinjector device is effective at reducing the peak magnitude of syringe acceleration/deceleration. This is expected to reduce the magnitude of the pressure and stress transients. Figures 7.3 and 7.4 are plots of the pressure and strains, equivalent to the plots shown in Figures 3.10 and 3.11 obtained without damping material.

Examining the pressure signal (Figure 7.3), the transients due to events 1, 2, and 3 are all visible, but the magnitude of the pressure remains below 2.0 MPa. This is a reduction of $50 \%$ compared to the undamped case. The rise time of the pressure pulses created in the syringe when damping material is present are also longer than without damping material, as expected. As an added benefit of damping, the syringe rebound is damped sufficiently to not create any measurable pressure transient. Another difference between the damped and undamped cases is in the final pressure measured at $t=15-20 \mathrm{~ms}$. In the damped case, this final pressure is approximately 0.6 MPa. In the undamped case, the final pressure is approximately $0.85 \mathrm{MPa}$. 


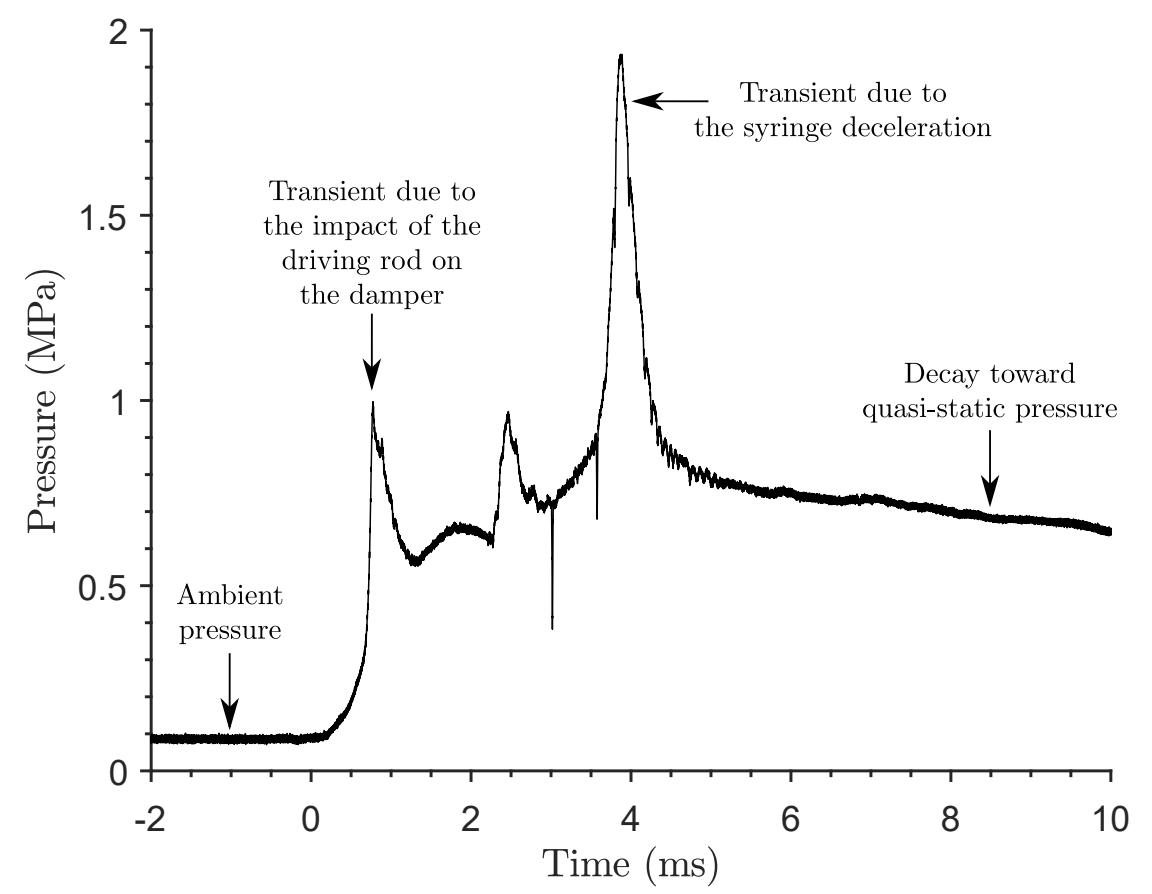

Figure 7.3: Liquid pressure in a SureClick autoinjector with damping material.

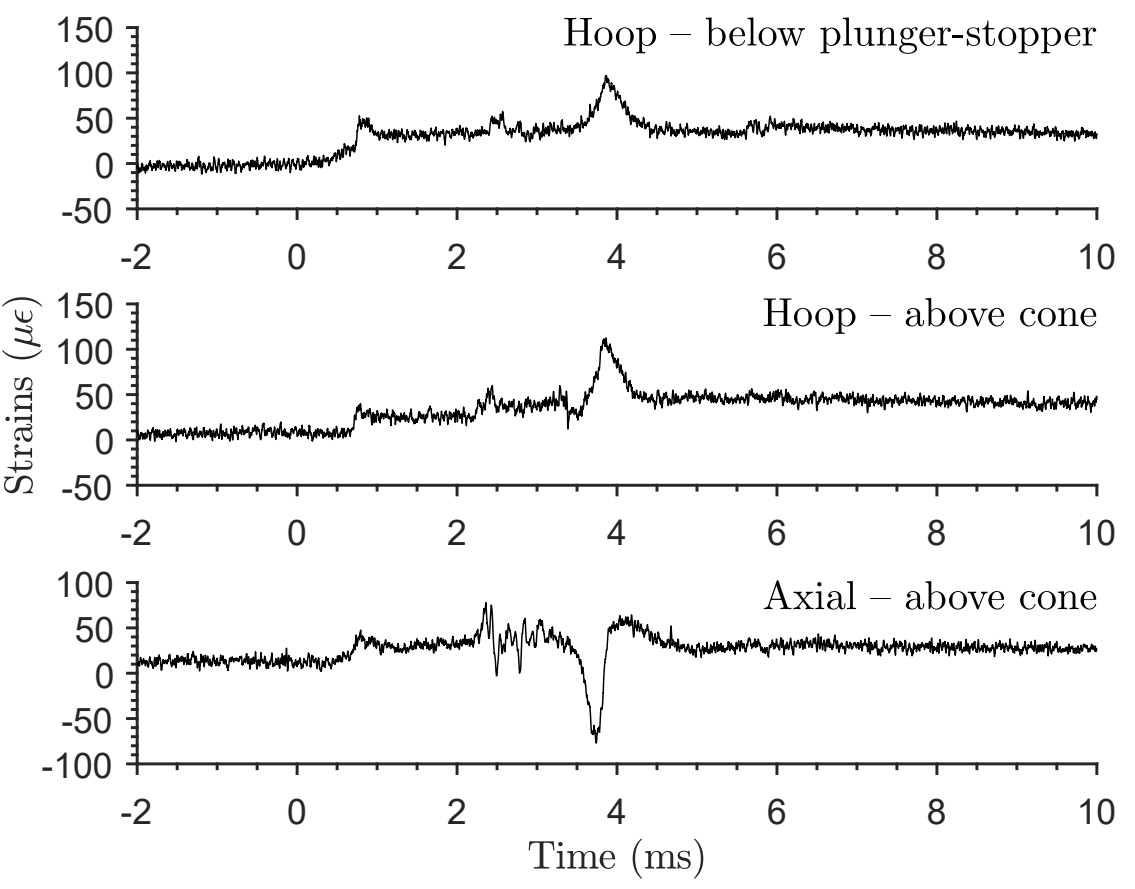

Figure 7.4: Hoop and axial strains on the barrel of the syringe in a SureClick autoinjector with damping material. 
Examining the strain signals (Figure 7.4), the effectiveness of the dampers at mitigating the transient events is clear. The transient events around $0 \mathrm{~ms}$ (events 1 and 3 ) are hardly noticeable. The peak magnitude of the strains created by the syringe deceleration (event 2) is drastically reduced compared to the undamped case. With the damping material, the maximum magnitude of the hoop strains is approximately $116 \mu \epsilon$, a reduction of $64 \%$ compared to the undamped case. The maximum magnitude of the axial strains is approximately $78 \mu \epsilon$, a reduction of $69 \%$ compared to the undamped case.

Preliminary testing suggests the presence of damping material does not significantly increase the time needed to extrude the syringe content through the needle. An increase of no more than $10 \%$ in the extrusion time is measured for a syringe which contains silicone oil (viscosity of $5 \mathrm{cP}$ ). Further testing and optimization of the damping system (geometry, material, placement, etc.) is needed to further reduce the magnitude of the transient events, and to avoid the reduction in quasi-static pressure.

In summary, the results in this section have shown that viscoelastic or memory foam can be used to effectively mitigate the pressure and stress transients created by device actuation. Small foam dampers located between the driving rod and the plungerstopper, and between the syringe tip and the shell proved capable of reducing the peak pressure and strains by $50 \%$ or more. The damping mechanism described and studied in this section has not been optimized. More testing and optimization is needed to further reduce the magnitude of the transient events without affecting the primary function of the autoinjector.

\subsection{Maximum Allowable Syringe Acceleration}

Ideally, the syringe acceleration needs to be limited to preclude cavitation from occurring. For a type 1 or impulsive acceleration (see Chapter 2), the constraint is on the change in velocity $\Delta u$ of the syringe during the acceleration event; if the change in velocity is small enough, cavitation does not occur. As discussed in Chapter 2, cavitation is not expected if:

$$
\Delta u<\frac{P_{0}-P_{v a p}}{\rho_{l} c_{l}} .
$$

The acceleration of the syringe in a SureClick autoinjector device is in regime 2. When this is the case, the model developed in Chapter 2 indicates the constraint is on the magnitude $a$ of syringe acceleration rather than on the change in velocity $\Delta u$. 
If the magnitude of syringe acceleration is small enough, cavitation is not expected. The analytical solution of the model given in Chapter 2 suggests that cavitation is not expected if:

$$
a<\frac{P_{0}-P_{v a p}}{2 \rho_{L} L_{l}} .
$$

The estimate above is a special case of the theoretical solution obtained in Chapter 2 using separation of variables. A constant acceleration (i.e., a linear velocity profile) was assumed to obtain this analytic solution.

Experiments were performed using the dynamic, large-scale model autoinjector from Chapter 4 to confirm the validity of Equation 7.2. The outer projectile is used to impact on the syringe wall and create the syringe acceleration. No buffer is present, and the syringe is filled with water. The clear, polycarbonate syringe is used in order to determine visually whether or not cavitation occurs. The length of the water column $L_{l}$ is approximately $0.3 \mathrm{~m}$. Equation 7.2 indicates the acceleration of the syringe must be less than $169 \mathrm{~m} / \mathrm{s}^{2}$ to preclude cavitation from occurring inside the syringe.

A varying amount of damping material - some eggshell memory foam - is introduced between the outer projectile and the syringe wall of the large-scale model to control the peak magnitude of syringe acceleration. As expected, increasing the amount of damping material results in a decrease of the peak acceleration of the syringe.

A summary of the results obtained with a flat tip is shown in Table 7.1. The result for each test is binary: cavitation occurs or does not occur. Cavitation is detected visually using a high-speed camera, and using the pressure signal recorded with a piezoelectric transducer mounted into the tip of the syringe.

The results indicate the maximum allowable acceleration in order to avoid cavitation is between 340 and $450 \mathrm{~m} / \mathrm{s}^{2}$, a threshold which is substantially larger than the predicted value of $169 \mathrm{~m} / \mathrm{s}^{2}$. The discrepancy is explained by the acceleration profile of the syringe; the syringe acceleration is not constant. The velocity profile for test case 7 is shown in Figure 7.5, confirming the velocity profile is not linear.

Equation 7.2 was obtained by assuming the magnitude of the acceleration is constant. When this happens, all the tensile waves created in the syringe through the acceleration of the tip have the exact same magnitude. This is a special case because the tensile waves at the bottom of the syringe can interfere destructively with the compression waves resulting from the reflection of tensile waves on the free surface located on the other end. Because the tensile waves and compression waves have 
Table 7.1: Experimental verification of the maximum allowable model syringe acceleration to eliminate cavitation - large-scale model syringe with a flat tip.

\begin{tabular}{ccc}
\hline Test & $\begin{array}{c}\text { Max acceleration } \\
\mathrm{m} / \mathrm{s}^{2}\end{array}$ & Cavitation \\
\hline 1 & 4974 & yes \\
2 & 1424 & yes \\
3 & 580 & yes \\
4 & 530 & yes \\
5 & 450 & yes \\
6 & 340 & no \\
7 & 275 & no \\
\hline
\end{tabular}

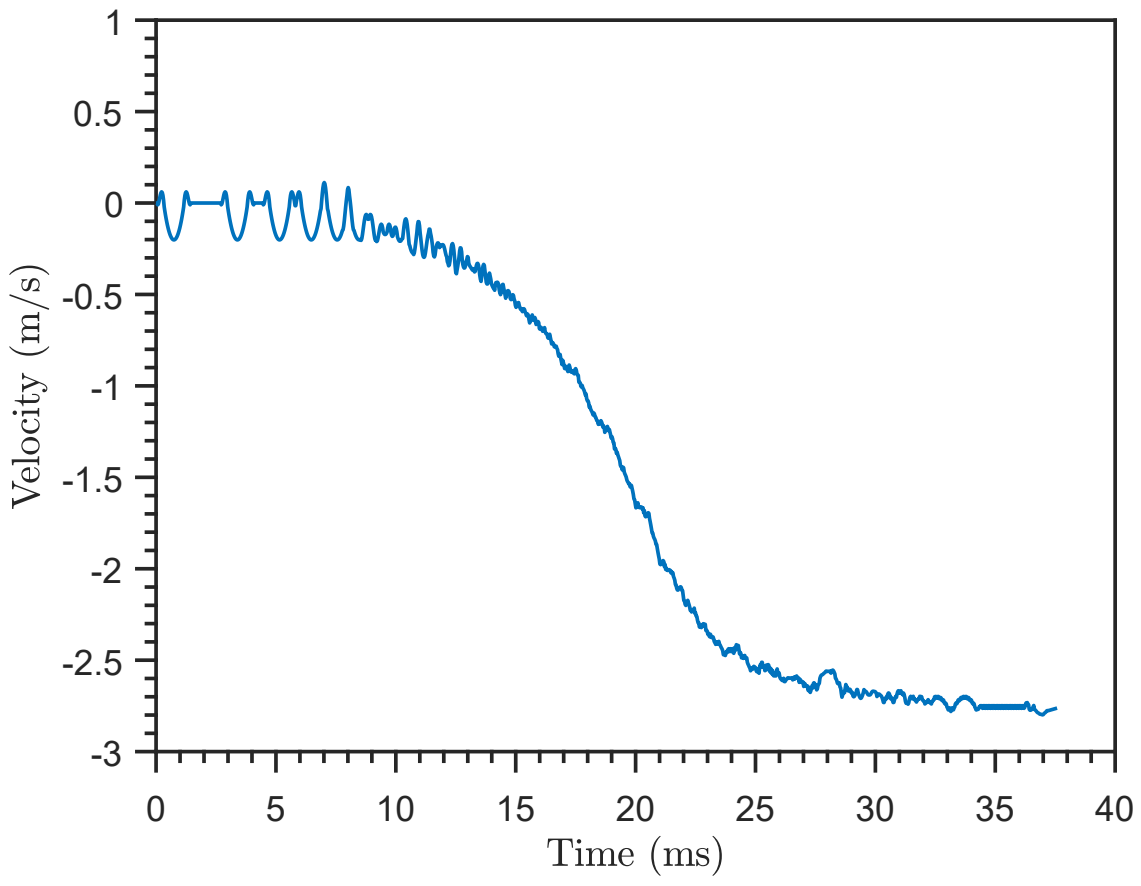

Figure 7.5: Syringe model acceleration with memory foam between the outer projectile and the large-scale syringe model wall. The profile shown is for test case 7 from Table 7.1. 
Table 7.2: Experimental verification of the maximum allowable model syringe acceleration to eliminate cavitation - large-scale model syringe with a conical tip.

\begin{tabular}{ccc}
\hline Test & $\begin{array}{c}\text { Max acceleration } \\
\mathrm{m} / \mathrm{s}^{2}\end{array}$ & Cavitation \\
\hline 1 & 720 & yes \\
2 & 532 & yes \\
3 & 475 & yes \\
5 & 363 & yes \\
6 & 308 & no \\
7 & 262 & no \\
\hline
\end{tabular}

equal magnitude, the destructive interference is such that the pressure can periodically return to ambient pressure in the vicinity of the tip. This is what explains the factor of 2 in the denominator of Equation 7.2.

If the syringe acceleration is not constant, the complete destructive interference of the waves at the bottom wall does not occur, and Equation 7.2 can't be used. It is possible, instead, to directly use Newton's second law of motion to estimate the maximum allowable syringe acceleration:

$$
a<\frac{P_{0}-P_{v a p}}{\rho_{L} L} .
$$

This equation corresponds to $a<F / m$ with both the numerator and the denominator divided by the inner cross-section of the syringe. There is only one difference between Equations 7.2 and 7.3: the factor of 2 in the denominator. Equation 7.3 indicates the maximum allowable acceleration is $338 \mathrm{~m} / \mathrm{s}^{2}$, a threshold which is in better agreement with the experimental results from Table 7.1.

The geometry of the tip is not expected to have an effect on this result. This is confirmed with the results shown in Table 7.2, obtained with a conical tip. The maximum allowable acceleration is between 308 and $363 \mathrm{~m} / \mathrm{s}^{2}$, in agreement with the results obtained with the flat tip, and in agreement with the theoretical estimate obtained using Equation 7.3.

It is possible to estimate the maximum allowable acceleration in a BD Hyflow $1 \mathrm{~mL}$ syringe using Equation 7.3. The length of the liquid column $L_{l}$ is approxi- 
mately $4 \mathrm{~cm}$, which results in a maximum allowable acceleration of approximately $2500 \mathrm{~m} / \mathrm{s}^{2}$.

With an air gap, the syringe acceleration in a SureClick autoinjector is as large as $7000 \mathrm{~m} / \mathrm{s}^{2}$. Without an air gap, accelerations as large as $15,000 \mathrm{~m} / \mathrm{s}^{2}$ have been measured. The results indicate the syringe acceleration needs to be reduced substantially in order to avoid cavitation.

In summary, the experimental results in this section demonstrate that it is possible to eliminate cavitation by decreasing the peak magnitude of syringe acceleration. Equation 7.2 is a more restrictive bound on the maximum allowable acceleration, obtained by assuming a constant acceleration. Equation 7.3 is a less restrictive bound, obtained through a direct application of Newton's second law of motion. The experimental results are in better agreement with the latter.

The results were specialized to the case of a BD Hyflow $1 \mathrm{~mL}$ pre-filled syringe. The results indicate the peak magnitude of acceleration should be limited to approximately $2500 \mathrm{~m} / \mathrm{s}^{2}$ to avoid cavitation inside the pre-filled syringe. Note that this result is independent of the syringe material, all other factors being the same. 
Chapter 8

\section{SUMMARY \& FUTURE WORK}

\subsection{Summary}

The transient events that can potentially occur during device actuation were investigated in this thesis. Some aspects of the transient events were studied experimentally in a real autoinjector device using novel in situ measurement techniques: miniature pressure transducers and strain gauges are used to measure the pressure and strains during the actuation phase, along with digital high-speed cameras to study the motion of the internal components. Other aspects of the transients events were studied experimentally using a large-scale model autoinjector which uses a syringe with a diameter which is approximately 6 times larger than the diameter of typical pre-filled syringe. It is the first time a scaled-up model is used to study the mechanics of autoinjector devices. One version of the large-scale autoinjector does not include the syringe motion, and another version includes the motion. Advanced numerical simulations were also performed in order to learn key information about physical phenomena which are difficult to measure experimentally, such as stresses in the syringe wall, the magnitude of shock focusing in the syringe cone, and the magnitude of shock waves created by cavitation events.

\section{Autoinjector Dynamics}

The dynamic events which can occur inside an autoinjector device such as a SureClick were described in detail in this thesis. To the author's knowledge, this is the first time a detailed study of the dynamic events and the resulting pressure and stress transients has been carried out and reported in the open literature.

One dynamic event is the syringe pressurization created by the impact of the springactuated driving rod on the plunger-stopper. When there is no air gap between the plunger-stopper and the syringe liquid content, the pressurization occurs rapidly through relatively sharp pressure waves. The magnitude of the pressure wave created by the motion of the plunger-stopper into the syringe can be estimated using acoustic theory. When an air gap is located between the plunger-stopper and the syringe liquid content, the syringe is pressurized through the relatively slow isentropic compression of the gap, and the pressure pulses are not sharp. 
Another dynamic event is the syringe acceleration. When there is no air gap between the plunger-stopper and the syringe liquid content, the syringe acceleration is created by the additive effect of the liquid pressure applied on the bottom wall of the syringe, and the friction between the plunger-stopper and the syringe. The syringe pressurization and the syringe acceleration occur almost simultaneously, and no cavitation occurs inside the syringe. When an air gap is initially located between the plunger-stopper and the syringe liquid content, the pressurization of the syringe occurs slowly. The syringe acceleration is primarily caused by the friction force between the plunger-stopper and the syringe, and this occurs before the liquid content becomes pressurized through air gap compression. This situation generally results in transient cavitation inside the syringe. The cavitation event is caused by the tension waves created by the rapid syringe acceleration. This is the first time cavitation in the syringe of an autoinjector was reported.

The last dynamic event discussed in this thesis is the syringe deceleration upon reaching the right penetration depth for the needle. The syringe deceleration creates a pressure pulse inside the liquid, and a stress wave inside the syringe wall. The magnitude of both can be estimated with reasonable fidelity using simple estimates developed and validated in this thesis using Newton's second law of motion: the peak magnitude of the pressure and stress waves is directly related to the magnitude of the syringe deceleration.

\section{Syringe Material}

Plastic syringes using a dry lubricant between the plunger-stopper and the syringe behave differently than glass syringes with silicone oil as the lubricant. The friction between the plunger-stopper and the syringe during the transient events is substantially reduced with the plastic syringe. This has the effect of synchronizing the syringe pressurization and the syringe acceleration. This has the benefit of suppressing cavitation, even when an air gap is initially present between the plunger-stopper and the syringe liquid content.

\section{Shock Focusing in the Syringe Cone}

The dynamic events can create sharp pressure waves inside the syringe. This is particularly true when there is no air gap between the plunger-stopper and the liquid content, or when cavitation occurs inside the syringe. Past research on shock focusing has shown that shock waves can be enhanced inside a cone. 
The amplification of acoustic pressure waves in a cone was investigated in this thesis. To the author's knowledge, this is the first time the magnitude of the focusing effect is quantified parametrically as a function of the rise time of the pressure pulse, the half-angle of the cone, and the acoustic impedance of the cone. It is also the first time the focusing effect in real syringe geometries is studied.

This thesis has shown that amplification of acoustic pressure pulses is possible only if the pulse is sufficiently sharp: the rise time needs to be less than one acoustic transit time of the waves within the straight cone. Also, the magnitude of the amplification inside the cone depends strongly on the half-angle and the acoustic impedance of the cone. For rigid cones, smaller half-angles result in higher amplification of the pressure pulse. When the acoustic impedance of the wall is reduced, the relation between the amplification factor and the half-angle of the cone becomes more complex. This is because the half-angle of the cone affects the depth of the cone which, in turn, affects the number of radial reverberation of the incident pressure pulse. Each radial reverberation results in a fraction of the pressure pulse being transmitted in the solid wall. As expected, no amplification occurs if the acoustic impedance of the wall is less than the acoustic impedance of the liquid.

The results indicate that shock focusing is likely to occur in glass syringes. Simulation results have shown that infinitely sharp pressure waves could be amplified by a factor of 4.5 to 5 within the cone of a BD Hyflow $1 \mathrm{~mL}$ pre-filled glass syringe. Plastic syringes have a lower acoustic impedance, resulting in no or only a small amount of amplification of the waves within the cone. Syringes terminated with a flat wall or a cone with a large half-angle, regardless of the syringe material, result in no or only a small amount of amplification.

When an air gap is initially present between the plunger-stopper and the liquid content, the pressure waves created by the motion of the plunger-stopper into the syringe has a long rise time, and no shock focusing of the incident pressure pulse created by the impact of the driving rod on the plunger-stopper is possible.

\section{Cavitation}

When cavitation occurs, large bubbles forming inside the syringe cone are consistently observed. The bubbles typically fill the entire cone, meaning they do not have a spherical shape. The collapse of the bubbles is rapid, creating a substantial pressure transient. Pressures as large as $10 \mathrm{MPa}$ were measured inside a pre-filled syringe 
during actuation. Pressures as large as $50 \mathrm{MPa}$ were measured in the large-scale model autoinjector.

To the author's knowledge, no experimental or numerical study has been published to date on the collapse of cavitation bubbles inside a straight cone. Researchers previously reported on the collapse of bubbles in the vicinity of a straight wall or inside a narrow channel. A preliminary numerical study of the collapse of bubbles in a straight cone was discussed in this thesis. More work is needed to develop a full understanding of bubble collapse in a cone.

Advanced numerical simulations of the collapse of initially spherical bubbles inside a cone suggest the pressure created on the syringe wall could be as large as $100 \mathrm{MPa}$ upon bubble collapse. The simulation results also indicate the cone can substantially reduce the collapse time of a bubble inside a cone. The corner waves focusing on the axis of symmetry of the syringe are responsible for increasing the rate at which the bubble collapses.

\section{Potential Failure Mechanism}

The potential for syringe failure was studied through numerical simulations using LS-DYNA. The author is aware that numerical studies have been performed by some device manufacturers, but the result of those studies have not been published in the open literature. Therefore, this is the first time a study of the potential for failure resulting from the dynamic events is made available to the public.

The results indicate the pressurization of the syringe and the rapid syringe deceleration create a potential for failure. Those transient events could result in syringe failures origination in the vicinity of the syringe shoulder, and/or the junction between the flange and the barrel. Despite the presence of shock focusing, the cone region is not the area which is most likely to fail because the cone and tip is where the syringe wall is typically the thickest. The results also indicate that supporting the syringe using its flange increases the potential for syringe failure substantially, meaning that it is preferable to use the syringe shoulder to support the syringe.

Numerical simulations have shown that the rapid collapse of cavitation bubbles can create highly localized, but relatively large stresses in the vicinity of the bubble. Bubbles collapsing in the syringe cone are likely capable to create a low but detectable failure rate, suggesting that cavitation in the syringe should be avoided in order to minimize the likelihood of glass failure. 


\section{Mitigation}

The pressure and stress transients can be mitigated though various design modifications, such as:

- a reduction of the impact velocity between the components;

- a reduction of the peak acceleration/deceleration of the moving components;

- elimination of cavitation.

A simple model validated using experiments was developed to predict the maximum magnitude of syringe acceleration which is allowable in order to preclude cavitation inside the syringe. This new and simple model could be used by device manufacturer to determine the maximum allowable syringe acceleration. It is advisable to make necessary design adjustments to maintain the syringe acceleration well below the estimated threshold. In a $1 \mathrm{~mL}$ pre-filled syringe, the maximum acceleration should be no more than approximately $2500 \mathrm{~m} / \mathrm{s}^{2}$.

It is possible to use damping in autoinjector devices which use stiff springs. The novel experimental techniques from this thesis were used to demonstrate that dampers are an effective way of reducing the peak magnitude of the pressure and stress transients. This, in turn, has the effect of reducing the potential for device failure. The user experience is also improved because damping reduces the jerking motion of the device upon release of the stiff spring.

\subsection{Future Work}

The work reported in this thesis can be extended in several ways to develop a deeper understanding and study new aspects of the pressure and stress transients in autoinjector devices. The results from the preceding chapters have highlighted several key elements which deserve to be investigated further in order to improve upon current designs. Some suggested future work is indicated below.

A second version of the large-scale model autoinjector could be designed; the information learned in this thesis should be used to improve the design of the model autoinjector. The new setup should allow for a better control of the timing between pressurization and acceleration of the syringe, an important parameter, and the effect of the timing between both should be investigated systematically. The mechanism used to initiate the transient events should also be revised: there should be a constant force applied on the buffer throughout the transient events, equivalent to the spring 
force applied on the plunger-stopper of a syringe. The physical results reported and discussed in this thesis along with the simple models discussed should also be used to scale more effectively the newer, large-scale model autoinjector test setup.

The large-scale model autoinjector could also be used to investigate further the effect of thoroughly degassing the liquid contained within the syringe on the cavitation events. Careful processing of the inner surface of the syringe to reduce the size and number of nucleation sites could be investigated. Degassing the liquid together with reducing the number of nucleation sites at the wall could maybe reduce the cavitation threshold (i.e., increase the magnitude of the tensile waves that are necessary to initiate cavitation), but this has not been confirmed.

The breakage of glass and plastic syringes as a result of cavitation events should be studied in greater detail. Experimental evidence (i.e., breakage of a beer bottle through column separation) have shown that cavitation can indeed damage glass. The collapse of bubbles inside a syringe creates pressure pulses of very short duration due to the small size of the bubbles. The effect of the relatively small impulse and the large strain rate need to be accounted for in determining the potential for damage. Using a Weibull curve to study syringe glass failure is an oversimplified approach, and concepts of fracture mechanics need to be used to study this problem more systematically.

Experiments could be designed to gather useful information to develop a better understanding of failures created by collapsing bubbles. For example, laser-induced bubbles could be created in close proximity of a glass/plastic surface to study stresses, erosion, crack propagation, etc. It would be possible to consider creating laser-induced bubbles directly inside the cone of a pre-filled syringe, and perhaps reproduce the cone failures observed by Amgen during clinical trials. The effect of collapsing bubbles on the delicate potent molecules should also be considered.

The effect of the syringe cone on collapsing bubbles should be studied more extensively though numerical simulations. In a pre-filled syringe, the bubble is not spherical, and this can affect substantially the collapse process. Bubble placement inside the cone is another factor which can influence the collapse. In particular, the collapse of bubbles located away from the axis of symmetry should be investigated as those could create a re-entrant jet oriented toward the syringe wall rather than the channel leading to the needle. The natural collapse of bubbles (i.e., no shock wave) should also be investigated because the effect of the cone could be significantly different than for a shock-induced collapse. 
More sophisticated damping methods could be considered and tested to mitigate the pressure and stress transients in autoinjector devices. An autoinjector device could be modified to include viscous dampers or dashpots. In situ measurements could be performed to assess the efficacy of those at reducing the magnitude of the pressure and stress waves created upon device actuation.

More in situ experiments should be performed with different syringe models (both glass and plastic syringes) and with different autoinjector devices. Using a device other than a SureClick needs to be considered as this might reveal new information about the dynamic events inside spring-actuated autoinjectors.

Lastly, the modeling aspect of the transient events should be investigated further. Numerical models which can be used to rapidly assess new device designs need to be developed. Effective numerical models should be used during the design phase to obtain key information and guide the device engineers in their work. Ultimately, the appropriate use of well designed numerical models to obtain relevant input early in the design phase will improve the devices, reduce the likelihood of device failure due to glass breakage, improve the user experience, and, more importantly, improve adherence to prescribed therapies. 


\section{BIBLIOGRAPHY}

Adler, M. (2012). Challenges in the Development of Pre-filled Syringes for Biologics from a Formulation Scientist's Point of View. On the WWW. http : //www . americanpharmaceuticalreview. com/. Accessed April 6, 2018.

Akers, M. J. (2010). Sterile Drug Products: Formulation, Packaging, Manufacturing and Quality. 1st ed. Drugs and the Pharmaceutical Sciences. Boca Raton, FL: CRC Press. ISBN: 9780849339936.

American Glass Research (2016). Analysis of Syringe Breakage. Tech. rep. C329-16. Butler, PA.

Amgen (2016). Instructions for Use-Enbrel (etanercept) injection for subcutaneous use Single-dose Prefilled SureClick Autoinjector. https: //pi . amgen . com/ $\sim$ /media / amgen / repositorysites / pi - amgen - com / enbrel / enbrel_ scifu.pdf. Thousand Oaks, CA.

Apazidis, N. and Eliasson, V. (2019). Shock Focusing Phenomena: High Energy Density Phenomena and Dynamics of Converging Shocks. 1st ed. Shock Wave and High Pressure Phenomena. Springer International. ISBN: 9783319758640. DOI: $10.1007 / 978-3-319-75866-4$.

Arendt-Nielsen, L., Egekvist, H., and Bjerring, P. (2006). "Pain following controlled cutaneous insertion of needles with different diameters". In: Somatosensory \& Motor Research 23 (1-2), pp. 37-43. DoI: 10. 1080/08990220600700925.

Bergant, A. and Simpson, A.R. (1999). "Pipeline Column Separation Flow Regimes". In: ASCE Journal of Hydraulic Engineering 125 (8), pp. 835-848. DOI: 10.106 1/(ASCE) 0733-9429(1999) $125: 8$ (835).

Bergant, A., Simpson, A.R., and Tijsseling, A.S. (2006). "Water hammer with column separation: A historical review". In: Journal of Fluids and Structures 22 (2), pp. 135-171. DoI: 10.1016/j . jfluidstructs.2005.08.008.

Bitter, N.P. and Shepherd, J.E. (2013). "On the Adequacy of Shell Models for Predicting Stresses and Strains in Thick-Walled Tubes Subjected to Detonation Loading". In: Proceedings of the ASME 2013 Pressure Vessels and Piping Conference. (Paris, France). Vol. 5. Paper PVP2013-97148. Dor: 10. 1115/PVP2013-97148.

Bond, C. et al. (2009). "Shock focusing in a planar convergent geometry: experiment and simulation". In: Journal of Fluid Mechanics 641, pp. 297-333. Dor: 10.101 7/S0022112009991492.

Bourne, N.K. and Field, J.E. (1992). "Shock-induced collapse of single cavities in liquids". In: Journal of Fluid Mechanics 244, pp. 225-240. DoI: 10.1017/S002 2112092003045.

Bower, A.F. (2009). Applied Mechanics of Solids. 1st ed. Boca Raton, FL: CRC Press. ISBN: 9781439802472. 
Brennen, C.E. (1995). Cavitation and Bubble Dynamics. Oxford Engineering Science Series. Oxford University Press. IsBN: 9780195094091.

Cadranel, J.F. et al. (2007). "Satisfaction of patients treated for chronic hepatitis $\mathrm{C}$ with the peginterferon alfa-2b pen device: the VISA observational study". In: Gastroenterologie Clinique et Biologique 31 (2), pp. 180-184. DoI: GCB-02-20 07-31-2-0399-8320-101019-200520011.

Callister, W.D. and Rethwisch, D.G. (2014). Materials Science and Engineering: an Introduction. 9th ed. Hoboken, NJ: John Wiley and Sons. ISBN: 9781118324578.

Cole, R. H. (1948). Underwater Explosions. Princeton, NJ: Princeton University Press.

Coralic, V. and Colonius, T. (2014). "Finite-volume WENO scheme for various compressible multicomponent flows". In: Journal of Computational Physics 274, pp. 95-121. Dor: 10.1016/j.jcp.2014.06.003.

Courant, R. and Friedrichs, K.O. (1976). Supersonic Flow and Shock Waves. 1st ed. Vol. 21. Applied Mathematical Sciences. Springer-Verlag New York. ISBN: 9780387902326.

Daily, D.J. et al. (2012). Catastrophic Cracking Courtesy of Quiescent Cavitation. eprint: arXiv: 1210.3764 .

Davis, J.L (1988). Wave Propagation in Solids and Fluids. 1st ed. Springer-Verlag New York. ISBN: 9781461283904. Dor: 10.1007/978-1-4612-3886-7.

Eliasson, V. et al. (2010). "Experimental investigation of converging shocks in water with various confinment materials". In: Shock Waves 20, pp. 395-408. DoI: 10. $1007 / \mathrm{s} 00193-010-0276-9$.

Franc, J.P. and Michel, J.M. (2005). Fundamentals of Cavitation. 1st ed. Vol. 76. Fluid Mechanics and Its Applications. Springer Netherlands. ISBN: 9781402022326. DOI: $10.1007 / 1-4020-2233-6$.

French, D.L. and Collins, J.J. (2010). "Advances in parenteral injection devices and aids". In: Pharmaceutical Dosage Forms: Parenteral Medications. Ed. by S. Nema and J.D. Ludwig. 3rd ed. Vol. 3: Regulations, Validation and the Future. Boca Raton, FL: CRC Press. Chap. 5, pp. 71-85. ISBN: 9781420086478.

Fry, A. (2014). "Injecting Highly Viscous Drugs”. In: Pharmaceutical Technology 38 (11).

Fujikawa, S. and Akamatsu, T. (1980). "Effects of non-equilibrium condensation of vapor on the pressure wave produced by the collapse of a bubble in a liquid". In: Journal of Fluid Mechanics 97 (3), pp. 481-512. DOI: 10. 1017/S00221120800 Q2662. 
Future Market Insights (2017). Auto-Injectors Market: Rising Incidence of Anaphylaxis Attack Among the Adult Population Expected to Elevate the Demand for Auto-Injectors: Global Industry Analysis and Opportunity Assessment 20162026. Tech. rep. REP-GB-1642. uRL: https: //www . futuremarketinsights . com/reports/auto-injectors-market.

Greenberg, M. (1998). Advanced Engineering Mathematics. 2nd ed. Pearson. IsBN: 9780133214314.

Haas, J.F. and Sturtevant, B. (1987). "Interaction of weak shock waves with cylindrical and spherical gas inhomogeneities". In: Journal of Fluid Mechanics 181, pp. 41-76. DOI: 10.1017/S0022112087002003.

Hallquist, J.O. (2016). LS-DYNA : THEORY MANUAL. http : / Www . lstc . com. Livermore Software Technology Corporation. Livermore, CA.

Hannah, R.L. and Reed, S.E. (1992). Strain Gage Users' Handbook. 1st ed. London, UK: Chapman \& Hall. IsBN: 9780412537202.

Harlow, F. and Amsden, A. (1971). Fluid Dynamics. Tech. rep. LANL Monograph LA-4700. Los Alamos National Labs.

Harvinder, G.S. and Prausnitz, M.R. (2007). “Does Needle Size Matter?” In: Journal of Diabetes Science and Technology 1 (5), pp. 725-729. Dor: 10.1177/193229 680700100517.

Heise, T. et al. (2014). "Impact of injection speed and volume on perceived pain during subcutaneous injections into the abdomen and thigh: a single-centre, randomized controlled trial”. In: Diabetes, Obesity \& Metabolism 16 (10), pp. 971976. DoI: $10.1111 /$ dom. 12304 .

Hibbeler, R.C. (2010). Mechanics of Materials. 8th ed. Pearson Education Canada. ISBN: 9789810685096.

Inaba, K. and Shepherd, J.E. (2010). "Dynamics of Cavitating Flow and Flexural Waves in Fluid-Filled Tubes Subject to Axial Impact". In: Proceedings of the ASME 2010 Pressure Vessels \& Piping Division. (Bellevue, WA). Vol. 4. Paper PVP2010-25989. DOI: 10.1115/PVP2010-25989.

Johnsen, E. and Colonius, T. (2009). "Numerical simulations of non-spherical bubble collapse". In: Journal of Fluid Mechanics 629, pp. 231-262. DoI: 10 . 1017 /S0022112009006351.

Johnson, K. L. et al. (2014). “Geometric Effects on Stress Wave Propagation”. In: Journal of Biomechanical Engineering-Transactions of the ASME 136 (2). DoI: 10.1115/1.4026320.

Jones, N. (1989). Structural Impact. 1st ed. Cambridge, U.K.: Cambridge University Press. ISBN: 9780521628907. 
Jorgensen, J.T. et al. (1996). "Pain Assessment of Subcutaneous Injections". In: The Annals of Pharmacotherapy 30 (7-8), pp. 729-732. DoI: 10.1177/1060028096 03000703.

Kojima, T. et al. (2017). "Dynamics of Wave Propagation Across Solid-Fluid Movable Interface in Fluid-Structure Interaction". In: Journal of Pressure Vessel Technology 139 (3). DOI: 10.1115/1. 4035376.

Kolsky, H. (1953). Stress Waves in Solids. London, U.K.: Oxford University Press.

Kundu, P.K., Cohen, I.M., and Dowling, D.R. (2012). Fluid Mechanics. 5th ed. Oxford, U.K.: Elsevier. ISBN: 9780123821003.

Lange, J. and Thompson, I. (2013). "Self-Injection Devices". In: Encyclopedia of Pharmaceutical Science and Technology. Ed. by J. Swarbrick. 4th ed. Taylor \& Francis, pp. 3132-3143. ISBN: 9781841848198.

Lauterborn, W. and Bolle, H. (1975). "Experimental investigations of cavitationbubble collapse in the neighbourhood of a solid boundary". In: Journal of Fluid Mechanics 72 (2), pp. 391-399. DOI: 10.1017/S0022112075003448.

Li, L. et al. (2014). “Concentration Dependent Viscosity of Monoclonal Antibody Solutions: Explaining Experimental Behavior in Terms of Molecular Properties". In: Pharmaceutical Research 31 (11), pp. 3161-3178. DoI: 10.1007/s11095-0 14-1409-0.

Lide, D. R. (1990). CRC Handbook of Chemistry and Physics. 71st ed. CRC Press. ISBN: 9780849304712.

Liepmann, H.W. and Roshko, A. (2002). Elements of Gasdynamics. Mineola, NY: Dover Publications. ISBN: 9780486419633.

Limmroth, V. and Gerbershagen, K. (2014). "Single-use autoinjector for onceweekly intramuscular injection of IFN $\beta$-1a". In: Expert Opinion on Drug Delivery 11 (12), pp. 1969-1978. DoI: 10.1517/17425247.2014.943181.

Liou, J.C.P. (2000). "Numerical Properties of the Discrete Gas Cavity Model for Transients". In: Journal of Fluids Engineering 122 (3), pp. 636-639. DoI: 10. 11 15/1. 1287037.

McLellan, G.W. and Shand., E.B. (1984). Glass Engineering Handbook. 3rd ed. McGraw-Hill. IsBN: 9780070448230.

Meng, J.C. (2016). "Numerical Simulations of Droplet Aerobreakup". PhD thesis. Pasadena, CA: California Institute of Technology.

Pierce, A.D. (1989). Acoustics : an Introduction to its Physical Principles and Applications. 2nd ed. Woodbury, NY: Acoustical Society of America. IsBN: 9780883186121.

Plesset, M.S. and Chapman, R.B. (1971). "Collapse of an initially spherical vapour cavity in the neighbourhood of a solid boundary". In: Journal of Fluid Mechanics 47 (2), pp. 283-290. DOI: 10. 1017/S0022112071001058. 
Pozzilli, C. et al. (2011). "Supportive strategies to improve adherence to IFN $\beta$-1b in multiple sclerosis - results of the $\beta$ Plus observational cohort study". In: Journal of the Neorological Sciences 307 (1-2), pp. 120-126. DoI: 10.1016/j . jns . 201 1.04 .026$.

Proovost, G.A. (1976). "Investigation into cavitation in a prototype pipeline caused by water hammer". In: Proceedings of the Second International Conference on Pressure Surges, BHRA. (London, UK), pp. 13-29.

Rayleigh, J.W.S. (1917). "On the pressure developed in a liquid during the collapse of a spherical cavity". In: The London, Edinburgh, and Dublin Philosophical Magazine and Journal of Science 34 (200). DOI: 10 . 1080/1478644080863568 1.

Rayleigh, J.W.S. (1945). The Theory of Sound. 2nd ed. Vol. 1 \& 2. New York, NY: Dover.

Royer, D., Morgan, D.P., and Dieulesaint, E. (2000). Elastic Waves in Solids I: Free and Guided Propagation. 1st ed. Advanced Texts in Physics. Springer-Verlag Berlin Heidelberg. ISBN: 9783642085215.

Russel, D. A. (1967). "Shock-wave strengthening by area convergence”. In: Journal of Fluid Mechanics 27 (2), pp. 305-314. Dor: 10.1017/S0022112067000333.

Sacha, G., Rogers, J.A., and Miller, R.L. (2015). "Pre-filled syringes: a review of the history, manufacturing and challenges". In: Pharmaceutical Development and Technology 20 (1), pp. 1-11. DOI: 10.3109/10837450.2014.982825.

Schiff, M. et al. (2014). "New autoinjector technology for the delivery of subcutaneous methotrexate in the treatment of rheumatoid arthritis". In: Expert Review of Medical Devices 11 (5), pp. 447-455. Dor: 10.1586/17434440. 2014 . 929492.

Schmidmayer, K., Marty, A., et al. (2018). "ECOGEN, an open-source tool dedicated to multiphase compressible multiphysics flows". In: Proceedings of the 53rd $3 A F$ International Conference on Applied Aerodynamics. (Salon-de-Provence, France). Paper FP49-AERO2018-daniel. eprint: arXiv: 1805.01363.

Schmidmayer, K., Petitpas, F., and Daniel, E. "Simplified adaptive mesh refinement algorithm based on dual cell-boundary trees for multiphase compressible flows". In: Journal of Computational Physics. Under review. DoI: 10.13140/RG . 2 . 2 . 1 8739.53280 .

Selfridge, A. R. (1985). "Approximate Material Properties in Isotropic Materials". In: IEEE Transactions on Sonics and Ultrasonics 32 (3), pp. 381-394. DoI: 10.1 109/T-SU . 1985 . 31608.

Setchell, R. E., Storm, E., and Sturtevant, B. (1972). "Investigation of Shock Strengthening in a Conical Convergent Channel". In: Journal of Fluid Mechanics 56, pp. 505-522. DOI: 10. 1017/S0022112072002484. 
Shepherd, J. E. and Pintgen, F. (2007). Elastic and Plastic Structural Response of Tubes to Deflagration-to-Detonation Transition. Tech. rep. FM2006-005. Graduate Aerospace Laboratories of the California Institute of Technology. URL: http: //shepherd.caltech.edu/EDL/publications/reprints/galcit_fm200 6-005.pdf.

Shepherd, J.E. and Inaba, K. (2010). "Shock Loading and Failure of Fluid-filled Tubular Structures". In: Dynamic Failure of Materials and Structures. Ed. by A. Shukla, G. Ravichandran, and Y.D.S. Rajapakse. 1st ed. Springer US. Chap. 6, pp. 153-190. ISBN: 978-1-4899-8420-3. DOI: 10 . 1007/978-1-4419-0446-1.

Shire, S.J., Shahrokh, Z., and Liu, J. (2004). "Challenges in the development of high protein concentration formulations". In: Journal of Pharmaceutical Sciences 93 (6), pp. 1390-1402. DOI: 10.1002/jps. 20079.

Simpson, A.R. and Bergant, A. (1994). "Numerical Comparison of Pipe-ColumnSeparation Models". In: ASCE Journal of Hydraulic Engineering 120 (3), pp. 361377. DOI: 10.1061/ (ASCE) 0733-9429 (1994) 120:3 (361).

Singh, R., Singh, S., and Lillard, J.W. (2008). "Past, Present, and Future Technologies for Oral Delivery of Therapeutic Proteins". In: Journal of Pharmaceutical Sciences 97 (7), pp. 2497-2523. DoI: 10.1002/jps. 21183.

Smith, P. D. and Hetherington, J. G. (1994). Blast and Ballistic Loading of Structures. 1st ed. Taylor and Francis. ISBN: 9780750620246.

Stout, D. and Vilivalam, V. (2009). "Plastic Prefilled Syringes: A Better Fit for Autoinjector Systems". In: Pharmaceutical Technology Volume 2009 Supplemental (6).

Streeter, V.L. (1969). "Water Hammer Analysis". In: ASCE Journal of the Hydraulics Division 95 (6), pp. 1959-1972.

Sturtevant, B. and Kulkarny, V.A. (1976). "The focusing of weak shock waves". In: Journal of Fluid Mechanics 73 (4), pp. 651-671. Dor: 10.1017/S00221120760 Q1559.

Supponen, O. et al. (2017). "Shock waves from nonspherical cavitation bubbles". In: Physical Review Fluids 2. Dor: 10.1103/PhysRevFluids . 2.093601.

Thompson, I. (2006). "Self-Injection Technology and Trends". In: Journal of Innovations in Pharmaceutical Technology 20 (6), pp. 60-63.

Thompson, I. and Lange, J. (2013). "Pen and Autoinjector Drug Delivery Devices". In: Sterile Product Development: Formulation, Process, Quality and Regulatory Considerations. Ed. by P. Kohle, M. Shah, and N. Rathore. 1st ed. Vol. 6. AAPS Advances in the Pharmaceutical Sciences Series. Springer-Verlag New York. Chap. 13, pp. 331-356. IsBN: 978-1-4614-7978-9. Dor: 10. 1007/978-1-4614 -7978-9_13.

Thompson, P.A. (1972). Compressible Fluid Dynamics. 1st ed. Advanced engineering series. New York, NY: McGraw-Hill. IsBN: 9780070644052. 
Trevana, D.H. (1987). Cavitation and tension in liquids. 1st ed. CRC Press. ISBN: 9780852744543.

Turner, M.R. and Balu-Iyer, S.V. (2018). "Challenges and Opportunities for the Subcutaneous Delivery of Therapeutic Proteins". In: Journal of Pharmaceutical Sciences 107 (5), pp. 1247-1260. DoI: 10.1016/j . xphs .2018.01.007.

Veilleux, J.-C., Jazayeri, J., et al. (2017). "Primary Packaging \& Delivery Systems Interaction". In: Presented at the 10th Workshop on Monoclonal Antibodies of the PDA. DOI: 10.13140/RG.2.2.10549.37601.

Veilleux, J.-C. and Shepherd, J.E. (2017). "Dampers and Methods for Performing Measurements in an Autoinjector". Patent application 20180015224 (US). URL: https://www . lens.org/lens/patent/123-673-900-361-650.

Vishay - Micro-Measurement (2010a). Installation Verification. 01-04-10. Application Note VMM8, Document 11171, http: //www . vi shaypg . com/docs/11 171/VMM- 8.pdf.

Vishay - Micro-Measurement (2010b). Installing Gages with Option P. 01-04-10. Application Note VMM-9, Document 11172, http : //www . vishaypg . com/ docs/11172/VMM-9.pdf.

Vishay - Micro-Measurement (2010c). Optimizing Strain Gage Excitation Levels. 01-11-10. Technical Note TN-502, Document 11052, http://www . vishaypg. com/docs/11052/tn502.pdf.

Vishay - Micro-Measurement (2014). Surface Preparation for Strain Gage Bonding. 08-07-14. Instruction Bulletin B-129-8, Document 11129, http: //www . visha ypg.com/docs/11129/11129B129.pdf.

Vishay - Micro-Measurement (2018). Strain Gage Installations with M-Bond 200 Adhesive. 02-12-18. Instruction Bulletin B-127, Document 11127, http: //www . vishaypg . com/docs/11127/11127B127 . pdf.

Wang, C. and Eliasson, V. (2012). "Shock wave focusing in water inside convergent structures". In: International Journal of Multiphysics 6 (3), pp. 267-281.

Warne, N.W. (2011). "Development of high concentration protein biopharmaceuticals: the use of platform approaches in formulation development". In: European Journal of Pharmaceutics and Biopharmaceutics 78 (2), pp. 208-212. DoI: 10.1 016/j.ejpb.2011.03.004.

Watters, G.Z. (1984). Analysis and Control of Unsteady Flow in Pipelines. 2nd ed. Stoneham, MA: Butterworths. ISBN: 9780250404926.

Weibull, W. (1951). "A statistical distribution function of wide applicability". In: Journal of Applied Mechanics 18, pp. 293-297. 
West Pharmaceutical Services. When Performance Matters Most-Daikyo Crystal Zenith. Document 8427C 0814, https: //www . westpharma.com/products/ prefillable - systems / daikyo - syringe - systems / daikyo - crystal zenith-insert-needle.

Whitham, G.B. (1999). Linear and Nonlinear Waves. New York, NY: Wiley Interscience. ISBN: 9780471359425.

Wiggert, D.C. and Tijsseling, A.S. (2001). "Fluid transients and fluid-structure interaction in flexible liquid-filled piping". In: Applied Mechanics Reviews 54 (5), pp. 455-481. DOI: 10.1115/1.1404122.

Wilkins, J. and Simpson, I. (2012). Mathematical Modeling for Faster Autoinjector Design. On the WWW. http://www . drug-dev. com/. Accessed April 6, 2018.

Wu, Z., Zong, Z., and Sun, L. (2014). “A Mie-Grüneisen mixture Eulerian model for underwater explosion”. In: Engineering Computations 31 (3), pp. 425-452. DOI: $10.1108 / \mathrm{EC}-03-2012-0065$.

Wylie, E. B. (1984). "Simulation of Vaporous and Gaseous Cavitation". In: Journal of Fluids Engineering 106 (3), pp. 307-311. DoI: 10.1115/1.3243120.

Wylie, E.B. and Streeter, V.L. (1993). Fluid Transients in Systems. Pearson. ISBN: 9780139344237.

Yadav, S., Shire, S.J., and Kalonia, D.S. (2010). "Factors affecting the viscosity in high concentration solutions of different monoclonal antibodies". In: Journal of Pharmaceutical Sciences 99 (12), pp. 4912-4829. DOI: 10.1002/jps . 22190.

Young, W.C. and Budynas, R.G. (2001). Roark's Formulas for Stress and Strain. 7th ed. McGraw-Hill. IsBN: 9780070725423. 
Appendix A

\section{ACOUSTICS AND METHOD-OF-CHARACTERISTICS}

\section{A.1 Introduction}

Acoustics began as the study of sounds in the air created by natural speech, musical instruments or mechanical vibrations like a tuning fork. Rayleigh's investigations combined the approach of natural philosophy with the mathematical methods developed in the 18th century to provide the first comprehensive mathematical theory of sound (Rayleigh, 1945). In modern usage, acoustics is the study of any small amplitude motions in a compressible material, which could be a gas, liquid or solid (Thompson, 1972).

The meaning of small amplitude is that changes in the pressure $\Delta P$ produce motions in the fluid with velocities $\Delta u$ much smaller than the speed of sound $c$. The speed of sound is defined by the thermodynamic relationship along the isentrope $s=$ constant:

$$
c=\sqrt{\left(\frac{\partial P}{\partial \rho}\right)_{s}} .
$$

This can be used to relate small changes in pressure to changes in density:

$$
\Delta P=c^{2} \Delta \rho .
$$

As it will be shown in the next section, the followings are all equivalent definitions of the meaning of small amplitude:

$$
\Delta u \ll c, \quad \Delta P \ll \rho c^{2}, \quad \Delta \rho<\rho .
$$

In a gas at initial pressure $P_{0}$, the restriction on pressure changes reduces to $\Delta P \ll$ $P_{0}$. In liquids and solids it is possible that $\Delta P \gg P_{0}$ and the acoustic approximation is still valid. For example, $\rho c^{2}=2.25 \mathrm{GPa}$ in water, so that pressure wave peak amplitudes as high as $100 \mathrm{MPa}$ can still be reasonably treated with the methods of acoustics. 


\section{A.2 Acoustics and Fluid Mechanics}

In general, fluid mechanics does not make the assumption of small amplitude motions, and the motion is described mathematically by a set of partial differential equations that describe the conservation of mass, momentum, and energy:

$$
\begin{aligned}
& \frac{\partial \rho}{\partial t}+u \frac{\partial \rho}{\partial x}=-\rho \frac{\partial u}{\partial x}, \\
& \frac{\partial u}{\partial t}+u \frac{\partial u}{\partial x}=-\frac{1}{\rho} \frac{\partial P}{\partial x}, \\
& \frac{\partial e}{\partial t}+u \frac{\partial e}{\partial x}=-\frac{P}{\rho} \frac{\partial u}{\partial x} .
\end{aligned}
$$

These equations have been simplified by omitting the effect of viscosity or other transport processes like thermal conduction. In this form, the governing equations are referred to as the Euler equations, and are the most common approximation used in modeling compressible flows.

The Euler equations describe how the quantities $(\rho, u, e)$ vary with time to an observer that is traveling with the local fluid velocity. This is the meaning of the common derivative on the left-hand side of Equations A.4 to A.5:

$$
\frac{\partial(\cdot)}{\partial t}+u \frac{\partial(\cdot)}{\partial x}=\frac{\mathrm{d}(\cdot)}{\mathrm{d} t} \quad \text { on } \quad \frac{\mathrm{d} x}{\mathrm{~d} t}=u .
$$

On the right-hand side of Equations A.5 and A.6 is the pressure $P$, which must be computed by supplying a thermodynamic relationship between internal energy $e$, density $\rho$, and pressure $P$ in the form of an equation of state:

$$
P=P(e, \rho) .
$$

For gases, this equation of state is often approximated by $e=\gamma P / \rho$, which leads to the classical equations of gas dynamics discussed by Liepmann and Roshko (2002). For liquids, the methods of thermodynamics have to be used to construct an appropriate relationship.

The fundamental relationship of thermodynamics:

$$
\mathrm{d} e=T \mathrm{~d} s+P \frac{1}{\rho^{2}} \mathrm{~d} \rho,
$$

provides an additional constraint on the relationship between changes in energy and density. Combining Equations A.4 and A.6, one finds that the equations of motion imply that:

$$
\frac{\mathrm{d} e}{\mathrm{~d} t}=P \frac{1}{\rho^{2}} \frac{\mathrm{d} \rho}{\mathrm{d} t} .
$$


Interpreting the changes in Equation A.9 as following the fluid motion in the sense of Equation A.7, an equivalent form of the energy equation is to specify that entropy is unchanged along the fluid motion paths:

$$
\frac{\partial s}{\partial t}+u \frac{\partial s}{\partial x}=0
$$

This relationship only works for smoothly varying compressible flows. When there are shock waves which result in rapid jumps in the properties across the wave front, additional considerations are needed.

Finally, using the definition of sound speed (see Equation A.1), for isentropic flows in any fluid it is possible to relate pressure and density changes along the fluid paths:

$$
\frac{\mathrm{d} P}{\mathrm{~d} t}=c^{2} \frac{\mathrm{d} \rho}{\mathrm{d} t} .
$$

This relationship is a generalization of Equation A.2 to the situation of a nonuniform fluid which may have different values of entropy and sound speed at different locations. Combining Equations A.2 and A.11, density can be eliminated, and the energy equation can be replaced by the entropy relationship to obtain the following version of the equations of fluid motion:

$$
\begin{aligned}
& \frac{\partial P}{\partial t}+u \frac{\partial P}{\partial x}=-\rho c^{2} \frac{\partial u}{\partial x} \\
& \frac{\partial u}{\partial t}+u \frac{\partial u}{\partial x}=-\frac{1}{\rho} \frac{\partial P}{\partial x} \\
& \frac{\partial s}{\partial t}+u \frac{\partial s}{\partial x}=0
\end{aligned}
$$

which are the most useful starting point for discussing the method-of-characteristics and acoustic approximations.

These equations, like Equations A.4 to A.6, have to be supplemented by a thermodynamic relationship $s(P, \rho)$ when the entropy is spatially nonuniform. The special case of $s=$ constant, or homentropic flow, enables the elimination of Equation A.15 from the equation set, and the only thermodynamic relationship that is required is knowledge of the isentrope $P=P(\rho ; s=$ constant $)$. 


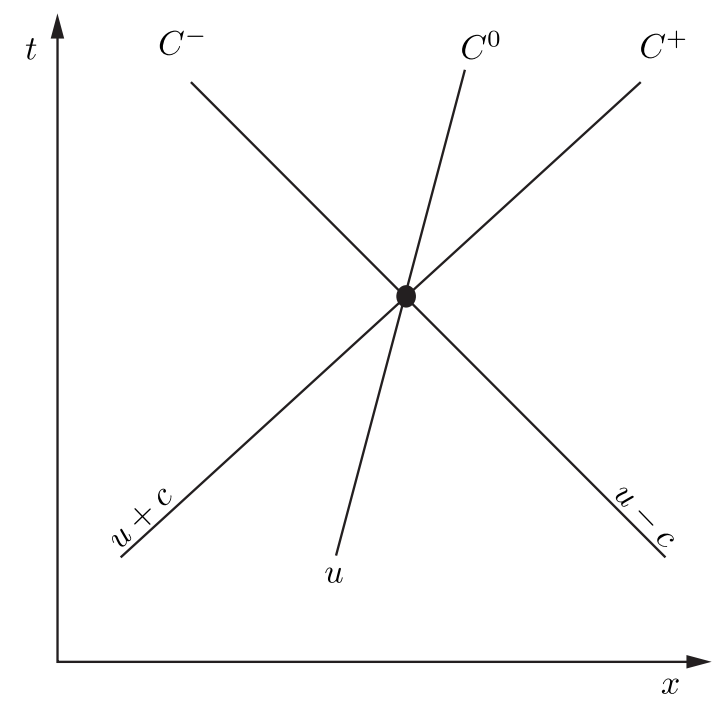

Figure A.1: Characteristic directions at a point in the flow. Labels correspond to the corresponding speeds $\mathrm{d} x / \mathrm{d} t$.

\section{A.3 Characteristics}

The method-of-characteristics is a solution method for solving Equations A.13 to A.15 using a transformation of coordinates and variables. The key idea is based on the recognition that the equations of compressible flow represent the propagation of disturbances in space with characteristic speeds of $u$ for changes in entropy, and $u \pm c$ for isentropic changes in pressure and velocity as shown in Figure A.1.

To rewrite the equations of motion in terms of these characteristic speeds, multiply Equation A.14 by $\rho c$, and add/substract from Equation A.13 to obtain two equivalent equations for $P$ and $u$. The system of equations becomes:

$$
\begin{aligned}
\left(\frac{\partial}{\partial t}+(u+c) \frac{\partial}{\partial x}\right) P+\rho c\left(\frac{\partial}{\partial t}+(u+c) \frac{\partial}{\partial x}\right) u & =0, \\
\left(\frac{\partial}{\partial t}+(u-c) \frac{\partial}{\partial x}\right) P-\rho c\left(\frac{\partial}{\partial t}+(u-c) \frac{\partial}{\partial x}\right) u & =0, \\
\left(\frac{\partial}{\partial t}+u \frac{\partial}{\partial x}\right) s & =0 .
\end{aligned}
$$

These equations are in characteristic form, which can be more concisely written as:

$$
\begin{aligned}
\frac{1}{\rho c} \frac{\mathrm{d} P}{\mathrm{~d} t} \pm \frac{\mathrm{d} u}{\mathrm{~d} t} & =0 & & \text { along } C^{ \pm}: & \frac{\mathrm{d} x}{\mathrm{~d} t} & =u \pm c \\
\frac{\mathrm{d} s}{\mathrm{~d} t} & =0 & & \text { along } C^{0}: & \frac{\mathrm{d} x}{\mathrm{~d} t} & =u .
\end{aligned}
$$




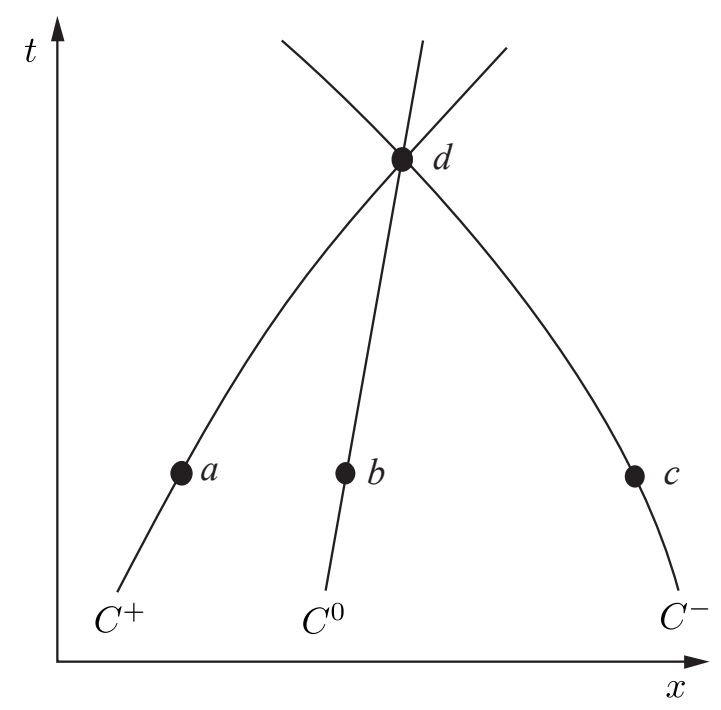

Figure A.2: Geometry of characteristics. The values of the variables at locations $a, b$ and $c$ can be used to determine the values later in time at location $d$ where all three characteristics intersect.

The paths $C^{ \pm}$are the characteristics, and in general form a network of curves in a space-time or $x$ - $t$ diagram, as shown in Figure A.2, as the slopes of the characteristics change with time and location within the flow.

Now consider the special case of $s=$ constant for which it is possible to define a unique function $F$ such that:

$$
\mathrm{d} F=\frac{\mathrm{d} P}{\rho c}, \quad F=\int \frac{\mathrm{d} P}{\rho c},
$$

where the integration is carried out along the isentrope. With this definition, the characteristic version of the equations becomes:

$$
\frac{\mathrm{d}}{\mathrm{d} t}(F \pm u)=0 \quad \text { on } \quad C^{ \pm}: \frac{\mathrm{d} x}{\mathrm{~d} t}=u \pm c
$$

The functions $F \pm u$ are known as the Riemann invariants, $\mathcal{P}$ and $\mathcal{Q}$, defined as:

$$
F+u=\mathcal{P} \quad F-u=Q .
$$

These quantities are referred to as invariants since Equation A.22 implies that $\mathcal{P}=$ constant on $C^{+}$, and $Q=$ constant on $C^{-}$. This property can be used to construct an algorithm to find the state of the motion at a later time given the solution at an earlier one. Referring to Figure A.2, $\mathcal{P}$ is evaluated at point $a$ and $d$ and $Q$ is evaluated at point $c$ and $d$ to obtain a pair of equations:

$$
\begin{gathered}
F_{a}+u_{a}=F_{d}+u_{d}, \\
F_{c}-u_{c}=F_{d}-u_{d},
\end{gathered}
$$


which can be solved for the state at $d$ :

$$
\begin{aligned}
F_{d} & =\frac{1}{2}\left(F_{a}+F_{c}\right)+\frac{1}{2}\left(u_{a}-u_{c}\right), \\
u_{d} & =\frac{1}{2}\left(F_{a}-F_{c}\right)+\frac{1}{2}\left(u_{a}+u_{c}\right) .
\end{aligned}
$$

This procedure can be implemented numerically to find values of $(F, u)$ for a series of locations $d$ in order to advance the solution in time.

There are two significant issues with implementing the method-of-characteristics as a numerical algorithm. First, given a point $d$, the location of points $a$ and $c$ are unknown. Finding these points accurately requires carrying out an implicit construction of the characteristic paths which depend on the solution that we are seeking because it is needed to integrate Equation A.22. Second, the method-ofcharacteristics algorithm, Equations A.26 and A.27, yields values of $F$, not $c$ or $P$. An implicit solution of Equation A.21 is needed at each point on the characteristic path.

The value of the method-of-characteristics as a numerical tool for gas dynamics is greatly diminished because of these issues, as well as the availability of robust alternative methods of numerical solution. However, it is still quite valuable as an analytical tool and an aid to constructing numerical solutions in the more restrictive framework of acoustics.

\section{A.4 The Wave Equation}

Wave propagation in acoustics is usually described mathematically as a solution to the wave equation which is obtained by simplifying Equations A.13 and A.14 by assuming small amplitude motion in a stationary fluid with uniform entropy. The key simplification is obtained by expressing all variables as small changes about initial values, $P=P_{0}+\Delta P, \rho=\rho_{0}+\Delta \rho$ and $u=\Delta u\left(u_{0}=0\right)$, assuming the initial state is uniform, substituting in the equations and dropping all quantities that represent products of small quantities (e.g., $\Delta P \Delta u$ ). The resulting equations are:

$$
\begin{aligned}
& \frac{\partial \Delta P}{\partial t}=-\rho_{0} c_{0}^{2} \frac{\partial \Delta u}{\partial x}, \\
& \frac{\partial \Delta u}{\partial t}=-\frac{1}{\rho_{0}} \frac{\partial \Delta P}{\partial x},
\end{aligned}
$$

which can be further simplified by taking $\partial / \partial t$ of Equation A.28 and $\partial / \partial x$ of Equation A.29, and summing to obtain the wave equation:

$$
\frac{\partial^{2} \Delta P}{\partial t^{2}}-c_{0}^{2} \frac{\partial^{2} \Delta P}{\partial x^{2}}=0 .
$$


Repeating the procedure that leads to Equation A.30, but eliminating $\Delta P$ instead of $\Delta u$, leads to an identical wave equation for $\Delta u$, and Equation A.2 leads to an identical wave equation for $\Delta \rho$.

Equations A.28 and A.29 are exactly satisfied if an acoustic potential $\phi$ is defined. The acoustic potential has the following properties:

$$
\begin{aligned}
\Delta P & =-\rho_{0} \frac{\partial \phi}{\partial t}, \\
\Delta u & =\frac{\partial \phi}{\partial x},
\end{aligned}
$$

and it follows from Equation A.2 that

$$
\Delta \rho=-\frac{\rho_{0}}{c_{0}^{2}} \frac{\partial \phi}{\partial t} .
$$

The potential satisfies the wave equation:

$$
\frac{\partial^{2} \phi}{\partial t^{2}}-c_{0}^{2} \frac{\partial^{2} \phi}{\partial x^{2}}=0
$$

which can be written as:

$$
\underbrace{\left(\frac{\partial}{\partial t}-c_{0} \frac{\partial}{\partial x}\right)}_{C^{-}} \underbrace{\left(\frac{\partial}{\partial t}+c_{0} \frac{\partial}{\partial x}\right)}_{C^{+}} \phi=0 .
$$

The term in the first brace corresponds to the derivative along the direction $C^{-}$in the limit of $u=0$, and the second term corresponds to the derivative along the direction $C^{+}$in the limit $u=0$. Analogous to the characteristic solutions to the nonlinear Equation A.22, one possible solution is a function $f(\alpha)$ that is constant along a given $C^{+}$characteristic $\alpha=x-c_{0} t$ and another is a function $g(\beta)$ that is constant along a given $C^{-}$characteristic $\beta=x+c_{0} t$. The most general solution is the sum of these two functions:

$$
\phi=f\left(x-c_{0} t\right)+g\left(x+c_{0} t\right) .
$$

This is known as d'Alembert's solution of the wave equation. In simple situations, this can be used to obtain analytical solutions to acoustic problems as discussed in Whitham (1999). The computation of $f$ and $g$ quickly becomes very cumbersome for finite domains where multiple reflections and/or complex boundary conditions are involved. 
The solutions represented by the function $f$ correspond to waves propagating to the right with speed $\mathrm{d} x / \mathrm{d} t=+c_{0}$, and the solutions represented by the function $g$ correspond to waves propagating to the left with speed $\mathrm{d} x / \mathrm{d} t=-c_{0}$. Substituting Equation A.36 into Equations A.31 and A.32, one finds for right-propagating waves $(g=0)$ :

$$
\Delta P=\rho_{0} c_{0} \Delta u,
$$

and for the left-propagating waves $(f=0)$ :

$$
\Delta P=-\rho_{0} c_{0} \Delta u \text {. }
$$

The property $\rho c$ is known as the acoustic impedance, and together with the sound speed is a key physical property in terming acoustic response. In the case of small amplitude wave propagation inside of a slightly flexible tube, the fluid sound speed $c$ should be replaced by the Korteweg speed $c_{K}$, as discussed by Shepherd and Inaba (2010):

$$
c_{K}=\frac{c}{\sqrt{1+\beta}}, \quad \beta=\frac{K D}{E h},
$$

where $K$ is the bulk modulus of the liquid, and $E$ and $h$ are respectively the Young's modulus and thickness of the solid walls.

\section{A.5 The Acoustic Limit of the Method-of-Characteristics}

For a special choice of discrete space and time increments, the acoustic limit of the method-of-characteristics results in a simple set of linear equations for describing acoustic motions in a uniform, one-dimensional (planar) situation. Start by choosing a spatial increment $\Delta x$ and divide the domain of length $L$ into $n$ spatial nodes separated by equal increments, $\Delta x=L /(n-1)$. The characteristic slope:

$$
\frac{\mathrm{d} x}{\mathrm{~d} t}= \pm c_{0},
$$

is constant in the acoustic limit and can be used to define a time increment:

$$
\Delta t=\frac{\Delta x}{c_{0}} .
$$

The resulting network of characteristics is shown in Figure A.3.

In the acoustic limit, the function $F$ defined by Equation A.21 has the simple form:

$$
F=\frac{\Delta P}{\rho_{0} c_{0}},
$$




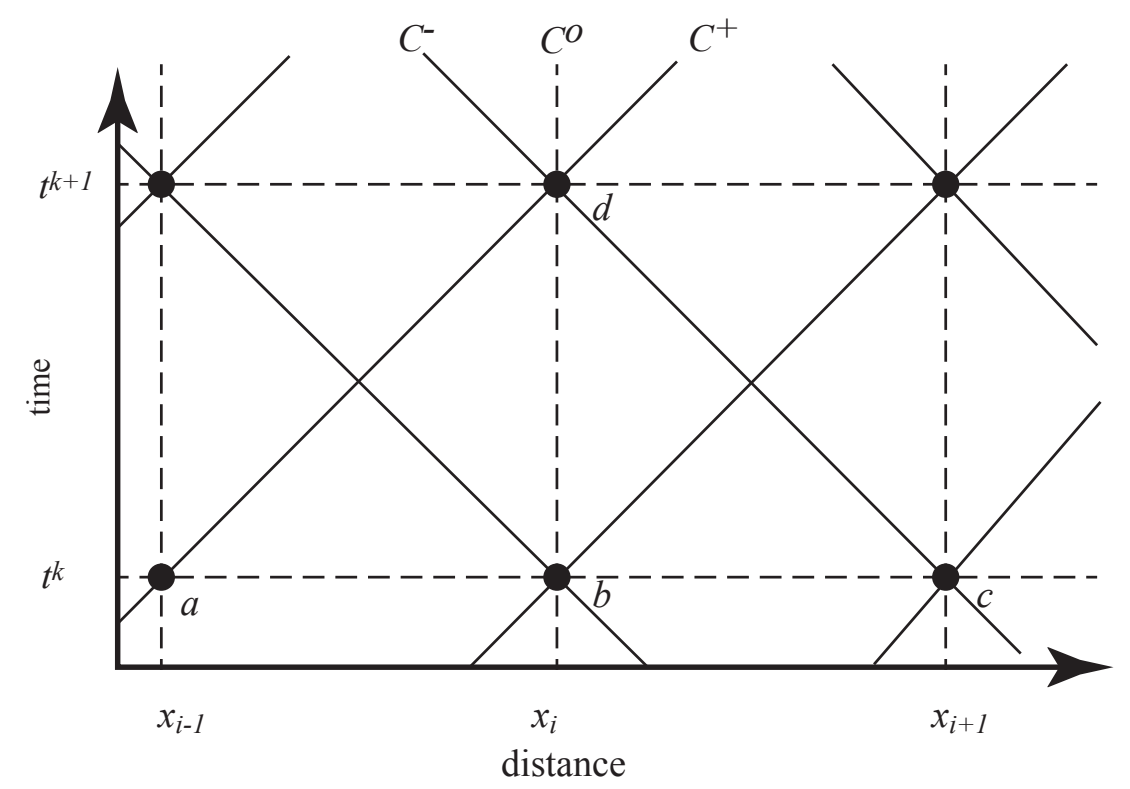

Figure A.3: Network of characteristics in the acoustic limit. The slope of the characteristics of provided by Equation A.41, and the spatial locations $x_{i}$ and temporal locations $t^{k}$ have been selected so that the characteristic network formed by $C^{+}, C^{0}$, $C^{-}$and the $x$ - $t$ network formed by the space-time grid have common intersections.

and the method-of-characteristic equations can be written as:

$$
\begin{aligned}
& \frac{\Delta P}{\rho_{0} c_{0}}+\Delta u=\mathcal{P} \quad \text { on } \quad C^{+}: \frac{\mathrm{d} x}{\mathrm{~d} t}=c_{0}, \\
& \frac{\Delta P}{\rho_{0} c_{0}}-\Delta u=Q \quad \text { on } \quad C^{-}: \frac{\mathrm{d} x}{\mathrm{~d} t}=-c_{0} .
\end{aligned}
$$

The method-of-characteristics algorithm, Equations A.26 and A.27, simplifies to:

$$
\begin{aligned}
\Delta P_{d} & =\frac{1}{2}\left(\Delta P_{a}+\Delta P_{c}\right)+\frac{\rho_{0} c_{0}}{2}\left(\Delta u_{a}-\Delta u_{c}\right), \\
\Delta u_{d} & =\frac{1}{2 \rho_{0} c_{0}}\left(\Delta P_{a}-\Delta P_{c}\right)+\frac{1}{2}\left(\Delta u_{a}+\Delta u_{c}\right) .
\end{aligned}
$$

On the rectangular grid shown in Figure A.3, the solution is defined at regular increments in space $x_{i}$ and time $t_{k}$, where $t_{k+1}-t_{k}=\Delta t$ and $x_{i+1}-x_{i}=\Delta x$, and the increments are related through Equation A.41. If the grid has $N$ spatial nodes over the domain $0 \leq x \leq L$, then the individual grid points are located at:

$$
x_{i}=(i-1) \Delta x \quad 1 \leq i \leq N \quad t_{k}=k \Delta t .
$$


The variables are defined at these locations (e.g., $\left.P_{i}^{k}=P\left(x_{i}, t_{k}\right)\right)$ and the method-ofcharacteristics equations become the set of equations:

$$
\begin{aligned}
P_{i}^{k+1} & =\frac{1}{2}\left(P_{i-1}^{k}+P_{i+1}^{k}\right)+\frac{\rho_{0} c_{0}}{2}\left(u_{i-1}^{k}-u_{i+1}^{k}\right), \\
u_{i}^{k+1} & =\frac{1}{2 \rho_{0} c_{0}}\left(P_{i-1}^{k}-P_{i+1}^{k}\right)+\frac{1}{2}\left(u_{i-1}^{k}+u_{i+1}^{k}\right) .
\end{aligned}
$$

For simplicity in notation, the $\Delta$ notation on the variables $u$ and $P$ was dropped.

Given a set of values for $\left(P_{i}^{k}, u_{i}^{k}\right)$ for all the spatial locations, $1 \leq i \leq N$, the difference relations can be used to compute values $\left(P_{i}^{k+1}, u_{i}^{k+1}\right)$ at the next time step $t^{k+1}=t^{k}+\Delta t$. The only complication is how to handle the variable updates at the boundaries of the domain, $i=1$ and $i=N$; this is discussed in the next section.

This method of solution of the characteristic equations was formulated and used extensively to solve problems in the field of hydraulics, particularly water hammers, the study of fluid transients in pipelines. A comprehensive discussion with extensions of the method to treat networks of piping, friction and gravity can be found in the monographs of Wylie and Streeter (1993) or Watters (1984).

\section{A.5.1 Boundary Conditions}

At the boundaries of the spatial domain (i.e., nodes $i=1$ and $i=N$ ), special considerations are required for updating the fluid properties. Equations A.48 and A.49 at these points cannot be directly applied as these involve the undefined properties at nodes outside the domain (i.e., nodes $i=0$ and $i=N+1$ ). Instead, the use of Equations A.48 and A.49 is limited to the interior of the domain $2 \leq i \leq N-1$ and for the boundary points, use only the information at the boundary together with the interior characteristic data. At the right-hand side of the domain, evaluating the Riemann invariant $\mathcal{P}$ on the $C^{+}$characteristic at $i=N$ yields:

$$
\frac{P_{N}^{k+1}}{\rho_{0} c_{0}}+u_{N}^{k+1}=\frac{P_{N-1}^{k}}{\rho_{0} c_{0}}+u_{N-1}^{k} .
$$

At the left-hand side of the domain, evaluating the Riemann invariant $Q$ on the $C^{-}$ characteristic at $i=1$ yields:

$$
\frac{P_{1}^{k+1}}{\rho_{0} c_{0}}-u_{1}^{k+1}=\frac{P_{2}^{k}}{\rho_{0} c_{0}}-u_{2}^{k} .
$$

There are two common situations: a) specified velocity; b) specified pressure. For example, suppose that the right-hand boundary is fixed so that $u_{N}=0$; substituting 
this into Equation A.50 and rearranging, the following is obtained:

$$
P_{N}^{k+1}=P_{N-1}^{k}+\rho_{0} c_{0} u_{N-1}^{k} .
$$

Suppose that at the opposite end, the left-hand boundary, the pressure $P_{1}(t)$ is specified. Rearranging Equation A.51 yields:

$$
u_{1}^{k+1}=\frac{P_{1}^{k+1}-P_{2}^{k}}{\rho_{0} c_{0}}+u_{2}^{k} .
$$

A special case of this is a free surface, which has $P_{1}=0$, which yields:

$$
u_{1}^{k+1}=-\frac{P_{2}^{k}}{\rho_{0} c_{0}}+u_{2}^{k} .
$$

The examples given are for specific conditions on the left and right sides, but these choices are just for illustration. In general, the specific boundary conditions will depend on the application and can be obtained by evaluating the Riemann invariant on the appropriate characteristic.

\section{A.5.2 Initial Conditions}

In addition to the boundary conditions, it is necessary to specify the values of $P$ and $u$ at the beginning of the computation at time $t^{1}=0$. A common situation is that the pressure and velocity have constant values with the simplest variant being a stationary, uniform fluid:

$$
\begin{aligned}
& P_{i}^{1}=0, \\
& u_{i}^{1}=0,
\end{aligned}
$$

for $1 \leq i \leq N$. The motion set up by suddenly bringing a column of fluid moving uniformly at speed $U_{o}$ to rest can be simulated by setting:

$$
\begin{aligned}
P_{i}^{1}=0 & \\
u_{i}^{1}=U_{0} & (1 \leq i \leq N), \\
u_{N}^{1}=0 . &
\end{aligned}
$$

This simulates instantaneous impact against the right-hand boundary. A softer impact can be simulated by prescribing $u_{N}(t)$ to decrease to zero over a specified period of time. 


\section{A.6 Cavitation Model for the Method-of-Characteristics}

Motivated by the water hammer events in piping systems, simple cavitation models which can be used within the acoustic version of the method-of-characteristics algorithm were developed and used. According to Bergant, Simpson, and Tijsseling (2006), there are three types of models: 1) the discrete single-cavity model; 2) the discrete multiple-cavity model; 3 ) the discrete gas cavity model. The most popular model is the discrete multiple-cavity model (DVCM) due to its simplicity and ability to account moderately well for both column separation and distributed cavitation.

Column separation corresponds to the formation of a large cavity within the liquid column. The cavity typically occupies most of the cross-sectional area $A$ of the tube or pipe. Locally, the void fraction $\alpha$ is close to unity. Distributed cavitation corresponds to the formation of multiple cavities of small size which are randomly distributed throughout the water column. The local void fraction $\alpha$ remains small, close to zero.

The monograph of Wylie and Streeter (1993) extensively describes the idea behind the discrete multiple-cavity model along with its implementation. An introduction to the model and its implementation is included in this appendix.

\section{A.6.1 Qualitative Description of DVCM}

The idea behind the discrete multiple-cavity model is that a vapor cavity is allowed to form at each computational grid point. A vapor cavity forms if, and only if, the cavitation model becomes active, which occurs whenever the pressure drops below the vapor pressure $P_{v a p}$ of the liquid. The cavitation model can become active at a grid point, while being inactive at the adjacent grid points. When a cavity forms,

the pressure inside remains equal to the vapor pressure of the liquid. Pressure $P_{i}^{k}$ is not an unknown anymore, and the method-of-characteristics algorithm needs to be updated to account for this.

Suppose the cavitation model is active at node $i$. The velocity of the liquid on the left-hand side (i.e., velocity of the liquid from node $i-1$ to node $i$ ), and the velocity of the liquid on the right-hand side (i.e., velocity of the liquid from node $i$ to node $i+1)$ do not need to be equal. The difference between the velocities on either side of the cavity governs the growth and collapse of the cavity; there can be a net inflow or outflow into the cavity. The time integration of the inflow/outflow makes it possible to keep track of the time evolution of the cavity volume. Whenever the volume of the cavity is predicted to become zero or negative (impossible), these are 


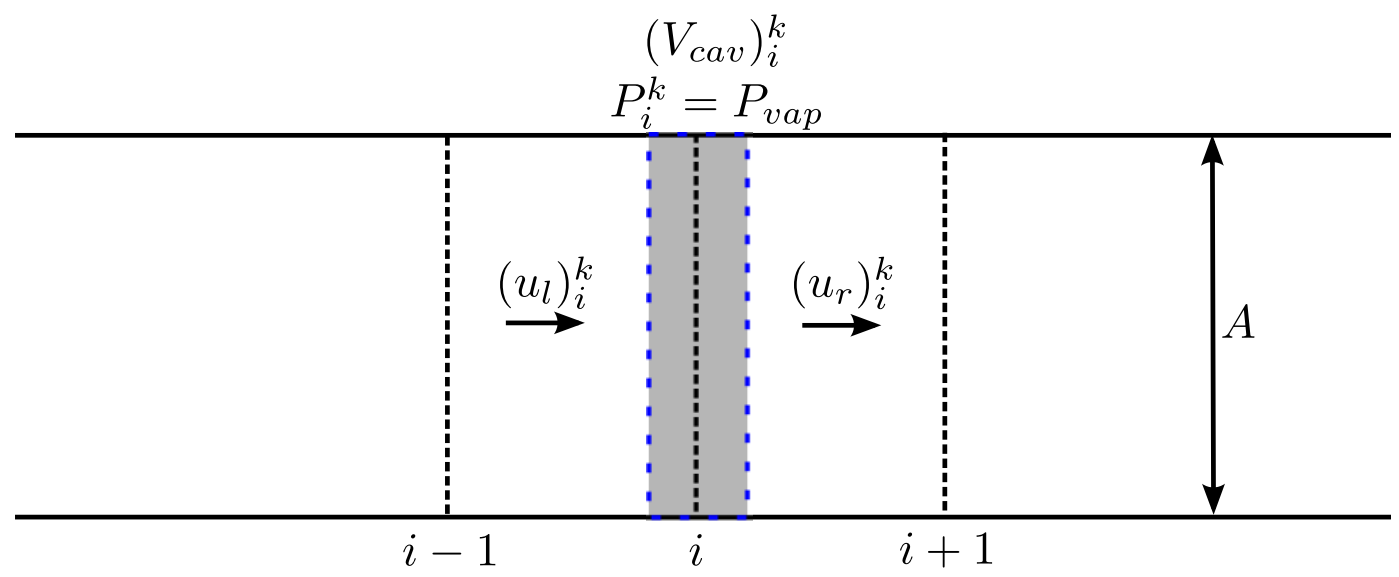

Figure A.4: Schematic representing the idea behind the DVCM model. The cavitation model is active at grid point $i$, where a vapor cavity of volume $V_{c a v}$ is present.

the indications that cavitation is not present anymore, and the cavitation model is locally deactivated.

The cavitation model affects the wave dynamics in different ways. First, the model prevents the liquid pressure from being less than the vapor pressure of the liquid. Second, the presence of a vapor cavity at a computational grid point effectively behaves like an internal constant pressure boundary condition, where incident pressure waves can reflect. This is what creates the slowing of the pressure waves in the liquid, mimicking the propagation of pressure waves in a bubbly mixture (Bergant, Simpson, and Tijsseling, 2006; Wylie, 1984).

\section{A.6.2 Mathematical Formulation of DVCM}

The mathematical formulation of the cavitation model is introduced using Figure A.4. The cavitation model is assumed to be active at node $i$. A vapor cavity of volume $\left(V_{\text {cav }}\right)_{i}^{k}$ is present, and the pressure is $P_{i}^{k}=P_{\text {vap }}$. The two unknowns at node $i$ are the velocities $\left(u_{l}\right)_{i}^{k}$ and $\left(u_{r}\right)_{i}^{k}$ to the left and to the right of the vapor cavity.

In the general case, $\left(u_{l}\right)_{i}^{k} \neq\left(u_{r}\right)_{i}^{k}$. Note that in all cases, $\left(u_{l}\right)_{i}^{k}=\left(u_{r}\right)_{i-1}^{k}$ for the model to respect conservation of mass. When the cavitation model is inactive, then $\left(u_{l}\right)_{i}^{k}=\left(u_{r}\right)_{i}^{k}$.

Solving for the fluid motion at node $i$ for time $t^{k+1}$ is relatively straightforward. First, the pressure is not an unknown:

$$
P_{i}^{k+1}=P_{\text {vap }}
$$


Solving for $u_{l, i}^{k+1}$ and $u_{r, i}^{k+1}$ requires using the invariants $\mathcal{P}$ and $Q$ on the characteristic lines. The definition of the invariants is different from before to account for the two velocities at each node:

$$
\begin{aligned}
& \frac{P_{i-1}^{k}}{\rho_{0} c_{0}}+u_{r, i-1}^{k}=\frac{P_{i}^{k+1}}{\rho_{0} c_{0}}+u_{l, i}^{k+1}=\mathcal{P} \quad \text { on } \quad C^{+}: \frac{\mathrm{d} x}{\mathrm{~d} t}=c_{o} \\
& \frac{P_{i+1}^{k}}{\rho_{0} c_{0}}-u_{l, i+1}^{k}=\frac{P_{i}^{k+1}}{\rho_{0} c_{0}}-u_{r, i}^{k+1}=\mathcal{Q} \quad \text { on } \quad C^{-}: \frac{\mathrm{d} x}{\mathrm{~d} t}=-c_{o} .
\end{aligned}
$$

Solving for $u_{l, i}^{k+1}$ and $u_{r, i}^{k+1}$, and using Equation A.60 to eliminate $P_{i}^{k+1}$ yields:

$$
\begin{aligned}
& u_{l, i}^{k+1}=\frac{P_{i-1}^{k}-P_{v a p}}{\rho_{0} c_{0}}+u_{l, i-1}^{k}, \\
& u_{r, i}^{k+1}=\frac{P_{v a p}-P_{i+1}^{k}}{\rho_{0} c_{0}}+u_{l, i+1}^{k} .
\end{aligned}
$$

The volume $V_{c a v}$ of the vapor cavity is obtained from the time integration of the net mass flux into the vapor cavity:

$$
V_{c a v}=\int_{0}^{t^{k+1}} A\left(u_{r}-u_{l}\right) \mathrm{d} t
$$

which can be evaluated numerically:

$$
V_{c a v, i}^{k+1}=V_{c a v, i}^{k}+A\left\{\psi\left(u_{r, i}^{k+1}-u_{l, i}^{k+1}\right)+(1-\psi)\left(u_{r, i}^{k}-u_{l, i}^{k}\right)\right\} \Delta t,
$$

where $\mathrm{A}$ is the cross-sectional area of the water column, and $\psi$ is a weighting factor which takes values ranging between 0 and 1 . When $\psi=0.5$ the above scheme is the well-known trapezoidal rule. Some authors have reported that it is sometimes better to use a value of $\psi=1.0$ to eliminate spurious, unphysical pressure spikes from the solution (Bergant and Simpson, 1999; Proovost, 1976; Streeter, 1969). In any case, it is generally recommended to keep the value of $\psi$ between 0.5 and 1.0 (Liou, 2000; Simpson and Bergant, 1994). The results shown in this thesis were all obtained with $\psi$ between 0.5 .

\section{A.6.3 Numerical Implementation of DVCM}

The numerical implementation of the DVCM model is introduced using pseudocode. An inner node not located on the boundary of the domain is first considered. The pseudo-code is provided as two separate algorithms. Algorithm 1 deals with the case where the cavitation model is active at time $t^{k}$. Algorithm 2 deals with the case where the cavitation model is inactive at time $t^{k}$. 


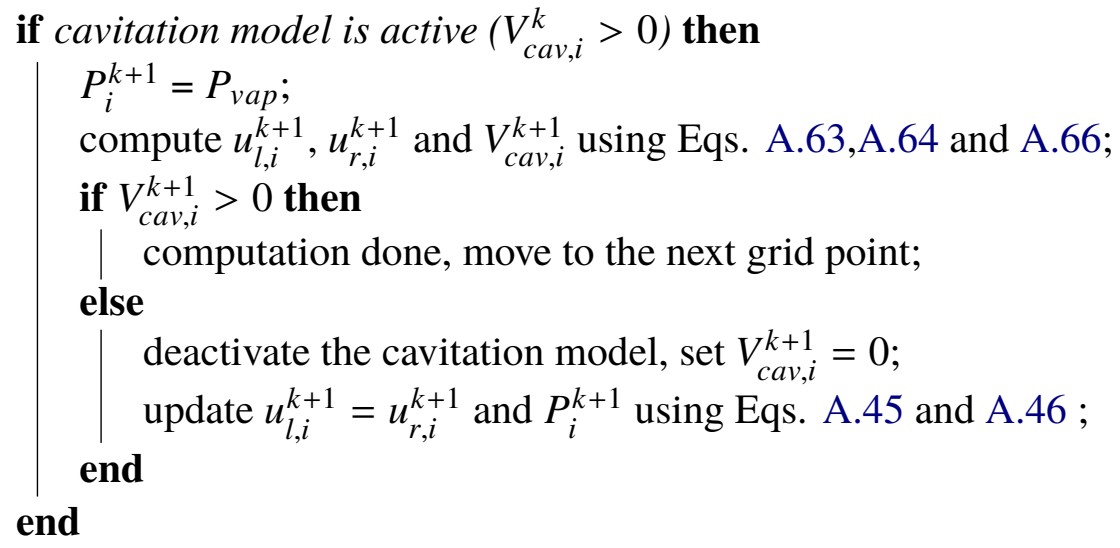

Algorithm 1: Pseudo-code for an inner computational grid when the cavitation model is initially active.

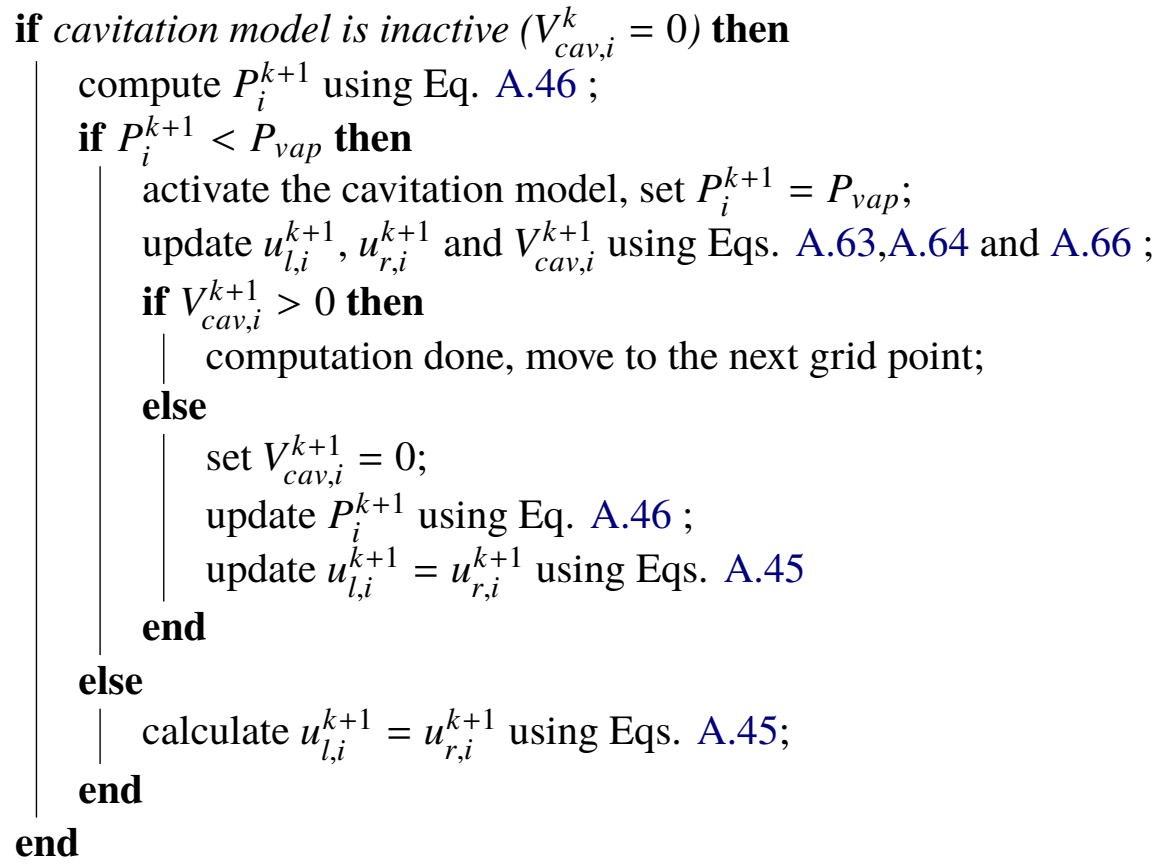

Algorithm 2: Pseudo-code for an inner computational grid when the cavitation model is initially inactive.

There is a third level of nested else-if statement in Algorithm 2. This extra check is needed to handle cases where the calculation can't easily determine whether or not the cavitation model should be active. In such cases, Equation A.46 predicts a pressure below $P_{\text {vap }}$, but Equation A.66 predicts $V_{\text {cav }, i}^{k+1} \leq 0$. When this happens, the third level of nested else-if statement deactivates the cavitation model, and the pressure is momentarily allowed to take a value below $P_{v a p}$. This choice has less detrimental consequences on the remainder of the computation. 
The boundary conditions are treated similarly to what was done earlier with the standard method-of-characteristics. The pseudo-code from Algorithms 1 and 2 remains unchanged, but Equations A.45, A.46, A.63, and A.64 need to be updated adequately using the $\mathcal{P}$ and $Q$ invariants, respectively on the right and left boundary of the domain. Examples are shown below.

The grid point on the left-hand side of the domain is considered (i.e., $i=1$ ), where a pressure $P_{B C}$ is prescribed. The method discussed herein is general, and pressure $P_{B C}$ can be a function of time. It is assumed that $P_{B C} \geq P_{v a p}$. As a result, Equations A.63 and A.64 are not needed because the cavitation model can't become active at this node. Equations A.45 and A.46 become:

$$
\begin{aligned}
& P_{1}^{k+1}=P_{B C}, \\
& u_{l, 1}^{k+1}=u_{r, 1}^{k+1}=\frac{P_{B C}^{k+1}-P_{2}^{k}}{\rho_{0} c_{0}}+u_{2}^{k} .
\end{aligned}
$$

The grid point on the right-hand side of the domain is considered (i.e., $i=N$ ), where a velocity $u_{B C}$ is prescribed. No assumption is made about $u_{B C}$, which can be a function of time. The pressure on this boundary could potentially drop below $P_{v a p}$, which indicates the cavitation model could become active. The updated version of Equations A.45, A.46, A.63, and A.64 is:

$$
\begin{aligned}
& P_{N}^{k+1}=P_{N-1}^{k}+\rho_{o} c_{o}\left(u_{l, N-1}^{k}-u_{B C}\right), \\
& u_{l, N}^{k+1}=u_{r, N}^{k+1}=u_{B C}, \\
& u_{l, N}^{k+1}=\frac{P_{N-1}^{k}-P_{v a p}}{\rho_{0} a_{0}}+u_{r, N-1}^{k}, \\
& u_{r, N}^{k+1}=u_{B C} .
\end{aligned}
$$

\section{A.7 Impact Between Solid Parts}

The actuation of an autoinjector device involves contacts or impacts between the moving components (see Chapter 2). This is also true about the large-scale model autoinjector test setups described in Chapter 4. For example, in the static large-scale model autoinjector test setup the transient events are initiated by the impact of a projectile on the buffer. The impact events need to be accounted for in developing a model for the transient events using the method-of-characteristics.

One alternative to model the impact between moving components is to use the method-of-characteristics to predict the formation and propagation of stress waves 


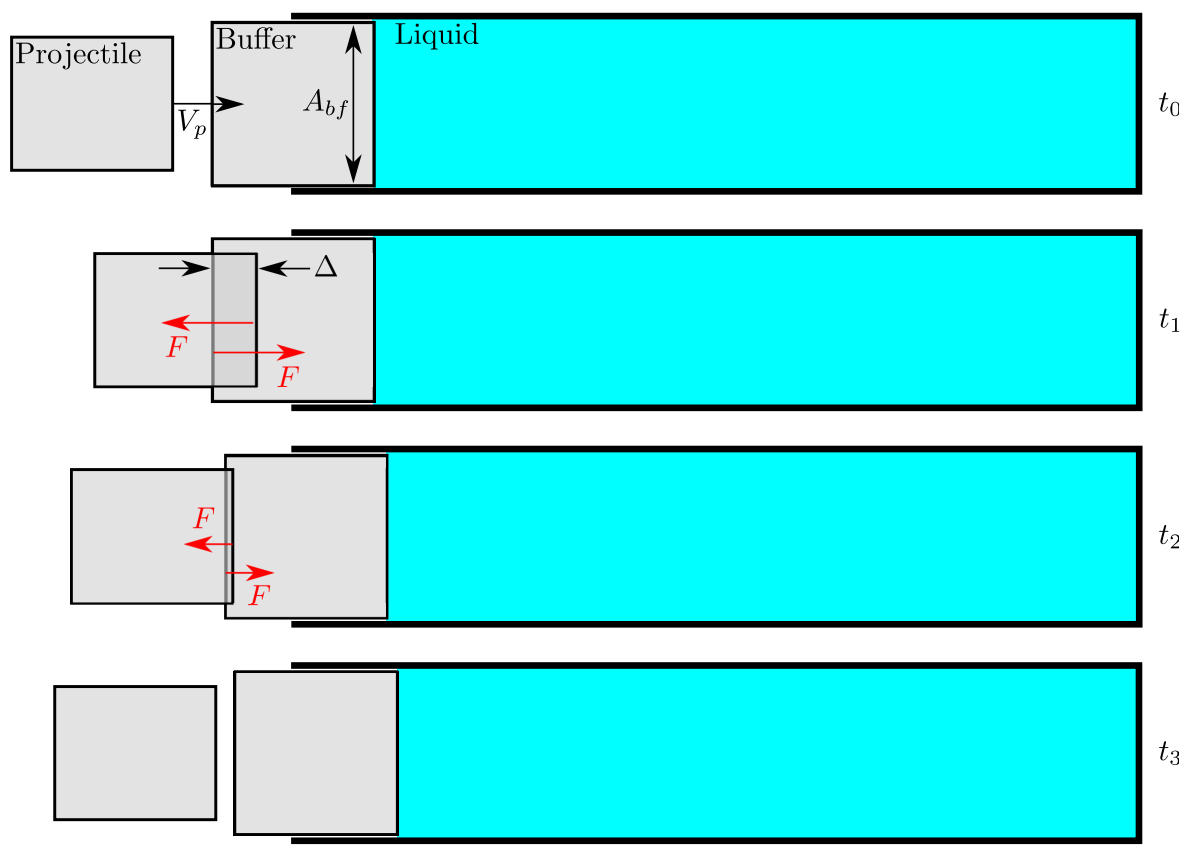

Figure A.5: Representation of the penalty coupling approach to model impact events.

in each solid part, an approach which can rapidly become complex. Another alternative is to model the impacts as being instantaneous using conservation of energy and conservation of linear momentum. However, the reality is that impact events occur over a finite time due to the compliance of the materials. The duration of the impact events substantially affects the formation and propagation of pressure waves in the liquid, and this often can't be neglected.

A simplified alternative that makes it possible to model impact events over a finite time is to use a penalty coupling approach. The penalty coupling approach is illustrated using Figure A.5, where the projectile is initially traveling to the right, and the buffer is initially stationary. The buffer is in contact with the liquid contained inside a cylinder. At time $t_{1}$ the projectile has travelled to the right, resulting in the penetration of the projectile into the buffer by a distance $\Delta$. A force of magnitude $F$ is then applied on both the buffer and the projectile, as illustrated in Figure A.5. The magnitude of $F$ is proportional to the interpenetration distance $\Delta$ :

$$
F=k \Delta,
$$

where $k$ is the penalty coupling parameter or stiffness of the contact. Note that force $F$ is zero when there is no interpenetration (i.e., when the contact is not active). 
In this example, the equation of motion of the projectile is:

$$
m_{p} \frac{\mathrm{d} V_{p}}{\mathrm{~d} t}=-F,
$$

where $m_{p}$ is the mass of the projectile, and $V_{p}$ is the velocity of the projectile. The equation of motion of the buffer is:

$$
m_{b f} \frac{\mathrm{d} V_{b f}}{\mathrm{~d} t}=F-P A_{b f},
$$

where $m_{b f}, V_{b f}$ and $A_{b f}$ are respectively the mass, the velocity and the crosssectional area of the buffer, and $P$ is the liquid pressure applied on the buffer. Note that pressure $P$ is obtained from solving the wave propagation problem in the liquid using the method-of-characteristics.

At time $t_{2}$, the interpenetration between the buffer and projectile has decreased, resulting in a reduction in the magnitude of force $F$. At time $t_{3}$ there is no interpenetration between the projectile and buffer (i.e., the impact event is over), and the projectile is traveling away from the buffer.

Increasing the magnitude of the penalty coupling parameter $k$ results in a stiffer contact: the duration of the impact event becomes shorter. The magnitude of $k$ in the model can be adjusted empirically to match as closely as possible the duration of the impacts in the experiments.

Note that this approach is equivalent to introducing a spring of stiffness $k$ between the projectile and the buffer to model the impact event. The stiffness of the spring is however 0 when the contact is inactive. 
Appendix B

\section{TEST MATRICES}

The tables in this appendix summarize the successful experiments performed with the in situ methods, the static large-scale autoinjector, and the dynamic large-scale autoinjector. Several abbreviations are used:

- S1H, S1A, S2H, S2A, etc. : "S" is for strain gauge, the number (1, 2 or 3) indicates the station or axial location of the gage, and the last letter $(\mathrm{A}$ or $\mathrm{H})$ indicates the strain component which is measured (axial or hoop);

- P: pressure transducer;

- HSV: high speed video;

- $\mathrm{Al}$ : aluminum;

- PC: polycarbonate;

- inf: infinite, used to indicate the absence of a buffer;

- I. on S.: impact on syringe, used to indicate this is an experiment where the outer projectile only impacts on the syringe wall;

- I. on. B.: impact on buffer, used to indicate this is an experiment where the reversed outer projectile only impacts on the buffer. 
Table B.1: Test matrix of the successful in situ experiments.

\begin{tabular}{|c|c|c|c|c|c|c|c|}
\hline Test & Date & Syringe & Liquid & Air gap & Damping & Instruments & Comment \\
\hline SC-001 & $4 / 7 / 2016$ & Glass & Water & $0 \mathrm{~mm}$ & None & S1H, HSV & \\
\hline SC-002 & $4 / 8 / 2016$ & Glass & Water & $0 \mathrm{~mm}$ & None & S1H, S1A, S2H, S2A, HSV & $\mathrm{S} 2 \mathrm{H}$ failed \\
\hline SC-003 & $4 / 8 / 2016$ & Glass & Water & $0 \mathrm{~mm}$ & None & $\mathrm{S} 1 \mathrm{H}, \mathrm{S} 2 \mathrm{H}, \mathrm{HSV}$ & \\
\hline SC-004 & $4 / 13 / 2016$ & Glass & Water & $0 \mathrm{~mm}$ & None & $\mathrm{S} 1 \mathrm{H}, \mathrm{S} 2 \mathrm{H}, \mathrm{HSV}$ & \\
\hline SC-005 & $4 / 13 / 2016$ & Glass & Water & $0 \mathrm{~mm}$ & None & $\mathrm{S} 1 \mathrm{H}, \mathrm{S} 2 \mathrm{H}, \mathrm{HSV}$ & \\
\hline SC-006 & $4 / 13 / 2016$ & Glass & Water & $0 \mathrm{~mm}$ & None & $\mathrm{S} 1 \mathrm{H}, \mathrm{S} 2 \mathrm{H}, \mathrm{S} 3 \mathrm{H}, \mathrm{HSV}$ & \\
\hline SC-007 & $4 / 13 / 2016$ & Glass & Water & $0 \mathrm{~mm}$ & None & $\mathrm{S} 1 \mathrm{H}, \mathrm{S} 2 \mathrm{H}, \mathrm{HSV}$ & \\
\hline SC-008 & $4 / 13 / 2016$ & Glass & Water & $0 \mathrm{~mm}$ & None & $\mathrm{S} 1 \mathrm{H}, \mathrm{S} 2 \mathrm{H}, \mathrm{HSV}$ & \\
\hline SC-009 & $4 / 13 / 2016$ & Glass & Water & $0 \mathrm{~mm}$ & None & $\mathrm{S} 1 \mathrm{H}, \mathrm{S} 2 \mathrm{H}, \mathrm{HSV}$ & \\
\hline SC-010 & $4 / 13 / 2016$ & Glass & Water & $0 \mathrm{~mm}$ & None & $\mathrm{S} 1 \mathrm{H}, \mathrm{S} 2 \mathrm{H}, \mathrm{HSV}$ & \\
\hline SC-011 & $4 / 13 / 2016$ & Glass & Water & $0 \mathrm{~mm}$ & None & $\mathrm{S} 1 \mathrm{H}, \mathrm{S} 2 \mathrm{H}, \mathrm{HSV}$ & \\
\hline SC-014 & $4 / 14 / 2016$ & Glass & Water & $0 \mathrm{~mm}$ & None & S1A, S2A, S3A, HSV & \\
\hline SC-015 & $4 / 14 / 2016$ & Glass & Water & $0 \mathrm{~mm}$ & None & S1A, S2A, HSV & S2A failed \\
\hline SC-016 & $4 / 14 / 2016$ & Glass & Water & $0 \mathrm{~mm}$ & None & S1A, S2A, HSV & S2A failed \\
\hline SC-017 & $4 / 15 / 2016$ & Glass & Water & $0 \mathrm{~mm}$ & None & S1A, S2A, HSV & \\
\hline SC-025 & $5 / 13 / 2016$ & Glass & Water & $0 \mathrm{~mm}$ & None & $\mathrm{P}$ & \\
\hline SC-026 & $5 / 19 / 2016$ & Glass & Water & $0 \mathrm{~mm}$ & None & $\mathrm{P}$ & \\
\hline SC-027 & $5 / 31 / 2016$ & Glass & Silicone oil $(5 \mathrm{cP})$ & $0 \mathrm{~mm}$ & None & $\mathrm{P}$ & \\
\hline SC-029 & $5 / 31 / 2019$ & Glass & Silicone oil $(5 \mathrm{cP})$ & $0 \mathrm{~mm}$ & None & $\mathrm{P}, \mathrm{HSV}$ & \\
\hline SC-030 & $6 / 1 / 2016$ & Glass & Water & $0 \mathrm{~mm}$ & None & P, HSV & \\
\hline
\end{tabular}




\begin{tabular}{|c|c|c|c|c|c|c|c|}
\hline Test & Date & Syringe & Liquid & Air gap & Damping & Instruments & Comment \\
\hline SC-031 & $6 / 1 / 2016$ & Glass & Water & $0 \mathrm{~mm}$ & None & P, HSV & \\
\hline SC-032 & $6 / 1 / 2016$ & Glass & Water & $0 \mathrm{~mm}$ & None & $\mathrm{P}, \mathrm{HSV}$ & \\
\hline SC-033 & $6 / 1 / 2016$ & Glass & Water & $0 \mathrm{~mm}$ & None & S1H, S1A, S2H, P, HSV & \\
\hline SC-034 & $6 / 8 / 2016$ & Glass & Water & $0 \mathrm{~mm}$ & None & S1H, S1A, S2H, P, HSV & \\
\hline SC-038 & 6/9/2016 & Glass & Water & $0 \mathrm{~mm}$ & D2 & S1H, S1A, S2H, P, HSV & S1A failed \\
\hline SC-040 & $6 / 22 / 2016$ & Glass & Water & $0 \mathrm{~mm}$ & D1, D2 & S1H, S1A, S2H, P, HSV & \\
\hline SC-056 & $6 / 29 / 2016$ & Glass & Water & $0 \mathrm{~mm}$ & D1, D2 & S1H, S1A, S2H, P, HSV & \\
\hline SC-081 & $7 / 7 / 2016$ & Glass & Water & $0 \mathrm{~mm}$ & D1, D2 & S1H, S1A, S2H, P, HSV & \\
\hline SC-100 & $8 / 18 / 2016$ & Glass & Water & $0 \mathrm{~mm}$ & None & S1H, S1A, S2H, P, HSV & \\
\hline SC-101 & $8 / 18 / 2016$ & Glass & Water & $2 \mathrm{~mm}$ & None & $\mathrm{S} 1 \mathrm{H}, \mathrm{S} 1 \mathrm{~A}, \mathrm{~S} 2 \mathrm{H}, \mathrm{P}, \mathrm{HSV}$ & \\
\hline SC-102 & $8 / 18 / 2016$ & Glass & Water & $2 \mathrm{~mm}$ & None & S1H, S1A, S2H, P, HSV & \\
\hline SC-103 & $8 / 19 / 2016$ & Glass & Water & $2 \mathrm{~mm}$ & None & S1H, S1A, S2H, P, HSV & \\
\hline SC-104 & $8 / 19 / 2016$ & Glass & Water & $2 \mathrm{~mm}$ & None & S1H, S1A, S2H, P, HSV & \\
\hline SC-105 & $8 / 19 / 2016$ & Glass & Water & $2 \mathrm{~mm}$ & None & S1H, S1A, S2H, P, HSV & \\
\hline SC-106 & $8 / 19 / 2016$ & Glass & Water & $0 \mathrm{~mm}$ & None & S1H, S1A, S2H, P, HSV & $P$ failed \\
\hline SC-107 & $8 / 22 / 2016$ & Glass & Water & $2 \mathrm{~mm}$ & None & S1H, S1A, S2H, P, HSV & \\
\hline SC-108 & $8 / 22 / 2016$ & Glass & Water & $0 \mathrm{~mm}$ & None & S1H, S1A, S2H, P, HSV & $\mathrm{S} 1 \mathrm{H}, \mathrm{S} 2 \mathrm{H}$ and S1A failed \\
\hline SC-109 & $9 / 6 / 2016$ & Glass & Water & $2 \mathrm{~mm}$ & None & S1H, S1A, S2H, P, HSV & \\
\hline SC-110 & $9 / 6 / 2016$ & Glass & Water & $2 \mathrm{~mm}$ & None & S1H, S1A, S2H, P, HSV & \\
\hline SC-111 & $9 / 6 / 2016$ & Glass & Water & $0 \mathrm{~mm}$ & None & S1H, S1A, S2H, P, HSV & \\
\hline SC-113 & $9 / 16 / 2016$ & Glass & Water & $0 \mathrm{~mm}$ & None & S1H, S1A, S2H, P, HSV & \\
\hline SC-114 & $9 / 20 / 2016$ & Glass & Water & $1 \mathrm{~mm}$ & None & S1H, S1A, S2H, P, HSV & S1A failed \\
\hline
\end{tabular}




\begin{tabular}{|c|c|c|c|c|c|c|c|}
\hline Test & Date & Syringe & Liquid & Air gap & Damping & Instruments & Comment \\
\hline SC-115 & $9 / 20 / 2016$ & Glass & Water & $1 \mathrm{~mm}$ & None & S1H, S1A, S2H, P, HSV & \\
\hline SC-116 & $9 / 26 / 2018$ & Glass & Water & $4 \mathrm{~mm}$ & None & S1H, S1A, S2H, P, HSV & $P$ failed \\
\hline SC-117 & $9 / 26 / 2016$ & Glass & Water & $4 \mathrm{~mm}$ & None & S1H, S1A, S2H, P, HSV & \\
\hline SC-118 & $9 / 29 / 2016$ & Glass & Water & $4 \mathrm{~mm}$ & None & S1H, S1A, S2H, P, HSV & $\mathrm{S} 2 \mathrm{H}$ failed \\
\hline SC-119 & $10 / 3 / 2016$ & Glass & Water & $4 \mathrm{~mm}$ & None & S1H, S1A, S2H, P, HSV & \\
\hline SC-121 & $11 / 7 / 2016$ & Glass & Water & $6 \mathrm{~mm}$ & None & S1H, S1A, S2H, P, HSV & S1A failed \\
\hline SC-122 & $11 / 9 / 2016$ & Glass & Water & $3 \mathrm{~mm}$ & None & HSV & Al. plunger-stopper \\
\hline SC-123 & $11 / 9 / 2016$ & Glass & Water & $4 \mathrm{~mm}$ & None & HSV & Al. plunger-stopper \\
\hline SC-124 & $11 / 9 / 2016$ & Glass & Water & $1 \mathrm{~mm}$ & None & HSV & Al. plunger-stopper \\
\hline SC-125 & $11 / 9 / 2016$ & Glass & Water & $2 \mathrm{~mm}$ & None & HSV & \\
\hline SC-126 & $11 / 9 / 2016$ & Glass & Water & $2 \mathrm{~mm}$ & None & HSV & \\
\hline SC-127 & $11 / 9 / 2016$ & Glass & Water & $5 \mathrm{~mm}$ & None & HSV & \\
\hline SC-128 & $11 / 9 / 2016$ & Glass & Water & $3 \mathrm{~mm}$ & None & HSV & \\
\hline SC-129 & $11 / 9 / 2016$ & Glass & Water & $3 \mathrm{~mm}$ & None & HSV & \\
\hline SC-130 & $11 / 9 / 2016$ & Glass & Water & $3 \mathrm{~mm}$ & None & HSV & \\
\hline SC-131 & $11 / 9 / 2016$ & Glass & Water & $3 \mathrm{~mm}$ & None & HSV & \\
\hline SC-132 & $11 / 9 / 2016$ & Glass & Water & $3 \mathrm{~mm}$ & None & HSV & \\
\hline SC-133 & $11 / 9 / 2016$ & Glass & Water & $2 \mathrm{~mm}$ & None & HSV & \\
\hline SC-134 & $11 / 9 / 2016$ & Glass & Water & $4 \mathrm{~mm}$ & None & HSV & \\
\hline SC-135 & $11 / 9 / 2016$ & Glass & Water & $3 \mathrm{~mm}$ & None & HSV & \\
\hline SC-136 & $11 / 9 / 2016$ & Glass & Water & $3 \mathrm{~mm}$ & None & HSV & \\
\hline SC-137 & $11 / 9 / 2016$ & Glass & Water & $3 \mathrm{~mm}$ & None & HSV & \\
\hline
\end{tabular}




\begin{tabular}{|c|c|c|c|c|c|c|c|}
\hline Test & Date & Syringe & Liquid & Air gap & Damping & Instruments & Comment \\
\hline SC-138 & $11 / 9 / 2016$ & Glass & Water & $0 \mathrm{~mm}$ & None & HSV & \\
\hline SC-139 & $11 / 9 / 2016$ & Glass & Water & $0 \mathrm{~mm}$ & None & HSV & \\
\hline SC-140 & $11 / 9 / 2016$ & Glass & Water & $3 \mathrm{~mm}$ & D1 & HSV & \\
\hline SC-141 & $11 / 9 / 2016$ & Glass & Water & $3 \mathrm{~mm}$ & D1 & HSV & \\
\hline SC-142 & $11 / 9 / 2016$ & Glass & Water & $3 \mathrm{~mm}$ & None & HSV & \\
\hline SC-143 & $11 / 9 / 2016$ & Glass & Water & $3 \mathrm{~mm}$ & None & HSV & \\
\hline SC-144 & $11 / 9 / 2016$ & Glass & Water & $3 \mathrm{~mm}$ & None & HSV & \\
\hline SC-145 & $11 / 9 / 2016$ & Glass & Water & $3 \mathrm{~mm}$ & D1 & HSV & \\
\hline SC-146 & $11 / 9 / 2016$ & Glass & Water & $3 \mathrm{~mm}$ & None & HSV & Reduced spring force \\
\hline SC-147 & $11 / 21 / 2016$ & Glass & Water & $1 \mathrm{~mm}$ & None & HSV & Reduced spring force \\
\hline SC-148 & $11 / 21 / 2016$ & Glass & Water & $2 \mathrm{~mm}$ & None & HSV & Reduced spring force \\
\hline SC-149 & $11 / 21 / 2016$ & Glass & Water & $3 \mathrm{~mm}$ & None & HSV & Reduced spring force \\
\hline SC-150 & $11 / 21 / 2016$ & Glass & Water & $3 \mathrm{~mm}$ & None & HSV & Reduced spring force \\
\hline SC-151 & $11 / 21 / 2016$ & Glass & Water & $4 \mathrm{~mm}$ & None & HSV & Reduced spring force \\
\hline SC-152 & $11 / 21 / 2016$ & Glass & Water & $2 \mathrm{~mm}$ & None & $\mathrm{P}, \mathrm{HSV}$ & \\
\hline SC-153 & $11 / 21 / 2016$ & Glass & Water & $2 \mathrm{~mm}$ & None & $\mathrm{S} 1 \mathrm{H}, \mathrm{S} 1 \mathrm{~A}, \mathrm{~S} 2 \mathrm{H}, \mathrm{P}, \mathrm{HSV}$ & $\mathrm{S} 1 \mathrm{H}, \mathrm{S} 2 \mathrm{H}, \mathrm{S} 1 \mathrm{~A}$ failed \\
\hline SC-154 & $11 / 23 / 2016$ & Glass & Water & $2 \mathrm{~mm}$ & None & S1H, S1A, S2H, P, HSV & \\
\hline SC-155 & $12 / 5 / 2016$ & Glass & Water & $2 \mathrm{~mm}$ & None & HSV & \\
\hline SC-156 & $12 / 5 / 2016$ & Glass & Water & $2 \mathrm{~mm}$ & None & HSV & Non-siliconized syringe \\
\hline SC-157 & $12 / 5 / 2016$ & Glass & Water & $2 \mathrm{~mm}$ & None & HSV & Non-siliconized syringe \\
\hline SC-158 & $12 / 5 / 2016$ & Glass & Water & $2 \mathrm{~mm}$ & None & HSV & Non-siliconized syringe \\
\hline SC-159 & $2 / 13 / 2017$ & Glass & Drug & $2 \mathrm{~mm}$ & None & HSV & \\
\hline
\end{tabular}




\begin{tabular}{|c|c|c|c|c|c|c|c|}
\hline Test & Date & Syringe & Liquid & Air gap & Damping & Instruments & Comment \\
\hline SC-160 & $2 / 13 / 2017$ & Glass & Drug & $2 \mathrm{~mm}$ & None & HSV & \\
\hline SC-161 & $2 / 13 / 2017$ & Glass & Drug & $2 \mathrm{~mm}$ & None & HSV & \\
\hline SC-162 & $2 / 13 / 2017$ & Glass & Drug & $2 \mathrm{~mm}$ & None & HSV & \\
\hline SC-163 & $2 / 13 / 2017$ & Glass & Drug & $2 \mathrm{~mm}$ & None & HSV & \\
\hline SC-164 & $2 / 13 / 2017$ & Glass & Drug & $4 \mathrm{~mm}$ & None & HSV & \\
\hline SC-165 & $2 / 13 / 2017$ & Glass & Drug & $5 \mathrm{~mm}$ & None & HSV & \\
\hline SC-166 & $2 / 13 / 2017$ & Glass & Drug & $0 \mathrm{~mm}$ & None & HSV & \\
\hline SC-167 & $2 / 13 / 2017$ & Glass & Drug & $3 \mathrm{~mm}$ & None & HSV & \\
\hline SC-168 & $2 / 13 / 2017$ & Glass & Drug & $2 \mathrm{~mm}$ & None & HSV & \\
\hline SC-169 & $2 / 13 / 2017$ & Glass & Drug & $11 \mathrm{~mm}$ & None & HSV & \\
\hline SC-170 & $2 / 13 / 2017$ & Glass & Drug & $2 \mathrm{~mm}$ & None & HSV & \\
\hline SC-172 & $3 / 2 / 2017$ & Glass & Drug & $0 \mathrm{~mm}$ & None & $\mathrm{P}, \mathrm{S} 1 \mathrm{H}$ & \\
\hline SC-173 & $3 / 8 / 2017$ & Glass & Drug & $0 \mathrm{~mm}$ & None & $\mathrm{P}, \mathrm{S} 1 \mathrm{H}$ & \\
\hline SC-174 & $3 / 9 / 2017$ & Glass & Drug & $0 \mathrm{~mm}$ & None & S1H, S1A, S2H, P, HSV & \\
\hline SC-176 & $3 / 14 / 2017$ & Glass & Drug & $0 \mathrm{~mm}$ & None & S1H, S1A, S2H, P, HSV & \\
\hline SC-177 & $3 / 27 / 2017$ & Glass & Drug & $3 \mathrm{~mm}$ & None & S1H, S1A, S2H, P, HSV & \\
\hline SC-178 & $3 / 27 / 2017$ & Glass & Drug & $3 \mathrm{~mm}$ & None & S1H, S1A, S2H, P, HSV & \\
\hline SC-179 & $4 / 5 / 2017$ & Glass & Drug & $5 \mathrm{~mm}$ & None & S1H, S1A, S2H, P, HSV & \\
\hline SC- 180 & $4 / 12 / 2017$ & Glass & Drug & $3 \mathrm{~mm}$ & None & $\mathrm{S} 1 \mathrm{H}, \mathrm{S} 1 \mathrm{~A}, \mathrm{~S} 2 \mathrm{H}, \mathrm{P}, \mathrm{HSV}$ & Tip up \\
\hline SC-181 & $4 / 17 / 2017$ & Glass & Drug & $3 \mathrm{~mm}$ & None & HSV & Tip up \\
\hline SC-182 & $4 / 17 / 2017$ & Glass & Drug & $2 \mathrm{~mm}$ & None & HSV & Tip up \\
\hline SC-183 & $4 / 17 / 2017$ & Glass & Drug & $2 \mathrm{~mm}$ & None & HSV & Tip up \\
\hline
\end{tabular}




\begin{tabular}{|c|c|c|c|c|c|c|c|}
\hline Test & Date & Syringe & Liquid & Air gap & Damping & Instruments & Comment \\
\hline SC-184 & $4 / 17 / 2017$ & Glass & Drug & $1 \mathrm{~mm}$ & None & HSV & Tip up \\
\hline SC-185 & $4 / 17 / 2017$ & Glass & Drug & $0 \mathrm{~mm}$ & None & HSV & Tip up \\
\hline SC-186 & $4 / 17 / 2017$ & Glass & Drug & $0 \mathrm{~mm}$ & None & HSV & Tip up \\
\hline SC-187 & $4 / 17 / 2017$ & Glass & Drug & $7 \mathrm{~mm}$ & None & HSV & Tip up \\
\hline SC-188 & $4 / 17 / 2017$ & Glass & Drug & $1 \mathrm{~mm}$ & None & HSV & Tip up \\
\hline SC-189 & $4 / 17 / 2017$ & Glass & Drug & $0 \mathrm{~mm}$ & None & HSV & Tip up \\
\hline SC-190 & $4 / 17 / 2017$ & Glass & Drug & $1 \mathrm{~mm}$ & None & HSV & Tip up \\
\hline SC-191 & $4 / 17 / 2017$ & Glass & Drug & $2 \mathrm{~mm}$ & None & HSV & Tip up \\
\hline SC-192 & $4 / 17 / 2017$ & Glass & Drug & $5 \mathrm{~mm}$ & None & HSV & Tip up \\
\hline SC-193 & $4 / 17 / 2017$ & Glass & Drug & $1 \mathrm{~mm}$ & None & HSV & Tip up \\
\hline SC-194 & $4 / 17 / 2017$ & Glass & Drug & $1 \mathrm{~mm}$ & None & S1H, S1A, S2H, P, HSV & Tip up \\
\hline SC-195 & $5 / 18 / 2017$ & Glass & Drug & $1 \mathrm{~mm}$ & None & S1H, S1A, S2H, P, HSV & Tip up, S1A failed \\
\hline SC-196 & $5 / 18 / 2017$ & Glass & Drug & $0 \mathrm{~mm}$ & None & S1H, S1A, S2H, P, HSV & Tip up, S1A failed \\
\hline SC-197 & $5 / 25 / 2017$ & Glass & Drug & $2 \mathrm{~mm}$ & None & S1H, S1A, S2H, P, HSV & Tip up \\
\hline SC-200 & $11 / 20 / 2017$ & Plastic & Water & $0 \mathrm{~mm}$ & None & S1H, S1A, S2H, P, HSV & \\
\hline SC-201 & $11 / 20 / 2017$ & Plastic & Water & $4 \mathrm{~mm}$ & None & S1H, S1A, S2H, P, HSV & \\
\hline SC-202 & $11 / 21 / 2017$ & Plastic & Water & $0 \mathrm{~mm}$ & None & HSV & \\
\hline SC-203 & $11 / 21 / 2017$ & Plastic & Water & $0 \mathrm{~mm}$ & None & HSV & \\
\hline SC-204 & $11 / 21 / 2017$ & Plastic & Water & $3 \mathrm{~mm}$ & None & HSV & \\
\hline SC-205 & $11 / 21 / 2017$ & Plastic & Water & $3 \mathrm{~mm}$ & None & HSV & \\
\hline SC-206 & $11 / 21 / 2017$ & Plastic & Water & $7 \mathrm{~mm}$ & None & HSV & \\
\hline SC-207 & $12 / 11 / 2017$ & Plastic & Water & $0 \mathrm{~mm}$ & None & S1H, S1A, S2H, P, HSV & \\
\hline
\end{tabular}




\begin{tabular}{llllllll}
\hline Test & Date & Syringe & Liquid & Air gap & Damping & Instruments & Comment \\
\hline SC-208 & $12 / 11 / 2017$ & Plastic & Water & $3 \mathrm{~mm}$ & None & S1H, S1A, S2H, P, HSV & \\
SC-209 & $12 / 11 / 2017$ & Plastic & Water & $0 \mathrm{~mm}$ & None & HSV \\
SC-210 & $12 / 11 / 2017$ & Plastic & Water & $5 \mathrm{~mm}$ & None & HSV \\
SC-211 & $12 / 11 / 2017$ & Plastic & Water & $4 \mathrm{~mm}$ & None & HSV & \\
SC-212 & $12 / 11 / 2017$ & Plastic & Water & $12 \mathrm{~mm}$ & None & HSV & Syringe empty of liquid \\
SC-213 & $12 / 11 / 2017$ & Plastic & Air & N/A & None & HSV & Syringe empty of liquid \\
SC-214 & $12 / 11 / 2017$ & Plastic & Air & N/A & None & HSV & Syringe empty of liquid \\
\hline SC-215 & $12 / 11 / 2017$ & Glass & Air & N/A & None & HSV &
\end{tabular}


Table B.2: Test matrix for the experiments performed with the static, large-scale model autoinjector.

\begin{tabular}{|c|c|c|c|c|c|}
\hline Test & Date & Base fixture & Air gap & Impact velocity & Comment \\
\hline GG-0023 & $8 / 30 / 2016$ & Al. Flat & $0 \mathrm{~mm}$ & $4.3 \mathrm{~m} / \mathrm{s}$ & \\
\hline GG-0024 & $8 / 30 / 2016$ & Al. Flat & $0 \mathrm{~mm}$ & $3.2 \mathrm{~m} / \mathrm{s}$ & \\
\hline GG-0025 & $8 / 30 / 2016$ & Al. Flat & $0 \mathrm{~mm}$ & $2.1 \mathrm{~m} / \mathrm{s}$ & \\
\hline GG-0026 & $8 / 30 / 2016$ & Al. Flat & $0 \mathrm{~mm}$ & $1.6 \mathrm{~m} / \mathrm{s}$ & \\
\hline GG-0027 & $8 / 30 / 2016$ & Al. Flat & $0 \mathrm{~mm}$ & $1.4 \mathrm{~m} / \mathrm{s}$ & \\
\hline GG-0028 & $8 / 30 / 2016$ & Al. Flat & $0 \mathrm{~mm}$ & $1.0 \mathrm{~m} / \mathrm{s}$ & \\
\hline GG-0029 & $8 / 30 / 2016$ & Al. Flat & $0 \mathrm{~mm}$ & $5.1 \mathrm{~m} / \mathrm{s}$ & \\
\hline GG-0030 & $8 / 30 / 2016$ & Al. Flat & $0 \mathrm{~mm}$ & $5.7 \mathrm{~m} / \mathrm{s}$ & \\
\hline GG-0031 & $8 / 30 / 2016$ & Al. Flat & $2.0 \mathrm{~mm}$ & $6.3 \mathrm{~m} / \mathrm{s}$ & \\
\hline GG-0032 & $8 / 30 / 2016$ & Al. Flat & $7.0 \mathrm{~mm}$ & $5.7 \mathrm{~m} / \mathrm{s}$ & \\
\hline GG-0033 & $8 / 30 / 2016$ & Al. Flat & $3.6 \mathrm{~mm}$ & $5.5 \mathrm{~m} / \mathrm{s}$ & \\
\hline GG-0034 & $8 / 30 / 2016$ & Al. Flat & $11.6 \mathrm{~mm}$ & $5.6 \mathrm{~m} / \mathrm{s}$ & \\
\hline GG-0035 & $8 / 30 / 2016$ & Al. Flat & $0.5 \mathrm{~mm}$ & $6.4 \mathrm{~m} / \mathrm{s}$ & \\
\hline GG-0037 & $8 / 31 / 2016$ & Al. Flat & $0 \mathrm{~mm}$ & $5.4 \mathrm{~m} / \mathrm{s}$ & \\
\hline GG-0040 & $8 / 31 / 2016$ & Al. Flat & $0 \mathrm{~mm}$ & N/A & \\
\hline GG-0037 & $8 / 31 / 2016$ & Al. Flat & $0 \mathrm{~mm}$ & $5.4 \mathrm{~m} / \mathrm{s}$ & \\
\hline GG-0040 & $8 / 31 / 2016$ & Al. Flat & $0 \mathrm{~mm}$ & N/A & \\
\hline GG-0045 & $9 / 2 / 2016$ & Al. Flat & $0 \mathrm{~mm}$ & $5.6 \mathrm{~m} / \mathrm{s}$ & \\
\hline GG-0046 & $9 / 2 / 2016$ & Al. Flat & $15.0 \mathrm{~mm}$ & $5.1 \mathrm{~m} / \mathrm{s}$ & \\
\hline GG-0047 & $9 / 2 / 2016$ & Al. Flat & $20 \mathrm{~mm}$ & $5.1 \mathrm{~m} / \mathrm{s}$ & \\
\hline GG-0070 & $10 / 12 / 2016$ & Al. Cone & $0 \mathrm{~mm}$ & $3.2 \mathrm{~m} / \mathrm{s}$ & \\
\hline GG-0071 & $10 / 12 / 2016$ & Al. Cone & $0 \mathrm{~mm}$ & $2.5 \mathrm{~m} / \mathrm{s}$ & \\
\hline GG-0072 & $10 / 12 / 2016$ & Al. Cone & $0 \mathrm{~mm}$ & $1.6 \mathrm{~m} / \mathrm{s}$ & \\
\hline GG-0073 & $10 / 12 / 2016$ & Al. Cone & $0 \mathrm{~mm}$ & $3.7 \mathrm{~m} / \mathrm{s}$ & \\
\hline GG-0074 & $10 / 12 / 2016$ & Al. Cone & $0 \mathrm{~mm}$ & $4.3 \mathrm{~m} / \mathrm{s}$ & \\
\hline GG-0075 & $10 / 12 / 2016$ & Al. Cone & $0 \mathrm{~mm}$ & $4.8 \mathrm{~m} / \mathrm{s}$ & \\
\hline GG-0076 & $10 / 12 / 2016$ & Al. Cone & $0 \mathrm{~mm}$ & $4.2 \mathrm{~m} / \mathrm{s}$ & \\
\hline GG-0077 & $10 / 12 / 2016$ & Al. Cone & $0 \mathrm{~mm}$ & $5.0 \mathrm{~m} / \mathrm{s}$ & \\
\hline GG-0078 & $10 / 12 / 2016$ & Al. Cone & $0 \mathrm{~mm}$ & $5.0 \mathrm{~m} / \mathrm{s}$ & \\
\hline GG-0079 & $10 / 12 / 2016$ & Al. Cone & $0 \mathrm{~mm}$ & $5.3 \mathrm{~m} / \mathrm{s}$ & \\
\hline GG-0080 & $10 / 12 / 2016$ & Al. Cone & $0 \mathrm{~mm}$ & $6.2 \mathrm{~m} / \mathrm{s}$ & \\
\hline GG-0081 & $10 / 12 / 2016$ & Al. Cone & $0 \mathrm{~mm}$ & $6.1 \mathrm{~m} / \mathrm{s}$ & \\
\hline GG-0082 & $10 / 12 / 2016$ & Al. Cone & $0 \mathrm{~mm}$ & $4.1 \mathrm{~m} / \mathrm{s}$ & \\
\hline GG-0093 & $10 / 26 / 2016$ & Al. Cone & $0 \mathrm{~mm}$ & $6.4 \mathrm{~m} / \mathrm{s}$ & \\
\hline
\end{tabular}




\begin{tabular}{|c|c|c|c|c|c|}
\hline Test & Date & Base fixture & Air gap & Impact velocity & Comment \\
\hline GG-0094 & $10 / 26 / 2016$ & Al. Cone & $1.5 \mathrm{~mm}$ & $6.4 \mathrm{~m} / \mathrm{s}$ & \\
\hline GG-0095 & $10 / 26 / 2016$ & Al. Cone & $2.5 \mathrm{~mm}$ & $6.4 \mathrm{~m} / \mathrm{s}$ & \\
\hline GG-0096 & $10 / 26 / 2016$ & Al. Cone & $5.0 \mathrm{~mm}$ & $6.4 \mathrm{~m} / \mathrm{s}$ & \\
\hline GG-0097 & $10 / 26 / 2017$ & Al. Cone & $7.5 \mathrm{~mm}$ & $6.4 \mathrm{~m} / \mathrm{s}$ & \\
\hline GG-0098 & $10 / 26 / 2016$ & Al. Cone & $10 \mathrm{~mm}$ & $6.4 \mathrm{~m} / \mathrm{s}$ & \\
\hline GG-0099 & $10 / 26 / 2016$ & Al. Cone & $12.7 \mathrm{~mm}$ & $6.4 \mathrm{~m} / \mathrm{s}$ & \\
\hline GG-0100 & $10 / 26 / 2016$ & Al. Cone & $15.0 \mathrm{~mm}$ & $6.4 \mathrm{~m} / \mathrm{s}$ & \\
\hline GG-0101 & $10 / 26 / 2016$ & Al. Cone & $18.0 \mathrm{~mm}$ & $6.4 \mathrm{~m} / \mathrm{s}$ & \\
\hline GG-0102 & $10 / 26 / 2016$ & Al. Cone & $20.5 \mathrm{~mm}$ & $6.4 \mathrm{~m} / \mathrm{s}$ & \\
\hline GG-0103 & $10 / 26 / 2016$ & Al. Cone & $5.0 \mathrm{~mm}$ & $6.4 \mathrm{~m} / \mathrm{s}$ & \\
\hline GG-0104 & $10 / 26 / 2016$ & Al. Cone & $6.5 \mathrm{~mm}$ & $6.4 \mathrm{~m} / \mathrm{s}$ & \\
\hline GG-0105 & $10 / 26 / 2016$ & Al. Cone & $7.5 \mathrm{~mm}$ & $6.4 \mathrm{~m} / \mathrm{s}$ & \\
\hline GG-0106 & $10 / 26 / 2016$ & Al. Cone & $9.0 \mathrm{~mm}$ & $6.4 \mathrm{~m} / \mathrm{s}$ & \\
\hline GG-0107 & $10 / 26 / 2016$ & Al. Cone & $10 \mathrm{~mm}$ & $6.4 \mathrm{~m} / \mathrm{s}$ & \\
\hline GG-0200 & $8 / 8 / 2018$ & PC Cone & $0 \mathrm{~mm}$ & $3.9 \mathrm{~m} / \mathrm{s}$ & \\
\hline GG-0201 & $8 / 9 / 2018$ & PC Cone & $0 \mathrm{~mm}$ & $5.1 \mathrm{~m} / \mathrm{s}$ & \\
\hline GG-0202 & $8 / 9 / 2018$ & PC Cone & $0 \mathrm{~mm}$ & $5.7 \mathrm{~m} / \mathrm{s}$ & \\
\hline GG-0203 & $8 / 9 / 2018$ & PC Cone & $0 \mathrm{~mm}$ & $5.9 \mathrm{~m} / \mathrm{s}$ & \\
\hline GG-0204 & $8 / 9 / 2018$ & PC Cone & $0 \mathrm{~mm}$ & $6.2 \mathrm{~m} / \mathrm{s}$ & \\
\hline GG-0205 & $8 / 9 / 2018$ & PC Cone & $0 \mathrm{~mm}$ & $6.2 \mathrm{~m} / \mathrm{s}$ & \\
\hline GG-0206 & $8 / 9 / 2018$ & PC Cone & $0 \mathrm{~mm}$ & $6.0 \mathrm{~m} / \mathrm{s}$ & Degassed water \\
\hline GG-0207 & $8 / 9 / 2018$ & PC Cone & $0 \mathrm{~mm}$ & $5.7 \mathrm{~m} / \mathrm{s}$ & Degassed water \\
\hline GG-0208 & $8 / 9 / 2018$ & PC Cone & $0 \mathrm{~mm}$ & $5.5 \mathrm{~m} / \mathrm{s}$ & Degassed water \\
\hline GG-0209 & $8 / 9 / 2018$ & PC Cone & $0 \mathrm{~mm}$ & $5.5 \mathrm{~m} / \mathrm{s}$ & Degassed water \\
\hline GG-0210 & $8 / 9 / 2018$ & PC Cone & $0 \mathrm{~mm}$ & $5.6 \mathrm{~m} / \mathrm{s}$ & Degassed water \\
\hline GG-0211 & $8 / 10 / 2018$ & PC Cone & $0 \mathrm{~mm}$ & $5.6 \mathrm{~m} / \mathrm{s}$ & \\
\hline GG-0212 & $8 / 10 / 2018$ & PC Cone & $0 \mathrm{~mm}$ & $5.6 \mathrm{~m} / \mathrm{s}$ & \\
\hline GG-0213 & $8 / 10 / 2018$ & PC Cone & $0 \mathrm{~mm}$ & $5.6 \mathrm{~m} / \mathrm{s}$ & \\
\hline GG-0214 & $8 / 10 / 2018$ & PC Cone & $0 \mathrm{~mm}$ & $5.6 \mathrm{~m} / \mathrm{s}$ & \\
\hline GG-0215 & $8 / 10 / 2018$ & PC Cone & $0 \mathrm{~mm}$ & $5.6 \mathrm{~m} / \mathrm{s}$ & \\
\hline GG-0216 & $8 / 10 / 2018$ & PC Cone & $3.5 \mathrm{~mm}$ & $5.6 \mathrm{~m} / \mathrm{s}$ & \\
\hline GG-0217 & $8 / 10 / 2018$ & PC Cone & $3.5 \mathrm{~mm}$ & $5.6 \mathrm{~m} / \mathrm{s}$ & \\
\hline GG-0218 & $8 / 10 / 2018$ & PC Cone & $3.5 \mathrm{~mm}$ & $5.6 \mathrm{~m} / \mathrm{s}$ & \\
\hline GG-0219 & $8 / 10 / 2018$ & PC Cone & $3.5 \mathrm{~mm}$ & $5.6 \mathrm{~m} / \mathrm{s}$ & \\
\hline GG-0220 & $8 / 10 / 2018$ & PC Cone & $3.5 \mathrm{~mm}$ & $5.7 \mathrm{~m} / \mathrm{s}$ & \\
\hline GG-0221 & $8 / 10 / 2018$ & PC Cone & $3.5 \mathrm{~mm}$ & $5.6 \mathrm{~m} / \mathrm{s}$ & Degassed water \\
\hline GG-0222 & $8 / 10 / 2018$ & PC Cone & $3.5 \mathrm{~mm}$ & $5.6 \mathrm{~m} / \mathrm{s}$ & Degassed water \\
\hline
\end{tabular}




\begin{tabular}{llllll}
\hline Test & Date & Base fixture & Air gap & Impact velocity & Comment \\
\hline GG-0223 & $8 / 13 / 2018$ & PC Cone & $3.5 \mathrm{~mm}$ & $5.6 \mathrm{~m} / \mathrm{s}$ & Degassed water \\
\hline GG-0224 & $8 / 13 / 2018$ & PC Cone & $3.5 \mathrm{~mm}$ & $5.6 \mathrm{~m} / \mathrm{s}$ & Degassed water \\
\hline GG-0225 & $8 / 13 / 2018$ & PC Cone & $3.5 \mathrm{~mm}$ & $5.6 \mathrm{~m} / \mathrm{s}$ & Degassed water \\
\hline GG-0226 & $8 / 13 / 2018$ & PC Cone & $12.0 \mathrm{~mm}$ & $5.5 \mathrm{~m} / \mathrm{s}$ & \\
GG-0227 & $8 / 13 / 2018$ & PC Cone & $12.0 \mathrm{~mm}$ & $5.6 \mathrm{~m} / \mathrm{s}$ & \\
\hline GG-0228 & $8 / 13 / 2018$ & PC Cone & $12.0 \mathrm{~mm}$ & $5.6 \mathrm{~m} / \mathrm{s}$ & \\
GG-0229 & $8 / 13 / 2018$ & PC Cone & $12.0 \mathrm{~mm}$ & $5.6 \mathrm{~m} / \mathrm{s}$ & \\
\hline GG-0230 & $8 / 13 / 2018$ & PC Cone & $12.0 \mathrm{~mm}$ & $5.5 \mathrm{~m} / \mathrm{s}$ & \\
\hline GG-0231 & $8 / 13 / 2018$ & PC Cone & $15.0 \mathrm{~mm}$ & $5.7 \mathrm{~m} / \mathrm{s}$ & \\
\hline GG-0232 & $8 / 13 / 2018$ & PC Cone & $15.0 \mathrm{~mm}$ & $5.7 \mathrm{~m} / \mathrm{s}$ & \\
GG-0233 & $8 / 13 / 2018$ & PC Cone & $12.7 \mathrm{~mm}$ & $5.7 \mathrm{~m} / \mathrm{s}$ & \\
\hline GG-0234 & $8 / 13 / 2018$ & PC Cone & $12.7 \mathrm{~mm}$ & $5.7 \mathrm{~m} / \mathrm{s}$ & \\
\hline GG-0235 & $8 / 13 / 2018$ & PC Cone & $12.7 \mathrm{~mm}$ & $5.6 \mathrm{~m} / \mathrm{s}$ & \\
\hline GG-0236 & $8 / 13 / 2018$ & PC Cone & $12.7 \mathrm{~mm}$ & $5.6 \mathrm{~m} / \mathrm{s}$ & \\
\hline GG-0237 & $8 / 13 / 2018$ & PC Cone & $12.7 \mathrm{~mm}$ & $5.6 \mathrm{~m} / \mathrm{s}$ & \\
\hline GG-0238 & $8 / 13 / 2018$ & PC Cone & $12.7 \mathrm{~mm}$ & $5.6 \mathrm{~m} / \mathrm{s}$ & Degassed water \\
\hline
\end{tabular}


Table B.3: Test matrix for the experiments performed with the dynamic, large-scale model autoinjector.

\begin{tabular}{|c|c|c|c|c|c|c|}
\hline Test & Date & Syringe & Tip & Impact & Air gap & Comment \\
\hline LS-0155 & $4 / 10 / 2017$ & Aluminum & Cone (Al) & I. on $\mathrm{S}$. & $\inf$ & \\
\hline LS-0156 & $4 / 10 / 2017$ & Aluminum & Cone (Al) & I. on $\mathrm{S}$. & $\inf$ & \\
\hline LS-0189 & $6 / 8 / 2017$ & Aluminum & Flat & I. on $\mathrm{S}$. & inf & \\
\hline LS-0192 & $6 / 14 / 2017$ & Aluminum & Cone (Al) & I. on B. & $0 \mathrm{~mm}$ & \\
\hline LS-0193 & $6 / 14 / 2017$ & Aluminum & Cone (Al) & I. on B. & $15.2 \mathrm{~mm}$ & \\
\hline LS-0194 & $6 / 14 / 2017$ & Aluminum & Cone (Al) & I. on B. & $12.7 \mathrm{~mm}$ & \\
\hline LS-0195 & $6 / 14 / 2017$ & Aluminum & Cone (Al) & I. on B. & $10.2 \mathrm{~mm}$ & \\
\hline LS-0196 & $6 / 14 / 2017$ & Aluminum & Cone (Al) & I. on B. & $7.6 \mathrm{~mm}$ & \\
\hline LS-0197 & $6 / 14 / 2017$ & Aluminum & Cone (Al) & I. on B. & $5.1 \mathrm{~mm}$ & \\
\hline LS-0198 & $6 / 15 / 2017$ & Aluminum & Cone (Al) & I. on B. & $2.5 \mathrm{~mm}$ & \\
\hline LS-0199 & $6 / 15 / 2017$ & Aluminum & Cone (Al) & I. on B. & $0 \mathrm{~mm}$ & \\
\hline LS-0200 & $6 / 16 / 2017$ & Aluminum & Cone (Al) & I. on B. & $0 \mathrm{~mm}$ & \\
\hline LS-0201 & 6/16/2017 & Aluminum & Cone (Al) & I. on B. & $0 \mathrm{~mm}$ & \\
\hline LS-0202 & $6 / 16 / 2017$ & Aluminum & Cone (Al) & I. on B. & $0 \mathrm{~mm}$ & \\
\hline LS-0203 & $6 / 16 / 2017$ & Aluminum & Cone (Al) & I. on B. & $2.5 \mathrm{~mm}$ & \\
\hline LS-0204 & $6 / 16 / 2017$ & Aluminum & Cone (Al) & I. on B. & $2.5 \mathrm{~mm}$ & \\
\hline LS-0205 & $6 / 16 / 2017$ & Aluminum & Cone (Al) & I. on B. & $2.5 \mathrm{~mm}$ & \\
\hline LS-0206 & $6 / 16 / 2017$ & Aluminum & Cone (Al) & I. on B. & $2.5 \mathrm{~mm}$ & \\
\hline LS-0207 & $6 / 16 / 2017$ & Aluminum & Cone (Al) & I. on B. & $5.1 \mathrm{~mm}$ & \\
\hline LS-0208 & $6 / 16 / 2017$ & Aluminum & Cone (Al) & I. on B. & $5.1 \mathrm{~mm}$ & \\
\hline
\end{tabular}




\begin{tabular}{|c|c|c|c|c|c|c|}
\hline Test & Date & Syringe & Tip & Impact & Air gap & Comment \\
\hline LS-0209 & $6 / 16 / 2017$ & Aluminum & Cone $(\mathrm{Al})$ & I. on B. & $5.1 \mathrm{~mm}$ & \\
\hline LS-0210 & $6 / 16 / 2017$ & Aluminum & Cone $(\mathrm{Al})$ & I. on B. & $5.1 \mathrm{~mm}$ & \\
\hline LS-0211 & $6 / 16 / 2017$ & Aluminum & Cone $(\mathrm{Al})$ & I. on B. & $7.6 \mathrm{~mm}$ & \\
\hline LS-0212 & $6 / 16 / 2017$ & Aluminum & Cone $(\mathrm{Al})$ & I. on B. & $7.6 \mathrm{~mm}$ & \\
\hline LS-0213 & $6 / 16 / 2017$ & Aluminum & Cone $(\mathrm{Al})$ & I. on B. & $7.6 \mathrm{~mm}$ & \\
\hline LS-0214 & $6 / 16 / 2017$ & Aluminum & Cone $(\mathrm{Al})$ & I. on B. & $7.6 \mathrm{~mm}$ & \\
\hline LS-0215 & $6 / 19 / 2017$ & Aluminum & Cone $(\mathrm{Al})$ & I. on B. & $10.2 \mathrm{~mm}$ & \\
\hline LS-0216 & $6 / 19 / 2017$ & Aluminum & Cone $(\mathrm{Al})$ & I. on B. & $10.2 \mathrm{~mm}$ & \\
\hline LS-0217 & $6 / 19 / 2017$ & Aluminum & Cone $(\mathrm{Al})$ & I. on B. & $10.2 \mathrm{~mm}$ & \\
\hline LS-0218 & $6 / 19 / 2017$ & Aluminum & Cone $(\mathrm{Al})$ & I. on B. & $10.2 \mathrm{~mm}$ & \\
\hline LS-0219 & $6 / 19 / 2017$ & Aluminum & Cone $(\mathrm{Al})$ & I. on B. & $12.7 \mathrm{~mm}$ & \\
\hline LS-0220 & $6 / 19 / 2017$ & Aluminum & Cone $(\mathrm{Al})$ & I. on B. & $12.7 \mathrm{~mm}$ & \\
\hline LS-0221 & $6 / 19 / 2017$ & Aluminum & Cone $(\mathrm{Al})$ & I. on B. & $12.7 \mathrm{~mm}$ & \\
\hline LS-0222 & $6 / 19 / 2017$ & Aluminum & Cone $(\mathrm{Al})$ & I. on B. & $12.7 \mathrm{~mm}$ & \\
\hline LS-0223 & $6 / 19 / 2017$ & Aluminum & Cone $(\mathrm{Al})$ & I. on B. & $15.2 \mathrm{~mm}$ & \\
\hline LS-0224 & $6 / 19 / 2017$ & Aluminum & Cone $(\mathrm{Al})$ & I. on B. & $15.2 \mathrm{~mm}$ & \\
\hline LS-0225 & $6 / 19 / 2017$ & Aluminum & Cone $(\mathrm{Al})$ & I. on B. & $15.2 \mathrm{~mm}$ & \\
\hline LS-0226 & $6 / 19 / 2017$ & Aluminum & Cone $(\mathrm{Al})$ & I. on B. & $15.2 \mathrm{~mm}$ & \\
\hline LS-0227 & $6 / 21 / 2017$ & Aluminum & Cone $(\mathrm{Al})$ & I. on $\mathrm{S}$. & $15.2 \mathrm{~mm}$ & \\
\hline LS-0228 & $6 / 21 / 2017$ & Aluminum & Cone $(\mathrm{Al})$ & I. on $\mathrm{S}$. & $15.2 \mathrm{~mm}$ & \\
\hline LS-0229 & $6 / 21 / 2017$ & Aluminum & Cone $(\mathrm{Al})$ & I. on $\mathrm{S}$. & $15.2 \mathrm{~mm}$ & \\
\hline LS-0230 & $6 / 21 / 2017$ & Aluminum & Cone $(\mathrm{Al})$ & I. on $\mathrm{S}$. & $15.2 \mathrm{~mm}$ & \\
\hline
\end{tabular}




\begin{tabular}{|c|c|c|c|c|c|c|}
\hline Test & Date & Syringe & Tip & Impact & Air gap & Comment \\
\hline LS-0231 & $6 / 21 / 2017$ & Aluminum & Cone $(\mathrm{Al})$ & I. on $\mathrm{S}$. & $15.2 \mathrm{~mm}$ & \\
\hline LS-0232 & $6 / 21 / 2017$ & Aluminum & Cone $(\mathrm{Al})$ & I. on $\mathrm{S}$. & $12.7 \mathrm{~mm}$ & \\
\hline LS-0233 & $6 / 21 / 2017$ & Aluminum & Cone $(\mathrm{Al})$ & I. on $\mathrm{S}$. & $12.7 \mathrm{~mm}$ & \\
\hline LS-0234 & $6 / 21 / 2017$ & Aluminum & Cone $(\mathrm{Al})$ & I. on $\mathrm{S}$. & $12.7 \mathrm{~mm}$ & \\
\hline LS-0235 & $6 / 21 / 2017$ & Aluminum & Cone $(\mathrm{Al})$ & I. on $\mathrm{S}$. & $12.7 \mathrm{~mm}$ & \\
\hline LS-0236 & $6 / 21 / 2017$ & Aluminum & Cone $(\mathrm{Al})$ & I. on $\mathrm{S}$. & $12.7 \mathrm{~mm}$ & \\
\hline LS-0237 & $6 / 21 / 2017$ & Aluminum & Cone $(\mathrm{Al})$ & I. on $\mathrm{S}$. & $10.2 \mathrm{~mm}$ & \\
\hline LS-0238 & $6 / 21 / 2017$ & Aluminum & Cone $(\mathrm{Al})$ & I. on $\mathrm{S}$. & $10.2 \mathrm{~mm}$ & \\
\hline LS-0239 & $6 / 22 / 2017$ & Aluminum & Cone $(\mathrm{Al})$ & I. on $\mathrm{S}$. & $10.2 \mathrm{~mm}$ & \\
\hline LS- 0240 & $6 / 22 / 2017$ & Aluminum & Cone $(\mathrm{Al})$ & I. on $\mathrm{S}$. & $10.2 \mathrm{~mm}$ & \\
\hline LS-0241 & $6 / 22 / 2017$ & Aluminum & Cone $(\mathrm{Al})$ & I. on $\mathrm{S}$. & $10.2 \mathrm{~mm}$ & \\
\hline LS-0242 & $6 / 22 / 2017$ & Aluminum & Cone $(\mathrm{Al})$ & I. on $\mathrm{S}$. & $7.6 \mathrm{~mm}$ & \\
\hline LS- 0243 & $6 / 22 / 2017$ & Aluminum & Cone $(\mathrm{Al})$ & I. on $\mathrm{S}$. & $7.6 \mathrm{~mm}$ & \\
\hline LS-0244 & $6 / 22 / 2017$ & Aluminum & Cone $(\mathrm{Al})$ & I. on $\mathrm{S}$. & $7.6 \mathrm{~mm}$ & \\
\hline LS-0245 & $6 / 22 / 2017$ & Aluminum & Cone $(\mathrm{Al})$ & I. on $\mathrm{S}$. & $7.6 \mathrm{~mm}$ & \\
\hline LS-0246 & $6 / 22 / 2017$ & Aluminum & Cone $(\mathrm{Al})$ & I. on $\mathrm{S}$. & $7.6 \mathrm{~mm}$ & \\
\hline LS-0247 & $6 / 22 / 2017$ & Aluminum & Cone $(\mathrm{Al})$ & I. on $\mathrm{S}$. & $5.1 \mathrm{~mm}$ & \\
\hline LS-0248 & $6 / 22 / 2017$ & Aluminum & Cone $(\mathrm{Al})$ & I. on $\mathrm{S}$. & $5.1 \mathrm{~mm}$ & \\
\hline LS-0249 & $6 / 22 / 2017$ & Aluminum & Cone $(\mathrm{Al})$ & I. on $\mathrm{S}$. & $5.1 \mathrm{~mm}$ & \\
\hline LS- 0250 & $6 / 22 / 2017$ & Aluminum & Cone $(\mathrm{Al})$ & I. on $\mathrm{S}$. & $5.1 \mathrm{~mm}$ & \\
\hline LS-0251 & $6 / 22 / 2017$ & Aluminum & Cone $(\mathrm{Al})$ & I. on $\mathrm{S}$. & $5.1 \mathrm{~mm}$ & \\
\hline LS-0252 & $6 / 23 / 2017$ & Aluminum & Cone $(\mathrm{Al})$ & I. on $\mathrm{S}$. & $2.5 \mathrm{~mm}$ & \\
\hline
\end{tabular}




\begin{tabular}{|c|c|c|c|c|c|c|}
\hline Test & Date & Syringe & Tip & Impact & Air gap & Comment \\
\hline LS- 0253 & $6 / 23 / 2017$ & Aluminum & Cone (Al) & I. on $\mathrm{S}$. & $2.5 \mathrm{~mm}$ & \\
\hline LS-0254 & $6 / 23 / 2017$ & Aluminum & Cone (Al) & I. on $\mathrm{S}$. & $2.5 \mathrm{~mm}$ & \\
\hline LS-0255 & $6 / 23 / 2017$ & Aluminum & Cone (Al) & I. on $\mathrm{S}$. & $2.5 \mathrm{~mm}$ & \\
\hline LS-0256 & $6 / 23 / 2017$ & Aluminum & Cone (Al) & I. on $\mathrm{S}$. & $2.5 \mathrm{~mm}$ & \\
\hline LS-0257 & $6 / 23 / 2017$ & Aluminum & Cone (Al) & I. on $\mathrm{S}$. & $0 \mathrm{~mm}$ & \\
\hline LS-0258 & $6 / 23 / 2017$ & Aluminum & Cone (Al) & I. on $\mathrm{S}$. & $0 \mathrm{~mm}$ & \\
\hline LS-0259 & $6 / 23 / 2017$ & Aluminum & Cone (Al) & I. on $\mathrm{S}$. & $0 \mathrm{~mm}$ & \\
\hline LS- 0260 & $6 / 23 / 2017$ & Aluminum & Cone (Al) & I. on $\mathrm{S}$. & $0 \mathrm{~mm}$ & \\
\hline LS-0261 & $6 / 23 / 2017$ & Aluminum & Cone (Al) & I. on $\mathrm{S}$. & $0 \mathrm{~mm}$ & \\
\hline LS-0262 & $6 / 26 / 2017$ & Aluminum & Cone (Al) & I. on $\mathrm{S}$. & $\inf$ & \\
\hline LS-0263 & $6 / 26 / 2017$ & Aluminum & Cone (Al) & I. on $\mathrm{S}$. & $\inf$ & \\
\hline LS-0264 & $6 / 26 / 2017$ & Aluminum & Cone (Al) & I. on $\mathrm{S}$. & inf & \\
\hline LS-0265 & $6 / 26 / 2017$ & Aluminum & Cone (Al) & I. on $\mathrm{S}$. & $\inf$ & \\
\hline LS-0266 & $6 / 26 / 2017$ & Aluminum & Cone (Al) & I. on $\mathrm{S}$. & $\inf$ & \\
\hline LS-0267 & $6 / 26 / 2017$ & Aluminum & Cone (Al) & I. on $\mathrm{S}$. & $\inf$ & \\
\hline LS-0268 & $6 / 27 / 2017$ & Aluminum & Cone (PC) & I. on $\mathrm{S}$. & $5.1 \mathrm{~mm}$ & \\
\hline LS-0269 & $6 / 27 / 2017$ & Aluminum & Cone (PC) & I. on $\mathrm{S}$. & $5.1 \mathrm{~mm}$ & \\
\hline LS- 0270 & $6 / 27 / 2017$ & Aluminum & Cone (PC) & I. on $\mathrm{S}$. & $5.1 \mathrm{~mm}$ & \\
\hline LS-0271 & $6 / 27 / 2017$ & Aluminum & Cone (PC) & I. on $\mathrm{S}$. & $5.1 \mathrm{~mm}$ & \\
\hline LS- 0272 & $6 / 27 / 2017$ & Aluminum & Cone (PC) & I. on $\mathrm{S}$. & $5.1 \mathrm{~mm}$ & \\
\hline LS-0273 & $6 / 27 / 2017$ & Aluminum & Cone (PC) & I. on $\mathrm{S}$. & $\inf$ & \\
\hline LS-0274 & $7 / 10 / 2017$ & Aluminum & Cone (PC) & I. on $\mathrm{S}$. & $\inf$ & \\
\hline
\end{tabular}




\begin{tabular}{|c|c|c|c|c|c|c|}
\hline Test & Date & Syringe & Tip & Impact & Air gap & Comment \\
\hline LS-0275 & $7 / 10 / 2017$ & Aluminum & Cone (PC) & I. on $\mathrm{S}$. & $\inf$ & \\
\hline LS-0276 & $7 / 10 / 2017$ & Aluminum & Cone (PC) & I. on $\mathrm{S}$. & $\inf$ & \\
\hline LS-0277 & $7 / 10 / 2017$ & Aluminum & Cone (PC) & I. on $\mathrm{S}$. & $\inf$ & \\
\hline LS-0278 & $7 / 12 / 2017$ & Aluminum & Cone (PC) & I. on $\mathrm{S}$. & $0 \mathrm{~mm}$ & \\
\hline LS-0279 & $7 / 12 / 2017$ & Aluminum & Cone (PC) & I. on $\mathrm{S}$. & $0 \mathrm{~mm}$ & \\
\hline LS- 0280 & $7 / 12 / 2017$ & Aluminum & Cone (PC) & I. on $\mathrm{S}$. & $0 \mathrm{~mm}$ & \\
\hline LS-0281 & $7 / 12 / 2017$ & Aluminum & Cone (PC) & I. on $\mathrm{S}$. & $0 \mathrm{~mm}$ & \\
\hline LS-0282 & $7 / 12 / 2017$ & Aluminum & Cone (PC) & I. on $\mathrm{S}$. & $0 \mathrm{~mm}$ & \\
\hline LS-0283 & $7 / 13 / 2017$ & Aluminum & Cone (PC) & I. on $\mathrm{S}$. & $0 \mathrm{~mm}$ & \\
\hline LS-0284 & $7 / 13 / 2017$ & Aluminum & Cone (PC) & I. on $\mathrm{S}$. & $0 \mathrm{~mm}$ & \\
\hline LS-0285 & $7 / 13 / 2017$ & Aluminum & Cone (PC) & I. on $\mathrm{S}$. & $0 \mathrm{~mm}$ & \\
\hline LS-0286 & $7 / 13 / 2017$ & Aluminum & Cone (PC) & I. on B. & $15.2 \mathrm{~mm}$ & \\
\hline LS-0287 & $7 / 13 / 2017$ & Aluminum & Cone (PC) & I. on B. & $15.2 \mathrm{~mm}$ & \\
\hline LS-0288 & $7 / 13 / 2017$ & Aluminum & Cone (PC) & I. on B. & $15.2 \mathrm{~mm}$ & \\
\hline LS-0289 & $7 / 13 / 2017$ & Aluminum & Cone (PC) & I. on B. & $5.1 \mathrm{~mm}$ & \\
\hline LS-0290 & $7 / 13 / 2017$ & Aluminum & Cone (PC) & I. on B. & $5.1 \mathrm{~mm}$ & \\
\hline LS-0291 & $7 / 13 / 2017$ & Aluminum & Cone (PC) & I. on B. & $5.1 \mathrm{~mm}$ & \\
\hline LS-0292 & $7 / 13 / 2017$ & Aluminum & Cone (PC) & I. on B. & $5.1 \mathrm{~mm}$ & \\
\hline LS-0293 & $7 / 13 / 2017$ & Aluminum & Cone (PC) & I. on B. & $5.1 \mathrm{~mm}$ & \\
\hline LS-0294 & $8 / 22 / 2017$ & Aluminum & Flat & I. on $\mathrm{S}$. & $15.2 \mathrm{~mm}$ & \\
\hline LS-0295 & $8 / 22 / 2017$ & Aluminum & Flat & I. on $\mathrm{S}$. & $15.2 \mathrm{~mm}$ & \\
\hline LS-0296 & $8 / 22 / 2017$ & Aluminum & Flat & I. on $\mathrm{S}$. & $15.2 \mathrm{~mm}$ & \\
\hline
\end{tabular}




\begin{tabular}{|c|c|c|c|c|c|c|}
\hline Test & Date & Syringe & Tip & Impact & Air gap & Comment \\
\hline LS-0297 & $8 / 22 / 2017$ & Aluminum & Flat & I. on $\mathrm{S}$. & $15.2 \mathrm{~mm}$ & \\
\hline LS-0298 & $8 / 22 / 2017$ & Aluminum & Flat & I. on $\mathrm{S}$. & $12.7 \mathrm{~mm}$ & \\
\hline LS-0299 & $8 / 22 / 2017$ & Aluminum & Flat & I. on $\mathrm{S}$. & $12.7 \mathrm{~mm}$ & \\
\hline LS-0300 & $8 / 22 / 2017$ & Aluminum & Flat & I. on $\mathrm{S}$. & $12.7 \mathrm{~mm}$ & \\
\hline LS-0301 & $8 / 22 / 2017$ & Aluminum & Flat & I. on $\mathrm{S}$. & $12.7 \mathrm{~mm}$ & \\
\hline LS-0302 & $8 / 23 / 2017$ & Aluminum & Flat & I. on $\mathrm{S}$. & $10.2 \mathrm{~mm}$ & \\
\hline LS-0303 & $8 / 23 / 2017$ & Aluminum & Flat & I. on $\mathrm{S}$. & $10.2 \mathrm{~mm}$ & \\
\hline LS-0304 & $8 / 23 / 2017$ & Aluminum & Flat & I. on $\mathrm{S}$. & $10.2 \mathrm{~mm}$ & \\
\hline LS-0305 & $8 / 23 / 2017$ & Aluminum & Flat & I. on $\mathrm{S}$. & $10.2 \mathrm{~mm}$ & \\
\hline LS-0306 & $8 / 23 / 2017$ & Aluminum & Flat & I. on $\mathrm{S}$. & $7.6 \mathrm{~mm}$ & \\
\hline LS-0307 & $8 / 23 / 2017$ & Aluminum & Flat & I. on $\mathrm{S}$. & $7.6 \mathrm{~mm}$ & \\
\hline LS-0308 & $8 / 23 / 2017$ & Aluminum & Flat & I. on $\mathrm{S}$. & $7.6 \mathrm{~mm}$ & \\
\hline LS-0309 & $8 / 23 / 2017$ & Aluminum & Flat & I. on $\mathrm{S}$. & $7.6 \mathrm{~mm}$ & \\
\hline LS-0310 & $8 / 23 / 2017$ & Aluminum & Flat & I. on $\mathrm{S}$. & $5.1 \mathrm{~mm}$ & \\
\hline LS-0311 & $8 / 23 / 2017$ & Aluminum & Flat & I. on $\mathrm{S}$. & $5.1 \mathrm{~mm}$ & \\
\hline LS-0312 & $8 / 23 / 2017$ & Aluminum & Flat & I. on $\mathrm{S}$. & $5.1 \mathrm{~mm}$ & \\
\hline LS-0313 & $8 / 23 / 2017$ & Aluminum & Flat & I. on $\mathrm{S}$. & $5.1 \mathrm{~mm}$ & \\
\hline LS-0314 & $8 / 24 / 2017$ & Aluminum & Flat & I. on $\mathrm{S}$. & $2.5 \mathrm{~mm}$ & \\
\hline LS-0315 & $8 / 24 / 2017$ & Aluminum & Flat & I. on $\mathrm{S}$. & $2.5 \mathrm{~mm}$ & \\
\hline LS-0316 & $8 / 24 / 2017$ & Aluminum & Flat & I. on $\mathrm{S}$. & $2.5 \mathrm{~mm}$ & \\
\hline LS-0317 & $8 / 24 / 2017$ & Aluminum & Flat & I. on $\mathrm{S}$. & $2.5 \mathrm{~mm}$ & \\
\hline LS-0318 & $8 / 24 / 2017$ & Aluminum & Flat & I. on $\mathrm{S}$. & $0 \mathrm{~mm}$ & \\
\hline
\end{tabular}




\begin{tabular}{|c|c|c|c|c|c|c|}
\hline Test & Date & Syringe & Tip & Impact & Air gap & Comment \\
\hline LS-0319 & $8 / 24 / 2017$ & Aluminum & Flat & I. on $\mathrm{S}$. & $0 \mathrm{~mm}$ & \\
\hline LS- 0320 & $8 / 24 / 2017$ & Aluminum & Flat & I. on $\mathrm{S}$. & $0 \mathrm{~mm}$ & \\
\hline LS-0321 & $8 / 24 / 2017$ & Aluminum & Flat & I. on $\mathrm{S}$. & $0 \mathrm{~mm}$ & \\
\hline LS-0322 & $8 / 24 / 2017$ & Aluminum & Flat & I. on $\mathrm{S}$. & $\inf$ & \\
\hline LS-0323 & $8 / 24 / 2017$ & Aluminum & Flat & I. on $\mathrm{S}$. & $\inf$ & \\
\hline LS-0324 & $8 / 24 / 2017$ & Aluminum & Flat & I. on $\mathrm{S}$. & $\inf$ & \\
\hline LS-0325 & $8 / 24 / 2017$ & Aluminum & Flat & I. on $\mathrm{S}$. & inf & \\
\hline LS-0326 & $9 / 5 / 2017$ & Aluminum & Flat & I. on B. & $15.2 \mathrm{~mm}$ & \\
\hline LS-0327 & $9 / 5 / 2017$ & Aluminum & Flat & I. on B. & $15.2 \mathrm{~mm}$ & \\
\hline LS-0328 & $9 / 5 / 2017$ & Aluminum & Flat & I. on B. & $15.2 \mathrm{~mm}$ & \\
\hline LS-0329 & $9 / 5 / 2017$ & Aluminum & Flat & I. on B. & $15.2 \mathrm{~mm}$ & \\
\hline LS-0330 & $9 / 6 / 2017$ & Aluminum & Flat & I. on B. & $12.7 \mathrm{~mm}$ & \\
\hline LS-0331 & $9 / 6 / 2017$ & Aluminum & Flat & I. on B. & $12.7 \mathrm{~mm}$ & \\
\hline LS-0332 & $9 / 6 / 2017$ & Aluminum & Flat & I. on B. & $12.7 \mathrm{~mm}$ & \\
\hline LS-0333 & $9 / 6 / 2017$ & Aluminum & Flat & I. on B. & $12.7 \mathrm{~mm}$ & \\
\hline LS-0334 & $9 / 6 / 2017$ & Aluminum & Flat & I. on B. & $10.2 \mathrm{~mm}$ & \\
\hline LS-0335 & $9 / 6 / 2017$ & Aluminum & Flat & I. on B. & $10.2 \mathrm{~mm}$ & \\
\hline LS-0336 & $9 / 6 / 2017$ & Aluminum & Flat & I. on B. & $10.2 \mathrm{~mm}$ & \\
\hline LS-0337 & $9 / 6 / 2017$ & Aluminum & Flat & I. on B. & $10.2 \mathrm{~mm}$ & \\
\hline LS-0338 & $9 / 6 / 2017$ & Aluminum & Flat & I. on B. & $7.6 \mathrm{~mm}$ & \\
\hline LS-0339 & $9 / 6 / 2017$ & Aluminum & Flat & I. on B. & $7.6 \mathrm{~mm}$ & \\
\hline LS- 0340 & $9 / 6 / 2017$ & Aluminum & Flat & I. on B. & $7.6 \mathrm{~mm}$ & \\
\hline
\end{tabular}




\begin{tabular}{|c|c|c|c|c|c|c|}
\hline Test & Date & Syringe & Tip & Impact & Air gap & Comment \\
\hline LS-0341 & $9 / 6 / 2017$ & Aluminum & Flat & I. on B. & $7.6 \mathrm{~mm}$ & \\
\hline LS-0342 & $9 / 6 / 2017$ & Aluminum & Flat & I. on B. & $5.1 \mathrm{~mm}$ & \\
\hline LS-0343 & $9 / 6 / 2017$ & Aluminum & Flat & I. on B. & $5.1 \mathrm{~mm}$ & \\
\hline LS-0344 & $9 / 6 / 2017$ & Aluminum & Flat & I. on B. & $5.1 \mathrm{~mm}$ & \\
\hline LS-0345 & $9 / 6 / 2017$ & Aluminum & Flat & I. on B. & $5.1 \mathrm{~mm}$ & \\
\hline LS-0346 & $9 / 8 / 2017$ & Aluminum & Flat & I. on B. & $2.5 \mathrm{~mm}$ & \\
\hline LS-0347 & $9 / 8 / 2017$ & Aluminum & Flat & I. on B. & $2.5 \mathrm{~mm}$ & \\
\hline LS-0348 & $9 / 8 / 2017$ & Aluminum & Flat & I. on B. & $2.5 \mathrm{~mm}$ & \\
\hline LS-0349 & $9 / 8 / 2017$ & Aluminum & Flat & I. on B. & $2.5 \mathrm{~mm}$ & \\
\hline LS-0350 & $9 / 8 / 2017$ & Aluminum & Flat & I. on B. & $0 \mathrm{~mm}$ & \\
\hline LS-0351 & 9/8/2017 & Aluminum & Flat & I. on B. & $0 \mathrm{~mm}$ & \\
\hline LS-0352 & $9 / 8 / 2017$ & Aluminum & Flat & I. on B. & $0 \mathrm{~mm}$ & \\
\hline LS-0353 & $9 / 8 / 2017$ & Aluminum & Flat & I. on B. & $0 \mathrm{~mm}$ & \\
\hline LS-0354 & $9 / 15 / 2017$ & Polycarbonate & Flat & I. on $\mathrm{S}$. & $15.2 \mathrm{~mm}$ & \\
\hline LS-0355 & $9 / 15 / 2017$ & Polycarbonate & Flat & I. on $\mathrm{S}$. & $15.2 \mathrm{~mm}$ & \\
\hline LS-0356 & $9 / 20 / 2017$ & Polycarbonate & Flat & I. on $\mathrm{S}$. & $12.7 \mathrm{~mm}$ & \\
\hline LS-0357 & $9 / 20 / 2017$ & Polycarbonate & Flat & I. on $\mathrm{S}$. & $2.5 \mathrm{~mm}$ & \\
\hline LS-0358 & $9 / 20 / 2017$ & Polycarbonate & Flat & I. on $\mathrm{S}$. & $7.6 \mathrm{~mm}$ & \\
\hline LS-0359 & $9 / 20 / 2017$ & Polycarbonate & Flat & I. on $\mathrm{S}$. & $5.1 \mathrm{~mm}$ & \\
\hline LS-0360 & $9 / 20 / 2017$ & Polycarbonate & Flat & I. on $\mathrm{S}$. & $2.5 \mathrm{~mm}$ & \\
\hline LS-0361 & $9 / 20 / 2017$ & Polycarbonate & Flat & I. on $\mathrm{S}$. & $0 \mathrm{~mm}$ & \\
\hline LS-0362 & $9 / 20 / 2017$ & Polycarbonate & Flat & I. on $\mathrm{S}$. & $\inf$ & \\
\hline
\end{tabular}




\begin{tabular}{|c|c|c|c|c|c|c|}
\hline Test & Date & Syringe & Tip & Impact & Air gap & Comment \\
\hline LS-0363 & $9 / 21 / 2017$ & Polycarbonate & Flat & I. on $\mathrm{S}$. & $12.7 \mathrm{~mm}$ & \\
\hline LS-0364 & $9 / 21 / 2017$ & Polycarbonate & Flat & I. on $\mathrm{S}$. & $12.7 \mathrm{~mm}$ & \\
\hline LS-0365 & $9 / 21 / 2017$ & Polycarbonate & Flat & I. on $\mathrm{S}$. & $12.7 \mathrm{~mm}$ & \\
\hline LS-0366 & $9 / 21 / 2017$ & Polycarbonate & Flat & I. on $\mathrm{S}$. & $12.7 \mathrm{~mm}$ & \\
\hline LS-0367 & $9 / 21 / 2017$ & Polycarbonate & Flat & I. on $\mathrm{S}$. & $12.7 \mathrm{~mm}$ & \\
\hline LS-0368 & $9 / 13 / 2018$ & Polycabornate & Flat & I. on $\mathrm{S}$. & $12.7 \mathrm{~mm}$ & \\
\hline LS-0369 & $9 / 13 / 2018$ & Polycabornate & Flat & I. on $\mathrm{S}$. & $12.7 \mathrm{~mm}$ & \\
\hline LS-0370 & $9 / 13 / 2018$ & Polycabornate & Flat & I. on $\mathrm{S}$. & $12.7 \mathrm{~mm}$ & \\
\hline LS-0371 & $9 / 13 / 2018$ & Polycabornate & Flat & I. on $\mathrm{S}$. & $12.7 \mathrm{~mm}$ & \\
\hline LS-0372 & $9 / 13 / 2018$ & Polycabornate & Flat & I. on $\mathrm{S}$. & $12.7 \mathrm{~mm}$ & \\
\hline LS-0373 & $9 / 13 / 2018$ & Polycabornate & Flat & I. on $\mathrm{S}$. & $12.7 \mathrm{~mm}$ & \\
\hline LS-0374 & $9 / 13 / 2018$ & Polycabornate & Flat & I. on $\mathrm{S}$. & $12.7 \mathrm{~mm}$ & \\
\hline LS-0375 & $9 / 13 / 2018$ & Polycabornate & Flat & I. on $\mathrm{S}$. & $12.7 \mathrm{~mm}$ & \\
\hline LS-0376 & $9 / 13 / 2018$ & Polycabornate & Flat & I. on $\mathrm{S}$. & $12.7 \mathrm{~mm}$ & \\
\hline LS-0377 & $9 / 13 / 2018$ & Polycabornate & Flat & I. on $\mathrm{S}$. & $12.7 \mathrm{~mm}$ & \\
\hline LS-0378 & $9 / 13 / 2018$ & Polycabornate & Flat & I. on $\mathrm{S}$. & $12.7 \mathrm{~mm}$ & \\
\hline LS-0379 & $9 / 13 / 2018$ & Polycabornate & Flat & I. on $\mathrm{S}$. & $12.7 \mathrm{~mm}$ & Degassed for 1 hour \\
\hline LS-0380 & $9 / 13 / 2018$ & Polycabornate & Flat & I. on $\mathrm{S}$. & $\inf$ & \\
\hline LS-0381 & $9 / 14 / 2018$ & Polycabornate & Flat & I. on $\mathrm{S}$. & $\inf$ & Replaced water \\
\hline LS-0382 & $9 / 14 / 2018$ & Polycabornate & Flat & I. on $\mathrm{S}$. & $\inf$ & \\
\hline LS-0383 & $9 / 14 / 2018$ & Polycabornate & Flat & I. on $\mathrm{S}$. & $\inf$ & \\
\hline LS-0384 & $9 / 14 / 2018$ & Polycabornate & Flat & I. on $\mathrm{S}$. & inf & \\
\hline
\end{tabular}




\begin{tabular}{lllllll}
\hline Test & Date & Syringe & Tip & Impact & Air gap & Comment \\
\hline LS-0385 & $9 / 14 / 2018$ & Polycabornate & Flat & I. on S. & inf & \\
\hline LS-0386 & $9 / 14 / 2018$ & Polycabornate & Flat & I. on S. & inf & \\
\hline LS-0387 & $9 / 14 / 2018$ & Polycabornate & Flat & I. on S. & inf & \\
\hline LS-0388 & $9 / 14 / 2018$ & Polycabornate & Flat & I. on S. & inf & \\
\hline LS-0389 & $9 / 14 / 2018$ & Polycabornate & Flat & I. on S. & inf & \\
\hline LS-0390 & $9 / 14 / 2018$ & Polycabornate & Flat & I. on S. & inf & Damping: multiple O-rings \\
\hline LS-0391 & $9 / 14 / 2018$ & Polycabornate & Flat & I. on S. & inf & Damping: foam \\
\hline LS-0392 & $9 / 17 / 2018$ & Polycabornate & Flat & I. on S. & inf & Damping: foam \\
\hline LS-0393 & $9 / 17 / 2018$ & Polycabornate & Flat & I. on S. & inf & Damping: foam \\
\hline LS-0394 & $9 / 17 / 2018$ & Polycabornate & Flat & I. on S. & inf & Damping: foam \\
\hline LS-0395 & $9 / 17 / 2018$ & Polycabornate & Flat & I. on S. & inf & Damping: foam \\
\hline LS-0396 & $9 / 20 / 2018$ & Polycabornate & Cone PC & I. on S. & inf & \\
\hline LS-0397 & $9 / 20 / 2018$ & Polycabornate & Cone PC & I. on S. & inf & \\
\hline LS-0398 & $9 / 20 / 2018$ & Polycabornate & Cone PC & I. on S. & inf & \\
\hline LS-0399 & $9 / 20 / 2018$ & Polycabornate & Cone PC & I. on S. & inf & \\
\hline LS-0400 & $9 / 20 / 2018$ & Polycabornate & Cone PC & I. on S. & inf & \\
\hline LS-0401 & $9 / 20 / 2018$ & Polycabornate & Cone PC & I. on S. & inf & \\
\hline LS-0402 & $9 / 20 / 2018$ & Polycabornate & Cone PC & I. on S. & inf & \\
\hline LS-0403 & $9 / 20 / 2018$ & Polycabornate & Cone PC & I. on S. & inf & \\
\hline LS-0404 & $9 / 20 / 2018$ & Polycabornate & Cone PC & I. on S. & inf & \\
\hline LS-0405 & $9 / 20 / 2018$ & Polycabornate & Cone PC & I. on S. & inf & \\
\hline LS-0406 & $9 / 20 / 2018$ & Polycabornate & Cone PC & I. on S. & inf & \\
\hline & & & & & & \\
\hline
\end{tabular}




\begin{tabular}{lllllll}
\hline Test & Date & Syringe & Tip & Impact & Air gap & Comment \\
\hline LS-0407 & $9 / 20 / 2018$ & Polycabornate & Cone PC & I. on S. & inf & \\
LS-0408 & $9 / 20 / 2018$ & Polycabornate & Cone PC & I. on S. & inf & \\
\hline LS-0409 & $10 / 1 / 2018$ & Polycabornate & Cone PC & I. on S. & inf & \\
\hline LS-0410 & $10 / 1 / 2018$ & Polycabornate & Cone PC & I. on S. & inf & Damping: foam \\
LS-0411 & $10 / 1 / 2018$ & Polycabornate & Cone PC & I. on S. & inf & Damping: foam \\
\hline LS-0412 & $10 / 1 / 2018$ & Polycabornate & Cone PC & I. on S. & inf & Damping: foam \\
\hline LS-0413 & $10 / 1 / 2018$ & Polycabornate & Cone PC & I. on S. & inf & Damping: foam \\
\hline LS-0414 & $10 / 1 / 2018$ & Polycabornate & Cone PC & I. on S. & inf & Damping: foam \\
\hline LS-0415 & $10 / 1 / 2018$ & Polycabornate & Cone PC & I. on S. & inf & Damping: foam \\
\hline LS-0416 & $10 / 1 / 2018$ & Polycabornate & Cone PC & I. on S. & inf & Damping: foam \\
\hline LS-0417 & $10 / 31 / 2018$ & Polycabornate & Flat & I. on S. & $15.2 \mathrm{~mm}$ & \\
\hline LS-0420 & $11 / 8 / 2018$ & Polycabornate & Cone PC & I. on B. & $7.6 \mathrm{~mm}$ & \\
\hline LS-0421 & $11 / 8 / 2018$ & Polycabornate & Flat & I. on B. & $7.6 \mathrm{~mm}$ & \\
\hline LS-0422 & $11 / 8 / 2018$ & Polycabornate & Flat & I. on B. & $0 \mathrm{~mm}$ & \\
\hline
\end{tabular}

